\title{
Argonne
}

ANL/ESD-21/9

\section{A Detailed Vehicle Simulation Process to Support CAFE Standards for the MY 2024-2026 Analysis}

Energy Systems Division 


\section{About Argonne National Laboratory}

Argonne is a U.S. Department of Energy laboratory managed by UChicago Argonne, LLC under contract DE-AC02-06CH11357. The Laboratory's main facility is outside Chicago, at 9700 South Cass Avenue, Lemont, Illinois 60439. For information about Argonne and its pioneering science and technology programs, see www.anl.gov.

\section{DOCUMENT AVAILABILITY}

Online Access: U.S. Department of Energy (DOE) reports produced after 1991 and a growing number of pre-1991 documents are available free at OSTI.GOV (http://www.osti.gov/), a service of the US Dept. of Energy's Office of Scientific and Technical Information.

\section{Reports not in digital format may be purchased by the public} from the National Technical Information Service (NTIS):

U.S. Department of Commerce

National Technical Information Service

5301 Shawnee Road

Alexandria, VA 22312

www.ntis.gov Phone:

(800) 553-NTIS (6847) or (703) 605-6000

Fax: (703) 605-6900

Email: orders@ntis.gov

Reports not in digital format are available to DOE and DOE contractors from the Office of Scientific and Technical Information (OSTI):

U.S. Department of Energy

Office of Scientific and Technical Information

P.O. Box 62

Oak Ridge, TN 37831-0062

www.osti.gov

Phone: (865) 576-8401

Fax: (865) 576-5728

Email: reports@osti.gov

\section{Disclaimer}

This report was prepared as an account of work sponsored by an agency of the United States Government. Neither the United States Government nor any agency thereof, nor UChicago Argonne, LLC, nor any of their employees or officers, makes any warranty, express or implied, or assumes any legal liability or responsibility for the accuracy, completeness, or usefulness of any information, apparatus, product, or process disclosed, or represents that its use would not infringe privately owned rights. Reference herein to any specific commercial product, process, or service by trade name, trademark, manufacturer, or otherwise, does not necessarily constitute or imply its endorsement, recommendation, or favoring by the United States Government or any agency thereof. The views and opinions of document authors expressed herein do not necessarily state or reflect those of the United States Government or any agency thereof, Argonne National Laboratory, or UChicago Argonne, LLC.

Preferred citation:

Islam, E. S., A. Moawad, N. Kim, R. Vijayagopal, and A. Rousseau. A Detailed Vehicle Simulation Process to Support CAFE Standards for the MY 2024-2026 Analysis. ANL/ESD-21/9. DOI \#10.2172/1810319 https:// doi.org/ 


\section{A Detailed Vehicle Simulation Process to Support CAFE Standards for the MY 2024-2026 Analysis}

By

Ehsan Sabri Islam, Ayman Moawad, Namdoo Kim, Ram Vijayagopal,

Aymeric Rousseau

Energy Systems Division, Argonne National Laboratory

August 5, 2021 


\section{Table of Contents}

ACRONYMS AND ABBREVIATIONS xvi

UNITS OF MEASURE xix

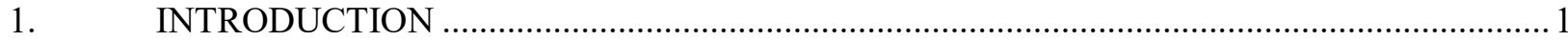

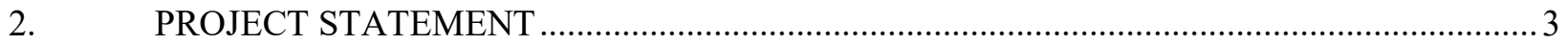

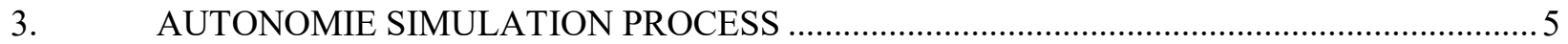

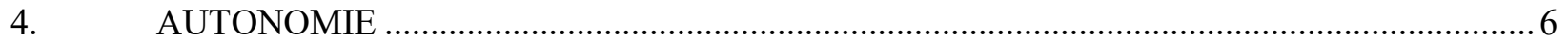

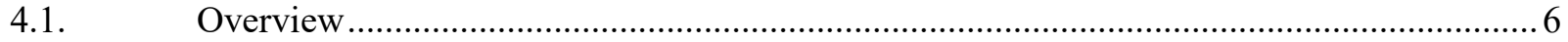

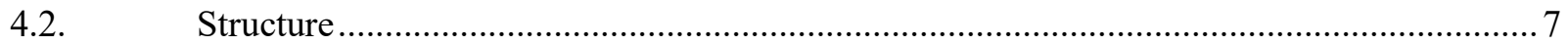

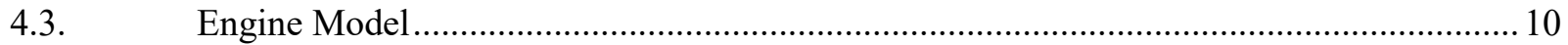

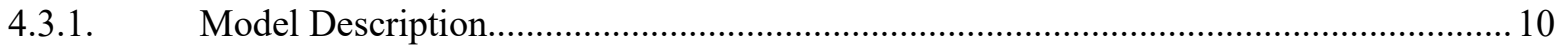

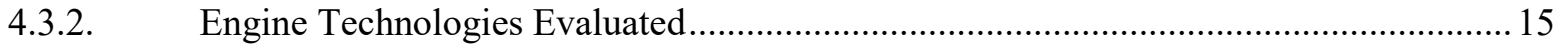

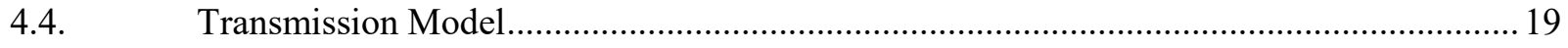

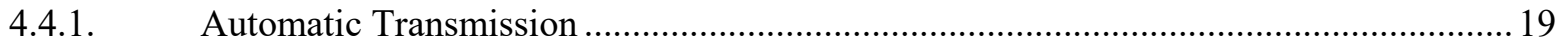

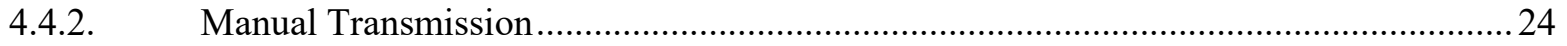

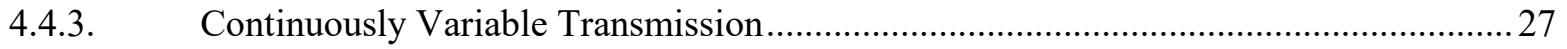

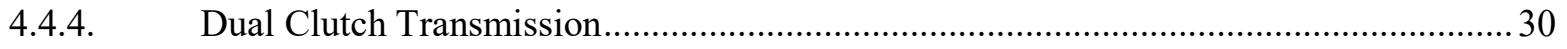

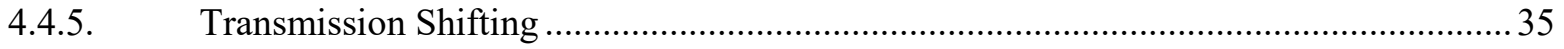

4.4.6. Engine Start Control for Pre-Transmission HEVs ........................................................ 44

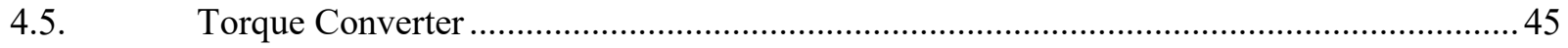

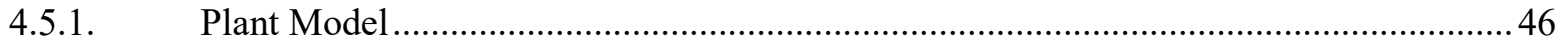

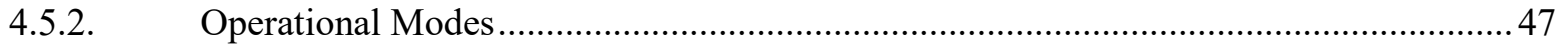

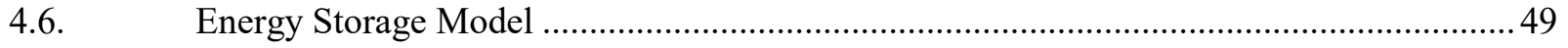

4.6.1. High-Power Battery Model (Used for Hybrid Electric Vehicles) ..................................50

4.6.2. High Energy Battery Model (Used for PHEVs and Battery Electric Vehicles)..............52

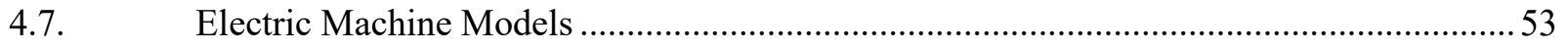

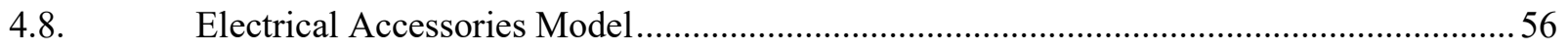

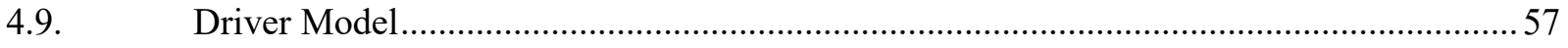

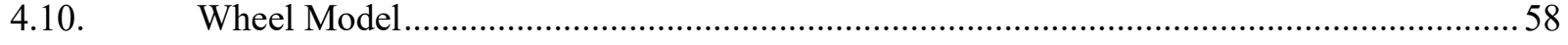

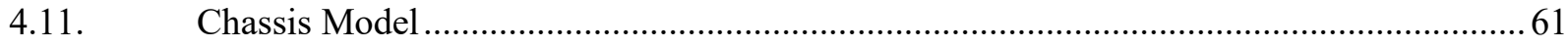

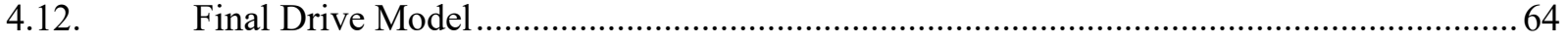

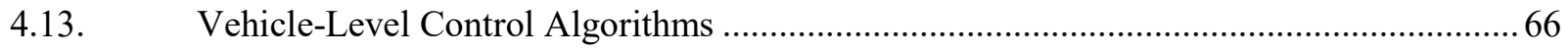

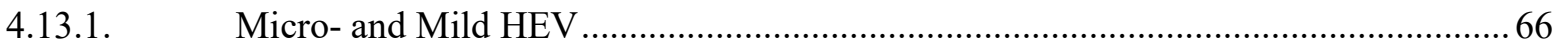

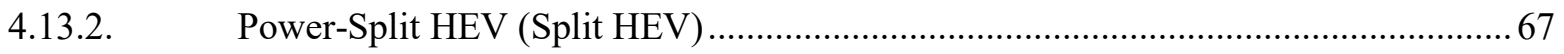




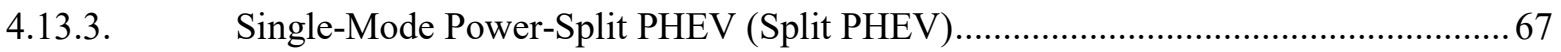

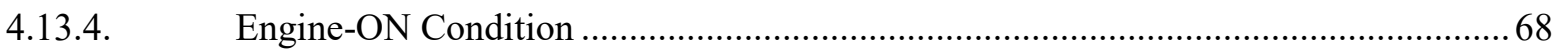

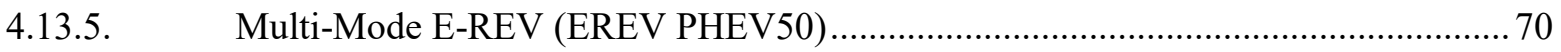

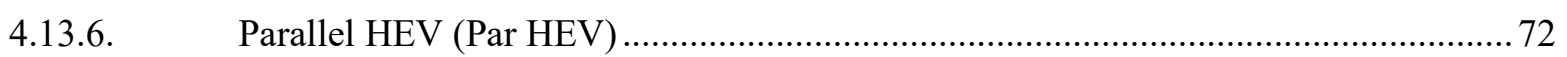

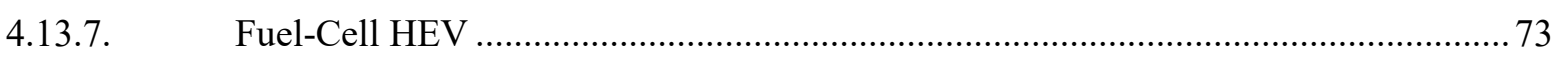

4.14. High-Level Overview of Autonomie Controllers ....................................................... 74

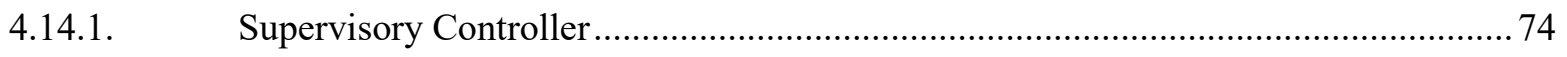

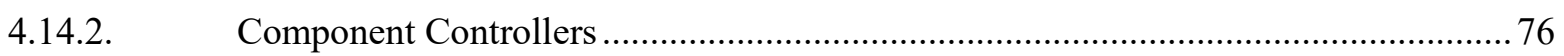

4.15. Low-Level Controller Summary for Engine and Transmission Models ............................ 79

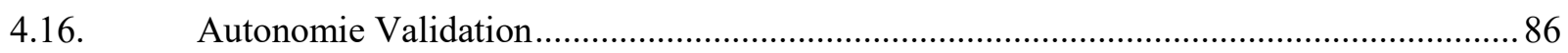

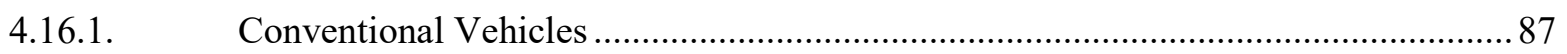

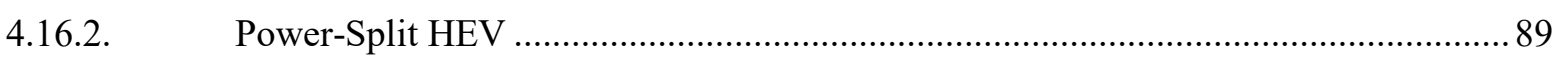

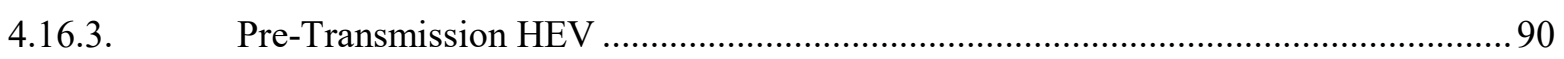

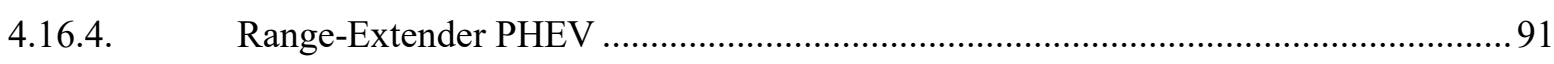

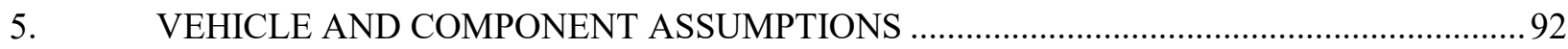

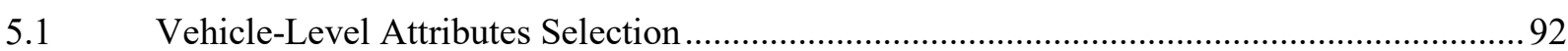

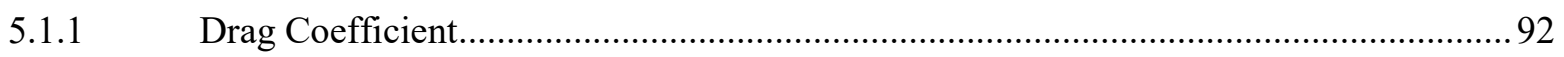

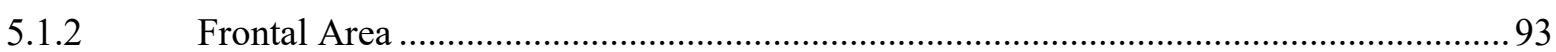

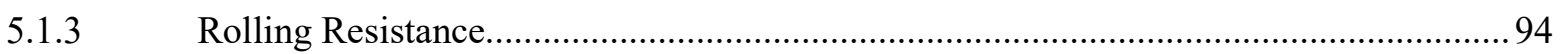

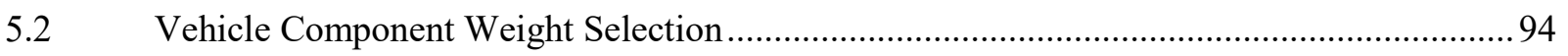

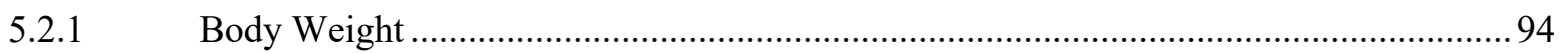

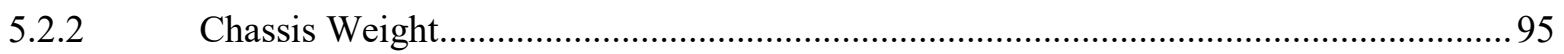

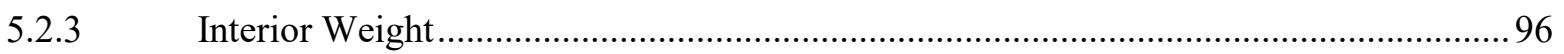

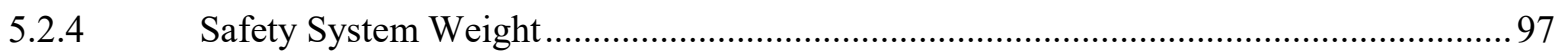

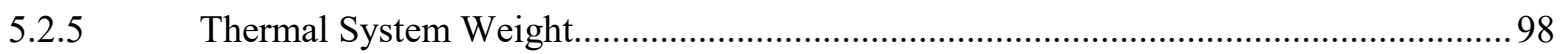

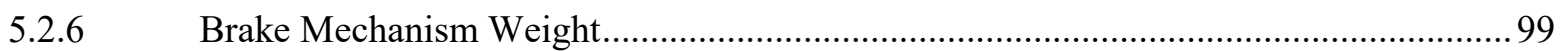

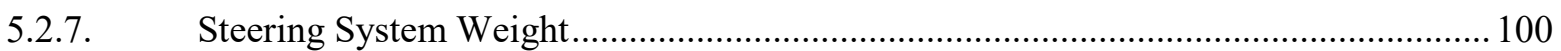

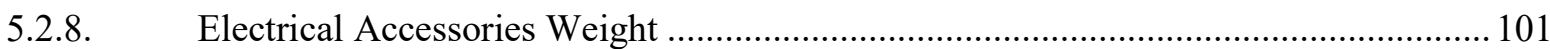

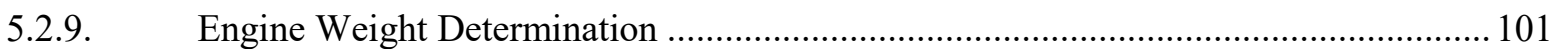

5.2.10. Electric Machines System Weight ........................................................................ 103

5.3. Transmission Assumptions ....................................................................................... 104

5.3.1. Transmission Technology Definitions .................................................................... 104

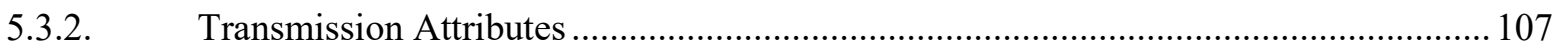

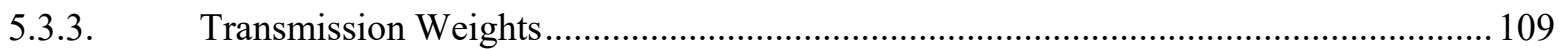

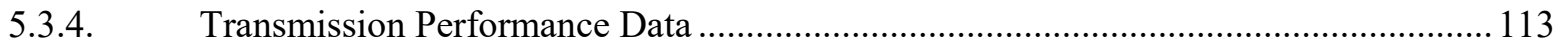




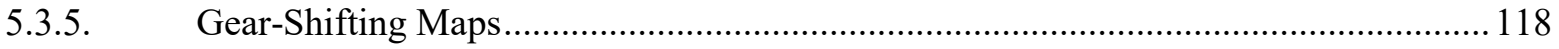

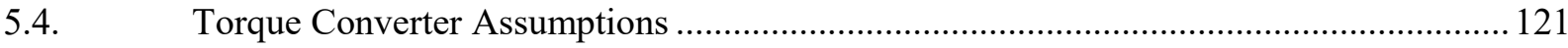

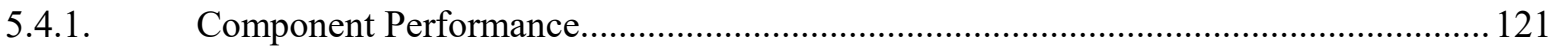

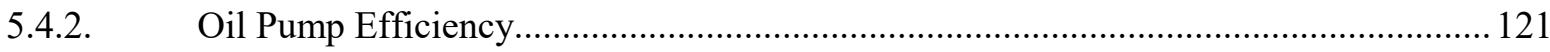

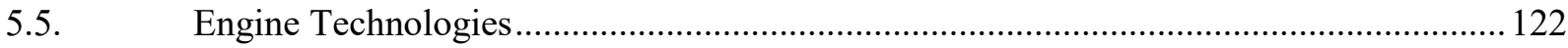

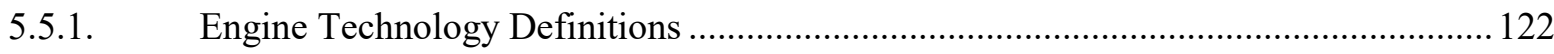

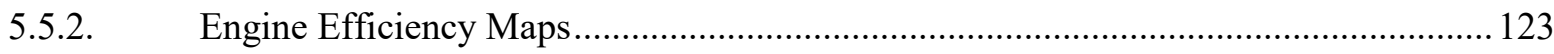

5.5.3. Incremental BSFC and Thermal Efficiency Difference of Engines............................ 139

5.5.4. Engine Efficiency Map Comparison With and Without DEAC Capability................... 145

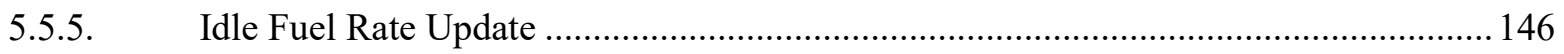

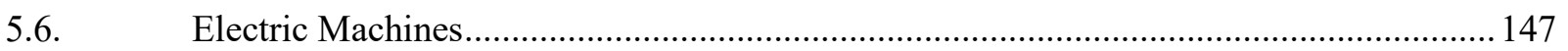

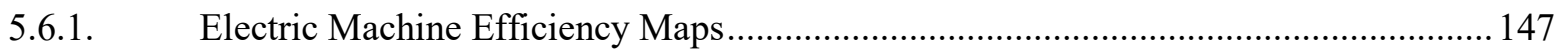

5.6.2. Electric Machine Peak Efficiency Scaling ............................................................... 148

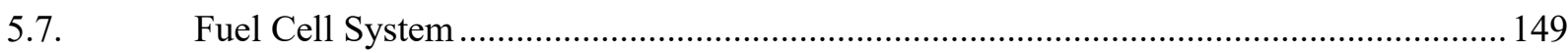

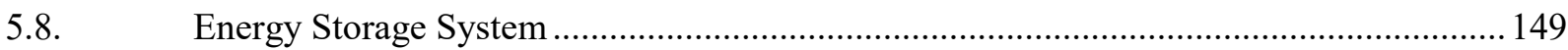

5.9. Battery Performance and Cost Model (BatPaC) …....................................................... 150

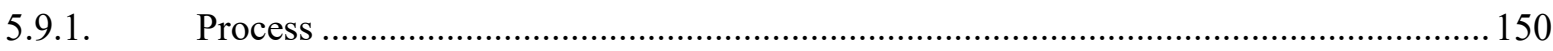

5.9.2. BatPaC Examples from Existing Vehicles in the Market ........................................... 152

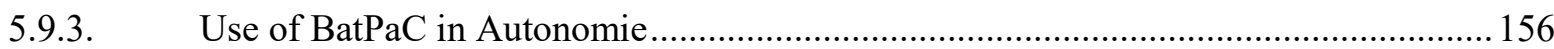

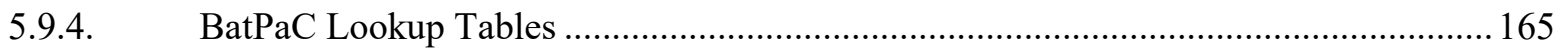

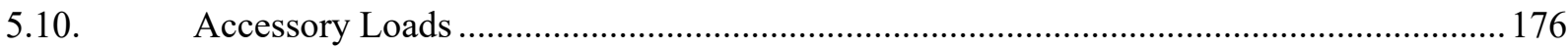

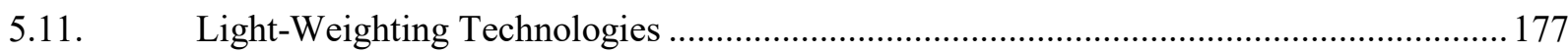

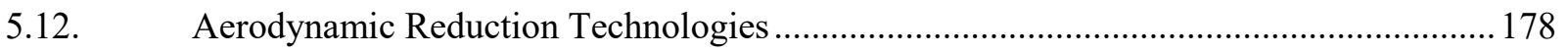

5.13. Rolling Resistance Reduction Technologies............................................................. 178

6. TEST PROCEDURE AND ENERGY CONSUMPTION CALCULATIONS ........................ 179

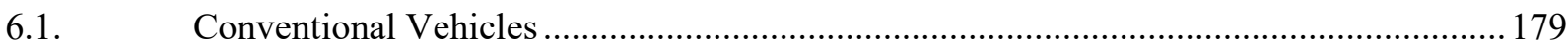

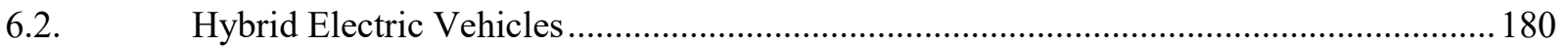

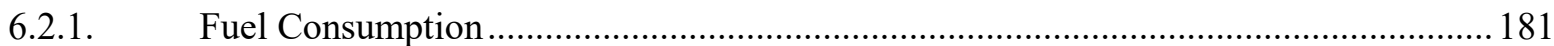

6.3. Plug-in Hybrid Electric Vehicles ............................................................................... 182

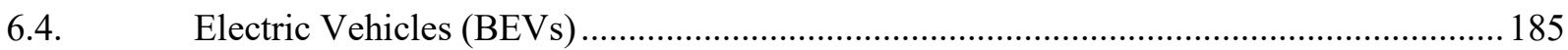

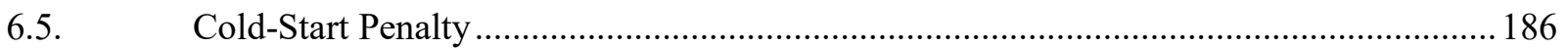

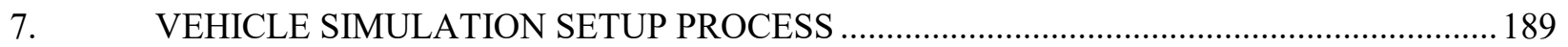

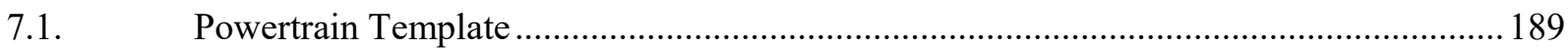

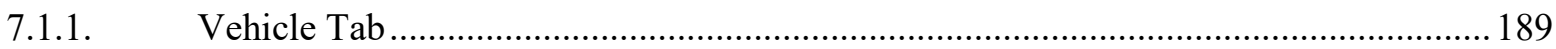

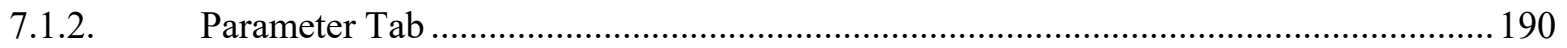




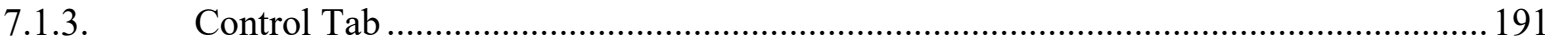

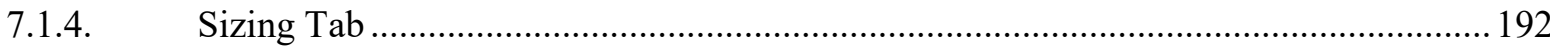

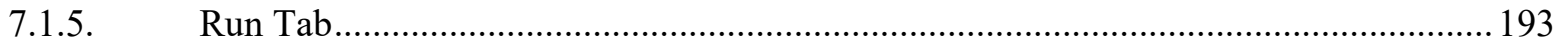

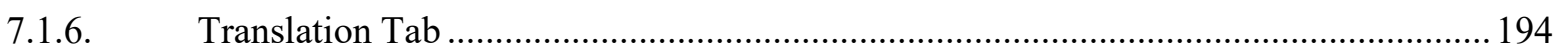

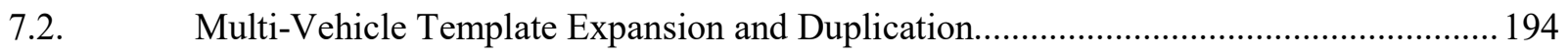

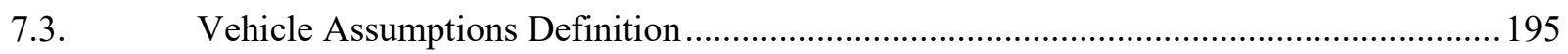

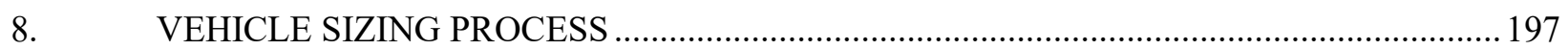

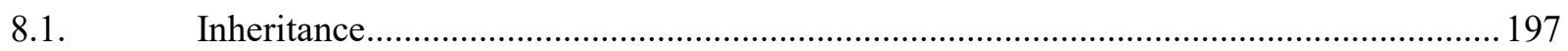

8.1.1. Conventional Powertrain (Conventional/Micro-12V/Mild Hybrid BISG) ................... 197

8.1.2. Hybrid Powertrains (Split HEV/Split PHEV/EREV PHEV/Fuel Cell HEV/BEV)...... 198

8.1.3. Parallel Hybrid Powertrains ........................................................................................ 200

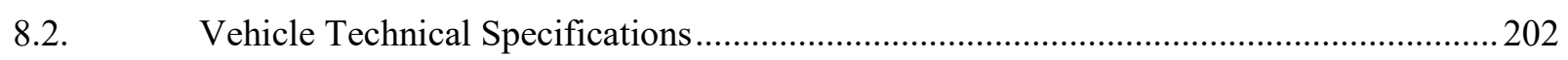

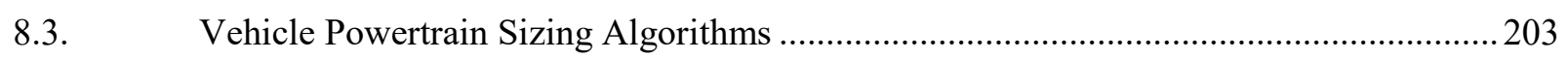

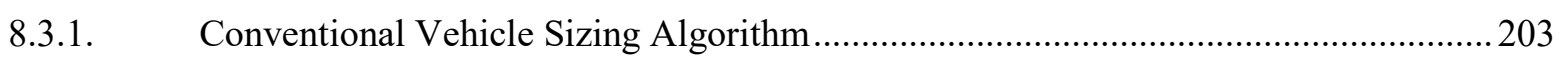

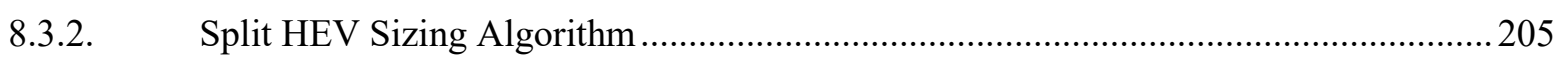

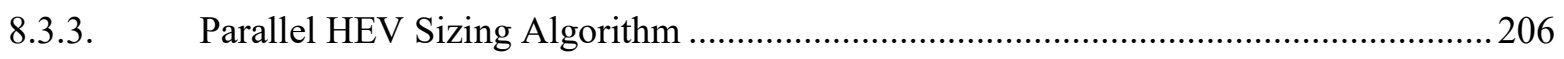

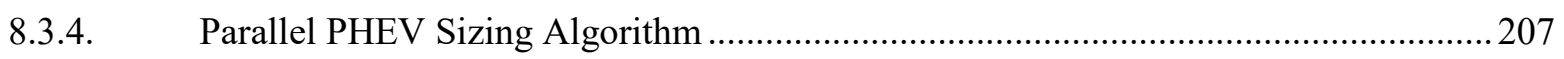

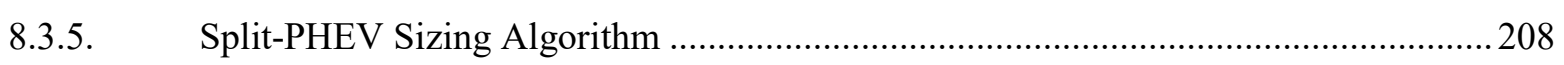

8.3.6. Voltec PHEV (Extended Range) Vehicle Sizing Algorithm ....................................... 209

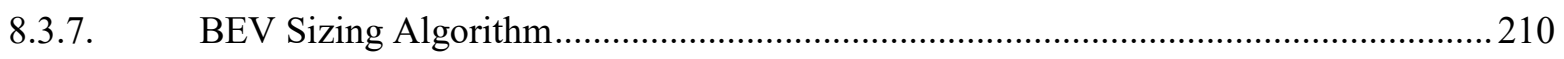

8.3.8. Fuel Cell Series HEV Sizing Algorithm .................................................................. 211

8.3.9. Selection of Drive Cycles for Electric Machine Sizing for PHEVs........................... 211

8.4. Determining the Relationship Between Engine Displacement and Number of Cylinders 213

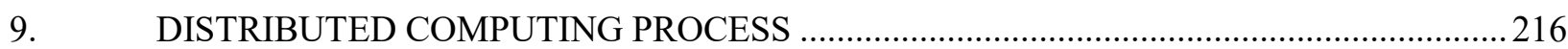

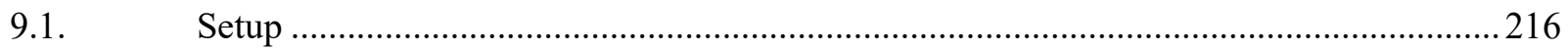

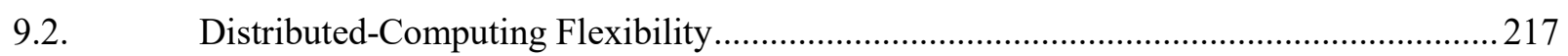

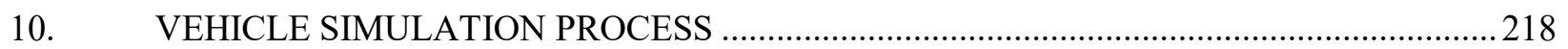

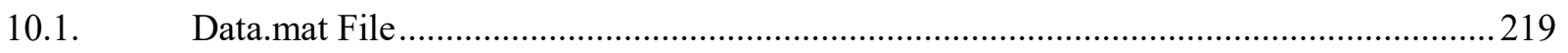

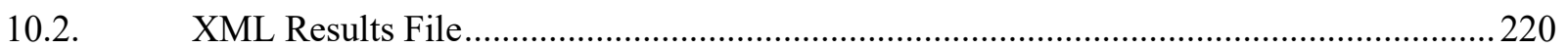

11. SIMULATION RESULTS ANALYSIS AND DETAILED VALIDATION PROCESSES.... 221

11.1. Engine Operating Points Across Transmissions .......................................................... 221

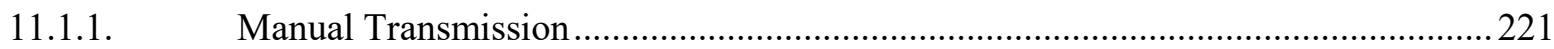

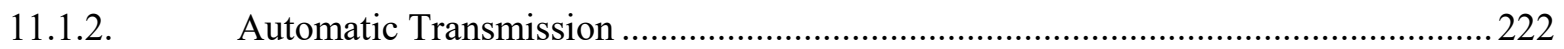

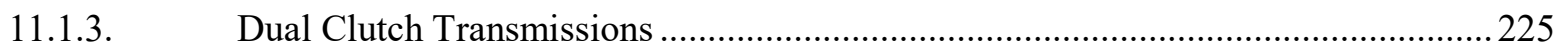

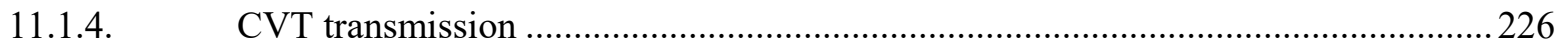




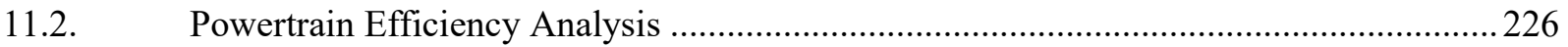

11.3. Total Number of Shifting Events with Different Transmission Types and Numbers of Gears ...233

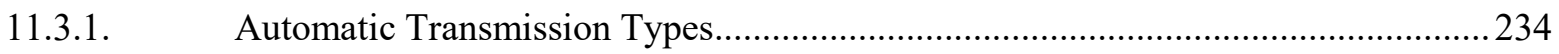

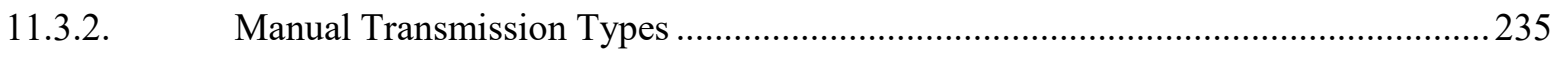

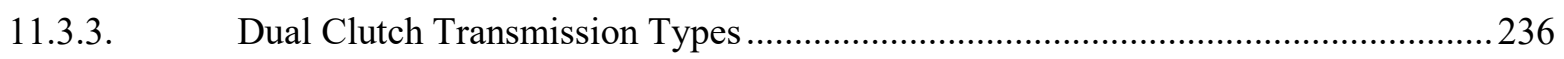

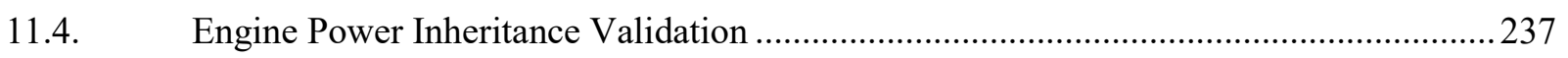

11.5. Validation Against Vehicle Technology Database .....................................................2 242

11.5.1. BEV Weight, Range and Battery Energy Analysis................................................. 242

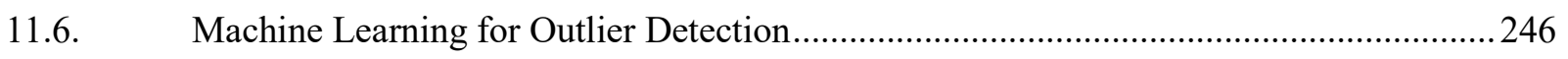

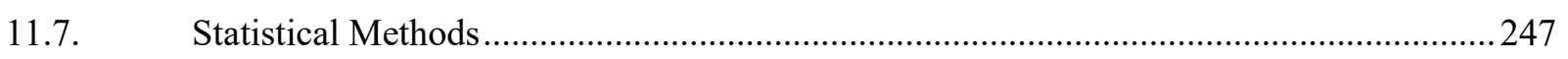

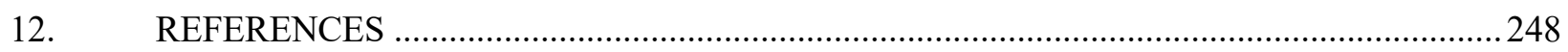

\section{Figures}

Figure 1. Technologies considered for the Argonne Autonomie for this analysis................................... 4

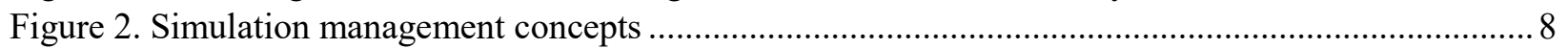

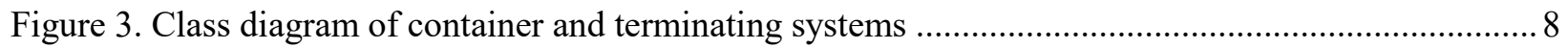

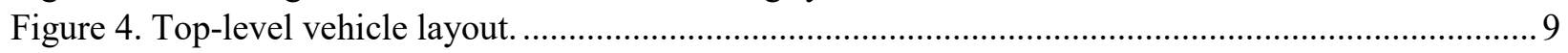

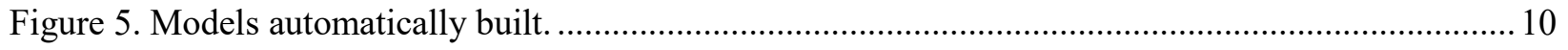

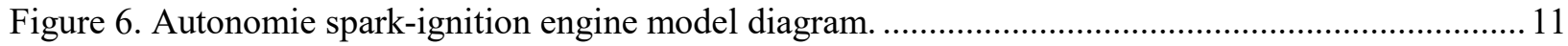

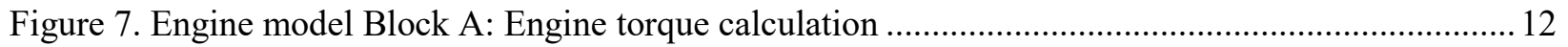

Figure 8. Engine model, Block B: Thermal model ......................................................................... 13

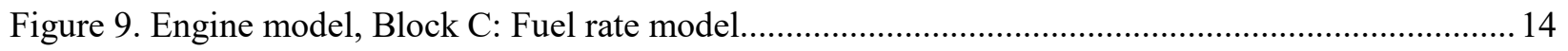

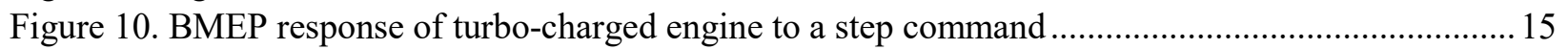

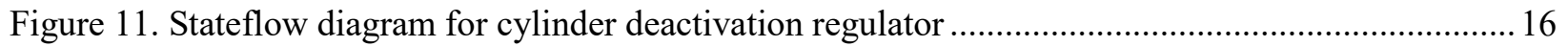

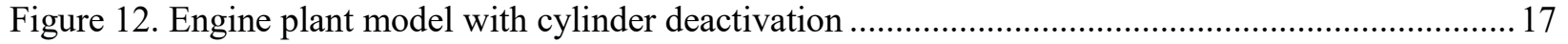

Figure 13. Stateflow diagram showing the ability to switch cylinder deactivation fuel maps.................... 17

Figure 14. Engine operating regions for throttled engines (WOT = wide-open throttle) ......................... 18

Figure 15. Engine operating region for unthrottled engines ........................................................ 18

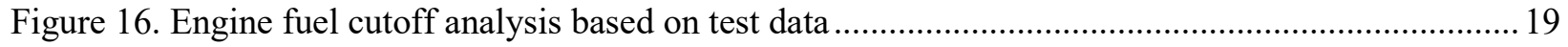

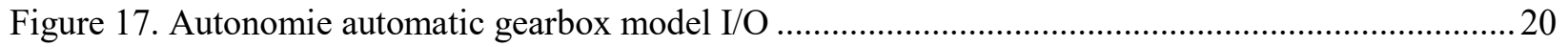

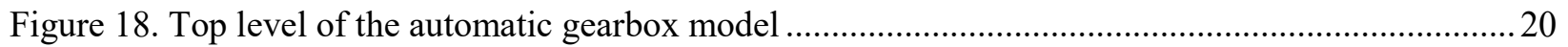

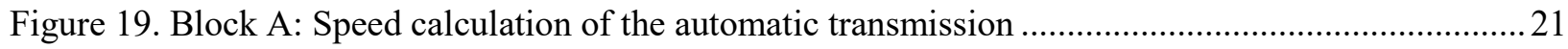

Figure 20. Block A1: Speed calculation when the gearbox is not in neutral ......................................... 21

Figure 21. Block A2: Speed calculation when the gearbox is in neutral .............................................. 22

Figure 22. Block A2-1: When the gearbox is not in neutral, with free-wheeling input shaft losses .......... 22

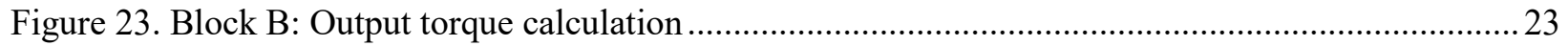

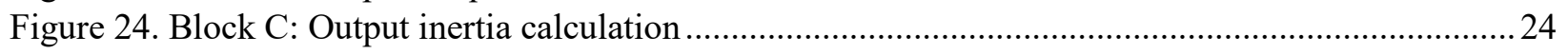

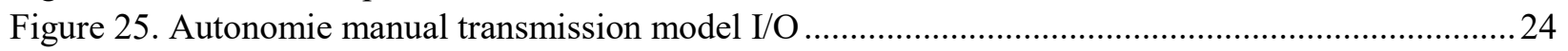

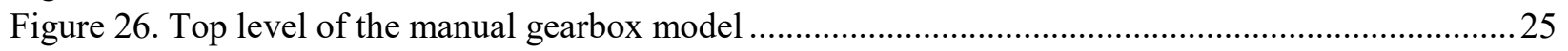

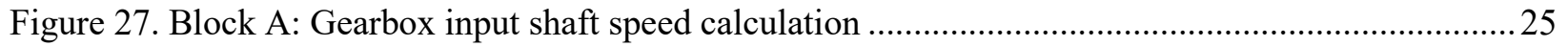

Figure 28. Block A1: Torque loss calculation when the gearbox is in neutral .....................................26

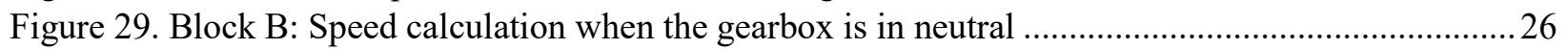


Figure 30. Block C: Output inertia calculation

Figure 31. Hydraulic pump loss and mechanical loss for metal v-belt continuously variable transmission

[35] 28

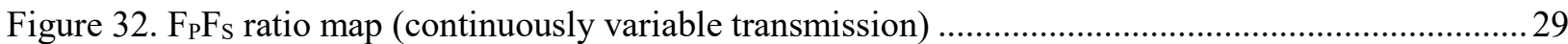

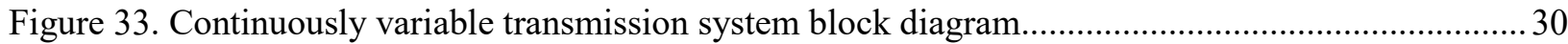

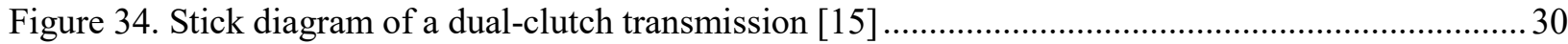

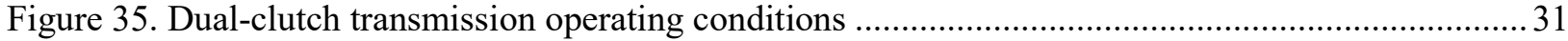

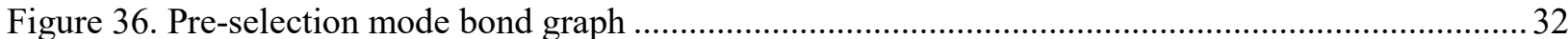

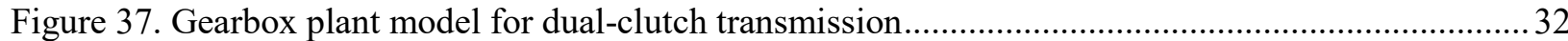

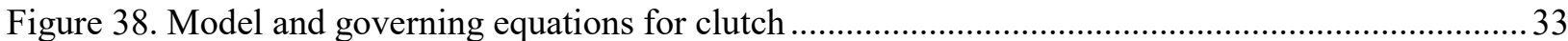

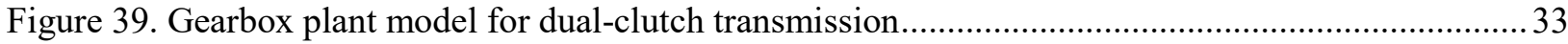

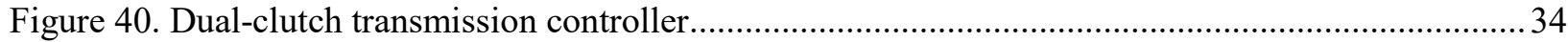

Figure 41. State diagram representation of the control strategy for a dual-clutch transmission .................34

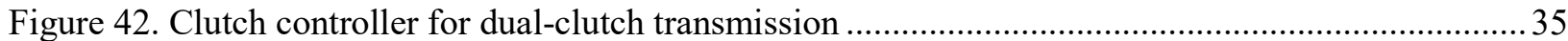

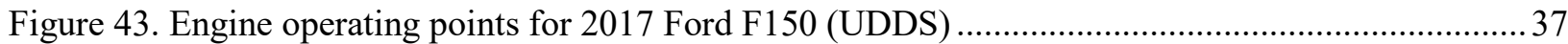

Figure 44. Example of engine operating conditions for 5-speed and 10-speed transmissions on UDDS

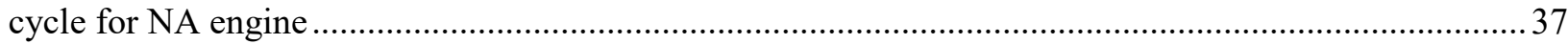

Figure 45. Upshifting gear map (left); upshifting vehicle speeds (right) .............................................. 38

Figure 46. Example of engine speed range in economical driving and economical shift........................38

Figure 47. Maximum engine torque at wheels and performance upshift speeds ....................................40

Figure 48. Design of upshifting and downshifting speed curves for two adjacent gears........................... 40

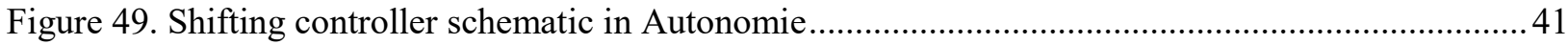

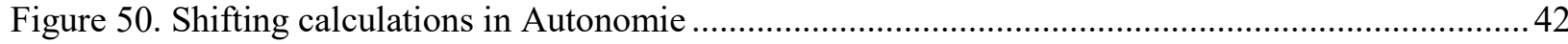

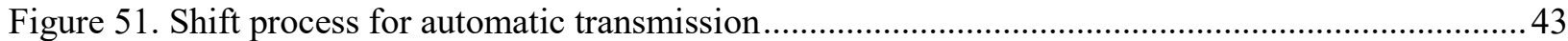

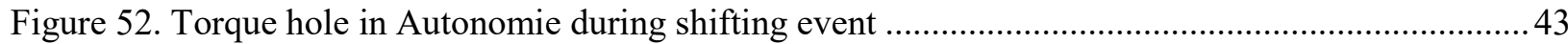

Figure 53. Engine start transient control using electric machine ............................................................... 45

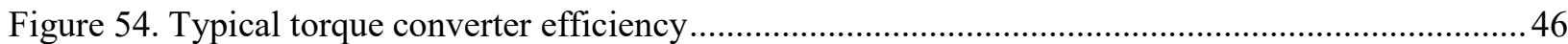

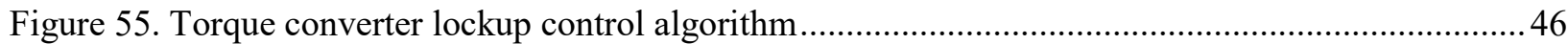

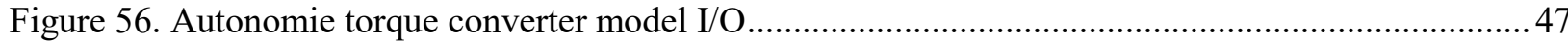

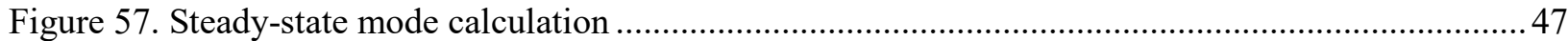

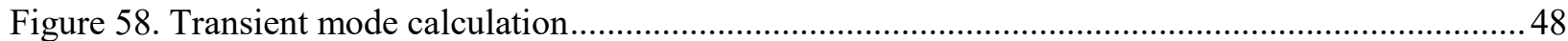

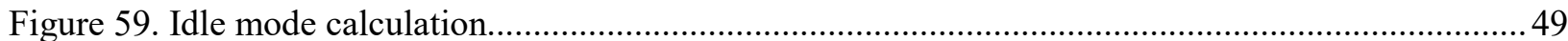

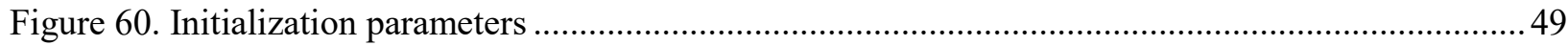

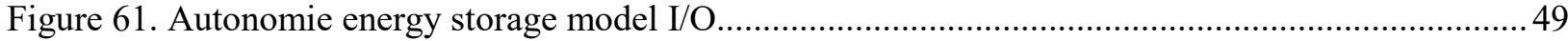

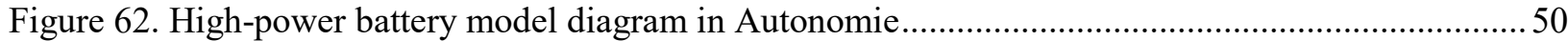

Figure 63. Block A: High-power battery model output voltage calculation........................................51

Figure 64. Block B: High-power battery model current calculation......................................................52

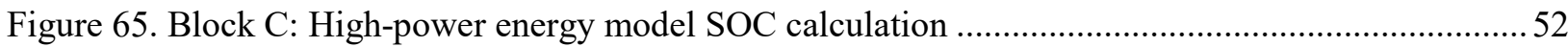

Figure 66. High-energy battery model diagram in Autonomie ............................................................5 53

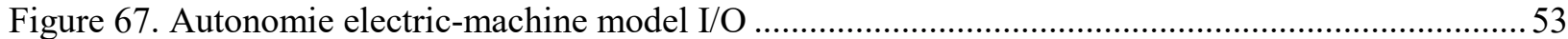

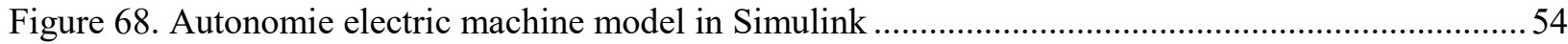

Figure 69. Block A: Electric machine model max torque calculation ..................................................... 55

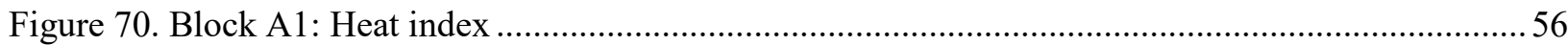

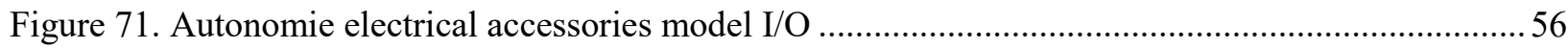

Figure 72. Top-level Simulink diagram of electrical accessories model ..............................................5 57

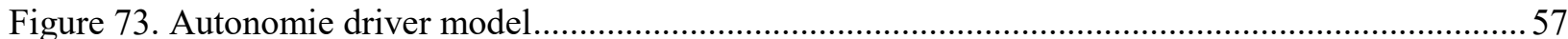

Figure 74. Top-level Simulink diagram of the look-ahead driver model...................................................5 58 


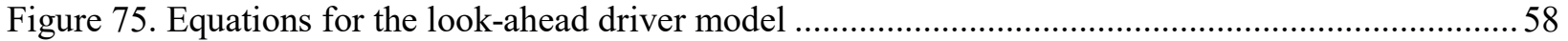

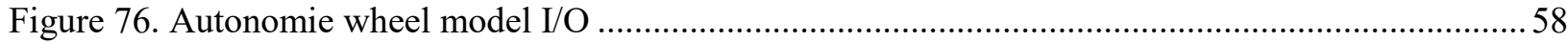

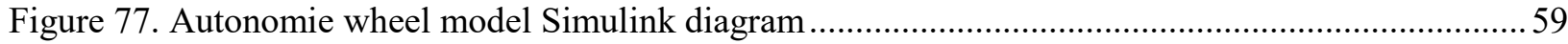

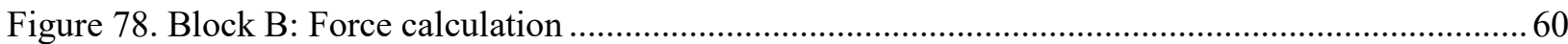

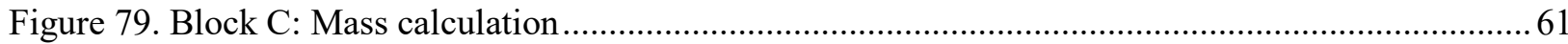

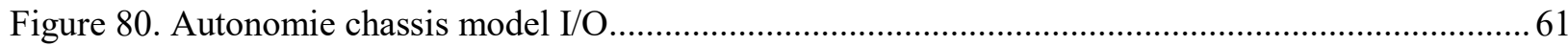

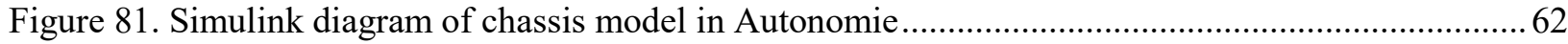

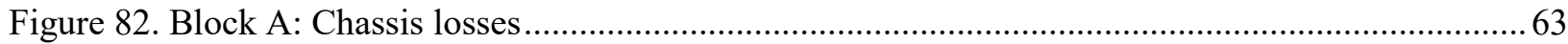

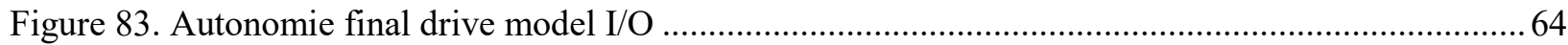

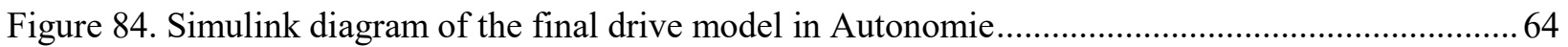

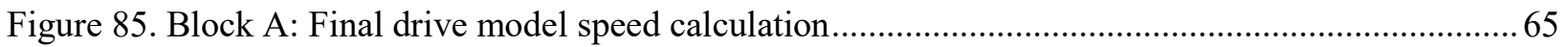

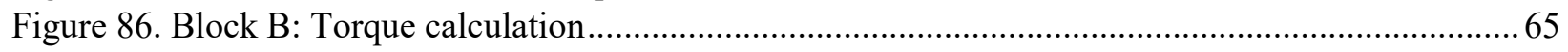

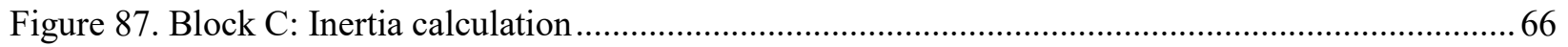

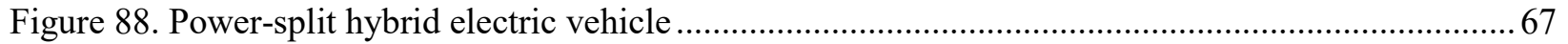

Figure 89. Engine-ON condition-2017 Prius Prime example based on 25 test cycles ........................... 68

Figure 90. SOC regulation algorithm—2017 Prius Prime example based on 25 test cycles ..................... 69

Figure 91. Example of engine operating target -2017 Prius Prime example based on 25 test cycles ....... 70

Figure 92. Configuration of the Chevrolet Volt 2016 powertrain system ............................................ 71

Figure 93. Summary of control analysis for the second-generation Voltec system................................. 72

Figure 94. Wheel torque vs. vehicle speed of 2013 VW Jetta HEV ................................................... 73

Figure 95. Component operating conditions of a fuel-cell vehicle on the urban European drive cycle

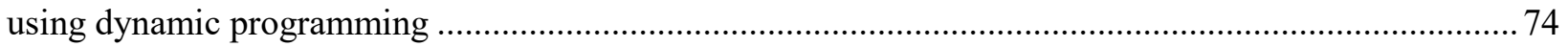

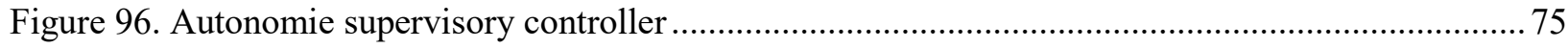

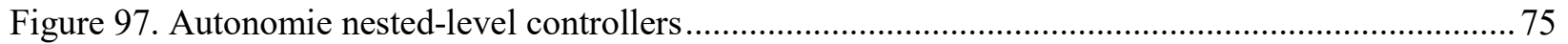

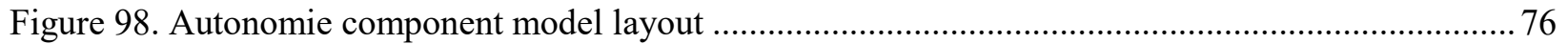

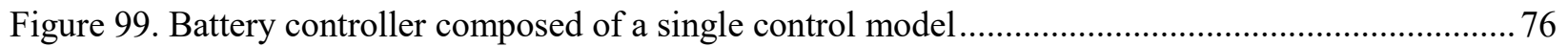

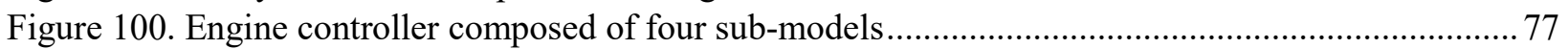

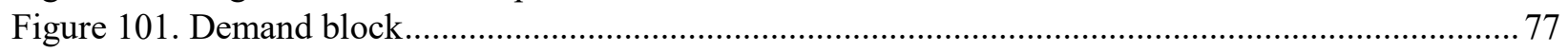

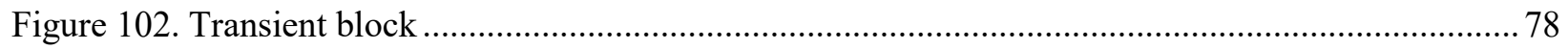

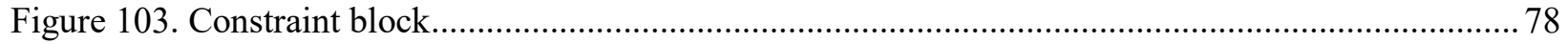

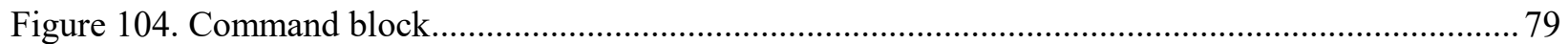

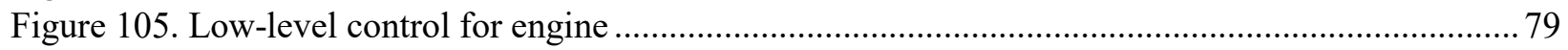

Figure 106. Low-level control for transmission..................................................................................... 80

Figure 107. Simulation and testing results on UDDS cycle (0-505 s) for 2013 Sonata conventional 6ATX

Figure 108. Shifting algorithm validation for 2013 Sonata conventional 6ATX (left) and 2013 Chrysler

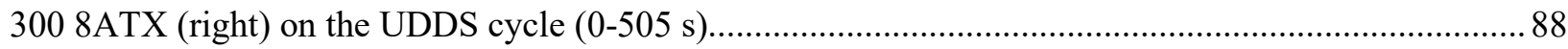

Figure 109. Shifting algorithm validation for 2012 Fusion conventional 6ATX (left) and 2013 Chrysler

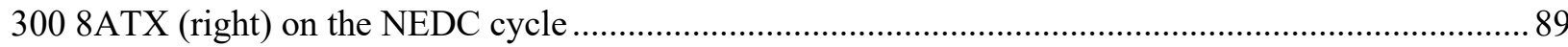

Figure 110. Comparison of simulation and test data for 2012 Honda Civic CVT HEV on UDDS (left) and

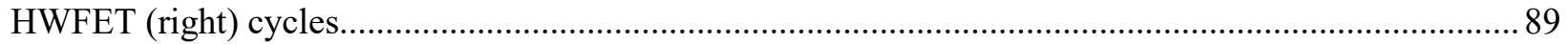

Figure 111. Results of simulation and testing on UDDS cycle for 2010 Toyota Prius HEV ................... 90

Figure 112. Results of simulation and testing on UDDS cycle for 2013 Jetta DCT hybrid ......................91

Figure 113. Results of simulation and testing on UDDS cycle for 2012 Chevrolet Volt PHEV ...............91

Figure 114. Distribution of drag coefficient values of compact (base) vehicle class .............................. 93

Figure 115. Body weight selection for compact (base) vehicles......................................................... 94

Figure 116. Chassis weight selection for compact (base) vehicles ..........................................................95 
Figure 117. Interior weight selection for compact (base) vehicles ................................................... 96

Figure 118. Safety system weight selection for compact (base) vehicle .............................................. 97

Figure 119. Thermal system weight selection for compact (base) vehicle ............................................ 98

Figure 120. Brake mechanism weight selection for compact (base) vehicle .............................................99

Figure 121. Steering system weight selection for compact (base) vehicle ........................................... 100

Figure 122. Electrical accessories weight selection for compact (base) vehicle .................................. 101

Figure 123. Engine weight $(\mathrm{kg})$ vs. engine power $(\mathrm{kW})$ of gasoline engines across different aspiration

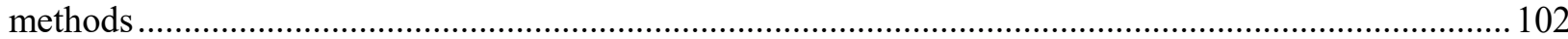

Figure 124. Engine weight (kg) vs. engine power $(\mathrm{kW})$ of diesel engines........................................ 103

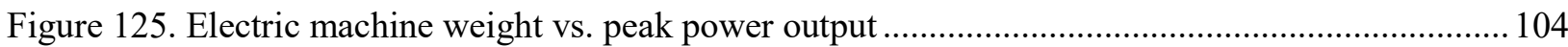

Figure 126. Efficiency for direct drive, for the range of temperatures and speeds considered................ 106

Figure 127. Vehicle attribute analysis for gear span of 6AU (Source: Argonne Vehicle Database)........ 107

Figure 128. Vehicle attribute analysis for final drive ratio of 6AU (Source: Argonne Vehicle Database)

108

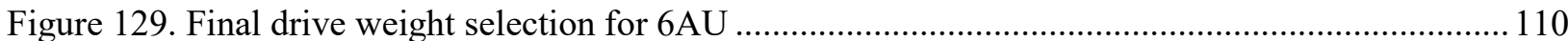

Figure 130. Gearbox weight selection for 6AU for midsize vehicles .................................................. 111

Figure 131. Automatic (AU/AU+/AU++) transmission efficiency maps for different numbers of gears 114

Figure 132. Manual (DM) transmission efficiency maps for different number of gears ........................ 115

Figure 133. DCT Efficiency maps for different numbers of gears …............................................. 115

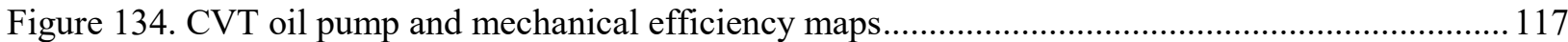

Figure 135. CVT+ oil pump and mechanical efficiency maps ......................................................... 118

Figure 136. Maps of transmission upshifting (solid lines) and downshifting (dotted lines)................... 120

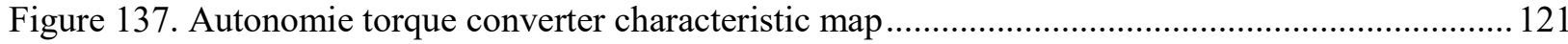

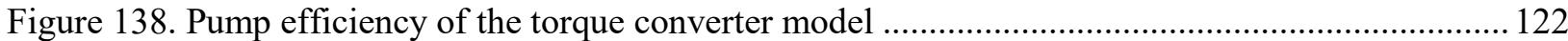

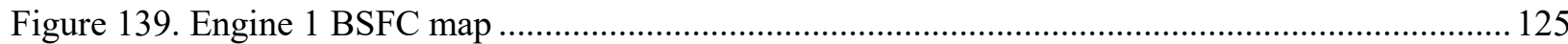

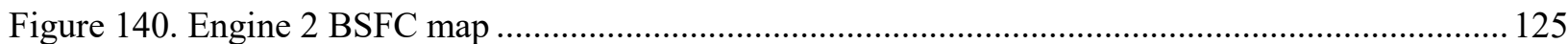

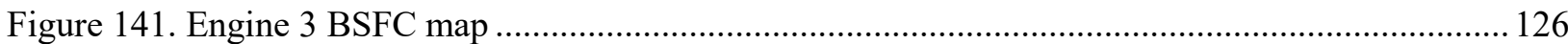

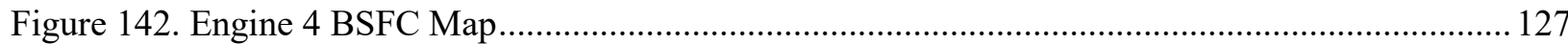

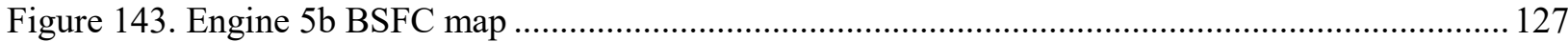

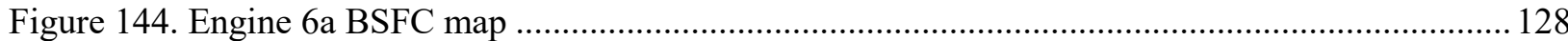

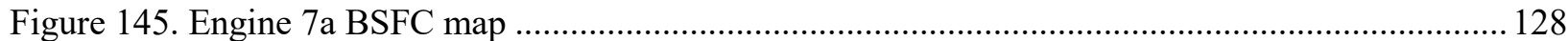

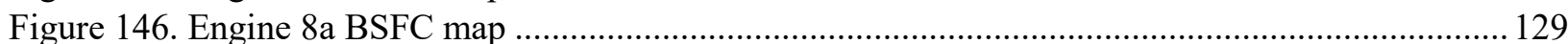

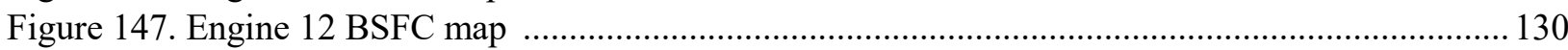

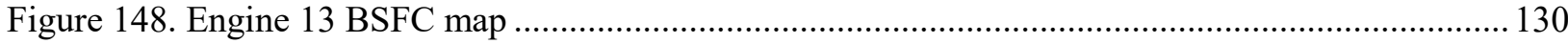

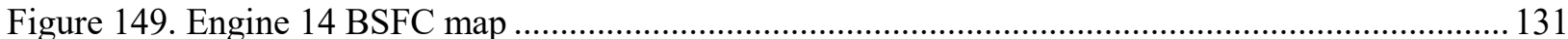

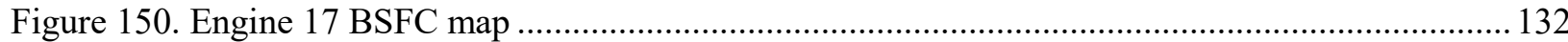

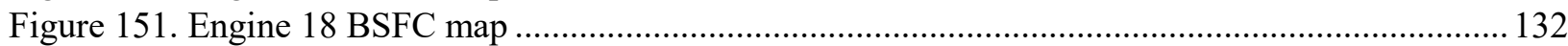

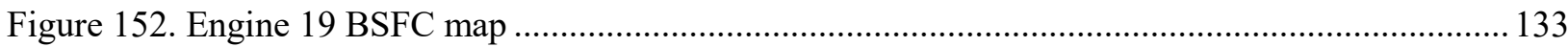

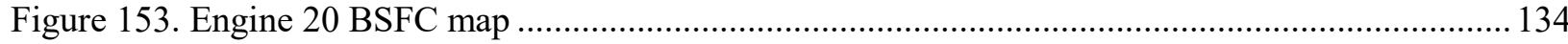

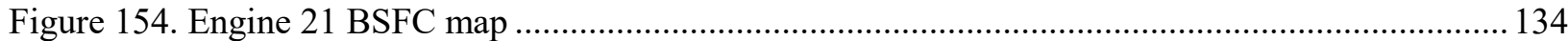

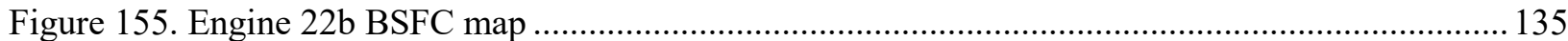

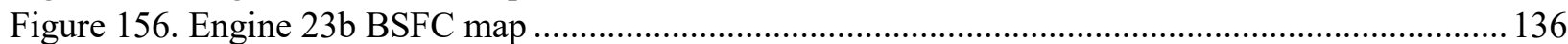

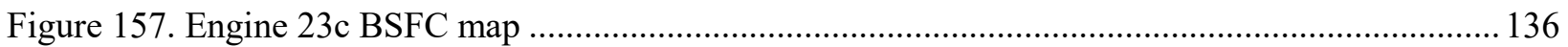

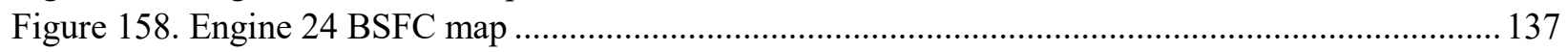

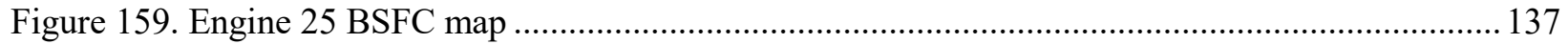

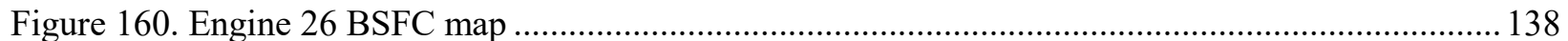

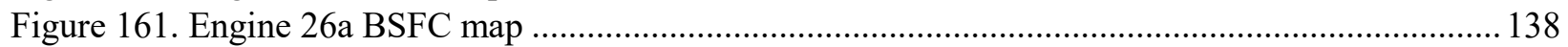


Figure 162. Incremental BSFC and efficiency differences among engines ........................................ 144

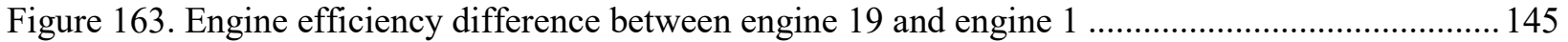

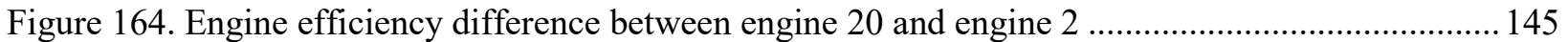

Figure 165. Engine efficiency difference between engine 21 and engine 18 ......................................... 146

Figure 166. Electric machines efficiency maps for different powertrains ............................................. 148

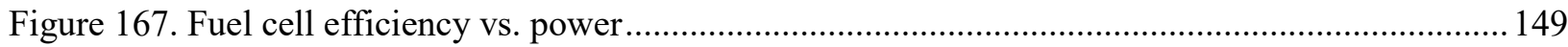

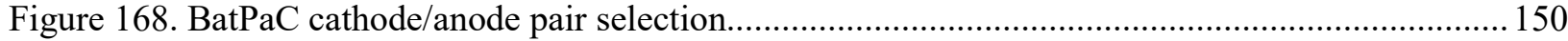

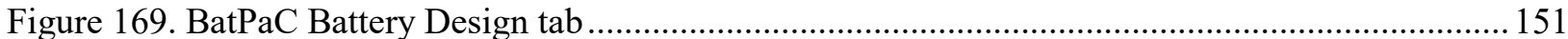

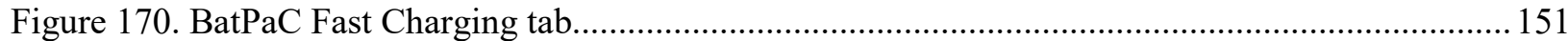

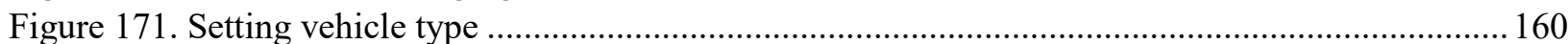

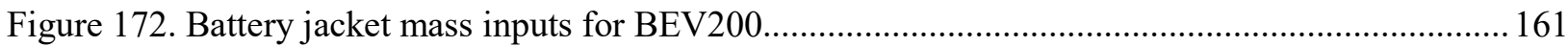

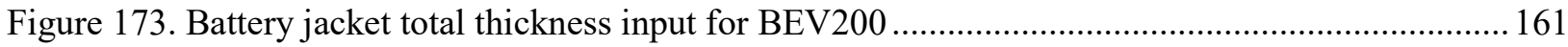

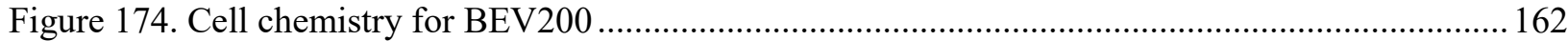

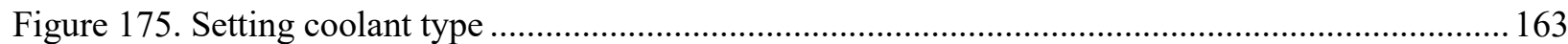

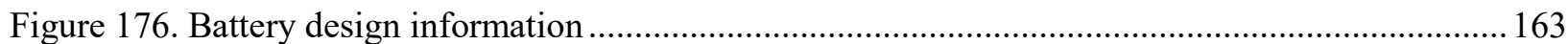

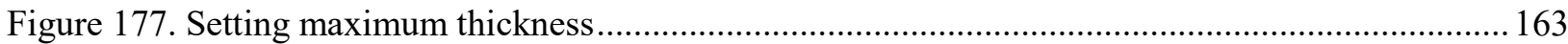

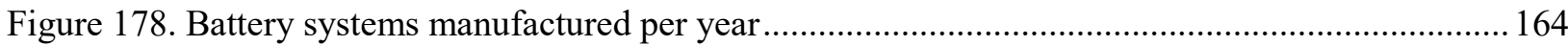

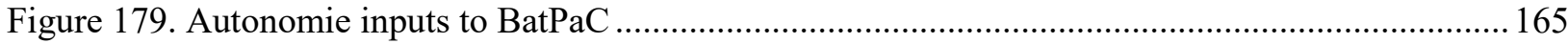

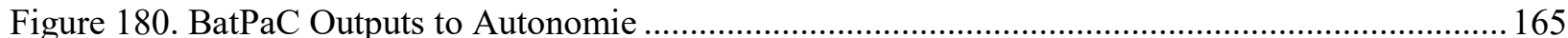

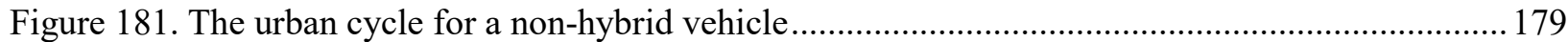

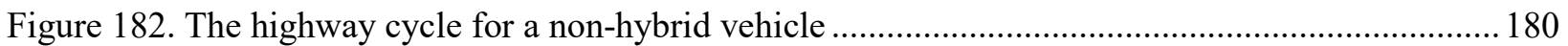

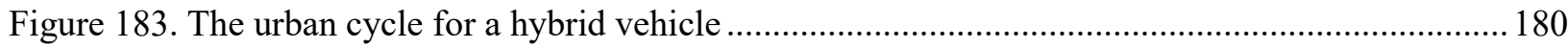

Figure 184. The highway cycle for a hybrid vehicle (only the results from the second cycle were used)

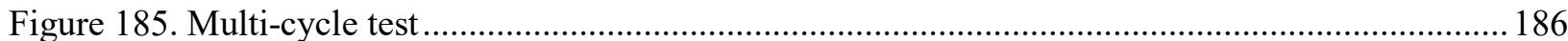

Figure 186. Cold-start penalty on Bag 1 across different engine types ................................................ 187

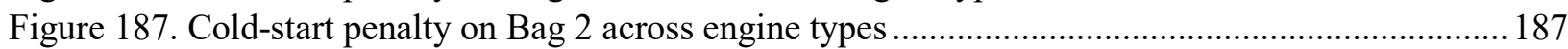

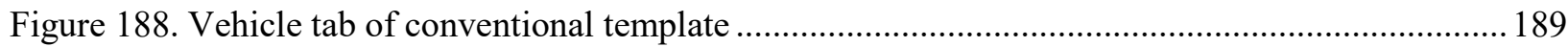

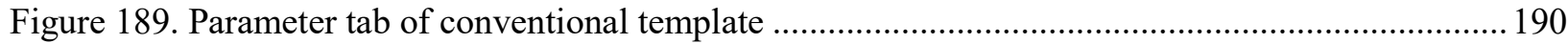

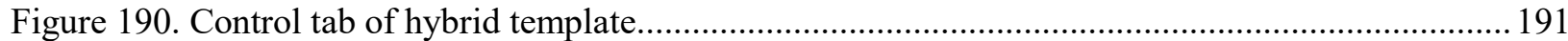

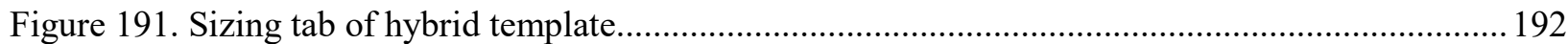

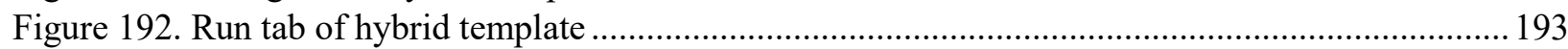

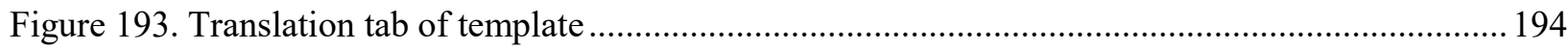

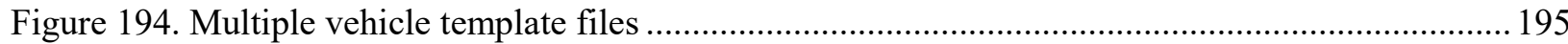

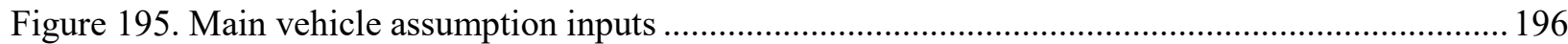

Figure 196. Conventional powertrain inheritance flowchart for eng01 ........................................... 197

Figure 197. Inheritance algorithm for conventional vehicle ................................................................ 198

Figure 198. Hybrid powertrain vehicle inheritance flowchart ............................................................ 198

Figure 199. Inheritance algorithm for battery-electric vehicles....................................................... 199

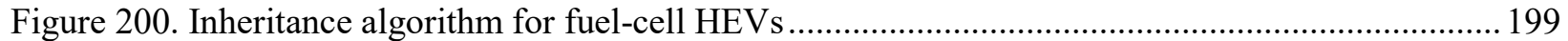

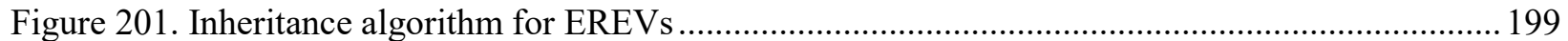

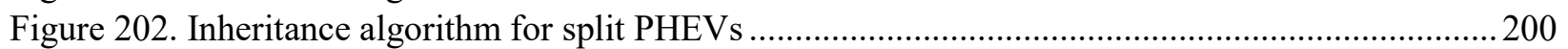

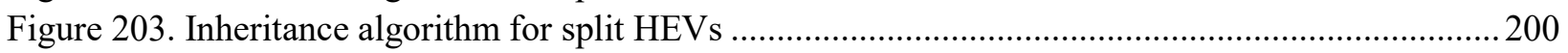

Figure 204. Parallel HEVs: Inheritance from reference baseline vehicles ........................................... 200

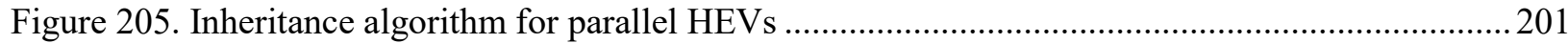

Figure 206. Parallel PHEVs: Inheritance from reference baseline vehicles ........................................ 201

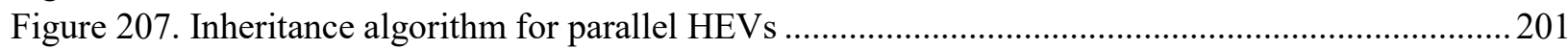




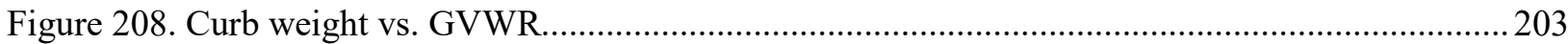

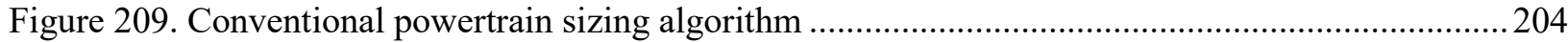

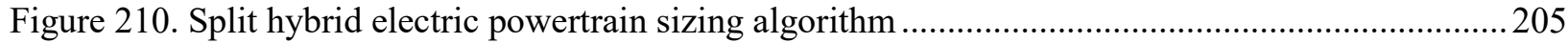

Figure 211. Parallel hybrid electric powertrain sizing algorithm ........................................................206

Figure 212. Parallel plug-in hybrid (Par PHEV20) electric vehicle powertrain sizing............................207

Figure 213. Parallel plug-in hybrid (Par PHEV50) electric vehicle powertrain sizing ...........................207

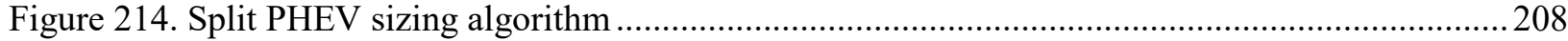

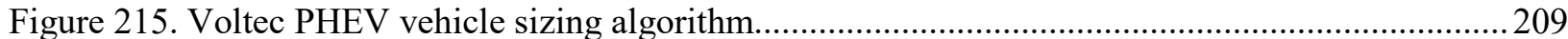

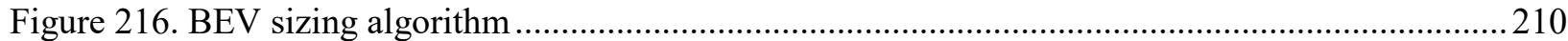

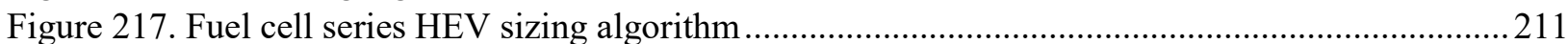

Figure 218. Electric machine $(\mathrm{kW})$ vs. battery energy $(\mathrm{kWh})$ for PHEV20s .....................................2 212

Figure 219. Electric machine $(\mathrm{kW})$ vs. battery energy $(\mathrm{kWh})$ for PHEV50s ...................................... 212

Figure 220. Electric machine $(\mathrm{kW})$ vs. battery energy $(\mathrm{kWh})$ for BEVs..........................................2213

Figure 221. Relationship between engine displacement and number of engine cylinders......................2214

Figure 222. Method for computing engine displacement and number of cylinders ...............................2 215

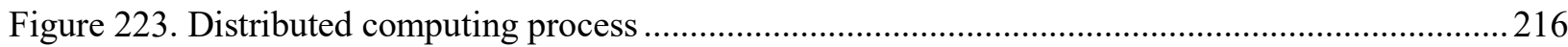

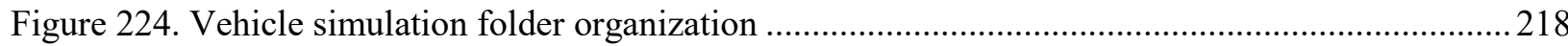

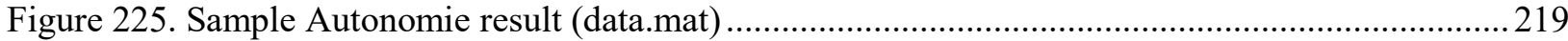

Figure 226. XML Autonomie results file (simulation.a result) ......................................................222

Figure 227. Engine operating points for 5- and 6-speed manual transmissions ...................................221

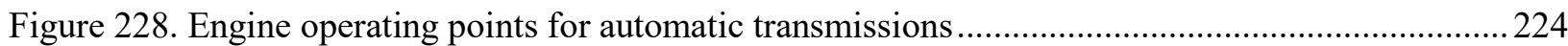

Figure 229. Engine operating points for 6- and 8-speed DCT transmissions $\quad$.......................................225

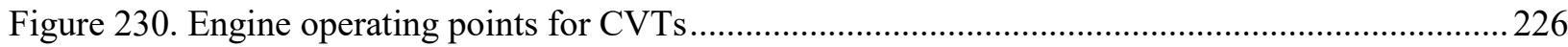

Figure 231. Powertrain efficiency values of different engine types with automatic transmissions and

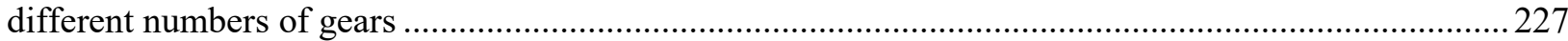

Figure 232. Powertrain efficiency values of different engine types with AU+ transmissions with different

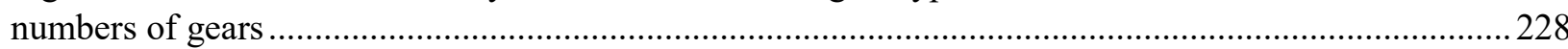

Figure 233. Powertrain efficiency values of different engine types with AU++ transmissions with

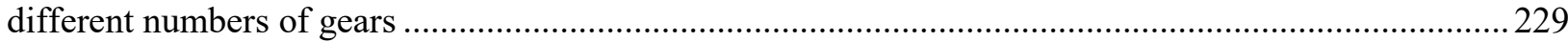

Figure 234. Powertrain efficiency values of different engine types with DCTs with different numbers of

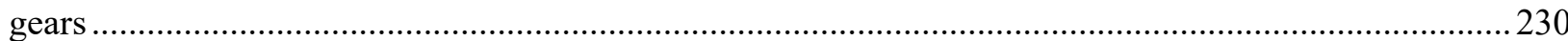

Figure 235. Powertrain efficiency values of different engine types with manual transmissions with

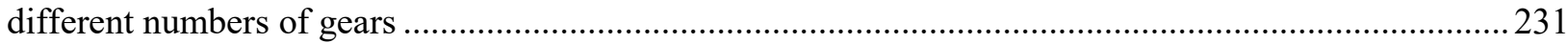

Figure 236. Powertrain efficiency values of different engine types with CVT ....................................2232

Figure 237. Powertrain efficiency values of different engine types with CVTps................................233

Figure 238. Total number of shifting events for AU/AU+/AU++ transmissions during UDDS cycle.....234

Figure 239. Total number of shifting events for AU/AU+/AU++ transmissions during US06 cycle ......234

Figure 240. Total number of shifting events for DM transmissions during UDDS cycle .....................2235

Figure 241. Total number of shifting events for DM transmissions during US06 cycle ......................2236

Figure 242. Total number of shifting events for DCTs during UDDS cycle .......................................2236

Figure 243. Total number of shifting events for DCTs during US06 cycle.........................................237

Figure 244. Engine power vs. mass reduction step (compact, non-performance) ...................................2237

Figure 245. Engine power vs. mass reduction step (compact, performance).........................................2238

Figure 246. Engine power vs. mass reduction step (midsize, non-performance) ..................................238

Figure 247. Engine power vs. mass reduction step (midsize, performance) ..........................................239

Figure 248. Engine power vs. mass reduction step (small SUV, non-performance) .............................2239

Figure 249. Engine power vs. mass reduction step (small SUV, performance) ....................................2 240 
Figure 250. Engine power vs. mass reduction step (midsize SUV, non-performance) ..........................240

Figure 251. Engine power vs. mass reduction step (midsize SUV, performance) .................................2241

Figure 252. Engine power vs. mass reduction step (pickup, non-performance) .....................................241

Figure 253. Engine power vs. mass reduction step (pickup, performance) .............................................2242

Figure 254. Motor-specific power vs. acceleration time (BEV200) ...................................................243

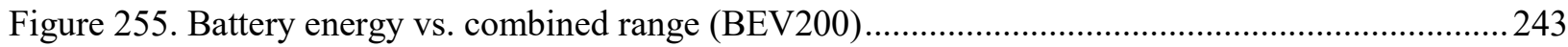

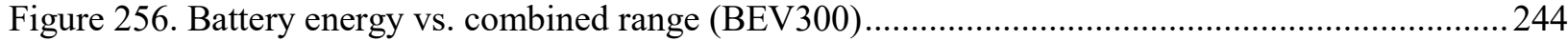

Figure 257. Motor-specific power vs. acceleration time (BEV300) …...............................................24

Figure 258. Battery energy vs. vehicle curb weight (BEV200) ........................................................245

Figure 259. Battery energy vs. vehicle curb weight (BEV300) ..........................................................245

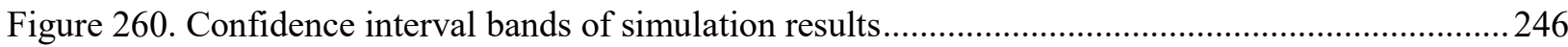

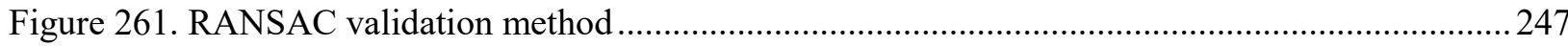

Figure 262. QQ plot to confirm the normal distribution of results ................................................... 247

\section{Tables}

Table 1. Engine Lugging Limits for Different Transmissions (Naturally Aspirated [NA] Engines) .........36

Table 2. Engine Lugging Limits for Different Transmissions (Turbo Engines).......................................36

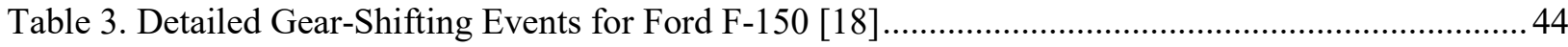

Table 4. Argonne Simulated Gear-Shifting Events for 10-Speed Transmission .....................................4 44

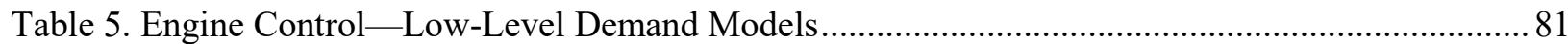

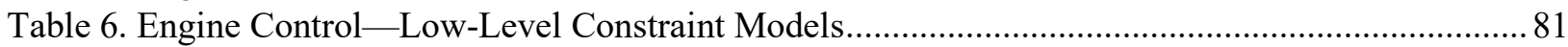

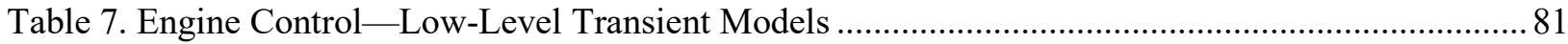

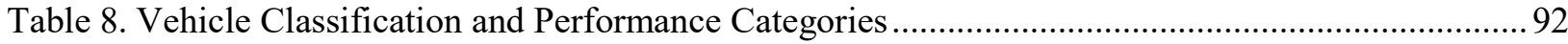

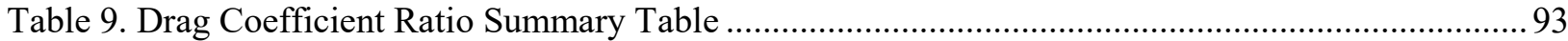

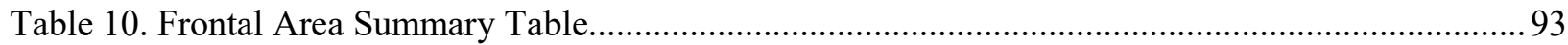

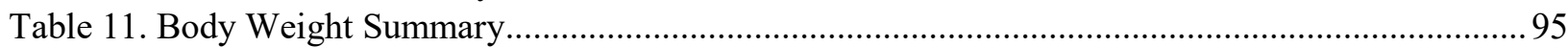

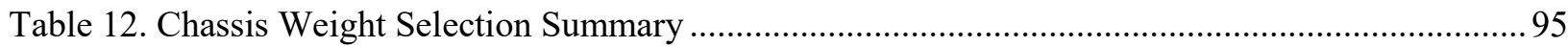

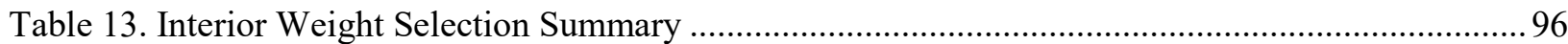

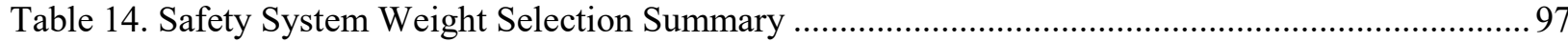

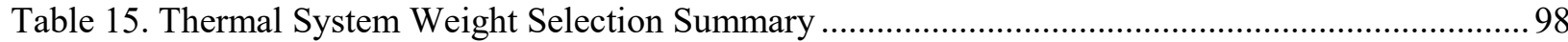

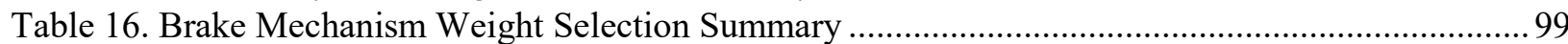

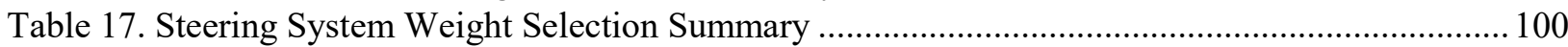

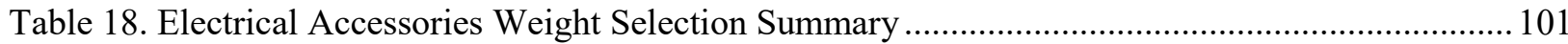

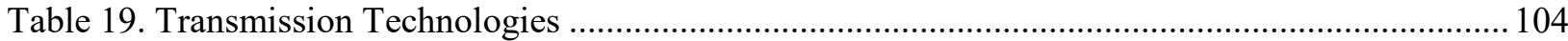

Table 20. Gear Ratios, Gear Span and Final Drive Ratio Selected for Different Transmissions ............. 109

Table 21. Final Drive Weight Summary for All Transmission Types .................................................. 112

Table 22. Gearbox Weight Summary for All Transmission Types.......................................................... 112

Table 23. Engine Technologies with Reference Peak Power and Reference Displacement..................... 122

Table 24. Characteristics of Fuel Used for Modeling Engines ........................................................... 124

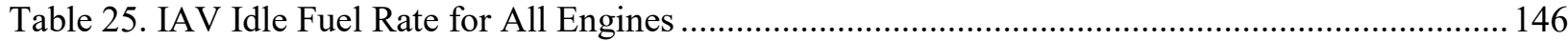

Table 26. Electric Machine Efficiency Map Sources for Different Powertrain Configurations............... 147

Table 27. Efficiency Scaling of Electric Machines............................................................................. 148

Table 28. BatPaC Input Details for BatPaC 4.0 October 01, 2020 ….................................................... 150

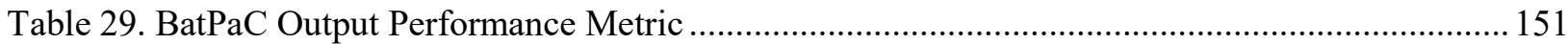

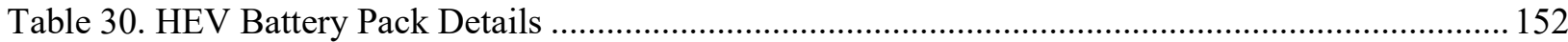

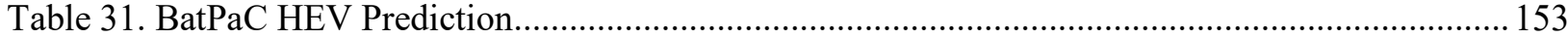




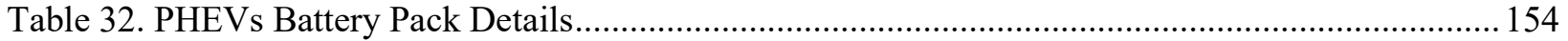

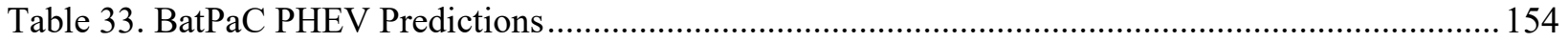

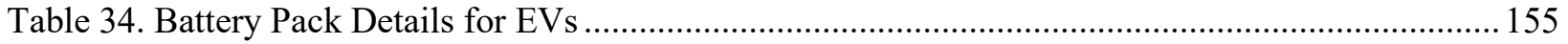

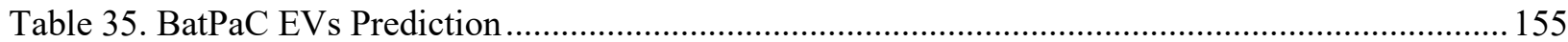

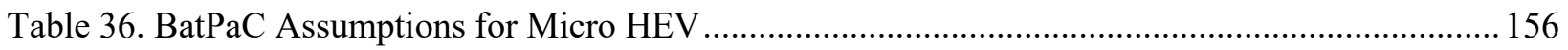

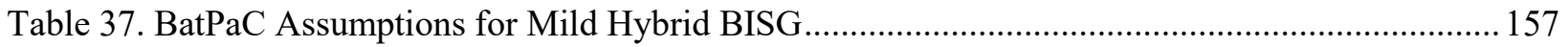

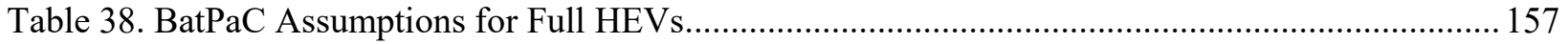

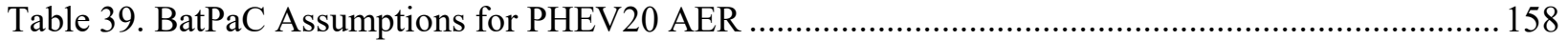

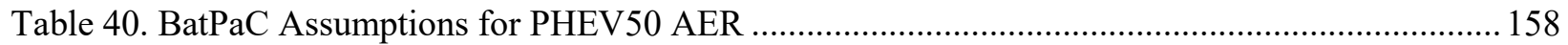

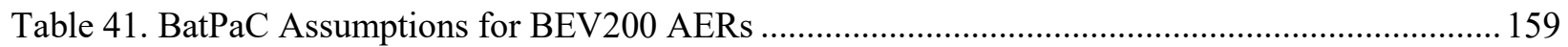

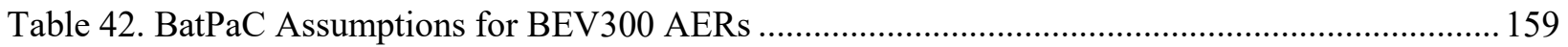

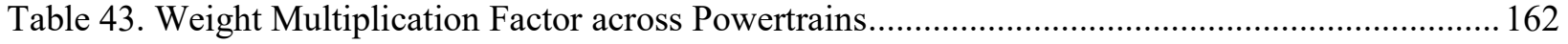

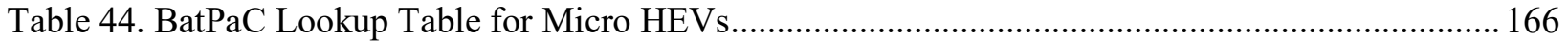

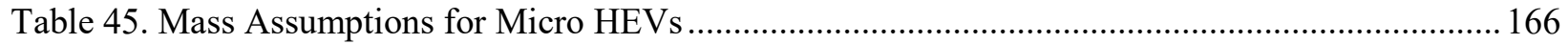

Table 46. BatPaC Lookup Table for Mild Hybrid BISGs ...................................................................... 167

Table 47. BatPaC Lookup Table for Full Hybrids (Compact/Midsize) .................................................... 168

Table 48. BatPaC Lookup Table for Full Hybrids (SUVs/Pickups) ...................................................... 168

Table 49. BatPaC Lookup Table for PHEV20 AERs (Compact/Midsize) .............................................. 169

Table 50. BatPaC Lookup Table for PHEV20 AERs (SUVs/Pickup) ................................................ 170

Table 51. BatPaC Lookup Table for PHEV50 AERs (Compact/Midsize) .............................................. 171

Table 52. BatPaC Lookup Table for PHEV50 AERs (SUVs/Pickups) ................................................ 172

Table 53. BatPaC Lookup Table for BEV200 AERs (Compact/Midsize).............................................. 173

Table 54. BatPaC Lookup Table for BEV200 AERs (SUVs/Pickup) .................................................. 174

Table 55. BatPaC Lookup Table for BEV300 AERs (Compact/Midsize) .............................................. 175

Table 56. BatPaC Lookup Table for BEV300 AERs (SUVs/Pickups)............................................. 176

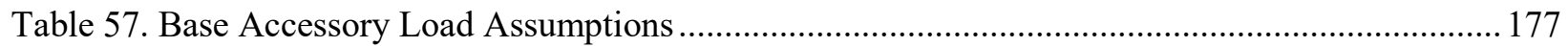

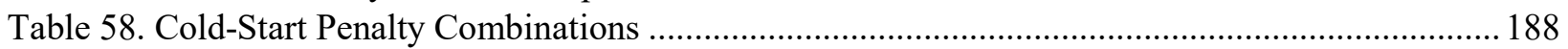

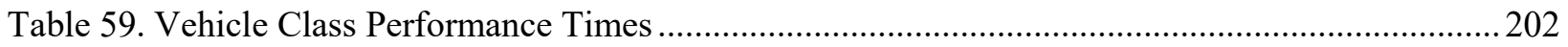

Table 60. Thresholds for Engine Displacement vs. Number of Engine Cylinders .................................214 


\section{ACRONYMS AND ABBREVIATIONS}

AER all-electric range

Argonne Argonne National Laboratory

AMTL Advanced Mobility Technology Laboratory

AU

automatic transmission

BEV battery electric vehicle

BISG belt-integrated starter generator

BMEP brake mean effective pressure

BSFC brake-specific fuel consumption

CAFE corporate average fuel economy

CAFE model CAFE Compliance and Effects Modeling System

CAN controller area network bus

CD charge-depleting

CISG crankshaft-integrated starter generator

CS charge-sustaining

CVT continuously variable transmission

DCT dual-clutch transmission

DEAC cylinder deactivation

DI direct injection

DM manual transmission

DOE U.S. Department of Energy

DOHC dual overhead camshaft valvetrain

DOT U.S. Department of Transportation

EGR exhaust gas recirculation

EPA U.S. Environmental Protection Agency

EPCA Energy Policy and Conservation Act

EREV extended range electric vehicle

EV electric vehicle 


$\begin{array}{ll}\text { FMEP } & \text { friction mean effective pressure } \\ \text { FRIA } & \text { Final Regulatory Impact Analysis } \\ \text { FRM } & \text { Final Rulemaking } \\ \text { FTP } & \text { Federal Test Procedure } \\ \text { GVW } & \text { gross vehicle weight } \\ \text { HEV } & \text { hybrid electric vehicle } \\ \text { HFTO } & \text { DOE Hydrogen and Fuel Cell Technologies Office } \\ \text { HIL } & \text { hardware-in-the-loop } \\ \text { HWFET } & \text { Highway Fuel Economy Test } \\ \text { ICE } & \text { internal combustion engine } \\ \text { IMEP } & \text { indicated mean effective pressure } \\ \text { I/O } & \text { input(s)/output(s) } \\ \text { IVM } & \text { initial vehicle movement } \\ \text { LHV } & \text { lower heating value } \\ \text { MR } & \text { pumping mean effective pressure } \\ \text { PHEV } & \text { plugged-in electrified vehicle (PHEV and BEV) } \\ \text { PEV } & \text { model year } \\ \text { NA } & \text { naturally aspirated } \\ \text { NEDC } & \text { New European Driving Cycle } \\ \text { NHTSA } & \text { National Highway Traffic Safety Administration } \\ \text { NPRM } & \text { Notice of Proposed Rulemaking } \\ \text { NVH } & \text { noise, vibration and harness } \\ \text { PHional-integral }\end{array}$


QQ quantile-quantile

RANSAC random sample consensus algorithm

RCP rapid-control prototyping

SAE Society of Automotive Engineers

SIL software-in-the-loop

SOC state of charge

SUV sport utility vehicle

TAR Technical Assessment Report

UDDS Urban Dynamometer Driving Schedule

VPA vehicle propulsion architecture

VVL variable valve lift

VVT variable valve timing

XML extensible markup language 


\section{UNITS OF MEASURE}

$\begin{array}{ll}\text { Ah } & \text { ampere-hour(s) } \\ \mathrm{h} & \text { hour(s) } \\ \mathrm{kg} & \text { kilogram(s) } \\ \mathrm{km} & \text { kilometer(s) } \\ \mathrm{kW} & \text { kilowatt(s) } \\ \mathrm{L} & \text { liter(s) } \\ \mathrm{m}^{2} & \text { square meter(s) } \\ \mathrm{mpg} & \text { mile(s) per gallon } \\ \mathrm{mph} & \text { mile(s) per hour } \\ \text { rad } & \text { radian(s) } \\ \text { rpm } & \text { rotation(s) per minute } \\ \mathrm{s}, \mathrm{sec} & \text { second(s) } \\ \mathrm{V} & \text { volt(s) } \\ \text { W } & \text { watt(s) } \\ \text { Wh } & \text { watt-hour(s) } \\ & \end{array}$




\section{INTRODUCTION}

In 1975, Congress passed the Energy Policy and Conservation Act (EPCA), requiring standards for corporate average fuel economy (CAFE), and charging the U.S. Department of Transportation (DOT) with the establishment and enforcement of these standards. The Secretary of Transportation has delegated these responsibilities to the National Highway Traffic Safety Administration (NHTSA).

NHTSA has contracted the DOT Volpe National Transportation Systems Center (Volpe Center) to provide analytical support for NHTSA's regulatory and analytical activities related to fuel economy standards. Unlike long-standing safety and criteria pollutant emissions standards, fuel economy standards apply to manufacturers' overall fleets rather than to individual vehicle models. In developing the standards, NHTSA made use of the CAFE Compliance and Effects Modeling System (the CAFE model), which was developed by DOT's Volpe Center for the 2005-2007 CAFE rulemaking and has been continually updated since. The model is the primary tool used by the agency to evaluate potential CAFE stringency levels by applying technologies incrementally to each manufacturer's fleet until the requirements under consideration are met. The CAFE model relies on numerous technology-related and economic inputs, such as market forecasts and technology cost and effectiveness estimates. These inputs are categorized by vehicle classification, technology synergies, phase-in rates, cost learning curve adjustments, and technology decision trees. The Volpe Center assists NHTSA in the development of the engineering and economic inputs to the CAFE model by analyzing the application of potential technologies to the current automotive industry vehicle fleet to determine the feasibility of future CAFE standards, the associated costs, and the benefits of the standards.

Part of the CAFE model's function is to estimate CAFE improvements that a given manufacturer could achieve by applying additional technologies to specific vehicles in its product line. Because CAFE standards apply to the average fuel economy across manufacturers' entire fleets of new passenger cars and light trucks, the model, when simulating manufacturers' potential application of technology, considers the entire range of each manufacturer's product line. This typically involves accounting for more than 1,000 distinct vehicle models and variants, many more than can be practically examined using full vehicle simulation (or the other techniques mentioned above). Instead, the model uses estimates of the effectiveness of specific technologies for a representative vehicle in each vehicle class, and arranges technologies in decision trees defining logical progressions from lower to higher levels of cost, complexity, development requirements, and/or implementation challenges.

All inputs to CAFE's decision tree model are related to the effectiveness (fuel consumption reduction) of each fuel-saving technology. Although vehicle testing could be used to estimate these factors, vehicle testing that spans many vehicle types and technology combinations could be prohibitively resourceintensive. Another alternative, either as a substitute for or as a complement to vehicle testing, is to make greater use of vehicle simulation. Full vehicle simulation tools use physics-based mathematical equations, engineering characteristics (e.g., engine maps, transmission shift points, hybrid vehicle control strategies), and explicit drive cycles to predict the effectiveness of individual fuel-saving technologies as well as their combinations.

Argonne National Laboratory, a U.S. DOE national laboratory, has developed a full vehicle simulation tool, Autonomie, which has become one of the industry's standard tools for analyzing vehicle 
performance, energy consumption, and technology effectiveness. Through an Inter Agency Agreement, the DOE Argonne Site Office and Argonne National Laboratory have been tasked with conducting full vehicle simulation to support NHTSA CAFE rulemaking.

This report describes the process that Argonne used to conduct full vehicle simulation for the MY 2024 2026 CAFE notice of proposed rulemaking. 


\section{PROJECT STATEMENT}

Through an inter-agency agreement, the DOE Argonne Site Office (ASO), Argonne National Laboratory, will perform full vehicle simulation through the Strategic Partnership Project (SPP) program to support NHTSA's regulatory and analytic activities related to fuel economy. NHTSA's Office of International Policy, Fuel Economy and Consumer Programs oversees this project. The several tasks will include:

1. Performing a full suite of full vehicle simulation of light-duty vehicles, including a wide variety of technologies and vehicle classes, to generate effectiveness inputs for the CAFE model.

2. Developing a comprehensive database of all full vehicle simulation output that could be referenced by the CAFE model.

3. Performing full vehicle simulation to support research for rulemakings related light-duty vehicles.

The CAFE model currently relies on multiple decision trees to represent the component technology options identified below:

- Powertrain

- Engine

- Transmission

- $\quad$ Light-weighting

- Aerodynamics

- Rolling resistance

Figure 1 shows the variety of technology combinations adapted to represent current and potential future technologies that are simulated for the final rule. 


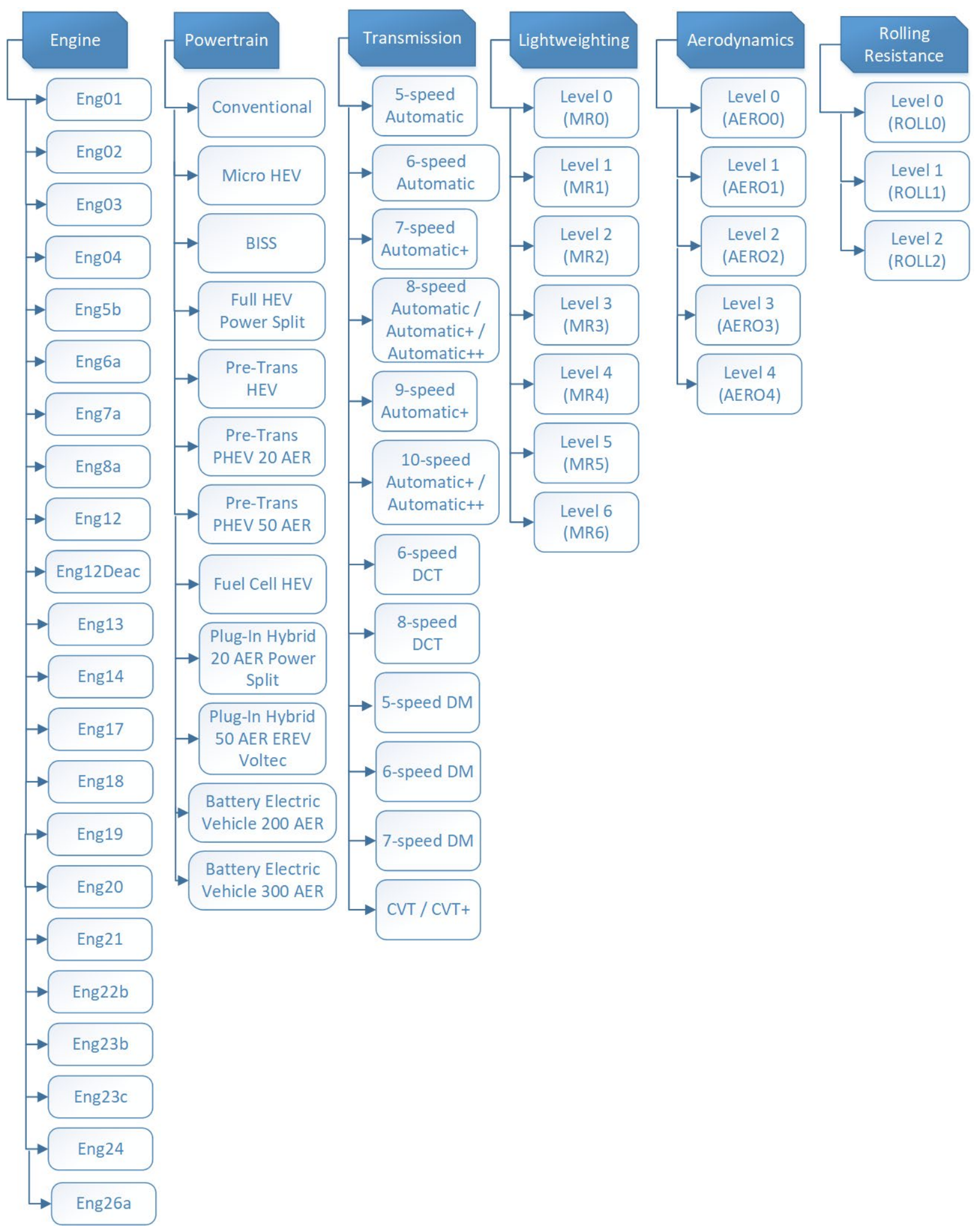

Figure 1. Technologies considered for the Argonne Autonomie for this analysis 


\section{AUTONOMIE SIMULATION PROCESS}

NHTSA directed the Argonne simulation team to update inputs and processes based on an extensive review of technical publications and meetings with stakeholders. In order to address the comments in the draft TAR, performance categories for the initial five vehicle classes simulated were added, with various performance times and estimated curb weights (and towing capacities for the pickup class) that would span a greater variety of vehicles on the road. The component weights were also updated, using the A2Mac1 database [1] across vehicle classes.

As mentioned, individual classes and performance categories have been simulated for every combination of vehicle, powertrain, and component technology. The combinations include the following:

- Five vehicle classes (compact, midsize, small SUV, midsize SUV, pickup)

- Two performance categories for each vehicle class: non-performance (base) and performance (premium)

- Twenty-two engine technologies

- Sixteen transmission technologies

- Seven light-weighting levels

- Five aerodynamic reduction levels

- Three rolling resistance reduction levels

- Four battery electric vehicle ranges

Along with the increased number of technology combinations, vehicle models were also updated. Changes in the process also included technology inheritance (platform-sharing) for engines and transmissions.

The process developed includes the following steps:

1. Specify the vehicle technology specifications (VTS).

2. Select component assumptions.

3. Build the vehicle models.

4. Size the reference powertrains to meet the desired performance.

5. Build all the different vehicle combinations, including "inheritance."

6. Simulate individual vehicles on U.S. standard driving cycles.

7. Perform QA/QC checks on the simulation results.

8. Create the CAFE model database. 


\section{AUTONOMIE}

\subsection{Overview}

Autonomie is a Mathworks ${ }^{\circledR}$-based software environment and framework for automotive controlsystem design, simulation, and analysis. The tool, sponsored by the DOE Vehicle Technologies Office (VTO), is designed for rapid and easy integration of models with varying levels of detail (low to high fidelity), abstraction (from subsystems to systems to entire architectures), and processes (e.g., calibration, validation). Developed by Argonne in collaboration with General Motors, Autonomie was designed to serve as a single tool that can be used to meet the requirements of automotive engineers throughout the development process - from modeling to control. Autonomie was built to:

- Estimate the energy, performance, and cost impact of advanced vehicle and powertrain technologies

- Support proper methods, from model-in-the-loop, software-in-the-loop (SIL), and hardware-in-theloop (HIL) to rapid-control prototyping (RCP)

- Integrate math-based engineering activities through all stages of development—-from feasibility studies to production release

- Promote re-use and exchange of models industry-wide through its modeling architecture and framework

- Support users' customization of the entire software package, including system architecture, processes, and post-processing

- Mix and match models with different levels of abstraction to facilitate execution efficiency with higher-fidelity models, for which analysis and high-detail understanding are critical

- Link with commercial off-the-shelf software applications, including GT-POWER, AMESim ${ }^{\mathrm{TM}}$, and $\mathrm{CarSim}^{\circledR}$, for detailed, physically based models

- Protect proprietary models and processes

By building models automatically, Autonomie enables simulation of an unparalleled number of component technologies and powertrain configurations. Autonomie offers the following capabilities:

- Simulate subsystems, systems, or entire vehicles

- Predict and analyze energy efficiency and performance

- Perform analyses and tests for virtual calibration, verification, and validation of hardware models and algorithms

- Support system hardware and software requirements

- Link to optimization algorithms

- Supply libraries of models for propulsion architectures of conventional powertrains, as well as electric-drive vehicles

In this study, Autonomie is used to assess the energy consumption of advanced powertrain technologies across vehicle classes and configurations. Autonomie has been validated for all powertrain configurations on multiple validated vehicles currently in the market, using dynamometer test data from Argonne's Advanced Mobility Technology Laboratory (AMTL), formerly known as APRF (Argonne Research Powertrain Facility) [2].

With hundreds of pre-defined powertrain configurations and controllers, Autonomie is a unique tool for analyzing the impact of different technologies (e.g., powertrains, components, controls, etc.) across different vehicle classes. Autonomie allows users to evaluate the impact of component sizing on energy consumption for different technologies, as well as to define the component requirements (e.g., power, 
energy) to maximize fuel displacement for a specific application. This is important for the current study because the use of validated plant models, vehicle controls, and complete vehicle models is critical to properly evaluating the benefit of any specific technology. The vehicle-level control algorithms (e.g., engine ON/OFF logic, component operating conditions algorithm) are crucial for properly quantifying technology impact, especially for electric drives. Argonne has been developing shifting algorithms for conventional vehicles based on different component characteristics (e.g., engine fuel flow rate, gear ratios), as well as developing vehicle-level controls for electrified powertrains, for more than 20 years.

The ability to simulate a large number of powertrain configurations, component technologies, and vehicle-level controls over numerous drive cycles has been used to support a large number of studies focusing on energy efficiency, cost-benefit analysis, or greenhouse gases. ${ }^{*}$ More than 250 companies and research entities, including major automotive companies, suppliers and research organizations, have licensed Autonomie to support advanced vehicle development programs.

\subsection{Structure}

Autonomie was designed for full plug-and-play support. Models in the standard format create building blocks, which are assembled at run time into a simulation model of a vehicle, system, or subsystem. All parts of the user interface are designed to be flexible to support architectures, systems, subsystems, and processes not yet envisioned. The software can be molded to individual uses, so it can grow as requirements increase and technical knowledge expands. This flexibility also allows for implementation of legacy models, including plant and controls.

Autonomie is based on standardized modeling architecture, on-demand model building, associated extensible markup language (XML) definition files, and user interfaces for managing models, including a file-versioning database, as seen in Figure 2.

* Lee, H., Lee, B., Kim, S., Kim, N., Rousseau, A., "Model-based fuel economy technology assessment," SAE 2017-01-0532, SAE World Congress, Detroit, April 2017. R. Vijayagopal, N. Shidore, M. Reynolds, C. Folkerts, A. Rousseau, "Estimating the Fuel Displacement Potential of a Thermoelectric Generator in a Conventional Vehicle Using Simulation,” EVS27, Oct 2013, Barcelona. Jeong, J., Kim, N., Stutenberg, K., Rousseau, A., “Analysis and Model Validation of the Toyota Prius Prime." SAE 2019-01-0369, SAE World Congress, Detroit, April 2019. Kim, N, Jeong, J. Rousseau, A. and Lohse-Busch, H. "Control Analysis and Thermal Model Development of PHEV," SAE 2015-01-1157, SAE World Congress, Detroit, April15, 2015. Kim, N., Rousseau, A. and Lohse-Busch, H. "Advanced Automatic Transmission Model Validation Using Dynamometer Test Data," SAE 2014-01-1778, SAE World Congress, Detroit, April 14, 2014.; Lee, D. Rousseau, A. and Rask, E. "Development and Validation of the Ford Focus BEV Vehicle Model," 2014-01-1809, SAE World Congress, Detroit, April 14, 2014. Kim, N., Rousseau, A., and Duoba, M. "Validating Volt PHEV Model with Dynamometer Test Data using Autonomie," SAE 2013-01-1458, SAE World Congress, Detroit, April 13, 2013. Kim, N., Rousseau, A., and Rask, E. "Autonomie Model Validation with Test Data for 2010 Toyota Prius," SAE 2012-01-1040, SAE World Congress, Detroit, April 12, 2012. Karbowski, D., Rousseau, A, Pagerit, S., and Sharer, P. "Plug-in Vehicle Control Strategy-From Global Optimization to Real Time Application," 22th International Electric Vehicle Symposium (EVS22), October 2006, Yokohama. 


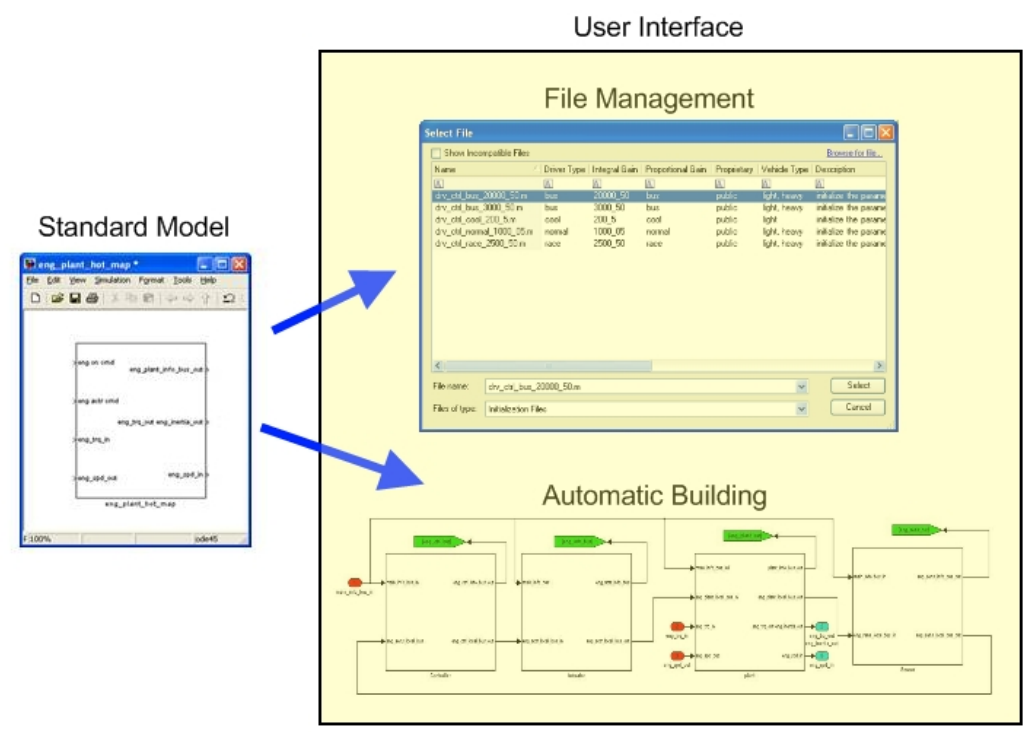

Figure 2. Simulation management concepts

All systems in the vehicle architecture can be logically categorized as either "containing systems" or "terminating systems" (Figure 3). Containing systems consist of one or more subsystems, as well as optional files to define that system. They do not contain models; they only describe the structure of the interconnections among systems and subsystems. Terminating systems consist of a model that defines the behavior of the system and any files needed to provide inputs or calculate outputs. Terminating system models contain the equations that describe the mathematical functions of a system or subsystem.

Both types of systems are arranged in a hierarchical fashion to define the vehicle to be simulated. To avoid confusion, it is a best practice to mimic the structure of the hardware as much as possible. For example, low-level component controllers should be grouped with the components that they control, at different levels of the hierarchy (where applicable). Only systems that appear in the vehicle should be represented; in other words, there is no need for unused components or empty controllers. In addition to simplifying the architecture, this philosophy will allow for easy transfer of systems among users and will fully support HIL, SIL, and RCP.
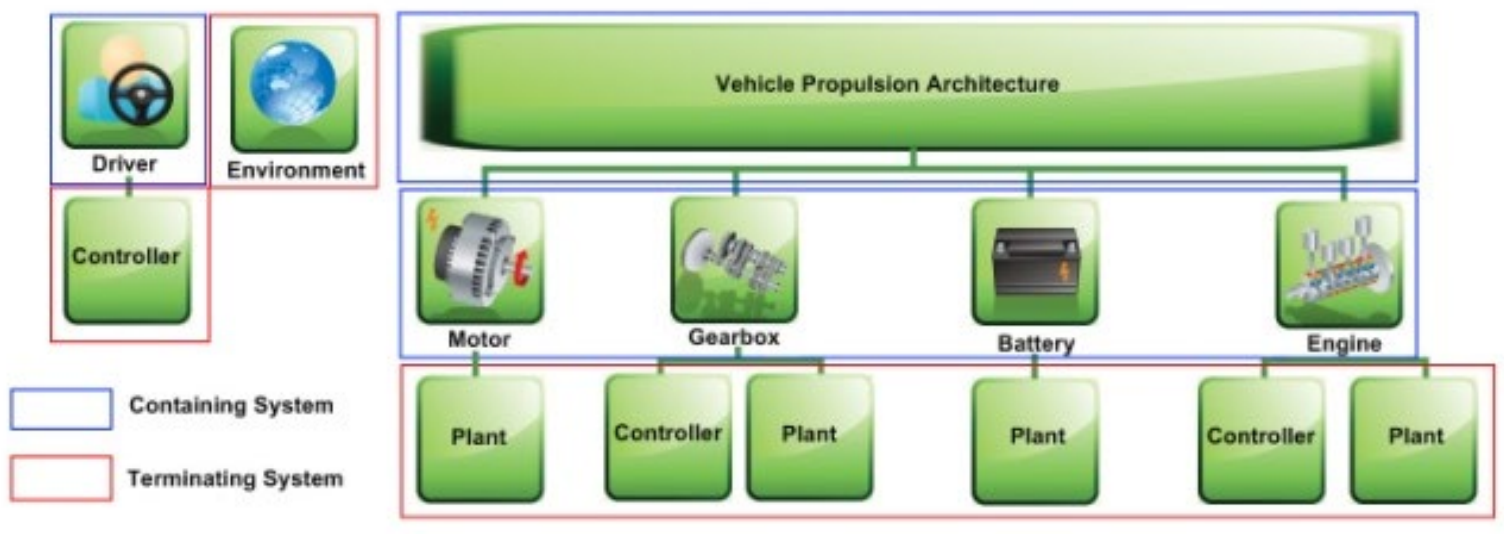

Figure 3. Class diagram of container and terminating systems 
At the top level is a vehicle system containing the following systems: environment, driver, vehicle propulsion controller for advanced powertrain vehicles, such as hybrid electric vehicles (HEVs) or plug-in hybrid electric vehicles (PHEVs) that require a vehicle level controller, and vehicle propulsion architecture (VPA) (Figure 4). The VPA system will contain the powertrain components that are required to simulate the vehicle, such as engine, battery, and wheels.

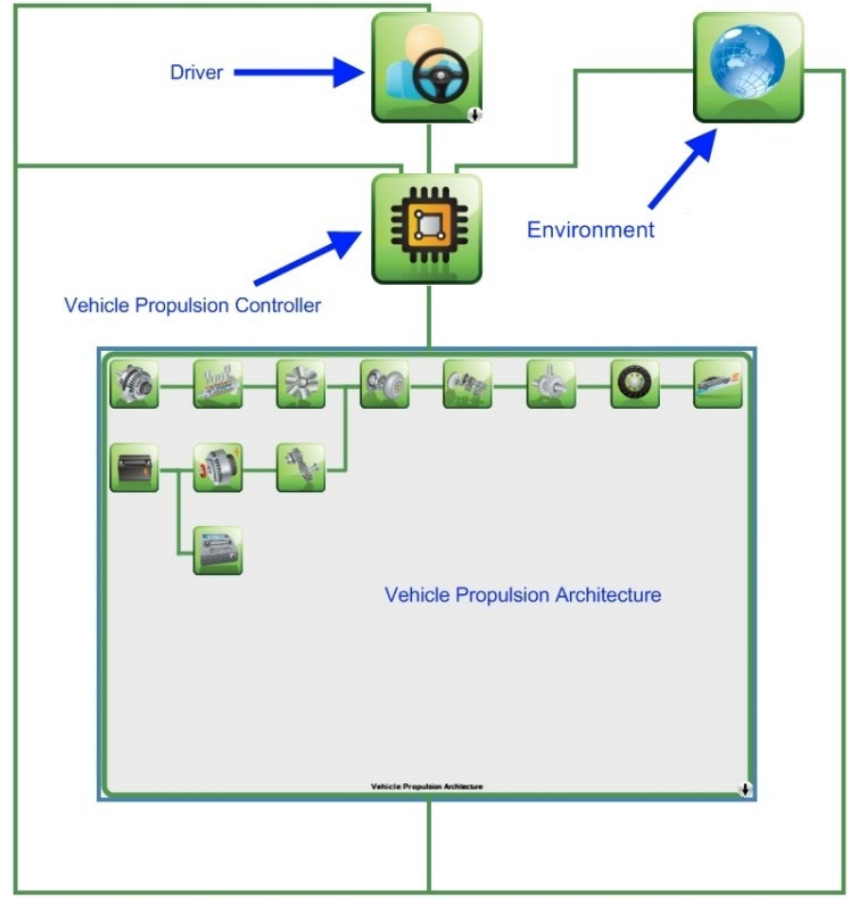

Figure 4. Top-level vehicle layout.

The model files created for the terminating systems are combined to allow simulation in Simulink ${ }^{\circledR}$. One option is to create every possible combination of the systems and save each complete vehicle as a separate model file. Because of the staggering number of possible combinations, this option is not feasible. Combinations involve not only many different components, but also different levels of fidelity and model versions for each component. Changing the version of a single component model would result in a new version of the entire vehicle. This method is clearly storage-intensive and impractical.

A second option is to save every model in its own file and manage a library of the models. This would be an improvement over the first option; however, it still presents some difficulties. When users wish to create a new vehicle, they must select all the appropriate models from the library and connect them by hand into a vehicle context. Not only is this manual process time-consuming, but it introduces many opportunities for error. Consider an engine control unit model for auto code generation that can have more than 2,000 inputs and outputs (I/Os). Manually connecting all I/Os almost guarantees errors. It also requires some outside solution for model library management (e.g., searching, versioning, and ensuring compatibility).

Autonomie uses a patented approach that combines the second option with an automated building process, giving the user the flexibility of saving and versioning models independently without the potential pitfalls of manual connections. Users select the desired files in a user interface, and the automatic building process uses metadata associated with the models to create the correct connections, as shown in Figure 5. 


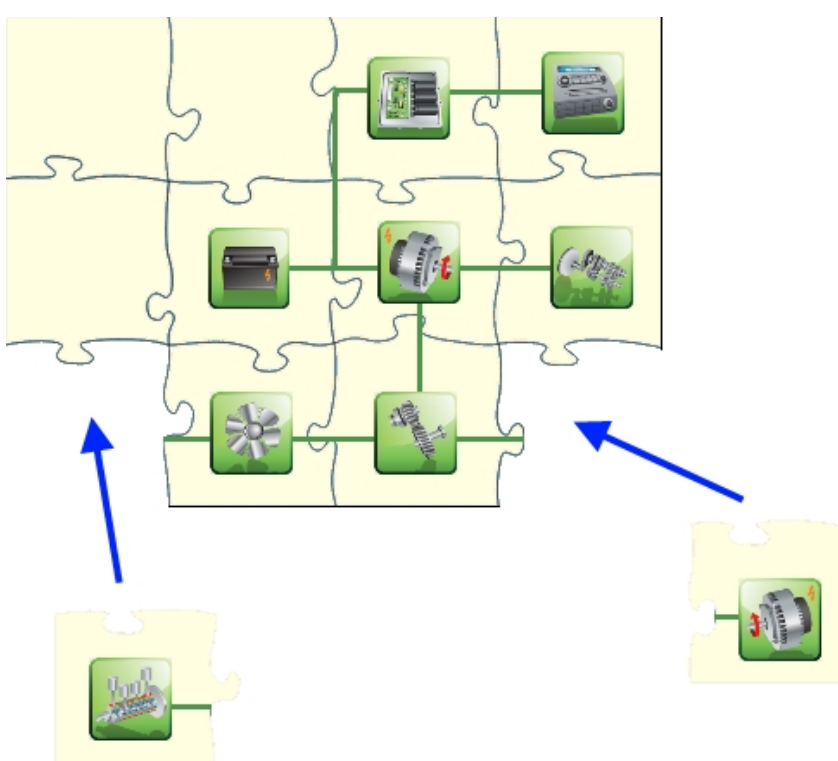

Figure 5. Models automatically built.

The ability to automatically build entire vehicle models is critical to the current study, as more than 1.5 million different vehicle models are considered.

\subsection{Engine Model}

Autonomie uses different engine models to support specific technologies (cylinder deactivation, turbocharging, etc.). For this study, five different engine models are used:

- Gasoline engine model

- Diesel engine model

- Gasoline turbocharged engine model

- Cylinder deactivation engine model

- Gasoline turbocharged with cylinder deactivation engine model

This section describes the baseline Autonomie engine model.

\subsubsection{Model Description}

The engine model simulates engine torque production, fuel consumption, etc. In this model, the fuel rate is expressed as a function of the engine's brake torque and speed.

The engine model is divided into three blocks: engine torque calculation, engine thermal calculation, and engine fuel rate calculation. Figure 6 shows the Autonomie spark-ignition engine model diagram, with these blocks outlined in red. 


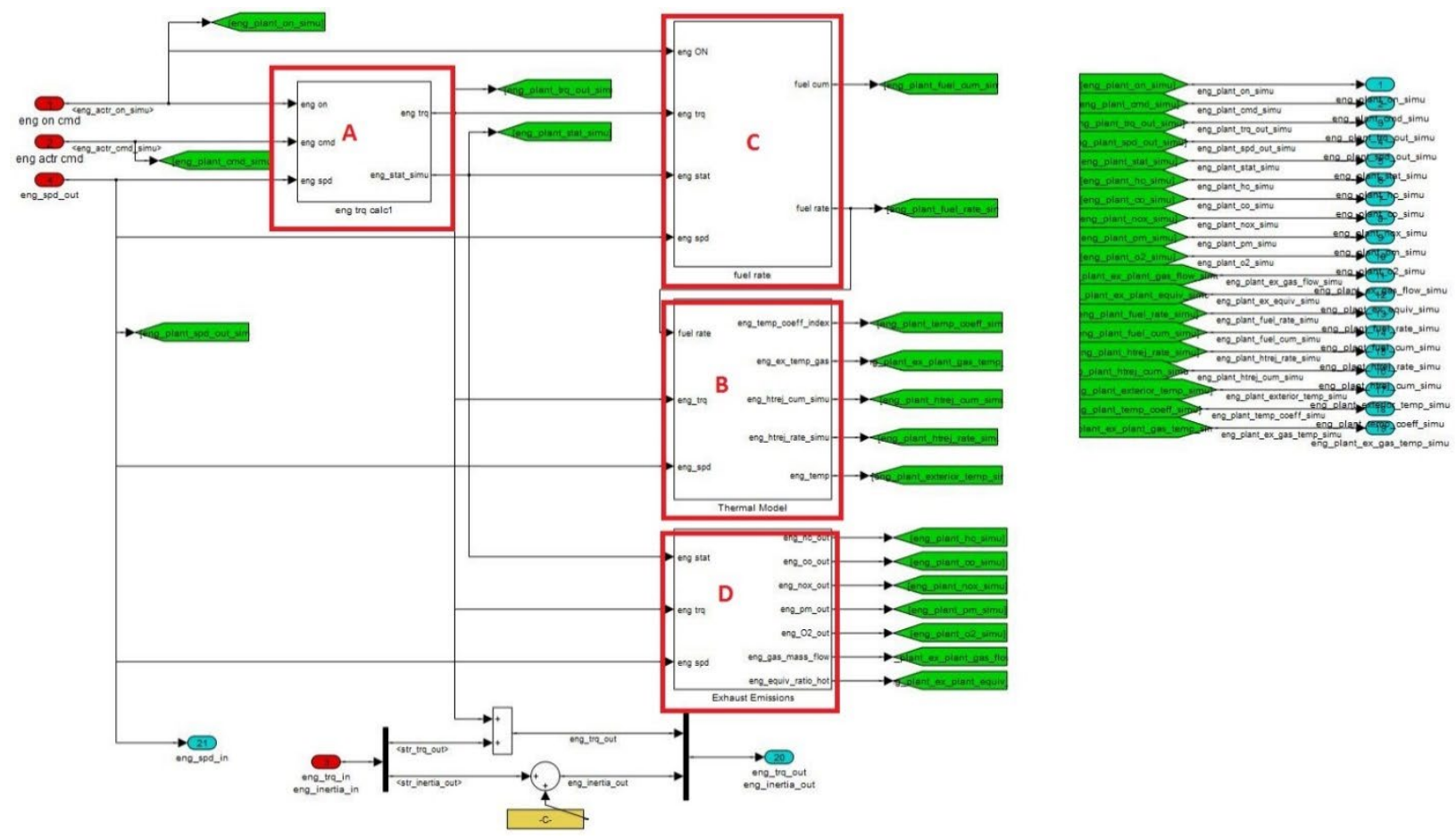

Figure 6. Autonomie spark-ignition engine model diagram.

Block A (Figure 7) calculates the engine torque by interpolating between the maximum and minimum torque curves, using the engine command from the controller. 


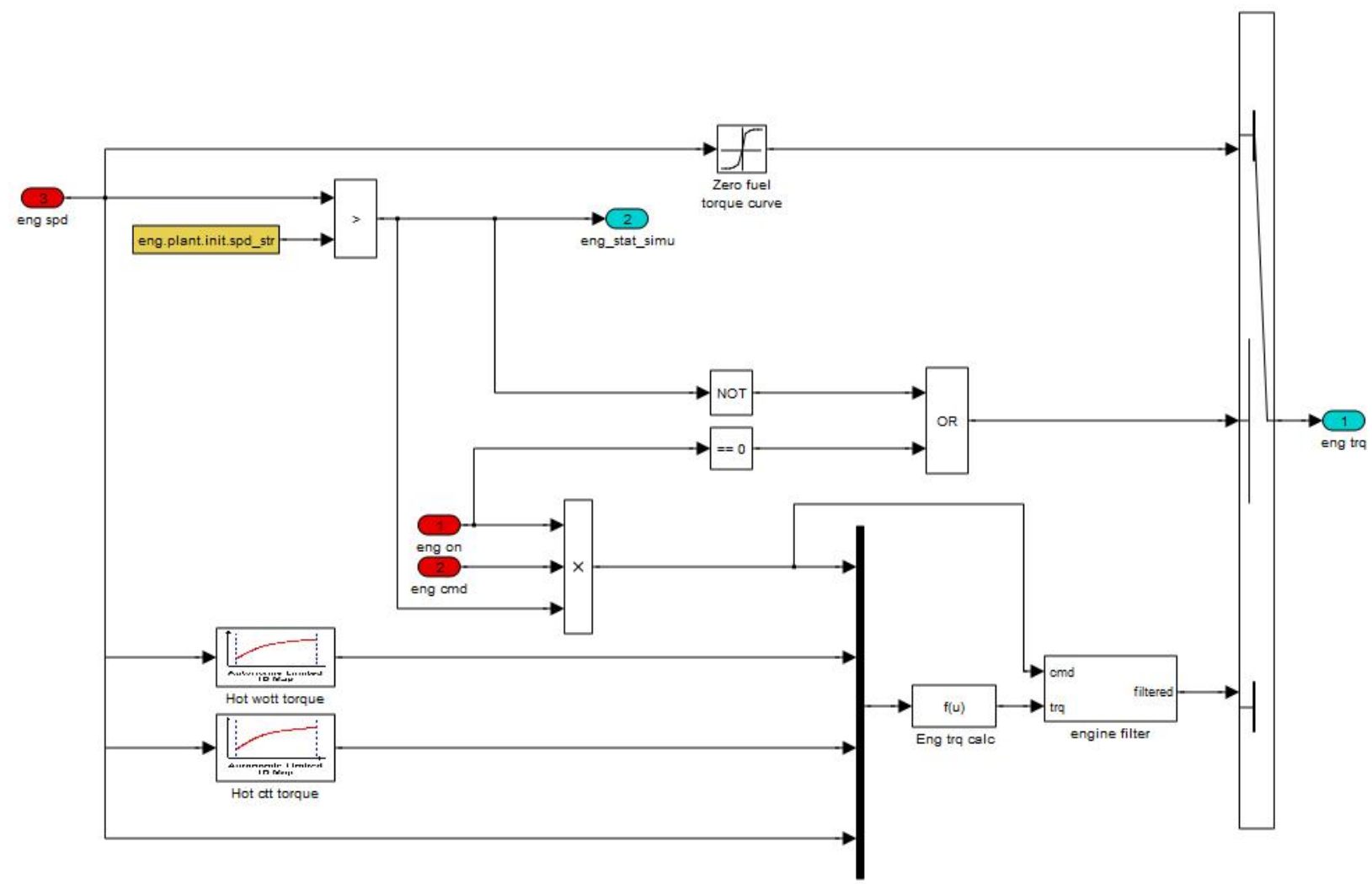

Figure 7. Engine model Block A: Engine torque calculation

If $\mathrm{T}_{\text {cmd }}>0$ and $\omega_{\text {eng }}>0$ or $\mathrm{T}_{\text {cmd }}$ equals 0 and $\omega_{\text {eng }}>0$, then

(1) $T_{\text {out }}=\left(1-T_{c m d}\right) \times T_{C T T}+\left(T_{c m d} \times T_{W O T}\right)$

Otherwise, if $\mathrm{T}_{\mathrm{cmd}}$ equals 0 and $\omega_{\text {eng }}$ equals 0 , then

(1a) $T_{\text {out }}=0$

(2) $T_{\text {cmd }}=P W M_{-}$cmd $\times E n g_{-} O N \times\left(W_{\text {eng }}>W_{\text {starting }}\right)$

Where

$\mathrm{T}_{C T T}=$ minimum torque curve of the engine as a function of speed (closed-throttle torque curve).

$\mathrm{T}_{W O T}=$ maximum torque curve of the engine as a function of speed (wide-open-throttle torque curve).

PWM_cmd = engine command from controller, not modified by any other signals.

$\mathrm{W}_{\text {starting }}=$ engine starting speed threshold (if the engine speed is above this value, combustion is assumed to be stable, and the engine is considered to consuming fuel and capable of producing torque).

As this engine model is only for hot operation, the engine temperature, exhaust gas temperature, and warm-up coefficient are all set to constant values, as shown in Block B (Figure 8). The engine cold-start penalties are taken into account after the simulations using adjustment factors developed from EPA test data. 


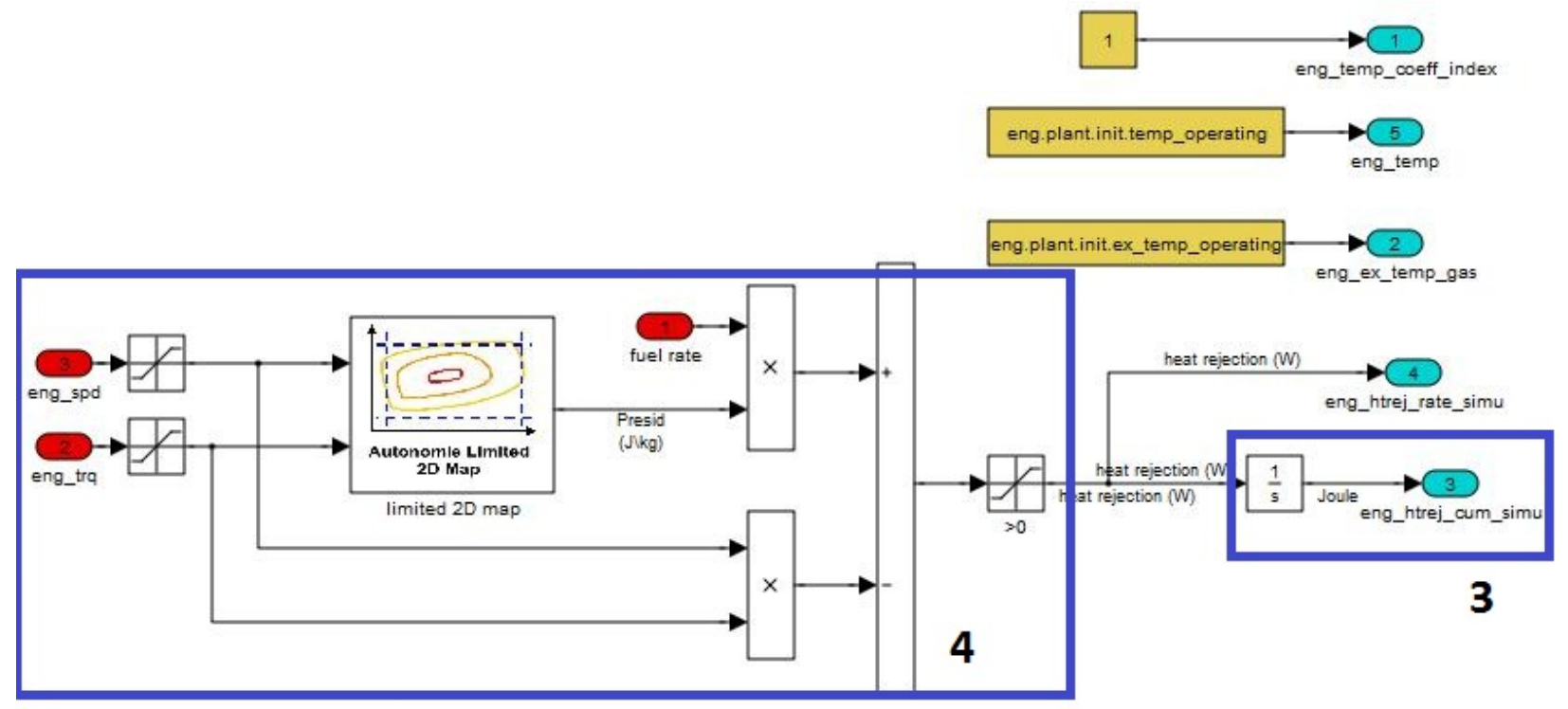

Figure 8. Engine model, Block B: Thermal model

(3) Instantaneous_Heat_Rejection $=($ Fuel_rate $\times$ Heat_rejected_per_unit_mass $)-$ $\left(W_{\text {eng }} \times T_{\text {eng }}\right)$

(4) Cumulative_heat_rejection $=\int$ Instantaneous_heat_rejection $\times d t$

Where

Fuel_rate $=$ mass flow rate of the engine fuel

Heat_rejected_per_unit_mass $=$ total energy released from the fuel by combustion per unit mass of fuel

Block C (Figure 9) calculates the fuel rate. Block $\mathrm{C} 1$ calculates the instantaneous fuel rate by using Equations 5 and 6. 


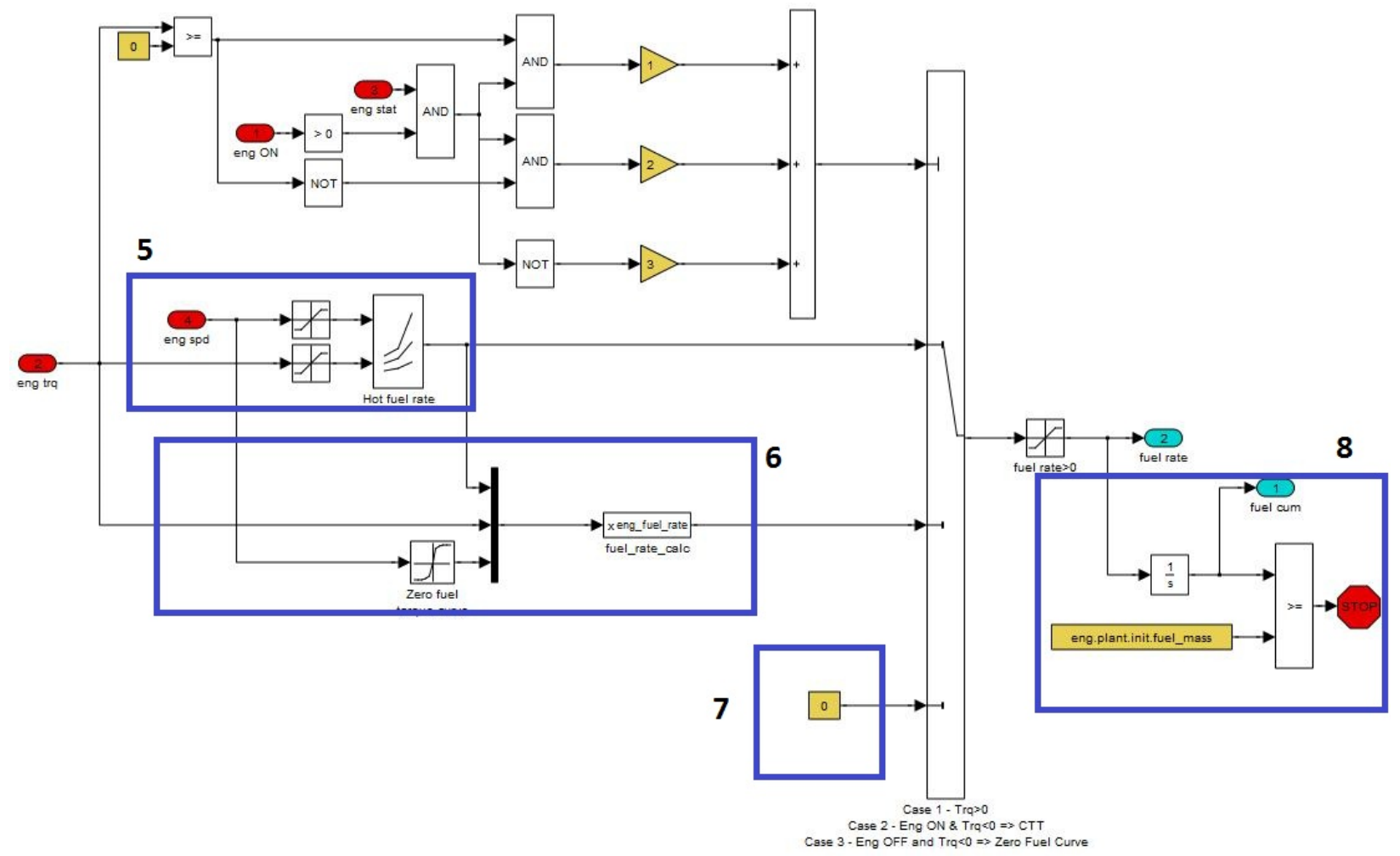

Figure 9. Engine model, Block C: Fuel rate model

Equation 6 interpolates the fuel rate when the engine is on and the torque delivered by the engine is below the torque boundary of the fuel rate map. Since no data are usually available in this region, the fuel rate is interpolated. When $\mathrm{T}_{\text {eng }}=\mathrm{T}_{\text {min_map }}$, the fuel rate at $\mathrm{T}_{\text {min_map }}$ is requested. When $\mathrm{T}_{\text {eng }}=\mathrm{T}_{C T T}$, the fuel rate is zero. The fuel rate is proportional to the engine torque fraction. Equation 7 ensures that no fuel is consumed when the engine is below its starting speed; the engine speed must be increased to starting speed by the starter motor before fuel can be injected. Note that the starting speed is lower than the engine idle speed.

If the engine is started and $\mathrm{T}_{\text {eng }}>\mathrm{T}_{\text {min_map }}$

(5) Instantaneous_fuel_rate $=$ function $\left(W_{\text {eng }}, T_{\text {eng }}\right)$

If the engine is started and $\mathrm{T}_{\text {eng }}<\mathrm{T}_{\text {min_map }}$

(6) Instantaneous_fuel_rate $=\frac{\text { Fuel }_{\text {rate }} \times\left(T_{\text {eng }}-T_{C T T}\right) \times\left(T_{\text {eng }} \times T_{C T T}\right)}{\text { Rmin_map }_{-} T_{C T T}}$

$\mathrm{T}_{\text {min_map }}=$ minimum torque index (minimum value in the vector eng.init.trq fuel_hot_index).

This is the torque boundary for the fuel rate map. Below this value, unless data are available the map must be interpolated.

Otherwise,

(7) Instantaneous_fuel_rate $=0$ 
Equation 8 calculates the total mass of fuel that went into the engine:

(8) Cumulative_fuel_rate $=\int$ Instantaneous_fuel_rate $\times d t$

\subsubsection{Engine Technologies Evaluated}

This section provides detail on the different engine technologies modeled in the gasoline and diesel engines.

\subsubsection{1. $\quad$ Friction Reduction}

Friction reduction has been shown to offer significant improvements in vehicle fuel consumption. Therefore, to evaluate the potential of friction reduction, engines can potentially be subjected to two levels of reduction in friction mean effective pressure (FMEP):

- A reduction in FMEP by 0.1 bar across the entire engine speed range.

- An extreme friction reduction (25\% FMEP) across the entire speed range.

For the current study, only the first level of friction reduction has been considered. A predictive FMEP equation was calibrated from test data to allow for a smooth and systemic friction study, but it may under-predict FMEP at high loads with late combustion phasing.

\subsubsection{2. $\quad$ Turbocharged Engines}

In addition to the naturally aspirated engines, turbo engines were also mapped using GT-POWER, a commercially available engine simulation tool with detailed cylinder modeling and combustion analysis. With turbo engines, there is a "lag" in torque delivery because of the operation of the turbo charger. This lag impacts vehicle performance and vehicle shifting on aggressive cycles. Turbo lag has been modeled in Autonomie for turbo systems based on principles of a first order delay, where the turbo lag kicks in after the naturally aspirated torque limit of the turbo engines has been reached.

Figure 10 shows the brake mean effective pressure (BMEP) response of the turbo engine model to a step command.

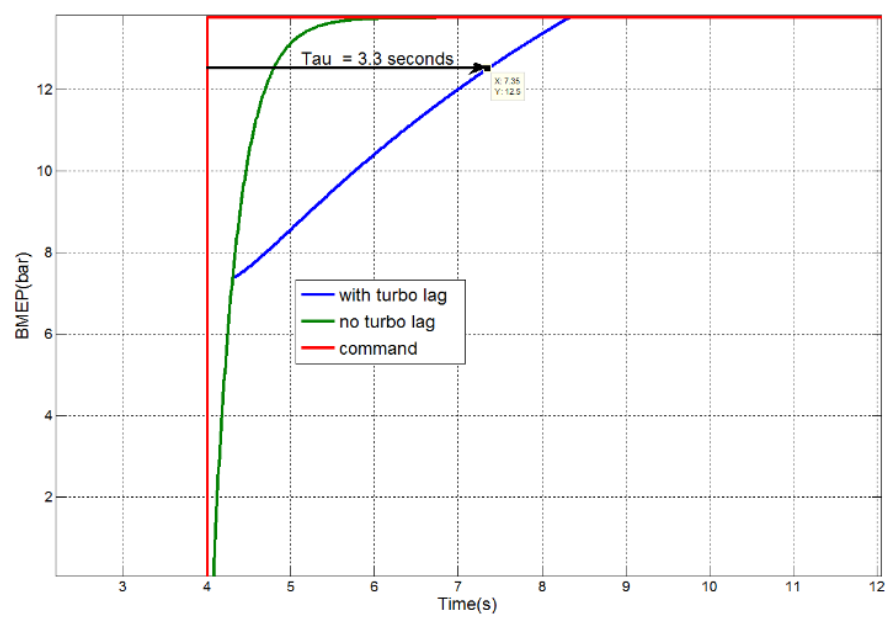

Figure 10. BMEP response of turbo-charged engine to a step command 
The turbo response varies with engine speed; i.e., at higher speeds, the turbo response is faster because of higher exhaust flow rates. It should be noted that the baseline engine maps for the naturally aspirated and the turbo engines were validated with test data. Maximum torque line on boosted engines is adjustable on the basis of boost pressure.

\subsubsection{Cylinder Deactivation and Advanced Cylinder Deactivation}

Autonomie also uses a specific engine model for cylinder deactivation, as this model uses an advanced fuel calculation subsystem with different maps. Due to noise, vibration and harness (NVH) considerations in production vehicles, cylinder deactivation operation is not performed during several vehicle operation modes, including vehicle warm-up, lower-gear operation, idle, and low engine speed. As a result, cylinder deactivation is disabled under the following conditions:

- If the engine idles or its speed is below 1000 RPM or above 3000 RPM

- If the vehicle is in the first or second gear

- If the engine load is above half the maximum BMEP of the engine (and a certain hysteresis is maintained to prevent constant activation and deactivation)

As noted above, cylinder deactivation is not typically performed during the vehicle warm-up phase, i.e., for a cold start. Since all the simulations considered in this study assume a "hot start," where the engine coolant temperature is steady at around $95^{\circ} \mathrm{C}$, the cold start condition was not considered for the simulations. In addition, changes in the transmission shifting calibration (like lugging speed limits) and additional torque converter slippage during cylinder deactivation have not been considered.

\subsubsection{Engine Cylinder Deactivation Methodology}

The cylinder deactivation state is implemented in a Stateflow ${ }^{\circledR}$ diagram as shown in Figure 11.

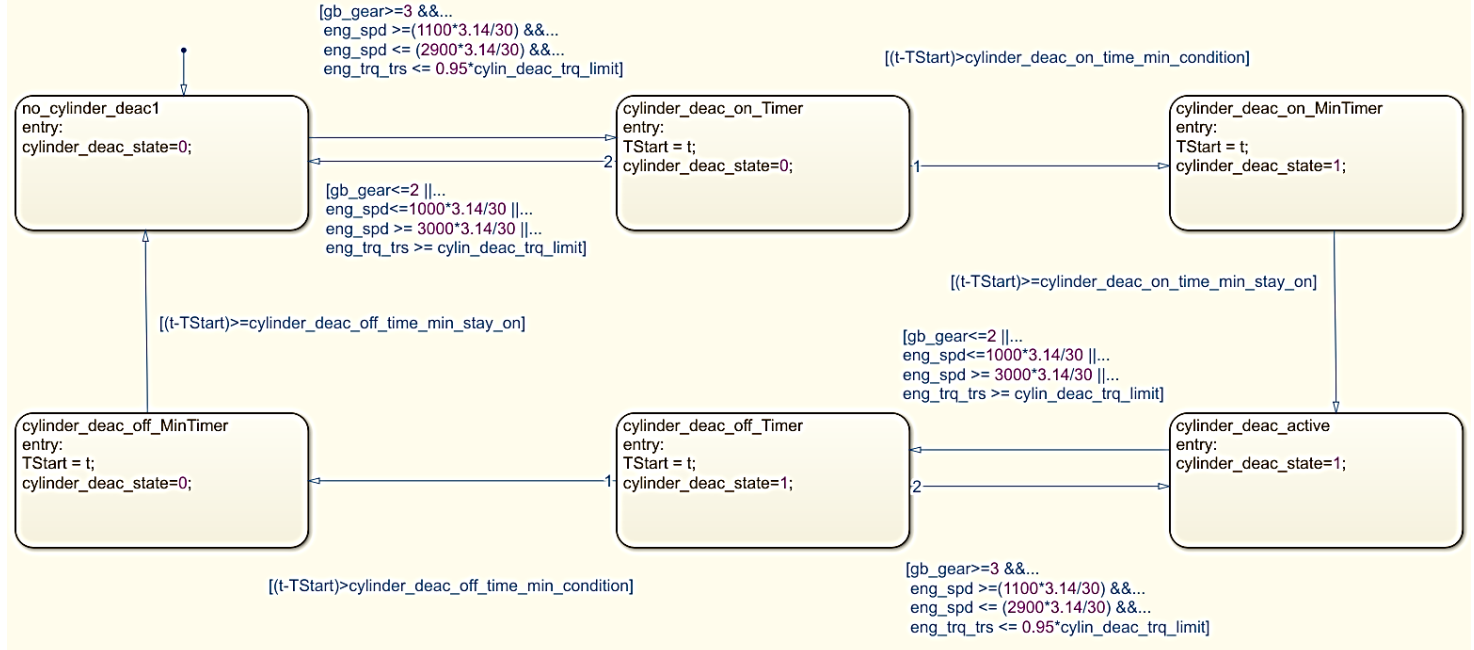

Figure 11. Stateflow diagram for cylinder deactivation regulator

In the engine plant model, the fuel rate maps for both cylinder deactivation and no cylinder deactivation are used by the Stateflow logic. Figure 12 shows the engine plant model with cylinder 
deactivation and shows how both fuel maps (with and without cylinder deactivation) are used by the Stateflow logic in the engine plant model.

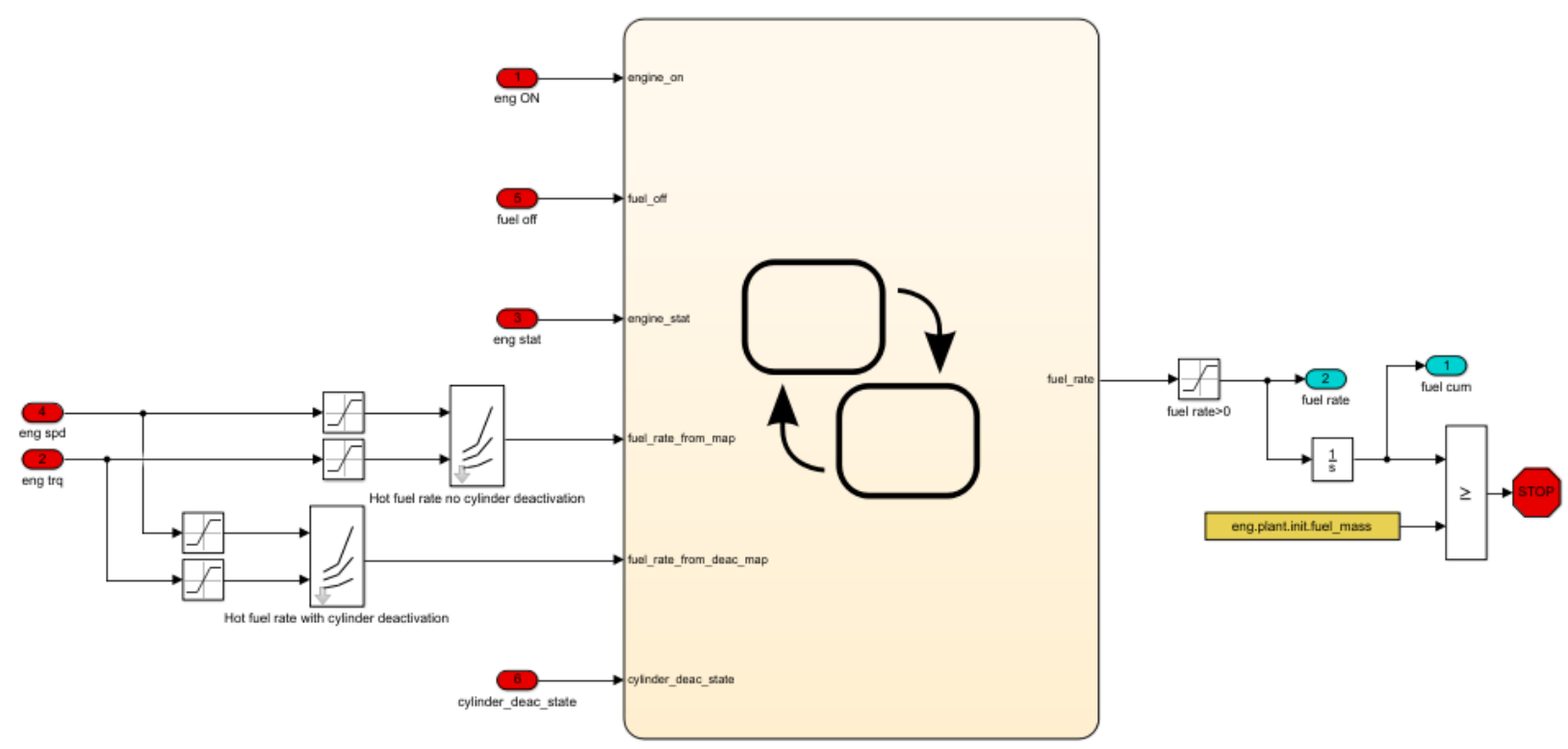

Figure 12. Engine plant model with cylinder deactivation

Figure 13 shows the Stateflow control that switches the fuel maps for the cylinder deactivation and no cylinder deactivation conditions on the basis of the cylinder deactivation signal.

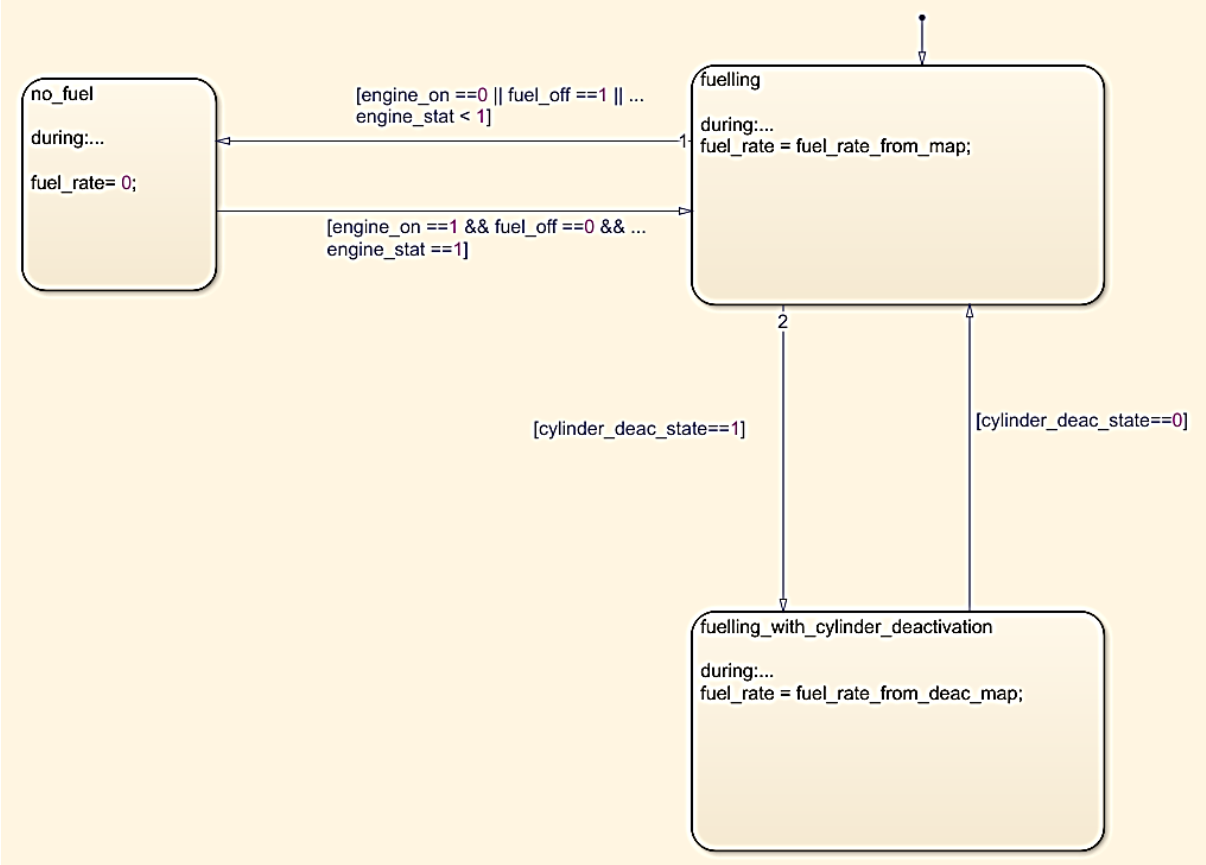

Figure 13. Stateflow diagram showing the ability to switch cylinder deactivation fuel maps 
As shown in Figure 13, a state diagram is used to switch between the two different engine maps (state fueling vs. fueling with cylinder deactivation) based on whether or not the cylinder deactivation state is active (cylinder_deac_state).

\subsubsection{Fuel Cutoff}

Autonomie's fuel cutoff model uses a specific torque calculation to calculate the torque loss when fuel is cut off during deceleration events. In general, engine models in Autonomie are of two types: throttled engines and unthrottled engines. As shown in Figure 14 and Figure 15, both types of models provide motoring torque when fuel is cut to the engine (e.g., when fuel is cut off during deceleration). With throttled engines, the motoring torque is a function of throttle position. Figure 14 shows the engine operating regions for throttled engines, and Figure 15 shows the engine operating regions for unthrottled engines.

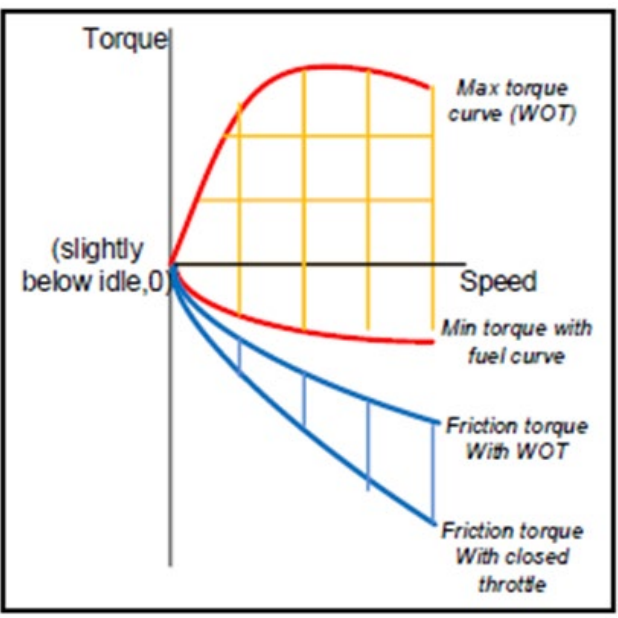

Figure 14. Engine operating regions for throttled engines (WOT = wide-open throttle)

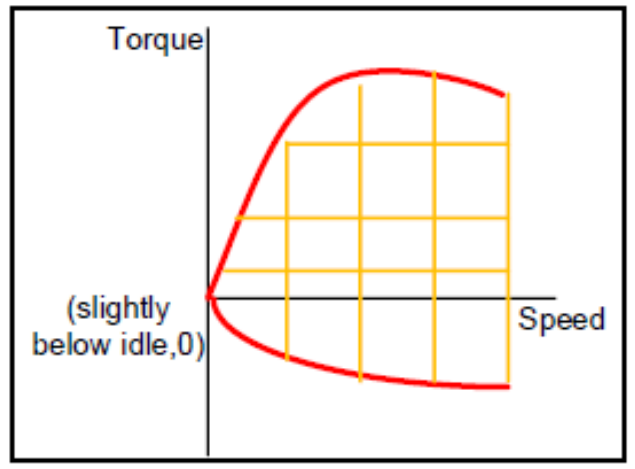

Figure 15. Engine operating region for unthrottled engines 
Engine fuel cutoff control algorithms used in the study have been developed on the basis of vehicle test data collected at AMTL. ${ }^{\dagger}$ The fuel cutoff controller is implemented for gasoline and diesel engines through analysis, as shown in Figure 16. In Autonomie, engine control and plant blocks are organized for idle fuel rate and fuel-off conditions. Engine fuel is cut off under the following conditions:

- Vehicle is actively braking for a certain minimum time.

- Engine speed is above a minimum threshold (e.g., 1000 RPM).
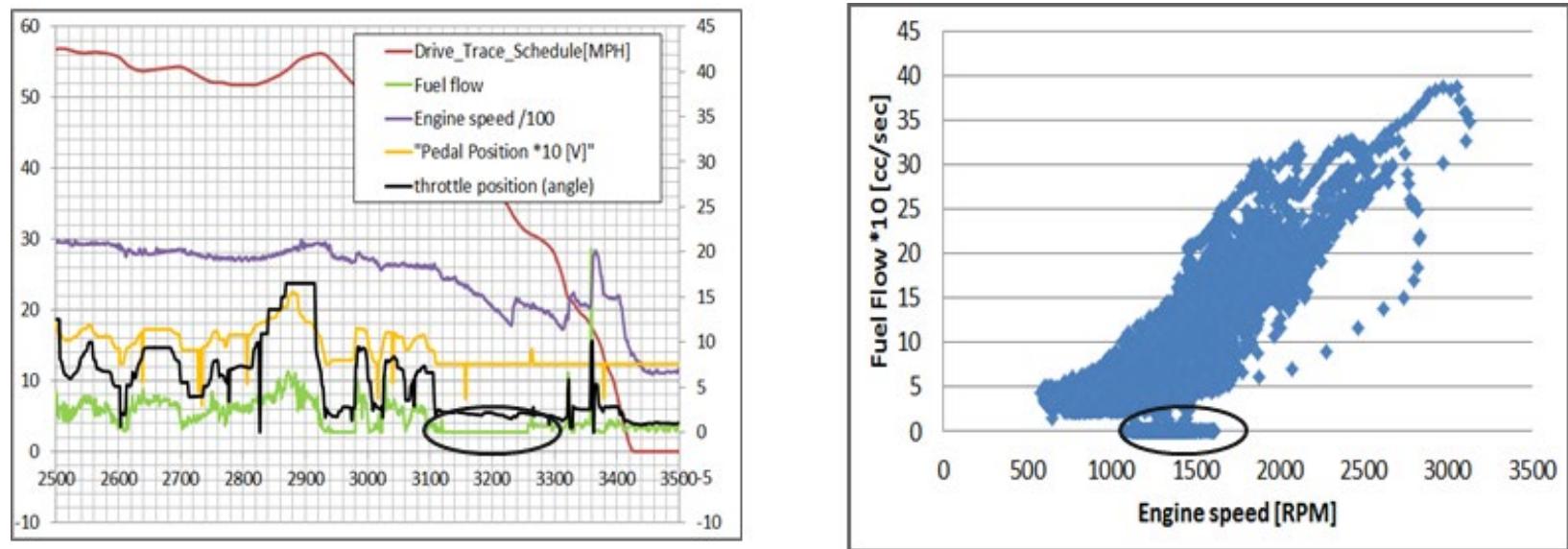

Figure 16. Engine fuel cutoff analysis based on test data

\subsection{Transmission Model}

The drivetrain is assumed to be rigidly attached to the wheels. Since the wheel speed and acceleration are calculated in the wheel model and propagated backward throughout the rest of the drivetrain model, the gearbox unit is modeled as a sequence of mechanical torque gains. The torque and speed are multiplied and divided, respectively, by the current ratio for the selected gear. Torque losses corresponding to the torque/speed operating point are subtracted from the torque input. Torque losses are defined on the basis of a three-dimensional efficiency lookup table (input shaft rotational speed, input shaft torque, and gear number). When a gear is selected, the input inertia is fed forward to the next component after being reflected to the output shaft using the square of the gear ratio. When the neutral gear is engaged, the input gearbox rotational speed is calculated on the basis of the input shaft inertia.

\subsubsection{Automatic Transmission}

For automatic transmissions, gear shifting occurs without having to pass through neutral and without a complete torque interruption at its output. The torque converter model is separate from the automatic gearbox model. Figure 17 shows the I/O of the automatic-transmission model.

$\dagger 2017$ Ford F150, 2016 Mazda CX9, 2014 Chevrolet Cruze Diesel, 2014 Mazda 3 iEloop, 2013 Dodge Ram 1500 HFE, 2013 Hyundai Sonata, 2013 Nissan Altima, 2013 Volkswagen Jetta TDI, 2012 Chrysler 300, 2012 Fiat 500 Sport, 2012 Ford F150 Ecoboost, 2012 Ford Focus, 2012 Ford Fusion V6, 2009 Volkswagen Jetta TDI. https://www.anl.gov/es/conventional-vehicle-testing 


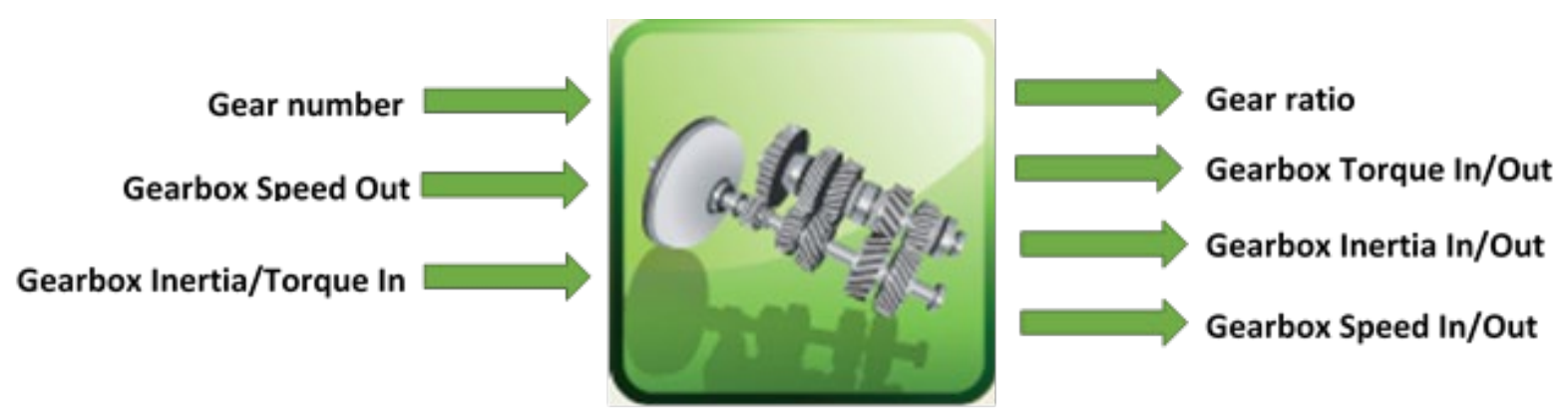

Figure 17. Autonomie automatic gearbox model I/O

The top-level diagram of the automatic gearbox is composed of three main subsystems: speed calculation (Block A); torque calculation (Block B); and inertia calculation (Block C). Figure 18 shows the top-level diagram of the automatic gearbox model.
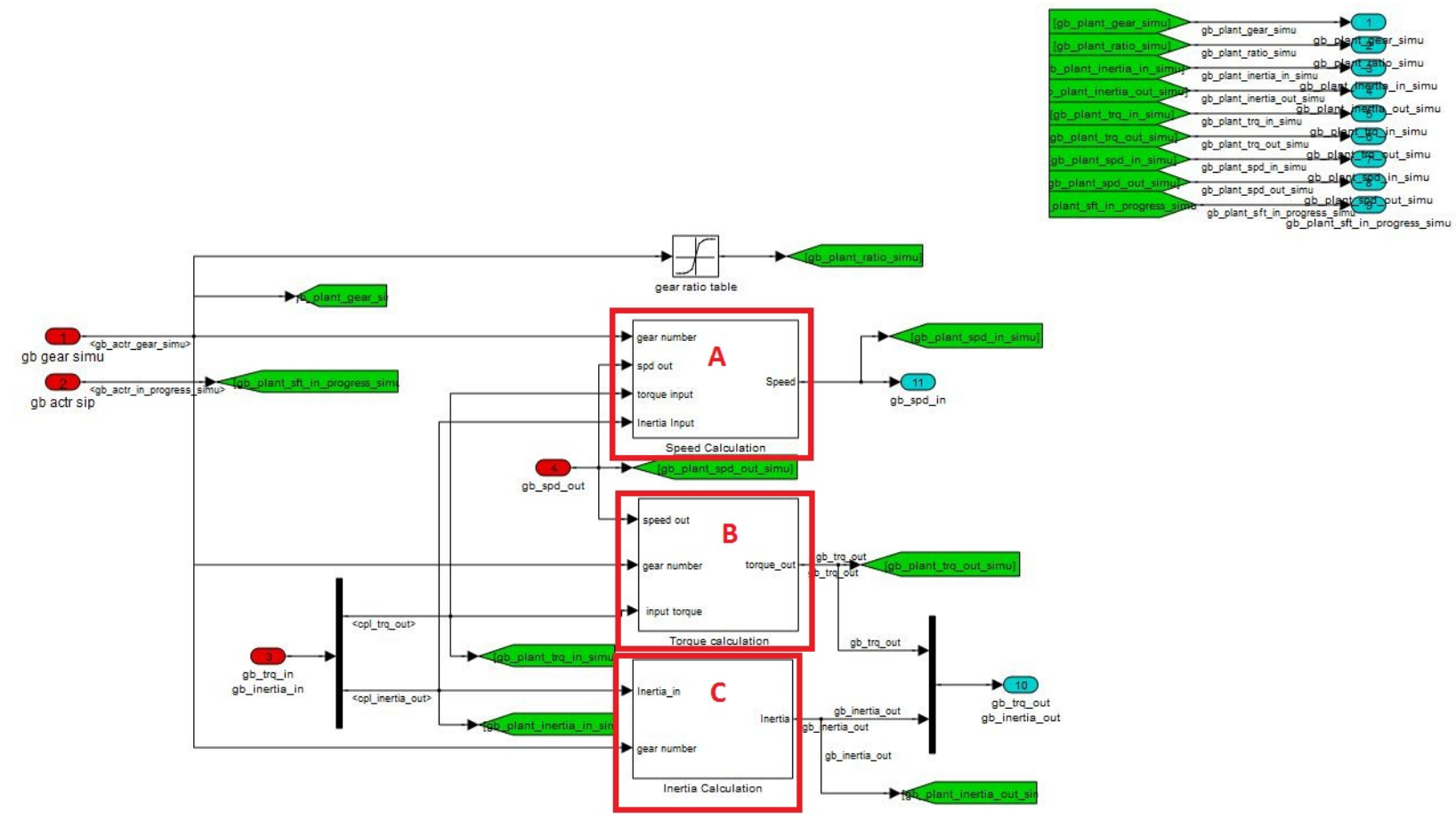

Figure 18. Top level of the automatic gearbox model

Block A (Figure 19) calculates the gearbox shaft input speed based on the output speed, gear ratio, and whether the gearbox is in neutral. If the gearbox is in first gear, Equation 9 is used to calculate the input speed of the gearbox.

(9) $W_{\text {in }}=K_{\text {Ratio }} \times W_{\text {out }}$ 


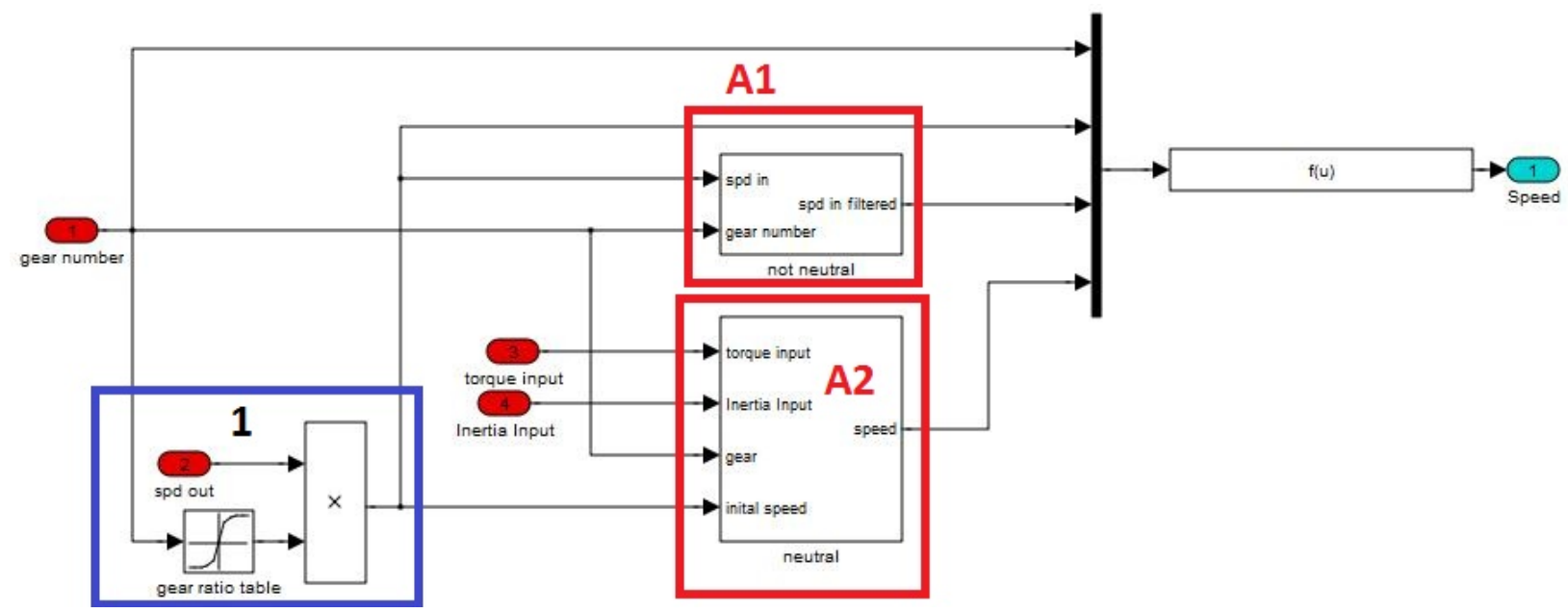

Figure 19. Block A: Speed calculation of the automatic transmission

In between gear choices during an automatic transmission shift, the output shaft speed lags the input shaft speed as one set of wet clutches opens and another closes, allowing for a smooth gear shift and no interruption in torque output. This speed characteristic of the automatic gearbox is modeled in Block A1 using a low-pass filter. However, this low-pass filter cannot be used during a shift from neutral into first gear, a shift that occurs during the performance test for a limited subset of hybrid configurations that are in neutral when the vehicle is stopped instead of being in first gear as a stopped conventional vehicle is. The low-pass filter interferes with the performance test, slowing the vehicle down and leading to exaggerated 0-to-60 $\mathrm{mph}$ times. To address this issue, the filter is not used during performance to ensure that when the vehicle is shifting from neutral into first, the input speed is proportional to the output speed.

Block A1 (Figure 20) calculates the input speed when the gearbox is not in neutral. Any gear changes will result in the input speed lagging the output speed. Equation 10 implements the phase difference between input and output speeds.

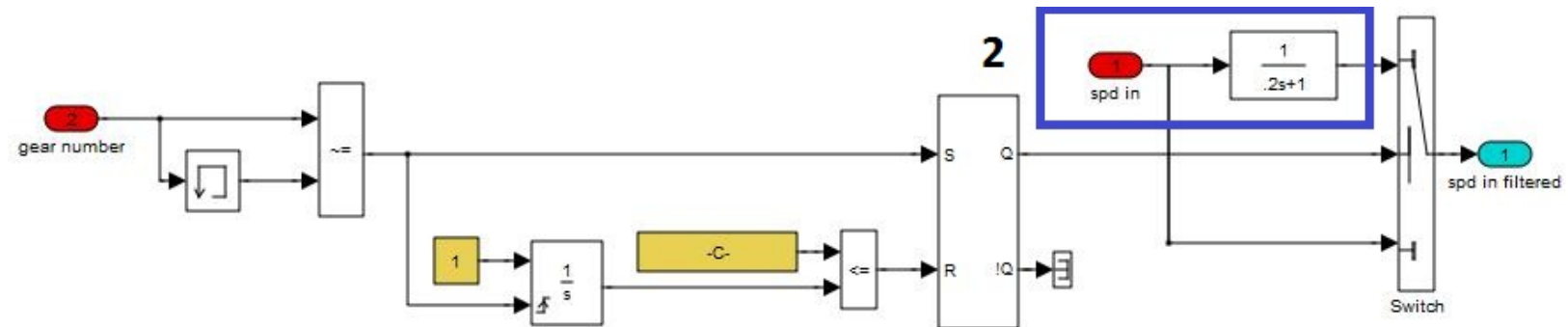

Figure 20. Block A1: Speed calculation when the gearbox is not in neutral

Equation 10 shows the calculation of the input speed when the gear number is higher than first.

$$
W_{\text {in }}=\frac{1}{1+\tau S} \times\left(K_{\text {Ratio }} \times W_{\text {out }}\right)
$$

Block A2 (Figure 21) calculates the input speed when the gearbox is in neutral. 


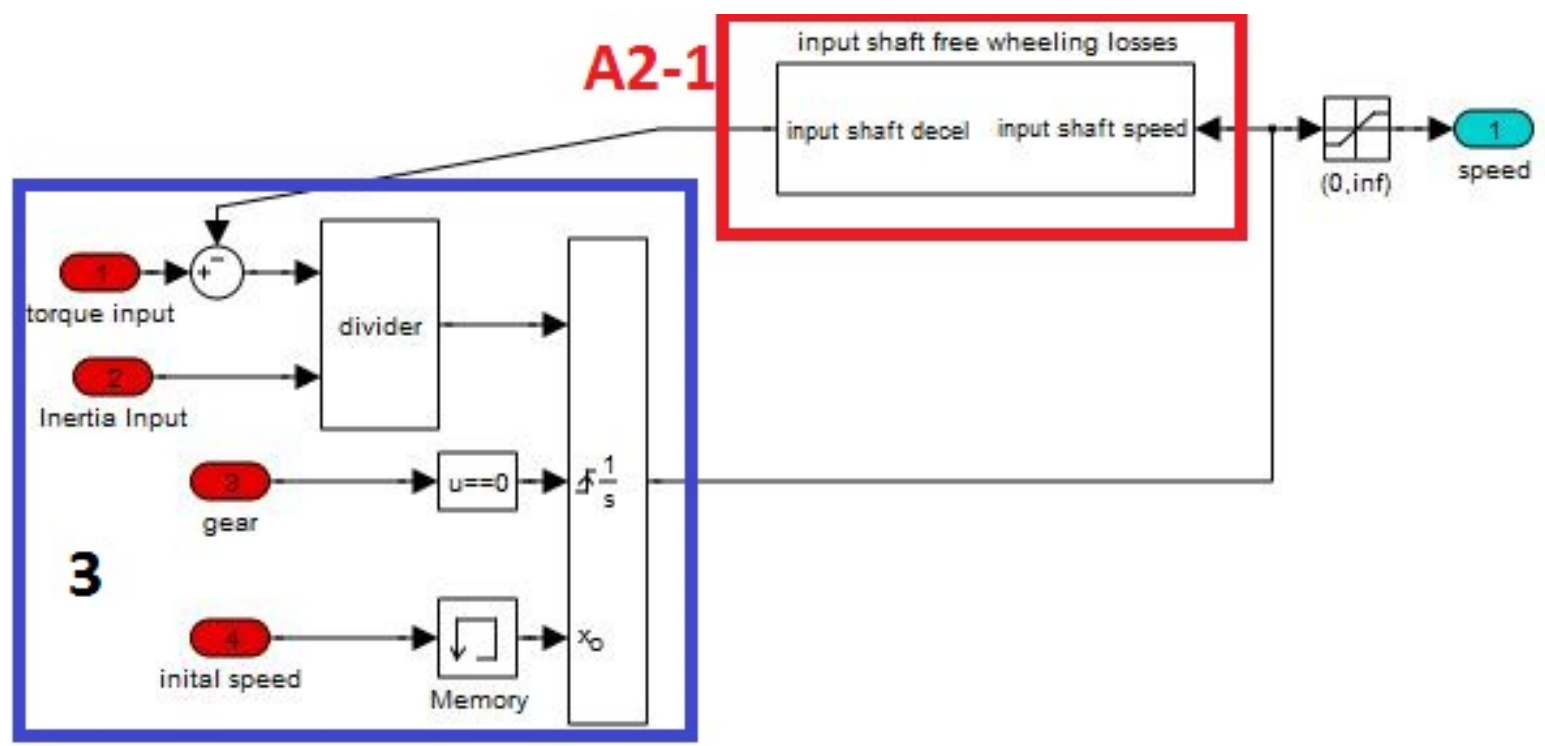

Figure 21. Block A2: Speed calculation when the gearbox is in neutral

When the gearbox is in neutral, the input shaft speed is calculated by using the first-order differential equation shown (Equation 11). If the input torque goes to 0 , the shaft will continue to spin until the drag torque brings it to rest.

$$
W_{\text {in }}=\int \frac{T_{\text {in }}-T_{\text {loss }}}{J_{\text {in }}}
$$

Block A2-1 (Figure 22) calculates the torque loss of the free-wheeling input shaft. The torque loss is proportional to the shaft speed, as illustrated by Equation 12 .

$$
T_{\text {loss }}=K_{\text {coeff }} \times W_{\text {in }}
$$

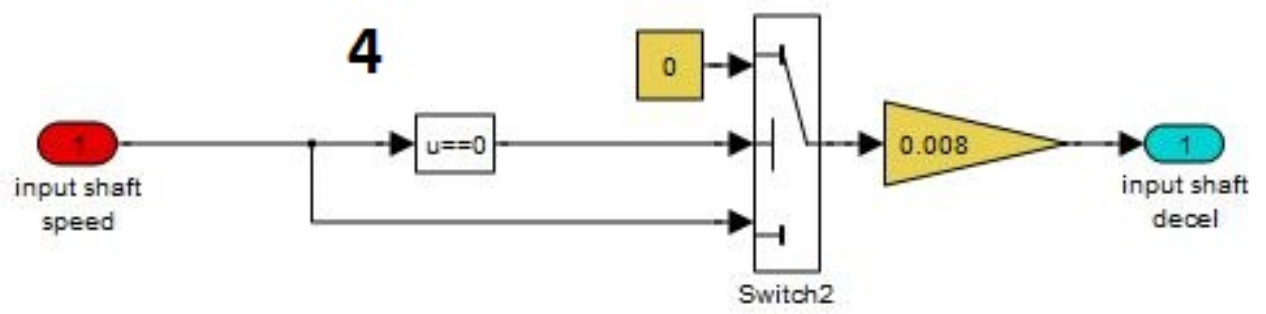

Figure 22. Block A2-1: When the gearbox is not in neutral, with free-wheeling input shaft losses

Block B (Figure 23) calculates the gearbox output torque using the gearbox ratio and torque loss. 


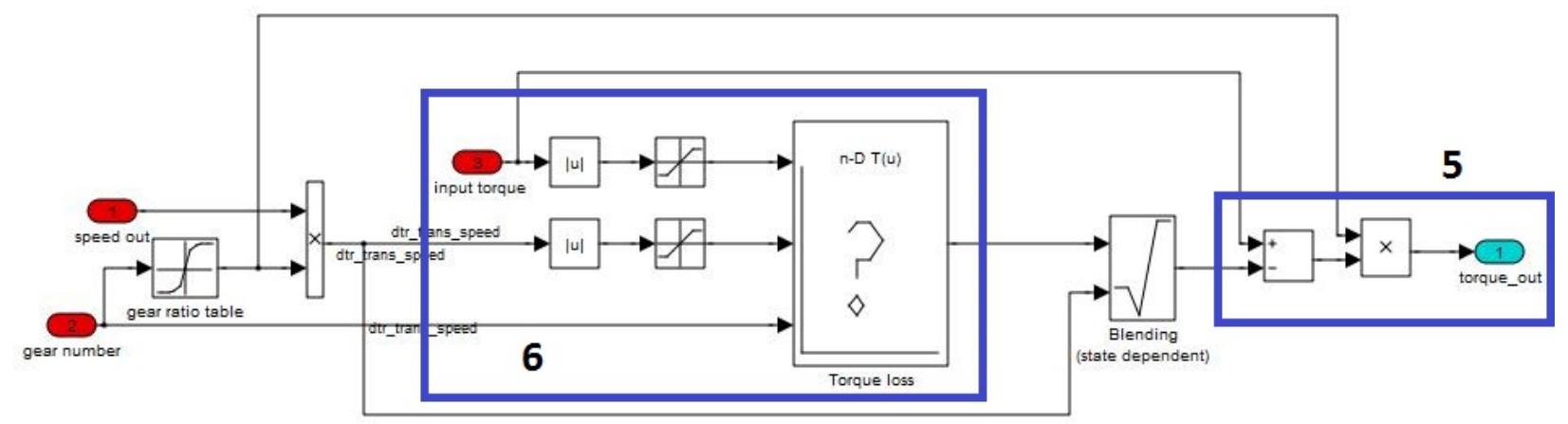

Figure 23. Block B: Output torque calculation

Equation 13 shows the output torque calculation based on input torque minus torque loss.

$$
T_{\text {out }}=T_{\text {in }}-T_{\text {loss }}
$$

Torque loss is a function of the gearbox input torque, speed, and ratio.

$$
T_{\text {loss }}=f\left(T_{\text {in }}, W_{\text {in }}, K_{\text {Ratio }}\right)
$$

When the gearbox is in neutral, the torque output is forced to 0 .

$$
T_{\text {loss }}=0
$$


Block C (Figure 24) gives the output inertia of the gearbox.

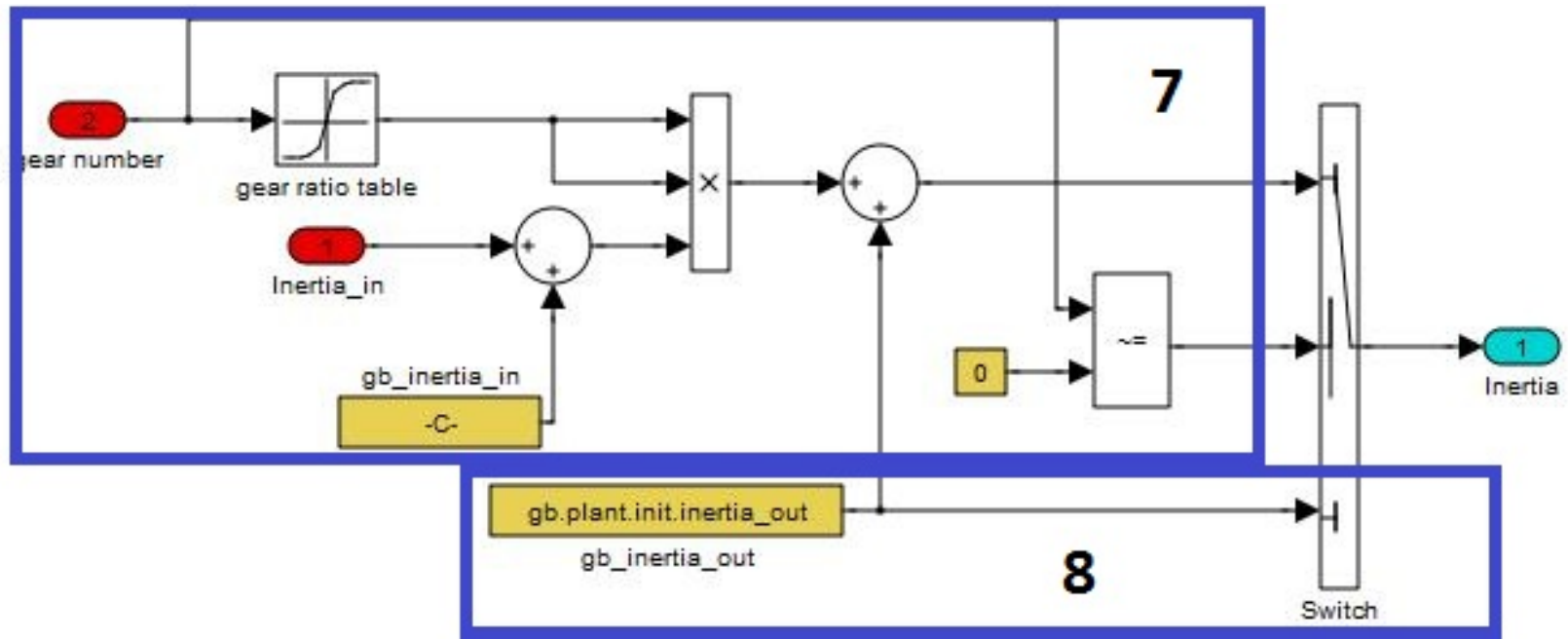

Figure 24. Block C: Output inertia calculation

The output inertia equals the upstream inertia reflected to the output shaft of the gearbox through the gear ratio plus the output shaft inertia of the gearbox.

$$
J_{\text {out }}=\left(J_{\text {in }}+J_{\text {input_shaft_tx }}\right) \times K_{\text {ratio }}^{2}+J_{\text {output_shaft_t }}
$$

When the gearbox is in neutral, Equation 17 demonstrates that the output inertia of the gearbox is the inertia of the output shaft.

$$
J_{\text {out }}=J_{\text {output_shaft_tx }}
$$

\subsubsection{Manual Transmission}

Figure 25 shows the main I/O of the manual transmission model.

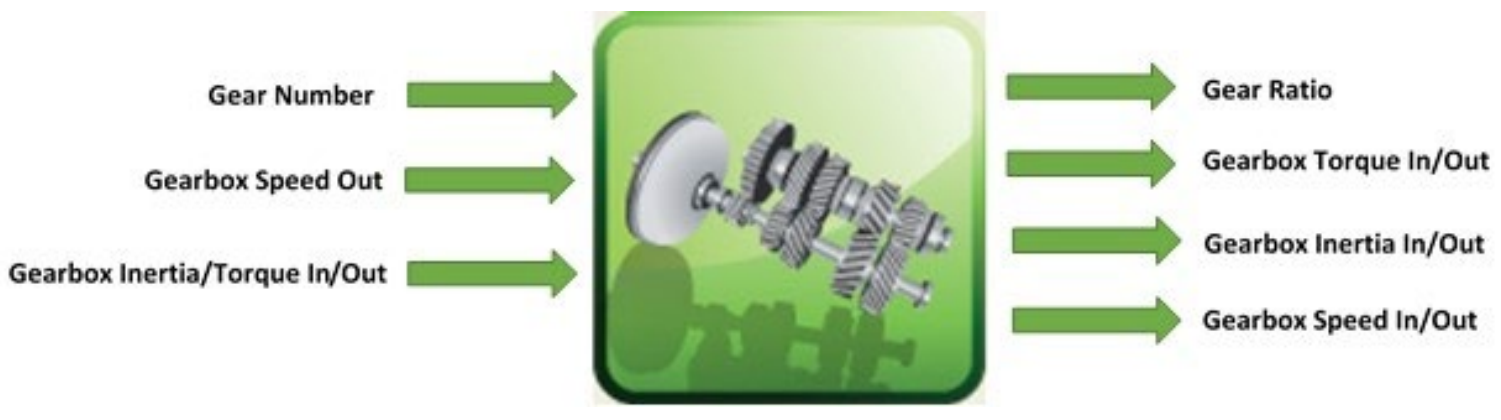

Figure 25. Autonomie manual transmission model $1 / O$

The top-level diagram of the manual gearbox is composed of three main subsystems: speed calculation (Block A), torque calculation (Block B), and inertia calculation (Block C). Figure 26 shows the top-level diagram of the manual gearbox model. 


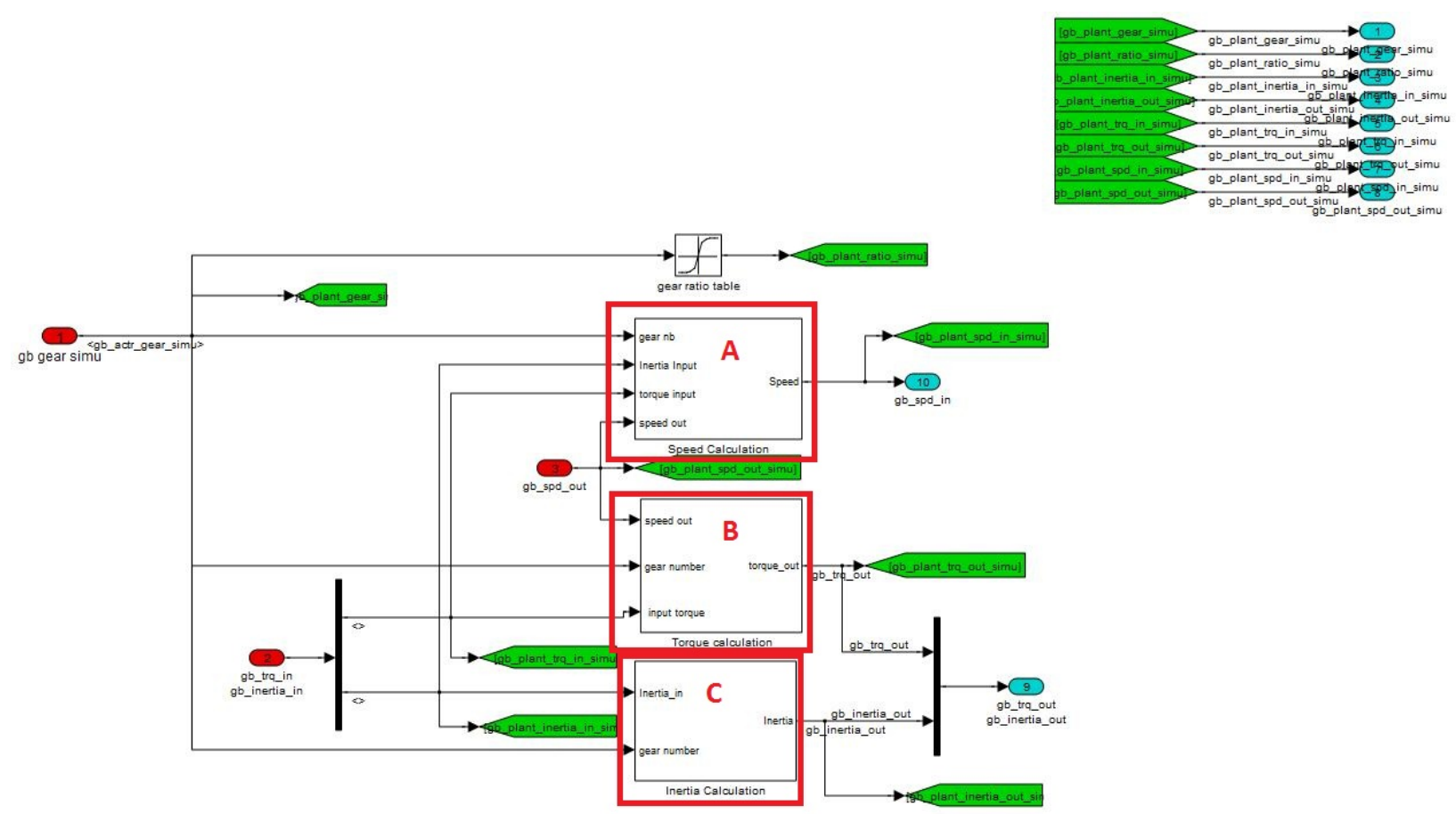

Figure 26. Top level of the manual gearbox model

Block A (Figure 27) calculates the input shaft speed of the gearbox on the basis of the current output shaft speed, the current gear ratio, and whether the gearbox is in neutral.

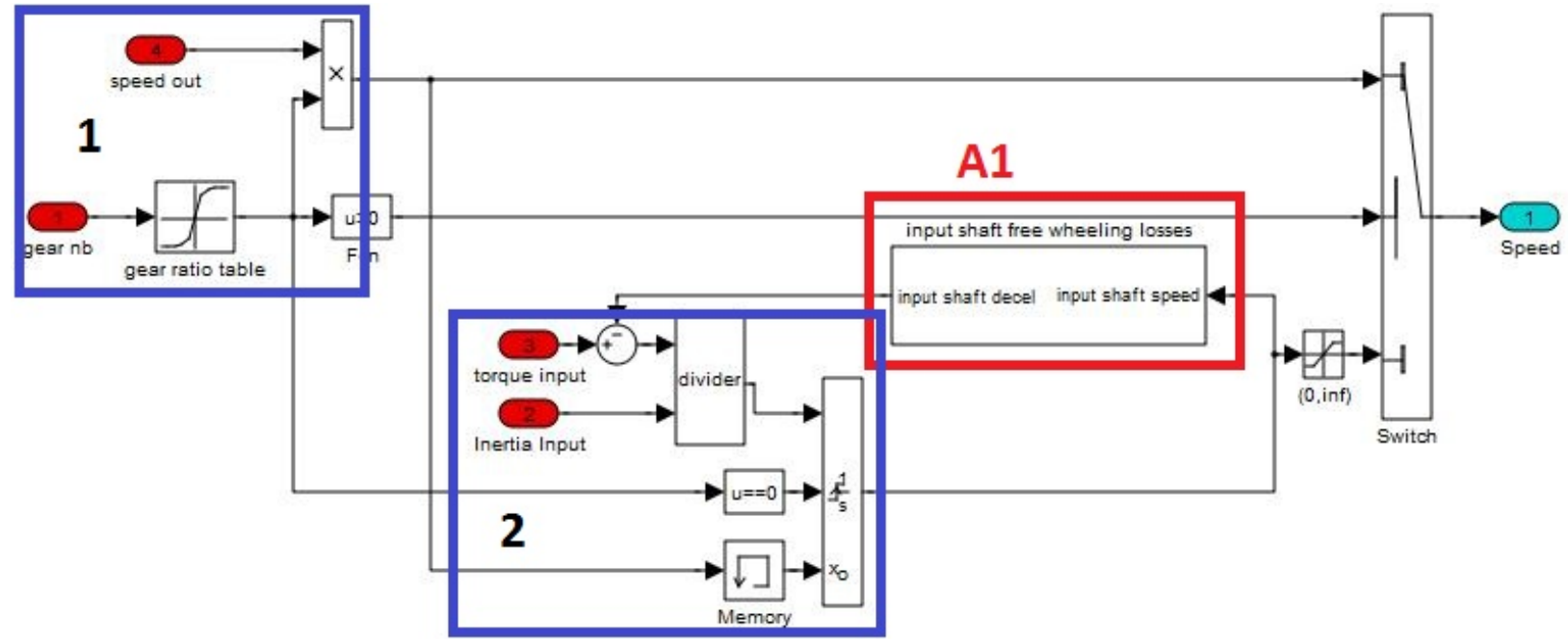

Figure 27. Block A: Gearbox input shaft speed calculation

If the gearbox is in gear, the input shaft speed is proportional to the output shaft speed. If the gearbox is in neutral, the input shaft is free to spin. The spinning shaft produces drag losses that are proportional to its speed. Equation 18 shows the speed calculation when the gearbox is in gear.

$$
W_{\text {in }}=K_{\text {Ratio }} \times W_{\text {out }}
$$


When the gearbox is in neutral, the input shaft speed is calculated by using the first-order differential equation shown below (Equation 19). If the input torque goes to 0 , the shaft will continue to spin until the drag torque brings it to rest.

$$
W_{\text {in }}=\int \frac{T_{\text {in }}-T_{\text {loss }}}{J_{\text {in }}}
$$

Block A1 (Figure 28) shows the torque loss calculation when the gearbox is in neutral.

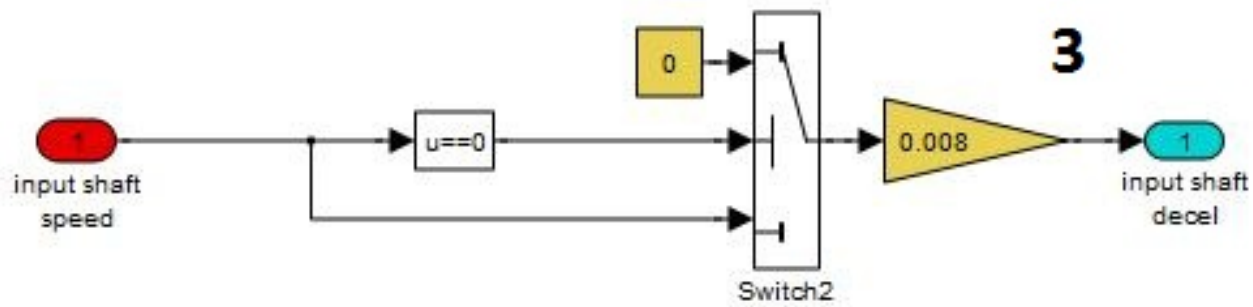

Figure 28. Block A1: Torque loss calculation when the gearbox is in neutral

The torque losses are proportional to the input speed, as shown in Equation 20.

$$
T_{\text {loss }}=K_{\text {coeff }} \times W_{\text {in }}
$$

Block B (Figure 29) calculates the torque output of the gearbox, accounting for the torque gain due to the ratio and the torque loss in the gearbox.

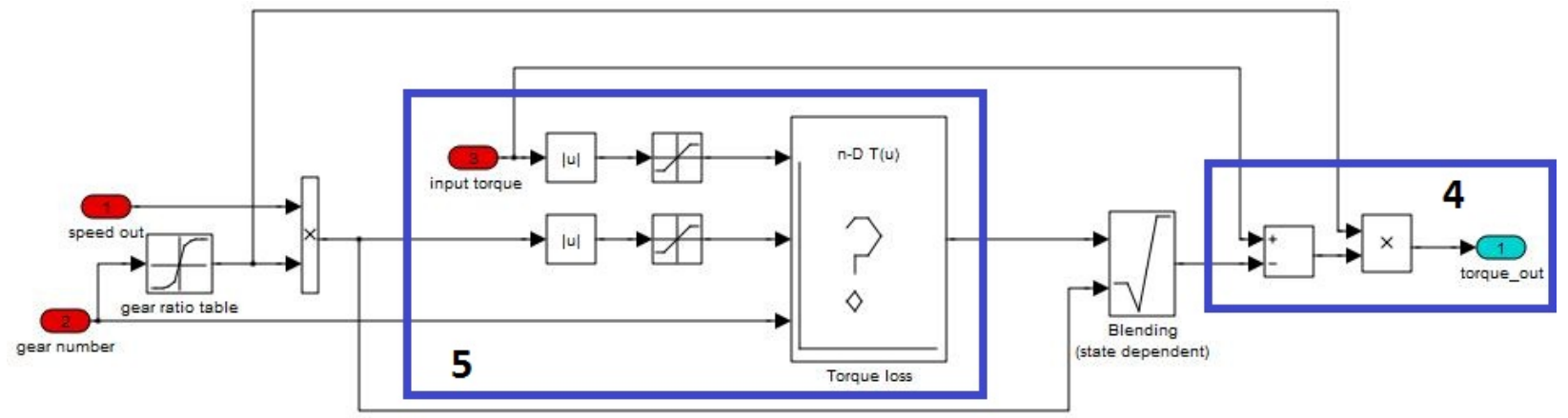

Figure 29. Block B: Speed calculation when the gearbox is in neutral

Equation 21 shows that the output torque before applying the ratio gain is the output torque minus the torque loss.

$$
T_{\text {out }}=T_{\text {in }}-T_{\text {loss }}
$$

When the gearbox is in neutral, the torque output is forced to 0 .

$$
T_{\text {out }}=0
$$

The torque loss is a function of the gearbox input torque, speed, and ratio.

$$
T_{\text {loss }}=f\left(T_{\text {in }}, W_{\text {in }}, K_{\text {ratio }}\right)
$$

Block C (Figure 30) gives the output inertia of the gearbox. 


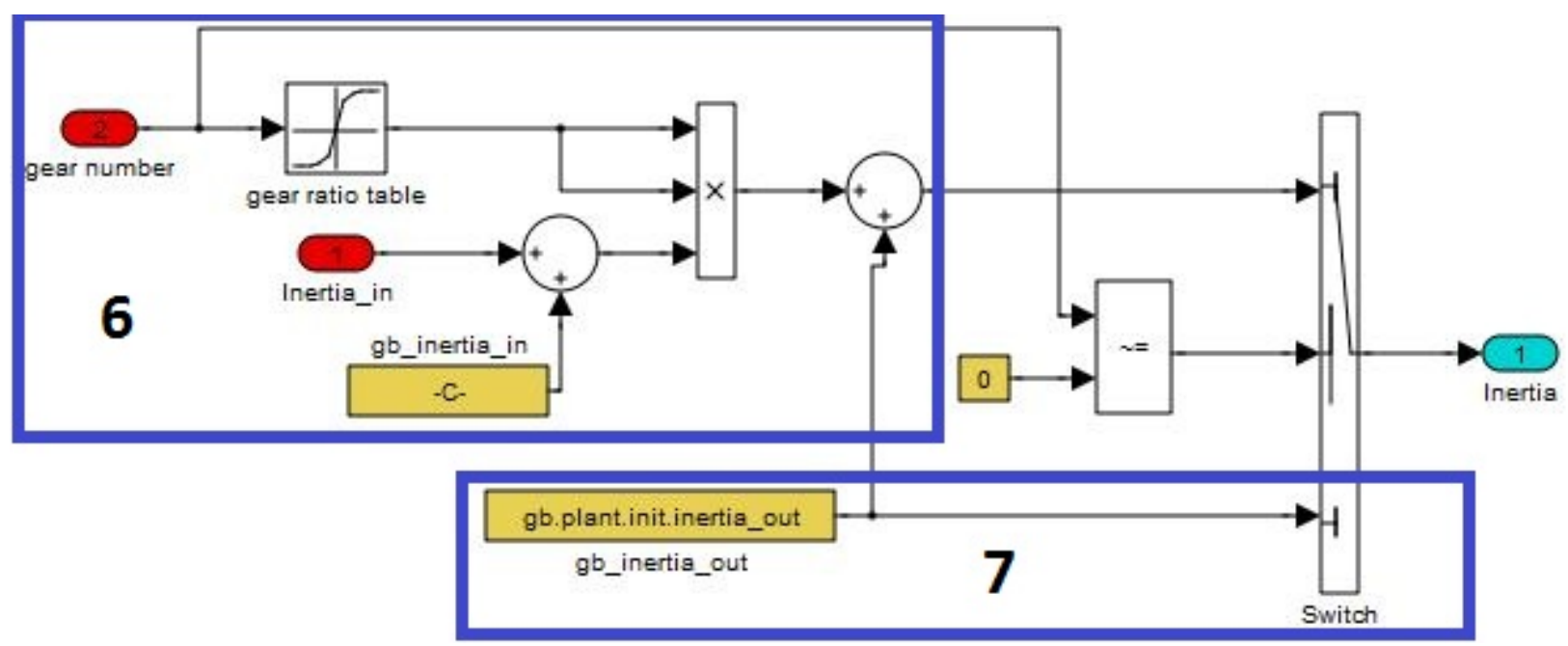

Figure 30. Block C: Output inertia calculation

The output inertia equals the upstream inertia reflected to the output shaft of the gearbox through the gear ratio plus the output shaft inertia of the gearbox.

$$
J_{\text {out }}=\left(J_{\text {in }}+J_{\text {input_shaft_t }}\right) \times K_{\text {ratio }}^{2}+J_{\text {output_shaft_tx }}
$$

When the gearbox is in neutral, Equation 25 demonstrates that the output inertia of the gearbox is the inertia of the output shaft.

$$
J_{\text {out }}=J_{\text {output_shaft_tx }}
$$

\subsubsection{Continuously Variable Transmission}

\subsubsection{1. $\quad$ Plant Model}

The metal V-belt continuously variable transmission (CVT) model is based on the concepts of hydraulic and mechanical loss. Hydraulic loss constitutes the majority of the total loss at low vehicle speeds, while mechanical loss is the main source of inefficiency at high speed, as shown in Figure 31. The CVT model considers oil pump efficiency to be a function of the line pressure and input speed. The mechanical loss map, collected from experimental data, is also taken to be a function of the speed ratio, input torque, and vehicle speed. 


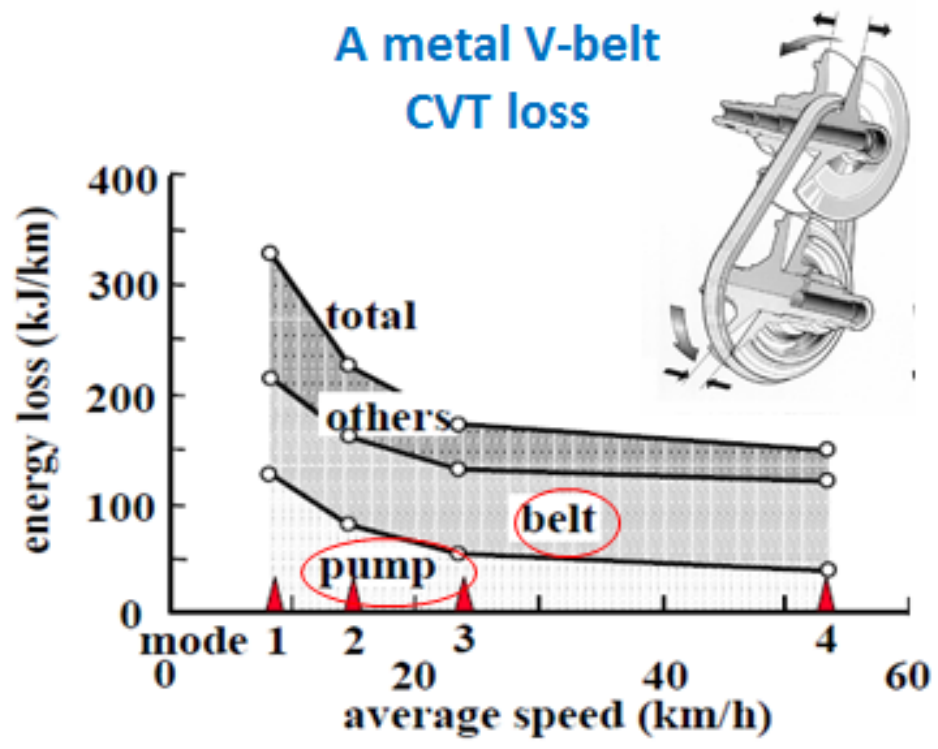

Figure 31. Hydraulic pump loss and mechanical loss for metal v-belt continuously variable transmission [37]

Generally, the operating conditions of the metal V-belt CVT system can be described by the following five parameters:

- $\quad$ Primary clamping force $\left(\mathrm{F}_{\mathrm{p}}\right)$ or primary pressure $\left(\mathrm{P}_{\mathrm{p}}\right)$

- Secondary clamping force $\left(\mathrm{F}_{\mathrm{s}}\right)$ or secondary pressure $\left(\mathrm{P}_{\mathrm{s}}\right)$

- Primary revolution speed $\left(\omega_{\mathrm{p}}\right)$

- Input torque $\left(\mathrm{T}_{\text {in }}\right)$

- Pulley ratio (i)

On both the primary and the secondary pulleys, the belt is clamped by the forces produced by the hydraulic pressures in the cylinders. These two clamping forces, $\mathrm{F}_{\mathrm{p}}$ and $\mathrm{F}_{\mathrm{s}}$, counteract each other. Therefore, when the pulley ratio is constant, $F_{p}$ and $F_{s}$ are balanced. A ratio change occurs when their balance is lost. In the next subsection, the relation between $F_{p}$ and $F_{s}$ in a state of balance is discussed, and a discussion of rate-of-change (di/dt) follows.

\subsubsection{2. $\quad$ Variator Clamping Force Model}

In the CVT system, the pulley ratio is controlled by the primary pulley; therefore, the state of balance is produced by the primary clamping force $\mathrm{F}_{\mathrm{P}}$. The necessary primary clamping force for counterbalance is denoted by $\mathrm{F}_{\mathrm{P}}{ }^{*}$, and the corresponding primary pressure is denoted by $\mathrm{P}_{\mathrm{P}}{ }^{*}$.

The secondary actuator pressure $\mathrm{P}_{\mathrm{S}}$ can be obtained for the given input torque $\mathrm{T}_{\text {in }}$ and CVT ratio $i$ from Fuji's formula:

$$
F_{S}=\frac{T_{I N}}{2 \mu R_{P}} \cos \frac{\alpha}{2}
$$

where $\mu$ is the friction coefficient between the belt and the pulley, and $R_{P}$ is the pitch radius of the primary pulley. In the CVT system design, Fs should be controlled to prevent slippage between the belt and the pulley. 
The primary pressure $\mathrm{P}_{\mathrm{P}}$ at steady state can be determined from the thrust ratio map. The thrust ratio shows the relationship between the steady-state primary force $F_{P}$ and the secondary force $F_{S}$ with respect to the speed ratio $i$ and the torque ratio. The thrust ratio $\mathrm{F}_{\mathrm{P}} \mathrm{F}_{\mathrm{S}}$ is represented as follows:

$$
F_{P} F_{S}=\frac{F_{P}}{F_{S}}=f\left(i_{C V T}, \frac{T_{I N}}{T_{\max }}\right)
$$

For the given speed ratio $i$ and the input torque $T_{i n}$, the secondary pressure $P_{S}$ is calculated from Equation 26. The primary actuator force $F_{S}$ is determined from the thrust ratio map for the given $i$ and the ratio $\mathrm{F}_{\mathrm{P}} \mathrm{F}_{\mathrm{S}}$. The primary pressure can be calculated from Equation 27. Figure 32 shows the $\mathrm{F}_{\mathrm{P}} \mathrm{F}_{\mathrm{S}}$ ratio map of the CVT.

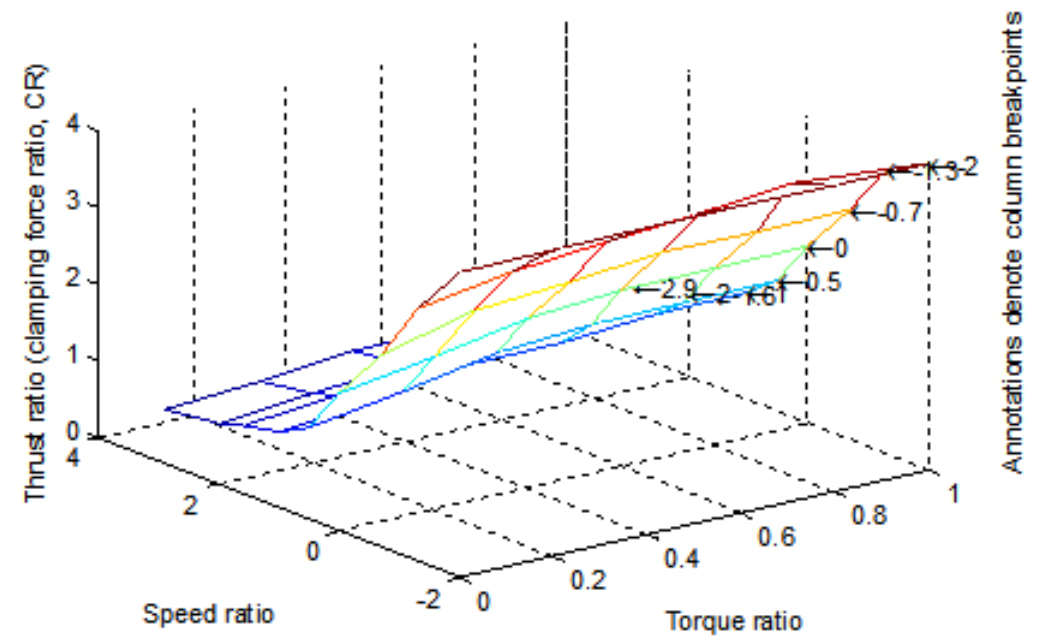

Figure 32. $F_{P} F_{S}$ ratio map (continuously variable transmission)

\subsubsection{3. $\quad$ CVT Shift Dynamics Model}

In order to obtain the dependency of the rate of changing speed di/dt on the five parameters mentioned above, we use Ide's formula [11], which is based on a test rig using only the belt and pulley set. The deviation of $\mathrm{P}_{\mathrm{P}}$ from $\mathrm{P}_{\mathrm{P}} *$ is denoted by $\Delta \mathrm{P}_{\mathrm{P}}$, and di/dt is expressed by the following equation:

$$
\frac{d i}{d t}=\beta(i) \times \omega_{P} \times\left(P_{P}-P_{P}^{*}\right)
$$

The parameters of Ide's formula were determined from experiments reported in the literature. The linear relation is also obtained for all pulley ratios. The effect of $\mathrm{P}_{\mathrm{P}}$ on $\mathrm{di} / \mathrm{dt}$ is obtained from the five parameters $\left(\mathrm{P}_{\mathrm{P}}, \mathrm{P}_{\mathrm{S}}, \omega_{\mathrm{p}}, \mathrm{T}_{\mathrm{in}}, \mathrm{i}\right)$. This formula shows that di/dt is in a proportional relation with $\Delta \mathrm{P}_{\mathrm{P}}$ under loaded conditions as well, and the slope $\beta$ barely changes even if $\mathrm{T}_{\text {in }}$ changes.

\subsubsection{Controller}

The CVT ratio control and clamping force control strategies, including the CVT shift dynamics, focus on the following:

- The demanded CVT ratio is determined from the engine best-efficiency line

- The secondary pressure is determined for the given input torque and CVT ratio

- The primary pressure needs to be controlled to meet the demanded CVT ratio 
Figure 33 shows a block diagram of the model-based ratio control and plan
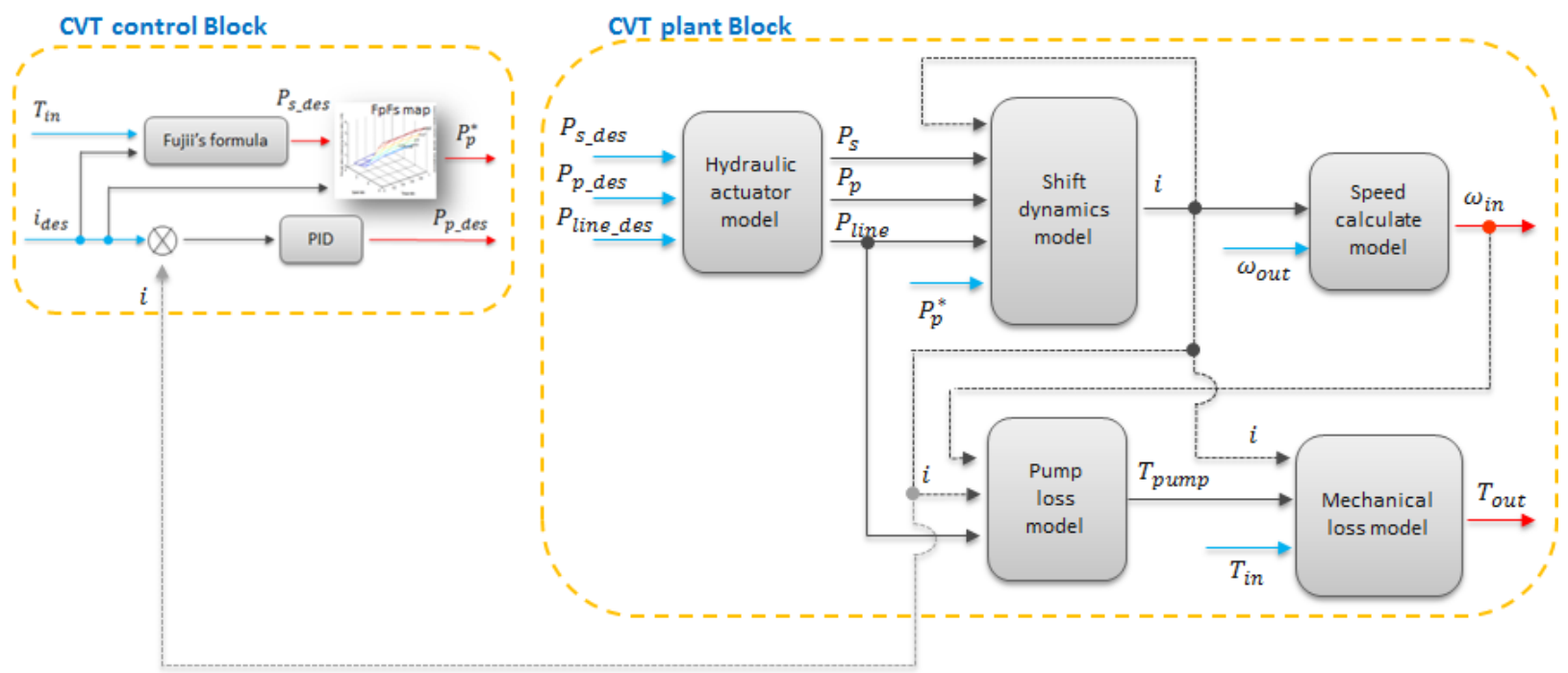

\section{CVT system block diagram}

Figure 33. Continuously variable transmission system block diagram

\subsubsection{Dual Clutch Transmission}

\subsubsection{Plant Model}

Dual clutch transmission (DCT) dynamic models include the clutch and gear train, as shown in Figure 34.

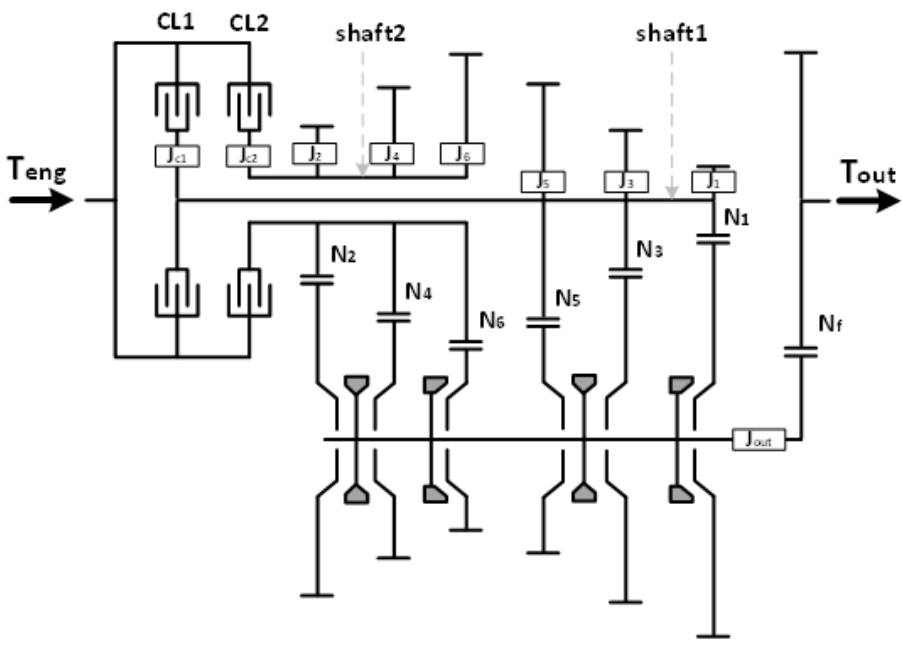

Figure 34. Stick diagram of a dual-clutch transmission [15]

The following assumptions are made in the model development of the shifting process: 
- All shafts within the powertrain are assumed to be rigid.

- The synchronization transition process of the synchronizer is ignored.

- The inertia of shaft 1 is combined with that of clutch1 (CL1), and the inertia of shaft 2 is combined with that of clutch2 (CL2).

\subsubsection{2. $\quad$ System Operating Conditions}

Gear pre-selection can be implemented based on the DCT operating conditions, as shown in Figure 35. For example, if the first synchronizer is at the first gear position, and the third through fifth synchronizers are at the neutral position (as they must be), then the gear ratio between shaft 1 and the output shaft is first gear. At the same time, the gear ratio between shaft 2 and the output shaft can be selected in the same manner for the pre-selection mode. To achieve a desired input-output gear ratio, the corresponding synchronizer and clutch must be applied.
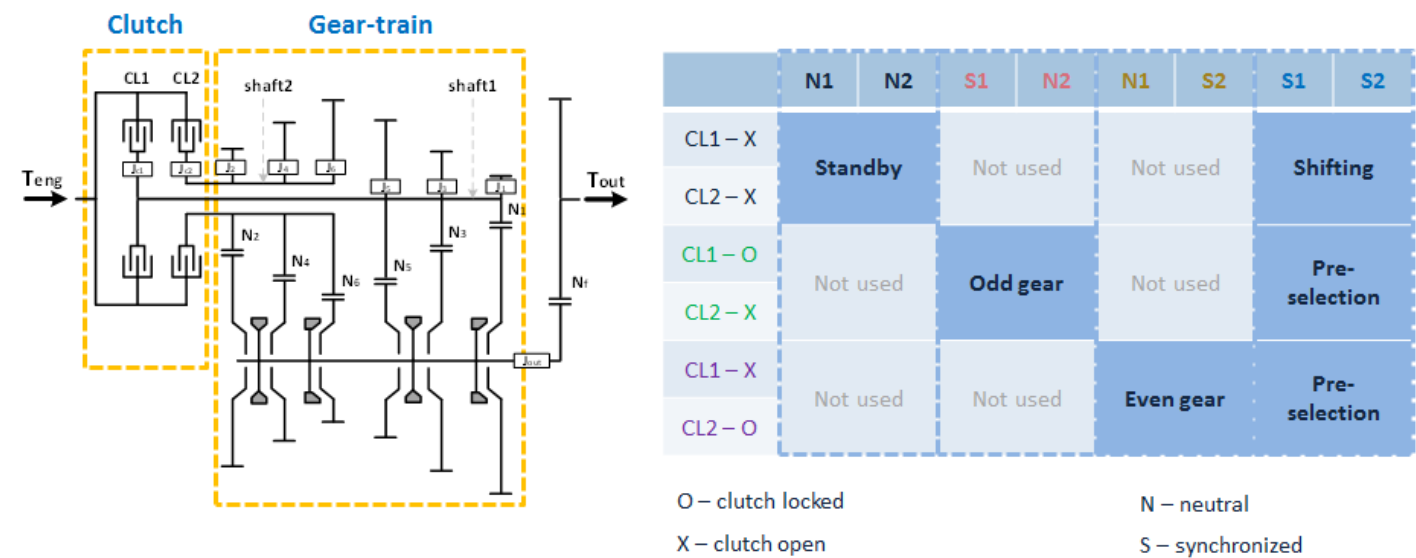

Figure 35. Dual-clutch transmission operating conditions

\section{Dynamic Modeling}

The schematic diagram in Figure 36 and the operating conditions in Figure 35 were used to generate the equations for each mode, as described below. 


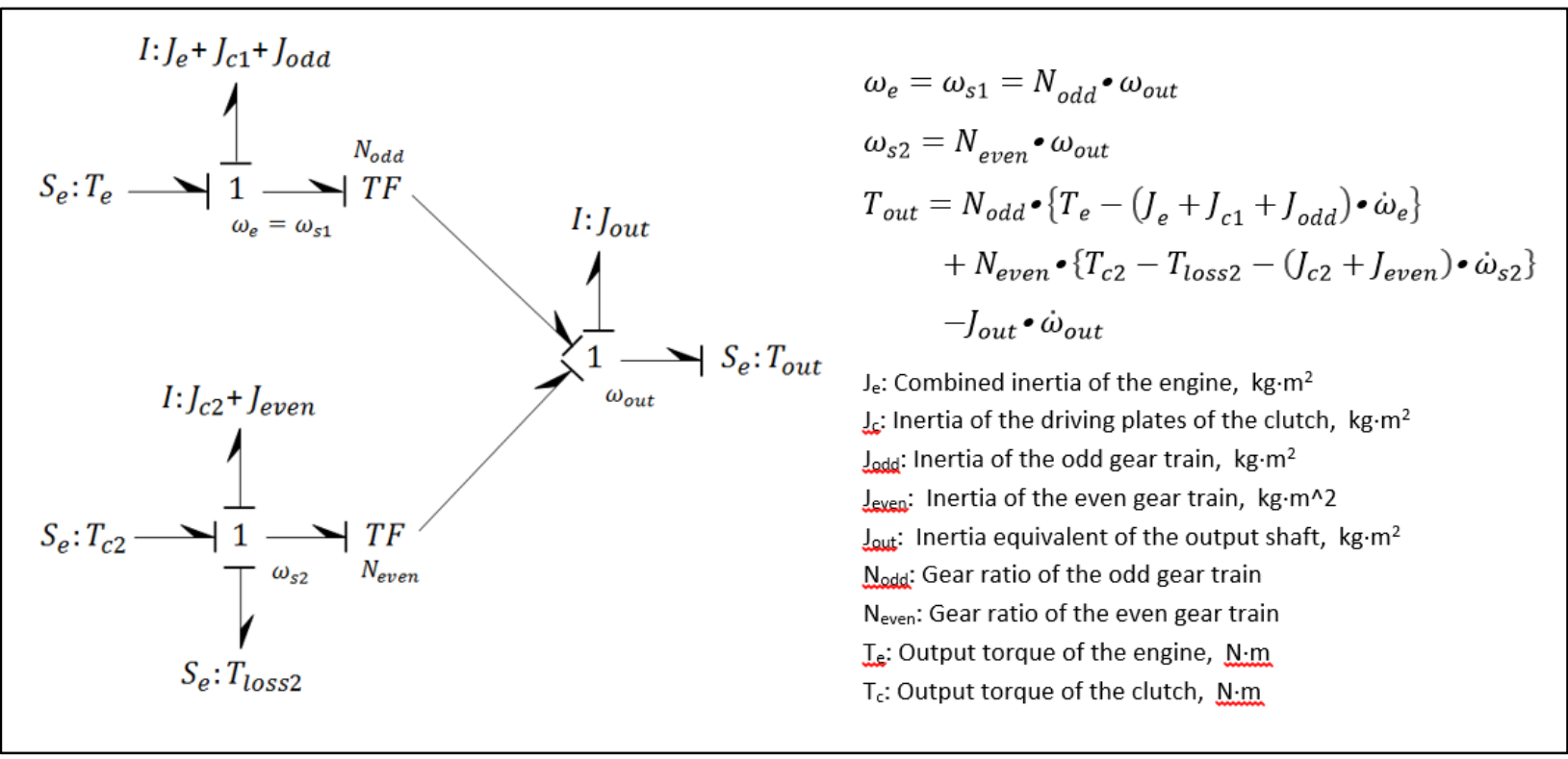

Figure 36. Pre-selection mode bond graph

The synchronizer of shaft 1 is at an odd gear position, and the synchronizer of shaft 2 is at an even gear position for the pre-selection. CL1 or CL2 is applied to connect the input power path into shaft1 or shaft2. Figure 36 shows the bond graph representation of the pre-selection mode when only CL1 is locked.

Figure 37 shows the details of the Autonomie gearbox plant model for the DCT.

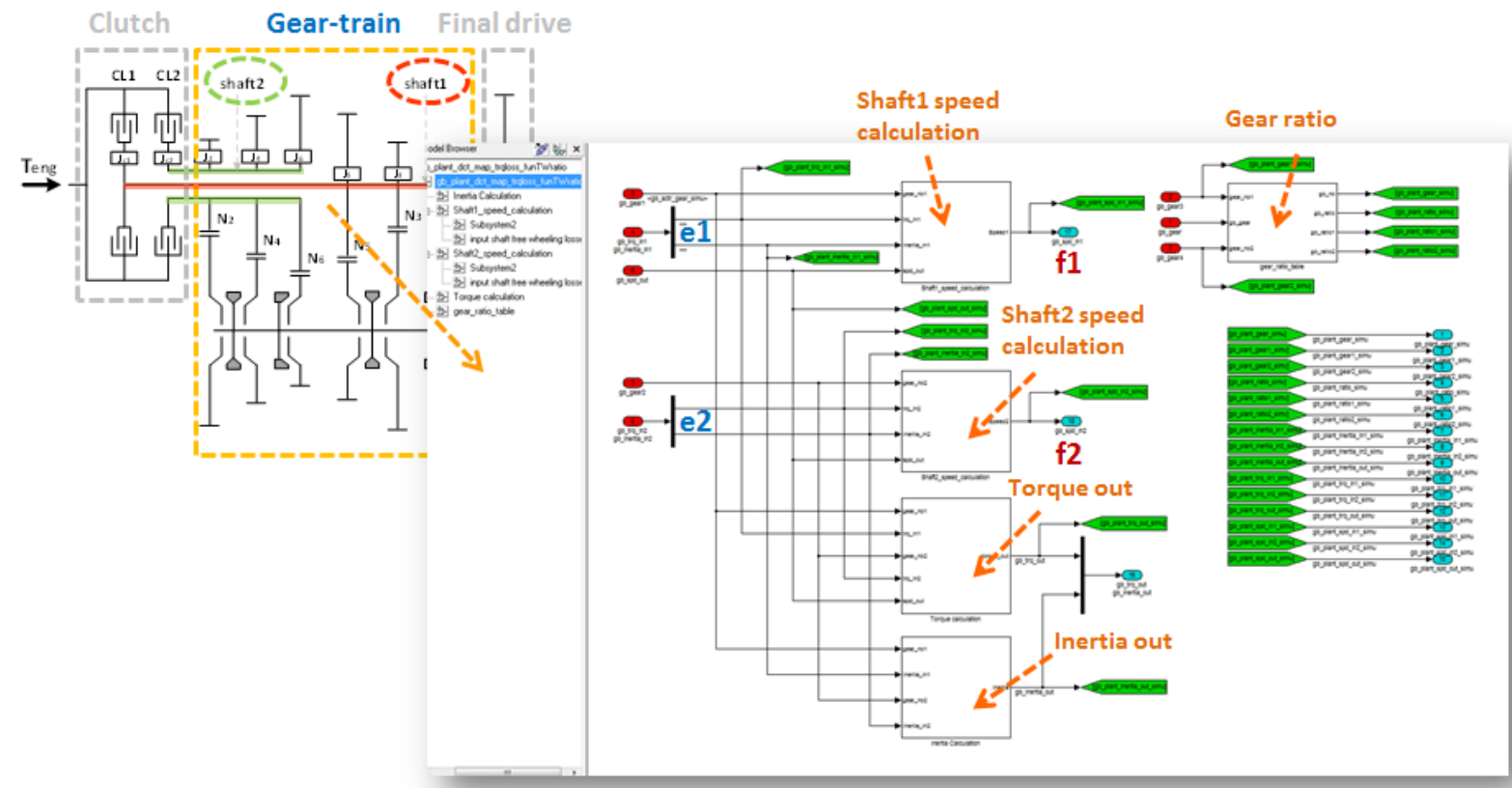

Figure 37. Gearbox plant model for dual-clutch transmission 
The model and the governing equations for the clutch are described below:

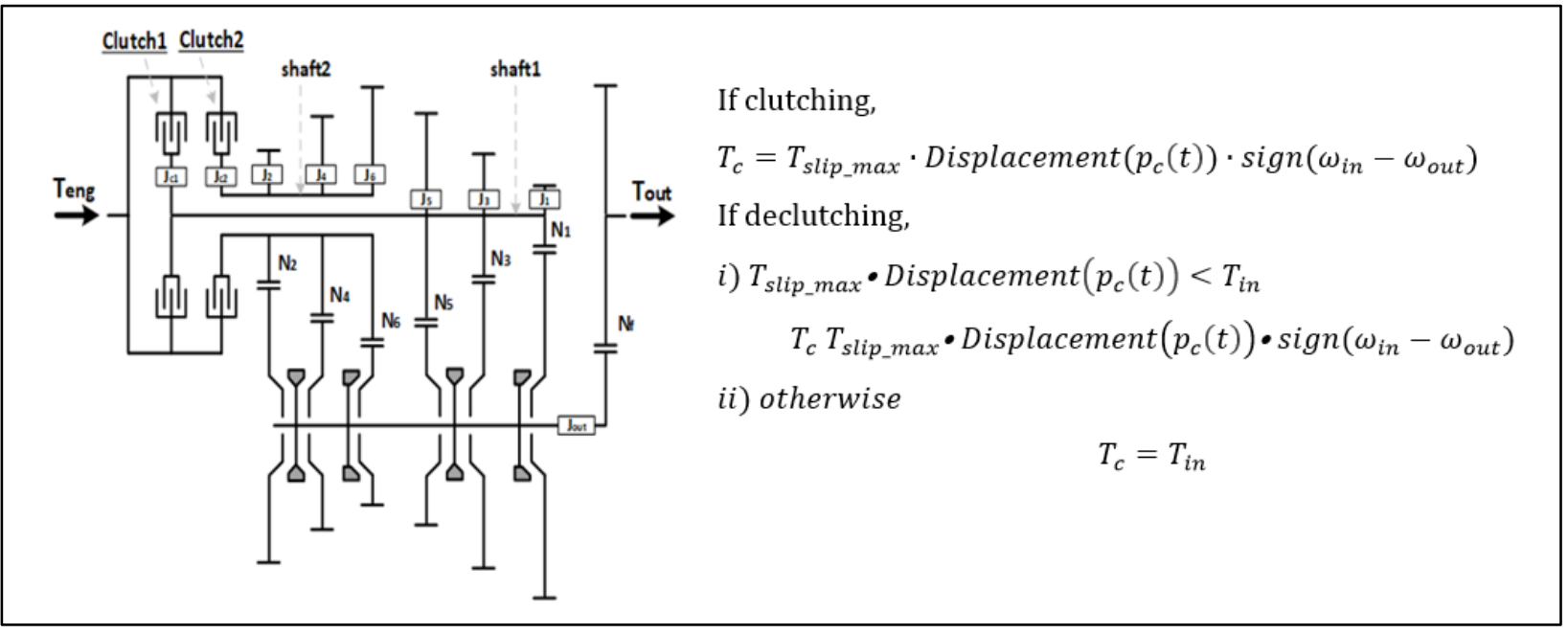

Figure 38. Model and governing equations for clutch

Figure 39 shows the high-level DCT model in Autonomie.

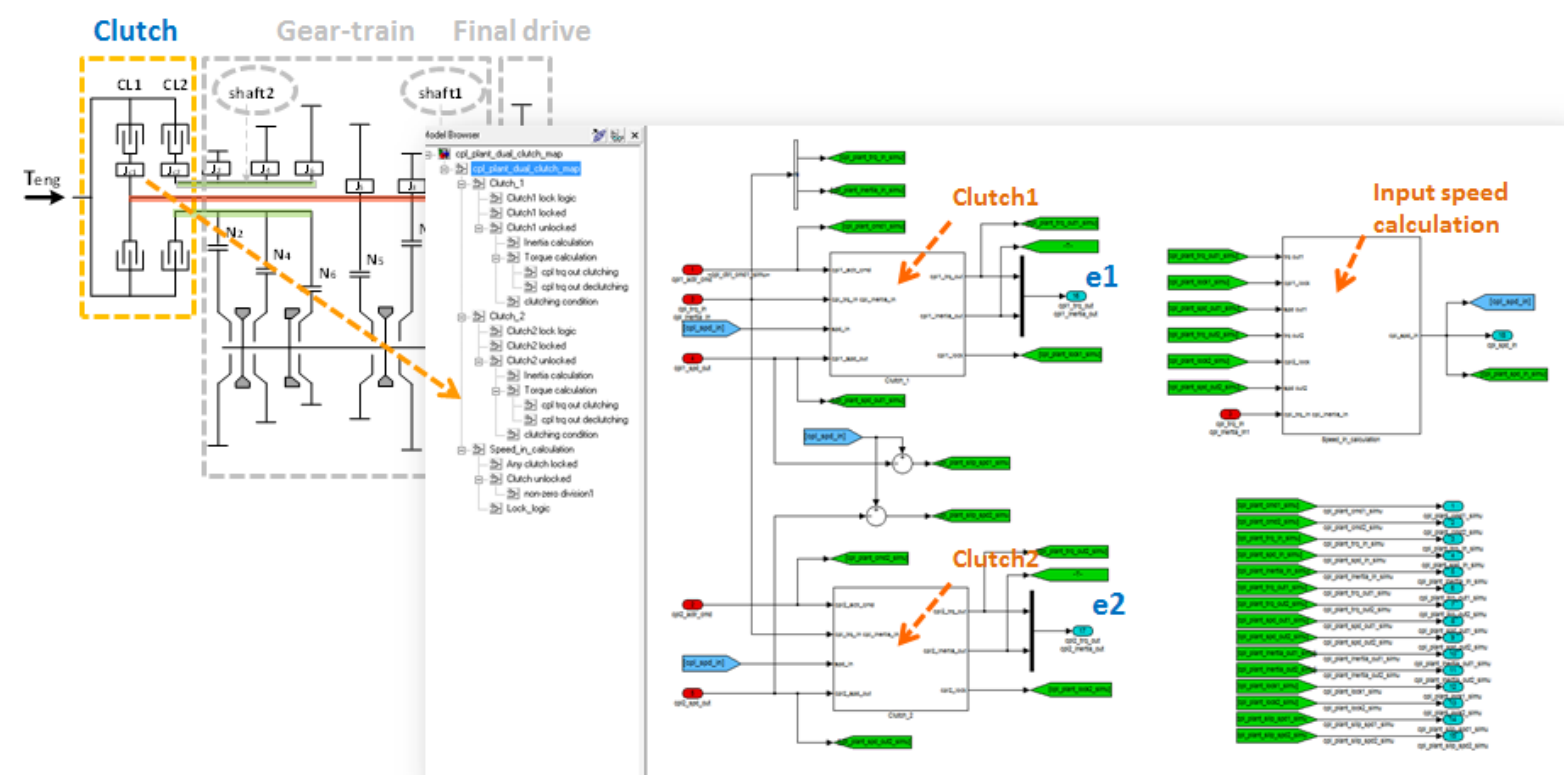

Figure 39. Gearbox plant model for dual-clutch transmission

\section{Controller}

The controller operating logic was defined based on the principle of DCT operation. The gearbox transient block coordinates all components during transient phases, as shown in Figure 40. 


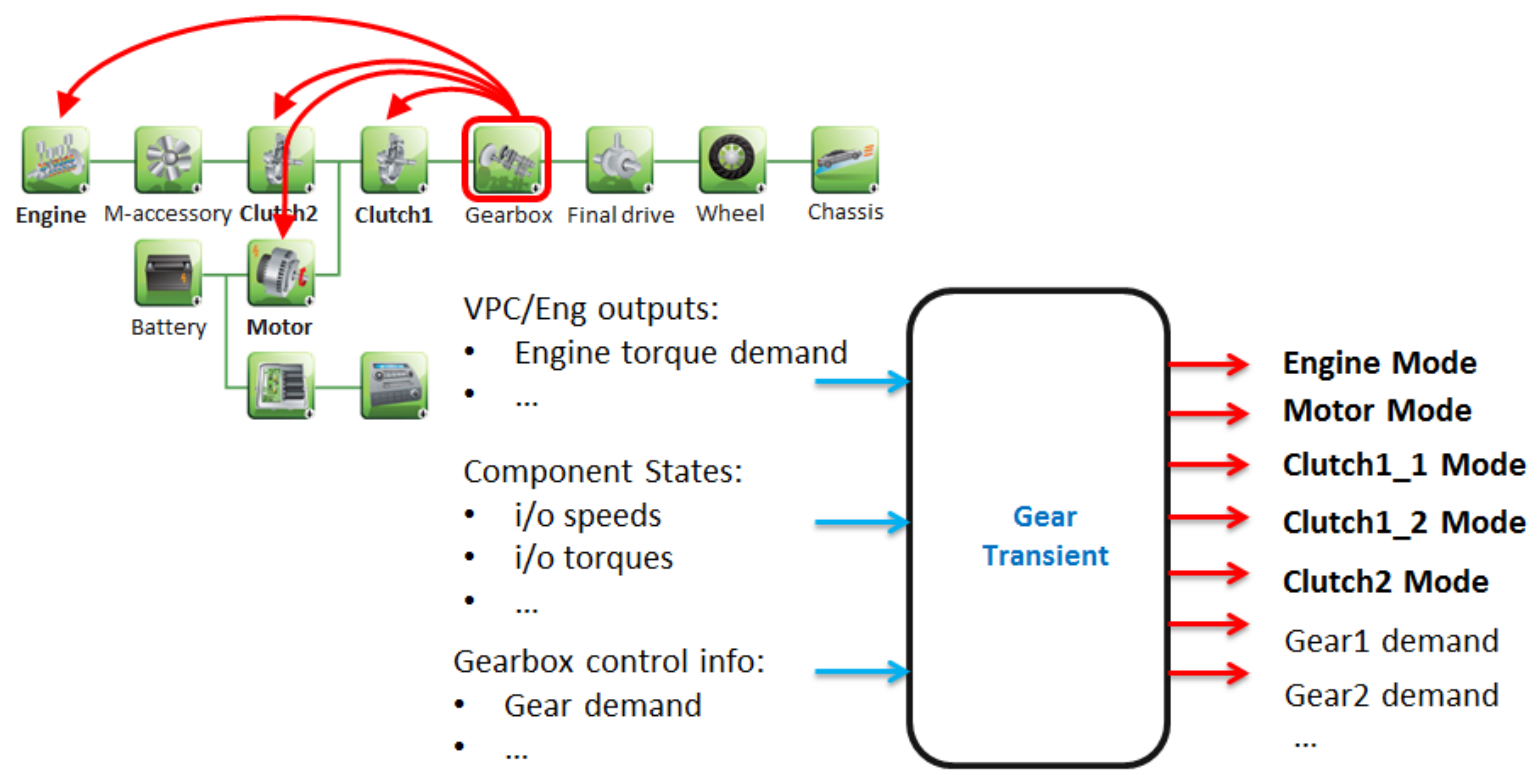

Figure 40. Dual-clutch transmission controller

\section{Gearbox Transient Controller}

The component modes (engine mode, clutch mode, and electric motor mode) and signals of gear demand (odd/even) are generated when a gear shift is required or the engine turns on. The gear selection control (synchronizer control) algorithm is unique to DCT systems (Figure 41). Once the gearshift schedule algorithm generates a new desired gear command, the gear selection control algorithm controls the synchronizer actuators to select the desired gear.

Example of gear shifting $: 2^{\text {nd }} \rightarrow 3^{\text {rd }}$

- Check gear dmd if odd or even?

EV driving or HEV driving?

- Pre-selection : synchronizing $3^{\text {rd }}$ in shaft1

- CL1 clutching / CL2 declutching

- CL1 locked / neutral of shaft2

- Finishing

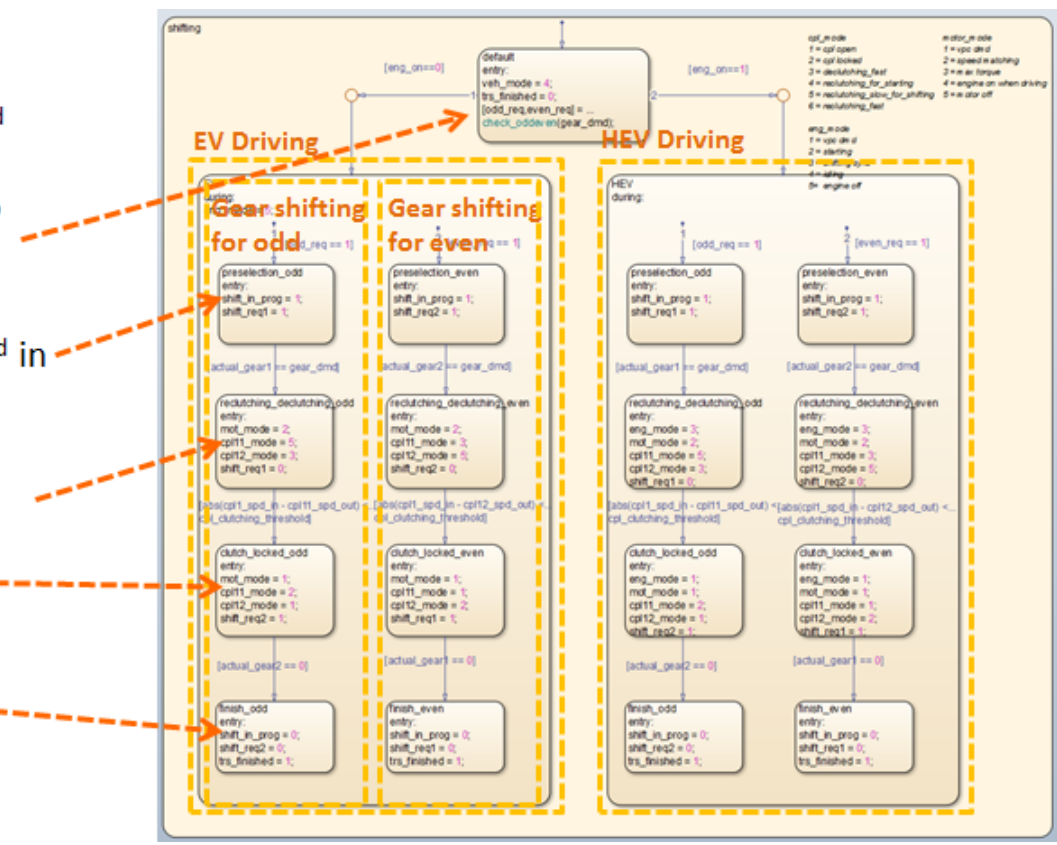

Figure 41. State diagram representation of the control strategy for a dual-clutch transmission 


\section{Clutch Controller}

The clutch controls use time-based clutch position maps, as shown in Figure 42.

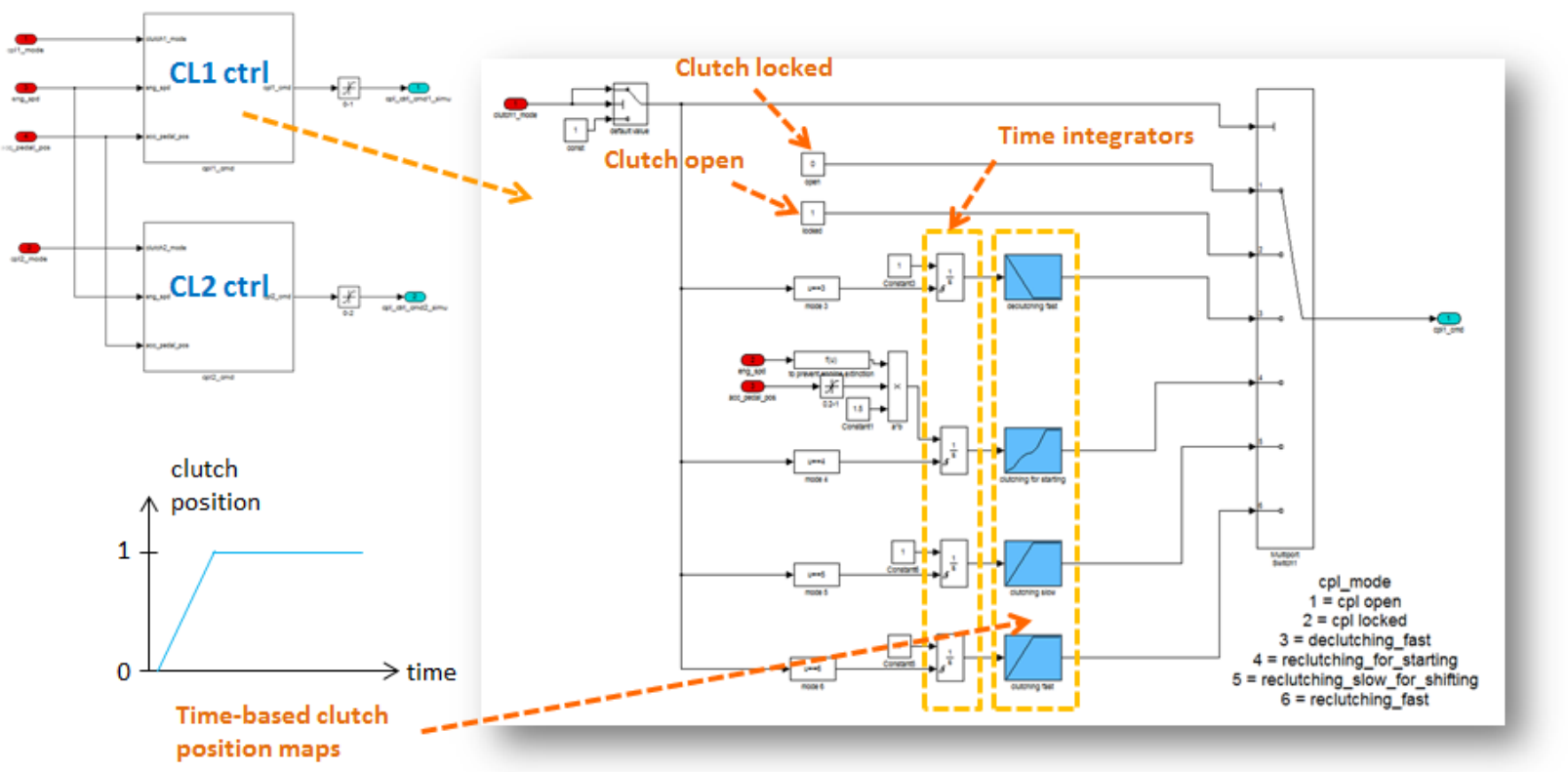

Figure 42. Clutch controller for dual-clutch transmission

\subsubsection{Transmission Shifting}

There are two parts to transmission shifting logic:

- Shift initializer

- The shift initializer logic, implemented in the vehicle model, decides when to change gears. In addition to the shift maps, there are different thresholds in place (i.e., gear upshift demand validation) to ensure proper drive quality. One of the key parameters of the shifting logic is the shift map. For a given transmission, the shifting logic is the same in all vehicles.

- Shift controller

The shift controller consists of the following:

- The shift map: There are two separate maps, one for upshift and one for downshift, which use vehicle speed and pedal position as inputs. Shift maps are developed for each individual vehicle/powertrain/component combination using a generic shifting algorithm.

- The shifting algorithm: The shifting algorithm uses the component characteristics (e.g., engine brake-specific fuel consumption (BSFC), gear ratios, final drive ratio, wheel radius, etc.) to develop the shift maps. While energy consumption is very important, other drive quality metrics are also taken into account (e.g., avoid low/high vehicle speeds, high torque demands, etc.)

Transmission shifting has a significant impact on vehicle energy consumption and should be carefully designed to maximize the powertrain efficiency while maintaining acceptable drive quality. The logic used in the simulated conventional light-duty vehicle models relies on two components: (1) the shifting controller, which provides the logic to select the appropriate gear during the simulation, and (2) the shifting initializer, the algorithm that defines the shift maps (i.e., the values of the parameters of the 
shifting controller) specific to a selected set of component assumptions. While the algorithm used to develop shift maps is similar across all vehicles, the shift maps are specific to each individual vehicle, as they are designed using component information such as gear and final drive ratios, BSFC, and so on.

\subsubsection{1. $\quad$ Engine Lugging Limits}

Engine lugging limit is a critical NVH parameter. The assumptions shown in Table 1 and Table 2 below describe the logic implemented in Autonomie to prevent lugging. The logic and values were developed based on AMTL vehicle test data analysis.

To ensure consistency with the different engine technologies using the vehicle test data, engine lugging limits for different geared transmissions are shown separately for naturally aspirated and turbocharged engines. Shift parameters are selected such that low-speed, high-torque operation is avoided. The selected shifting limits are based on test data observations relative to the number of gears available.

Table 1. Engine Lugging Limits for Different Transmissions (Naturally Aspirated [NA] Engines)

\begin{tabular}{|l|c|c|c|c|c|c|}
\hline & 5-speed & 6-speed & 7-speed & 8-speed & 9-speed & 10-speed \\
\hline Lugging speed (rad/s) & 140 & 130 & 120 & 110 & 110 & 110 \\
\hline Lugging speed (RPM) & 1337 & 1241 & 1241 & 1050 & 1050 & 1050 \\
\hline
\end{tabular}

Table 2. Engine Lugging Limits for Different Transmissions (Turbo Engines)

\begin{tabular}{|l|c|c|c|c|c|c|}
\hline & 5-speed & 6-speed & 7-speed & 8-speed & 9-speed & 10-speed \\
\hline Lugging speed (rad/s) & 140 & 130 & 130 & 130 & 130 & 130 \\
\hline Lugging speed (RPM) & 1337 & 1241 & 1241 & 1241 & 1241 & 1241 \\
\hline
\end{tabular}

Figure 43 shows the engine operating points for a 2017 Ford F-150 during an urban dynamometer driving schedule (UDDS) cycle [18]. 


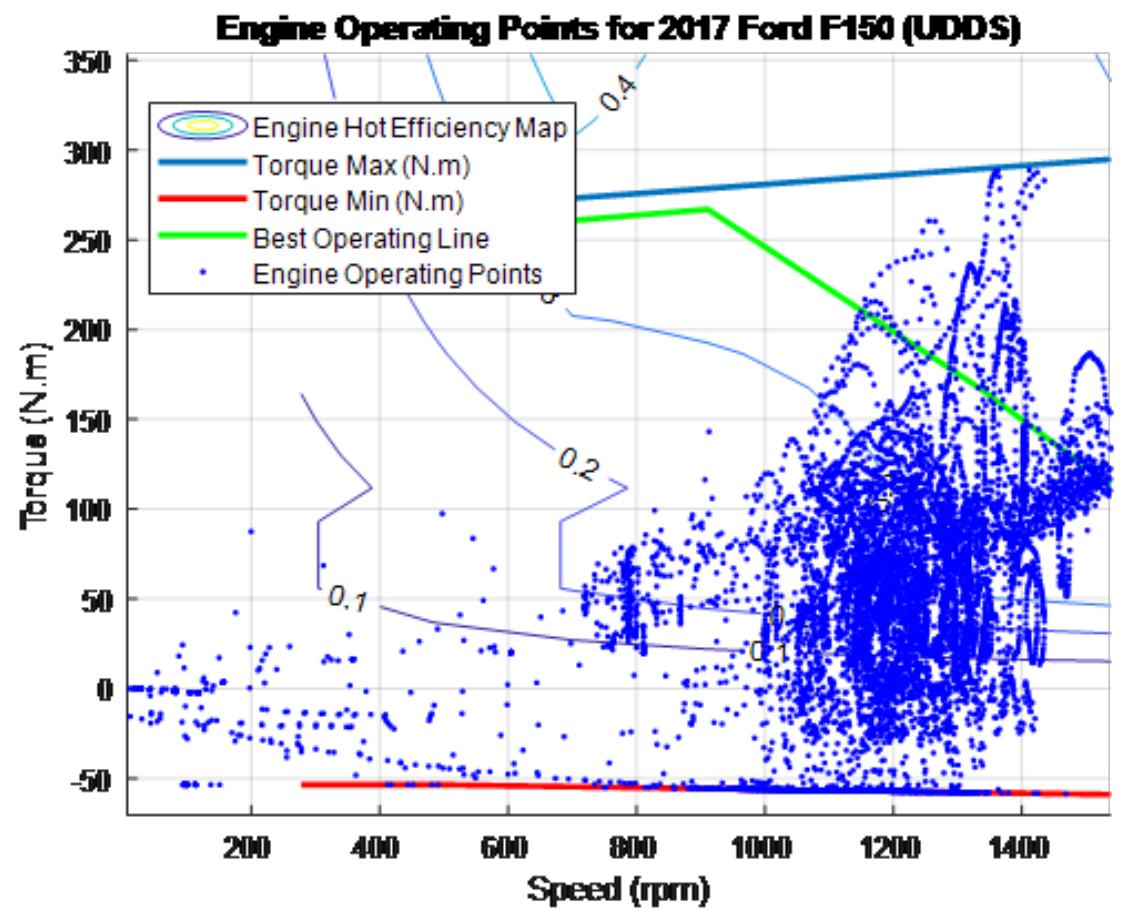

Figure 43. Engine operating points for 2017 Ford F150 (UDDS)

Figure 44 shows an example of how engine operating conditions are restricted to prevent lugging for 5 -speed and 10-speed automatic transmissions on the UDDS cycle for a naturally aspirated engine.
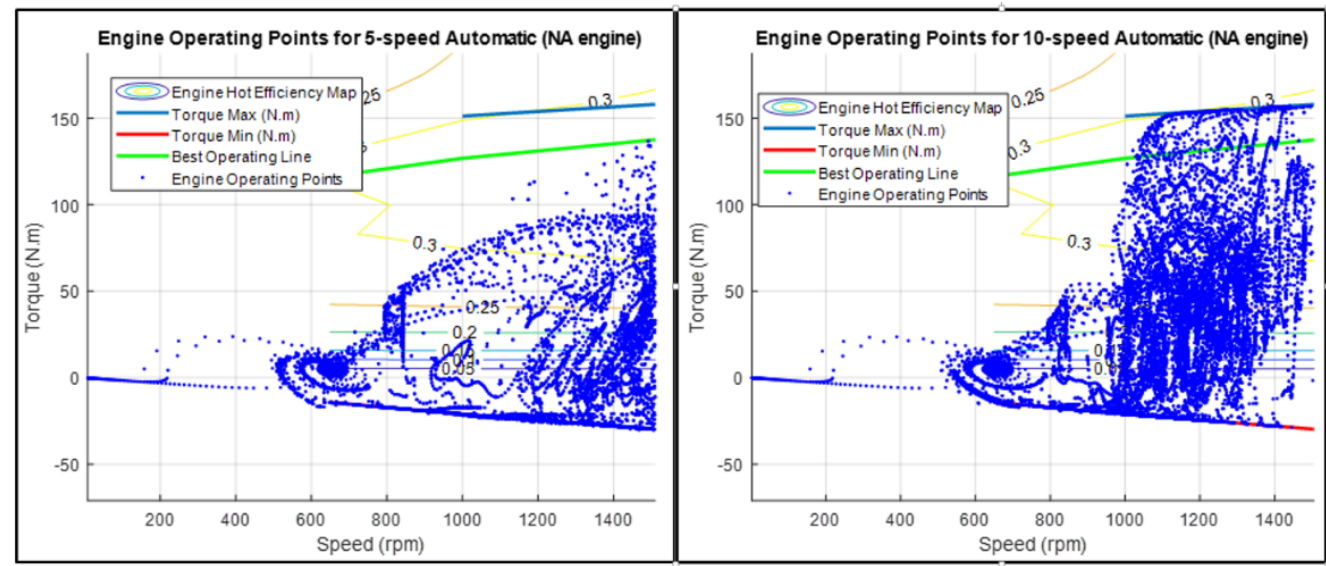

Figure 44. Example of engine operating conditions for 5-speed and 10-speed transmissions on UDDS cycle for NA engine

\subsubsection{Shifting Initializer}

The shifting controller uses shifting maps to compute the gear command. In the controller, the shift map is a two-dimensional (2D) lookup table indexed by vehicle speed and accelerator pedal position. Defining such a map is equivalent to defining the "boundaries" of each gear area; those boundaries are the shifting speeds. Figure 45 illustrates that equivalence. 

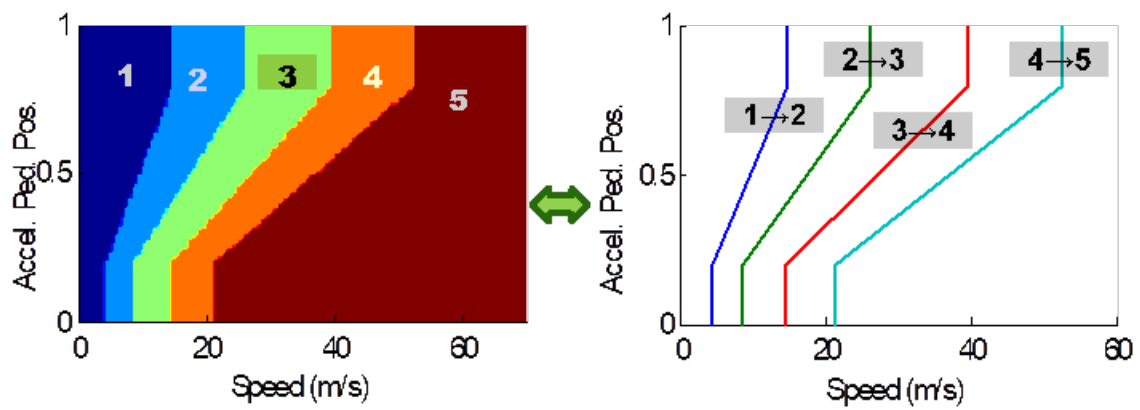

Figure 45. Upshifting gear map (left); upshifting vehicle speeds (right)

For each shifting curve, there are two key points: the "economical" shifting speed (at very low pedal position) and the "performance" shifting speed (at high pedal position). The objective of the control engineer is to combine both goals of the shifting control to fulfill the driver's expectations: minimization of energy consumption on the one hand and maximization of vehicle performance on the other.

The economical shifting speed for an upshift or a downshift is the speed at which the upshift/downshift occurs when the accelerator pedal is very lightly pressed: $V_{e c o}^{k \rightarrow k+1}$ is the economical vehicle speed for upshifting from gear $\mathrm{k}$ to gear $\mathrm{k}+1$, and $V_{e c o}^{k+1 \rightarrow k}$ is the downshifting speed for this same set of gears. The vehicle speed shift points are computed from the engine shift points $\omega_{e c o}^{k \rightarrow k+1}$ and $\omega_{e c o}^{k+1 \rightarrow k}$. Figure 46 shows the engine speed shift points for an engine associated with a 5 -speed transmission.

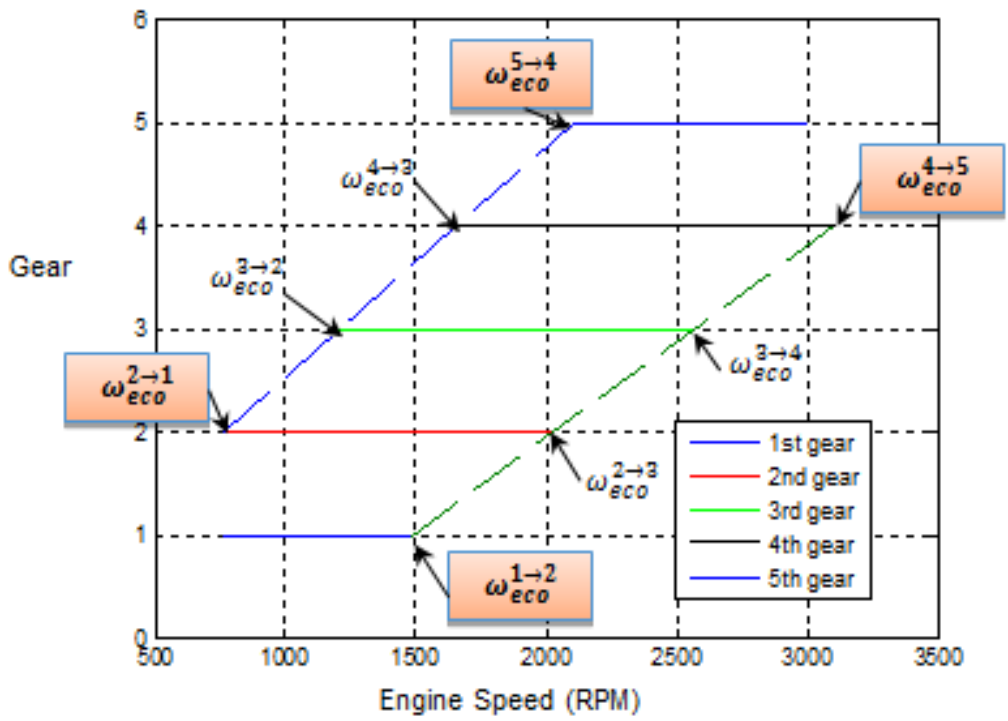

Figure 46. Example of engine speed range in economical driving and economical shift

The initializing algorithm for the shifting controller computes the upshifting and downshifting speeds at zero pedal position based on the four "extreme" shift points: upshifting from lowest gear $\left(\omega_{\text {eco }}^{1 \rightarrow 2}\right)$, upshifting into highest gear $\left(\omega_{e c o}^{N-1 \rightarrow N}\right)$, downshifting into lowest gear $\left(\omega_{e c o}^{2 \rightarrow 1}\right)$, and downshifting from highest gear $\left(\omega_{e c o}^{N \rightarrow N-1}\right)$, where $\mathrm{N}$ is the number of gears. The speeds can be set by the user or left at their default values. Below is a description of their default values in Autonomie: 
$\omega_{\text {eco }}^{2 \rightarrow 1}=\omega_{\text {idle }}+\omega_{\text {margin }}$ where $\omega_{\text {idle }}$ is the engine idle speed and $\omega_{\text {margin }}$ is the speed margin of $\approx 50-100 \mathrm{rpm}$

$\omega_{\text {eco }}^{1 \rightarrow 2}=\omega_{\text {idle }} \frac{k_{1}}{k_{2}}\left(1+\epsilon_{u d}\right)$ where $\mathrm{k} 1$ and $\mathrm{k} 2$ are gear ratios for gears 1 and 2 and $\epsilon \_\mathrm{ud}$ is the margin to avoid overlap, $\approx 0.05-0.1$

$\omega_{e c o}^{N-1 \rightarrow N}$ is the engine speed at which best efficiency can be achieved

$\omega_{e c o}^{N \rightarrow N-1}=\omega_{e c o}^{N-1 \rightarrow N}-\omega_{\Delta}$ where $\omega \Delta \approx 1,000 \mathrm{rpm}$

Once those four speeds are computed, the remaining ones are computed by linear interpolation to allow consistent shifting patterns that are acceptable to the driver. For example, any upshifting speed is given by Equation 29:

$$
\omega_{e c o}^{i \rightarrow i+1}=\frac{\omega_{e c o}^{N-1 \rightarrow N}-\omega_{e c o}^{1 \rightarrow 2}}{N-2} \times(i-1)+\omega_{e c o}^{1 \rightarrow 2} \text { where } 1 \leq \mathrm{i} \leq \mathrm{N}-1
$$

In a shifting map, the vehicle upshifting speed from gear $i$ to $i+1$ must be higher than the downshifting speed from gear $i+1$ to $i$. Otherwise, the downshifting speed will always request gear $i$ while gear $i+1$ is engaged and vice versa, resulting in oscillations between gears that would be unacceptable to the driver. For this study, the algorithm in the initialization file prevents that by making sure the following relation is true:

$$
\omega_{\text {eco }}^{i \rightarrow i+1}>\omega_{\text {eco }}^{i+1 \rightarrow i} \times \frac{k_{1}}{k_{2}}\left(1+\epsilon_{u d}\right) \text { where } 1 \leq \mathrm{i} \leq \mathrm{N}-1
$$

The values of the engine economical shifting speeds at lowest and highest gears are automatically defined on the basis of the engine and transmission characteristics.

Finally, the vehicle economical up- and downshifting speeds can be computed using the engine upand downshifting speeds, the gear ratio, the final drive ratio and the wheel radius:

$$
V_{e c o}^{i \rightarrow i+1}=\frac{\omega_{e c o}^{i \rightarrow i+1}}{k_{i} k_{F D}} \times R_{w h} \text { where } \mathrm{k}_{\mathrm{FD}} \text { is the final drive ratio and } \mathrm{R}_{\mathrm{wh}} \text { is the wheel radius }
$$

During performance, the gears are automatically selected to maximize the torque at the wheel. Figure 47 illustrates that gear selection, which consists of finding the point where the curve of engine peak torque (reported at the wheels) at gear $\mathrm{k}$ falls under the curve at gear $\mathrm{k}+1$. 


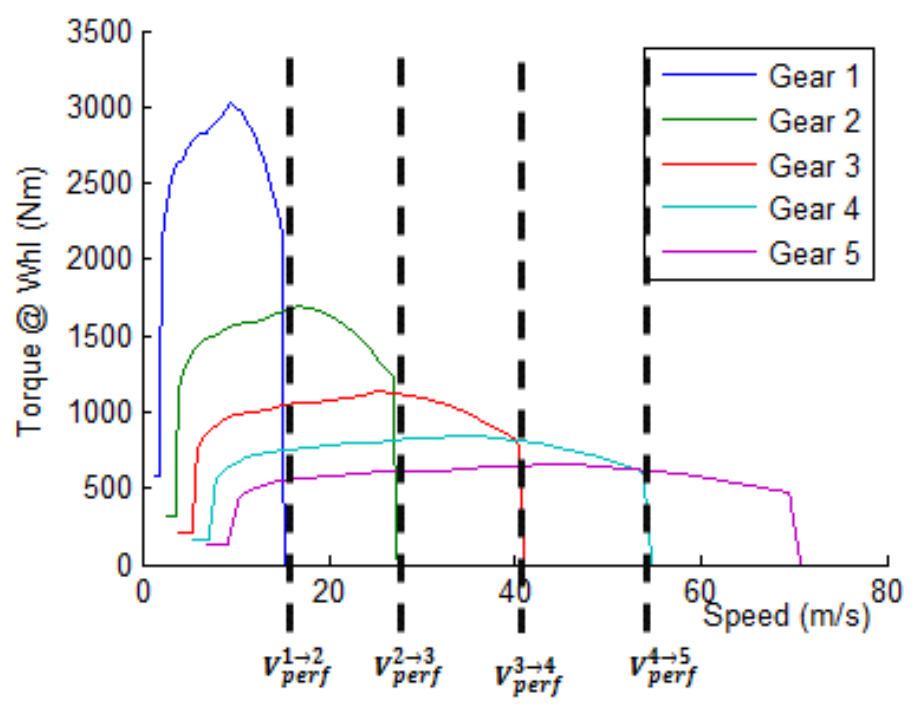

Figure 47. Maximum engine torque at wheels and performance upshift speeds

The performance downshifting speed is given by the performance upshifting speed and the difference between the economical shifting speeds:

$$
\Delta V_{\text {perf }}^{i}=\alpha_{p f, e c} \times \Delta V_{\text {eco }}^{i} \Leftrightarrow V_{\text {perf }}^{i \rightarrow i+1}-V_{\text {perf }}^{i+1 \rightarrow i}=\alpha_{p f, e c} \times\left(V_{\text {perf }}^{i \rightarrow i+1}-V_{\text {perf }}^{i+1 \rightarrow i}\right)
$$

The definition of the final shifting curves is critical to properly evaluating the benefits of technologies while maintaining acceptable performance. Figure 48 shows how a set of upshifting and downshifting curves for two adjacent gears is built, based on selected vehicle speeds and accelerator pedal positions. At low pedal positions (i.e., below $a_{\text {eco }}^{u p}$ ), the upshifting speed is the economical upshifting speed. Similarly, below $a_{e c o}^{d n}$, the downshifting speed is the economical downshifting speed. This approach ensures optimal engine operating conditions under gentle driving conditions. At high pedal positions (i.e., above $a_{\text {perf }}$ ), the shifting speed is the performance shifting speed, ensuring maximum torque at the wheels under aggressive driving conditions.

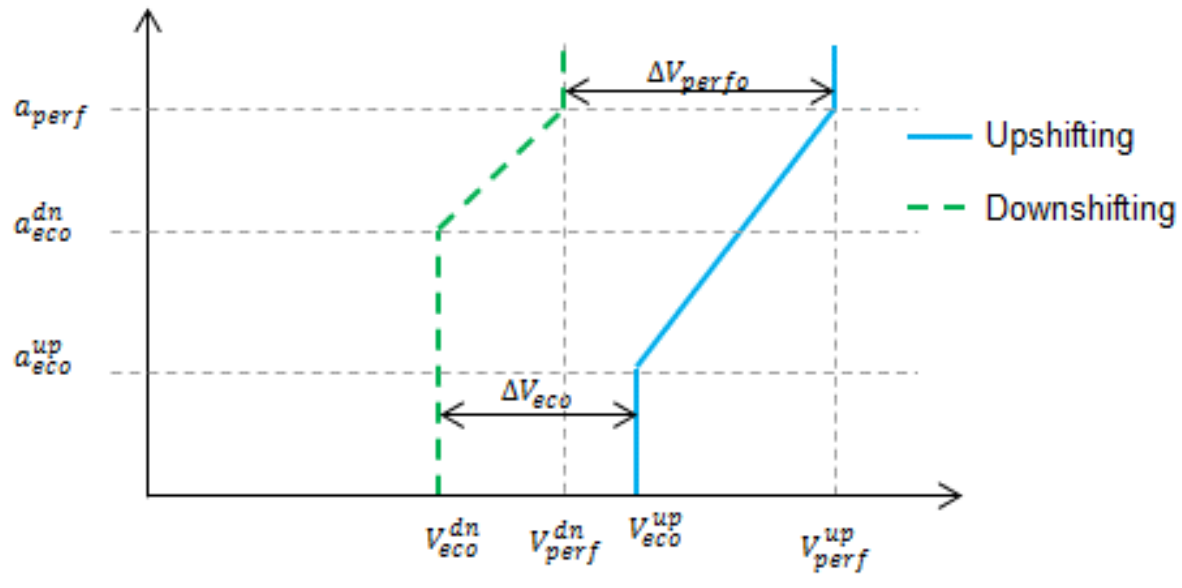

Figure 48. Design of upshifting and downshifting speed curves for two adjacent gears 


\subsubsection{3. $\quad$ Traditional Gear Shifting Controller}

The shifting controller determines the appropriate gear command at each simulation step using the shifting maps developed by the shifting initializer. A simplified schematic of the controller is shown in Figure 49. The letters and numbers in the discussion that follows correspond to those shown in circles in Figure 49.

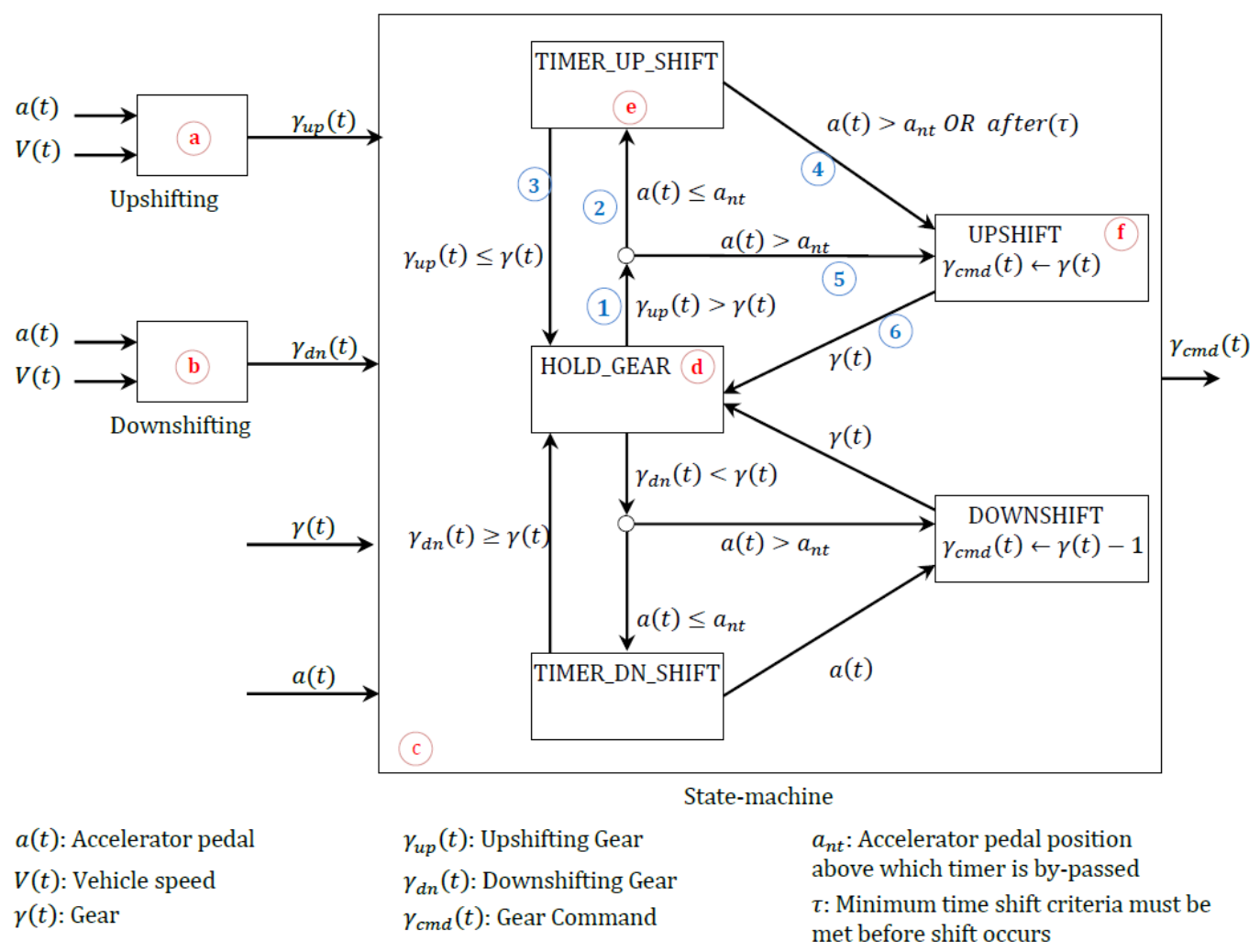

Figure 49. Shifting controller schematic in Autonomie

The controller is based on two main shifting maps - one for upshifting (a) or moving from a lower gear to a higher gear, and another one for downshifting (b) or moving from a higher gear to a lower gear - as well as a state-machine (c) that defines the status of the system (e.g., no shifting, upshifting). Each shifting map outputs a next-gear command $\gamma_{d n}(t)$ and $\gamma_{u p}(t)$ based on the current accelerator pedal position a $\mathrm{a}(\mathrm{t})$ and vehicle speed $\mathrm{V}(\mathrm{t})$. The state machine is composed of different states, of which only one is active at any time step; a change in state occurs whenever a transition condition from the active state becomes true (i.e., an upshift will occur only if a set of conditions is true).

The state that is active most of the time is the hold-gear state (d), which makes sense because, most of the time, for drivability reasons, the vehicle should be in gear and not shifting. An upshift occurs when the upshifting gear $\gamma_{u p}(t)$ is strictly higher than the current gear $\gamma(\mathrm{t})(1)$ (e.g., $\gamma_{u p}(t)=5$ and $\left.\gamma(t)=4\right)$.

For all vehicles, the shift does not necessarily happen instantly when the command to shift is given, depending on the current pedal position. In aggressive driving, i.e., at high accelerator-pedal positions (5), the shift happens as soon as the gear transition (1) becomes true, ensuring optimal performance. In contrast, in "normal" driving, i.e., at low pedal positions (2), there is an intermediate state (e) that allows 
the shift only when the gear condition (1) is true for a minimum time $\tau$. This constraint is imposed to avoid an excessive number of shifting events, which would lead to unacceptable drive quality and increased energy consumption. The upshifting itself is executed in state (f), in which the shift command $\gamma_{c m d}(t)$ is incremented (i.e., the next upper gear is selected); once the shifting is completed (6), the state machine comes back to the hold-gear state (d). Downshifting occurs in a similar way.

As an additional level of robustness in the Autonomie control algorithm, an upshift or downshift cannot occur if the resulting engine speed would be too low or too high, respectively. This approach ensures that the engine is not operated below idle or above its maximum rotational speed, as shown in Figure 50 .

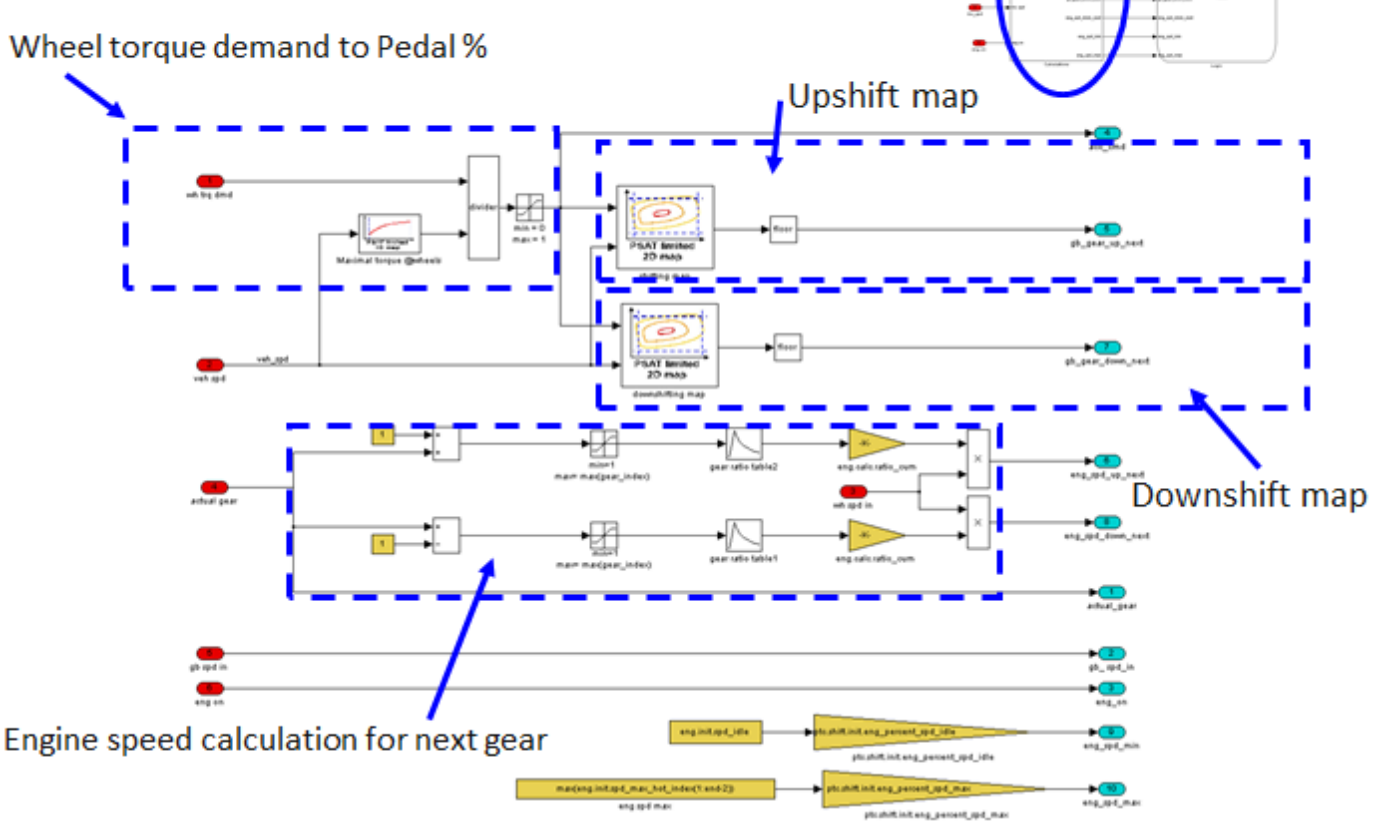

Figure 50. Shifting calculations in Autonomie

\subsubsection{4. $\quad$ Torque Control During Shifting Events for Automatic Transmission}

Figure 51 shows the transmission clutch pressure, output torque, and engine speed curves during a change from first to second gear. The output torque experiences both a trough period (lower than the torque in the original gear) and a crest period (higher than the torque in the original gear). The trough period is called a torque hole, while the crest period is called a torque overshoot. The torque hole is defined by depth and width, where the depth is the difference between minimum torque and the torque in previous gear, and the width is the half value of the maximum width of the torque hole. 


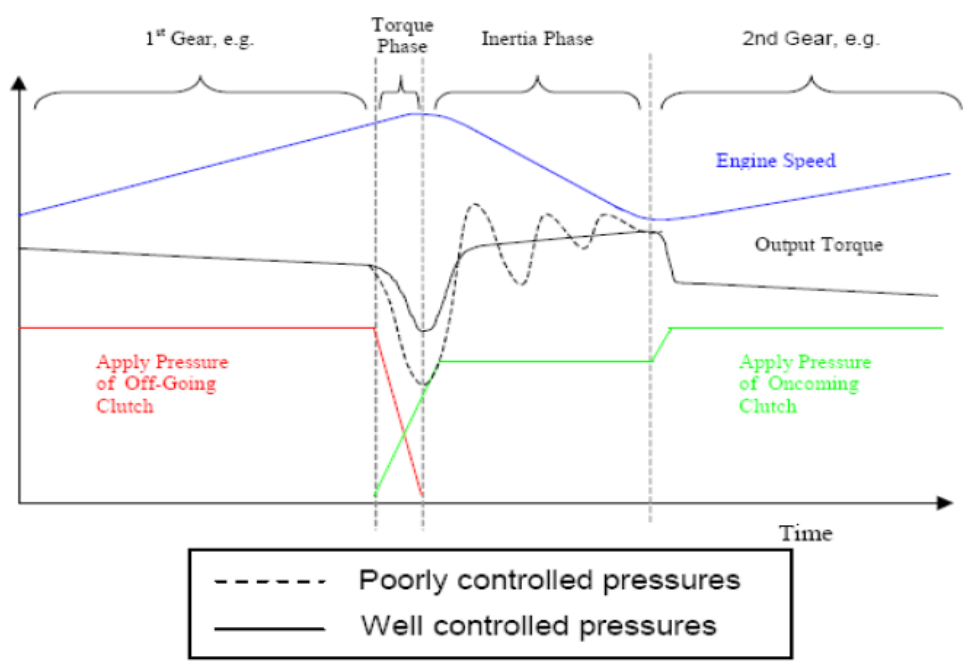

Figure 51. Shift process for automatic transmission

The bigger the torque hole, the larger the decrease of torque in torque phase, resulting in a more significant reduction in acceleration. Because the decrease in acceleration causes discomfort for both the driver and passengers, the torque hole should be as shallow and narrow as possible. Torque reduction behavior is a well-known phenomenon, observed during vehicle testing and referenced in several papers and presentations.

Autonomie integrates a low-level control algorithm that reproduces the torque hole phenomenon. Figure 52 illustrates, in detail, the behavior of the vehicle model for a short period of time ( $205 \mathrm{sec}$ to $205.8 \mathrm{sec}$ ). The area highlighted by the oval outline indicates the torque hole during a shifting event.

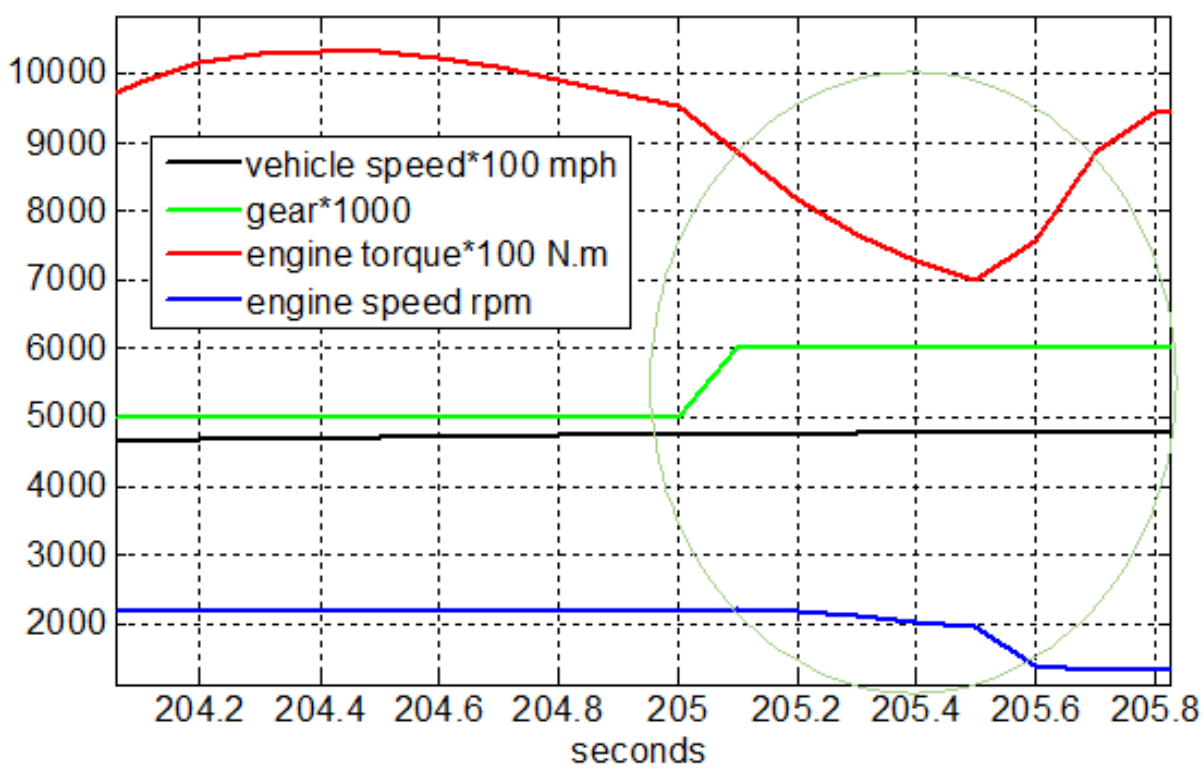

Figure 52. Torque hole in Autonomie during shifting event 


\subsubsection{Gear Skip-Shifting Strategy}

For the current set of runs, a gear skip-shifting strategy was developed and implemented for 10-speed transmissions using the Ford F-150 10-speed benchmarking [18].

Table 3 below is presented in the report outlined for F-150 benchmarking:

Table 3. Detailed Gear-Shifting Events for Ford F-150 [18]

\begin{tabular}{|l|c|c|c|c|c|c|c|c|c|c|c|c|c|c|}
\hline $\begin{array}{c}\text { \# of } \\
\text { shifts }\end{array}$ & $\mathbf{1 - 2}$ & $\mathbf{1 - 3}$ & $\mathbf{2 - 3}$ & $\mathbf{2 - 4}$ & $\mathbf{3 - 4}$ & $\mathbf{3 - 5}$ & $\mathbf{4 - 5}$ & $\mathbf{4 - 6}$ & $\mathbf{5 - 6}$ & $\mathbf{6 - 7}$ & $\mathbf{7 - 8}$ & $\mathbf{8 - 9}$ & $\mathbf{9 - 1 0}$ & Total \\
\hline UDDS & & 18 & & & 14 & 5 & 16 & 1 & 21 & 12 & 4 & 1 & 2 & $\mathbf{9 4}$ \\
\hline Highway & & 1 & & & 1 & & 1 & & 1 & 2 & 2 & 5 & 5 & $\mathbf{1 8}$ \\
\hline US06 & 1 & 6 & & 1 & & 7 & 1 & & 9 & 5 & 7 & 5 & 11 & $\mathbf{5 3}$ \\
\hline
\end{tabular}

From the table, a fairly consistent gear-skipping can be observed for the drive cycles covered in the study. A similar gear-skipping method was implemented for this analysis. Table 4 details the shifting events for the different cycles in simulation of pickup-nonperfo/micro hybrid/eng12/MR0/AERO0/ROLL0.

Table 4. Argonne Simulated Gear-Shifting Events for 10-Speed Transmission

\begin{tabular}{|l|c|c|c|c|c|c|c|c|c|c|c|c|}
\hline Cycles & $1-2$ & $\mathbf{1 - 3}$ & $\mathbf{2 - 3}$ & $\mathbf{3 - 4}$ & $\mathbf{3 - 5}$ & $\mathbf{4 - 5}$ & $\mathbf{5 - 6}$ & $\mathbf{6 - 7}$ & $\mathbf{7 - 8}$ & $\mathbf{8 - 9}$ & $\mathbf{9 - 1 0}$ & Total \\
\hline UDDS & & 18 & & & 18 & & 21 & 12 & 1 & 1 & 2 & $\mathbf{7 3}$ \\
\hline Highway & & 1 & & & 1 & & 1 & 2 & 2 & 4 & 3 & $\mathbf{1 3}$ \\
\hline US06 & & 7 & & & 8 & & 8 & 7 & 5 & 6 & 4 & $\mathbf{4 6}$ \\
\hline
\end{tabular}

\subsubsection{Engine Start Control for Pre-Transmission HEVs}

The vehicle speed, engine speed, and electric motor torque for engine start-up are compared with the vehicle test results for $\mathrm{t}=21-24 \mathrm{sec}$ in Figure 53. The single clutch (CPL2) is located inside the electric machine, between the engine and the electric machine. The clutch is engaged when starting the internal combustion engine (ICE). 

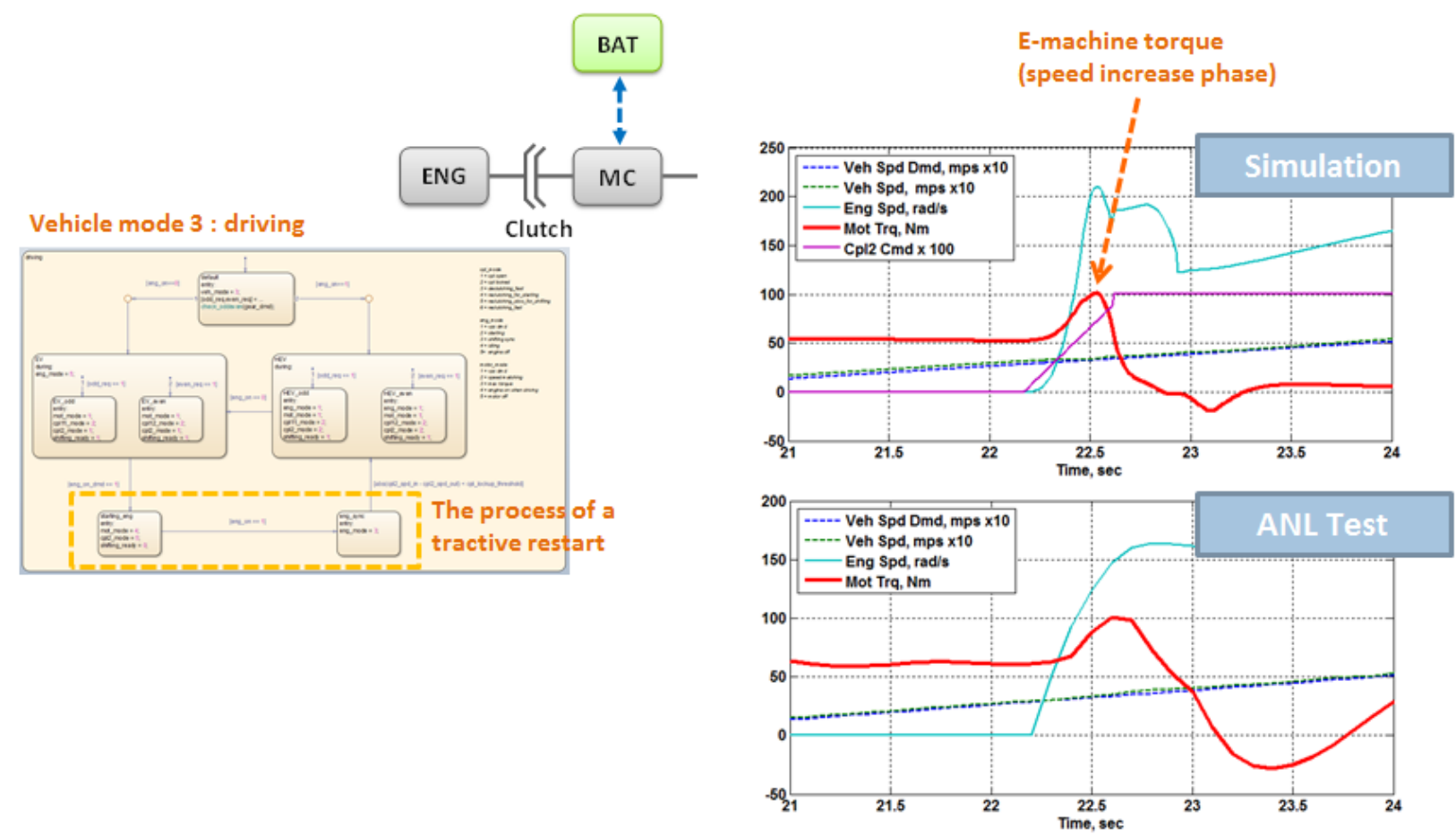

Figure 53. Engine start transient control using electric machine

The electric machine torque is also controlled to quickly synchronize the clutch input and output speed. The controller reacts by setting a torque-increasing intervention that is added to the torque of the electric machine in the speed increase phase.

\subsection{Torque Converter}

A torque converter is a hydrodynamic fluid coupling used to transfer rotating power from a prime mover, such as an ICE, to a rotating driven load. It consists of an impeller (drive element), a turbine (driven component), and a stator (a set of stationary windings that generate a magnetic field) that assists the torque converter function. The torque converter is filled with oil and transmits the engine torque by means of the flowing force of the oil. The device compensates for speed differences between the engine and the other drivetrain components and is therefore ideally suited for the start-up function.

The torque converter is modeled as two separate rigid bodies when the coupling is unlocked and as one rigid body when the coupling is locked. The downstream portion of the torque converter unit is treated as being rigidly connected to the drivetrain. Therefore, there is only one degree of dynamic freedom, and the model has only one integrator. This integrator is reset when the coupling is locked, which corresponds to the loss of the degree of dynamic freedom. Figure 54 shows the efficiency of the torque converter used for the study.

The effective inertias are propagated downstream until the point where actual integration takes place. When the coupling is unlocked, the engine inertia is propagated up to the coupling input, where it is used for calculating the rate of change of the input speed of the coupling. When the coupling is locked, the engine inertia is propagated all the way to the wheels. 


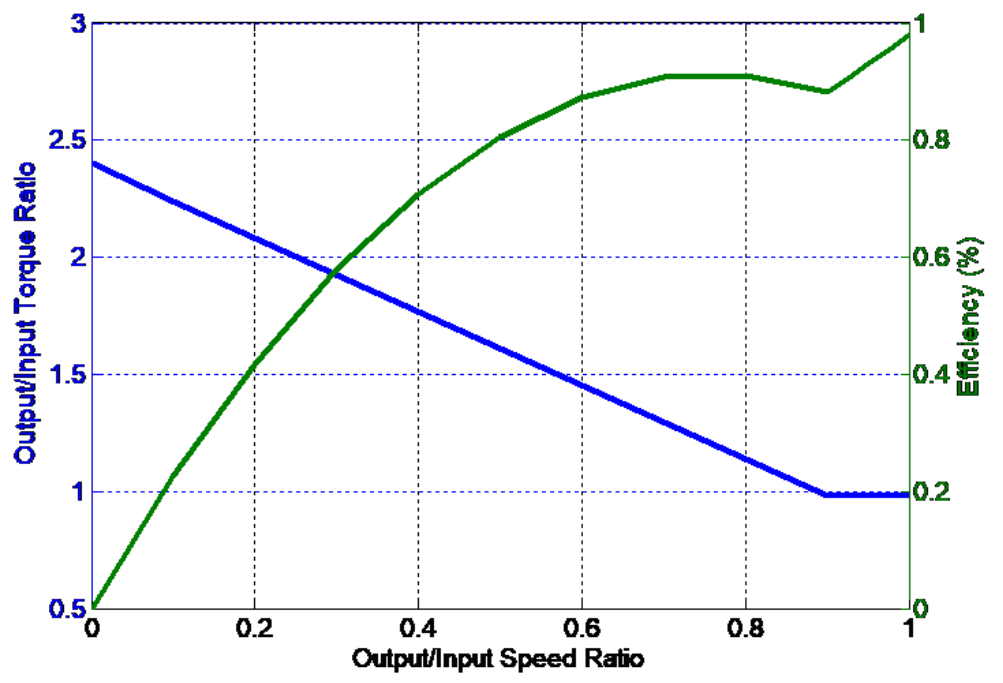

Figure 54. Typical torque converter efficiency

Figure 55 describes the conditions under which the torque converter will be locked. The same algorithm is used to represent current torque converter lockup logic as well as future aggressive lockup logic. The torque converter is used as a start-up device in first gear, with very low slip (torque ratio of 0.95) at higher speeds in first gear. Recent trends in torque-converter technology suggest operation in locked or controlled-slip mode in second and higher gears. In general, the torque converter is in controlled slip mode or mechanically locked, depending on vehicle speed and pedal position, for each gear apart from the first. To suggest advances in torque-converter technology, it was assumed that the torque converter would be in a mechanically locked state for the second and higher gears. This approach has been applied to all transmissions with six gears or more. The logic does not change between different automatic transmissions types (AU/AU+/AU++).

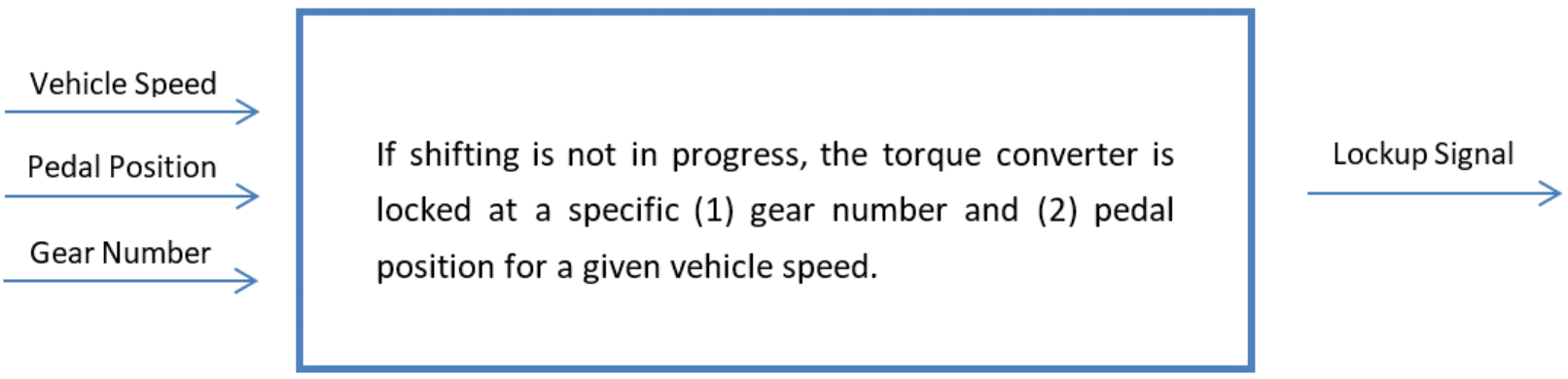

Figure 55. Torque converter lockup control algorithm

\subsubsection{Plant Model}

Figure 56 shows the main I/O of the torque converter model. The torque converter model is based on a lookup table, which determines the output torque depending on the lockup command. The upstream acceleration during slip and the downstream acceleration are taken into account in calculating the output speed. 


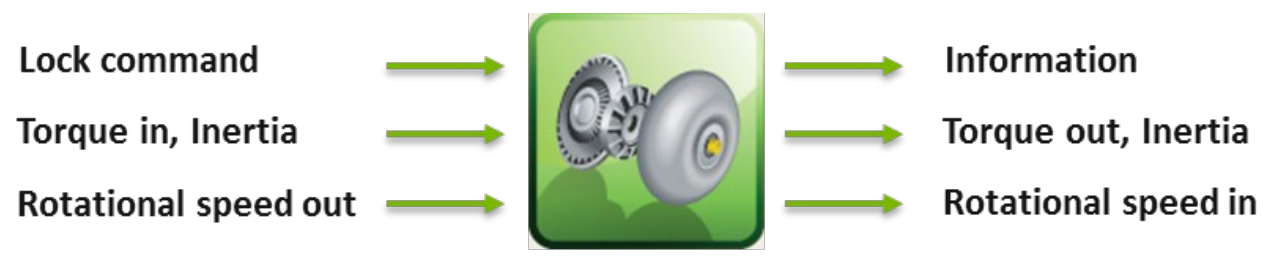

Figure 56. Autonomie torque converter model I/O

\subsubsection{Operational Modes}

The different operational modes are described below.

- Mode 1: Idle

We enter this mode if $\mathrm{W}_{\text {in }}<\mathrm{W}_{\text {eng_idle }}+$ Threshold. We quit the mode if $\mathrm{W}_{\mathrm{in}}>\mathrm{W}_{\text {eng_idle }}+$ Threshold and $\mathrm{T}_{\text {in }}$ is increasing and positive.

- Mode 2: Acceleration (transient calculation)

We are in this mode if $\mathrm{T}_{\text {in }}>0$ and Speed_Ratio $<0.1$ (meaning $\mathrm{W}_{\text {in }} \gg \mathrm{W}_{\text {out }}$ ).

- Mode 3: Steady state

We are in this mode if $\mathrm{W}_{\text {in }}>\mathrm{W}_{\text {eng_idle }}+$ Threshold and $\mathrm{W}_{\text {in }}$ is close to $\mathrm{W}_{\text {out }}$

OR Speed_Ratio $>0.8\left(\mathrm{~W}_{\text {in }}\right.$ close to $\left.\mathrm{W}_{\text {out }}\right)$

OR $\mathrm{T}_{\text {in }}<0$.

- Mode 4: Locked

We enter this mode if the torque converter command is 1 .

\subsubsection{Steady-State Calculation}

Figure 57 details the steady-state operational mode calculation of the torque converter model.

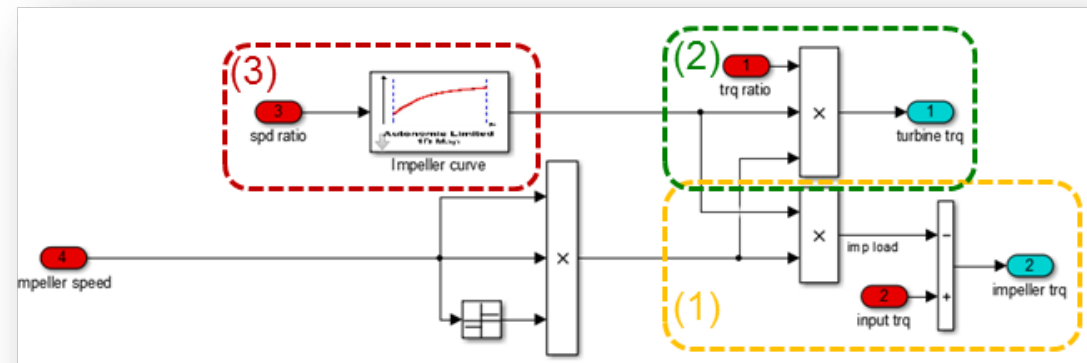

Figure 57. Steady-state mode calculation 


$$
\begin{aligned}
& T_{\text {impeller }}=T_{\text {in }}-\left[K_{\text {in }} \times \omega_{\text {impeller }}^{2} \times \operatorname{sign}\left(\omega_{\text {impeller }}\right)\right] \\
& T_{\text {turbine }}=T_{\text {ratio }} \times K_{\text {in }} \times \omega_{\text {impeller }}^{2} \times \operatorname{sign}\left(\omega_{\text {impeller }}\right) \\
& K_{\text {in }}=\frac{1}{\text { capacity_factor }^{2}}=f\left(\omega_{\text {ratio }}\right) \\
& T_{\text {ratio }}=f\left(\omega_{\text {ratio }}\right) \\
& \omega_{\text {impeller }}=\int \frac{T_{\text {impeller }}}{J_{\text {impeller }}} \\
& \omega_{\text {ratio }}=\frac{\omega_{\text {turbine }}}{\omega_{\text {impeller }}}
\end{aligned}
$$

\subsubsection{Transient Calculation}

Figure 58 details the transient operational mode calculation of the torque converter model.

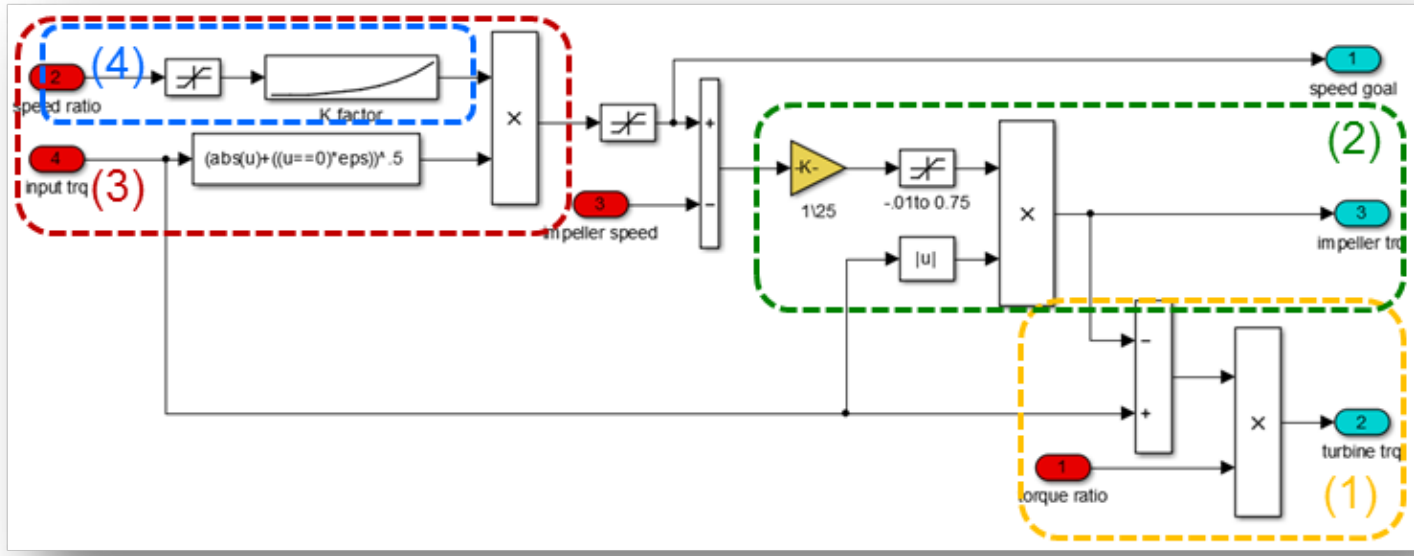

Figure 58. Transient mode calculation

(36)

(37) $T_{\text {impeller }}=\left|T_{\text {in }}\right| \times \frac{\omega_{\text {goal }}-\omega_{\text {impeller }}}{\text { gain }}$

$$
\begin{aligned}
& \omega_{\text {goal }}=\text { capacity_factor } \times T_{\text {in }}^{0.5} \\
& \text { capacity_factor }=\frac{\omega_{\text {impeller }}}{\sqrt{T_{\text {impeller }}}}=f\left(\omega_{\text {ratio }}\right)
\end{aligned}
$$

$$
\omega_{\text {impeller }}=\int \frac{T_{\text {impeller }}}{J_{\text {impeller }}}
$$




\subsubsection{Idle Calculation}

Figure 59 details the idle operational mode calculation of the torque converter model. Figure 60 details the list of initialization parameters for the torque converter model in Autonomie.

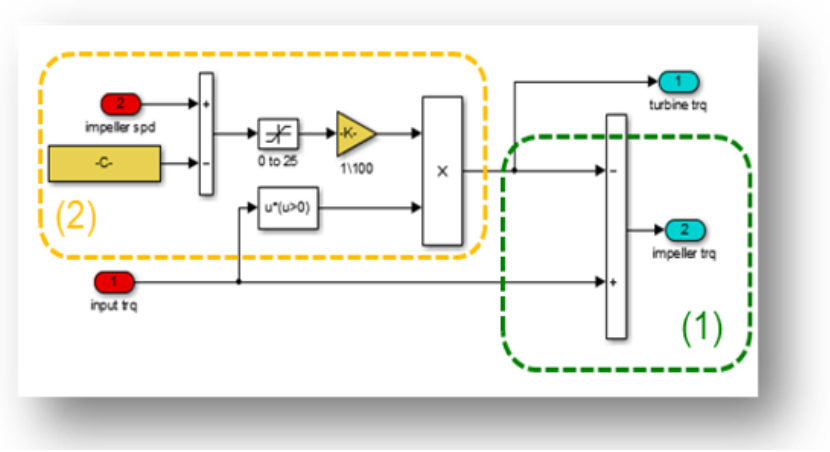

(1) $T_{\text {impeller }}=T_{\text {in }}-T_{\text {turbine }}$

(2) $T_{\text {turbine }}=T_{\text {in }} \cdot \frac{\omega_{\text {impeller }}-\omega_{\text {engine_idle }}}{\text { gain }}$

$\omega_{\text {impeller }}=\int \frac{T_{\text {impeller }}}{J_{\text {impeller }}}$

Figure 59. Idle mode calculation

\begin{tabular}{|c|c|c|c|}
\hline Name & Unit & '́ption & Size \\
\hline cpl.plant.init.trq_impeller.idx1_spd & $\mathrm{rad} / \mathrm{s}$ & Impeller speed index used to index imperler torque map; used in calculating the " $\mathrm{k}$ " factor & $1 * n$ \\
\hline cpl.plant.init.trq_impeller.map & $\mathrm{Nm}$ & Impeller torque map indexed by fímpeller speed index; used in calculating the "k" factor & $1 * n$ \\
\hline cpl.plant.init.k_factor.idx1_spd_ratio & - & Speed ratio yéctor used to index the torque ratio map or $\mathrm{k}$ factor & $1^{*} \mathrm{n}$ \\
\hline cpl.plant.init.k_factor.map & $\begin{array}{l}(\mathrm{rad} / \mathrm{s}) / \\
\mathrm{sqrt}(\mathrm{Nm})\end{array}$ & $\begin{array}{l}\text { Torque converter "k" value calculated from impeller speed index and impeller torque } \\
\text { map used in the transient calculation block to calculate the desired impeller speed }\end{array}$ & $1 * \mathrm{n}$ \\
\hline
\end{tabular}

Figure 60. Initialization parameters

\subsection{Energy Storage Model}

The energy storage system block models the battery pack as a charge reservoir and an equivalent circuit. The equivalent circuit accounts for the circuit parameters of the battery pack as if it were a perfect open-circuit voltage source in series with an internal resistance and two resistor-capacitor (RC) circuits that represent the polarization time constants. Figure 61 shows the main I/O of the energy storage model.

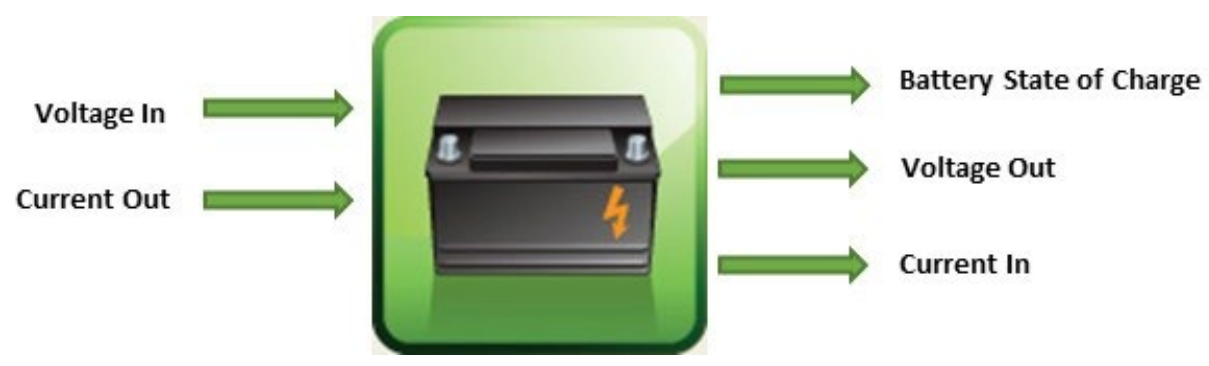

Figure 61. Autonomie energy storage model I/O

Autonomie includes several energy storage models for use in various high power and high energy applications: 
- High-power application: One battery model is used as a charge reservoir. An equivalent circuit, the parameters of which are a function of the remaining charge in the reservoir (also known as the state of charge $[\mathrm{SOC}]$ ), accounts for circuit parameters of the battery pack as if it were a perfect open-circuit voltage source in series with an internal resistance.

- High-energy application: Another battery model in Autonomie, used for high-energy batteries, uses two time constants to represent the polarization behavior of the battery pack. This lumped parameter model can represent internal resistance, capacitance, and open-circuit voltage - all maps based on SOC and in some cases temperature - for many different battery chemistries

The pulse power limits of the battery pack are another important aspect to consider for sizing. There are several different options to represent the maximum power of the battery. The main one represents maximum power as a function of SOC. Other models introduce a time constraint for the maximum power. These battery packs have different power limits for 10-second, 2-second, and continuous power. The model accounts for the duration of the pulse and limits power accordingly. This aspect is not necessarily a feature of the plant, but rather is handled by the low-level control and is dependent on the battery chemistry and plant's performance characteristics.

\subsubsection{High-Power Battery Model (Used for Hybrid Electric Vehicles)}

Figure 62 shows the top-level diagram of the high-power battery model.

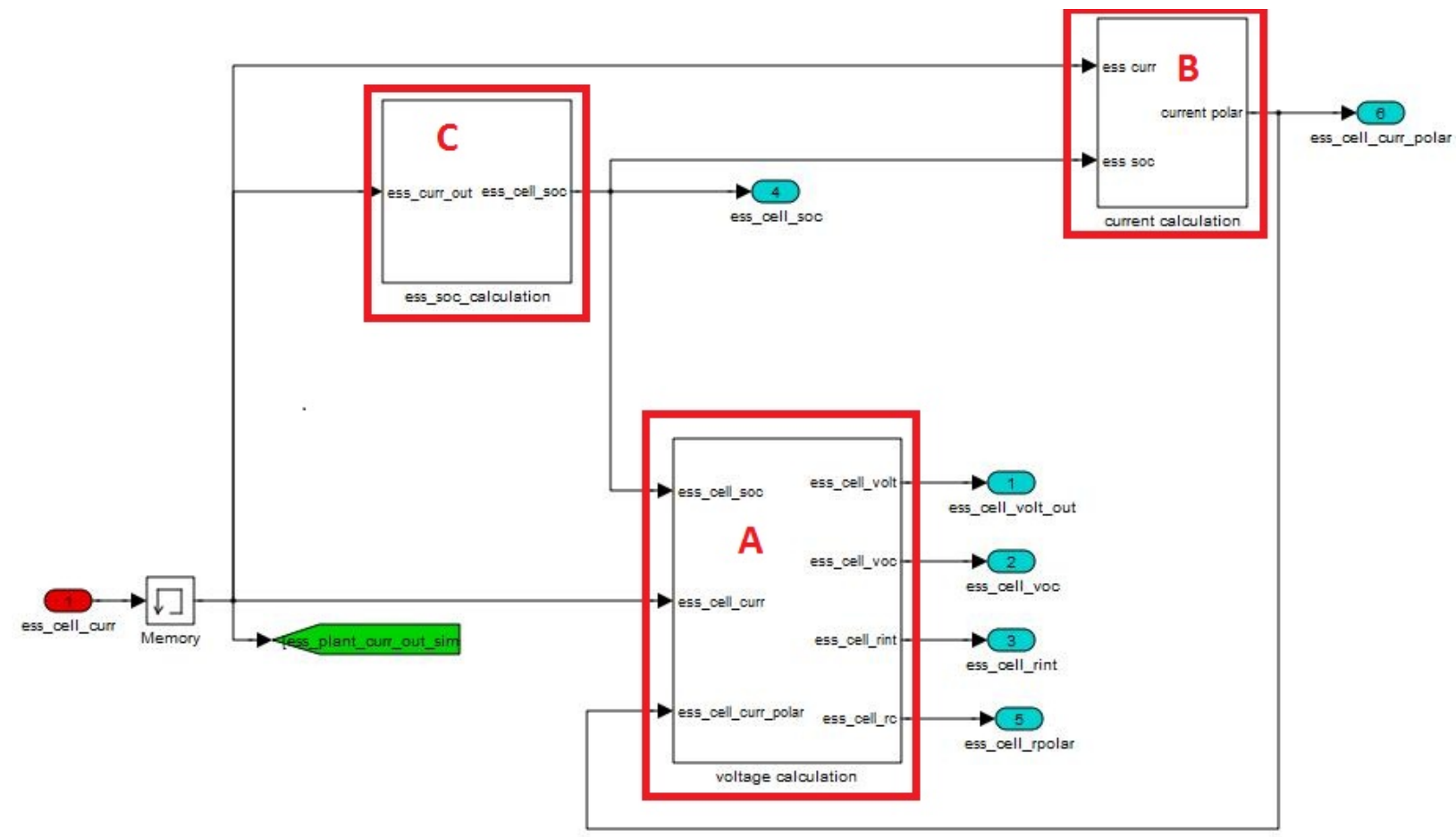

Figure 62. High-power battery model diagram in Autonomie

The top-level diagram of the high-power model consists of three main blocks: the voltage calculation (Block A), the current calculation (Block B), and the SOC calculation (Block C).

Block A (Figure 63) calculates the output voltage by taking the open-circuit voltage, which depends on SOC, and subtracting three terms: the voltage drop due to the internal resistance of each cell, the 
voltage drop due to the polarization resistance of each cell, and the voltage drop due to an effective series capacitance, modeling the variation of output voltage with the time integral of the current.

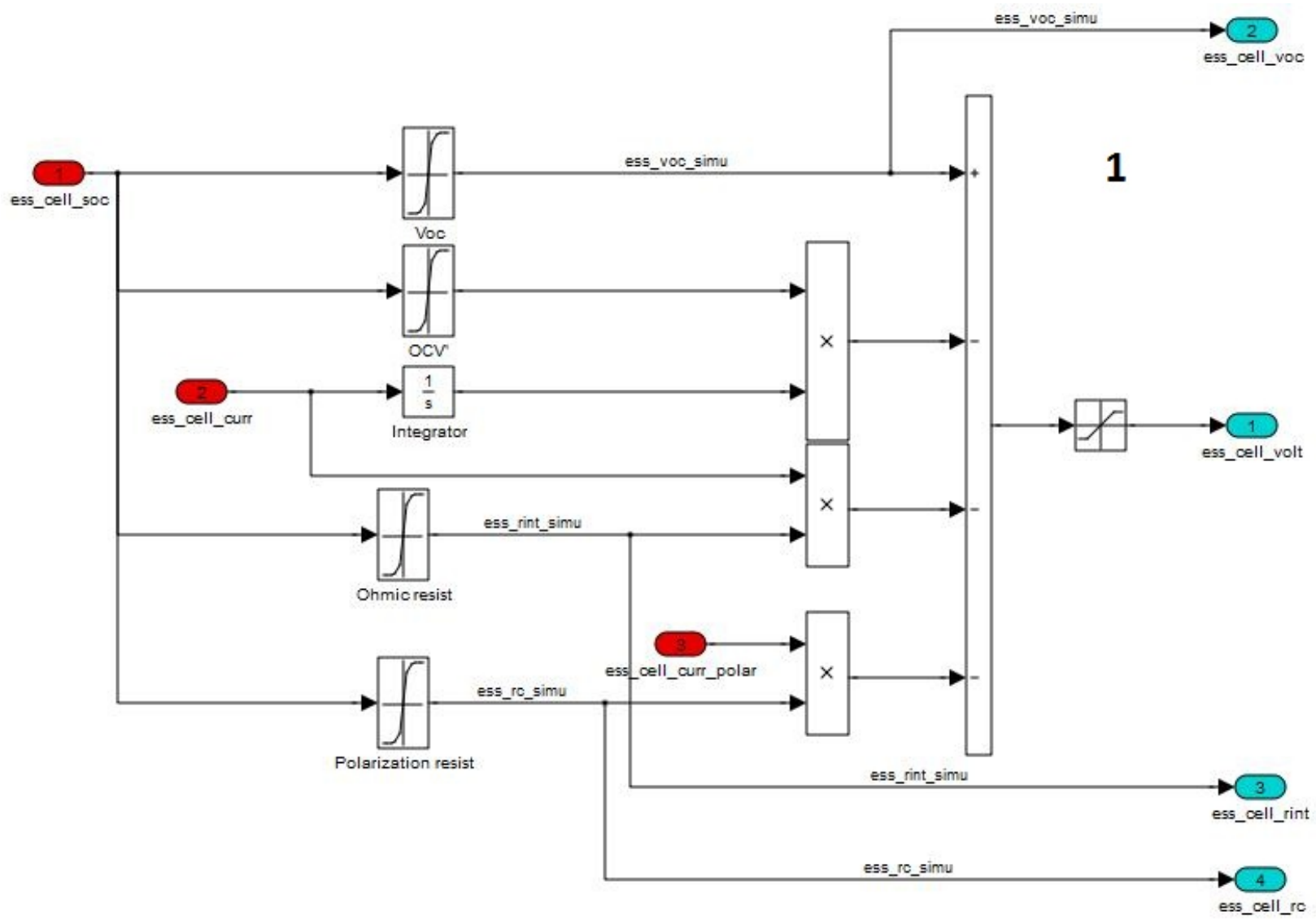

Figure 63. Block A: High-power battery model output voltage calculation

The polarization current causes an additional voltage drop at the terminals of the battery. As current continues to flow in one direction through the cell, this voltage drop increases. As the ions migrate in a lithium-ion cell, it takes more work to keep the current flowing in the same direction. However, when the current reverses, the migrated ions facilitate current flow in the reverse direction.

$$
V_{\text {out }}=V_{\text {oc }}-O C V \times \int I_{\text {load }} \times d t-R_{0} \times I_{\text {load }}-R_{p} \times I_{p}
$$

Where:

$\mathrm{I}_{\text {load }}=$ current flowing into the load, that is, the input current from the voltage bus

$\mathrm{OCV}=$ series capacitance of the battery modeling the variation of output voltage with the time integral of the current

$\mathrm{R}_{0}=$ series internal resistance of a cell

$\mathrm{R}_{\mathrm{p}}=$ polar resistance of a cell

$\mathrm{I}_{\mathrm{p}}=$ polar current in a cell

In Block B (Figure 64), the polarization current is calculated by solving differential Equation 41. 


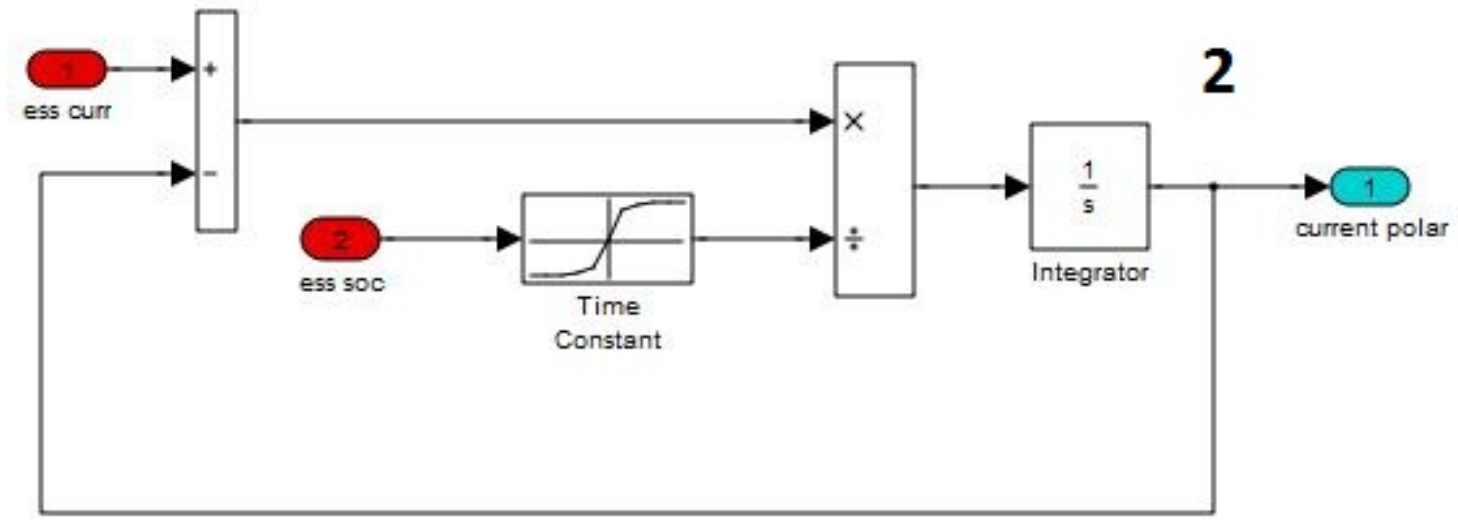

Figure 64. Block B: High-power battery model current calculation

$$
\tau_{p}(S O C) \times \frac{d I_{p}}{d t}+I_{p}=I_{l o a d}
$$

In Block C (Figure 65), the SOC for the battery is calculated. If the SOC drops too low, the stop block automatically stops the simulation.

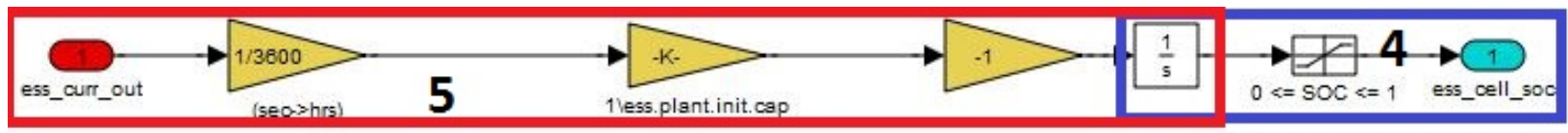

Figure 65. Block C: High-power energy model SOC calculation

$$
\begin{aligned}
& S O C=S O C_{\text {init }}+\triangle S O C \\
& \triangle S O C=-\int \frac{I_{\text {in }}}{\text { Capacity }_{\max }}
\end{aligned}
$$

Where

$\mathrm{SOC}_{\text {init }}=$ initial SOC (initial value of the integrator)

$\mathrm{I}_{\text {in }}=$ input current into the battery from the bus

Capacity_max $=$ maximum charge capacity

The SOC is calculated by determining the variation of charge in the battery and dividing it by the maximum coulombs the battery can store. A value of 0 is the unattainable state of having no charge remaining in the battery, while a value of 1 is the unattainable state of having a perfectly charged battery. Attempting to reach either of these values in practice would damage the battery and result in a short life cycle.

In practice, different values of minimum and maximum SOC values are used, depending on the battery chemistry and applications.

\subsubsection{High Energy Battery Model (Used for PHEVs and Battery Electric Vehicles)}

Unlike the high-power battery model, the high-energy battery model utilizes two polarization resistances. The top-level diagram of the high-energy battery model in Figure 66 comprises three main 
blocks: the voltage calculation (Block A), the current calculation (the two B blocks correspond to the two current polarizations), and the SOC calculation (Block C).

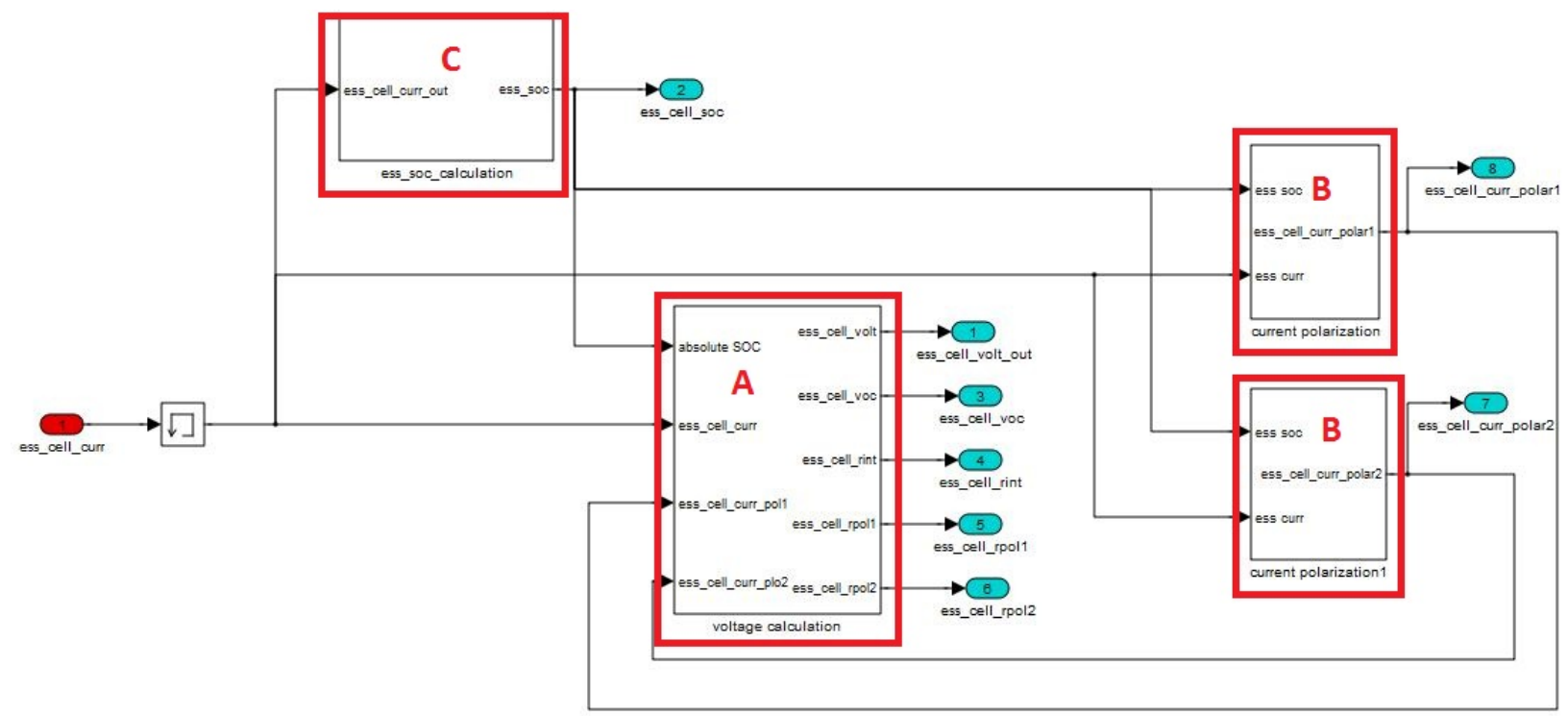

Figure 66. High-energy battery model diagram in Autonomie

\subsection{Electric Machine Models}

The electric machine transforms electrical power into mechanical power by creating a magnetic field that applies a force to current-carrying conductors. Electric machines that operate on this principle can be divided into two main categories: direct current (DC) electric machines and alternating current (AC) electric machines. DC electric machines can be further divided into electric machines with and without brushes. AC electric machines can also be divided into two main categories: synchronous and asynchronous. The AC electric machine categories can be even further subdivided based on the number of phases. Figure 67 shows the main I/O of the electric machine model in Autonomie.

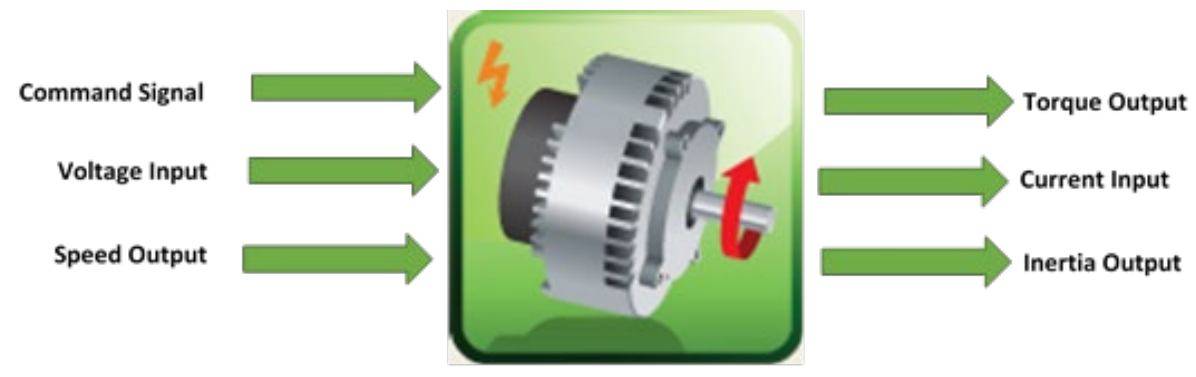

Figure 67. Autonomie electric-machine model I/O

All electric machines consist of two major parts: the stator, a set of stationary windings that generate a magnetic field that encompasses the rotor or armature, and the second part, the rotor or armature, which is the rotating part.

Electric machine plant models in Autonomie use torque or power as the command and produce a torque output. The electric machine operating speed is determined by components connected to the electric machine. In a vehicle, the vehicle speed and gear ratios determine the electric machine operating 
speed. The lookup table used in an electric machine model estimates operational losses over the entire operating region.

Typically, an electric machine has a continuous operating region (a region under the continuous torque curve) and a transient region in which the electric machine can operate for a short period of time. The peak torque capability of an electric machine is defined for a specific duration, such as 30 seconds. The maximum torque output gets de-rated to continuous torque levels when the electric machine's temperature increases. The electric machine model in Autonomie has this general logic built into it.

Figure 68 shows the general map-based electric machine model used in Autonomie. It has three essential maps:

- Continuous torque as a function of speed

- Maximum torque as a function of speed

- A four-quadrant efficiency map as a function of speed and torque

A warm-up-time constant is used to interpolate between the maximum and continuous torque curves of the electric machine. The maximum-torque curve and efficiency map do not depend on the electric machine input voltage. Except for the fuel-cell-only configuration, this is the default electric machine model used in all of configurations.

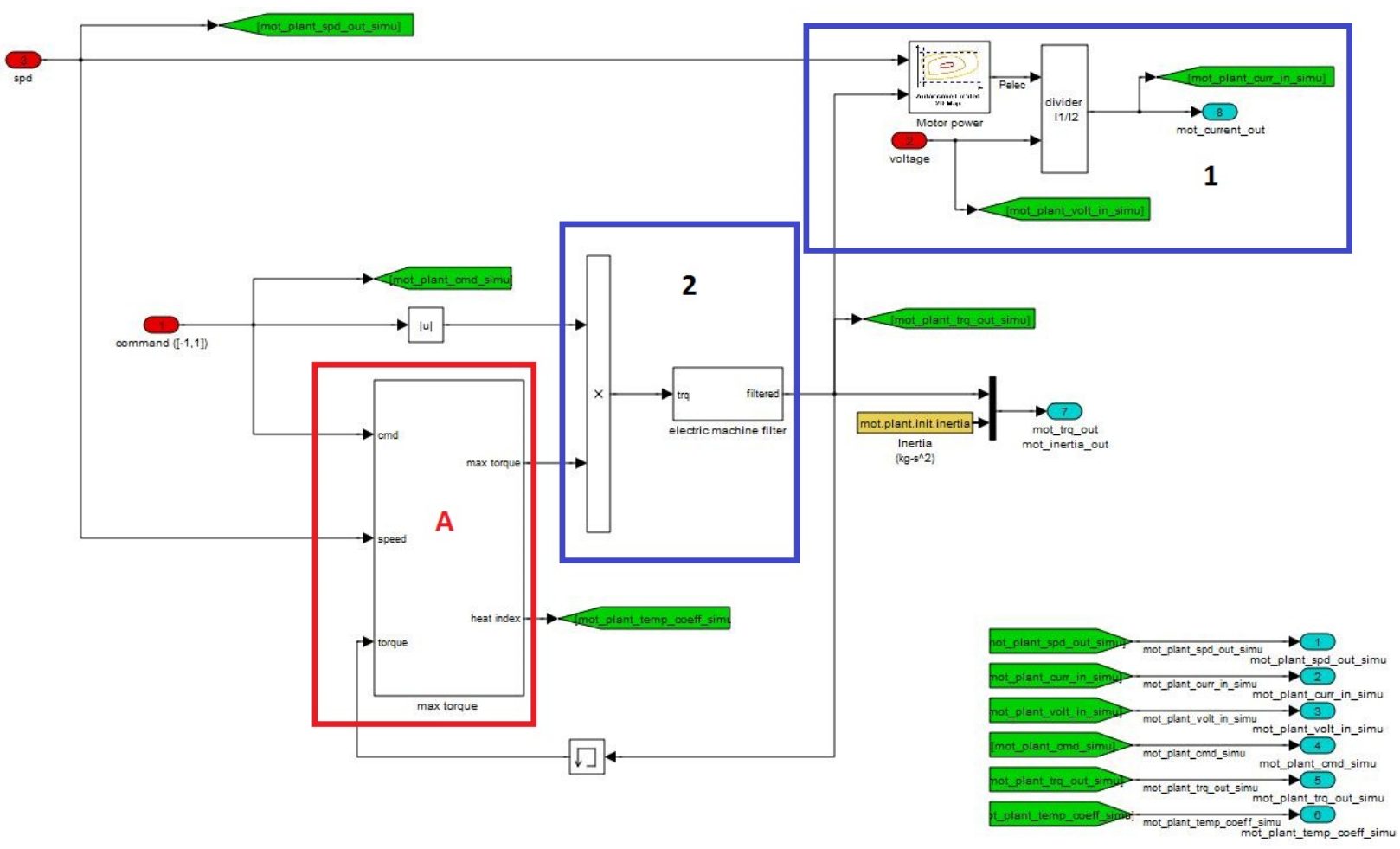

Figure 68. Autonomie electric machine model in Simulink

Equation 44 calculates the electric machine current:

$$
I_{\text {in }}=\frac{P_{\text {electrical }}}{V_{\text {in }}}
$$


Equation 45 computes the electric machine output torque by using the electric machine command and the maximum available torque of the electric machine at a given temperature and speed.

$$
T_{\text {out }}=T_{\max } \times P W M_{c m d}
$$

Figure 69 shows the diagram of Block A for the max torque calculation of the electric machine model.

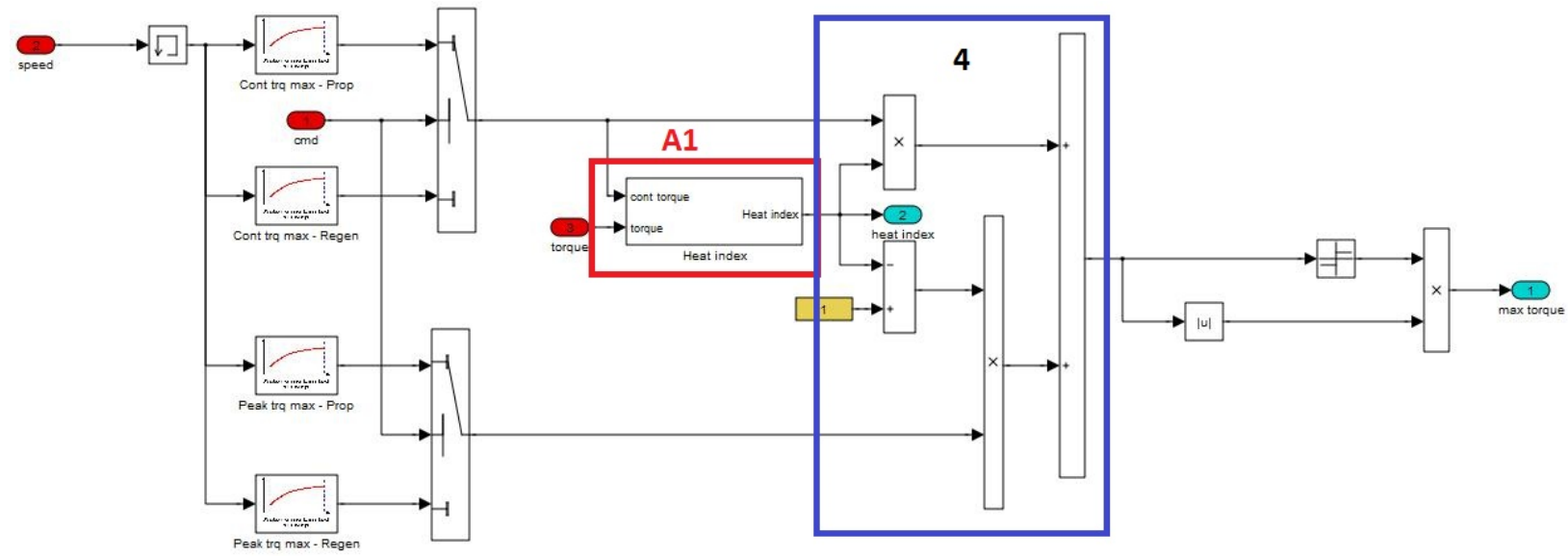

Figure 69. Block A: Electric machine model max torque calculation

Equation 46 interpolates between the continuous-torque curve and the maximum-torque curve by using the heat index. If the electric machine is hot, then the continuous-torque curve is used (that is, the heat index is 1). If the electric machine is at its operating temperature, then the maximum-torque curve is used.

$$
T_{\text {max }} \text { mechanicl }=T_{\text {cont }} \times P W M_{\text {heatindex }}+T_{\text {Peak }} \times\left(1-P W M_{\text {heatindex }}\right)
$$

Equations 47 through 51 show the dependence of the electric machine torque curves on the electric machine speed.

$$
\begin{aligned}
& T_{\text {cont }}=T_{\text {cont }_{\text {prop }}}=\operatorname{function}\left(W_{\text {in }}\right) \\
& T_{\text {Peak }}=T_{\text {peak }} \text { prop } \\
& T_{\text {cont }}=T_{\text {cont }_{\text {regen }}}=\operatorname{function}\left(W_{\text {in }}\right) \\
& T_{\text {peak }}=T_{\text {peak }} \text { regen } \\
& T_{\text {max }_{\text {electrical }}}=\operatorname{function}\left(W_{i n}\right)
\end{aligned}
$$

Figure 70 shows Block A1 for the heat index calculation of the electric machine model. 


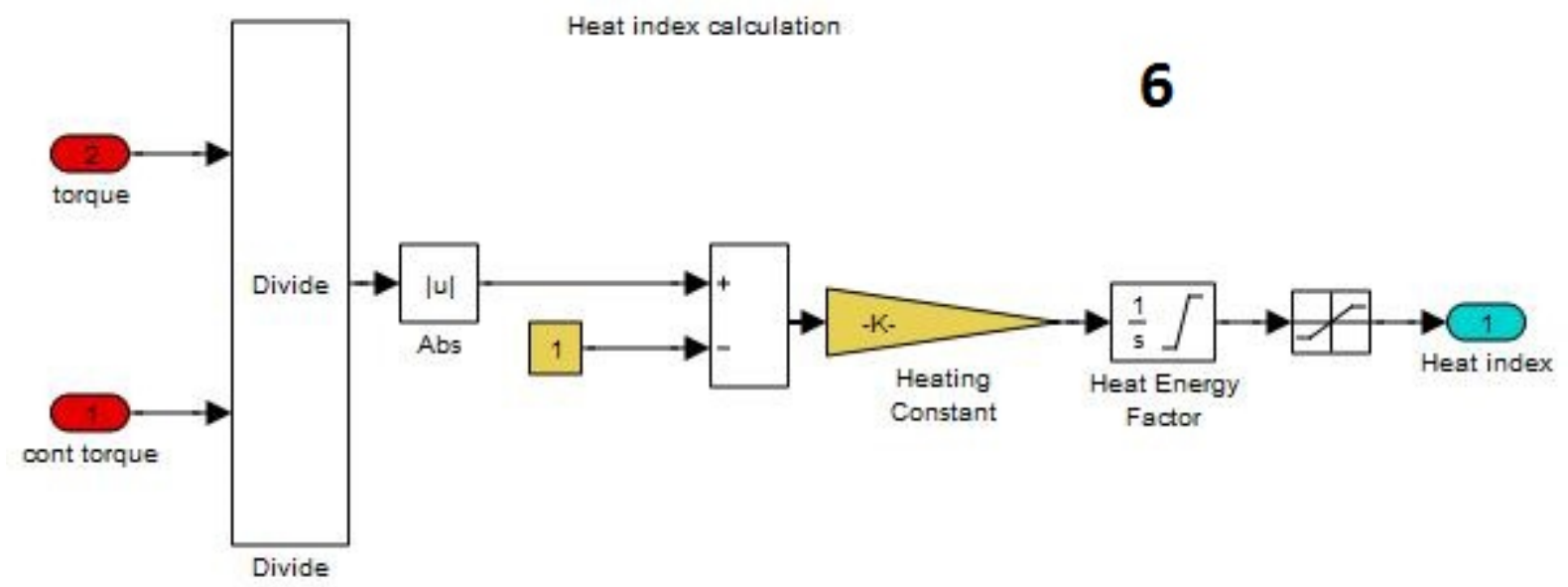

Figure 70. Block A1: Heat index

Equation 52 computes the heat index of the electric machine.

$$
P W M_{\text {Heatindex }}=-0.3+\int \frac{0.3}{\tau} \times\left(\frac{T_{\text {out }}}{T_{\text {cont }}}-1\right) \times d t
$$

\subsection{Electrical Accessories Model}

Electrical accessories, such as lamps, radiator fans, and wipers, obtain their energy from an electrical source. They represent dedicated auxiliary load systems.

The plant model of an electrical accessory loss uses a constant power draw. The model considers the electrical losses associated with the powertrain. The current losses are taken from the energy storage. While the accessory load varies under real-world driving conditions, it is fairly constant during standard driving cycles. Therefore, the approach of constant power draw is valid for the study. Figure 71 shows the main $\mathrm{I} / \mathrm{O}$ of the electrical accessories model.

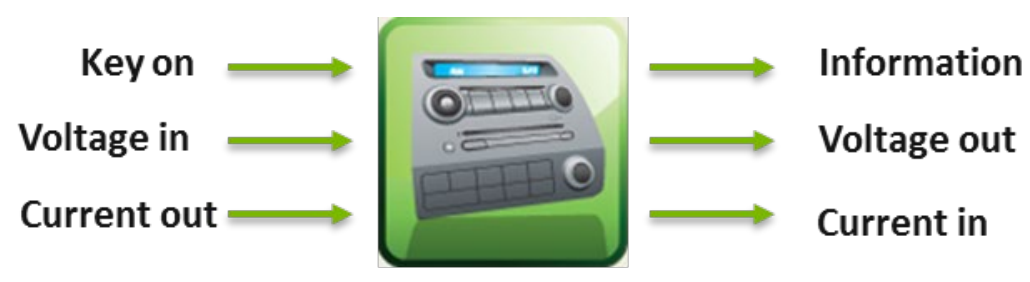

Figure 71. Autonomie electrical accessories model I/O

Figure 72 shows the Simulink diagram for the electrical accessories model. 


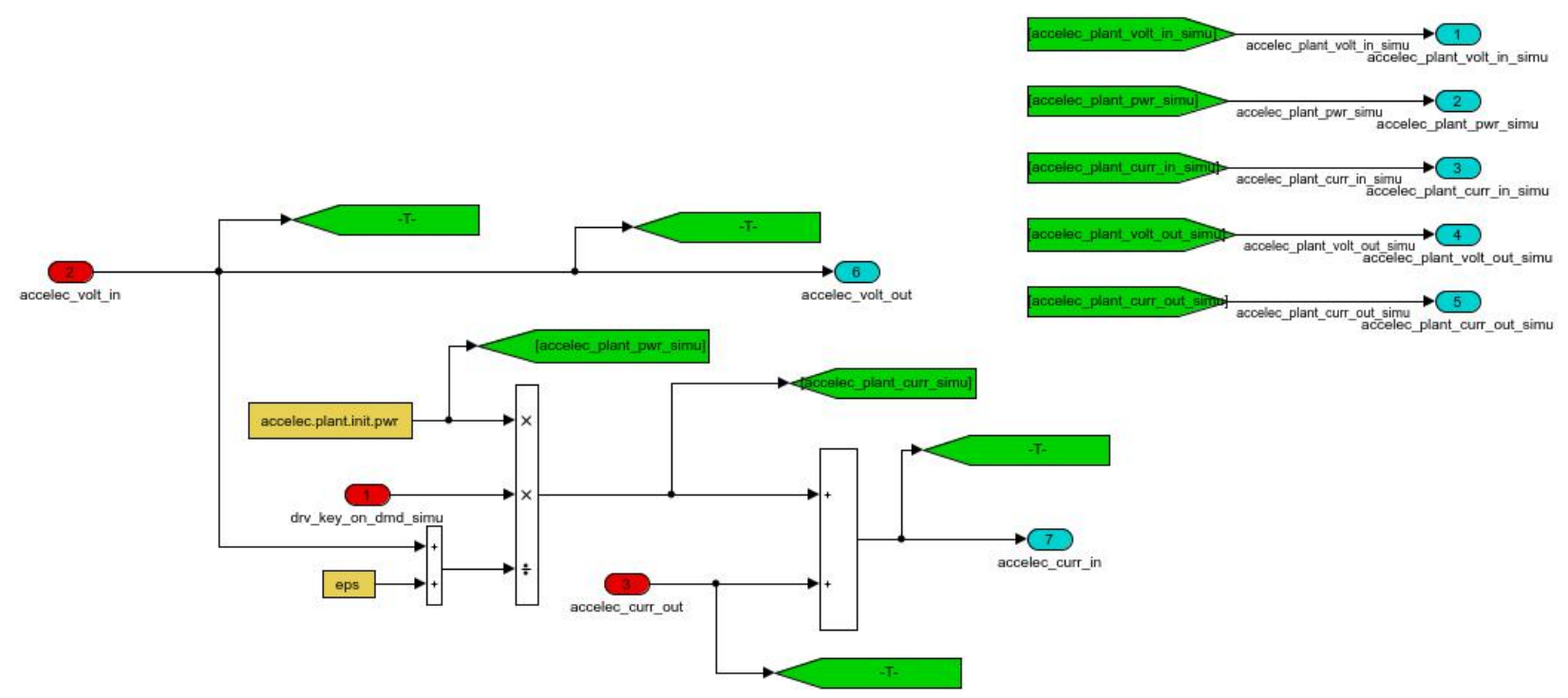

Figure 72. Top-level Simulink diagram of electrical accessories model

Two equations govern the electrical-accessories plant model:

$$
\begin{aligned}
& I_{\text {in }}=I_{\text {out }}+\frac{P_{\text {loss }}}{V_{\text {in }}} \\
& V_{\text {out }}=V_{\text {in }}
\end{aligned}
$$

The current drawn by the accessory load is the constant power loss constant divided by the bus voltage. As shown in Equation 53, the total current flowing in is the sum of the current sunk by the accessory load and the output current propagated back to this model.

Section 5.9 details the constant auxiliary loads used for this analysis.

\subsection{Driver Model}

The driver model uses a look-ahead controller to model the accelerator and brake pedals. The desired vehicle speed is compared with the current speed, and a controller is used to request more or less torque to the vehicle. Figure 73 shows the main I/O of the driver model.

\section{Look-ahead model}

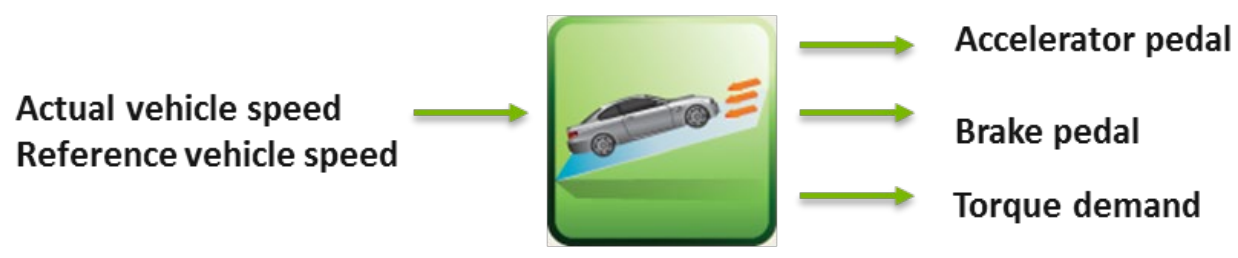

Figure 73. Autonomie driver model

The look-ahead model estimates the additional torque needed to meet the speed trace in the next $\Delta$ seconds in order to provide a more realistic model of driver behavior. This approach avoids unrealistic high torque demands during acceleration. Figure 74 shows the top-level diagram of the driver model in Autonomie. 


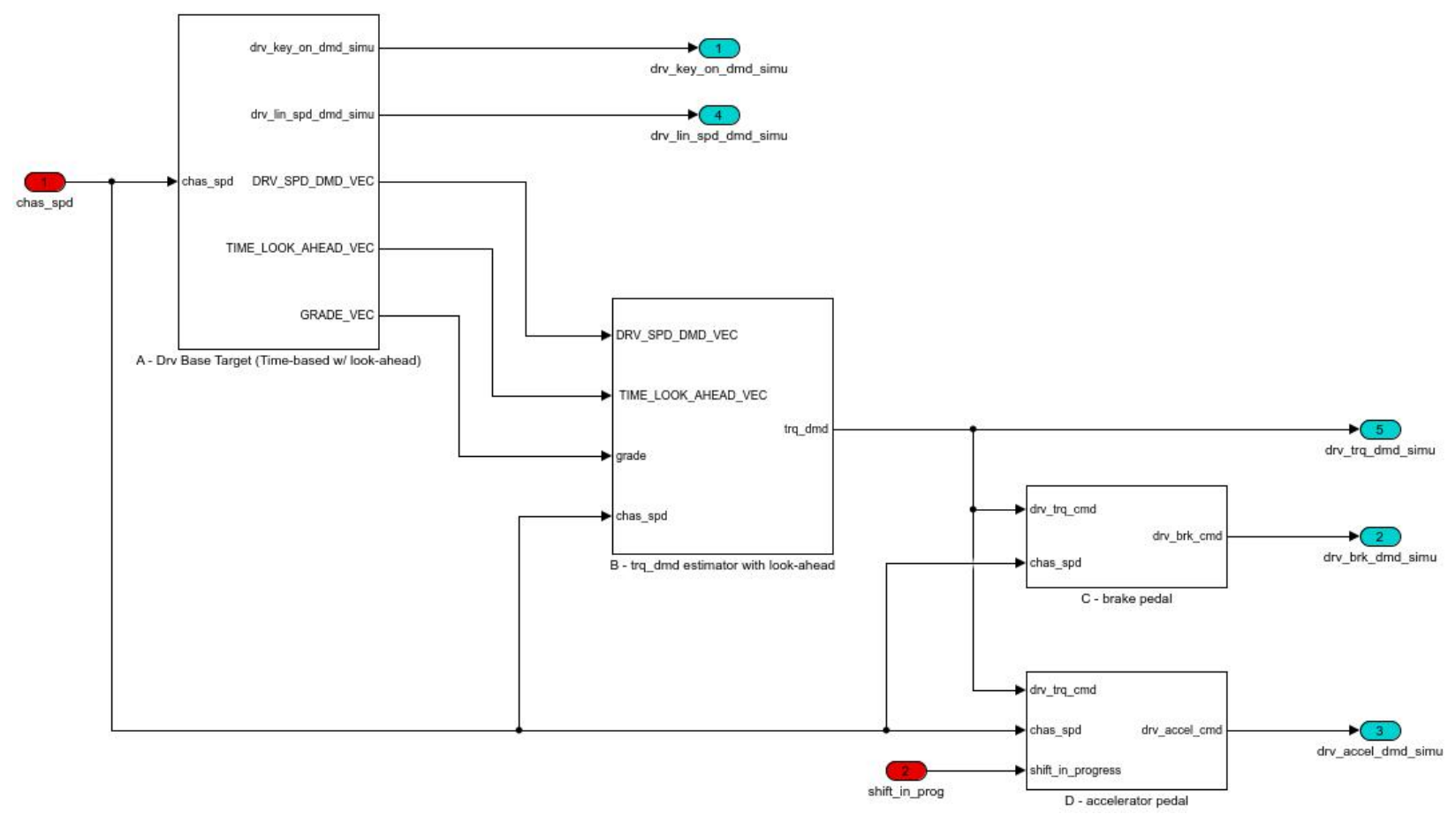

Figure 74. Top-level Simulink diagram of the look-ahead driver model

The equations involved in the look-ahead driver model are shown in Figure 75.

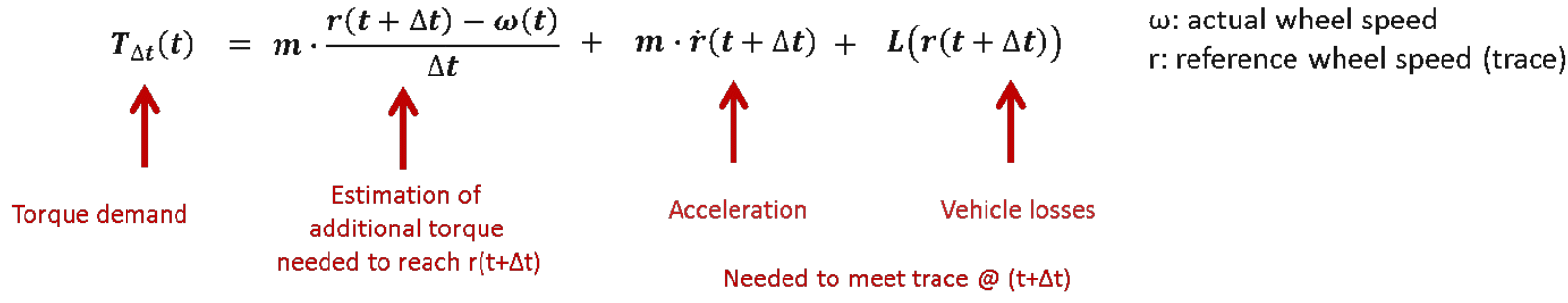

Figure 75. Equations for the look-ahead driver model

\subsection{Wheel Model}

The wheel model accounts for the braking force at each wheel and the added inertia to the drivetrain of all the wheels. Figure 76 shows the main I/O of the Autonomie wheel model.

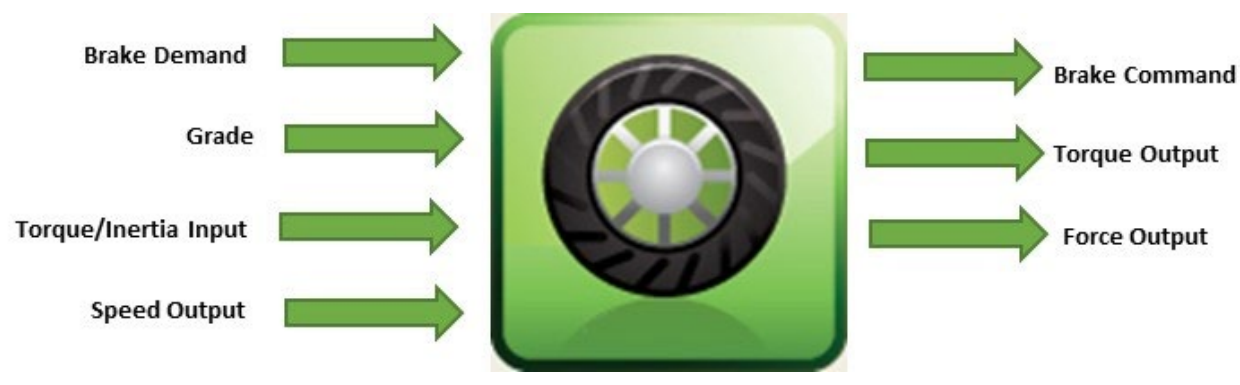

Figure 76. Autonomie wheel model I/O 
As shown in Figure 77, the wheel model can be divided into three subsystems: wheel angular speed calculation (Block A), output force calculation (Block B), and wheel mass calculation (Block C).
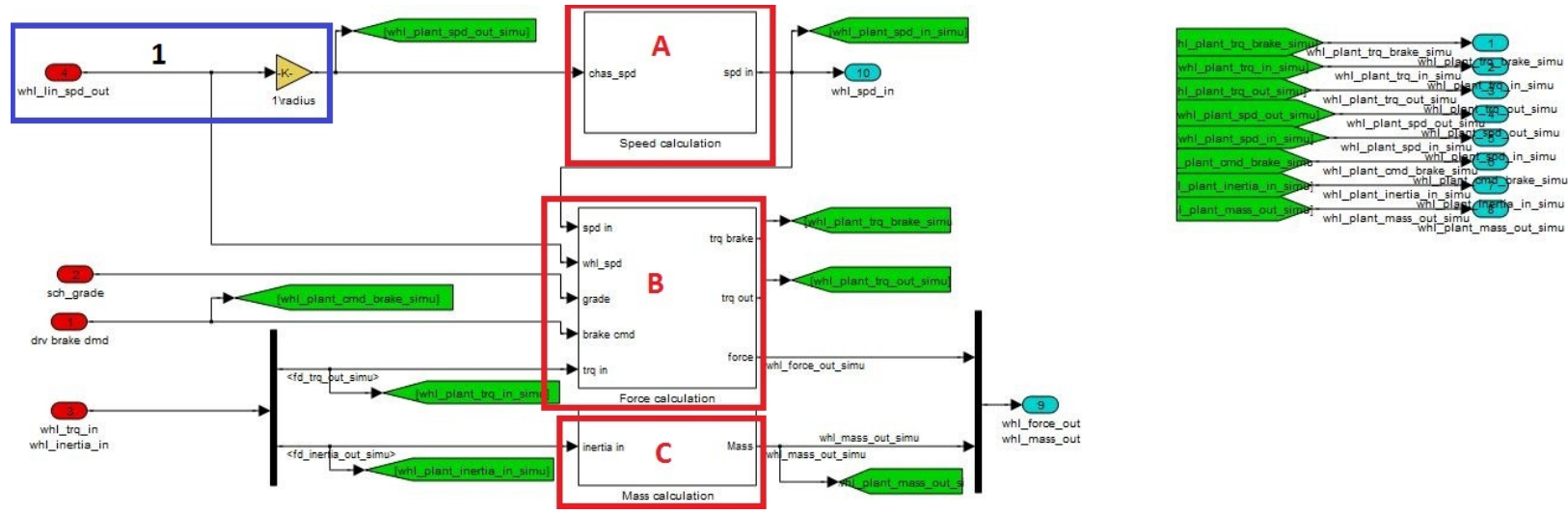

Figure 77. Autonomie wheel model Simulink diagram

The wheel angular speed is calculated by dividing the linear speed of the vehicle by the radius of the wheel.

$$
W_{\text {in }}=\frac{V_{\text {veh }}}{K_{\text {Radius }}}
$$

There is no slip calculation in the wheel model.

Block B (Figure 78) implements the wheel-force calculation. 


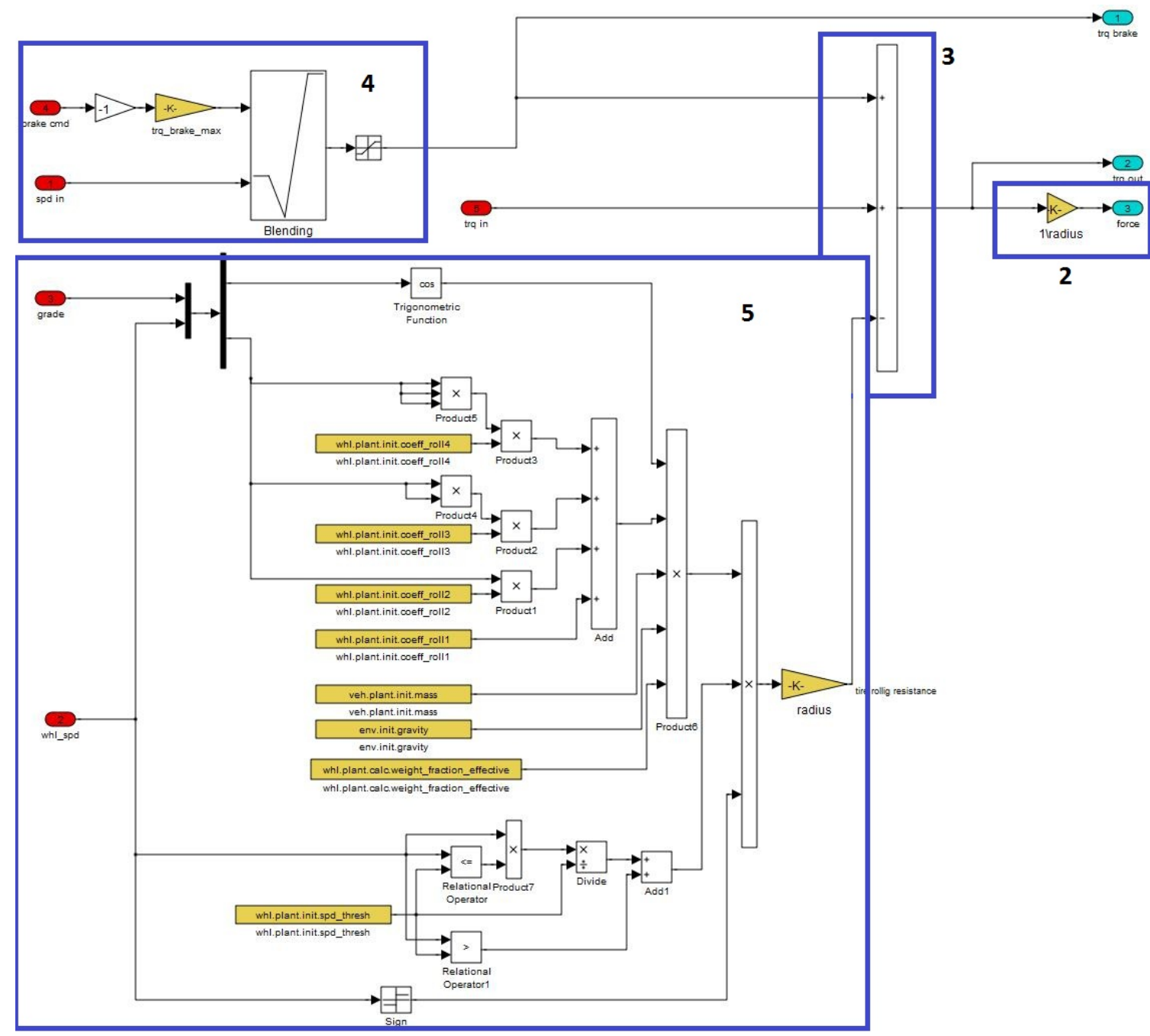

Figure 78. Block B: Force calculation

The wheel net torque is converted into a force by dividing by the wheel radius:

$$
F_{\text {out }}=\frac{T_{\text {out }}}{K_{\text {Radius }}}
$$

The wheel net torque equals the torque in from the drive axle minus the brake torque minus the rolling resistance losses:

$$
T_{\text {out }}=T_{\text {in }}-T_{\text {loss }_{\text {brakes }}}-T_{\text {loss }_{\text {tire }}}
$$

The maximum brake torque is calculated by multiplying the brake command from the controller by the maximum available brake torque. The maximum available brake torque is assumed to be a constant and is unchanged across different vehicle powertrains and classes:

$$
T_{\text {loss }_{\text {brake }}}=P W M_{\text {brake }} \times T_{\text {max }_{\text {brake }}}
$$


The wheel losses are modeled by a fourth-degree polynomial that is a function of vehicle speed:

$$
\begin{aligned}
& T_{\text {loss }_{\text {tire }}}=\left[\cos \left(\alpha_{\text {grade }}\right) \times\left(C_{1}+C_{2} \times V_{\text {veh }}+C_{3} \times V_{\text {veh }}^{2}+C_{4} \times V_{\text {veh }}^{3}\right) \times M_{\text {veh }} \times g \times\right. \\
& \left.\eta_{\text {weight }_{\text {traction }}} \times f\left(\frac{\omega}{\omega_{\text {threshold }}}\right)\right] \times R_{\text {wheel }}
\end{aligned}
$$

The powertrain rotating inertia is converted into an equivalent mass using the wheel radius, as seen in Figure 79.

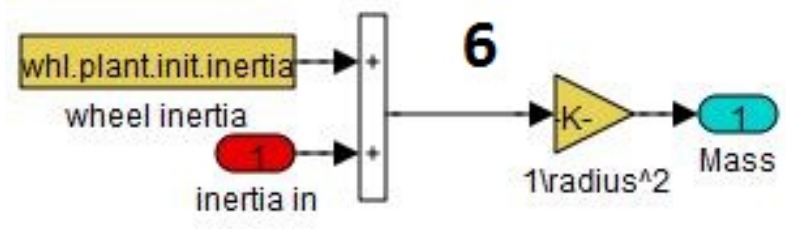

\section{Figure 79. Block C: Mass calculation}

The powertrain upstream inertia is added to the wheels inertia and then divided by the wheel radius squared to yield the equivalent powertrain mass:

$$
\text { Mass }_{\text {inertia }}=\frac{J_{\text {in }}+J_{w h}}{K_{\text {Radius }}^{2}}
$$

\subsection{Chassis Model}

The chassis model calculates speed based on the fed-forward upstream inertia of the drivetrain, the chassis mass, and the drag and grade losses. Figure 80 shows the main chassis model I/O.

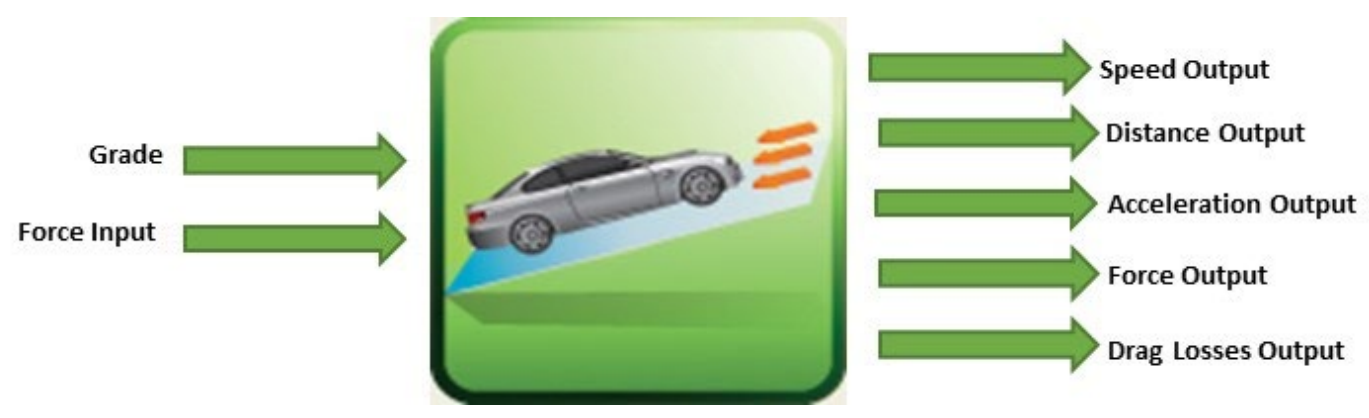

Figure 80. Autonomie chassis model I/O

This model uses the frontal area, drag coefficient and grade to calculate the losses. Since actual drag coefficient and frontal area values are used instead of the costdown coefficients, rolling resistance is accounted for in the wheel model. Figure 81 shows the chassis model Simulink diagram. 


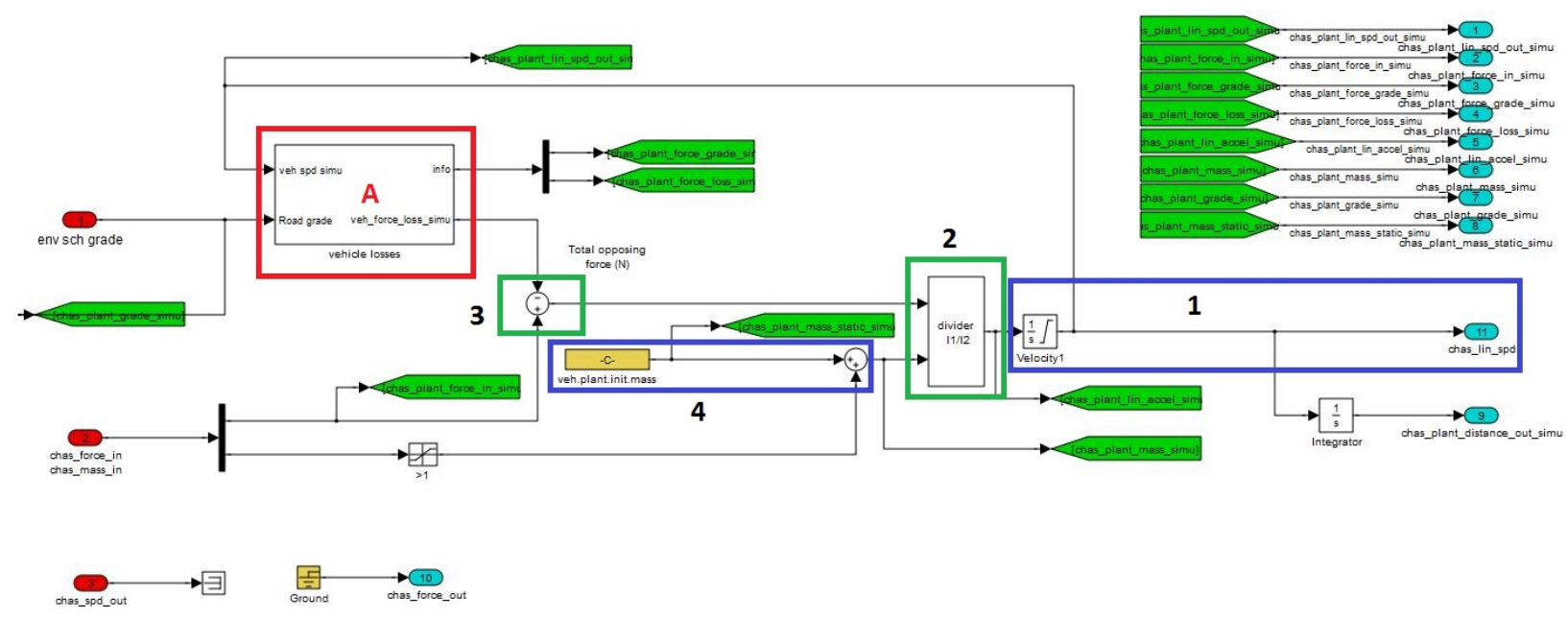

Figure 81. Simulink diagram of chassis model in Autonomie

The vehicle speed is calculated by:

$$
V_{v e h}=\int \text { Acceleration } \times d t
$$

The acceleration is calculated by dividing the net chassis force at the wheels by the chassis equivalent mass. This equivalent mass accounts for the translation acceleration of the chassis and the powertrain rotational acceleration.

$$
\text { Acceleration }=\frac{F_{\text {vehicle }}}{\text { Mass } \text { vehicle }}
$$

The net chassis force is the total force coming from the upstream powertrain components minus the chassis-level losses due to aerodynamic drag and grade:

$$
F_{\text {vehicle }}=F_{\text {in }}-F_{\text {loss }}
$$

The equivalent chassis mass is the sum of the static chassis mass and the equivalent powertrain mass. The powertrain inertia is converted into an equivalent mass in the wheel model by dividing by the square of the wheel radius.

$$
\text { Mass }_{\text {vehicle }}=\text { Mass }_{\text {staticframe }}+\sum J
$$

Block A (Figure 82) calculates the aerodynamic drag and grade loss. 


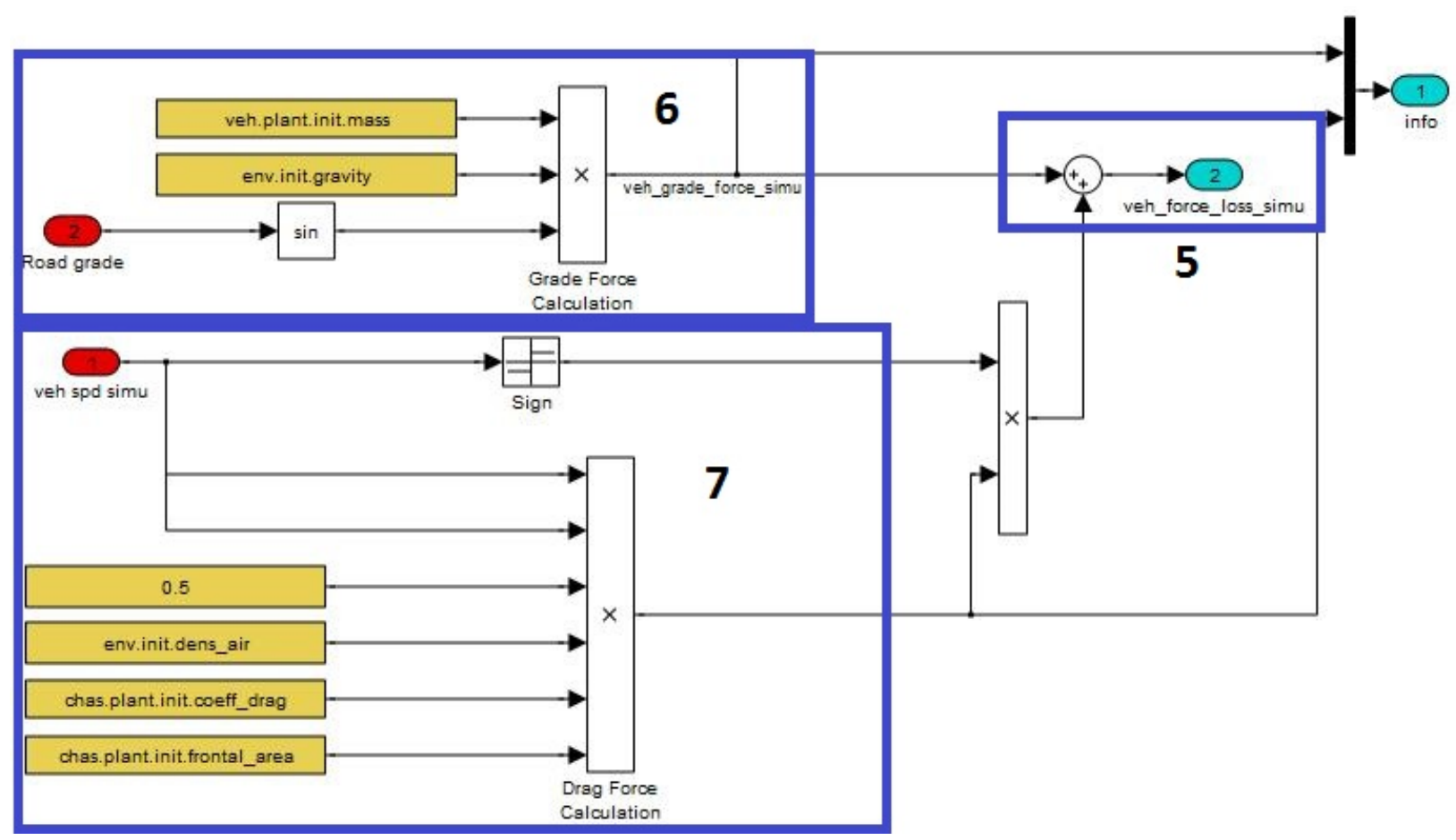

Figure 82. Block A: Chassis losses

The total chassis losses are the sum of the aerodynamic losses and the grade losses.

$$
F_{\text {loss }}=F_{\text {aerodynamics }}+F_{\text {grade }}
$$

The grade loss is computed when the grade angle and the weight of the chassis are known. The grade angle is the inclination that the chassis makes with the horizontal (parallel to the ground).

$$
F_{\text {grade }}=\text { Mass }_{\text {vehicle }} \times g \times \sin (\text { grade })
$$

The standard equation for aerodynamic drag is used. The sine function is used to ensure that the aerodynamic drag opposes chassis movement. The chassis should never move backwards in simulations, so this function is not necessarily needed.

$$
F_{\text {aerodynamics }}=-K h \times \rho_{\text {air }} \times c_{d} \times f_{a} \times V_{v e h}^{2} \times \operatorname{sign}\left(V_{v e h}\right)
$$

Equation 68 shows the variation of air density with altitude, due to the compressibility of air. This equation is not currently implemented in the chassis model.

$$
K h=\frac{\rho}{2 \times g}=\left\{\begin{array}{l}
0.6 @ 200 \text { meters } \\
0.57 @ 500 \text { meters } \\
0.5 @ 1500 \text { meters }
\end{array}\right.
$$

Equation 69 expresses air density as a function of air pressure and temperature. Like Equation 8, it is not currently implemented in the chassis model.

$$
\rho_{\text {air }}=1.225 \times \frac{P r}{101.325} \times \frac{288.16}{273.16+T_{r}}
$$


To estimate the frontal area of a chassis from track width and chassis height, Equation 70 is used:

$$
f_{a}=0.9 \times \text { Width }_{\text {veh }} \times \text { Height }_{\text {veh }}
$$

\subsection{Final Drive Model}

Final drive gears are incorporated into vehicle driving axles and transaxles for the following reasons:

- To provide a right-angled drive from either the propeller shaft or the gearbox layshaft to the driven wheels

- Alternatively, to provide a parallel drive from the gearbox layshaft to the driven wheels

- To permit an additional and constant gear reduction in the transmission system

The second requirement applies only in the case of passenger cars with transversely mounted engines (i.e., one lying parallel to the axle of the driven front or rear wheels).

Two basic types of final drive reduction gearing have generally been used in vehicles with longitudinally mounted engines: bevel drive and worm drive. In the modern vehicle, the bevel drive has proven to be lighter, more efficient, less expensive, and equally quiet-running. For cars with transversely mounted engines, the final drive reduction gears are of the helical pinion type.

Figure 83 shows the main final drive model I/O.

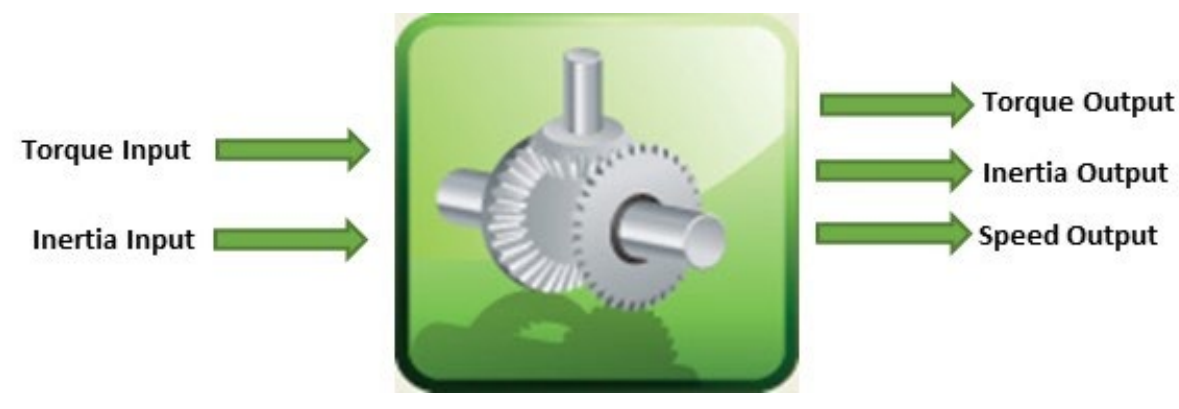

Figure 83. Autonomie final drive model $\mathrm{I} / \mathrm{O}$

Figure 84 shows the top-level Simulink diagram of the final drive model in Autonomie.
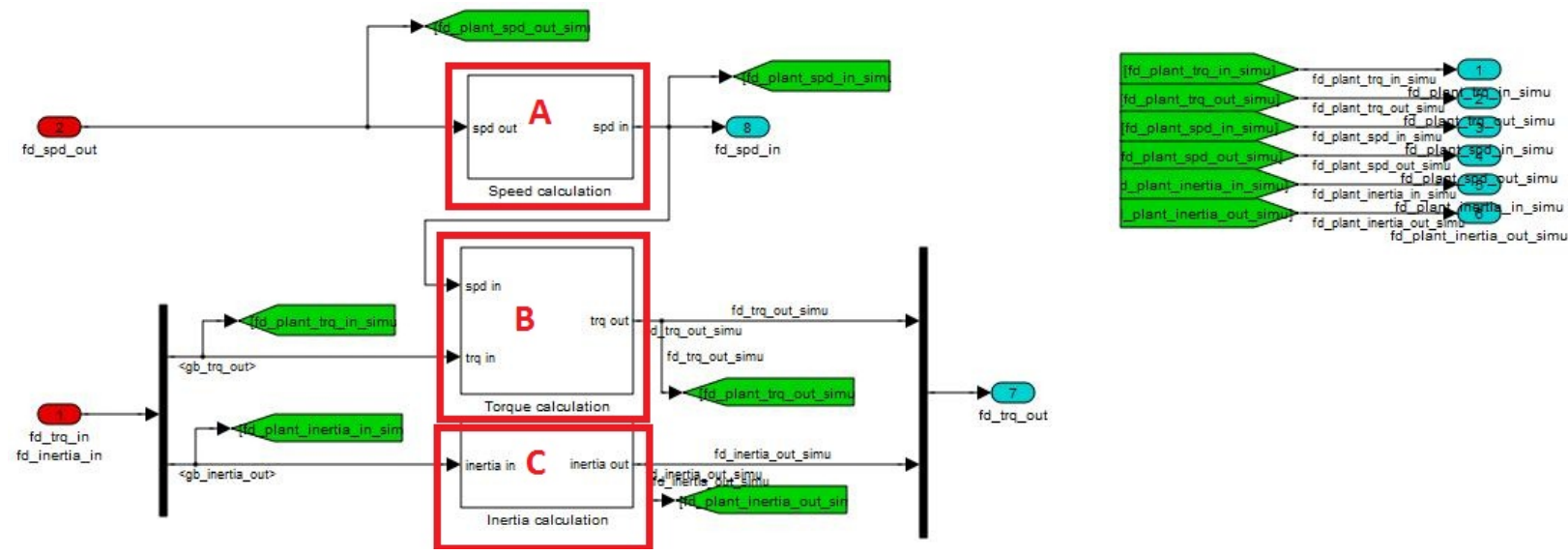

Figure 84. Simulink diagram of the final drive model in Autonomie 
The top-level diagram of the final drive model is divided into three blocks, highlighted in red in Figure 84. Block A calculates the input and output speeds of the final drive, as shown in Figure 85. Block $\mathrm{B}$ calculates the output torque, as shown in Figure 86, and Block $\mathrm{C}$ calculates the inertia that is fed forward, as shown in Figure 87.

The input speed is the product of the final drive ratio and the output speed of the final drive. The output speed of the final drive is calculated downstream and fed back.

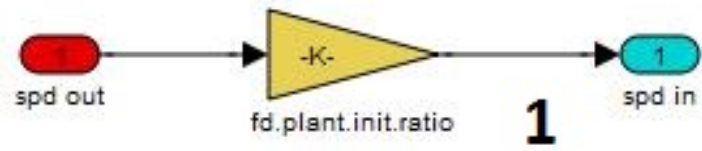

Figure 85. Block A: Final drive model speed calculation

$$
W_{\text {in }}=W_{\text {out }} \times K_{\text {finaldrive }}
$$

Where

$\mathrm{W}_{\text {in }}=$ final drive speed in

$\mathrm{W}_{\text {ou }} \mathrm{t}=$ final drive speed out

$\mathrm{K}_{\text {final_drive }}=$ reduction gear ratio for the final drive

Block B (Figure 86) determines the torque output of the final drive by first subtracting the torque loss, which is a function of input torque and input speed, and then multiplying the remainder by the final drive ratio. The blending block ensures that at zero speed the torque loss is zero; otherwise, a negative torque at zero vehicle speed would lead the vehicle model to give the erroneous result of a negative vehicle acceleration and, consequently, a negative vehicle speed. Essentially, the vehicle model cannot discriminate between negative loss torque and negative propulsive torque.

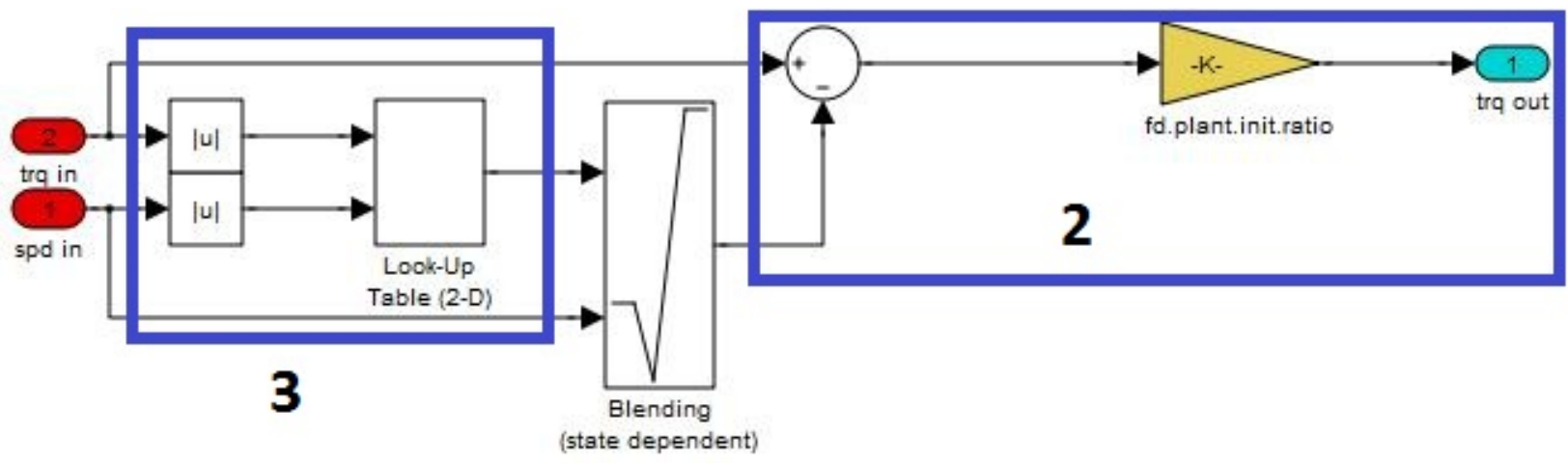

Figure 86. Block B: Torque calculation

$$
\begin{aligned}
& T_{\text {out }}=K_{\text {finaldrive }}\left(T_{\text {in }}-T_{\text {loss }}\right) \\
& T_{\text {loss }}=\text { function }\left(W_{\text {in }}, T_{\text {in }}\right)
\end{aligned}
$$

Where

$\mathrm{T}_{\text {out }}=$ final drive output torque speed 
$\mathrm{W}_{\text {in }}=$ final drive input shaft speed

$\mathrm{T}_{\text {in }}=$ final drive input shaft torque

$\mathrm{T}_{\text {loss }}=$ final drive input torque loss

$\mathrm{K}_{\text {final_drive }}=$ reduction gear ratio of the final drive

Block C (Figure 87) and Equation 74 reflect the product of multiplying the drivetrain inertia upstream of the final drive by the final drive ratio and adding its equivalent inertia at the output of the final drive. This equivalent inertia is fed forward on a path to the vehicle model, where the combined drivetrain inertia is used to calculate the vehicle speed. Drivetrain speeds are then fed back through the drivetrain model.

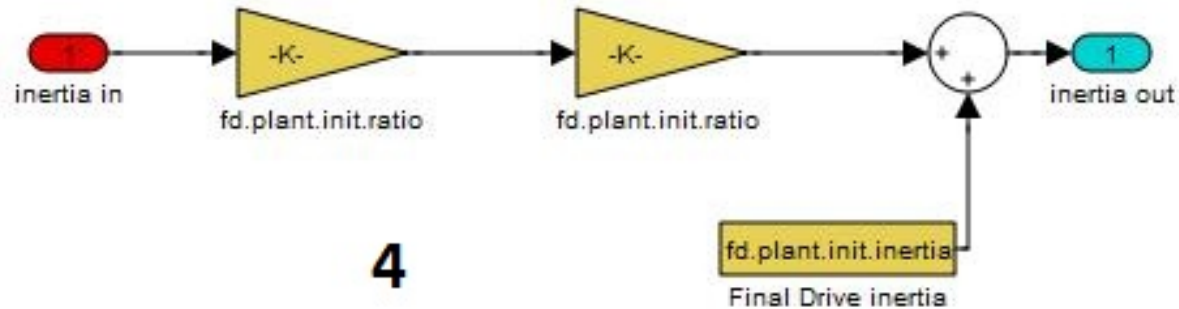

Figure 87. Block C: Inertia calculation

$$
J_{\text {out }}=J_{\text {in }} \times K_{\text {finaldrive }}^{2}+J_{F D}
$$

Where

$$
\begin{aligned}
& \mathrm{J}_{\text {in }}=\text { final drive input inertia from upstream components } \\
& \mathrm{J}_{\mathrm{out}}=\text { final drive output inertia fed forward to downstream components } \\
& \mathrm{J}_{\mathrm{FD}}=\text { inertia of the final drive } \\
& \mathrm{K}_{\text {final_drive }}=\text { reduction gear ratio of the final drive }
\end{aligned}
$$

\subsection{Vehicle-Level Control Algorithms}

All the vehicle-level control algorithms used in the study have been developed from vehicle test data collected at AMTL. It is important to note that while the logic for the vehicle-level control algorithms was developed based on test data, only the logic has been used for the present study, since the calibration parameters have been adapted for each vehicle to ensure fuel consumption minimization with acceptable drive quality (i.e., acceptable number of engine on/off conditions).

\subsubsection{Micro- and Mild HEV}

The vehicle-level control strategies of the micro- and mild (i.e., BISG) HEVs are similar in many aspects because of the low peak power and energy available from the energy storage system.

For the micro-HEV case, the engine is turned off as soon as the vehicle is fully stopped and restarted as soon as the brake pedal is released. No regenerative braking is considered in this controller. 
For the mild HEV cases, the engine is also turned off as soon as the vehicle is fully stopped. However, since some regenerative braking energy can be recovered, the vehicle is propelled by the electric machine during vehicle launch, allowing the engine to be restarted later. The electric machine also provides some limited assist during propelling to improve engine efficiency.

\subsubsection{Power-Split HEV (Split HEV)}

As shown in Figure 88, power-split hybrids combine the best aspects of both series and parallel hybrids to create an extremely efficient system. The most common configuration, called an input split, is composed of a power-split device (planetary gear transmission), two electric machines and an engine. Within this architecture, all these elements can operate separately. Indeed, the engine is not always ON, and the electricity from the generator may go directly to the wheels to help propel the vehicle or go through an inverter to be stored in the battery. The operational phases for an input split configuration are the following:

- During vehicle launch, when driving, or when the SOC of the battery is high enough, the ICE is not as efficient as electric drive, so the ICE is turned off and the electric machine alone propels the vehicle.

- During normal operation, the ICE output power is split, with part going to drive the vehicle and part used to generate electricity. The electricity goes either to the electric machine, which assists in propelling the vehicle, or to charge the energy storage system. The generator also acts as a starter for the engine.

- During full-throttle acceleration, the ICE and electric machine both power the vehicle, with the energy storage device (e.g., battery) providing extra energy.

During deceleration or braking, the electric machine acts as a generator, transforming the kinetic energy of the wheels into electricity to charge the energy storage system.

The vehicle control algorithms were based on both the Toyota and Ford implementations.

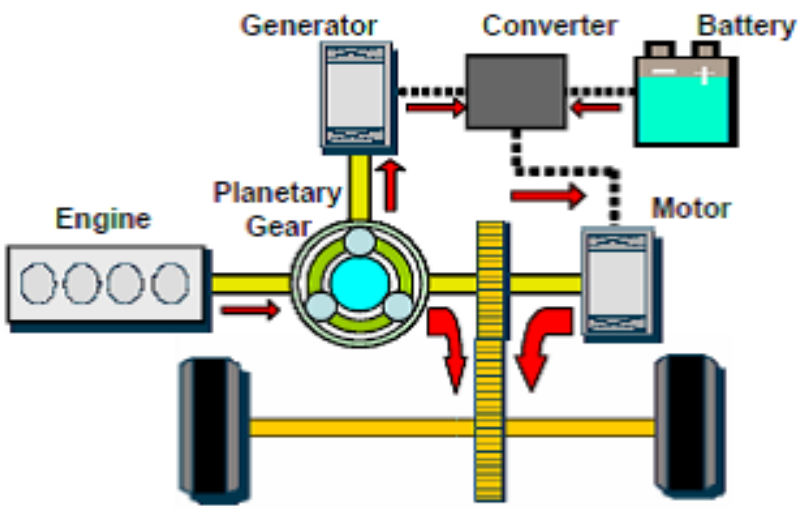

Figure 88. Power-split hybrid electric vehicle

\subsubsection{Single-Mode Power-Split PHEV (Split PHEV)}

The vehicle-level control strategy of a single-mode power-split PHEV was based on the Toyota Prius Prime [13]. The implemented control can be divided into three areas: engine-ON condition, battery SOC control, and engine operating condition. Each algorithm is described below. 


\subsubsection{Engine-ON Condition}

The engine operation determines the mode: pure electric vehicle (PEV) mode or HEV mode. The engine is turned ON when the driver's power demand exceeds a predefined threshold. As shown in Figure 89 , the engine is ON only when the battery SOC is under $17 \%$. This means that only electric energy is used when the battery SOC is over 17\%, i.e., in charge-sustaining (CS) mode. Once the operating mode, based on SOC, is determined, the engine is turned ON early if the driver's torque demand exceeds a predefined threshold, which means that the system changes from PEV mode to HEV mode to meet the power demand.
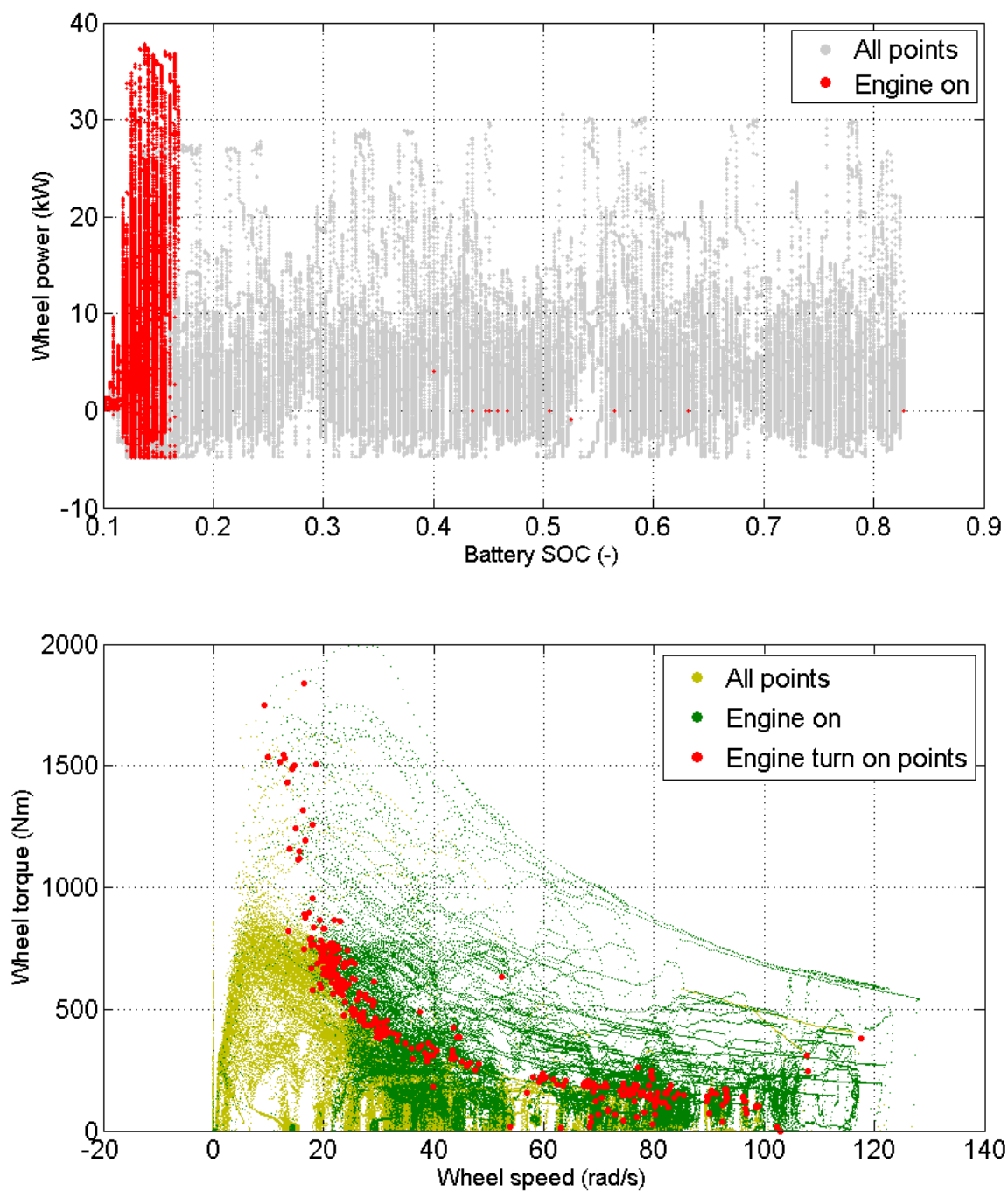

Figure 89. Engine-ON condition-2017 Prius Prime example based on 25 test cycles

\subsubsection{SOC Control in CS Mode}

The desired output power of the battery is closely related to the energy management strategy. When the vehicle is in HEV mode, the battery power is determined by the current SOC, as shown in Figure 90. The overall trend shows that the energy management strategy consists of bringing the SOC back to a regular value close to $14 \%$. When the battery SOC decreases to under $13.5 \%$, the battery is charged to 10 
$\mathrm{kW}$ to sustain the battery SOC. As battery SOC is increasing, the charging power is decreasing, and the battery is discharged when the battery SOC is over $14.5 \%$. If the battery output power is determined, engine output power can be calculated.

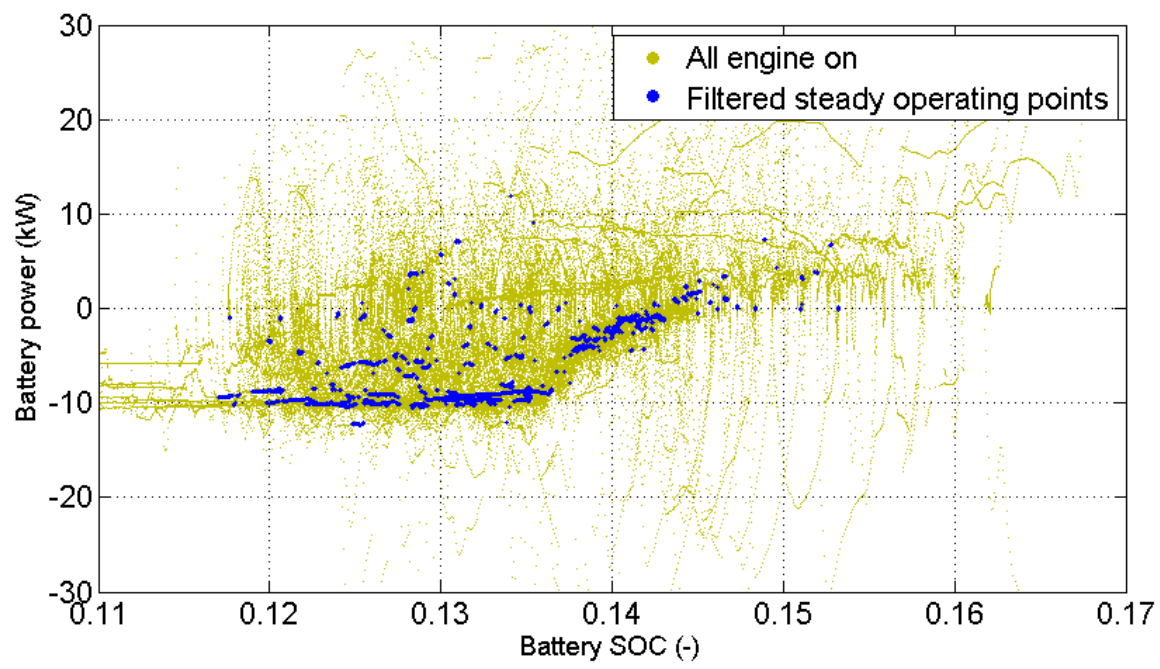

Figure 90. SOC regulation algorithm-2017 Prius Prime example based on 25 test cycles

\subsubsection{2. $\quad$ Engine Operation}

The two previously described control concepts determine the power-split ratio. The concepts do not, however, generate the target speed or torque of the engine, because the power-split system could have infinite control targets that produce the same power. Therefore, an additional algorithm is needed to determine the engine torque operating points according to the engine speed, as shown in Figure 91. An engine operating line is defined on the basis of the best efficiency curve to select the optimum engine speed for a specific engine power demand. 


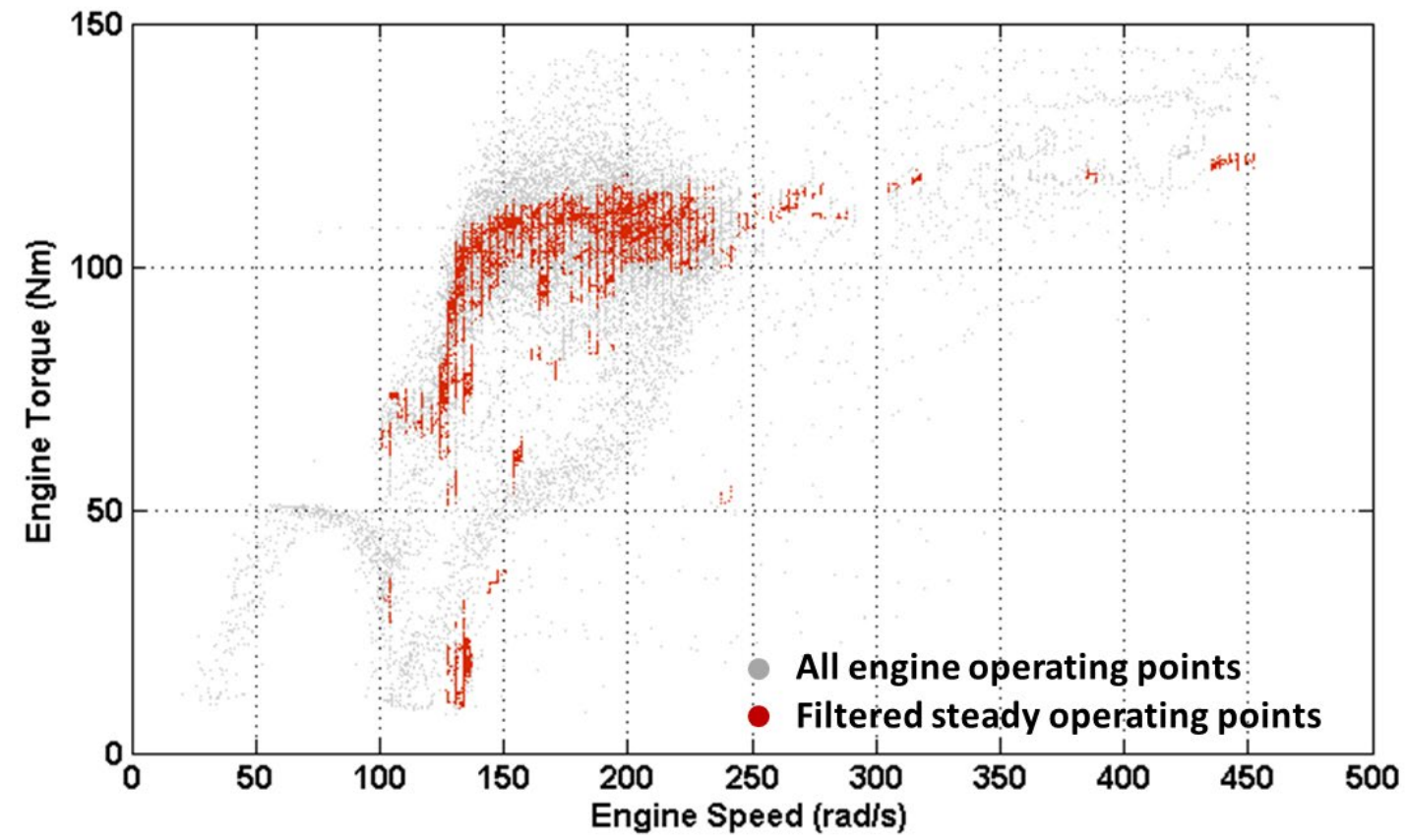

Figure 91. Example of engine operating target-2017 Prius Prime example based on 25 test cycles

In summary, the engine is turned $\mathrm{ON}$ based on the power demand at the wheel along with the battery SOC. If the engine is turned on, the desired output power of the battery is determined based on the current SOC, and the engine should provide appropriate power to drive the vehicle. The engine operating targets are determined by a predefined line, so the controller can produce required torque values for the motor and the generator based on the engine speed and torque target.

\subsubsection{Multi-Mode E-REV (EREV PHEV50)}

The multi-mode EREV control is based on the Voltec powertrain from General Motors [9] [19].

The second-generation Voltec PHEV has one engine, two motor-generators, and one battery. The two electric machines are connected to a main transmission shaft using an individual planetary gear set, as shown in Figure 92. By activating the brake (BK) and clutches, the vehicle can be driven in various modes. Normally, electric machine 1 (MG1) drives the vehicle only by holding the BK. When the BK and one-way clutch (OWC) are locked, both electric machines can provide the maximum torque, putting the vehicle into "two-motor electric vehicle" (EV) mode. An additional planetary gear set is used for a compound power-split mode in extended-range operation. The clutch $(\mathrm{CL})$ position or the BK activation status determines whether the mode is the input-split or the compound-split. The input-split mode is activated by the BK by holding the ring gear of the second planetary gear set. The compound-split mode is activated by the CL when it connects the sun gear of the first planetary gear set to the ring gear of the second gear set. 


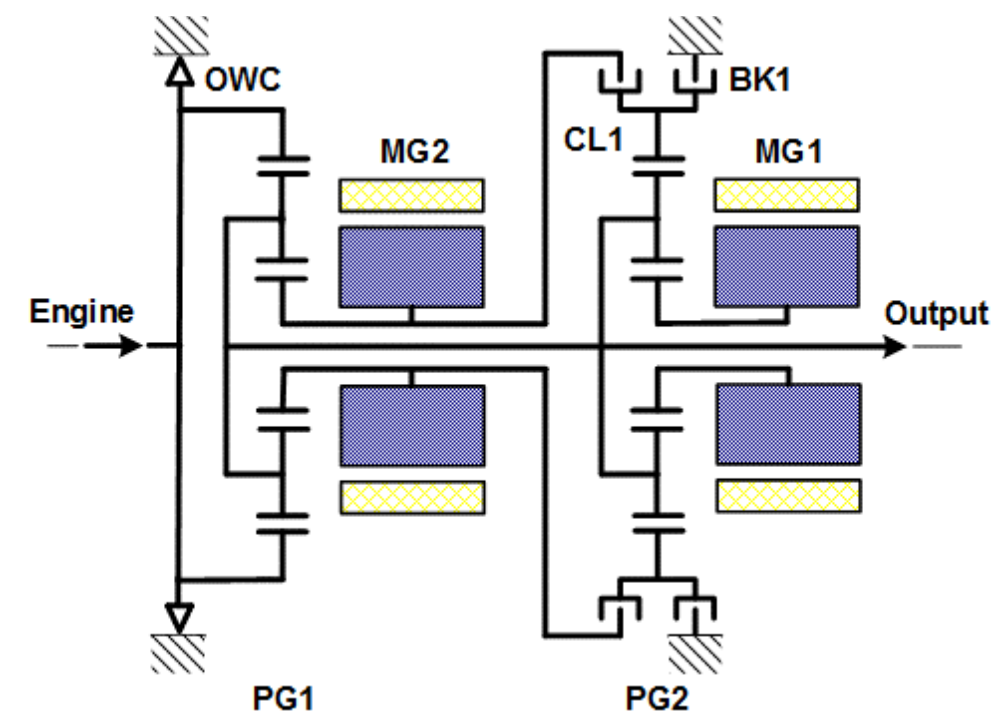

\begin{tabular}{|l|c|c|c|}
\hline Mode & BK1 & CL1 & OWC \\
\hline EV1 & closed & open & open \\
\hline EV2 & closed & open & closed \\
\hline Low extended range & closed & open & open \\
\hline Fixed ratio & closed & closed & open \\
\hline High extended range & open & closed & open \\
\hline
\end{tabular}

Figure 92. Configuration of the Chevrolet Volt 2016 powertrain system

Although a number of sophisticated control concepts were added to the supervisory control concepts, the main control flow of the vehicle, based on test data, can be summarized as shown in Figure 93. First, the engine ON/OFF control is determined by the battery SOC and the driver's power demand. During EV driving, the use of two electric machines allows for two EV driving modes to provide either maximum output torque or increased efficiency by torque distribution. If the engine is $\mathrm{ON}$ after most of the battery energy has been depleted by EV driving, the operational state of the clutch or brakes is defined to select the extended-range mode. Energy management between the engine and the battery is controlled, depending on the powertrain operation mode.

Once the operation mode is chosen, the battery power demand is determined by the proportional control power, which also determines the engine power demand by subtracting the battery power demand from the driver power demand. Then, each component operates according to an optimal target based on engine target and battery power demand. The entire powertrain model, including the vehicle-level controller, was implemented in Autonomie. 


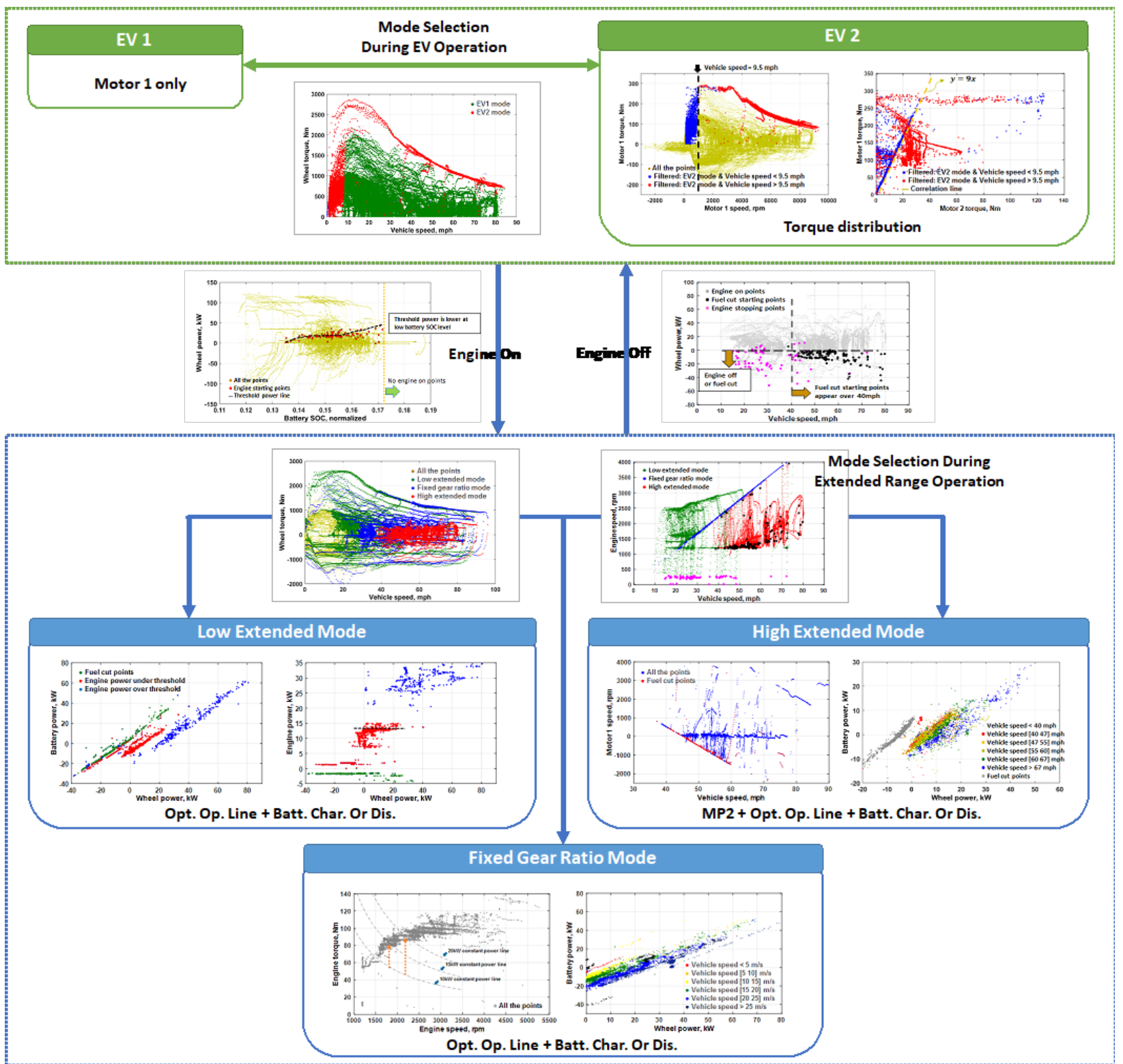

Figure 93. Summary of control analysis for the second-generation Voltec system

\subsubsection{Parallel HEV (Par HEV)}

In a parallel configuration, the vehicle can be directly propelled by either electrical or mechanical power. Direct connection between the power sources and the wheels leads to lower powertrain losses compared to the pure series configuration. However, since all of the components' speeds are linked to the vehicle's speed, the engine cannot consistently be operated close to its best efficiency curve. The pretransmission configuration used in this study has an electric machine between the engine and the transmission. The electric machine peak power usually ranges from 19 to $45 \mathrm{~kW}$ for light-duty applications, allowing the driver to propel the vehicle in electric-only mode as well as recover energy through regenerative braking.

The pre-transmission parallel HEV configuration can take advantage of different gear ratios that allow the electric machine to operate at higher efficiency and provide high torque for a longer operating 
range. This configuration allows operation in electric mode during low and medium power demands in addition to the ICE ON/OFF operation. The main challenge for these configurations is being able to maintain a good drive quality given the engine ON/OFF feature and the high component inertia during shifting events.

The vehicle-level control strategy for a parallel HEV is based on the 2013 Volkswagen Jetta HEV [15]. Vehicle control behaviors are interpreted on the basis of the analyzed results in order to understand the overall control behaviors.

\subsubsection{Engine Operation}

The upper panel in Figure 94 shows the vehicle speed and wheel demand torque when the engine is ON. The lower panel shows the operating area of pure electric driving in the same index.
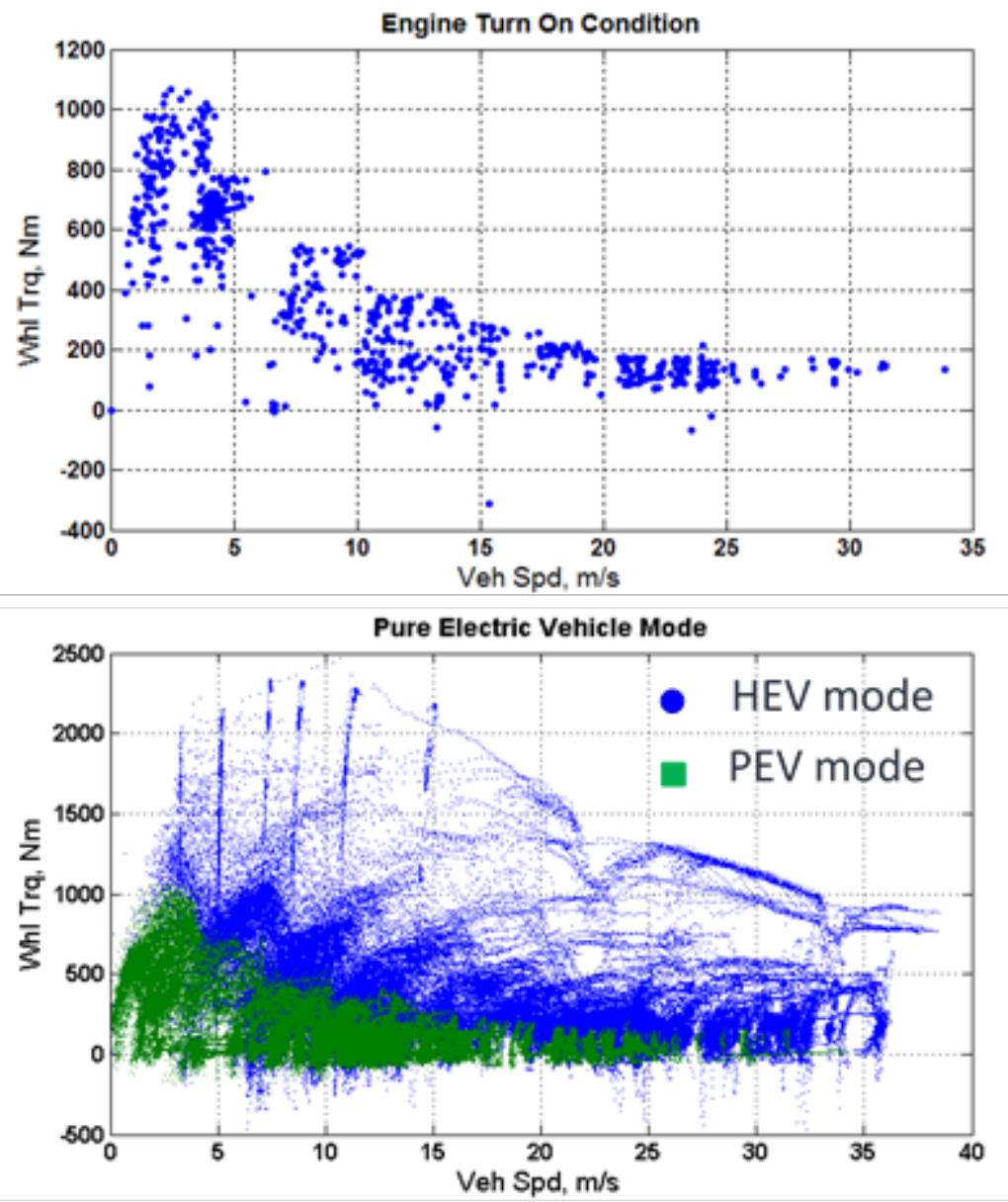

Figure 94. Wheel torque vs. vehicle speed of $2013 \mathrm{VW}$ Jetta HEV

\subsubsection{Fuel-Cell HEV}

Unlike the other vehicle-level control algorithms previously discussed, the algorithm for fuel-cell HEVs is not derived from test data, owing to the lack of test vehicles. Instead, dynamic programming was used to define the optimum vehicle-level control algorithms for a fuel-cell vehicle. A rule-based control is then implemented to represent the rules issued from the dynamic programming. 
Overall, owing to the high efficiency of the fuel-cell system, energy storage only regains energy during deceleration and propels the vehicle under low-load operations; the fuel-cell system does not recharge the battery. Unlike in electric-drive powertrains with an engine, the battery does not smooth the transient demands. An example of fuel-cell hybrid operations is shown in Figure 95.
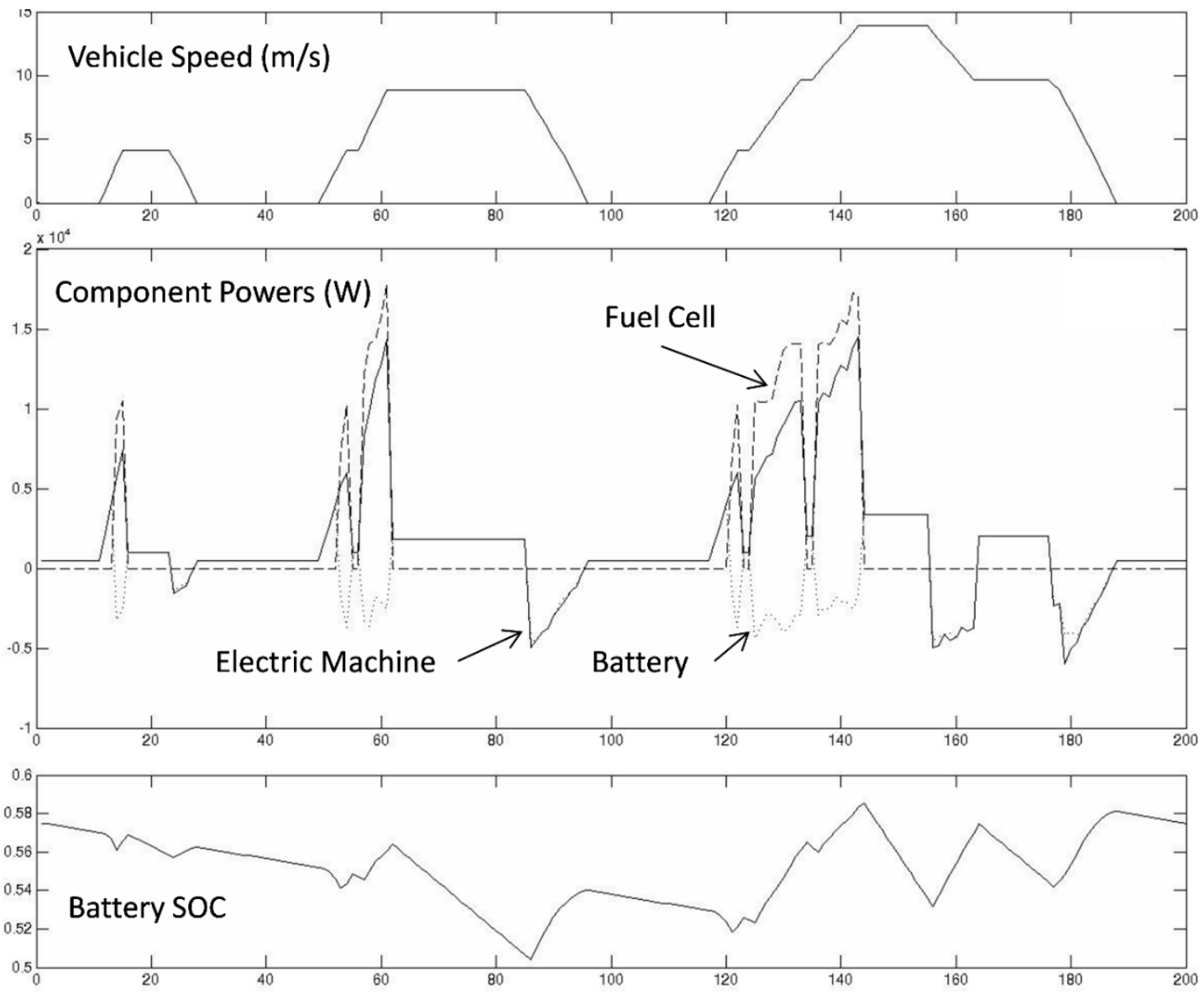

Figure 95. Component operating conditions of a fuel-cell vehicle on the urban European drive cycle using dynamic programming

\subsection{High-Level Overview of Autonomie Controllers}

\subsubsection{Supervisory Controller}

In Autonomie, the supervisory controller manages the vehicle-level decision-making. The main control decisions (gear shift demand, etc.) are sent to the vehicle propulsion architecture (VPA). The lower-level controls within the VPA execute the demands, such as different steps to perform a shifting event coordinated between the engine, gearbox, etc. The split of the control logic between the supervisory controller and component controllers depends on the vehicle configuration.

For conventional vehicles, all decisions are made at the component (including the driver) level.

For electrified vehicles, the supervisory controller manages most decisions, including engine ON/OFF strategy, engine demand, electric machine demand, and others, while the transients (for example, how to shift a gear) are handled at the component level. Figure 96 shows the Autonomie supervisory controller. The individual component controllers are part of the VPA. 


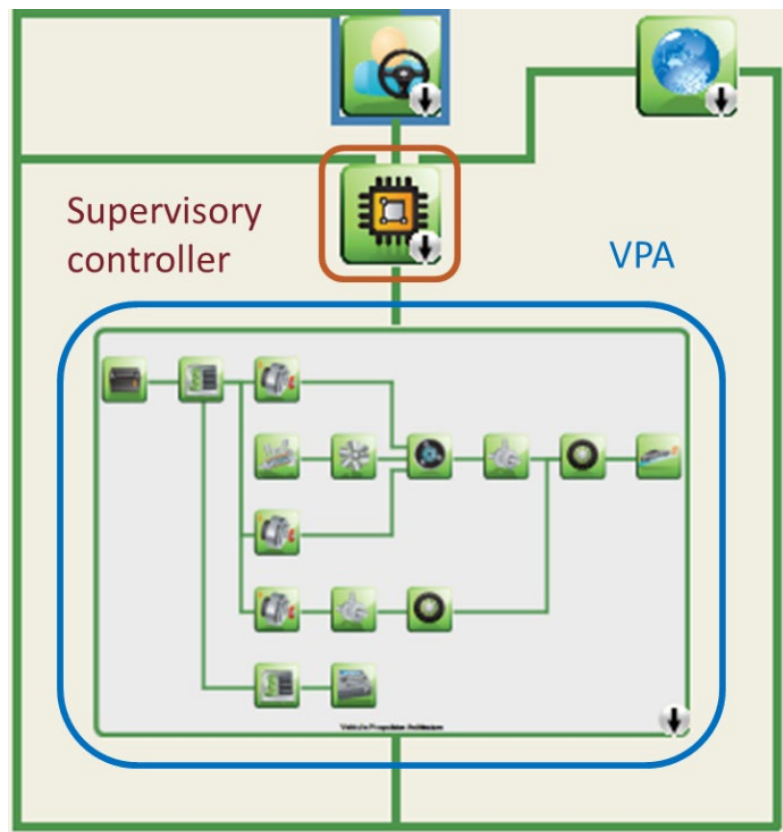

Figure 96. Autonomie supervisory controller

Figure 97 shows the structure inside the VPA.

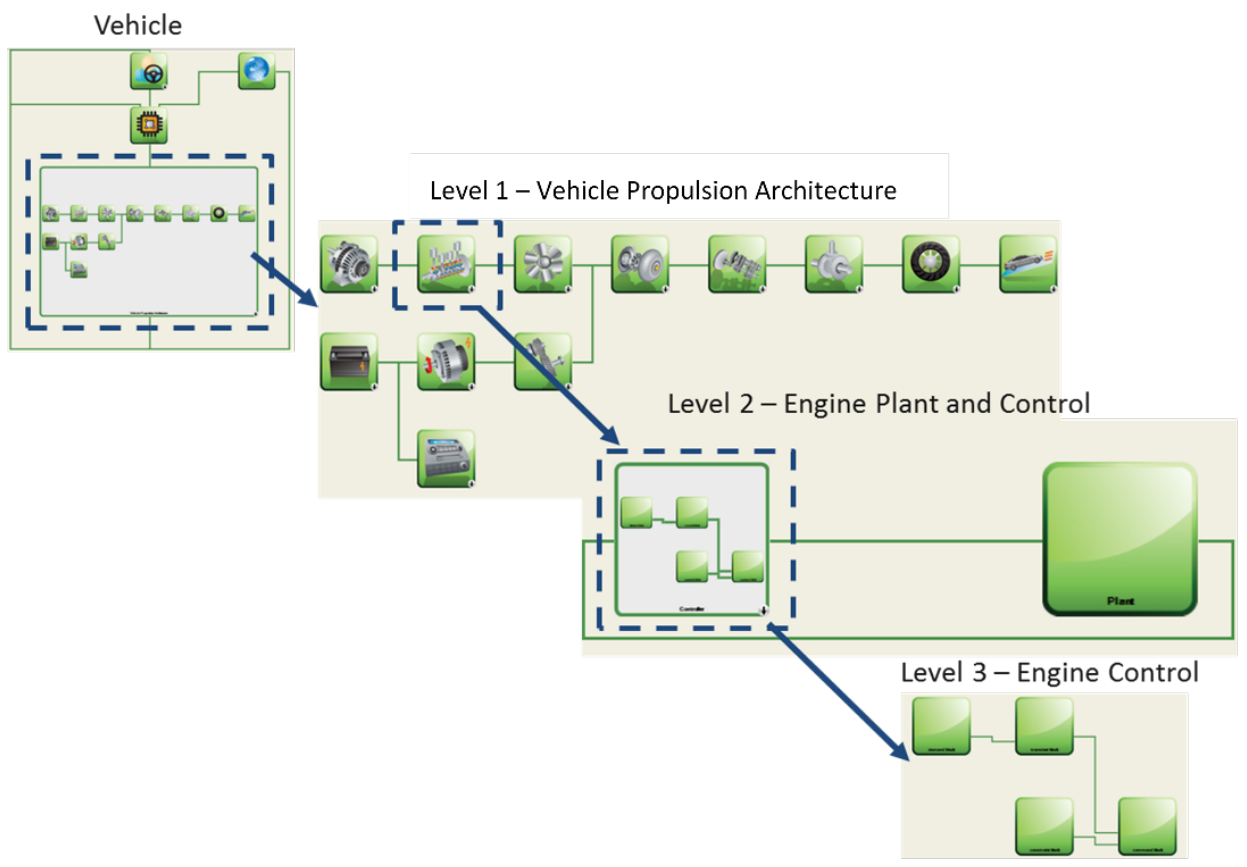

Figure 97. Autonomie nested-level controllers

Each of the component models in Autonomie may contain several subsystems, consisting of either plant model only or plant and controller models. Figure 98 shows the component model layout in Autonomie. 


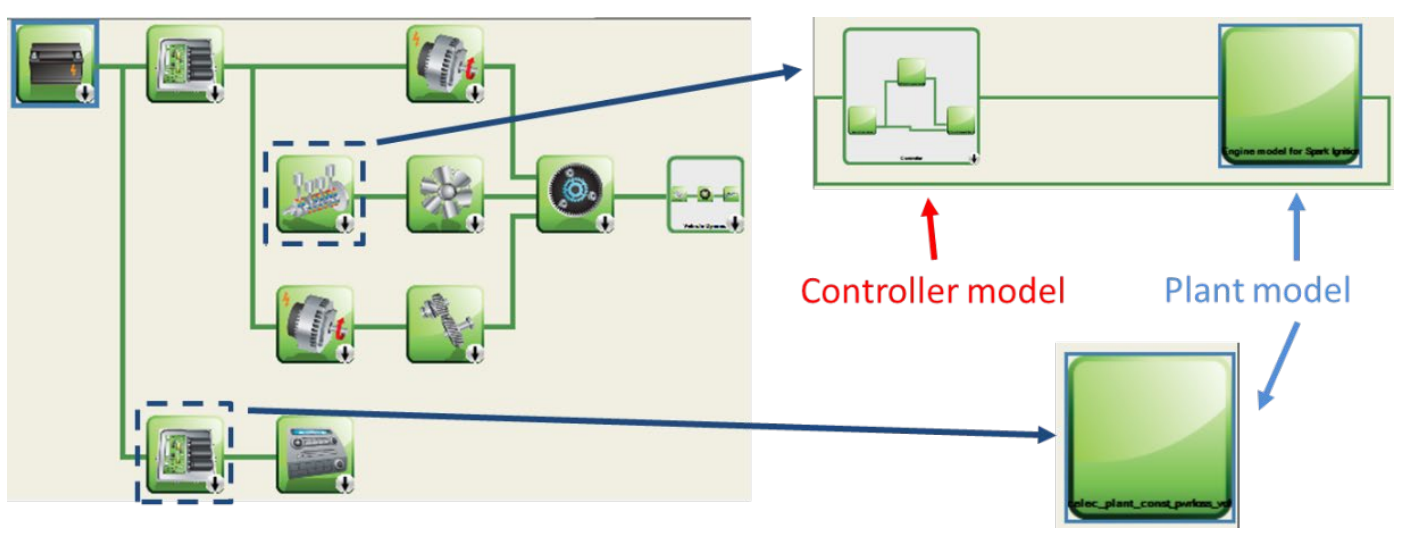

Figure 98. Autonomie component model layout

\subsubsection{Component Controllers}

A component controller can contain a single control model (ctrl) or a combination of two, three, or four of the following sub-models:

- Demand model (dmd)

- Transient model (trs)

- Constraint model (cstr)

- Command model (cmd)

For example, the battery controller in Figure 99 consists of a single control model (ctrl).

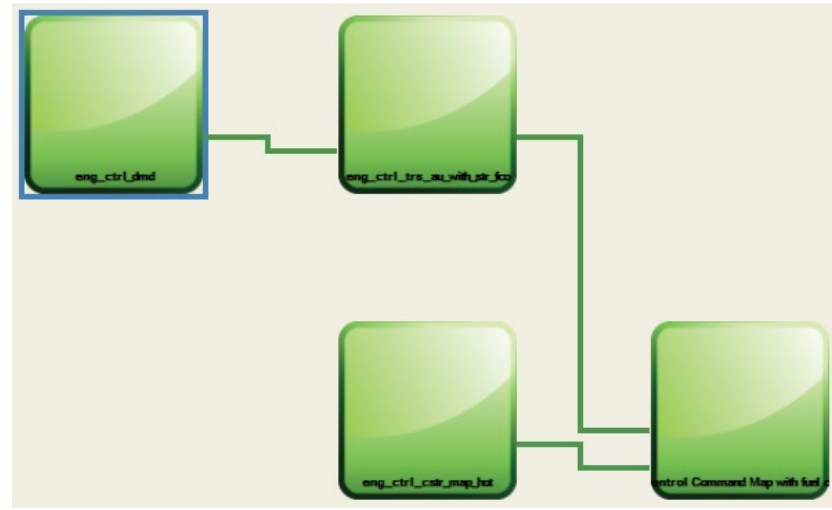

Figure 99. Battery controller composed of a single control model

In contrast, the engine controller in Figure 100 consists of four sub-models: dmd, trs, cstr and cmd. 


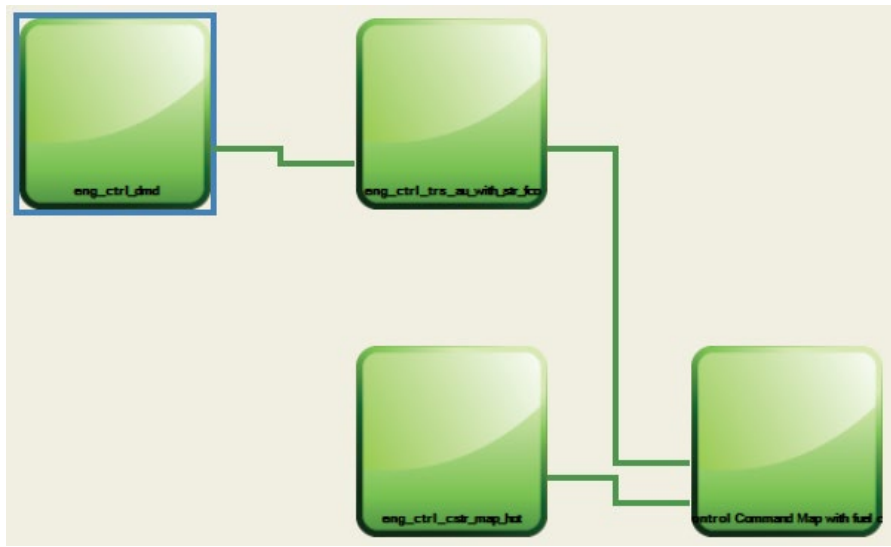

Figure 100. Engine controller composed of four sub-models

\subsubsection{The Demand Block-dmd}

The demand block in Figure 101 is the usual entry point of the low-level controllers. The request coming from the vehicle controller is translated into a request at the component level-for example, torque demand to pulse-width modulation (0-1). The request then feeds into the transient block.

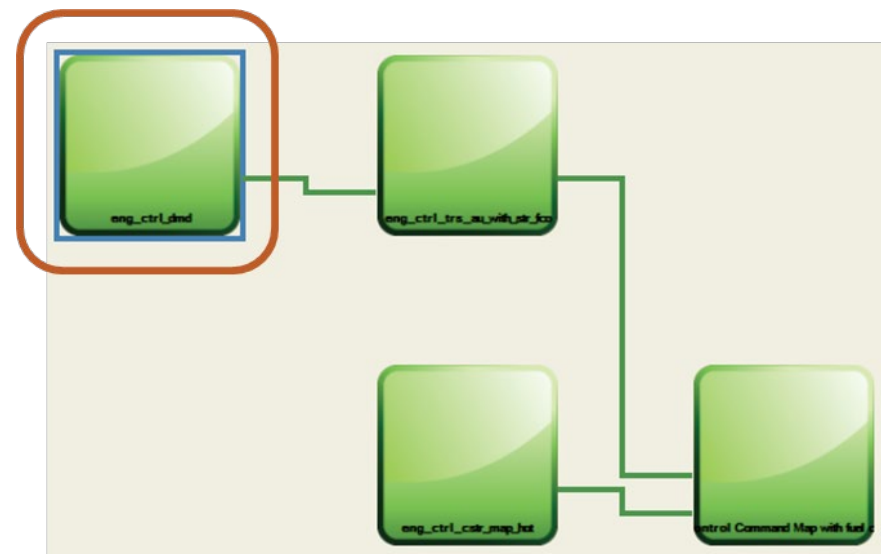

Figure 101. Demand block

\subsubsection{2. $\quad$ The Transient Block - trs}

The transient block in Figure 102 checks for conditions that may impact the request coming from prior blocks. For instance, if a shift is in progress, the engine torque may need to be reduced for the duration of the shift. 


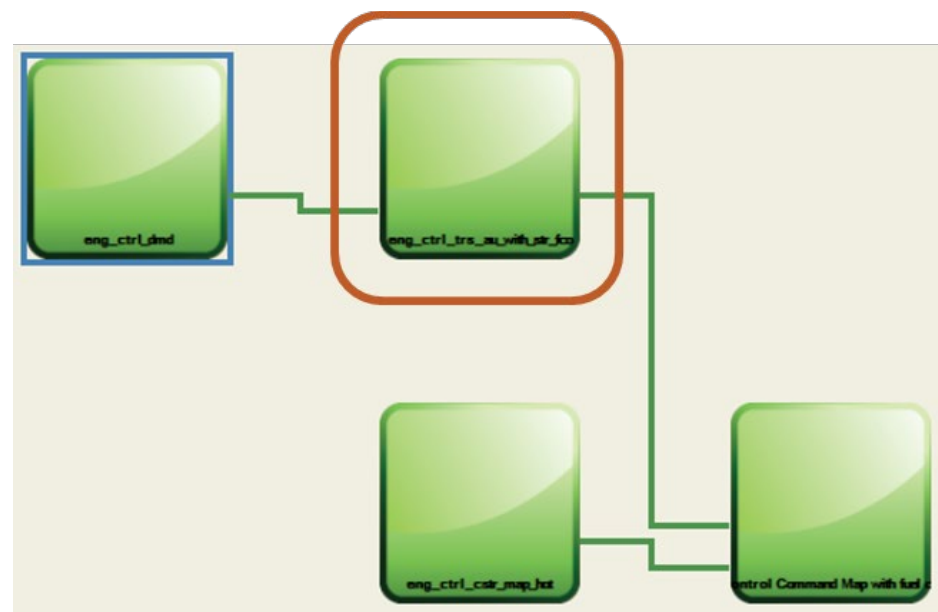

Figure 102. Transient block

\subsubsection{The Constraint Block - cstr}

The constraint block, shown in Figure 103, checks component limits at any given time. For example:

- The maximum engine torque available, given the current engine speed and engine condition

- The maximum battery charge and discharge current, given the current conditions (voltage, temperature, etc.)

The constraints are fed into the command block.

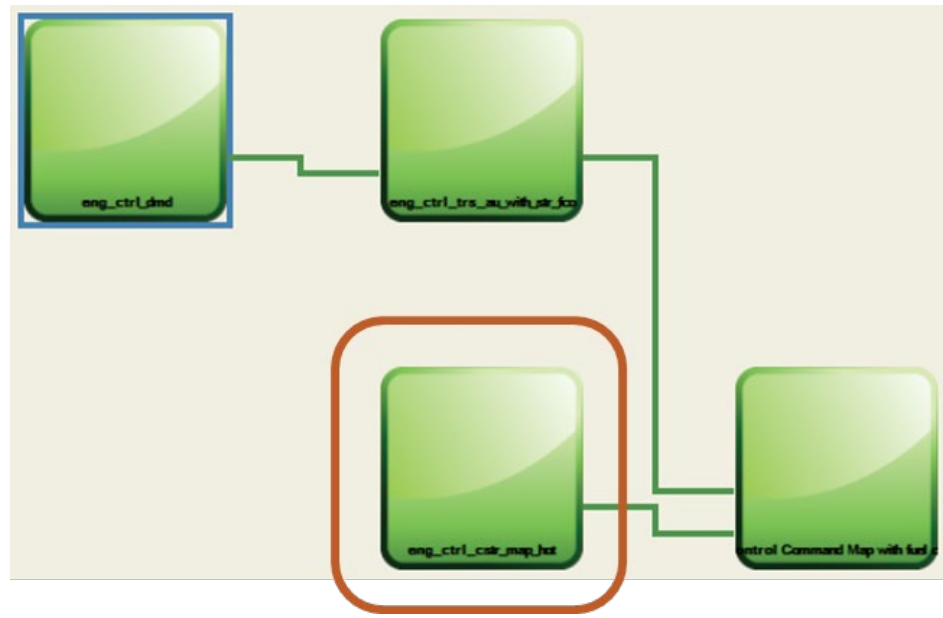

Figure 103. Constraint block

\subsubsection{The Command Block-cmd}

The command block, shown in Figure 104, receives inputs from the transient and constraint blocks and determines the final request that will be sent to the plant model. 


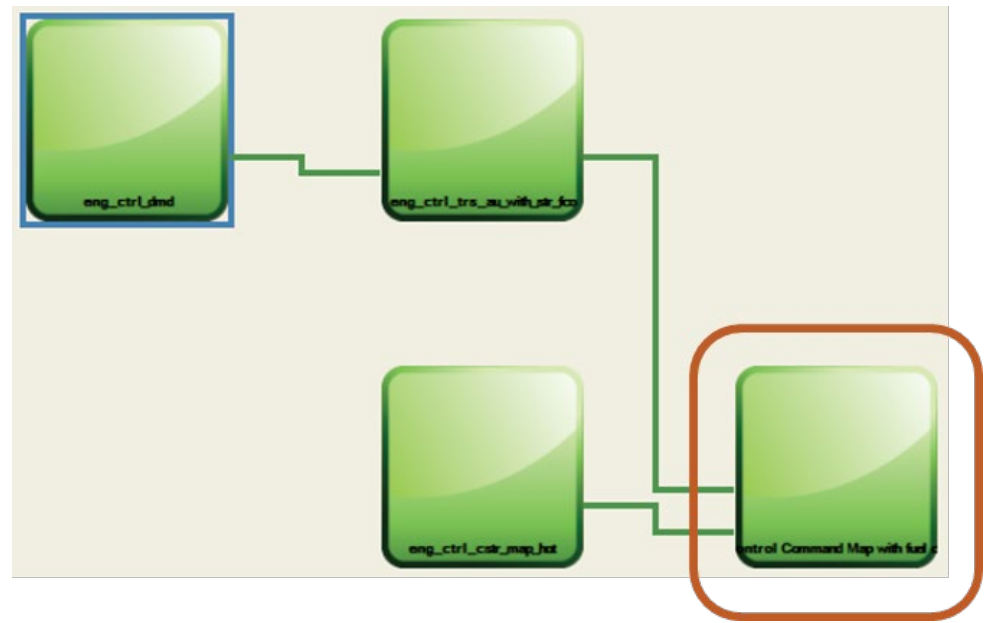

Figure 104. Command block

\subsection{Low-Level Controller Summary for Engine and Transmission Models}

Autonomie allows for the simulation of hundreds of powertrains and technology combinations. That being said, many control functionalities are common across architectures. The following section describes the list of low-level controller option for both engine and transmission and how some are shared across multiple vehicle powertrains.

Figure 105 shows how the engine low-level controls are organized by functionality (transient, command, constraint, and demand) and re-used across transmissions and powertrains.
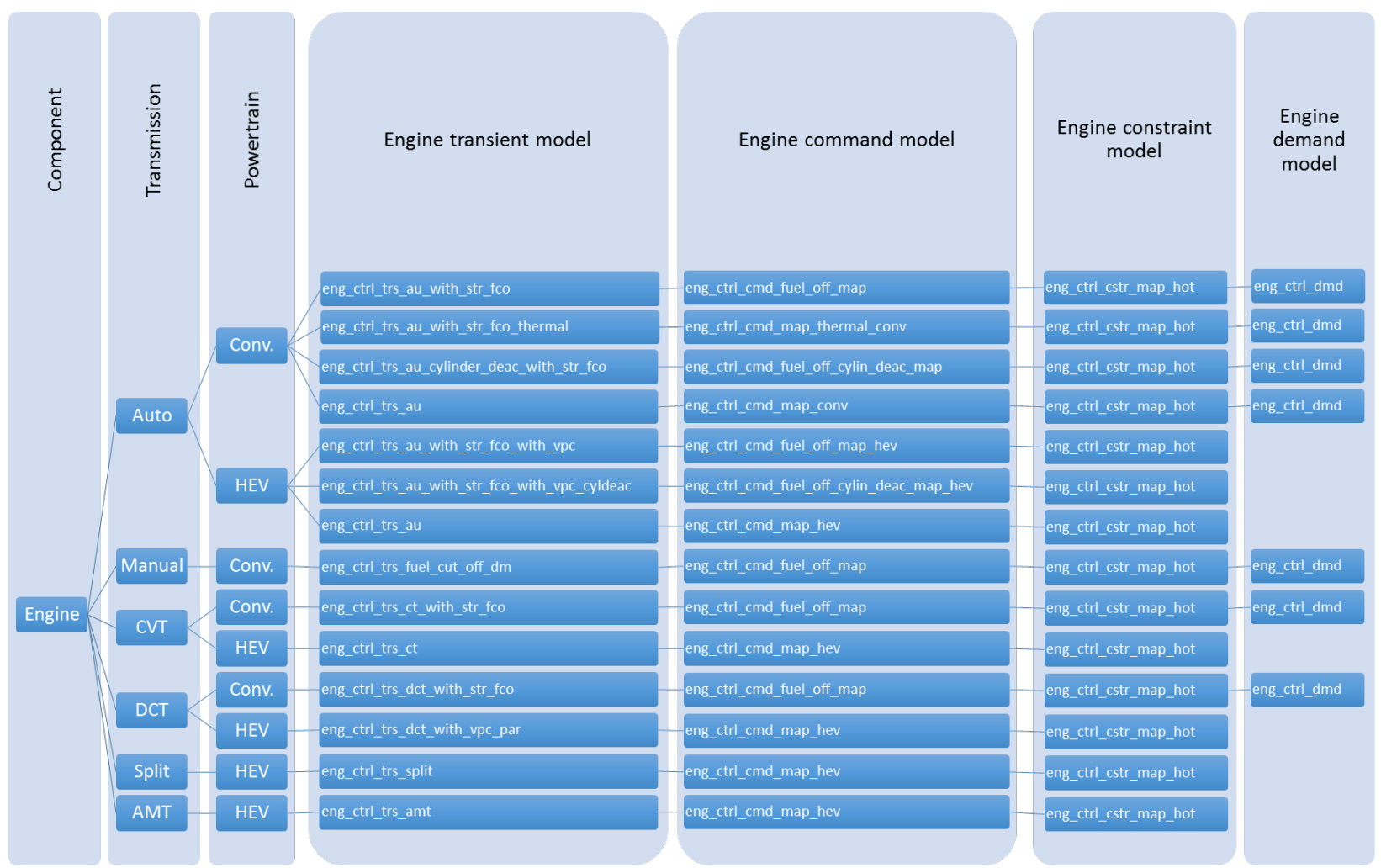

Figure 105. Low-level control for engine 
Figure 106 shows how the transmission low-level controls are organized by functionality (transient, demand, and command) and reused across transmission types and powertrains.

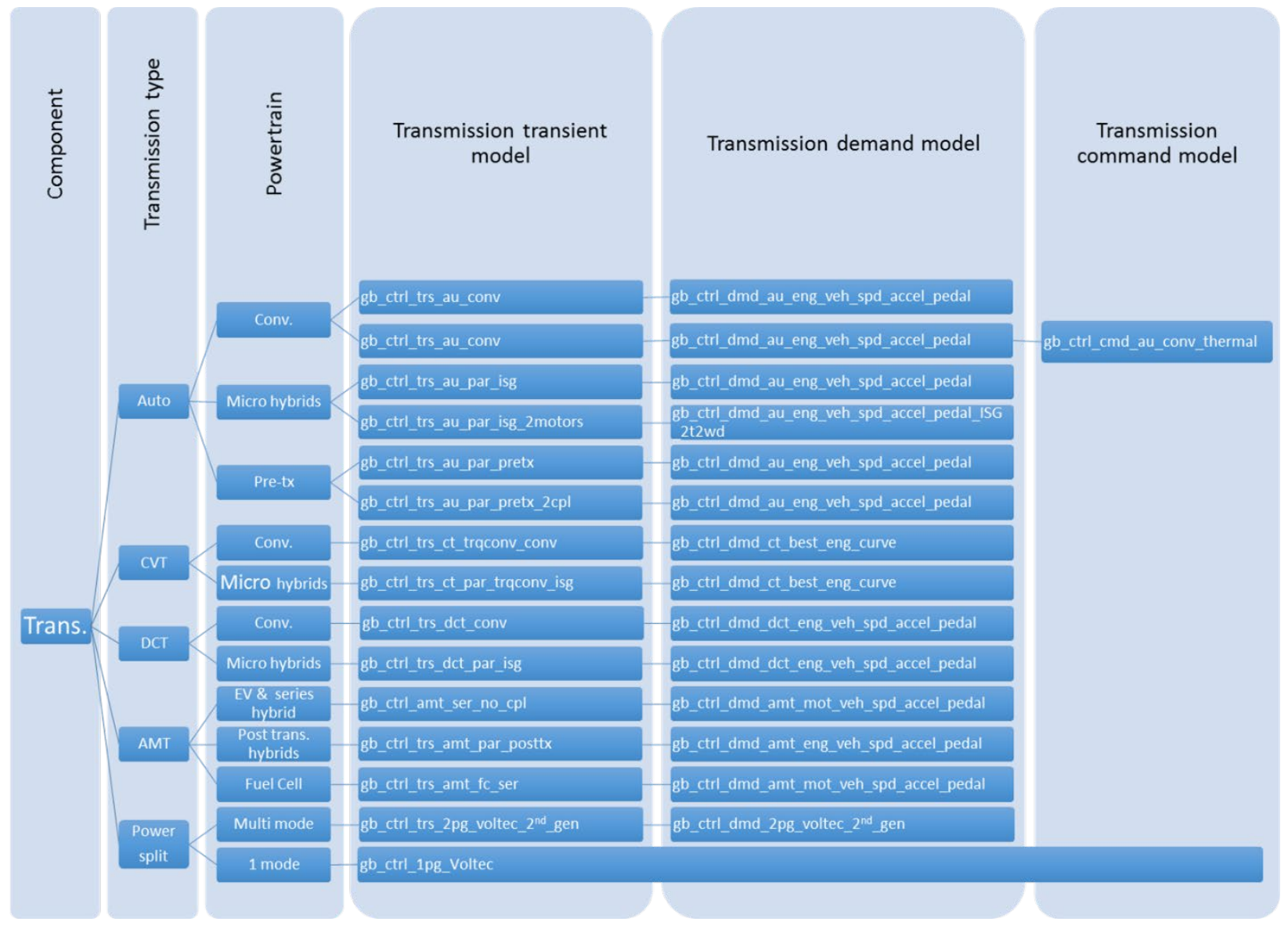

Figure 106. Low-level control for transmission

Table 5 through Table 7 provide short descriptions of the component control blocks and their $\mathrm{I} / \mathrm{O}$, as well as lists of compatible plant models. 
Table 5. Engine Control—Low-Level Demand Models

\begin{tabular}{|c|c|c|c|c|c|}
\hline Model Name & Description & Powertrains Used & Compatible Plant Models & Inputs & Outputs \\
\hline eng_ctrl_dmd & $\begin{array}{l}\text { Translates the } \\
\text { driver pedal } \\
\text { position into a } \\
\text { torque } \\
\text { demand }\end{array}$ & $\begin{array}{l}\text { Light-duty } \\
\text { conventional } \\
\text { powertrains with any } \\
\text { transmission }\end{array}$ & $\begin{array}{l}\text { eng_plant_hot_map_si_fuel_cut_off } \\
\text { eng_plant_hot_map_si_thermal } \\
\text { eng_plant_hot_map_ci } \\
\text { eng_plant_hot_map_si_fuel_cut_off_with } \\
\text { _cylinder_deac }\end{array}$ & $\begin{array}{l}\text { drv_accel_dmd_simu } \\
\text { gb_plant_ratio_simu } \\
\text { chas_plant_lin_spd_out_simu } \\
\text { eng_ctrl_cstr_trq_hot_max_simu } \\
\text { eng_ctrl_cstr_trq_hot_min_simu } \\
\text { eng_plant_spd_out_simu }\end{array}$ & eng_ctrl_trq_dmd_simu \\
\hline
\end{tabular}

Table 6. Engine Control—Low-Level Constraint Models

\begin{tabular}{|c|c|c|c|c|c|}
\hline Model Name & Description & Powertrains Used & Compatible Plant Models & Inputs & Outputs \\
\hline $\begin{array}{l}\text { eng_ctrl_cstr_m } \\
\text { ap_hot }\end{array}$ & $\begin{array}{l}\text { Provides min } \\
\text { and max } \\
\text { torque } \\
\text { capabilities } \\
\text { based on } \\
\text { engine speed }\end{array}$ & $\begin{array}{l}\text { Used in all } \\
\text { powertrain } \\
\text { configurations }\end{array}$ & $\begin{array}{l}\text { eng_plant_hot_map_si_fuel_cut_off } \\
\text { eng_plant_hot_map_si_thermal } \\
\text { eng_plant_hot_map_ci } \\
\text { eng_plant_hot_map_si_fuel_cut_off_with_cylinder_deac } \\
\text { eng_plant_hot_map_si } \\
\text { eng_plant_hot_map } \\
\text { eng_plant_hot_map_cs }\end{array}$ & $\begin{array}{l}\text { eng_plant_spd_out } \\
\text { _simu }\end{array}$ & $\begin{array}{l}\text { eng_ctrl_cstr_trq_hot_ma } \\
\text { x_simu } \\
\text { eng_ctrl_cstr_trq_hot_min } \\
\text { _simu }\end{array}$ \\
\hline
\end{tabular}

Table 7. Engine Control—Low-Level Transient Models

\begin{tabular}{|c|c|c|c|c|c|}
\hline Model Name & Description & Powertrains Used & Compatible Plant Models & Inputs & Outputs \\
\hline eng_ctrl_trs_au & $\begin{array}{l}\text { Determines } \\
\text { appropriate engine } \\
\text { torque on basis of } \\
\text { engine mode (idling, } \\
\text { shift in progress, } \\
\text { engine starting, etc.) }\end{array}$ & $\begin{array}{l}\text { Conventional and } \\
\text { hybrid powertrain } \\
\text { with automatic } \\
\text { transmission }\end{array}$ & $\begin{array}{l}\text { eng_plant_hot_map_ci } \\
\text { eng_plant_hot_map_si } \\
\text { eng_plant_hot_map_cs }\end{array}$ & $\begin{array}{l}\text { gb_ctrl_trs_eng_mode_simu } \\
\text { eng_ctrl_dmd_trq_simu } \\
\text { eng_ctrl_cmd_on_simu } \\
\text { eng_plant_spd_out_simu } \\
\text { accmech_plant_trq_simu } \\
\text { cpl plant cmd simu }\end{array}$ & $\begin{array}{l}\text { eng_ctrl_trs_trq_simu } \\
\text { eng_ctrl_trs_on_simu }\end{array}$ \\
\hline
\end{tabular}




\begin{tabular}{|c|c|c|c|c|c|}
\hline Model Name & Description & Powertrains Used & Compatible Plant Models & Inputs & Outputs \\
\hline & & & & $\begin{array}{l}\text { drv_accel_dmd_simu } \\
\text { gb_ctrl_trs_previous_gear_simu } \\
\text { gb_ctrlddmd_gear_simu } \\
\text { gb_ctrl_trs_sit_in_progress_simu }\end{array}$ & \\
\hline $\begin{array}{l}\text { eng_ctrl_trs_au_w } \\
\text { ith_str_fco }\end{array}$ & $\begin{array}{l}\text { Determines } \\
\text { appropriate engine } \\
\text { torque on basis of } \\
\text { engine mode (idling, } \\
\text { shift in progress, } \\
\text { engine starting, etc.) }\end{array}$ & $\begin{array}{l}\text { Conventional } \\
\text { powertrain with } \\
\text { automatic } \\
\text { transmission with } \\
\text { fuel cut-off }\end{array}$ & $\begin{array}{l}\text { eng_plant_hot_map_si_fuel_c } \\
\text { ut_off }\end{array}$ & $\begin{array}{l}\text { gb_ctrl_trs_eng_mode_simu } \\
\text { eng_ctrl_dmd_trq_simu } \\
\text { eng_ctrl_cmd_on_simu } \\
\text { eng_plant_spd_out_simu } \\
\text { accmeh_plant_tr_simu } \\
\text { cpl_plant_cmd_simu } \\
\text { drv_accel_dmd_simu } \\
\text { gb_plant_gear_simu } \\
\text { gb_ctrl_dmd_gear_simu } \\
\text { gb_ctrltrs_sft_in_progress_simu } \\
\text { drv_brk_dmd_simu } \\
\text { gen_plant_trq_out_simu } \\
\text { chas_plant_lin_spd_out_simu }\end{array}$ & $\begin{array}{l}\text { eng_ctrl_trs_trq_simu } \\
\text { eng_ctrl_trs_on_simu } \\
\text { eng_ctrlttrs_fuel_cut_off_simu }\end{array}$ \\
\hline $\begin{array}{l}\text { eng_ctrl_trs_au_w } \\
\text { ith_str_fco_with_v } \\
\text { pc }\end{array}$ & $\begin{array}{l}\text { Determines } \\
\text { appropriate engine } \\
\text { torque on the basis of } \\
\text { engine mode (idling, } \\
\text { shift in progress, } \\
\text { engine starting, etc.) }\end{array}$ & $\begin{array}{l}\text { Hybrid powertrain } \\
\text { with automatic } \\
\text { transmission with } \\
\text { fuel cut-off }\end{array}$ & $\begin{array}{l}\text { eng_plant_hot_map_si_fuel_c } \\
\text { ut_off }\end{array}$ & $\begin{array}{l}\text { eng_mode } \\
\text { vpc_eng_trq_dmd } \\
\text { vpc_eng_on_dmd } \\
\text { eng_spd } \\
\text { accmech_trq } \\
\text { cpl_position } \\
\text { drv_acc_pedal } \\
\text { previous_gear } \\
\text { gb_gear_dmd } \\
\text { shift_in_progress } \\
\text { drv_brk_dmd }\end{array}$ & $\begin{array}{l}\text { eng_ctrl_trs_trq_simu } \\
\text { eng_ctrl_trs_on_simu } \\
\text { eng_ctrlttrs_fuel_cut_off_simu }\end{array}$ \\
\hline $\begin{array}{l}\text { eng_ctrl_trs_au_w } \\
\text { ith_str_fco_with_v } \\
\text { pc_cyldeac }\end{array}$ & $\begin{array}{l}\text { Determines } \\
\text { appropriate engine } \\
\text { torque on the basis of } \\
\text { engine mode (idling, } \\
\text { shift in progress, } \\
\text { engine starting, etc.) }\end{array}$ & $\begin{array}{l}\text { Hybrid powertrain } \\
\text { with automatic } \\
\text { transmission with } \\
\text { fuel cut-off and } \\
\text { cylinder deactivation }\end{array}$ & $\begin{array}{l}\text { eng_plant_hot_map_si_fuel_c } \\
\text { ut_off_with_cylinder_deac }\end{array}$ & $\begin{array}{l}\text { eng_mode } \\
\text { vpc_eng_trq_dmd } \\
\text { vpc_eng_on_dmd } \\
\text { eng_spd } \\
\text { accmech_trq } \\
\text { cpl_position } \\
\text { drv_acc_pedal } \\
\text { previous_gear } \\
\text { gb_gear_dmd } \\
\text { shift_in_progress } \\
\text { drv_brk_dmd }\end{array}$ & $\begin{array}{l}\text { eng_ctrl_trs_trq_simu } \\
\text { eng_ctrl_trs_on_simu } \\
\text { eng_ctrl_trs_fuel_cut_off_simu } \\
\text { eng_ctrl_trs_cylin_deac_state } \\
\text { simu }\end{array}$ \\
\hline
\end{tabular}




\begin{tabular}{|c|c|c|c|c|c|}
\hline Model Name & Description & Powertrains Used & Compatible Plant Models & Inputs & Outputs \\
\hline $\begin{array}{l}\text { eng_ctrl_trs_au_w } \\
\text { ith_str_fco_therm } \\
\text { al }\end{array}$ & $\begin{array}{l}\text { Determines } \\
\text { appropriate engine } \\
\text { torque on the basis of } \\
\text { engine mode (idling, } \\
\text { shift in progress, } \\
\text { engine starting, etc.) }\end{array}$ & $\begin{array}{l}\text { Conventional } \\
\text { powertrain with } \\
\text { automatic } \\
\text { transmission and an } \\
\text { engine with a } \\
\text { temperature model }\end{array}$ & $\begin{array}{l}\text { eng_plant_hot_map_si_therm } \\
\text { al }\end{array}$ & $\begin{array}{l}\text { gb_ctrl_trs_eng_mode_simu } \\
\text { eng_ctrl_dmd_trq_simu } \\
\text { eng_ctrl_cmd_on_simu } \\
\text { eng_plant_spd_out_simu } \\
\text { accmeh_plant_trq_simu } \\
\text { cpl_plant_cmd_simu } \\
\text { drv_accel_dmd_simu } \\
\text { gb_plant_gear_simu } \\
\text { gb_ctrl_dmd_gear_simu } \\
\text { gb_ctrl_trs_sft_in_progress_simu } \\
\text { drv_brk_dmd_simu } \\
\text { gen_plant_trq_out_simu } \\
\text { chas_plant_lin_spd_out_simu } \\
\text { eng_plant_temp_simu }\end{array}$ & $\begin{array}{l}\text { eng_ctrl_trs_trq_simu } \\
\text { eng_ctrl_trs_on_simu } \\
\text { eng_ctrl_trs_fuel_cut_off_simu }\end{array}$ \\
\hline $\begin{array}{l}\text { eng_ctrl_trs_fuel_ } \\
\text { cut_off_dm }\end{array}$ & $\begin{array}{l}\text { Determines } \\
\text { appropriate engine } \\
\text { torque on the basis of } \\
\text { engine mode (idling, } \\
\text { shift in progress, } \\
\text { engine starting, etc.) }\end{array}$ & $\begin{array}{l}\text { Conventional } \\
\text { powertrain with } \\
\text { manual transmission } \\
\text { with fuel cut-off }\end{array}$ & $\begin{array}{l}\text { eng_plant_hot_map_si_fuel_c } \\
\text { ut_off }\end{array}$ & $\begin{array}{l}\text { gb_ctrl_trs_eng_mode_simu } \\
\text { eng_ctrl_dmd_trq_simu } \\
\text { drv_key_on_dmd_simu } \\
\text { eng_plant_spd_out_simu } \\
\text { accmeh_plant_trq_simu } \\
\text { gb_plant_gear_simu } \\
\text { cpl_plant_cmd_simu } \\
\text { gb_ctrl_dmd_gear_simu } \\
\text { drv_accel_dmd_simu } \\
\text { drv_brk_dmd_simu } \\
\text { cpl_plant_spd_out_simu }\end{array}$ & $\begin{array}{l}\text { eng_ctrl_trs_trq_simu } \\
\text { eng_ctrl_trs_on_simu } \\
\text { eng_ctrl_trs_fuel_cut_off_simu }\end{array}$ \\
\hline $\begin{array}{l}\text { eng_ctrl_trs_ct_wi } \\
\text { th_str_fco }\end{array}$ & $\begin{array}{l}\text { Determines } \\
\text { appropriate engine } \\
\text { torque on the basis of } \\
\text { engine mode (idling, } \\
\text { shift in progress, } \\
\text { engine starting, etc.) }\end{array}$ & $\begin{array}{l}\text { Conventional } \\
\text { powertrain with a } \\
\text { CVT and with fuel } \\
\text { cut-off }\end{array}$ & $\begin{array}{l}\text { eng_plant_hot_map_si_fuel_c } \\
\text { ut_off }\end{array}$ & $\begin{array}{l}\text { gb_ctrl_trs_eng_mode_simu } \\
\text { eng_ctrl_dmd_trq_simu } \\
\text { eng_ctrl_cmd_on_simu } \\
\text { eng_plant_spd_out_simu } \\
\text { accmeh_plant_trq_simu } \\
\text { cpl_plant_cmd_simu } \\
\text { drv_accel_dmd_simu } \\
\text { gb_ctrl_trs_cpl_mode_simu } \\
\text { drv_brk_dmd } \\
\text { gen_trq_out } \\
\text { veh_speed }\end{array}$ & $\begin{array}{l}\text { eng_ctrl_trs_trq_simu } \\
\text { eng_ctrl_trs_on_simu } \\
\text { eng_ctrl_trs_fuel_cut_off_simu }\end{array}$ \\
\hline
\end{tabular}




\begin{tabular}{|c|c|c|c|c|c|}
\hline Model Name & Description & Powertrains Used & Compatible Plant Models & Inputs & Outputs \\
\hline $\begin{array}{l}\text { eng_ctrl_trs_dct_ } \\
\text { with_str_fco }\end{array}$ & $\begin{array}{l}\text { Determines } \\
\text { appropriate engine } \\
\text { torque on the basis of } \\
\text { engine mode (idling, } \\
\text { shift in progress, } \\
\text { engine starting, etc.) }\end{array}$ & $\begin{array}{l}\text { Conventional } \\
\text { powertrain with a } \\
\text { DCT and with fuel } \\
\text { cut-off }\end{array}$ & $\begin{array}{l}\text { eng_plant_hot_map_si_fuel_c } \\
\text { ut_off }\end{array}$ & $\begin{array}{l}\text { gb_ctrl_trs_eng_mode_simu } \\
\text { eng_ctrl_dmd_trq_simu } \\
\text { eng_ctrl_cmd_on_simu } \\
\text { eng_plant_spd_out_simu } \\
\text { accmeh_plant_trq_simu } \\
\text { cpl_plant_cmd1_simu } \\
\text { cpl_plant_cmd2_simu } \\
\text { drv_accel_dmd_simu } \\
\text { gb_plant_spd_out_simu } \\
\text { gb_ctrl_dmd_gear_simu } \\
\text { gb_ctrltrs_sft_in_progress_simu } \\
\text { gen_plant_gear_simu } \\
\text { drv_brk_dmd_simu }\end{array}$ & $\begin{array}{l}\text { eng_ctrl_trs_trq_simu } \\
\text { eng_ctrl_trs_on_simu } \\
\text { eng_ctrl_trs_fuel_cut_off_simu }\end{array}$ \\
\hline eng_ctrl_trs_split & $\begin{array}{l}\text { Limits engine torque } \\
\text { to the max engine } \\
\text { capability }\end{array}$ & $\begin{array}{l}\text { Hybrid configuration } \\
\text { with a power-split } \\
\text { powertrain, including } \\
2 \text { times } 2 \text {-wheel } \\
\text { drive, extended } \\
\text { range, and multi } \\
\text { mode vehicles }\end{array}$ & $\begin{array}{l}\text { eng_plant_hot_map_si } \\
\text { eng_plant_hot_map_ci } \\
\text { eng_plant_hot_map_si_therm } \\
\text { al }\end{array}$ & $\begin{array}{l}\text { vpc_eng_trq_dmd_simu } \\
\text { eng_ctrl_cstr_trq_hot_max_simu } \\
\text { vpc_eng_on_dmd_simu }\end{array}$ & $\begin{array}{l}\text { eng_ctrl_trs_trq_simu } \\
\text { eng_ctrl_trs_on_simu }\end{array}$ \\
\hline eng_ctrl_trs_amt & $\begin{array}{l}\text { Determines } \\
\text { appropriate engine } \\
\text { torque on the basis of } \\
\text { engine mode (idling, } \\
\text { shift in progress, } \\
\text { engine starting, etc.) }\end{array}$ & $\begin{array}{l}\text { HEV and PHEV } \\
\text { powertrain with an } \\
\text { automated manual } \\
\text { transmission }\end{array}$ & eng_plant_hot_map_si & $\begin{array}{l}\text { gb_ctrl_trs_eng_mode_simu } \\
\text { eng_ctrl_dmd_trq_simu } \\
\text { eng_ctrl_cmd_on_simu } \\
\text { eng_plant_spd_out_simu } \\
\text { accmech_plant_trq_simu } \\
\text { cpl_plant_cmd_simu } \\
\text { drv_accel_dmd_simu } \\
\text { gb_plant_spd_out_simu } \\
\text { gb_ctrl_dmd_gear_simu } \\
\text { gb_ctrltrs_sft_in_progress_simu } \\
\text { gb_plant_gear_simu }\end{array}$ & $\begin{array}{l}\text { eng_ctrl_trs_trq_simu } \\
\text { eng_ctrl_trs_on_simu }\end{array}$ \\
\hline eng_ctrl_trs_ct & $\begin{array}{l}\text { Determines } \\
\text { appropriate engine } \\
\text { torque on the basis of } \\
\text { engine mode (idling, } \\
\text { shift in progress, } \\
\text { engine starting, etc.) }\end{array}$ & $\begin{array}{l}\text { Parallel hybrid } \\
\text { powertrain with CVT }\end{array}$ & eng_plant_hot_map_si & $\begin{array}{l}\text { eng_ctrl_trs_eng_mode_simu } \\
\text { eng_ctrl_dmd_trq_simu } \\
\text { eng_ctrl_cmd_on_simu } \\
\text { eng_plant_spd_out_simu } \\
\text { accmech_plant_trq_simu } \\
\text { cpl_plant_cmd_simu } \\
\text { drv_accel_dmd_simu } \\
\text { gb_ctrl_trs_cpl_mode_simu }\end{array}$ & $\begin{array}{l}\text { eng_ctrl_trs_trq_simu } \\
\text { eng_ctrl_trs_on_simu }\end{array}$ \\
\hline
\end{tabular}




\begin{tabular}{|c|c|c|c|c|c|}
\hline Model Name & Description & Powertrains Used & Compatible Plant Models & Inputs & Outputs \\
\hline $\begin{array}{l}\text { eng_ctrl_trs_au_c } \\
\text { ylinder_deac_with } \\
\text { _str_fco }\end{array}$ & $\begin{array}{l}\text { Determines } \\
\text { appropriate engine } \\
\text { torque on the basis of } \\
\text { engine mode (idling, } \\
\text { shift in progress, } \\
\text { engine starting, etc.) }\end{array}$ & $\begin{array}{l}\text { Conventional } \\
\text { powertrain with an } \\
\text { automatic } \\
\text { transmission, fuel } \\
\text { cut-off, and cylinder } \\
\text { deactivation }\end{array}$ & $\begin{array}{l}\text { eng_plant_hot_map_si_fuel_c } \\
\text { ut_off_with_cylinder_deac }\end{array}$ & $\begin{array}{l}\text { eng_mode } \\
\text { vpc_eng_trq_dmd } \\
\text { vpc_eng_on_dmd } \\
\text { eng_spd } \\
\text { accmech_trq } \\
\text { cpl_position } \\
\text { drv_acc_pedal } \\
\text { previous_gear } \\
\text { gb_gear_dmd } \\
\text { shift_in_progress } \\
\text { drv_brk_dmd } \\
\text { gen_trq_out } \\
\text { veh_speed }\end{array}$ & $\begin{array}{l}\text { eng_ctrl_trs_trq_simu } \\
\text { eng_ctrl_trs_on_simu } \\
\text { eng_ctrl_trs_fuel_cut_off_simu } \\
\text { eng_ctrl_trs_cylin_deac_state } \\
\text { simu }\end{array}$ \\
\hline
\end{tabular}




\subsection{Autonomie Validation}

Argonne has been validating vehicle models for almost 30 years, leveraging vehicle dynamometer test data from the AMTL. Test data have been collected at Argonne from more than 60 vehicles, ranging from model year 2000 to the present. Signals were collected on each vehicle for specific control analysis: component efforts (torque, current, etc.) and flow (rotational speed, linear speed, etc.), as well as temperatures and direct fuel-flow measurement collected using sensors and high-speed controller area networks (CANs). These measurements were integrated and aligned into a single data acquisition system. Some additional parameters were estimated on the basis of measured data and other advanced vehicle technology (e.g., electric machine current as estimated from measured speed, torque and voltage). Each individual model was then independently validated. Vehicle system model validation was quantified using normalized cross-correlation power (NCCP) [19], over a large number of cycles.

As a result, a large number of Autonomie vehicle models have been validated within test-to-test repeatability for a wide range of technologies and powertrain configurations. The following section provides some validation examples, using AMTL data.

Below is a subset of references for vehicle and component model validations in Autonomie over the years:

- $\quad$ Stutenberg, K., Kim, N., Russo, D. M., Islam, E., Kim, K., Lohse-Busch, H., Rousseau, A., Vijayagopal, R. (2021, July). Vehicle technology assessment, model development, and validation of a 2018 Honda Accord LX with a 1.5L I4 and continuously variable transmission (Report No. DOT HS 813 159). National Highway Traffic Safety Administration.

- Stutenberg, K., Kim, N., Russo, D. M., Islam, E., Kim, K., Lohse-Busch, H., Rousseau, A., \& Vijayagopal, R. (2021, July). Vehicle technology assessment, model development and validation of a 2018 Toyota Camry XLE with a 2.5L I4 and 8-speed automatic transmission (Report No. DOT HS 813 160). National Highway Traffic Safety Administration.

- Stutenberg, K., Kim, N., Russo, D. M., Islam, E., Lohse-Busch, H., Rousseau, A., \& Vijayagopal, R. (2021, July). Vehicle technology assessment, model development, and validation of a 2019 Acura MDX Sport Hybrid (Report No. DOT HS 813 161). National Highway Traffic Safety Administration.

- Jehlik, F., Kim, N., Islam, E., Lohse-Busch, H., Rousseau, A., Stutenberg, K., \& Vijayagopal, R. (2021, July). Vehicle technology assessment, model development, and validation of a 2019 Infiniti QX50 (Report No. DOT HS 813 162). National Highway Traffic Safety Administration.

- N. Kim, S. Choi, J. Jeong, R. Vijayagopal, K. Stutenberg and A. Rousseau, "Vehicle Level Control Analysis for Voltec Powertrain." 30th International Electric Vehicle Symposium and Exhibition (EVS30), Stuttgart, Germany, October 9-11, 2017

- J. Jeong, W. Lee, N. Kim, K. Stutenberg, et al., "Control Analysis and Model Validation for BMW i3 Range Extender.” SAE Technical Paper 2017-01-1152, 2017, DOI:10.4271/2017-01-1152

- H. Son, N. Kim, S. Ko, A. Rousseau, and H. Kim, "Development of Performance Simulator for a HEV with CVT and Validation with Dynamometer Test Data." 28th International Electric Vehicle Symposium (EVS28), Kintex, South Korea, 2015

- N. Kim, N. Kim, and A. Rousseau, "Thermal Model Developments for Electrified Vehicles.” 28th International Electric Vehicle Symposium (EVS28), Kintex, South Korea, 2015

- N. Kim, J. Jeong, A. Rousseau, and H. Lohse-Busch, "Control Analysis and Thermal Model Development of PHEV." SAE 2015-01-1157, SAE World Congress, Detroit, April 15, 2015

- N. Kim, A. Rousseau, and H. Lohse-Busch, "Advanced Automatic Transmission Model Validation Using Dynamometer Test Data.” SAE 2014-01-1778, SAE World Congress, Detroit, April 2014 
- N. Kim, E. Rask and A. Rousseau, "Control Analysis under Different Driving Conditions for Peugeot 3008 Hybrid 4.” SAE 2014-01-1818, SAE World Congress, Detroit, April 2014

- D. Lee, A. Rousseau, E. Rask, "Development and Validation of the Ford Focus BEV Vehicle Model." 2014-01-1809, SAE World Congress, Detroit, April 2014

- N. Kim, A. Rousseau, D. Lee, and H. Lohse-Busch, "Thermal Model Development \& Validation for the 2010 Toyota Prius." 2014-01-1784, SAE World Congress, Detroit, April 2014

- N. Kim, N. Kim, A. Rousseau, and M. Duoba, "Validating Volt PHEV Model with Dynamometer Test Data using Autonomie." SAE 2013-01-1458, SAE World Congress, Detroit, April 2013

- N. Kim, A. Rousseau, and E. Rask, "Autonomie Model Validation with Test Data for 2010 Toyota Prius.” SAE 2012-01-1040, SAE World Congress, Detroit, April 2012

- N. Kim, R. Carlson, F. Jehlik, and A. Rousseau, "Tahoe HEV Model Development in PSAT." SAE paper 2009-01-1307, SAE World Congress, Detroit, April 2009

- Q. Cao, S. Pagerit, R. Carlson, and A. Rousseau, "PHEV Hymotion Prius model validation and control improvements." 23rd International Electric Vehicle Symposium (EVS23), Anaheim, CA, December 2007

- $\quad$ Rousseau, P. Sharer, S. Pagerit, and M. Duoba, "Integrating Data, Performing Quality Assurance, and Validating the Vehicle Model for the 2004 Prius Using PSAT." SAE paper 2006-01-0667, SAE World Congress, Detroit, April 2006

- M. Pasquier, A. Rousseau, and M. Duoba, "Validating Simulation Tools for Vehicle System Studies Using Advanced Control and Testing Procedures." 18th International Electric Vehicle Symposium (EVS18), Berlin, Germany, October 2001

- Rousseau, B. Deville, G. Zini, J. Kern, J. Anderson, and M. Duoba, "Honda Insight Validation Using PSAT." 01-FTT49, Future Transportation Technology Conference, Costa-Mesa, August 2001

- Rousseau and M. Pasquier, "Validation of a Hybrid Modeling Software (PSAT) Using Its Extension for Prototyping (PSAT-PRO)." Global Powertrain Congress, Detroit, June 2001.

\subsubsection{Conventional Vehicles}

The main focus of conventional vehicles' validation is the shifting algorithm, torque converter lockup and fuel cutoff. First, the simulated vehicle speed, engine speed, and engine torque are compared with test results. For example, Figure 107 shows the comparison for an automatic transmission on the UDDS cycle:

- Initial calibration (simulation 1)

- Calibrated algorithm using test data (simulation 2) 

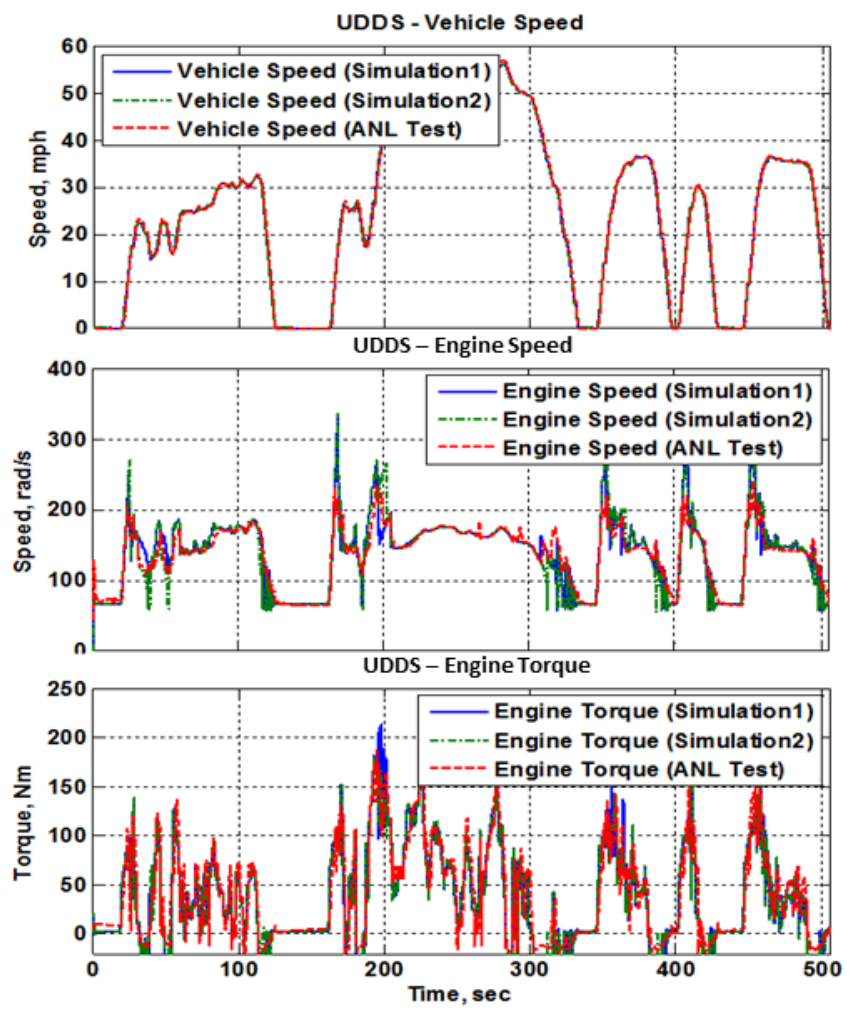

Figure 107. Simulation and testing results on UDDS cycle (0-505 s) for 2013 Sonata conventional $6 A T X$

In Figure 108, the gear numbers on the UDDS cycle are compared with the test results for both 6- and 8 -speed transmissions. Shifting performance in both simulations is close to the test results. Figure 109 demonstrates the robust calibration of the shifting algorithm in the new European driving cycle (NEDC).
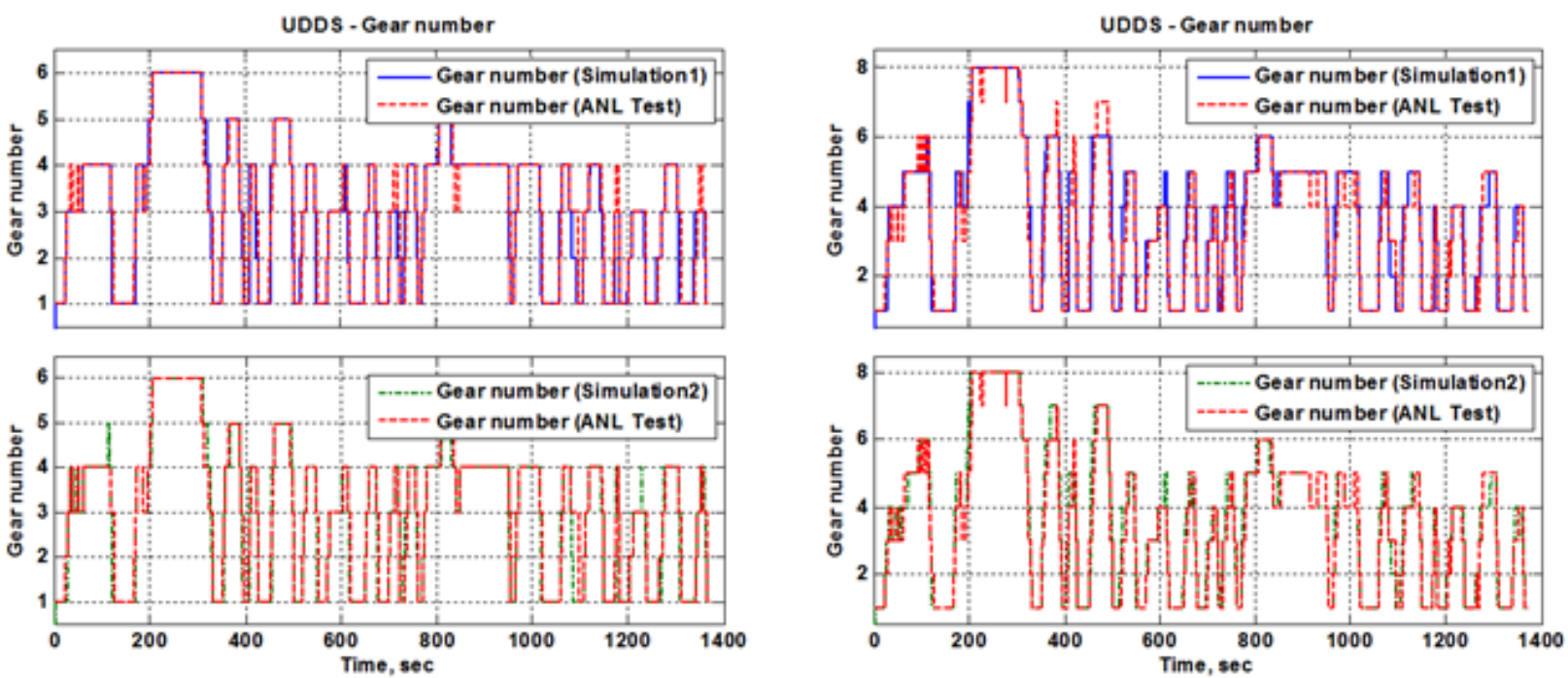

Figure 108. Shifting algorithm validation for 2013 Sonata conventional 6ATX (left) and 2013 Chrysler 300 8ATX (right) on the UDDS cycle (0-505 s) 

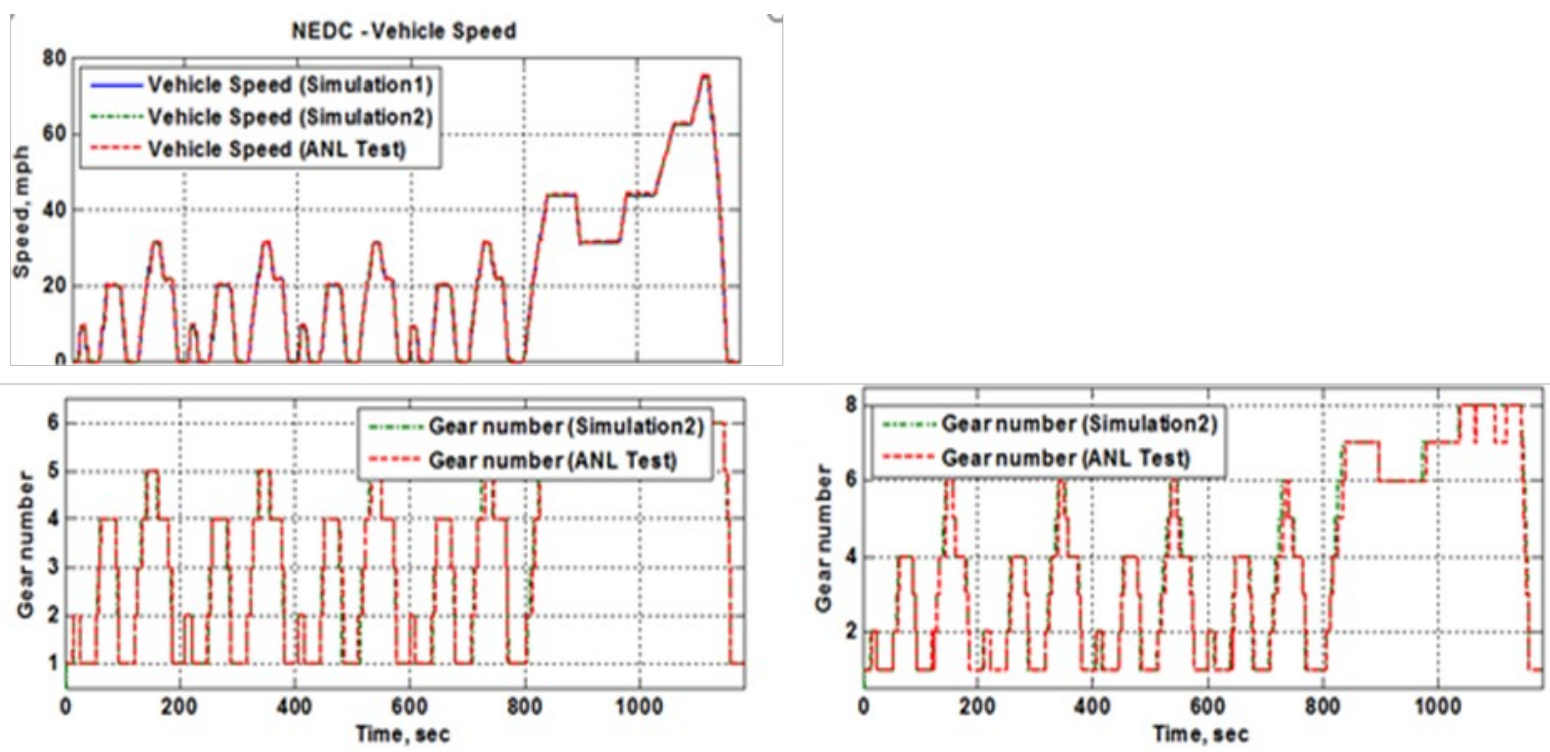

Figure 109. Shifting algorithm validation for 2012 Fusion conventional 6ATX (left) and 2013 Chrysler $3008 A T X$ (right) on the NEDC cycle

The CVT model and shifting control strategy developed in Autonomie were also validated by comparing the simulation results with the experimental results from Argonne's AMTL. Figure 110 shows the validation results for the 2012 Honda Civic HEV system on the UDDS and highway fuel economy (HWFET) cycles. The simulated vehicle speed, gear ratio, engine torque and battery SOC behave like the experimental results, demonstrating the validity of the simulation model and control strategy.
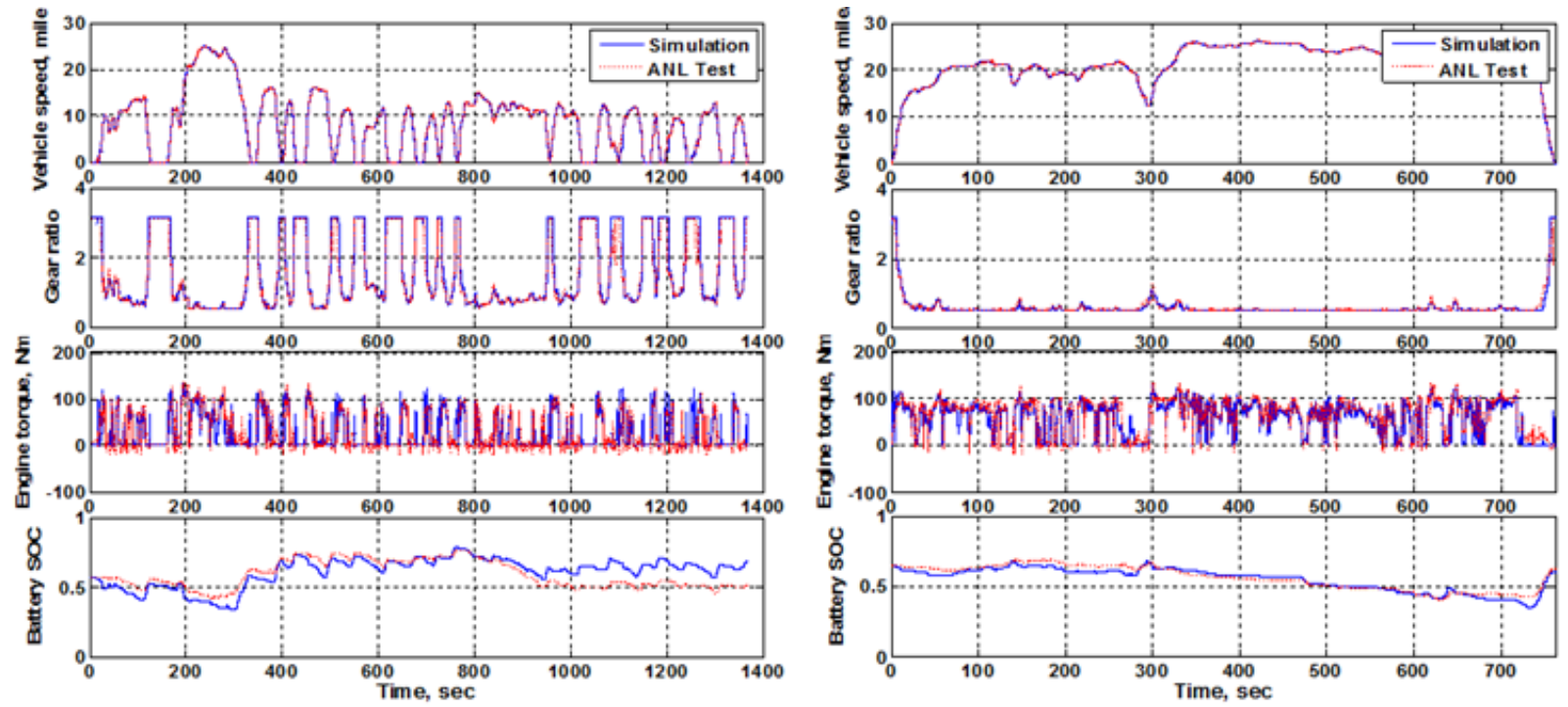

Figure 110. Comparison of simulation and test data for 2012 Honda Civic CVT HEV on UDDS (left) and HWFET (right) cycles

\subsubsection{Power-Split HEV}

Multiple versions of the power-split HEV have been tested and validated since early 2000. As with the other powertrains, we focus first on validating the component operating conditions throughout the 
driving cycles. For example, Figure 111 shows consistent engine ON/OFF, SOC control and component operating conditions (engine torque \& speed) for the 2010 Toyota Prius on the UDDS cycle. The latest Toyota Prius HEV has been similarly validated.
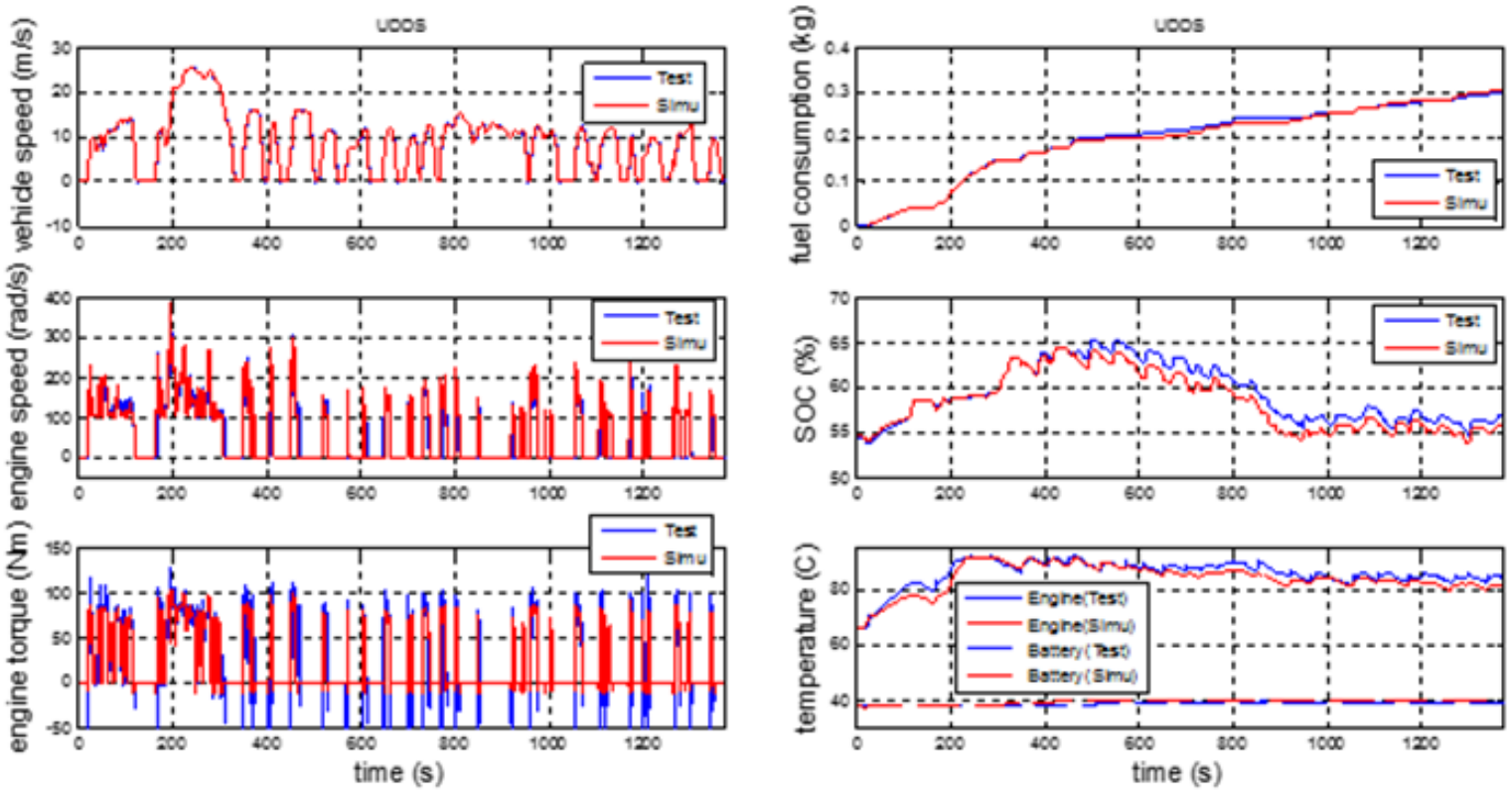

Figure 111. Results of simulation and testing on UDDS cycle for 2010 Toyota Prius HEV

\subsubsection{Pre-Transmission HEV}

The pre-transmission HEV control logic was validated using Argonne AMTL test data from the 2013 Jetta DCT hybrid. The simulation results for the vehicle speed, gear number, and battery SOC on the UDDS cycle, shown in Figure 112, showed good correlation with the test data.
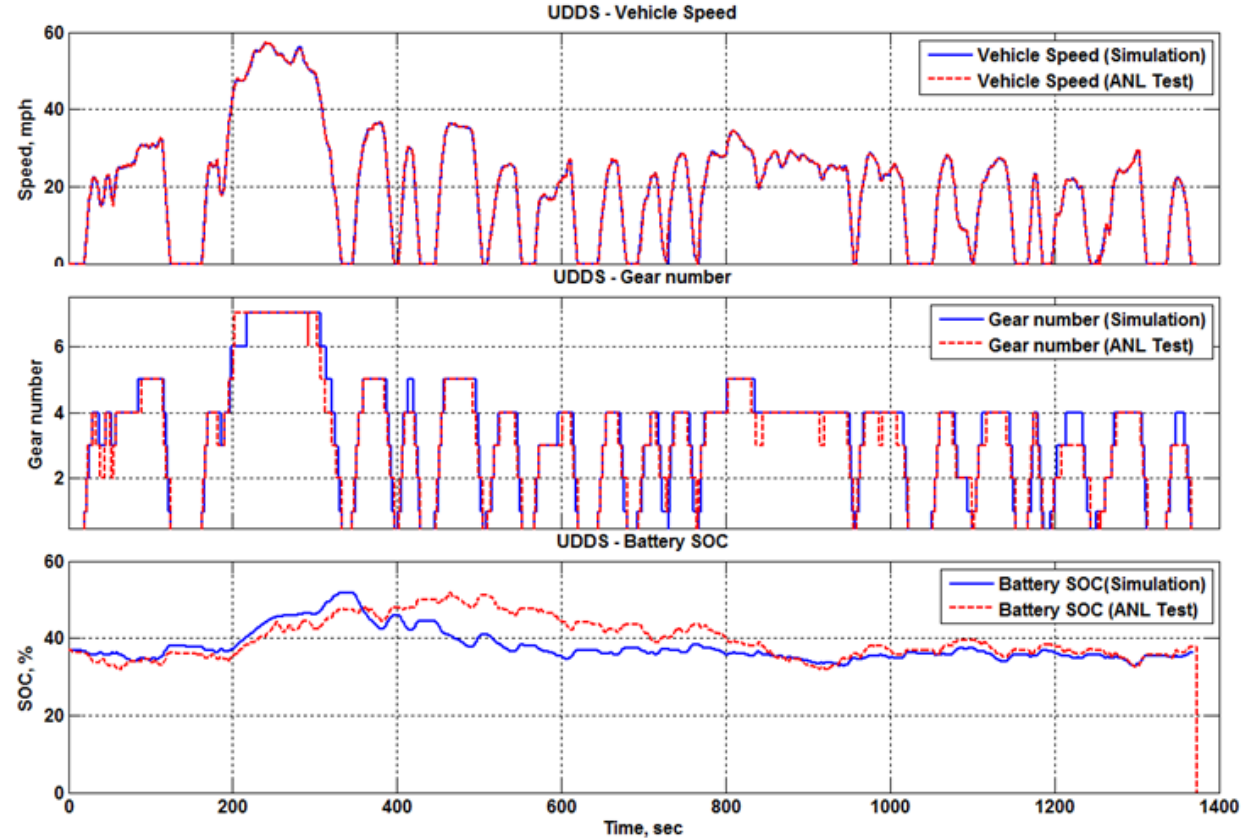
Figure 112. Results of simulation and testing on UDDS cycle for 2013 Jetta DCT hybrid

\subsubsection{Range-Extender PHEV}

The range-extender PHEV model was validated in different thermal conditions, using Argonne's AMTL test data from the second-generation Chevrolet Volt. The vehicle operating behavior, including vehicle speed, battery SOC, fuel consumption, and engine speed, torque, and temperature under ambient temperature were successfully compared with the testing results shown in Figure 113.
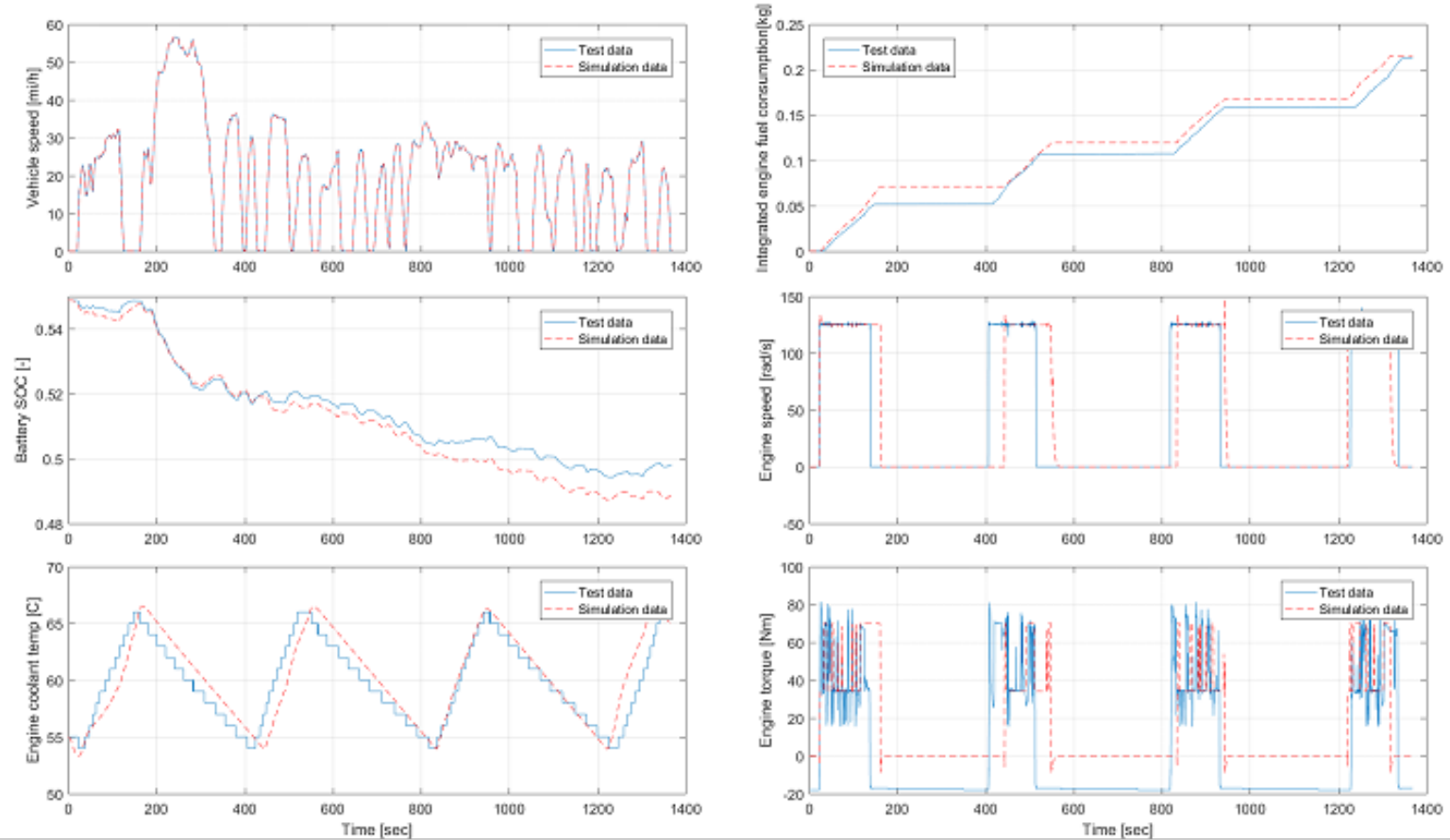

Figure 113. Results of simulation and testing on UDDS cycle for 2012 Chevrolet Volt PHEV

Additional configurations including start-stop, blended PHEV, and battery electric vehicles (BEVs) have also been validated. 


\section{VEHICLE AND COMPONENT ASSUMPTIONS}

Directed by NHTSA and based on the feedback received from reviewers on the midterm TAR, a number of steps were taken to improve the overall vehicle simulation process and consider additional vehicles to better replicate fleets in both existing and future markets.

The primary focus was to update the vehicle and component assumptions to represent the latest vehicles in the market. An extensive analysis was carried out on the existing vehicles in the market to evaluate vehicle parameters and component weights across the vehicle classifications considered. In addition to vehicle parameters (frontal area, drag coefficient, etc.), individual component weight assumptions (body, chassis, interior, etc.) have been analyzed. The vehicle attribute parameters have been updated using Argonne's internal vehicle technical database. Finally, the vehicle component weights have been updated using A2Mac1 [1] teardown analysis of different representative vehicles.

This section elaborates on the method used to select each attribute. The attributes were defined across different vehicle classes and performance categories, with different transmission types and number of gears. Table 8 shows the different vehicle classifications and the definition of the different performance categories.

Table 8. Vehicle Classification and Performance Categories

\begin{tabular}{|l|l|c|}
\hline Vehicle Class & \multicolumn{1}{|c|}{$\begin{array}{c}\text { Performance } \\
\text { Category }\end{array}$} & 0-60 mph Time (s) \\
\hline Compact & Base / Premium & $10 / 8$ \\
\hline Midsize & Base / Premium & $9 / 6$ \\
\hline Small SUV & Base / Premium & $9 / 7$ \\
\hline Midsize SUV & Base / Premium & $10 / 7$ \\
\hline Pickup & Base / Premium & $7 / 7$ \\
\hline
\end{tabular}

Additional performance metrics have been developed:

- Gradeability: 6\% grade at $65 \mathrm{mph}$

- Payload: $900 \mathrm{~kg}$ (pickup base/premium only)

- Towing: $3000 \mathrm{~kg}$ (pickup base) and $4350 \mathrm{~kg}$ (pickup premium)

\subsection{Vehicle-Level Attributes Selection}

The assumptions for each vehicle class and performance category have been defined individually.

\subsubsection{Drag Coefficient}

The following section shows the distribution of the drag coefficient using the Argonne internal vehicle technology database. "ANL Value" represents the parameter value selected for the reference vehicle in Autonomie. Figure 114 shows the distribution of drag coefficient values for the compact (base) vehicle class. 


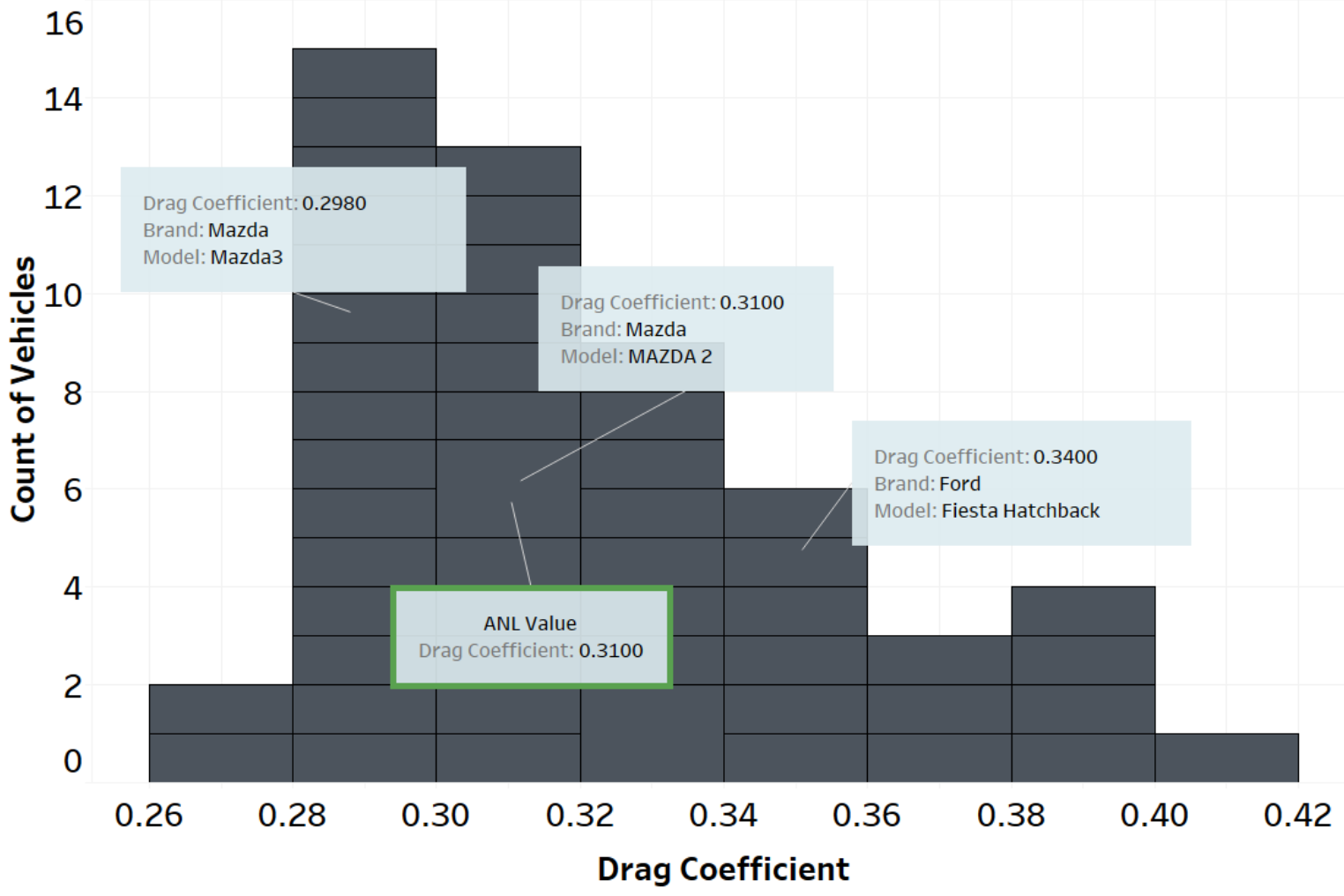

Figure 114. Distribution of drag coefficient values of compact (base) vehicle class

Such analyses were performed across the different vehicle classes and performance categories and the reference values selected. Table 9 summarizes the values defined for the drag coefficient of the reference vehicles for each vehicle class and performance category.

Table 9. Drag Coefficient Ratio Summary Table

\begin{tabular}{|l|l|c|}
\hline $\begin{array}{l}\text { Vehicle } \\
\text { Class }\end{array}$ & Performance Category & Reference Value \\
\hline Compact & Base/Premium & 0.31 \\
\hline Midsize & Base/Premium & 0.30 \\
\hline Small SUV & Base/Premium & 0.36 \\
\hline Midsize SUV & Base/Premium & 0.38 \\
\hline Pickup & Base/Premium & 0.42 \\
\hline
\end{tabular}

\subsubsection{Frontal Area}

A similar detailed analysis was performed to determine the frontal area values for the reference vehicle across different vehicle classifications and performance categories.

Table 10 summarizes values defined for the frontal area of the reference vehicles for the different vehicle classes and performance categories.

Table 10. Frontal Area Summary Table 


\begin{tabular}{|l|l|c|}
\hline \multicolumn{1}{|c|}{ Vehicle Class } & Performance Category & Reference Value $\mathbf{( m}^{\mathbf{2}} \mathbf{)}$ \\
\hline Compact & Base/Premium & 2.3 \\
\hline Midsize & Base/Premium & 2.35 \\
\hline Small SUV & Base/Premium & 2.65 \\
\hline Midsize SUV & Base/Premium & 2.85 \\
\hline Pickup & Base/Premium & 3.25 \\
\hline
\end{tabular}

\subsubsection{Rolling Resistance}

A constant value of 0.009 is used as the first coefficient term of the wheel rolling resistance for all classes and performance categories. The overall rolling resistance of the wheel is, however, speeddependent, with a factor of 0.00012 .

\subsection{Vehicle Component Weight Selection}

The vehicles in Autonomie are built from the ground up using individual component weights. Powertraindependent component weights (engine, motor, fuel cell system, high-power/energy battery, etc.) are updated as part of the sizing procedure. The following sections describe the process of selecting the nonpowertrain weights based on an analysis using the A2Mac1 database [1].

\subsubsection{Body Weight}

Figure 115 shows the distribution of body weights for the compact (base) vehicle class.

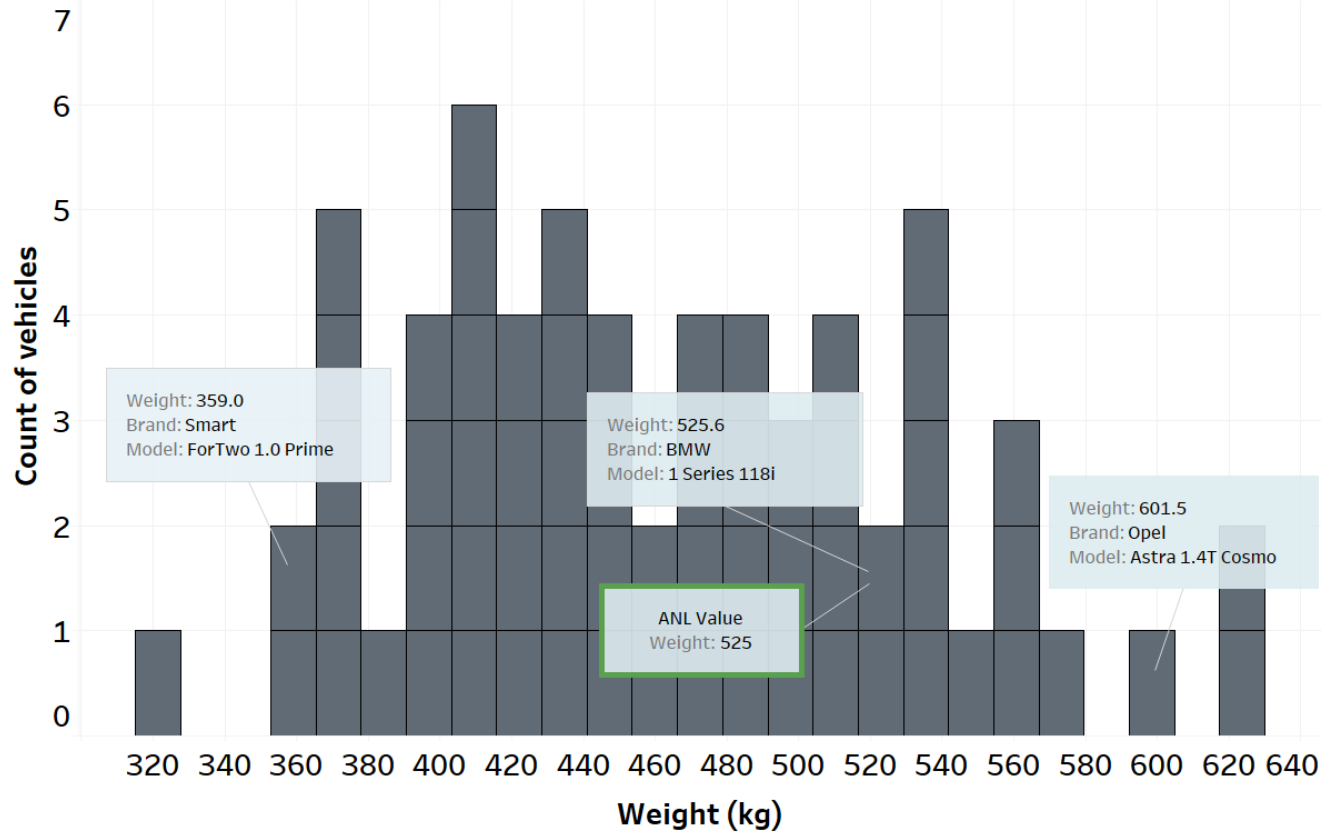

Figure 115. Body weight selection for compact (base) vehicles

Such analyses are performed across the different vehicle classes and performance categories and the reference values selected. 
Table 11 summarizes the values defined for the body weight of the reference vehicles for the different vehicle classes and performance categories.

Table 11. Body Weight Summary

\begin{tabular}{|l|l|c|}
\hline Vehicle Class & Performance Category & Reference value (kg) \\
\hline Compact & Base/Premium & 525 \\
\hline Midsize & Base/Premium & 650 \\
\hline Small SUV & Base/Premium & 650 \\
\hline Midsize SUV & Base/Premium & $650 / 750$ \\
\hline Pickup & Base/Premium & $650 / 800$ \\
\hline
\end{tabular}

\subsubsection{Chassis Weight}

Figure 116 shows the distribution of chassis weights for the compact (base) vehicle class.

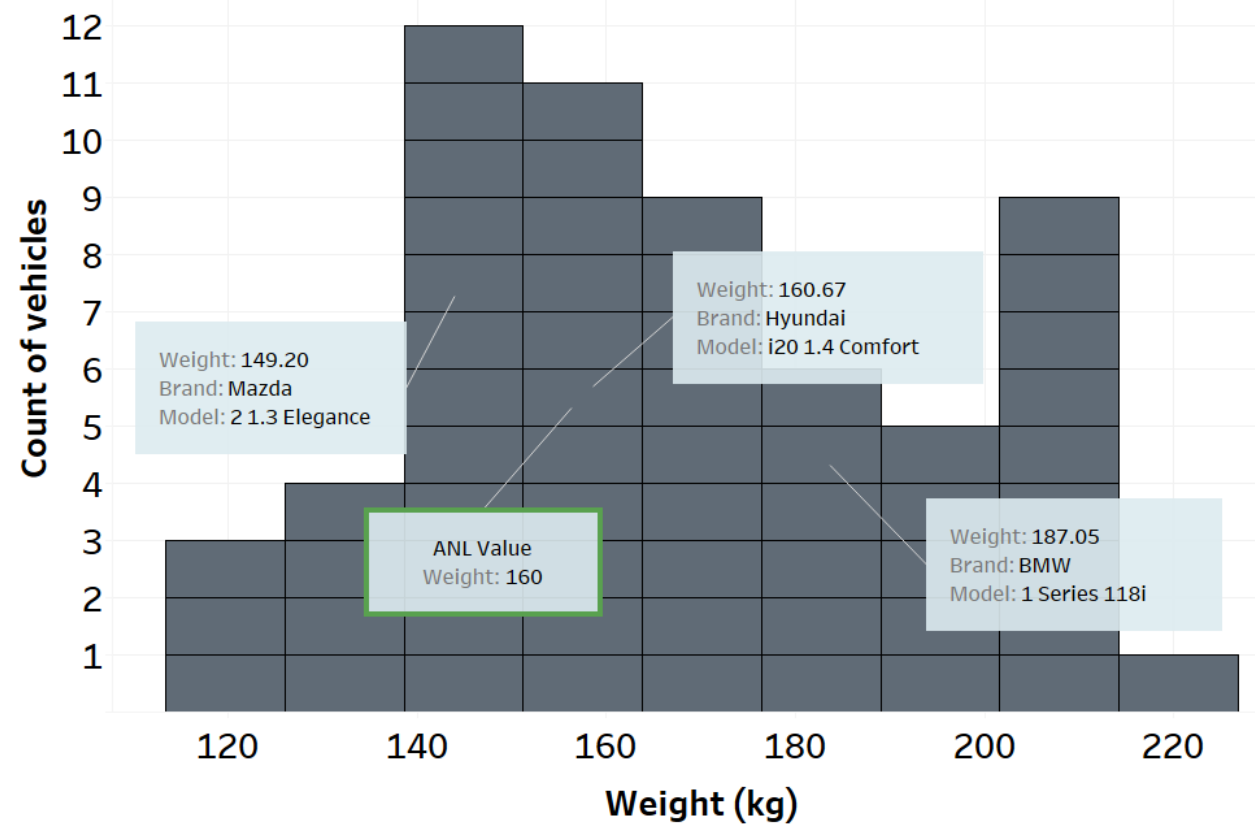

Figure 116. Chassis weight selection for compact (base) vehicles

These analyses are performed across the different vehicle classes and performance categories, and the reference values are selected.

Table 12 summarizes the values defined for the chassis weights of the reference vehicles for the different vehicle classes and performance categories.

Table 12. Chassis Weight Selection Summary

\begin{tabular}{|l|l|c|}
\hline Vehicle Class & Performance Category & Reference Value (kg) \\
\hline Compact & Base/Premium & 160 \\
\hline
\end{tabular}




\begin{tabular}{|l|l|c|}
\hline Vehicle Class & Performance Category & Reference Value (kg) \\
\hline Midsize & Base/Premium & 200 \\
\hline Small SUV & Base /Premium & 200 \\
\hline Midsize SUV & Base /Premium & $200 / 225$ \\
\hline Pickup & Base/Premium & $300 / 350$ \\
\hline
\end{tabular}

\subsubsection{Interior Weight}

Figure 117 shows the distribution of interior weights for the compact (base) vehicle class.

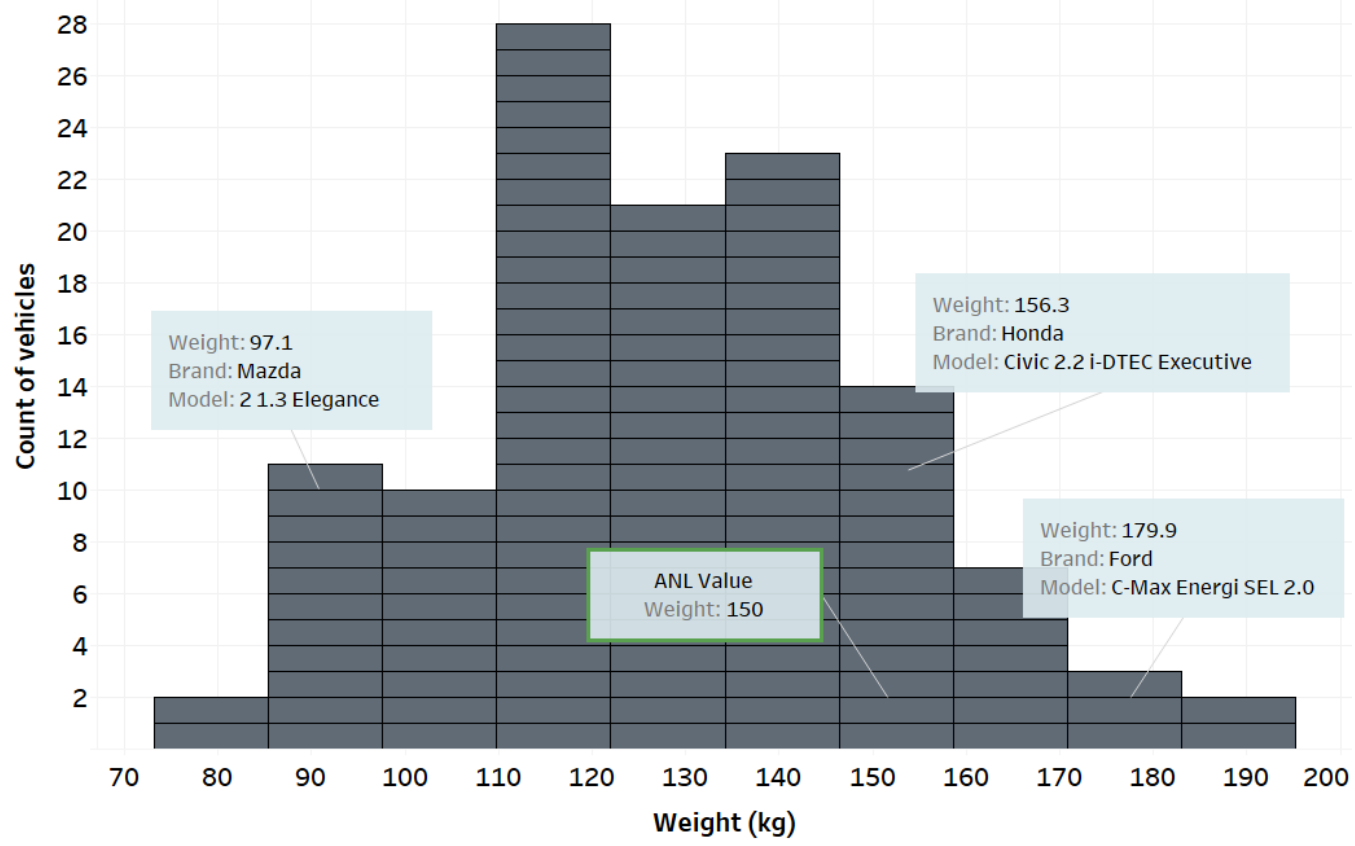

Figure 117. Interior weight selection for compact (base) vehicles

These analyses are performed across the different vehicle classes and performance categories, and the reference values are selected. Table 13 summarizes the values defined for the interior weights of the reference vehicles for the different vehicle classes and performance categories.

Table 13. Interior Weight Selection Summary

\begin{tabular}{|l|l|c|}
\hline Vehicle Class & Performance Category & Reference Value (kg) \\
\hline Compact & Base/Premium & $150 / 200$ \\
\hline Midsize & Base/Premium & $175 / 200$ \\
\hline Small SUV & Base/Premium & $180 / 220$ \\
\hline Midsize SUV & Base/Premium & $200 / 240$ \\
\hline Pickup & Base/Premium & $160 / 200$ \\
\hline
\end{tabular}




\subsubsection{Safety System Weight}

Figure 118 shows the distribution of safety-system weights for the compact (base) vehicle class.

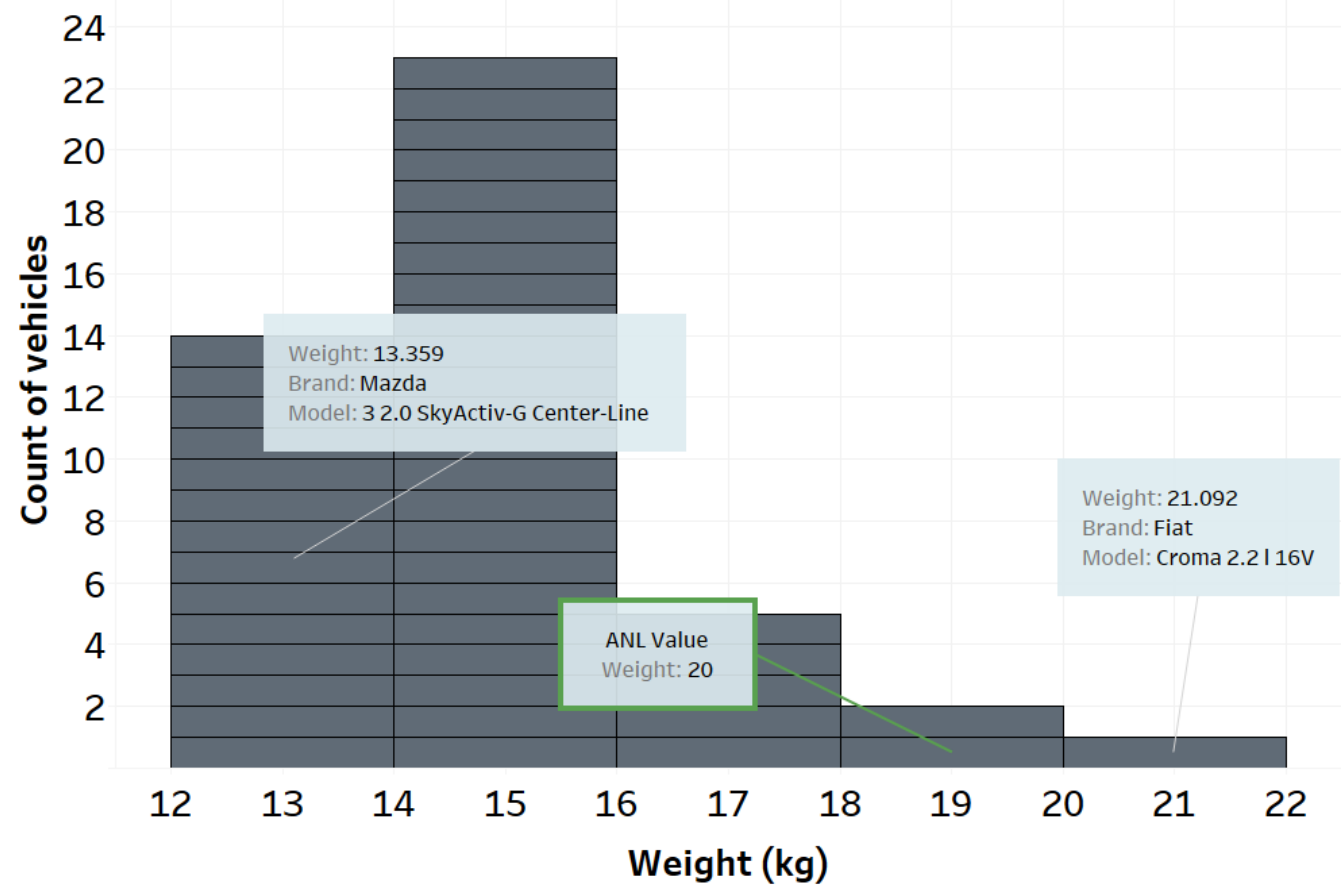

Figure 118. Safety system weight selection for compact (base) vehicle

These analyses are performed across the different vehicle classes and performance categories, and the reference values are selected. Table 14 summarizes the values defined for the safety-system weights of the reference vehicles for the different vehicle classes and performance categories.

Table 14. Safety System Weight Selection Summary

\begin{tabular}{|l|l|c|}
\hline Vehicle Class & Performance Category & Reference Value (kg) \\
\hline Compact & Base/Premium & $20 / 22$ \\
\hline Midsize & Base/Premium & $25 / 28$ \\
\hline Small SUV & Base/Premium & $25 / 28$ \\
\hline Midsize SUV & Base/Premium & 30 \\
\hline Pickup & Base/Premium & 30 \\
\hline
\end{tabular}




\subsubsection{Thermal System Weight}

Figure 119 shows the distribution of thermal-system weights for the compact (base) vehicle class.

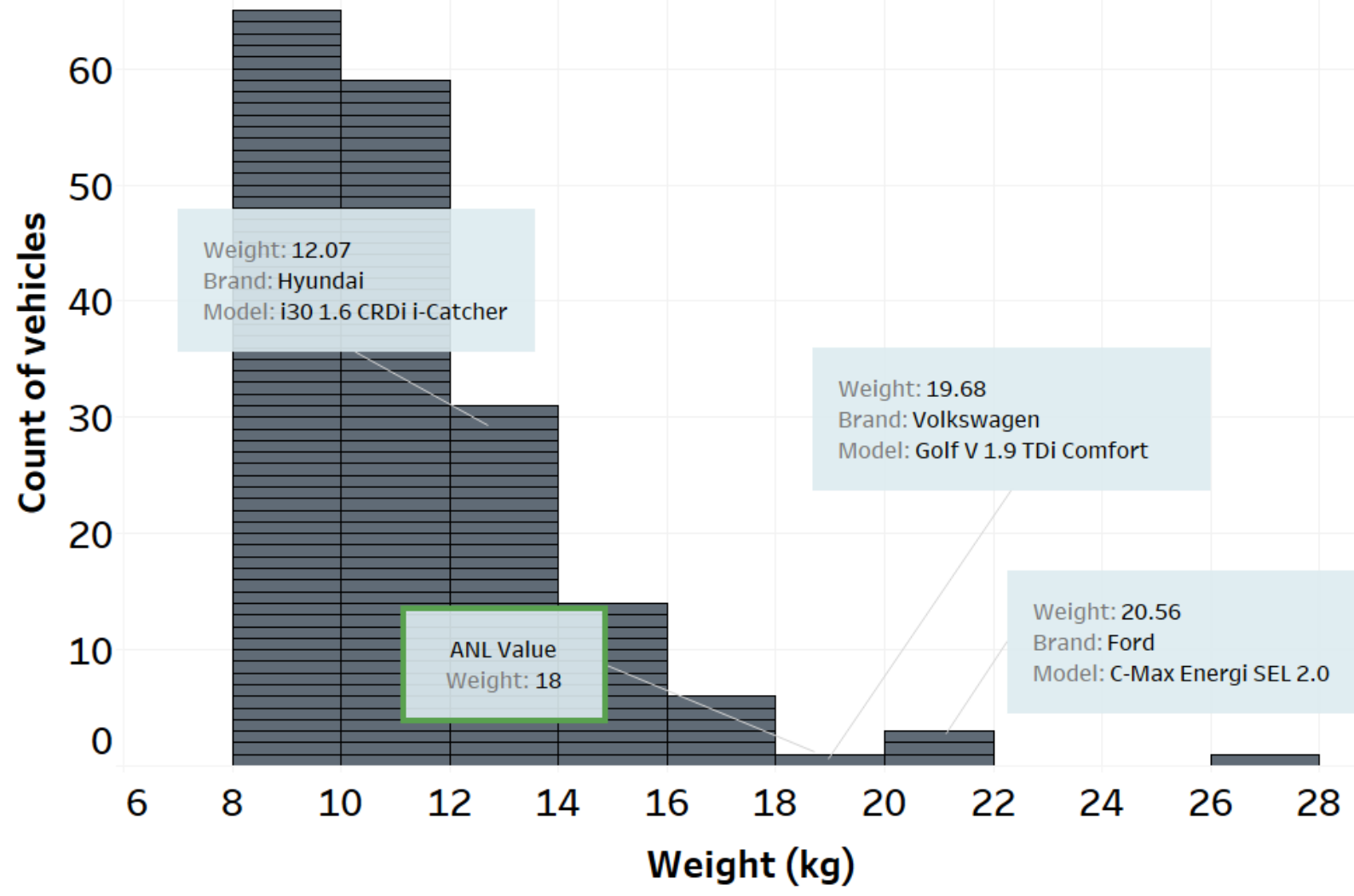

Figure 119. Thermal system weight selection for compact (base) vehicle

These analyses are performed across the different vehicle classes and performance categories, and the reference values are selected. Table 15 summarizes the values defined for the thermal system weights of the reference vehicles for the different vehicle classes and performance categories.

Table 15. Thermal System Weight Selection Summary

\begin{tabular}{|l|l|c|}
\hline Vehicle Class & Performance Category & Reference Value (kg) \\
\hline Compact & Base/Premium & $18 / 20$ \\
\hline Midsize & Base/Premium & $25 / 28$ \\
\hline Small SUV & Base/Premium & $25 / 28$ \\
\hline Midsize SUV & Base/Premium & 30 \\
\hline Pickup & Base/Premium & 30 \\
\hline
\end{tabular}




\subsubsection{Brake Mechanism Weight}

Figure 120 shows the distribution of brake mechanism weights for the compact (base) vehicle class.

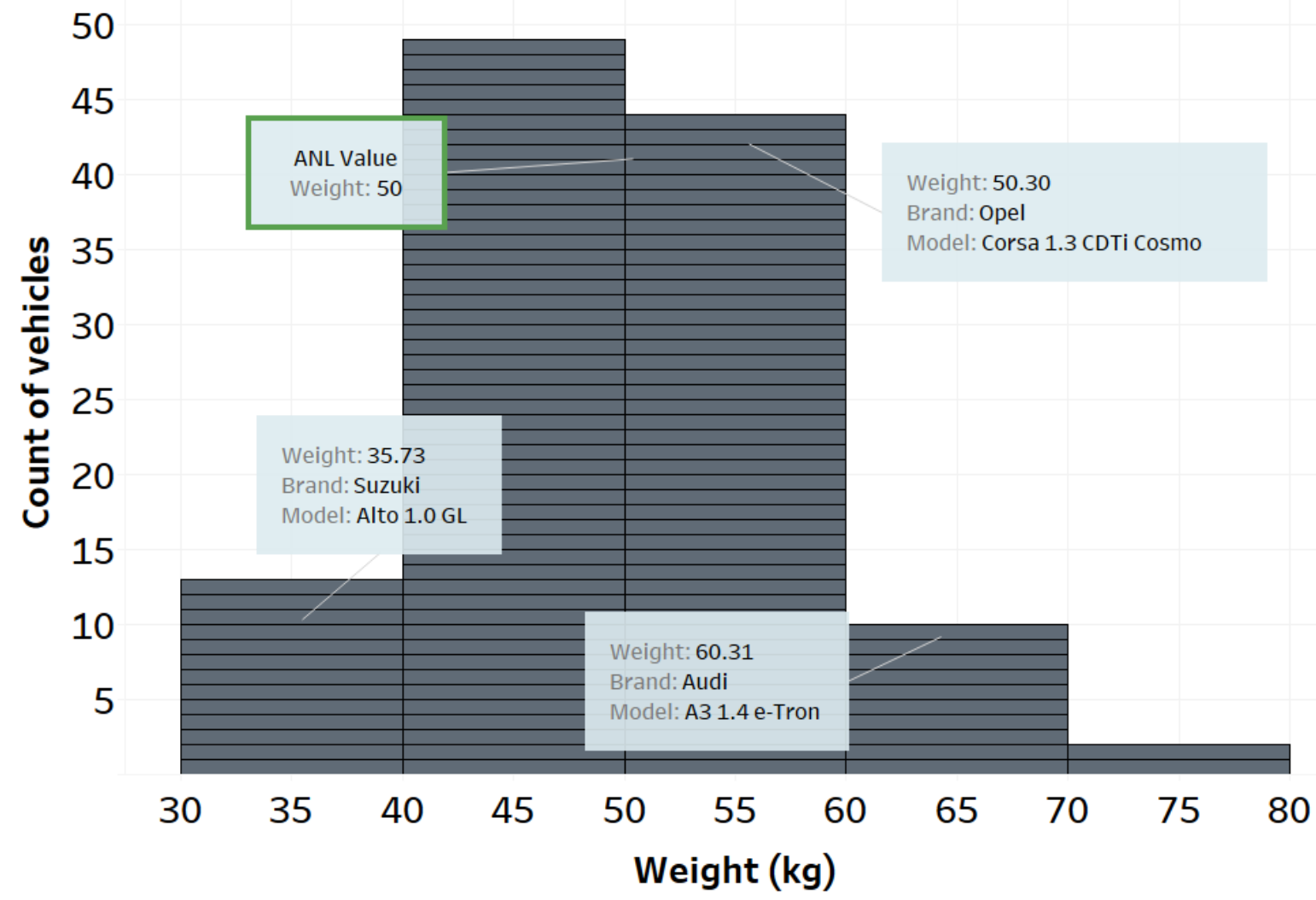

Figure 120. Brake mechanism weight selection for compact (base) vehicle

These analyses are performed across the different vehicle classes and performance categories, and the reference values are selected. Table 16 summarizes the values defined for the brake mechanism weights of the reference vehicles for the different vehicle classes and performance categories.

Table 16. Brake Mechanism Weight Selection Summary

\begin{tabular}{|l|l|c|}
\hline Vehicle Class & Performance Category & Reference Value (kg) \\
\hline Compact & Base/Premium & $50 / 55$ \\
\hline Midsize & Base/Premium & $60 / 65$ \\
\hline Small SUV & Base/Premium & $60 / 75$ \\
\hline Midsize SUV & Base/Premium & $70 / 75$ \\
\hline Pickup & Base/Premium & $90 / 95$ \\
\hline
\end{tabular}




\subsubsection{Steering System Weight}

Figure 121 shows the distribution of steering system weights for the compact (base) vehicle class.

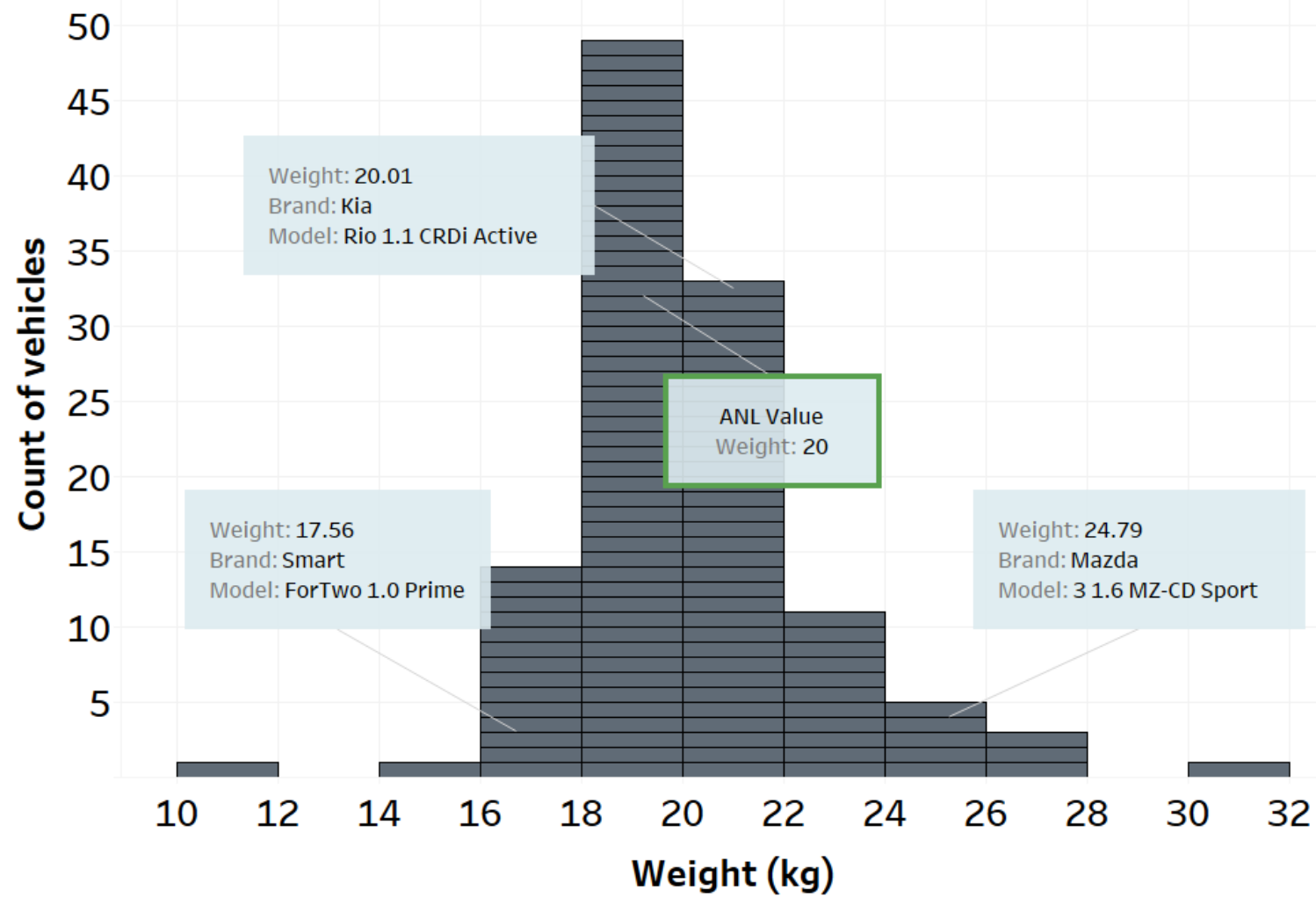

Figure 121. Steering system weight selection for compact (base) vehicle

These analyses are performed across the different vehicle classes and performance categories, and the reference values are selected. Table 17 summarizes the values defined for the steering system weights of the reference vehicles for the different vehicle classes and performance categories.

Table 17. Steering System Weight Selection Summary

\begin{tabular}{|l|l|c|}
\hline Vehicle Class & Performance Category & Reference Value (kg) \\
\hline Compact & Base/Premium & $20 / 22$ \\
\hline Midsize & Base/Premium & $25 / 28$ \\
\hline Small SUV & Base/Premium & $25 / 28$ \\
\hline Midsize SUV & Base/Premium & 30 \\
\hline Pickup & Base/Premium & 30 \\
\hline
\end{tabular}




\subsubsection{Electrical Accessories Weight}

Figure 122 shows the distribution of electrical accessories weights for the compact (base) vehicle class.

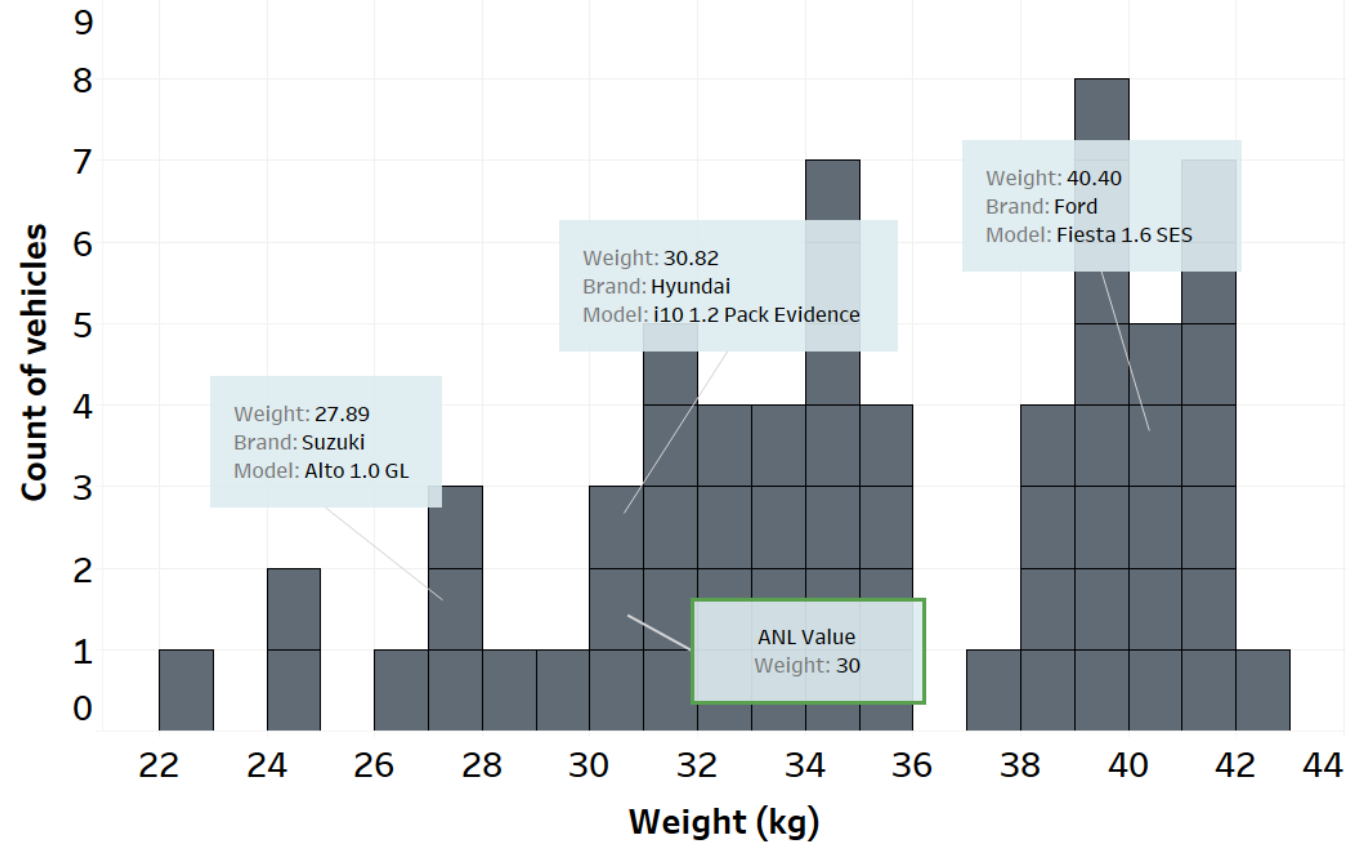

Figure 122. Electrical accessories weight selection for compact (base) vehicle

These analyses are performed across the different vehicle classes and performance categories, and the reference values are selected. Table 18 summarizes the values defined for the electrical accessories weights of the reference vehicles for the different vehicle classes and performance categories.

Table 18. Electrical Accessories Weight Selection Summary

\begin{tabular}{|l|l|c|}
\hline Vehicle Class & Performance Category & Reference Value (kg) \\
\hline Compact & Base/Premium & $30 / 35$ \\
\hline Midsize & Base/Premium & $30 / 40$ \\
\hline Small SUV & Base/Premium & $30 / 40$ \\
\hline Midsize SUV & Base/Premium & $30 / 50$ \\
\hline Pickup & Base/Premium & $80 / 100$ \\
\hline
\end{tabular}

\subsubsection{Engine Weight Determination}

For the current set of runs, a detailed analysis of engine weight was conducted on A2mac1. The analysis consisted of different engine technologies for the North American market in A2Mac1. Figure 123 shows the updated regression analysis performed across MY 2015-2020 in A2Mac1 for gasoline engines. 


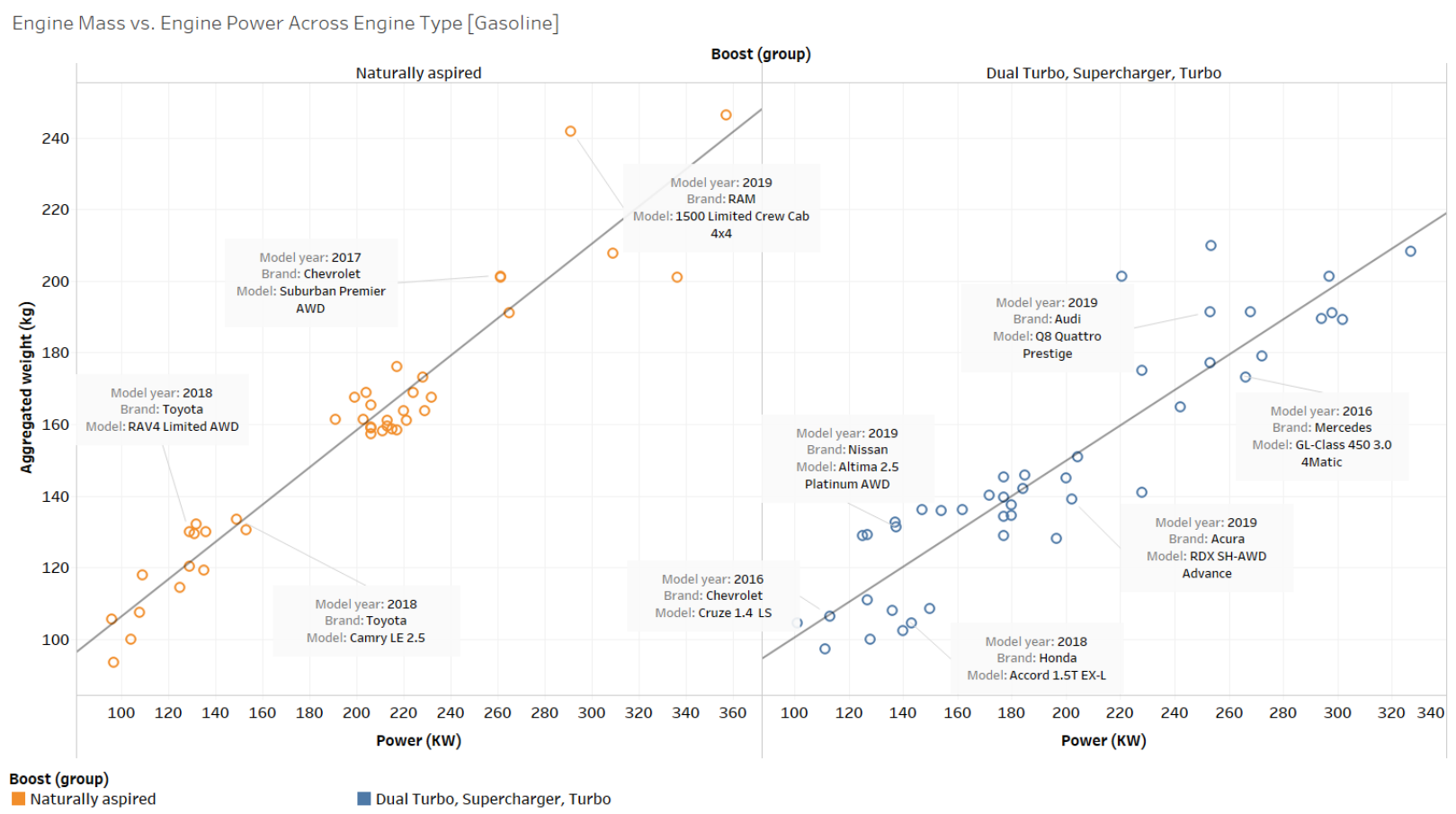

Figure 123. Engine weight (kg) vs. engine power $(\mathrm{kW})$ of gasoline engines across different aspiration methods

Figure 124 shows the updated regression analysis performed for diesel engines of vehicles across MY 2007-2020 from North America in A2Mac1. Using the updated regression analyses performed for vehicles in A2Mac1, Argonne is using the following equations for the current set of runs.

- Naturally aspirated, gasoline:

Weight $(\mathrm{kg})=0.520905 \times($ Engine Power $[\mathrm{kW}])+54.0967$

- Turbocharged engine, gasoline:

Weight $(\mathrm{kg})=0.493508 \times($ Engine Power $[\mathrm{kW}])+51.0354$

- Diesel engine:

Weight $(\mathrm{kg})=1.19554 \times($ Engine Power $[\mathrm{kW}])+65.1573$ 


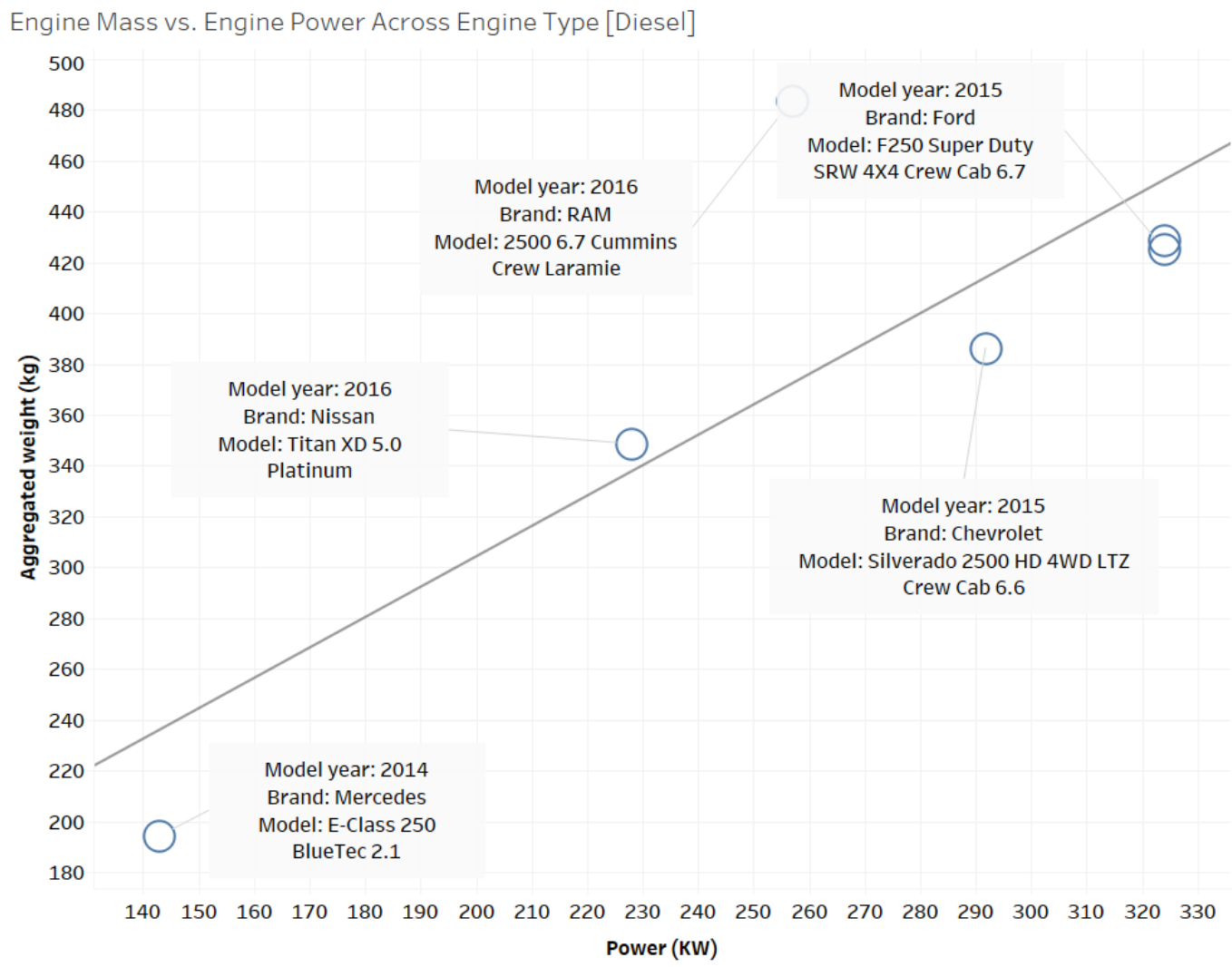

Figure 124. Engine weight (kg) vs. engine power $(\mathrm{kW})$ of diesel engines

\subsubsection{Electric Machines System Weight}

For the current set of runs, the electric machine weight computation was updating by analyzing the existing electric machine component and controller weights against the electric machine peak power output from A2mac1. Figure 125 shows the updated regression analysis performed from A2Mac1 data across different electrified vehicles. 


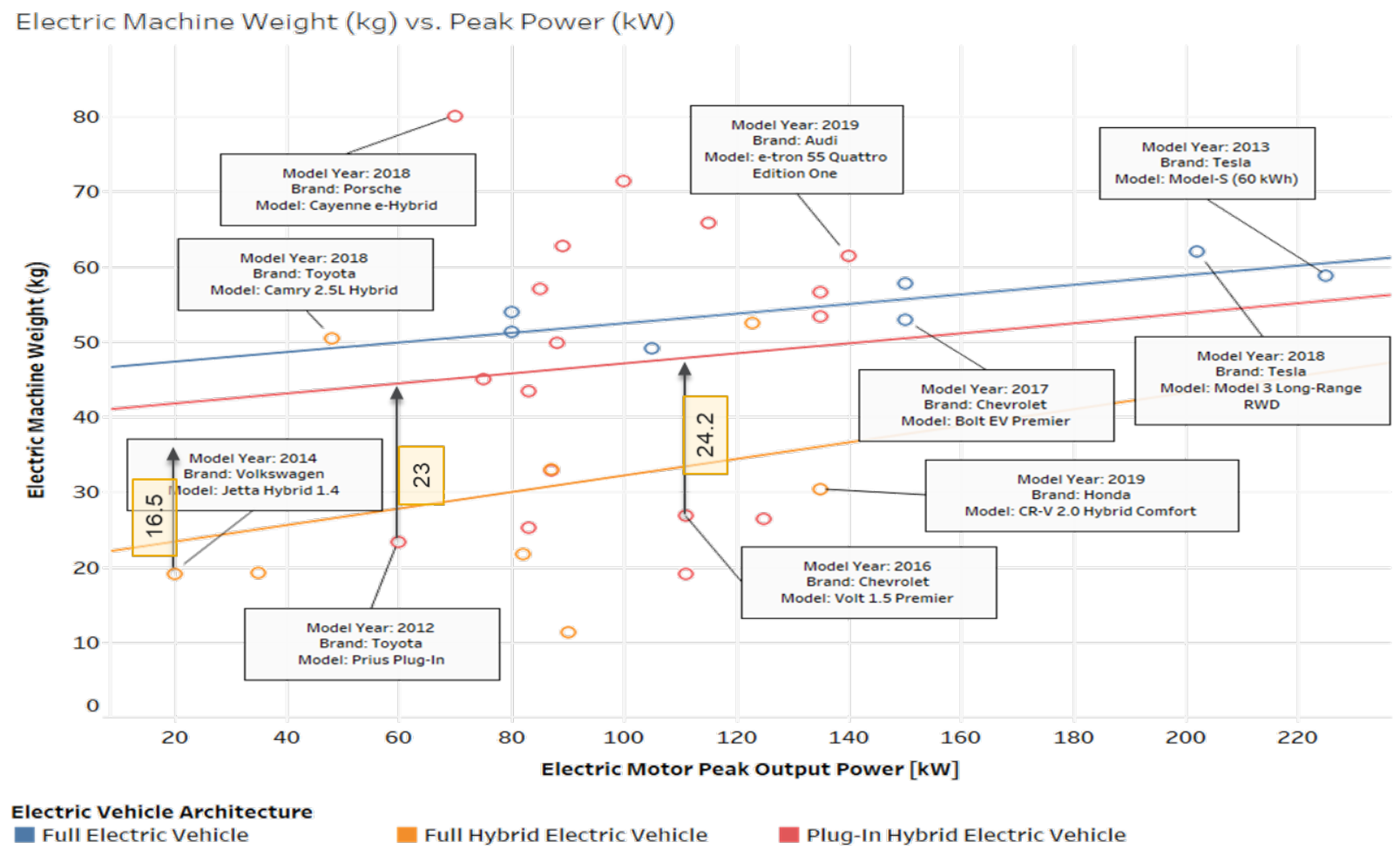

Figure 125. Electric machine weight vs. peak power output

It can be seen from the figure that, while accounting for the enclosure masses for the HEVs and PHEVs, the values are close to the EV trend line observed. Therefore, from the analysis above, Argonne recommends using the regression analysis performed for the EVs to develop a generalized equation:

(1) Weight $(\mathrm{kg})=0.0639176 \times$ Electric Motor Peak Output Power $(k W)+46.0497$

\subsection{Transmission Assumptions}

\subsubsection{Transmission Technology Definitions}

Table 19 details the different transmission technologies used in the study.

Table 19. Transmission Technologies

\begin{tabular}{|l|l|l|}
$\begin{array}{c}\text { Simulation } \\
\text { Name }\end{array}$ & \multicolumn{1}{|c|}{ Transmission Type } & \multicolumn{1}{c|}{ Description/Source } \\
\hline $5 \mathrm{AU}$ & $\begin{array}{l}\text { 5-speed automatic (base } \\
\text { class) }\end{array}$ & $\begin{array}{l}\text { 1:1 ratio efficiency from 6AU (base) and use rule to } \\
\text { generate the efficiency for other ratios }\end{array}$ \\
\hline $5 \mathrm{AU}$ & $\begin{array}{l}\text { 5-speed automatic } \\
\text { (premium class) }\end{array}$ & $\begin{array}{l}1: 1 \text { ratio efficiency from 6AU (premium) and use rule to } \\
\text { generate the efficiency for other ratios }\end{array}$ \\
\hline $6 \mathrm{AU}$ & $\begin{array}{l}\text { 6-speed automatic (base } \\
\text { class) }\end{array}$ & Transmission used for low-torque engines. [31] \\
\hline $6 \mathrm{AU}$ & $\begin{array}{l}\text { 6-speed automatic } \\
\text { (premium class) }\end{array}$ & Transmission used for high-torque engines [22] \\
\hline
\end{tabular}




\begin{tabular}{|c|c|c|}
\hline $\begin{array}{l}\text { Simulation } \\
\text { Name }\end{array}$ & Transmission Type & Description/Source \\
\hline 7AUp & 7-speed automatic+ & $\begin{array}{l}1: 1 \text { ratio efficiency from } 8 A U+\text { and use rule to generate } \\
\text { the efficiency for other ratios }\end{array}$ \\
\hline $8 A U$ & 8-speed automatic & Source: U.S. EPA test data - Ram 845RE [32] \\
\hline 8AUp & 8-speed automatic+ & 845RE (8AU) with improved efficiency [22] \\
\hline 8AUpp & 8-speed automatic++ & 845RE (8AU) with improved efficiency [22] \\
\hline 9AUp & 9-speed automatic+ & $\begin{array}{l}1: 1 \text { ratio efficiency from } 8 A U+\text { and use rule to generate } \\
\text { the efficiency for other ratios }\end{array}$ \\
\hline 10AUp & 10-speed automatic+ & MY 2017 Ford F-150 10R80 [38] \\
\hline 10AUpp & 10-speed automatic++ & 10R80 (10AUp) with improved efficiency \\
\hline 5DM & 5-speed manual & $\begin{array}{l}\text { 1:1 ratio efficiency from } 6 \mathrm{DCT} \text { and use rule to generate } \\
\text { the efficiency for other ratios }\end{array}$ \\
\hline 6DM & 6-speed manual & $\begin{array}{l}1: 1 \text { ratio efficiency from } 6 D C T \text { and use rule to generate } \\
\text { the efficiency for other ratios }\end{array}$ \\
\hline 7DM & $\begin{array}{l}\text { 7-speed manual (premium } \\
\text { class) }\end{array}$ & $\begin{array}{l}\text { 1:1 ratio efficiency from } 6 \mathrm{DCT} \text { and use rule to generate } \\
\text { the efficiency for other ratios }\end{array}$ \\
\hline 6DCT & 6-speed DCT & [16] \\
\hline 8DCT & 8-speed DCT & $\begin{array}{l}1: 1 \text { ratio efficiency from } 6 \mathrm{DCT} \text { and use rule to generate } \\
\text { the efficiency for other ratios }\end{array}$ \\
\hline CVT & CVT & [27] \\
\hline CVTp & CVT+ & CVT with improved efficiency [22] \\
\hline
\end{tabular}

Like engines, transmissions in the market always include multiple improvements from one generation to the next (such as increased gear number and efficiency). The objective of the transmission selection process was to separate the benefits of increased gear number from those of improved efficiency. For example, simulations 6AU to 8AU quantify the effectiveness of increased gear span and gear number, while $8 \mathrm{AU}$ to $8 \mathrm{AU}+$ quantify the impact of efficiency. As a result, while the test data were used to model several transmissions, a rule was used to develop some transmission models to ensure appropriate effectiveness value.

\subsubsection{Automatic transmission efficiency rule [35]}

In the equations below, $\tau$ is the normalized torque (torque/max rated input torque). In the specific data set that was used to generate these equations, the maximum torque was taken to be $450 \mathrm{Nm}$.

The maximum efficiency is given by:

(2) $\eta=100-1.385 \times \tau^{-1.0127}$

The temperature dependence is considered as a function of torque for temperatures ranging from $\mathrm{T}=38^{\circ} \mathrm{C}$ to $\mathrm{T}=93^{\circ} \mathrm{C}$ :

(3) $\Delta \eta=0.3612 \times \tau^{-0.9238}$

The speed dependence is a function of input torque for speeds ranging from $500 \mathrm{rpm}$ to $5000 \mathrm{rpm}$ :

(4) $\Delta \eta=0.6394 \times \tau^{-1.3068}$ 
The efficiency data is generated using the following steps:

- Start with the "maximum efficiency curve," which essentially represents the efficiency for direct drive $\left(1: 1\right.$ ratio) at $93^{\circ} \mathrm{C}$.

- The temperature offset is applied when calculating efficiency at $38^{\circ} \mathrm{C}$.

- The speed offset is applied.

- The gear ratio other than the direct drive is scaled.

Figure 126 shows the plot of direct drive efficiency for the range of temperatures and speeds considered. For other gears, the results are scaled down by a factor ranging between 0.97 and 1.0 .

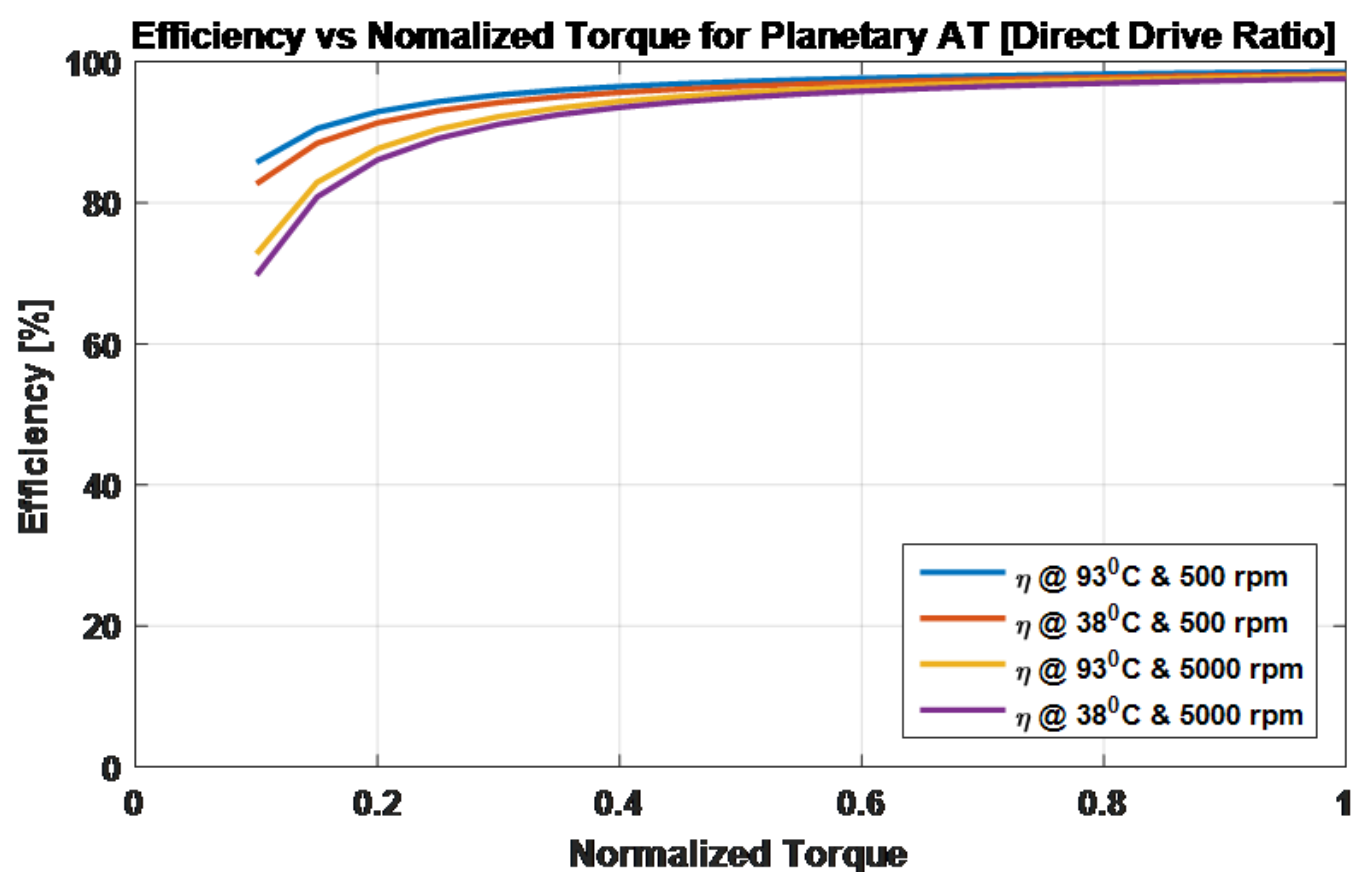

Figure 126. Efficiency for direct drive, for the range of temperatures and speeds considered

\subsubsection{Dual-clutch transmission efficiency rule}

The efficiency of the DCT is broken down into a speed-dependent term (spin loss) and a load dependent term (gear train mechanical efficiency).

For the speed-dependent part, the turning torque $(\mathrm{Nm})$ is given by the following equations through curve fit as a function of the overall gear ratio $\mathrm{R}$ :

At $93^{\circ} \mathrm{C}$ and $500 \mathrm{rpm}$ :

(5) $T=4.89 \times\left(\frac{1}{R}\right)^{2}+0.135 \times\left(\frac{1}{R}\right)+0.21$ 
At $93^{\circ} \mathrm{C}$ and $5000 \mathrm{rpm}$ :

(6) $T=23.5 \times\left(\frac{1}{R}\right)^{2}+1.4 \times\left(\frac{1}{R}\right)+1.7$

The turning torque is approximately linear between $500 \mathrm{rpm}$ and $5000 \mathrm{rpm}$.

The gear mechanical efficiency is very high, and can be assumed to be in the range of $99 \%$ to $99.5 \%$ per gear mesh. The mesh efficiency is higher when the meshing gears are of similar size.

The efficiency data is generated by the following steps:

- The torque loss is subtracted from the input torque.

- The additional torque loss due to constant mechanical efficiency is calculated by multiplying the difference between the input torque and the torque loss by (1 - efficiency).

- The efficiency is calculated by taking the sum of the (spin) torque loss and the loss due to mechanical efficiency and dividing it by the input torque.

The data set is based on a dual-clutch transmission with a rated input torque of up to $250 \mathrm{Nm}$.

\subsubsection{Transmission Attributes}

\subsubsection{Gear Span}

Figure 127 shows the analysis done on the existing vehicle attributes to determine the gear span of a 6-speed automatic transmission.

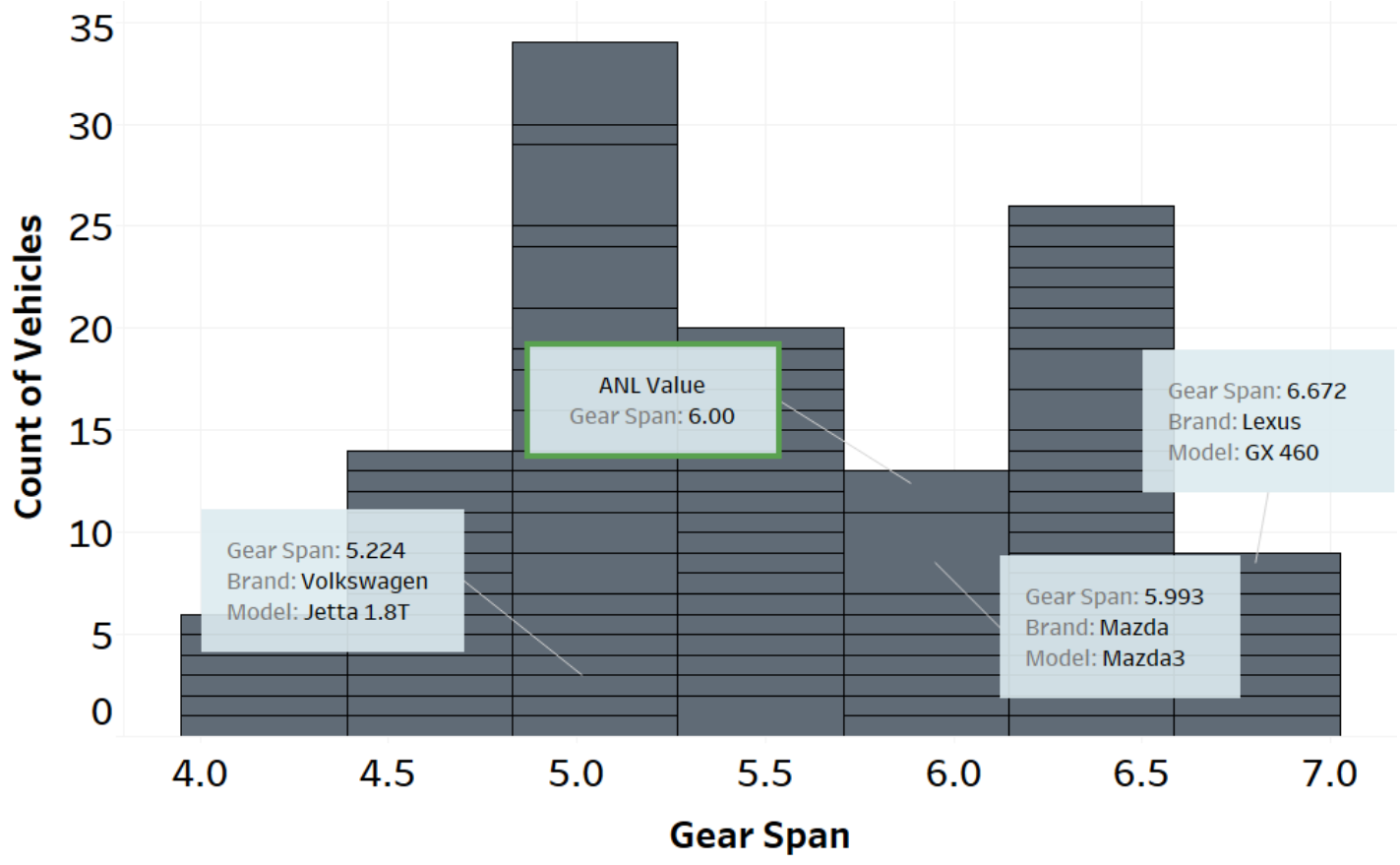

Figure 127. Vehicle attribute analysis for gear span of 6AU (Source: Argonne Vehicle Database)

A similar analysis was conducted on other transmission types and numbers of gears considered for the study. Table 20 summarizes the gear span values implemented across the different transmission types and gear numbers. 
While we recognize that some transmissions currently in the market have a higher gear span, the study focuses on selecting assumptions on the basis of the overall market. Separate studies will be conducted to quantify the impact of uncertainties related to variations of the assumptions.

\subsubsection{2. $\quad$ Final Drive Ratio}

Figure 128 shows the analysis done on the existing vehicle attributes to determine the final drive ratio of 6-speed automatic transmissions.

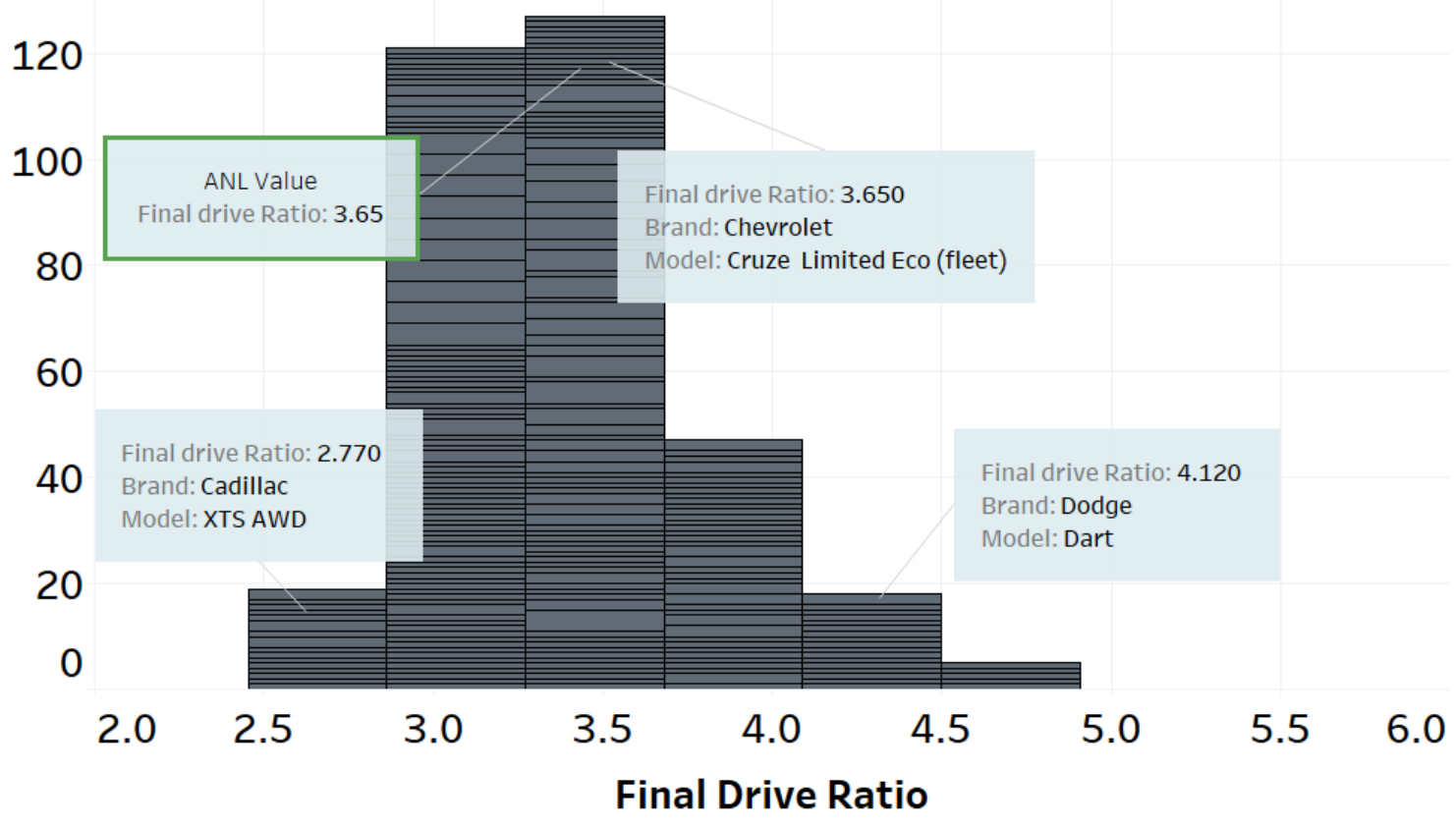

Figure 128. Vehicle attribute analysis for final drive ratio of $6 A U$ (Source: Argonne Vehicle Database)

A similar analysis was conducted on other transmission types and number of gears considered for the study. Table 20 summarizes the final drive ratio values implemented across the different transmission types and gear numbers.

\subsubsection{3. $\quad$ Gear Ratio Selected for Each Transmission}

Table 20 also summarizes the gear ratios selected for the different transmissions for the study. 
Table 20. Gear Ratios, Gear Span and Final Drive Ratio Selected for Different Transmissions

\begin{tabular}{|c|c|c|c|c|c|c|c|c|c|c|c|c|}
\hline \multirow{2}{*}{$\begin{array}{l}\text { Simulation } \\
\text { Name }\end{array}$} & \multicolumn{10}{|c|}{ Gear } & \multirow{2}{*}{$\begin{array}{l}\text { Gear } \\
\text { Span }\end{array}$} & \multirow{2}{*}{$\begin{array}{l}\text { Final } \\
\text { Drive }\end{array}$} \\
\hline & 1 & 2 & 3 & 4 & 5 & 6 & 7 & 8 & 9 & 10 & & \\
\hline $5 A U$ & 3.85 & 2.3262 & 1.5039 & 1.0403 & 0.77 & & & & & & 5 & 3.31 \\
\hline 5DM & 3.85 & 2.2714 & 1.4339 & 0.9685 & 0.7 & & & & & & 5.5 & 3.6 \\
\hline $6 \mathrm{AU}$ & 4.074 & 2.4867 & 1.6241 & 1.135 & 0.8487 & 0.679 & & & & & 6 & 3.65 \\
\hline $6 \mathrm{DM}$ & 4.074 & 2.4867 & 1.6241 & 1.135 & 0.8487 & 0.679 & & & & & 6 & 3.65 \\
\hline 6DCT & 4.074 & 2.4867 & 1.6241 & 1.135 & 0.8487 & 0.679 & & & & & 6 & 3.65 \\
\hline 7AU+ & 4.78 & 3.10 & 1.98 & 1.37 & 1.00 & 0.87 & 0.78 & & & & 6.16 & 3.13 \\
\hline 7DM & 4.298 & 2.624 & 1.7141 & 1.1981 & 0.8961 & 0.7171 & 0.614 & & & & 7 & 3.5 \\
\hline $8 \mathrm{AU} /+/++$ & 4.284 & 2.6593 & 1.7763 & 1.2553 & 0.9546 & 0.7768 & 0.6763 & 0.63 & & & 6.8 & 3.6 \\
\hline 8DCT & 4.284 & 2.6593 & 1.7763 & 1.2553 & 0.9546 & 0.7768 & 0.6763 & 0.63 & & & 6.8 & 3.6 \\
\hline $9 A U+$ & 4.69 & 2.902 & 1.9213 & 1.3611 & 1.0317 & 0.8368 & 0.7262 & 0.6743 & 0.67 & & 7 & 3.3 \\
\hline $10 \mathrm{AU}+$ & 4.7 & 2.99 & 2.15 & 1.8 & 1.52 & 1.28 & 1 & 0.85 & 0.69 & 0.64 & 7.34 & 3.31 \\
\hline 10AU++ & 5.25 & 3.27 & 2.19 & 1.6 & 1.3 & 1 & 0.78 & 0.65 & 0.58 & 0.52 & 10.10 & 3.55 \\
\hline CVT & \multicolumn{10}{|c|}{ Ratios from 0.529 to 3.172} & & 4.44 \\
\hline CVTp & \multicolumn{10}{|c|}{ Ratios from 0.45 to 3.6} & & 4.44 \\
\hline $\begin{array}{l}\text { Planetary } \\
\text { Gear }\end{array}$ & \multicolumn{10}{|c|}{ Sun $=30$, Ring $=78$} & & 3.267 \\
\hline Voltec & \multicolumn{10}{|c|}{ Sun $=37$, Ring $=83$} & & 3.02 \\
\hline
\end{tabular}

\subsubsection{Transmission Weights}

Like the vehicle component weights, the weights for transmission system components were updated after the midterm TAR comments. The weights have been evaluated from the A2Mac1 database [1] for different transmission types and numbers of gears for the different vehicle classes and performance categories.

\subsubsection{1. $\quad$ Final Drive Weight}

Figure 129 shows the distribution of final drive weights for 6-speed automatic transmissions for different vehicle classes. 


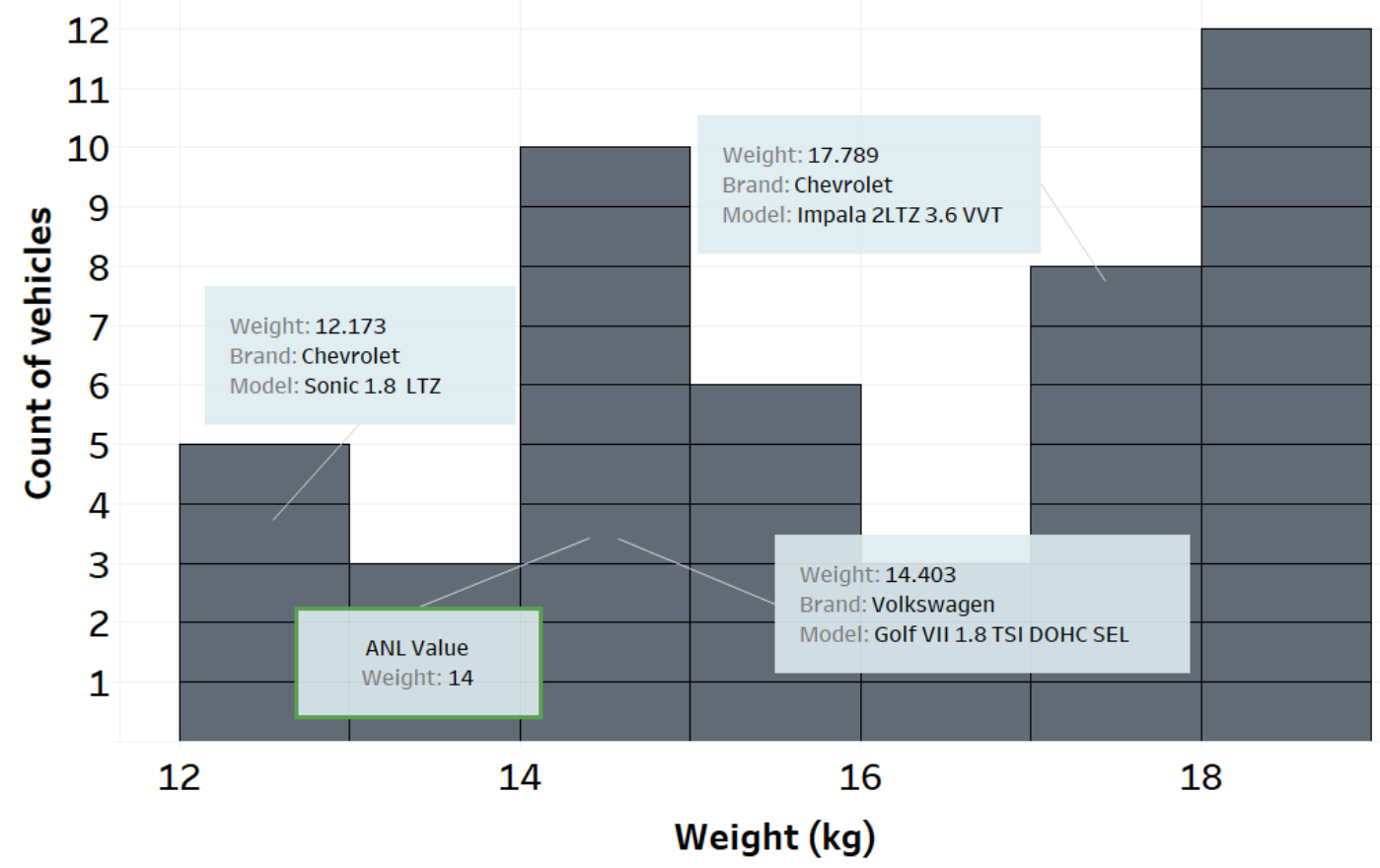

Figure 129. Final drive weight selection for $6 \mathrm{AU}$

These analyses were performed across different transmissions, numbers of gears, vehicle classes and performance categories, and the values for the reference vehicles were defined.

Table 21 (below) summarizes the values defined for the final drive weights of the reference vehicles with different transmissions across different numbers of gears, vehicle classes and performance categories.

\subsubsection{Gearbox Weight}

Gearbox weights have been re-evaluated from the A2Mac1 database [1] and have been assigned across the different vehicle classifications and performance categories for the different transmission types and numbers of gears.

Figure 130 shows the distribution of gearbox weight for 6-speed automatic transmissions for the midsize vehicle class. 


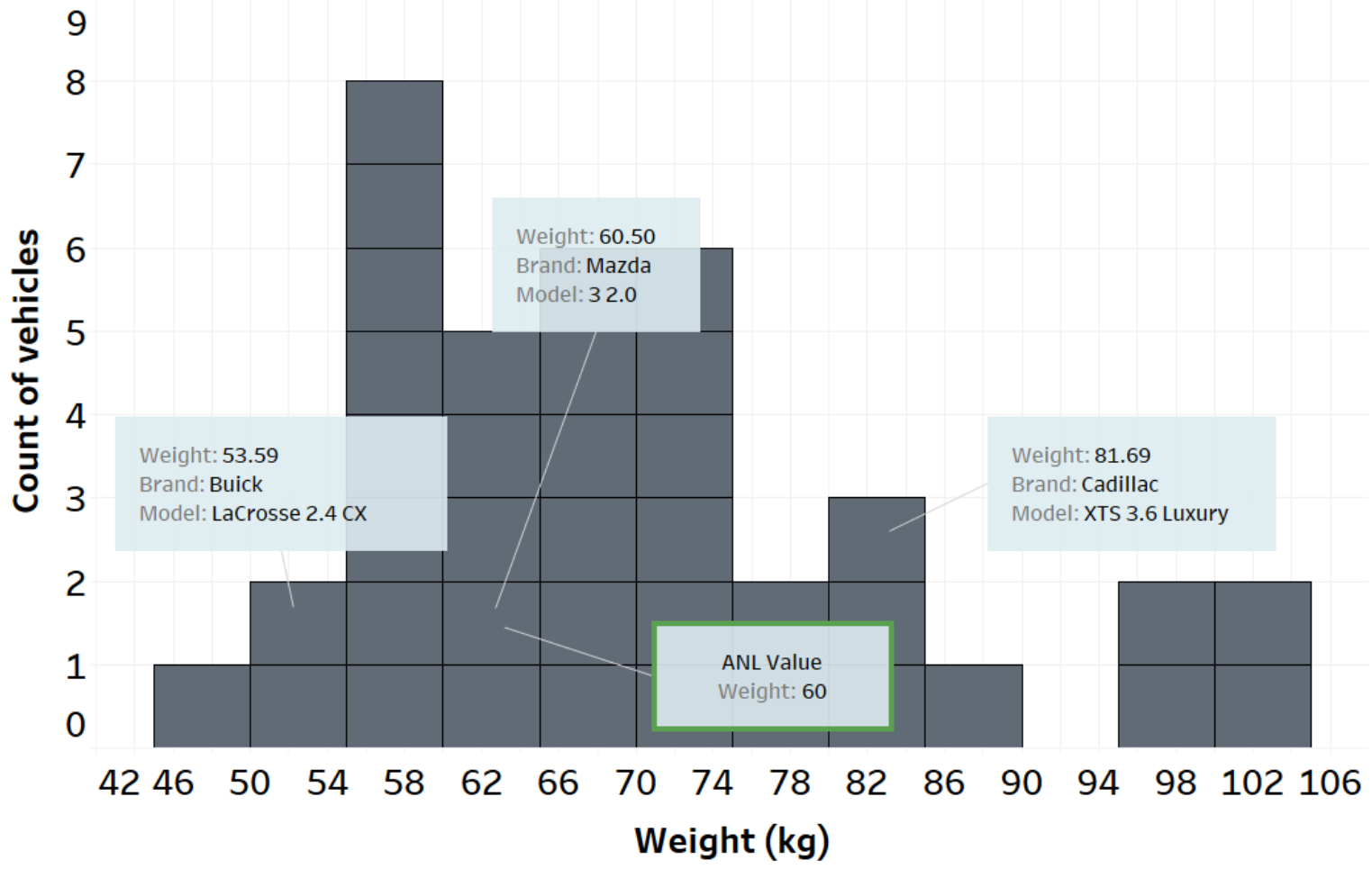

Figure 130. Gearbox weight selection for $6 A U$ for midsize vehicles

These analyses are performed across different transmissions, number of gears, vehicle classes and performance categories, and the values for the reference vehicles are defined. Table 22 summarizes the gearbox weights for different transmissions across different number of gears for the different vehicle classes and performance categories. 
Table 21. Final Drive Weight Summary for All Transmission Types

\begin{tabular}{|c|c|c|c|c|c|c|c|c|c|c|c|c|c|c|c|}
\hline \multirow{2}{*}{$\begin{array}{l}\text { Vehicle } \\
\text { Class }\end{array}$} & \multirow{2}{*}{$\begin{array}{l}\text { Performance } \\
\text { Category }\end{array}$} & \multicolumn{14}{|c|}{ Reference Value (kg) } \\
\hline & & $5 \mathrm{AU}$ & 6AU & $7 \mathrm{AU}$ & 8AU & 9AU & 10AU & 5DM & 6DM & 7DM & 6DCT & 8DCT & CVT/+ & Power-Split & EREV \\
\hline Compact & Base/Premium & 14 & 14 & 14 & 14 & 14 & 14 & 12 & 14 & 14 & 14 & 14 & 14 & 14 & 14 \\
\hline Midsize & Base/Premium & 17 & 17 & 17 & 17 & 17 & 17 & 12 & 14 & 14 & 14 & 14 & 14 & 14 & 14 \\
\hline Small SUV & Base/Premium & 20 & 22 & 22 & 22 & 22 & 22 & 24 & 24 & 24 & 24 & 24 & 24 & 24 & 24 \\
\hline Midsize SUV & Base/Premium & 25 & 30 & 30 & 30 & 30 & 30 & 35 & 35 & 35 & 35 & 35 & 35 & 35 & 35 \\
\hline Pickup & Base/Premium & 60 & 70 & 72 & 75 & 75 & 75 & 60 & 65 & 65 & 65 & 65 & 65 & 65 & 65 \\
\hline
\end{tabular}

Table 22. Gearbox Weight Summary for All Transmission Types

\begin{tabular}{|c|c|c|c|c|c|c|c|c|c|c|c|c|c|c|c|}
\hline \multirow{2}{*}{$\begin{array}{l}\text { Vehicle } \\
\text { Class }\end{array}$} & \multirow{2}{*}{$\begin{array}{l}\text { Performance } \\
\text { Category }\end{array}$} & \multicolumn{14}{|c|}{ Reference Value (kg) } \\
\hline & & 5AU & 6AU & 7AU & 8AU & 9AU & 10AU & 5DM & 6DM & 7DM & 6DCT & 8DCT & CVT/+ & $\begin{array}{l}\text { Power- } \\
\text { Split }\end{array}$ & EREV \\
\hline Compact & Base/Premium & 60 & 50 & 60 & 65 & 70 & 40 & 30 & 40 & 50 & 65 & 80 & 41 & 40 & 50 \\
\hline Midsize & Base/Premium & 65 & 60 & 70 & 80 & 85 & 50 & 35 & 45 & 50 & 70 & 90 & 51 & 40 & 50 \\
\hline Small SUV & Base/Premium & 70 & 65 & 72 & 80 & 90 & 55 & 45 & 50 & 50 & 75 & 90 & 56 & 50 & 60 \\
\hline Midsize SUV & Base/Premium & 80 & 65 & 72 & 80 & 90 & 75 & 45 & 50 & 70 & 80 & 90 & 56 & 50 & 60 \\
\hline Pickup & Base/Premium & 80 & 75 & 80 & 90 & 95 & 85 & 50 & 60 & 70 & 90 & 100 & 65 & 50 & 60 \\
\hline
\end{tabular}




\subsubsection{Transmission Performance Data}

This section details the transmission losses for different ratios for each transmission type considered in the study.

\subsubsection{1. $\quad$ Automatic Transmission}

Figure 131 shows the transmission efficiency maps for different numbers of gears for automatic (AU/AU+/AU++) transmissions.
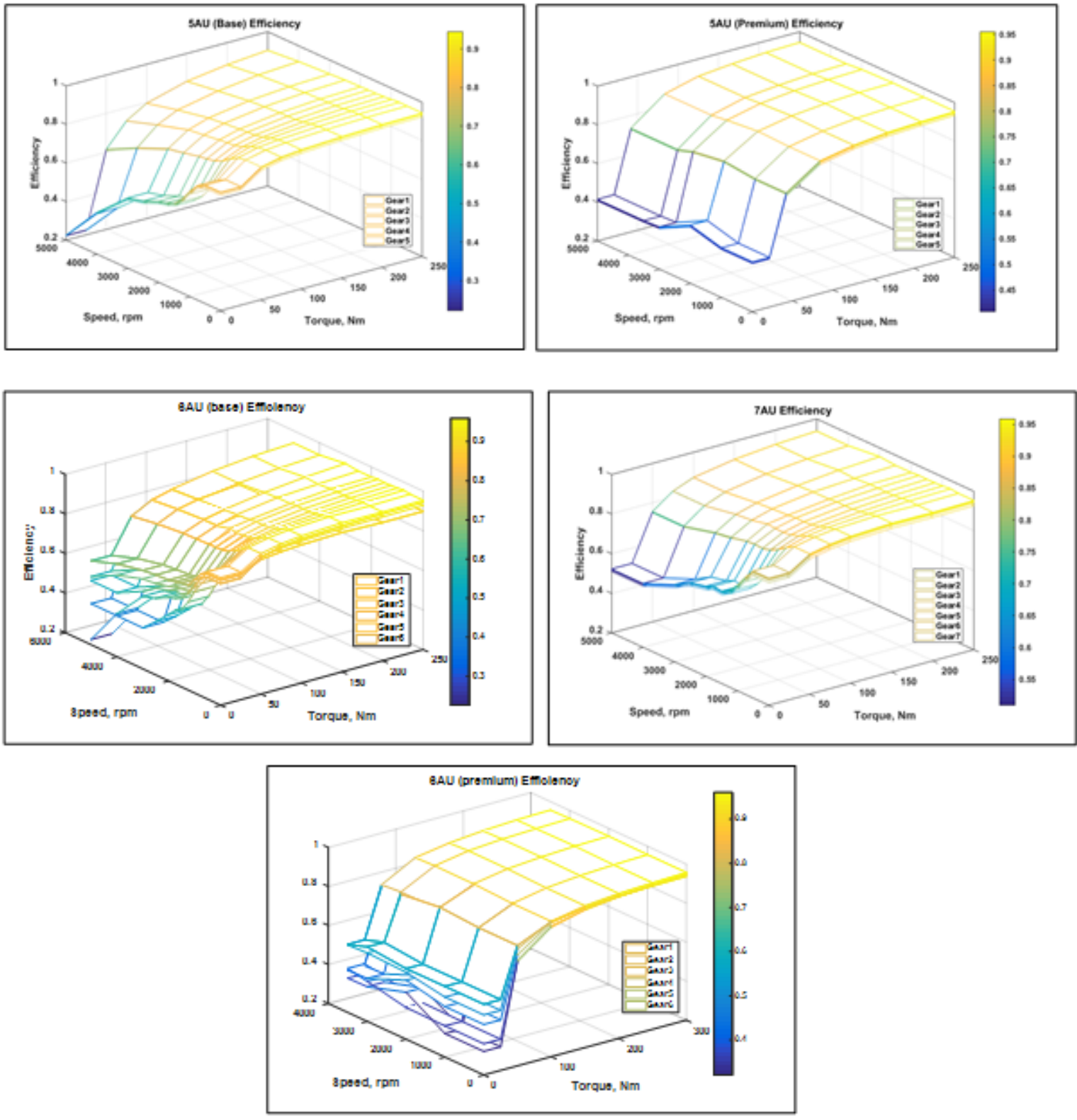

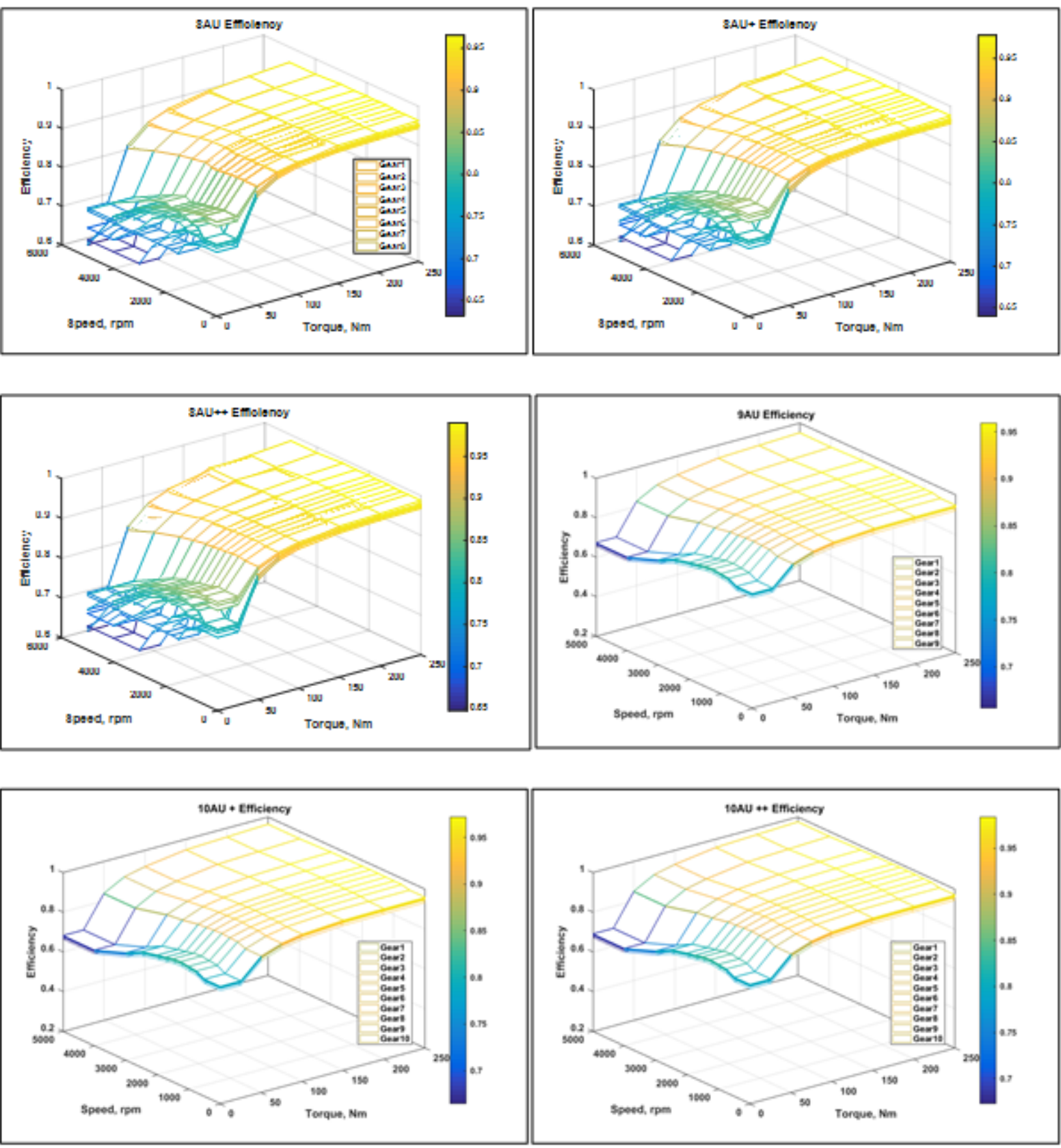

Figure 131. Automatic (AU/AU+/AU++) transmission efficiency maps for different numbers of gears

\subsubsection{2. $\quad$ Manual Transmissions}

Figure 132 shows the transmission efficiency maps for different number of gears for manual (DM) transmissions. 

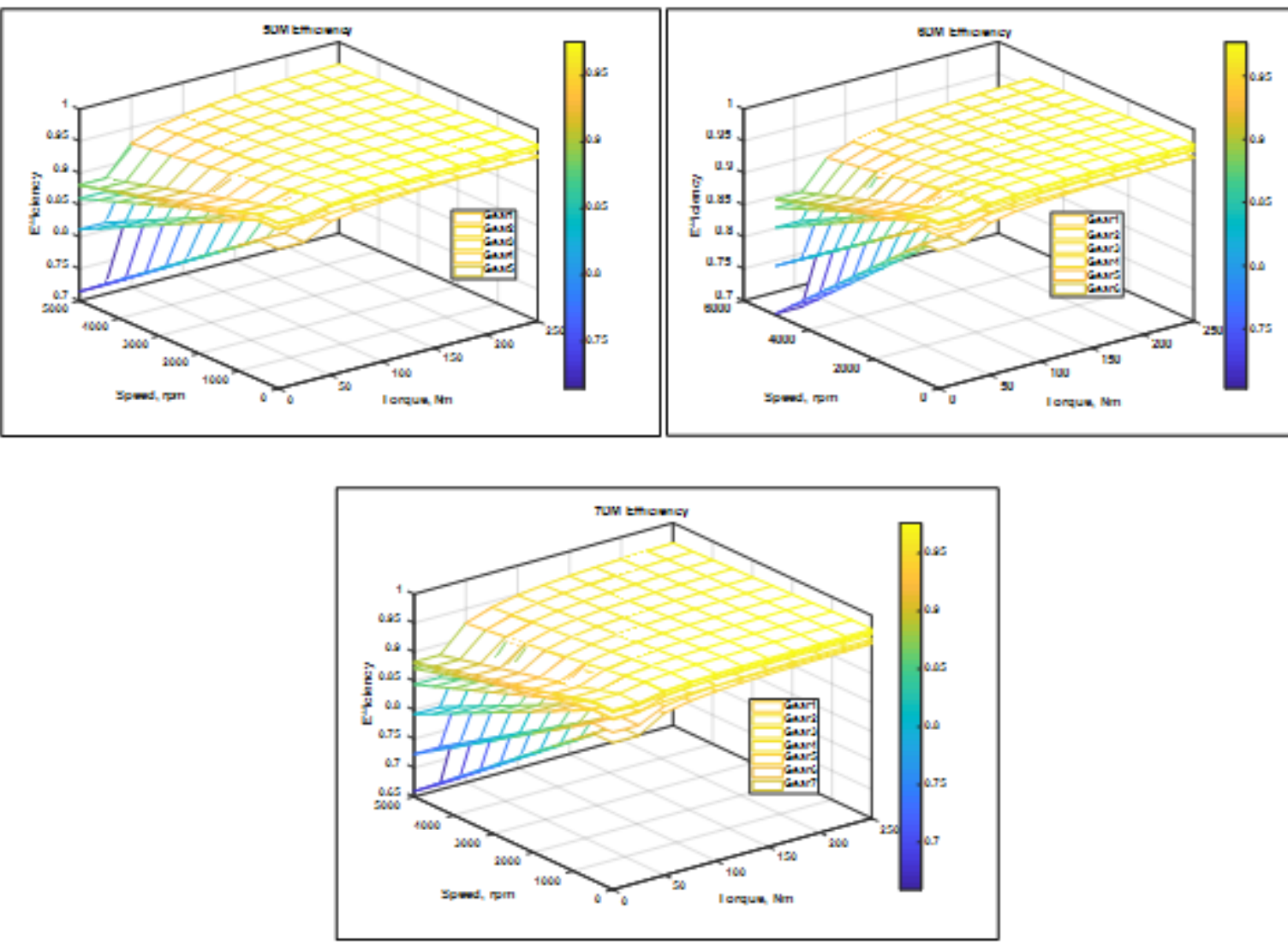

Figure 132. Manual (DM) transmission efficiency maps for different number of gears

\subsubsection{Dual-Clutch Transmission}

Figure 133 shows the transmission efficiency maps fort different numbers of gears for dual clutch transmissions (DCTs).
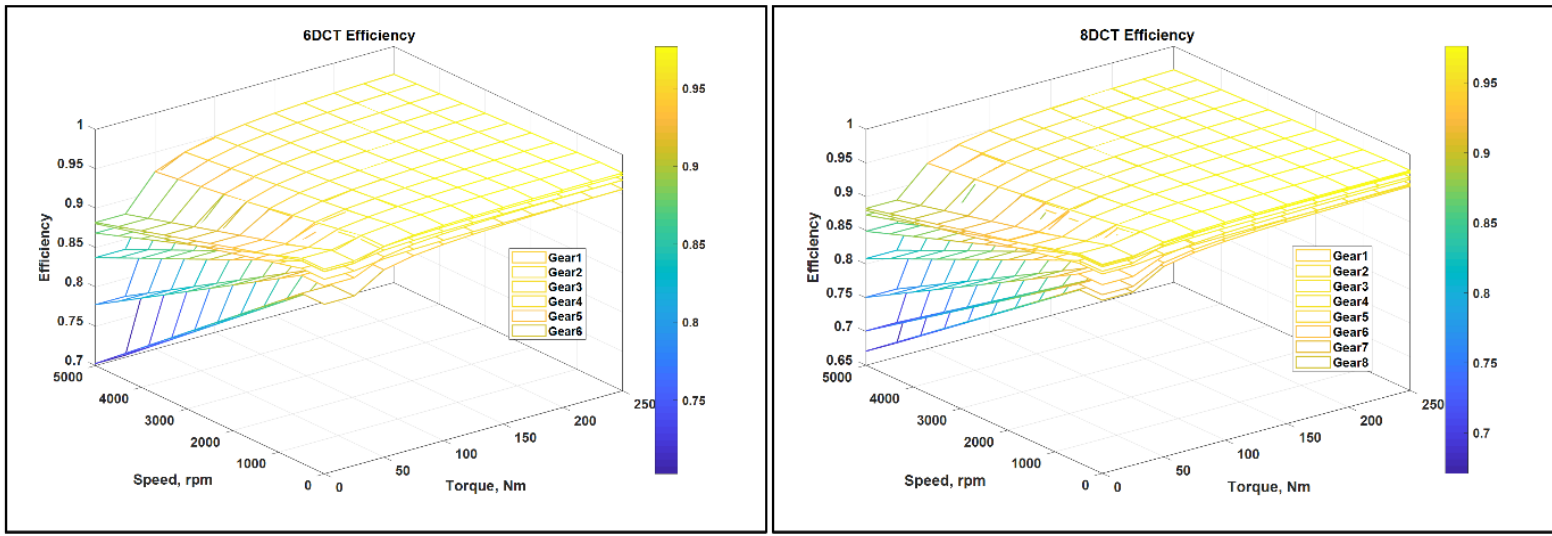

Figure 133. DCT Efficiency maps for different numbers of gears 


\subsubsection{Continuously Variable Transmission}

Continuously variable transmission (CVT) system loss consists of the hydraulic loss and the mechanical loss. The hydraulic loss comes from the pump loss in providing the CVT with clamping force when the pump generates line pressure. The mechanical loss is caused by the torque loss from the slip between pulley and belt. The hydraulic loss has a dominant influence on the total CVT system efficiency when the vehicle is driving at low speed, while the mechanical loss is the main part of the CVT system loss at high speed. The CVT system model was developed by considering both hydraulic and mechanical losses, using an experiment-based map data.

\subsection{CVT Efficiency Maps}

Figure 134 shows the oil pump efficiency map and the mechanical efficiency maps for different vehicle speeds for the CVT transmission type. 

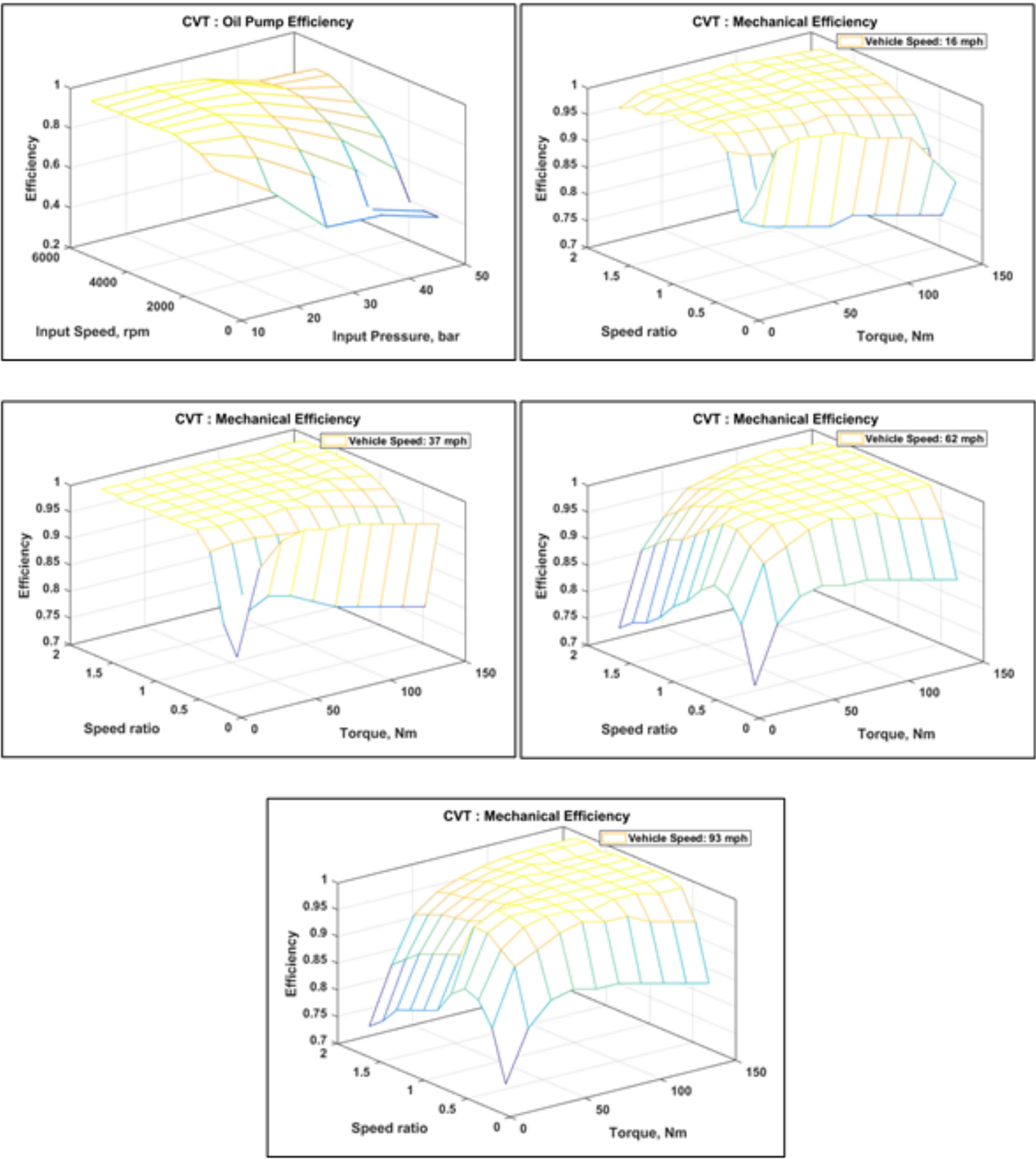

Figure 134. CVT oil pump and mechanical efficiency maps

\subsubsection{2. $\quad C V T+$ Efficiency Maps}

Figure 135 shows the oil pump efficiency map and the mechanical efficiency maps for different vehicle speeds for the CVT+ transmission type. 

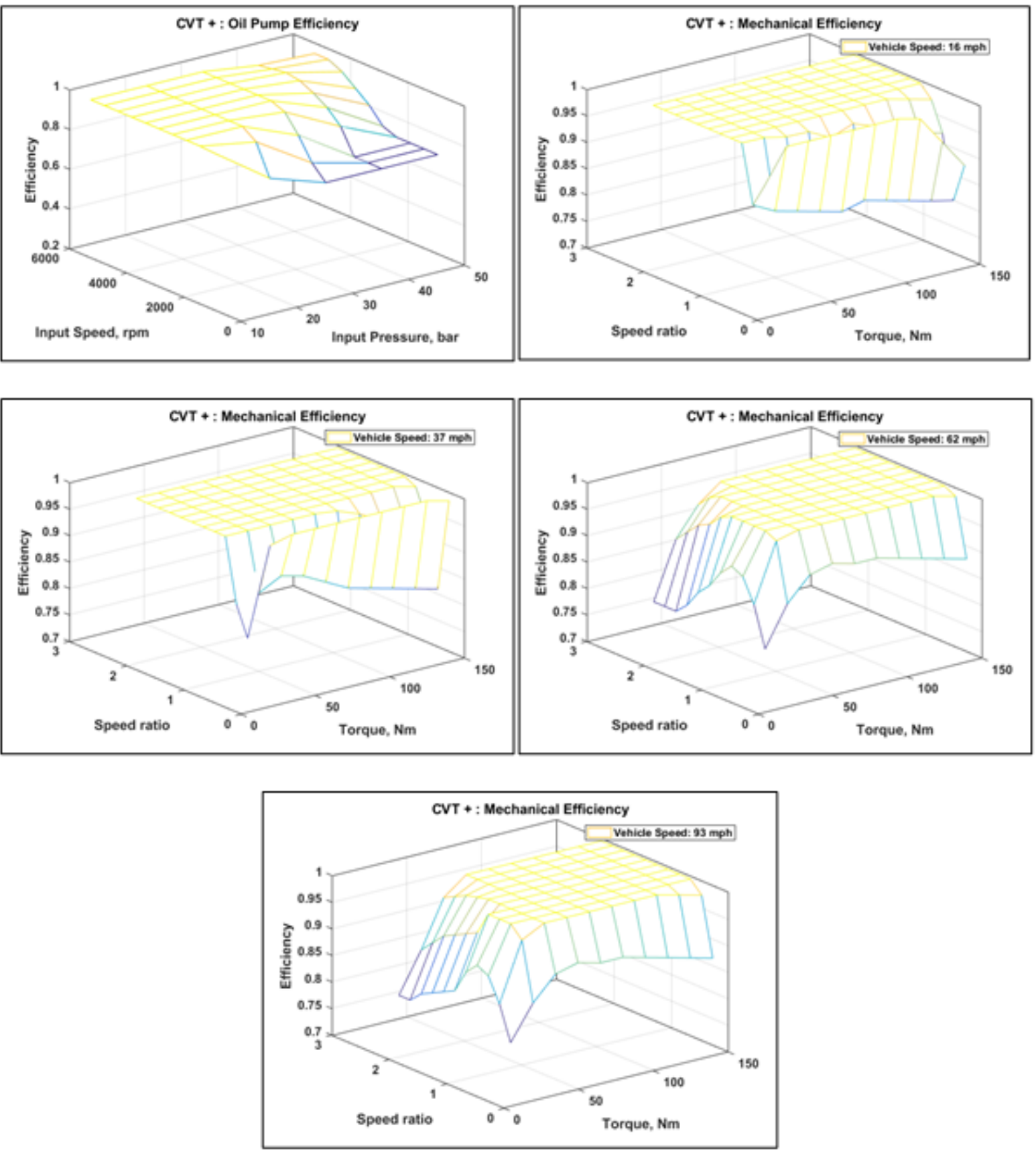

Figure 135. CVT+ oil pump and mechanical efficiency maps

\subsubsection{Gear-Shifting Maps}

Examples of shifting maps for the midsize (base) category are presented below in Figure 136. Shifting maps have been developed to ensure minimum energy consumption across all transmissions while maintaining an acceptable drivability. While plant models with a higher degree of fidelity would most accurately model the impact of each technology on drivability, using such models was not appropriate for the current study. As a result, the work related to drive quality focused on number of shifting events, time between shifting events, engine time response, and engine torque reserve. 
Figure 136 shows the upshifting and downshifting maps for the different transmission types and numbers of gears for conventional gasoline powertrains in midsize (base) vehicles.
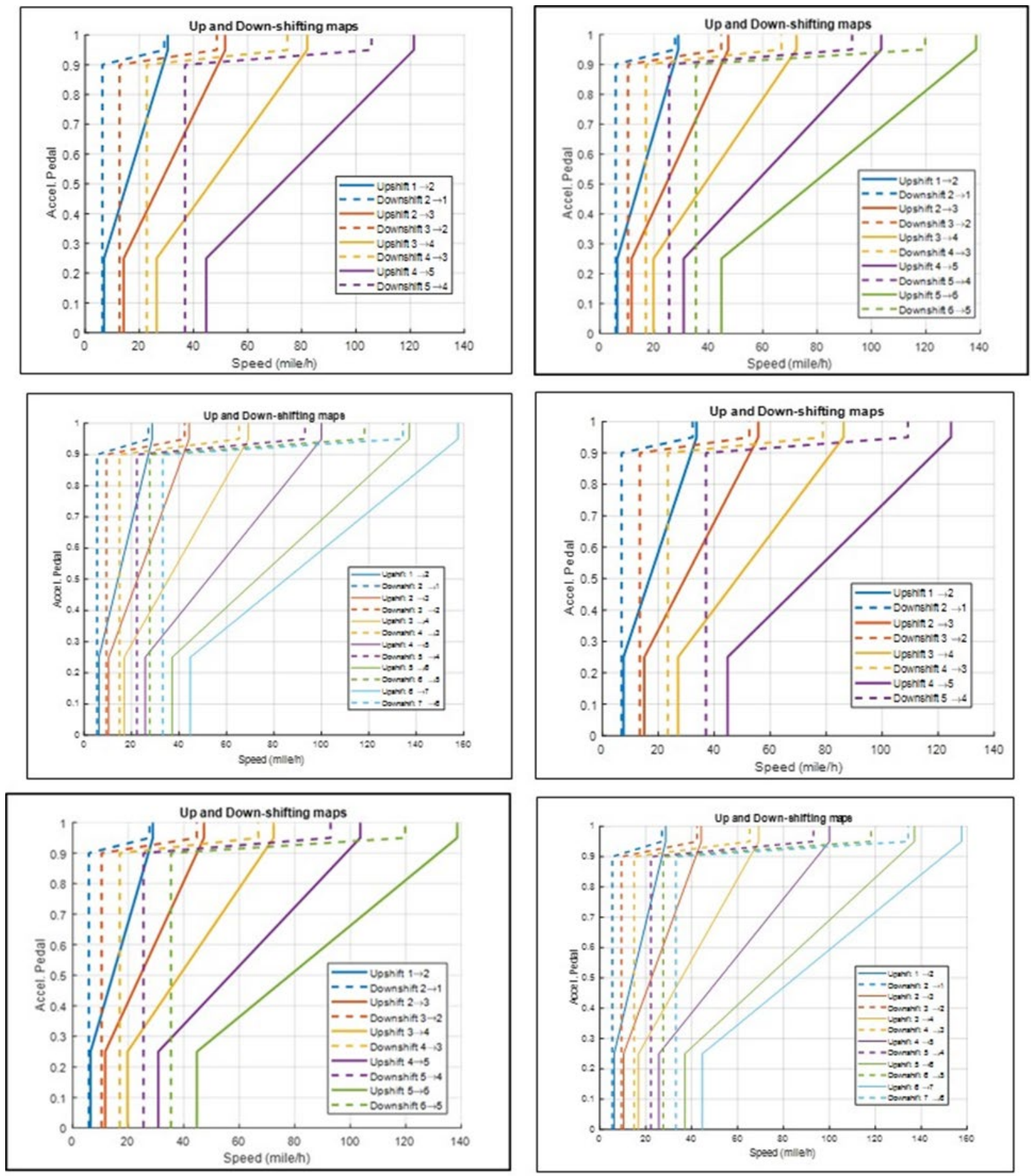

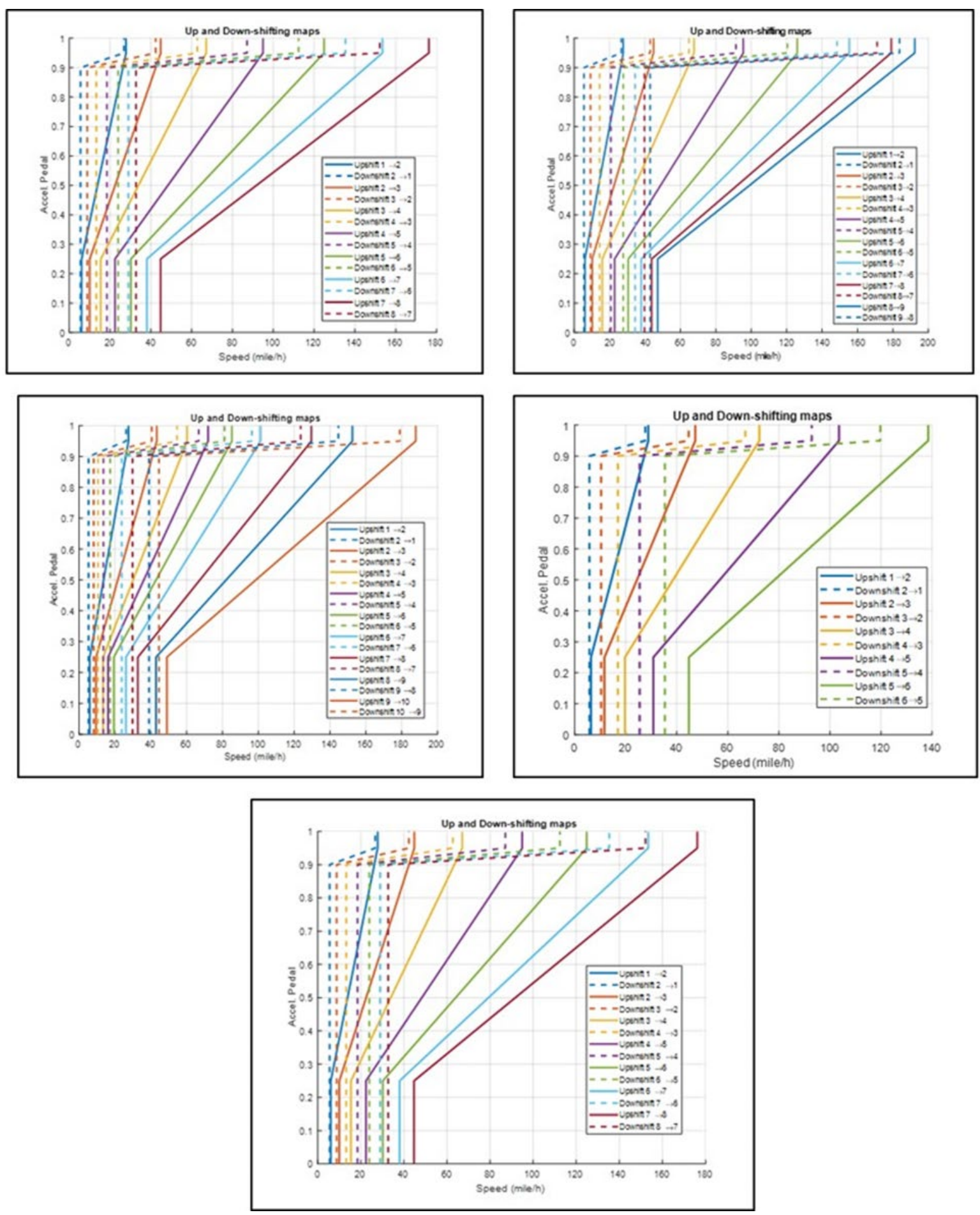

Figure 136. Maps of transmission upshifting (solid lines) and downshifting (dotted lines) 


\subsection{Torque Converter Assumptions}

\subsubsection{Component Performance}

Figure 137 shows the characteristic map for the torque converter data.

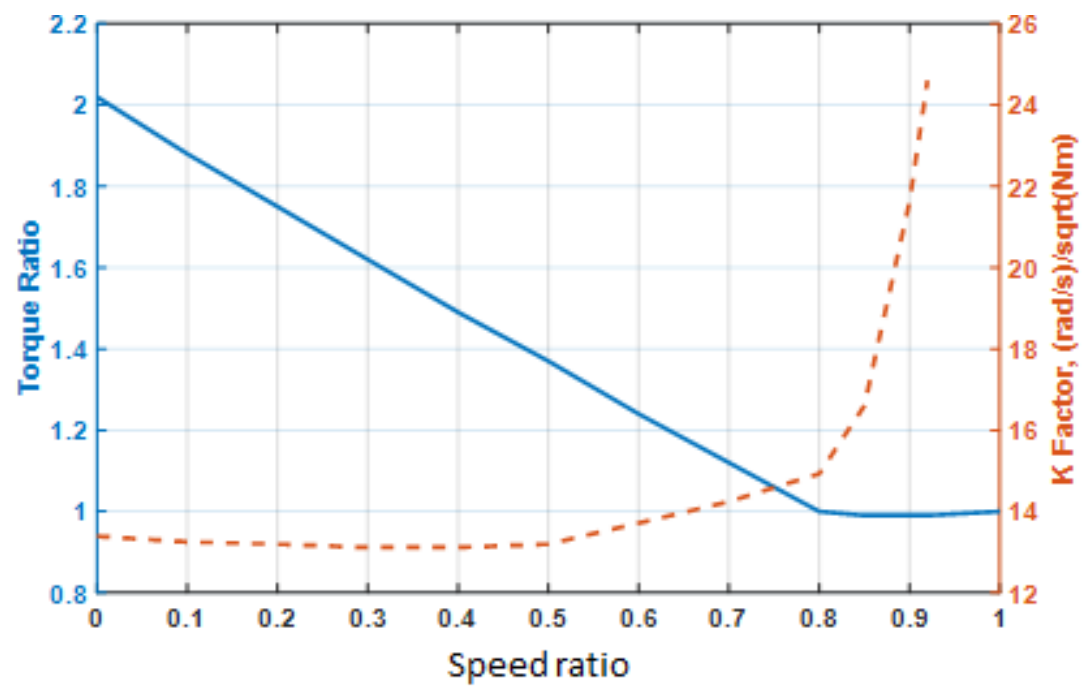

Figure 137. Autonomie torque converter characteristic map

The relationships of the different factors are as follows:

Speed ratio $=\frac{\text { output speed }}{\text { input speed }}$

Torque ratio $=\frac{\text { output torque }}{\text { input torque }}$

$K$ factor $=\frac{\text { input speed }}{\text { input torque }}$

\subsubsection{Oil Pump Efficiency}

The equation below calculates the pump torque loss map as a function of speed and torque for the torque converter.

(7) $T_{\text {loss_map }_{i, j}}=\left(1-\eta_{i, j}\right) T_{\text {loss_index }_{i}}$

This torque loss represents an external pump driving the hydraulic fluid through the torque converter, not for the turbine.

(8) $T_{\text {in }}=T_{\text {eng }}-T_{\text {loss }}$ pump

Figure 138 shows the pump efficiency map for the torque converter model. 


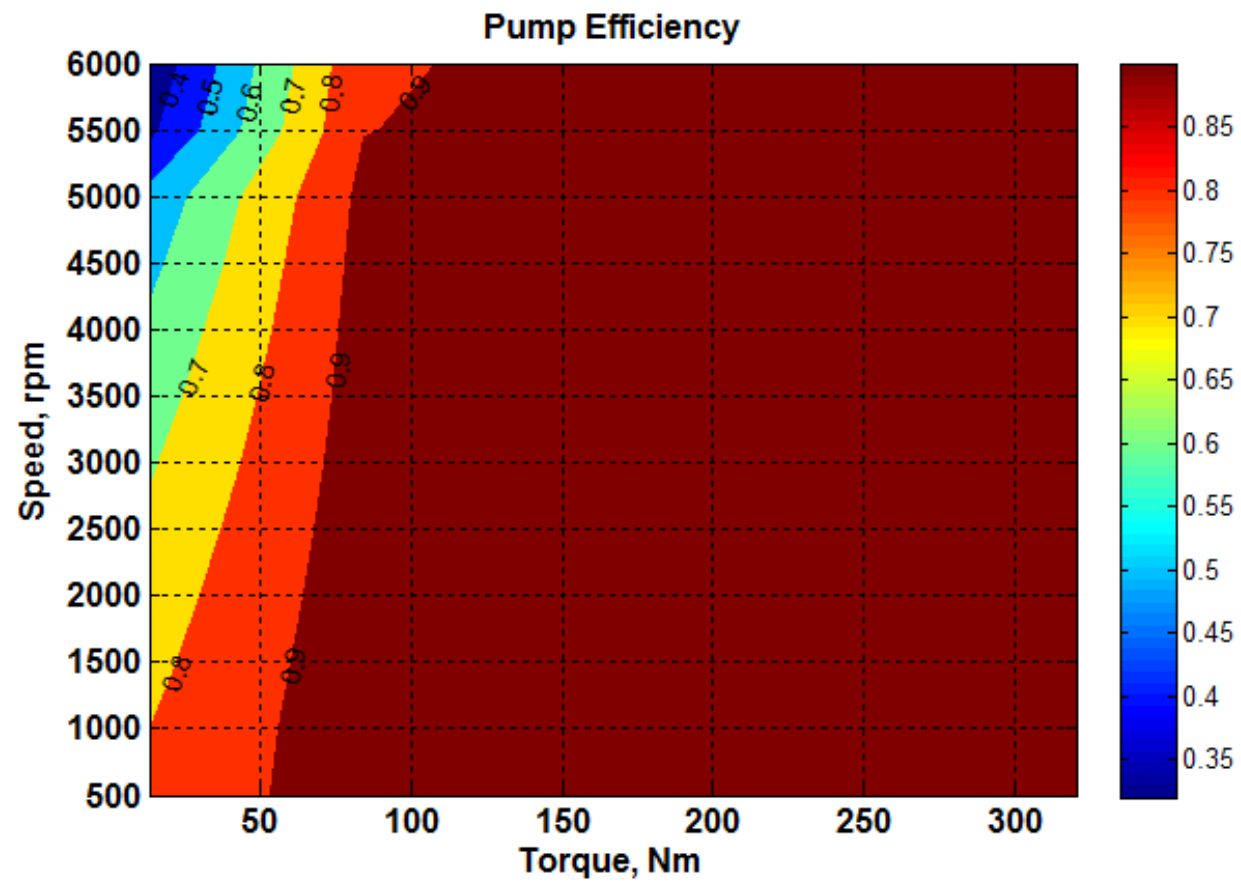

Figure 138. Pump efficiency of the torque converter model

\subsection{Engine Technologies}

\subsubsection{Engine Technology Definitions}

Table 23 shows the different engine technologies studied, along with the associated simulation names and the reference peak power. The engine performance values were provided by IAV Automotive Engineering, Inc., except for the eng24 and eng25 values, which were provided by the U.S. EPA, and eng26 values, which were derived from Argonne AMTL test data.

Table 23. Engine Technologies with Reference Peak Power and Reference Displacement

\begin{tabular}{|l|l|c|c|c|}
\hline $\begin{array}{c}\text { Engine } \\
\text { Simulation } \\
\text { Name }\end{array}$ & \multicolumn{1}{|c|}{ Engine Technology } & $\begin{array}{c}\text { Engine } \\
\text { Reference } \\
\text { Peak Power } \\
\mathbf{( k W )}\end{array}$ & $\begin{array}{c}\text { Engine } \\
\text { Reference } \\
\text { Displacement } \\
\mathbf{( L )}\end{array}$ & $\begin{array}{c}\text { Engine } \\
\text { Turbo Max } \\
\text { Boost Level }\end{array}$ \\
\hline eng01 & DOHC VVT & 108 & 2.0 & \\
\hline eng02 & DOHC VVT+VVL & 108 & 2.0 & \\
\hline eng03 & DOHC VVT+VVL+GDI & 113 & 2.0 & \\
\hline eng04 & DOHC VVT+VVL+GDI+DEAC & 113 & 2.0 & \\
\hline eng5b & SOHC VVT (level 1 reduced friction) & 109 & 2.0 & \\
\hline eng6a & SOHC VVT+VVL (level 1 reduced friction) & 109 & 2.0 & \\
\hline eng7a & $\begin{array}{l}\text { SOHC VVT+VVL+GDI (level 1 reduced } \\
\text { friction) }\end{array}$ & 114 & 2.0 & \\
\hline eng8a & $\begin{array}{l}\text { SOHC VVT+VVL+GDI+DEAC (level 1 } \\
\text { reduced friction) }\end{array}$ & 114 & 2.0 & \\
\hline eng12 & DOHC Turbo 1.6I 18bar & 132 & 1.6 & 1.5271 \\
\hline
\end{tabular}




\begin{tabular}{|l|l|c|c|c|}
\hline $\begin{array}{c}\text { Engine } \\
\text { Simulation } \\
\text { Name }\end{array}$ & \multicolumn{1}{|c|}{ Engine Technology } & $\begin{array}{c}\text { Engine } \\
\text { Reference } \\
\text { Peak Power } \\
\mathbf{( k W )}\end{array}$ & $\begin{array}{c}\text { Engine } \\
\text { Reference } \\
\text { Displacement } \\
\text { (L) }\end{array}$ & $\begin{array}{c}\text { Engine } \\
\text { Turbo Max } \\
\text { Boost Level }\end{array}$ \\
\hline eng13 & DOHC Turbo 1.2I 24bar & 133 & 1.2 & 2.0409 \\
\hline eng14 & DOHC Turbo 1.2l 24bar + cooled EGR & 133 & 1.2 & 2.0409 \\
\hline eng17 & Diesel & 140 & 2.2 & \\
\hline eng18 & DOHC VVT + SGDI & 113 & 2.0 & \\
\hline eng19 & DOHC VVT + DEAC & 113 & 2.0 & \\
\hline eng20 & DOHC VVT + VVL + DEAC & 113 & 2.0 & \\
\hline eng21 & DOHC VVT + SGDI + DEAC & 113 & 2.0 & \\
\hline eng24 & Current SkyActiv 2.0 93AKI [34] & 101 & 2.0 & \\
\hline eng25 & Future SkyActiv 2.0I CEGR 93AKI+DEAC & 101 & 2.0 & \\
\hline eng26 & Atkinson - PSHEV and PSPHEV only & 73 & 1.8 & \\
\hline eng12Deac & DOHC Turbo 1.6I 18bar + DEAC & 144 & 1.6 & 1.5271 \\
\hline eng22b & Atkinson 2.5L VVT PFI CR14 & 132 & 2.5 & \\
\hline eng23b & 2.0 Miller VTG + VVT + VVL + DI + cEGR + & 139 & 2.0 & \\
\hline eng23c & Miller eCharger + VVT + DI + eEGR + CR12 & 139 & 2.0 & \\
\hline eng26a & VCR VVT + DI + Turbo + cEGR + CR9/12 & 180 & 2.0 & 2.0870 \\
\hline
\end{tabular}

\subsubsection{Engine Efficiency Maps}

IAV Automotive Engineering modeled gasoline and diesel engine maps in GT-POWER and supplied those maps to Argonne for use in Autonomie.

IAV Automotive Engineering also provided wide-open-throttle engine performance values and BSFC maps for future engine concepts. To properly quantify the benefits of individual technologies, each incremental technology was modeled using GT-POWER and validated with existing dynamometer measurements for several engines. GT-POWER is used to predict engine performance quantities such as power, torque, airflow, volumetric efficiency, fuel consumption, turbocharger performance, and matching and pumping losses. The models were trained over the entire engine operating range and have predictive combustion capability. This is essential, since the BSFC prediction needs to be accurate, while the engine setup is subject to change.

Relevant engine geometries and parameters were measured and modeled with friction/flow losses, heat transfer, and other parameters and calibrated to match measurements. Displacement-normalized mechanical friction was modeled as a function of engine speed and specific load. A combustion model was trained to predict fuel heat release rates in response to physical effects such as cylinder geometries, pressure, temperature, turbulence, residual gas concentration and other parameters. A knock correlation based on in-cylinder conditions and fuel octane rating predicts whether knock will occur and at what intensity. A combustion stability threshold prediction was trained using covariance of indicated mean effective pressure (IMEP) data and is used for understanding exhaust gas recirculation (EGR) tolerance, especially at low loads. Load controllers were developed for fuel/air path actuators and targeting 
controllers to drive optimal and knock-limited combustion phasing, just as in a physical engine. Careful modeling practice was used to provide confidence that calibrations will scale and predict reasonable and reliable results as parameters are changed throughout the various technology concept studies.

IAV Automotive Engineering provided 20 engine maps in total, for 15 naturally aspirated gasoline engines, four turbocharged gasoline engines, and one diesel engine. Two naturally aspirated gasoline engine maps (for eng24 and eng25) were provided by the U.S. Environmental Protection Agency (EPA). EPA also benchmarked the Skyactiv 2.0L 13:1 compression ratio engine in an EPA engine test cell [34] . Finally, one Atkinson engine map, generated using Argonne test data, was used for electrified vehicles with power-split architecture. Thus, the total number of engine maps used in the study is 19 .

For all IAV Automotive Engineering engines, engine speed, BMEP, brake torque, fuel flow rate, pumping mean effective pressure (PMEP), and FMEP data were provided in a standardized format to Argonne. These channels were provided from 1,000 RPM to the maximum engine speed and from 0 bar BMEP to full load to provide a full operation map. Fuel flow rates at zero output torque were provided separately from 650 RPM (defined idle) to 6000 RPM.

The following fuel specifications are used by IAV when modeling the engines in GT-POWER:

- $\quad$ Gasoline LHV $=43.1 \mathrm{MJ} / \mathrm{kg}$

- $(\mathrm{R}+\mathrm{M}) / 2=87$

- Ambient temperature, $\mathrm{T} \neg \mathrm{amb}=25^{\circ} \mathrm{C}$

- Ambient pressure, $\mathrm{Pamb}=990 \mathrm{mbar}$

Table 24 lists the detailed characteristics of fuel used by IAV Automotive Engineering when modeling the engines in GT-POWER.

Table 24. Characteristics of Fuel Used for Modeling Engines

\begin{tabular}{|c|c|c|c|c|c|c|}
\hline $\begin{array}{l}\text { Type of } \\
\text { Fluid }\end{array}$ & Composition & \multicolumn{3}{|c|}{ Molecular Formula } & Density & Lower Heating \\
\hline & Mass fraction & C & H & 0 & $\mathrm{~kg} / \mathrm{m}^{3}$ & MJ/kg \\
\hline Hydrocarbon & 0.903712493 & 8 & 14.851265 & 0 & 741.9 & 43.19 \\
\hline Ethanol & 0.094801493 & 2 & 6 & 1 & 785 & 26.9 \\
\hline Water & 0.001486014 & 0 & 2 & 1 & 1002.5 & 0 \\
\hline
\end{tabular}

\subsubsection{Engine (eng01) $2 L \_p f i \_d o h c \_v v t \_e n g i n e 1 \_b a s e l i n e$}

Engine 1 is a naturally aspirated PFI 2.0 L gasoline engine from a MY 2013 vehicle with variable valve timing engine technology (VVT). A BSFC engine map was generated from dynamometer testing of the existing engine, which then served as the baseline BSFC map for all simulated naturally aspirated engines (Engines 1-8a, 18-21). The engine calibrations are fully optimized for best BSFC and maximum torque. Figure 139 shows the BSFC map for engine 1. 


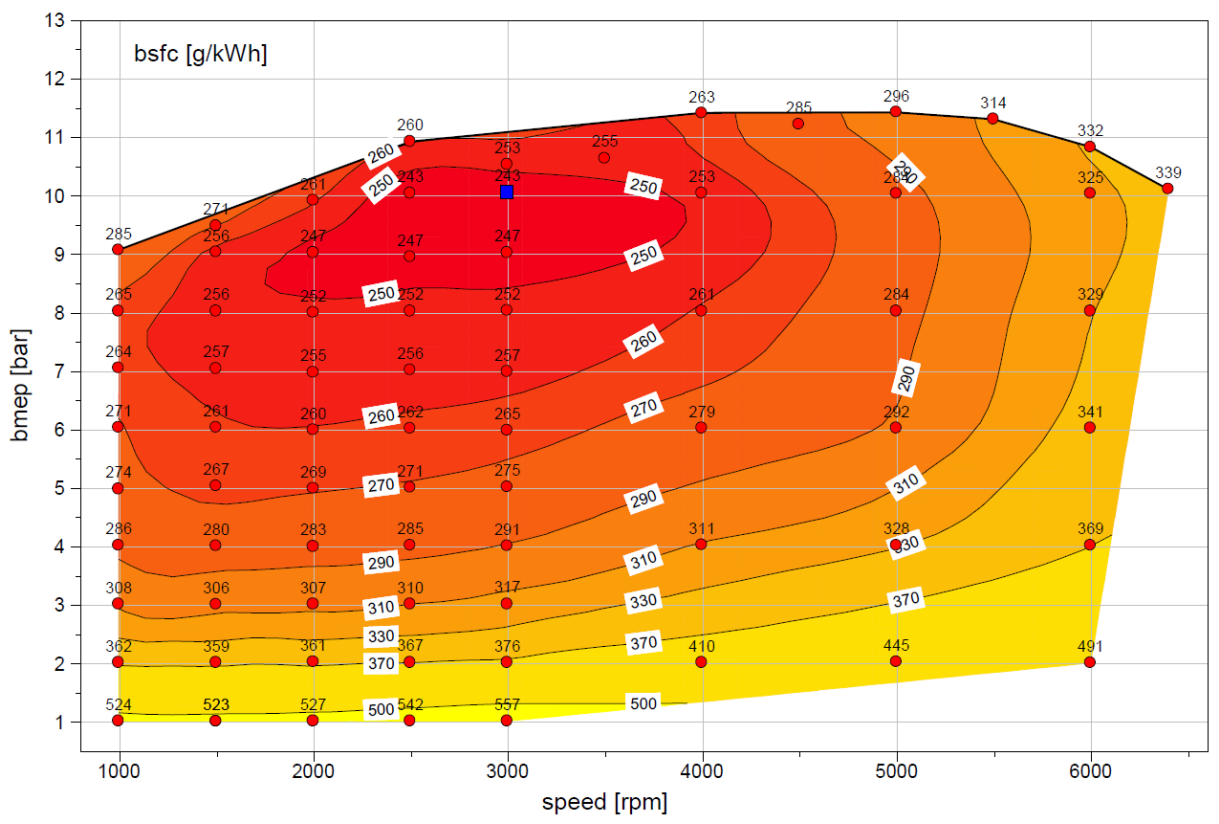

Figure 139. Engine 1 BSFC map

\subsubsection{2. $\quad$ Engine (eng02) $2 L \_p f i \_d o h c \_v v t \_v v l$ engine2}

For engine 2, a VVL system was added to the intake valves on engine 1 with valve lift and timing optimized. The additional benefit includes reduced pumping work at low torques and more torque at low speeds from reduced intake duration. Figure 140 shows the BSFC map for engine 2.

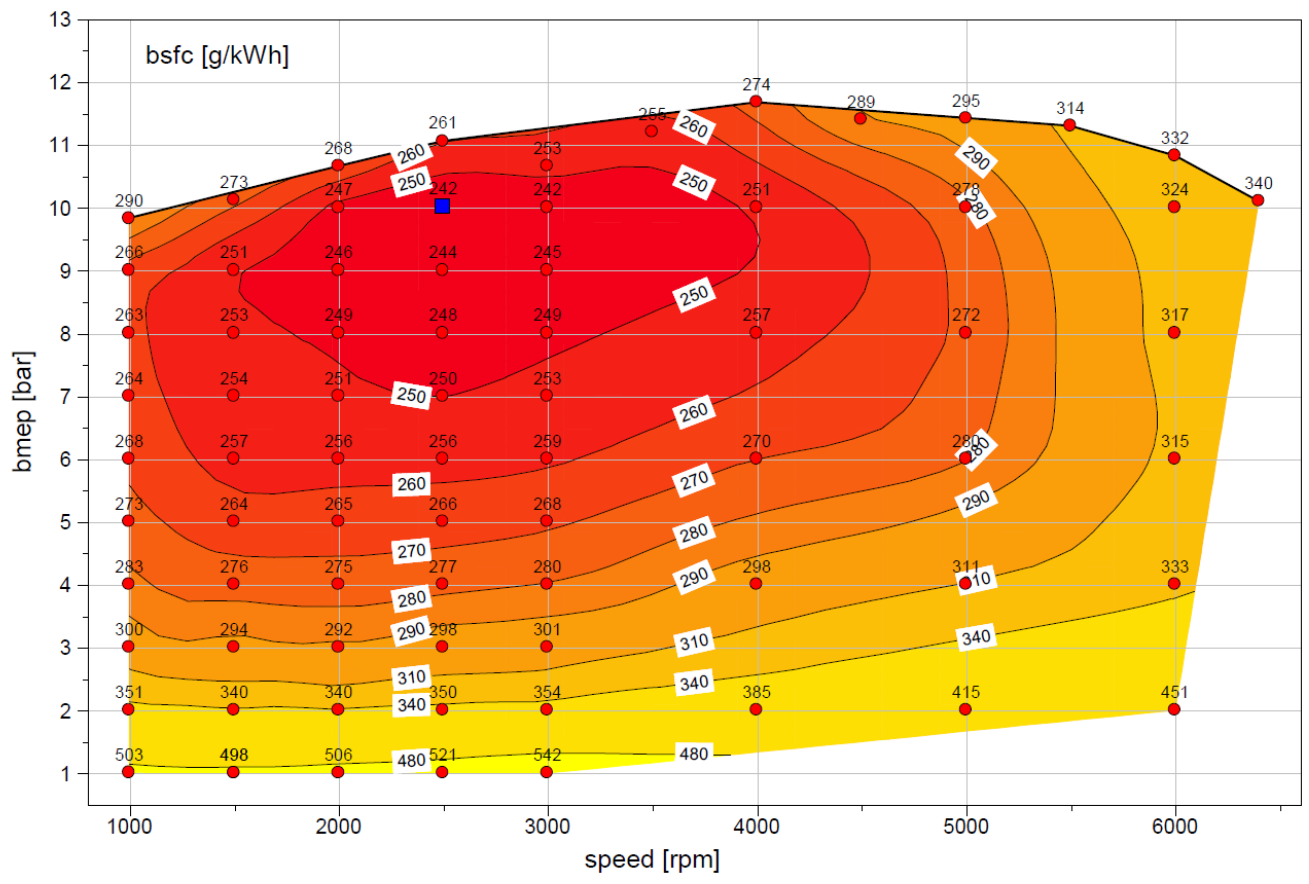

Figure 140. Engine 2 BSFC map 


\subsubsection{3. $\quad$ Engine (eng03) $2 L_{-}$di_engine3}

For engine 3, the port fuel injection (PFI) from engine 2 was converted to direct injection (DI), and the compression ratio was raised from 10.2 to 11.0 , with injection timing optimized. The benefit of this technology is that DI provides greater knock tolerance, allowing higher compression ratios and increased efficiency over the entire map. Figure 141 shows the BSFC map for engine 3.

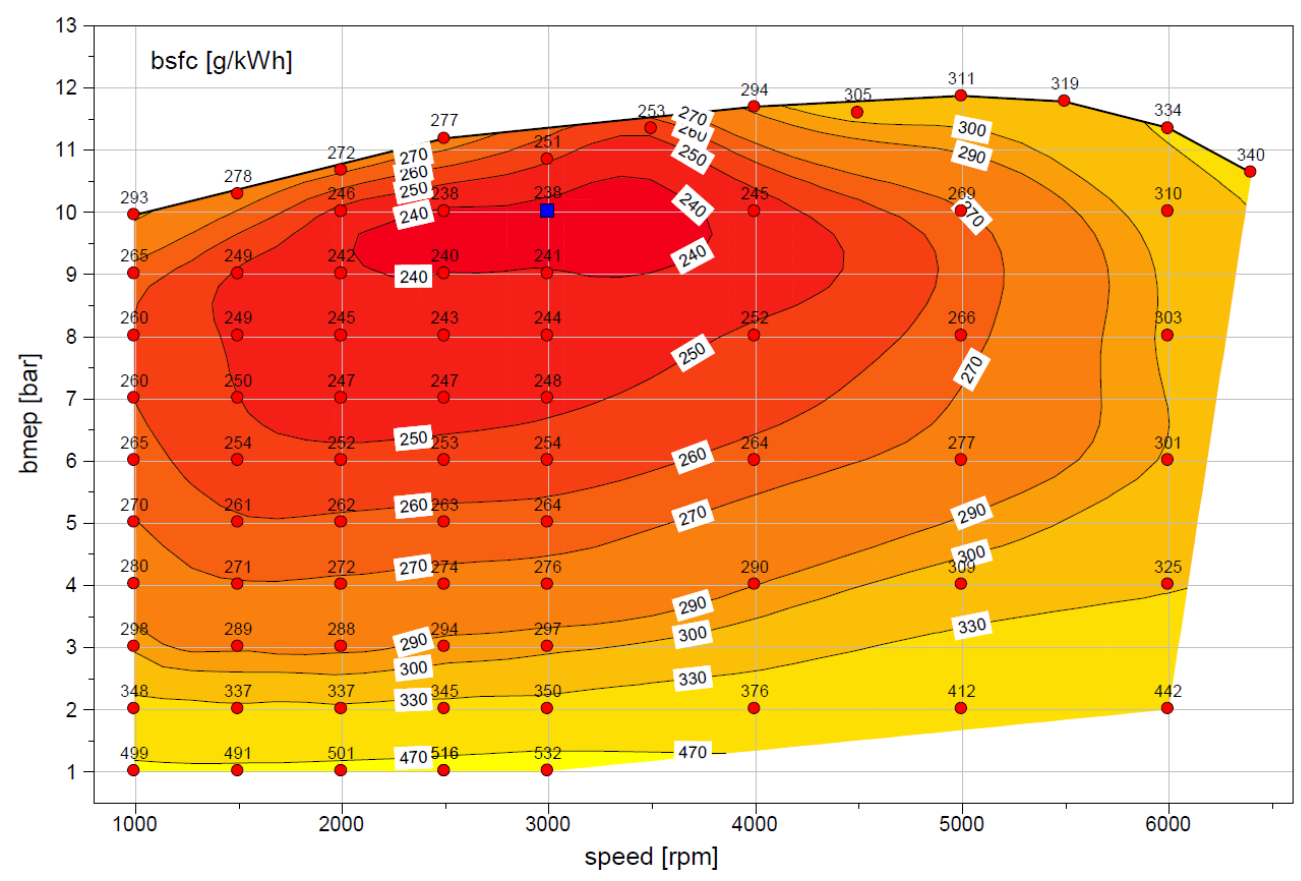

Figure 141. Engine 3 BSFC map

\subsubsection{Engine (eng04) $2 L_{-}$cylinder_deac_engine4}

For engine 4, cylinder deactivation capabilities were added to engine 3 . The engine fires only two cylinders at low loads and speeds below 3000 RPM by deactivating the valves on two cylinders. The additional benefit of this technology is that the effective load doubles on two cylinders, providing less pumping work and higher efficiency. Figure 142 shows the BSFC map for engine 4. 


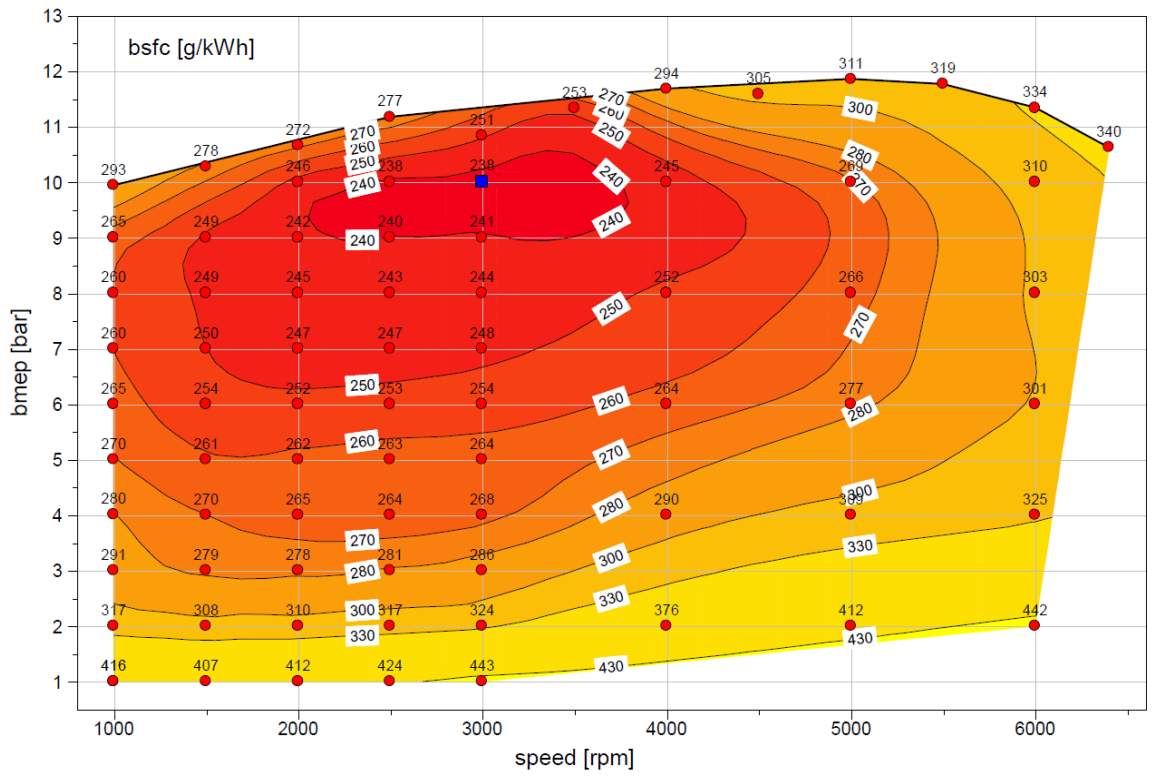

Figure 142. Engine 4 BSFC Map

\subsubsection{Engine (eng5b) 2L_engine5b_SOHC_low_friction}

For engine $5 \mathrm{~b}$, the valve timing was optimized for a fixed-overlap camshaft with a standard friction model from dual overhead camshaft (DOHC) concepts. The engine FMEP was reduced by 0.1 bar over the entire operation range to evaluate the friction benefit from a single overhead camshaft (SOHC). The benefit of this technology is reduced friction, which improves efficiency at all load points and raises the full-load line. Figure 143 shows the BSFC map for engine 5b.

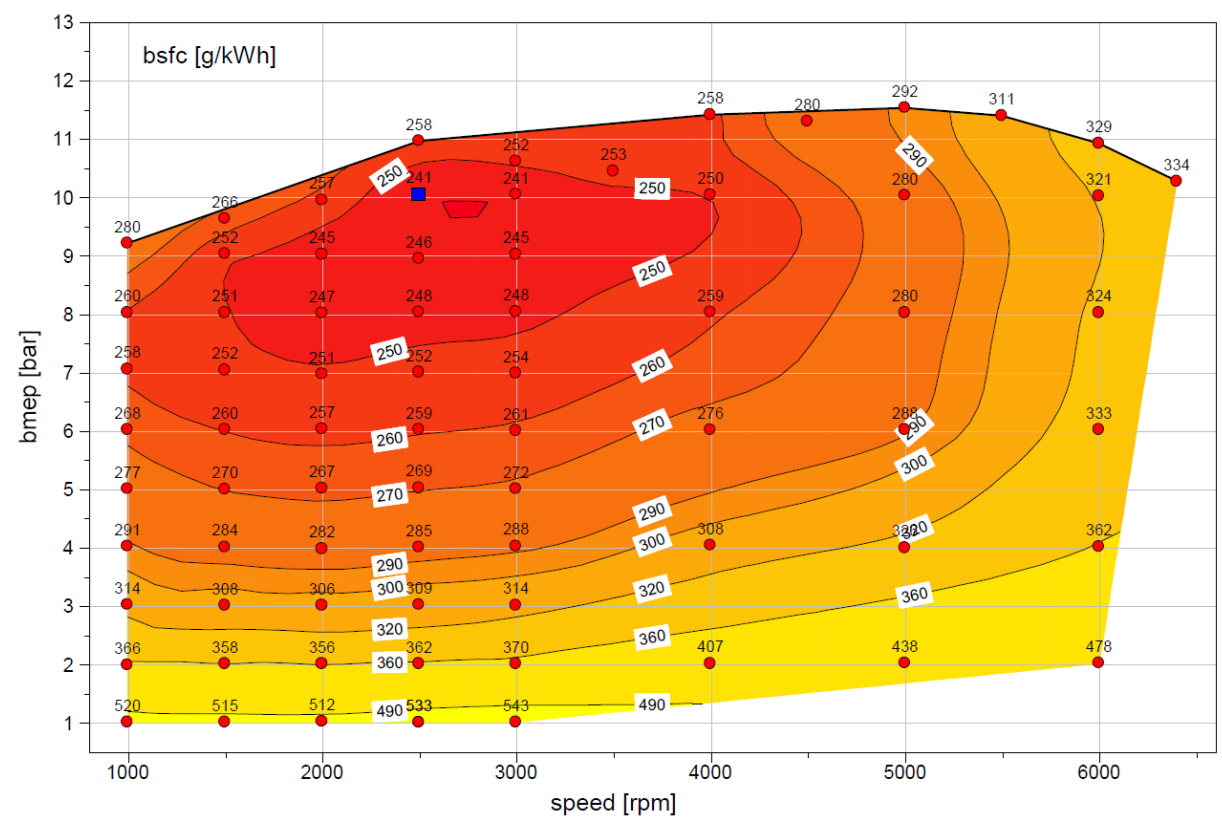

Figure 143. Engine 5b BSFC map 


\subsubsection{6. $\quad$ Engine (eng6a) $2 L \_$engine6a_VVL_low_friction}

For engine $6 \mathrm{a}$, the engine 2 FMEP was reduced by 0.1 bar over the entire operation range. The benefit of this change is similar to that for engine 5b, with improved efficiency at all load points and the full-load line raised compared with engine 2. Figure 144 shows the BSFC map for engine $6 a$.

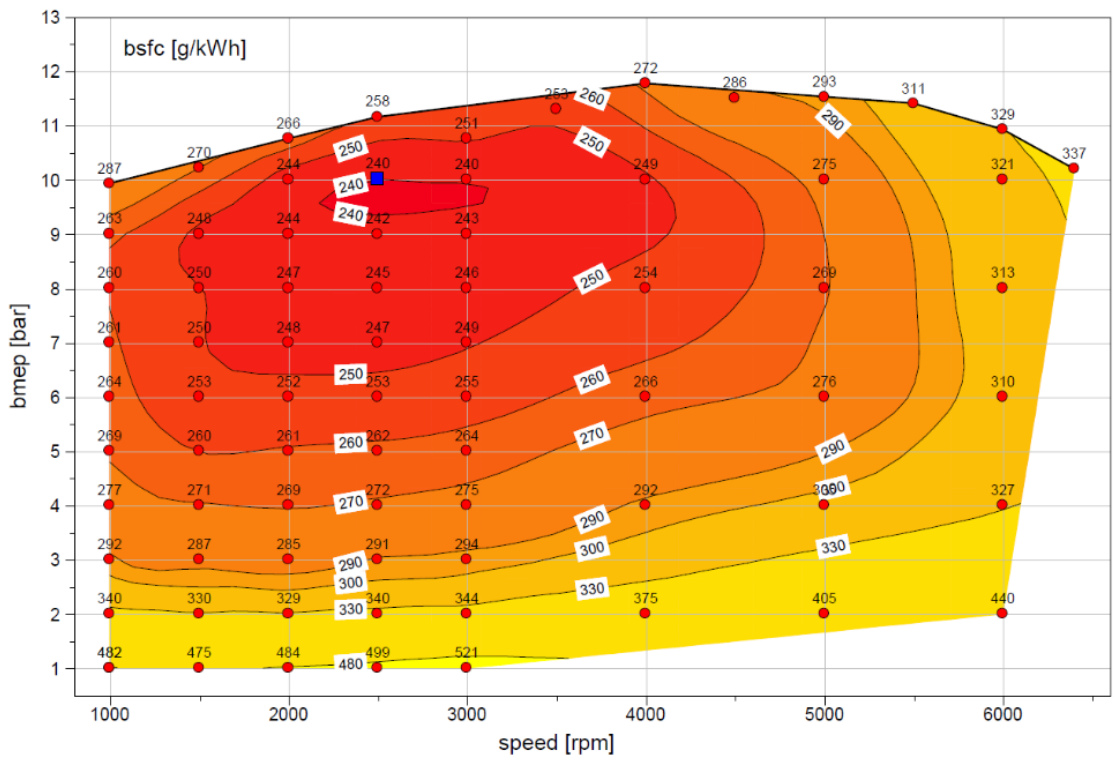

Figure 144. Engine 6a BSFC map

\subsubsection{Engine (eng7a) 2L_engine7a_DI_low_friction}

For engine $7 \mathrm{a}$, the engine 3 FMEP was reduced by 0.1 bar over the entire operation range. The reduced friction improves efficiency at all load points and raises the full-load line. Figure 145 shows the BSFC map for engine $7 \mathrm{a}$.

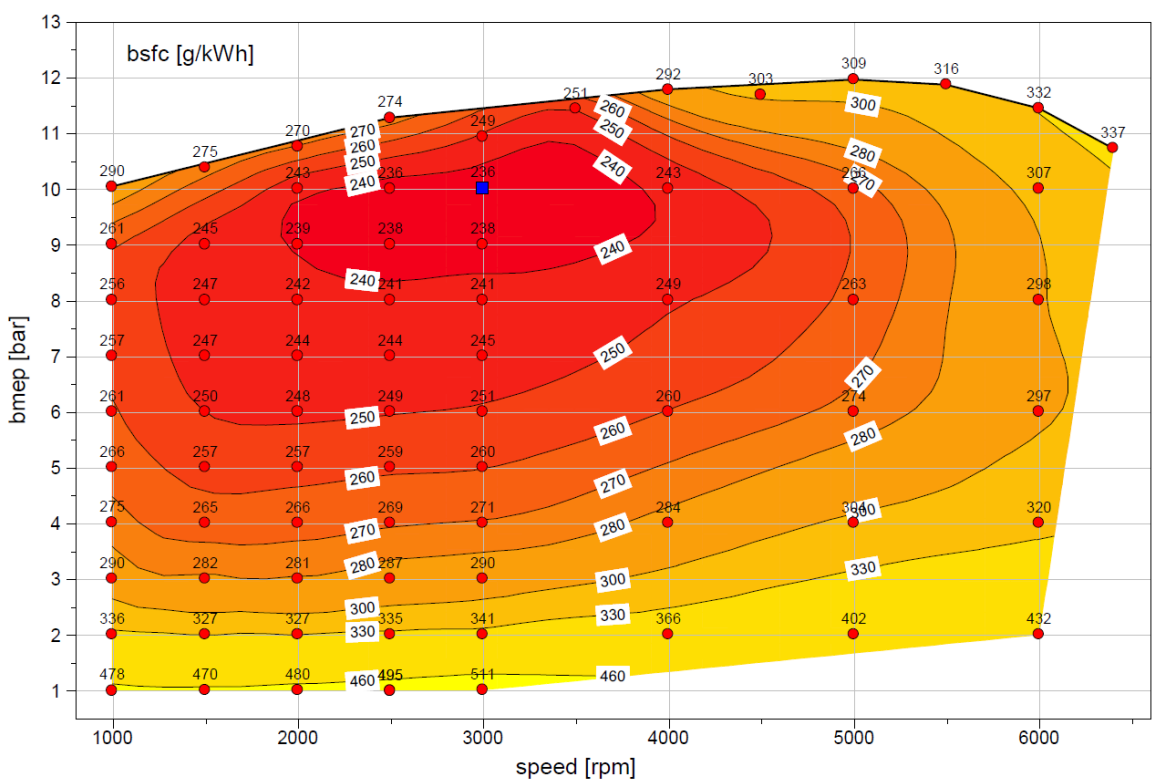

Figure 145. Engine 7a BSFC map 


\subsubsection{Engine (eng8a) $2 L \_$engine8a_cylinder_deac_low_friction}

For engine $8 \mathrm{a}$, the engine 4 FMEP was reduced by 0.1 bar over the entire operation range. The reduced friction improves efficiency at all loads and raises the full-load line. Figure 146 shows the BSFC map for engine 8a.

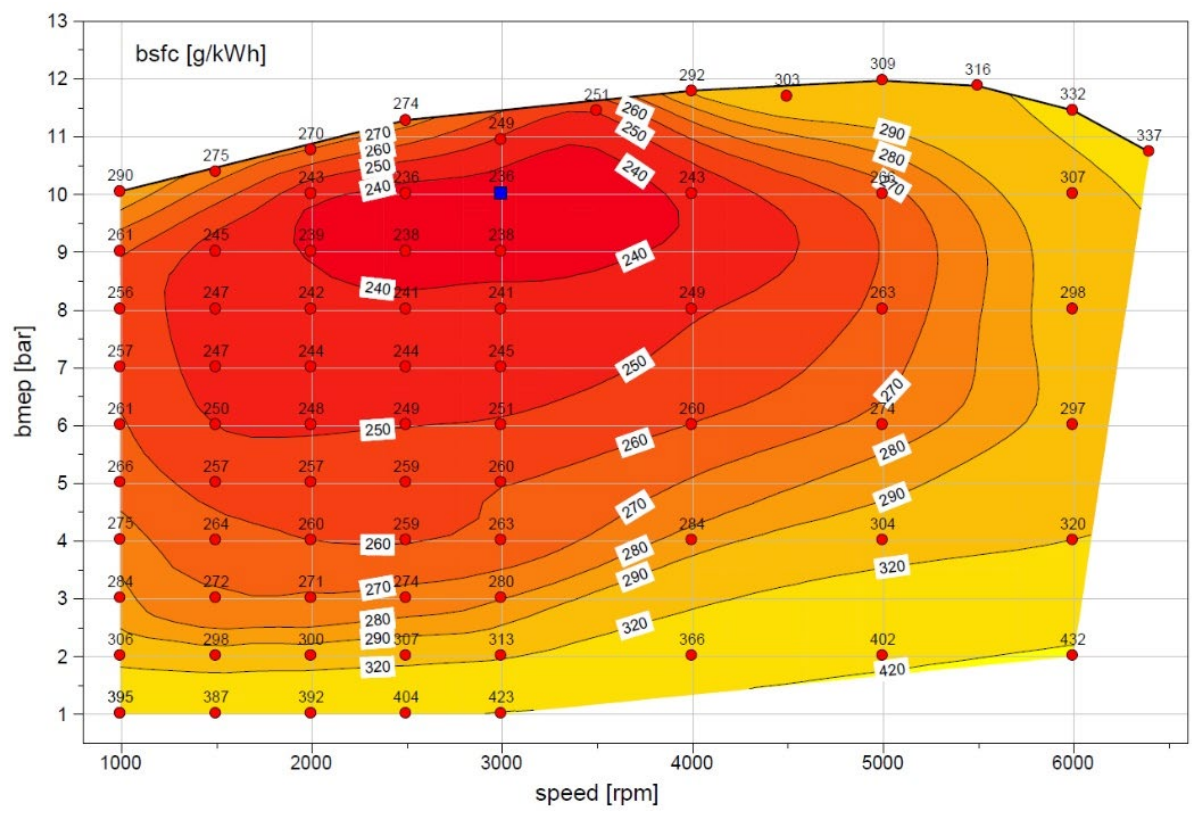

Figure 146. Engine 8a BSFC map

\subsubsection{9. $\quad$ Engine (eng12) 1pt6L_engine12_turbo_DI_DOHC_VVT_VVL}

Engine 12 is a $1.6 \mathrm{~L}$, 4-cylinder, turbocharged gasoline engine with DI, DOHC, dual-cam variable valve timing (VVT), and intake variable valve lift (VVL). The calibrations for the engine are fully optimized for the best BSFC. Figure 147 shows the BSFC map for engine 12. 


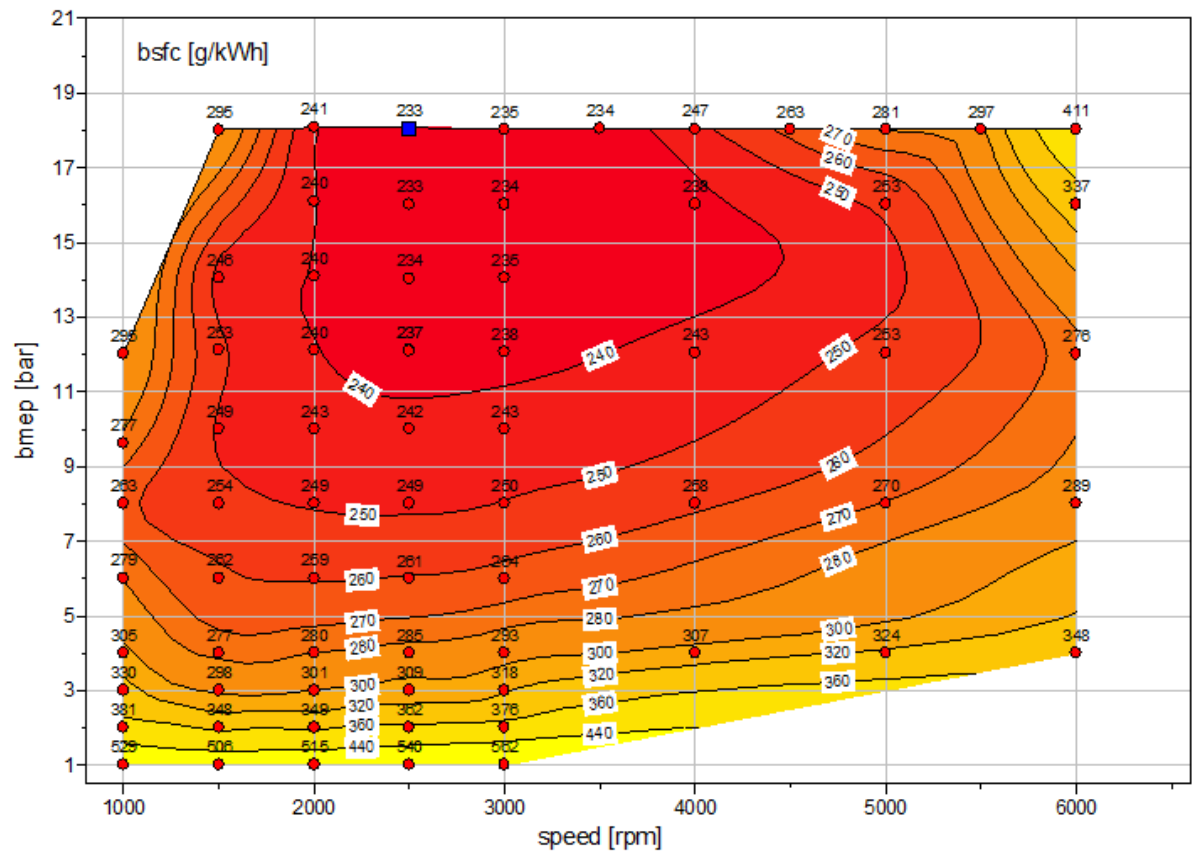

Figure 147. Engine 12 BSFC map

\subsubsection{Engine (eng13) 1pt2L_engine13_turbo_DI_DOHC_VVT_VVL}

For engine 13, engine 12 was downsized to $1.2 \mathrm{~L}$. The turbocharger maps were scaled to improve torque at low engine speeds. The downsizing allows for operation at a higher engine load point (increased efficiency) at a given vehicle torque demand. Figure 148 shows the BSFC map for engine 13.

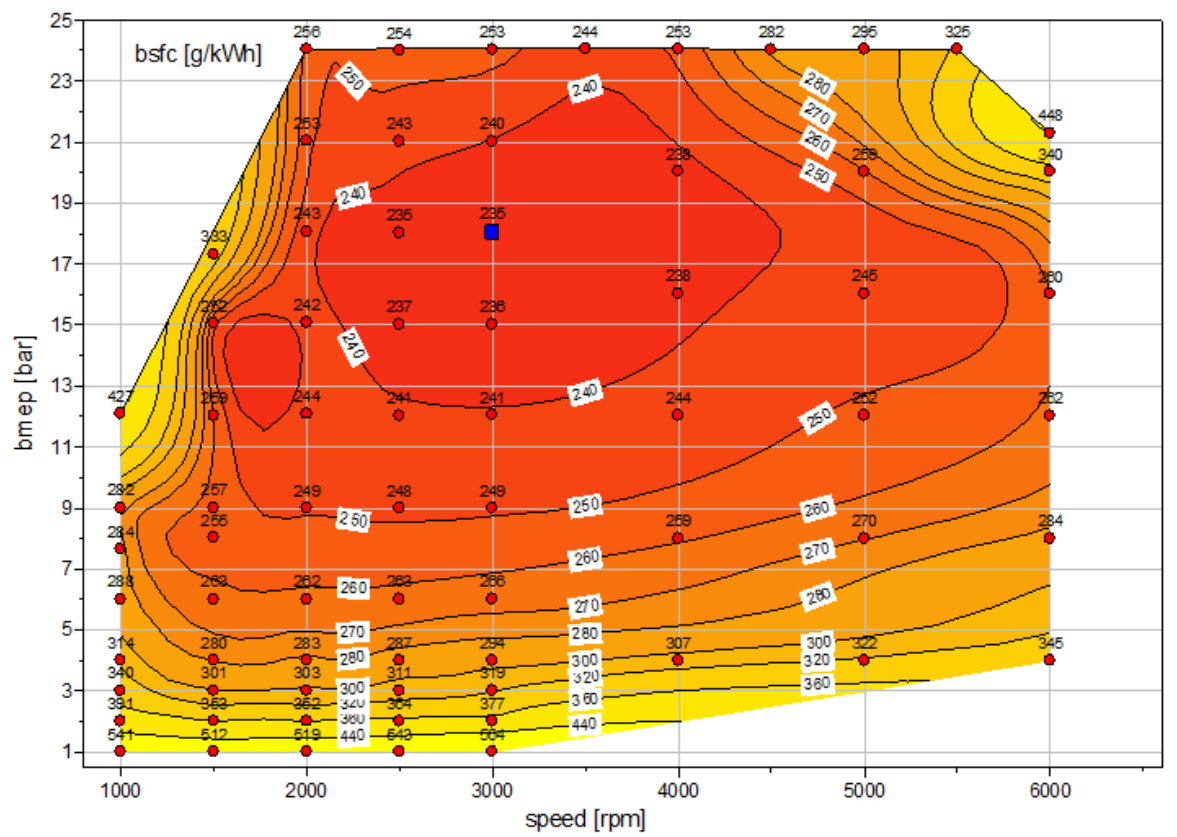

Figure 148. Engine 13 BSFC map 


\subsubsection{Engine (eng14) 1pt2L_engine14_turbo_external_cool_EGR}

For engine 14, high-pressure cooled exhaust gas recirculation (EGR) was added to engine 13 [4]. The cooled EGR target set points were further optimized. There are multiple benefits from the application of cooled EGR:

- The cooled burned gas lowers in-cylinder temperatures, reducing the knock tendency and thus improving combustion phasing.

- Reduced in-cylinder temperatures lead to reduced exhaust temperatures and therefore a reduced need for enrichment to protect exhaust components.

Figure 149 shows the BSFC map for engine 14.

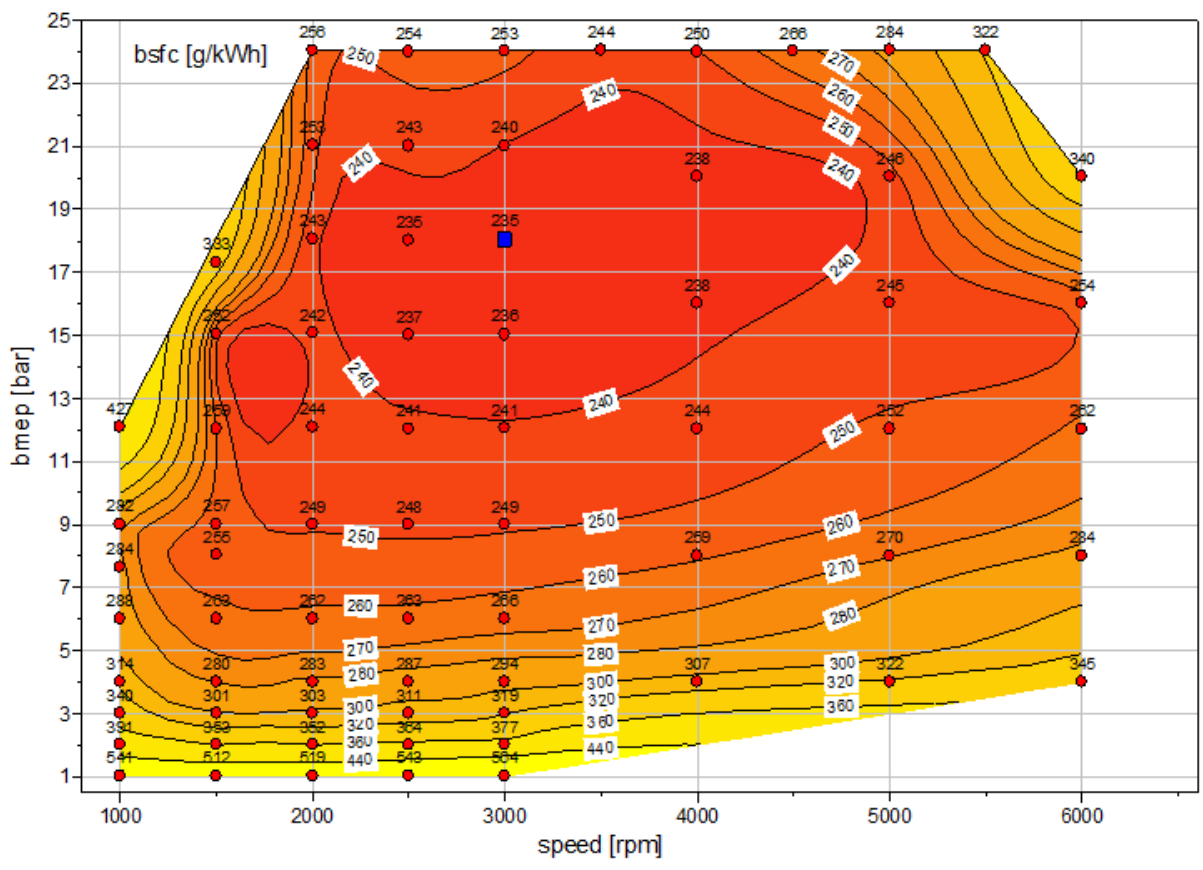

Figure 149. Engine 14 BSFC map

\subsubsection{Engine (eng17) eng_plant_ci_2pt2L_IAV_Engine17}

Engine 17 is a $2.2 \mathrm{~L}$, 4-cylinder diesel engine. Figure 150 shows the BSFC map for engine 17. 


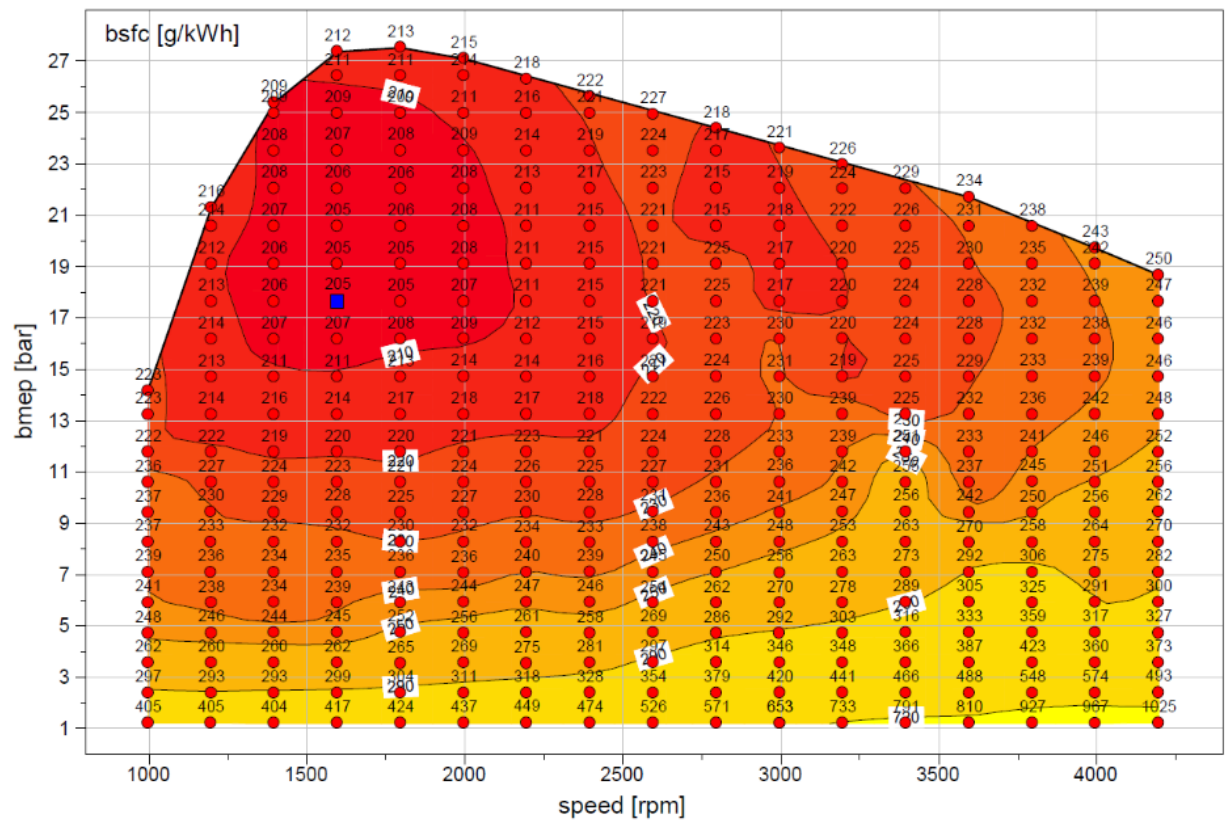

Figure 150. Engine 17 BSFC map

\subsubsection{Engine (eng18) 2L_di_dohc_vvt_engine18}

Engine 18 is a 2.0 L, 4-cylinder, naturally aspired gasoline engine with DOHC, dual VVT, and DI. The engine was developed from engine 1 , with increased knock resistance and volumetric efficiency due to in-cylinder vaporization of the fuel. Open-valve injection and homogeneous operation were assumed [10]. Figure 151 shows the BSFC map for engine 18.

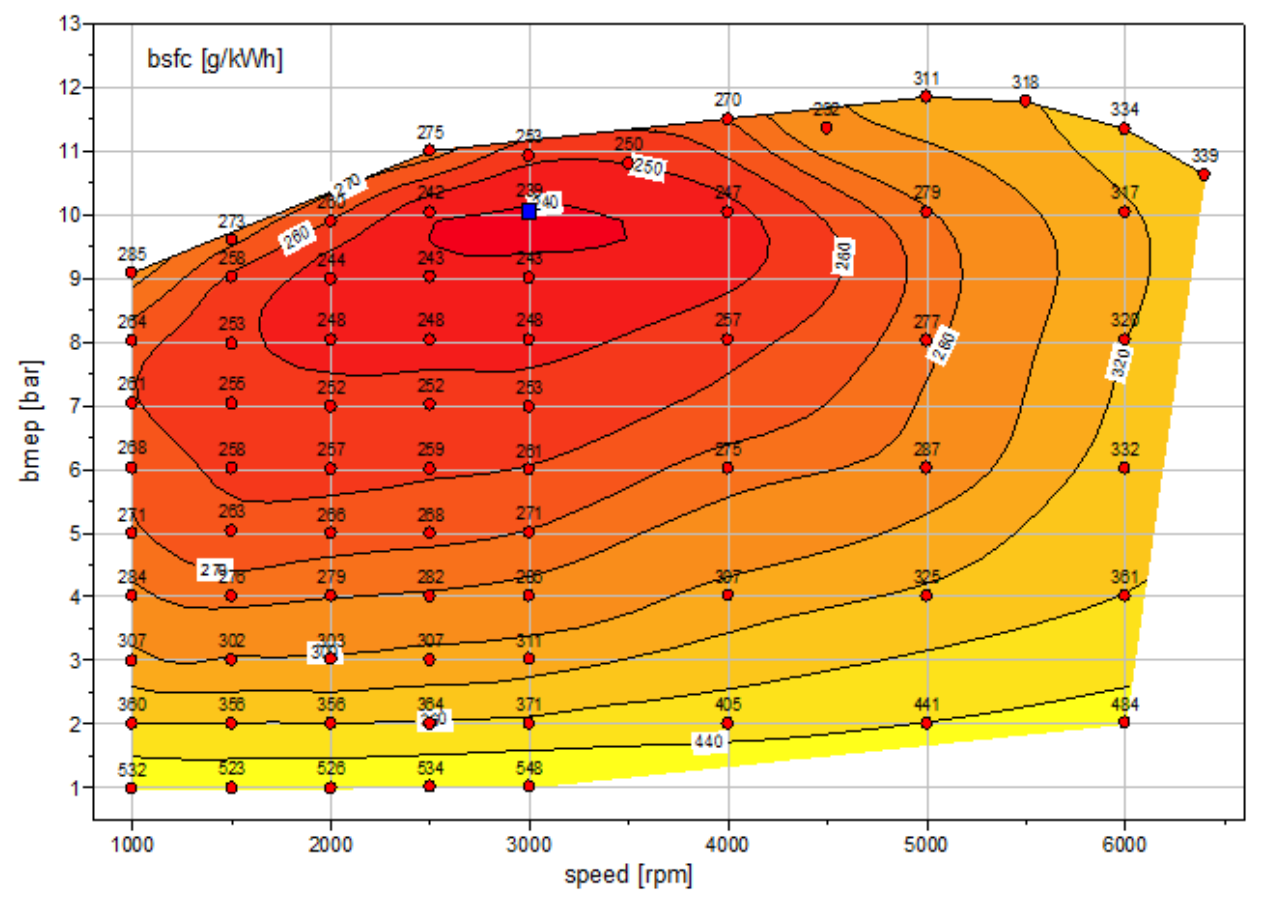

Figure 151. Engine 18 BSFC map 


\subsubsection{Engine (eng19) $2 L \_c y l i n d e r+d e a c \_e n g i n e 19$}

Engine 19 is a 2.0 L, 4-cylinder, naturally aspired PFI gasoline engine with DOHC, dual VVT, and cylinder deactivation (DEAC) capability. The engine was developed from engine 1, with the VVT timing map of active cylinders based on the cylinder IMEP of engine 1 [10]. Figure 152 shows the BSFC map for engine 19 .

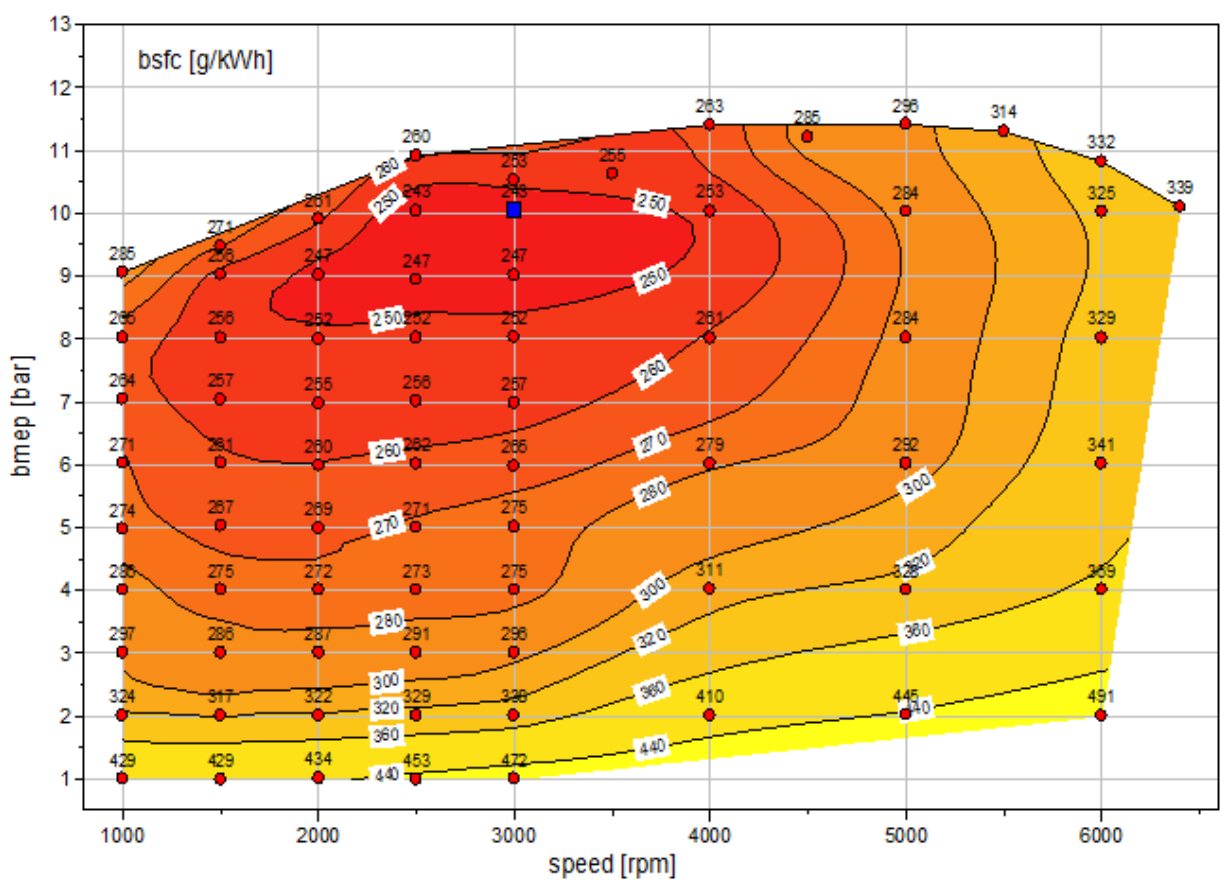

Figure 152. Engine 19 BSFC map

\subsubsection{Engine (eng20) $2 L \_c y l i n d e r \_d e a c \_e n g i n e 20$}

Engine 20 is a $2.0 \mathrm{~L}$, 4-cylinder, naturally aspired PFI engine with DOHC, dual VVT, intake VVL, and DEAC abilities. The engine was developed from engine 2. The VVT maps and intake valve map lift of active cylinders are based on the cylinder IMEP of engine 2 [10]. Figure 153 shows the BSFC map for engine 20 . 


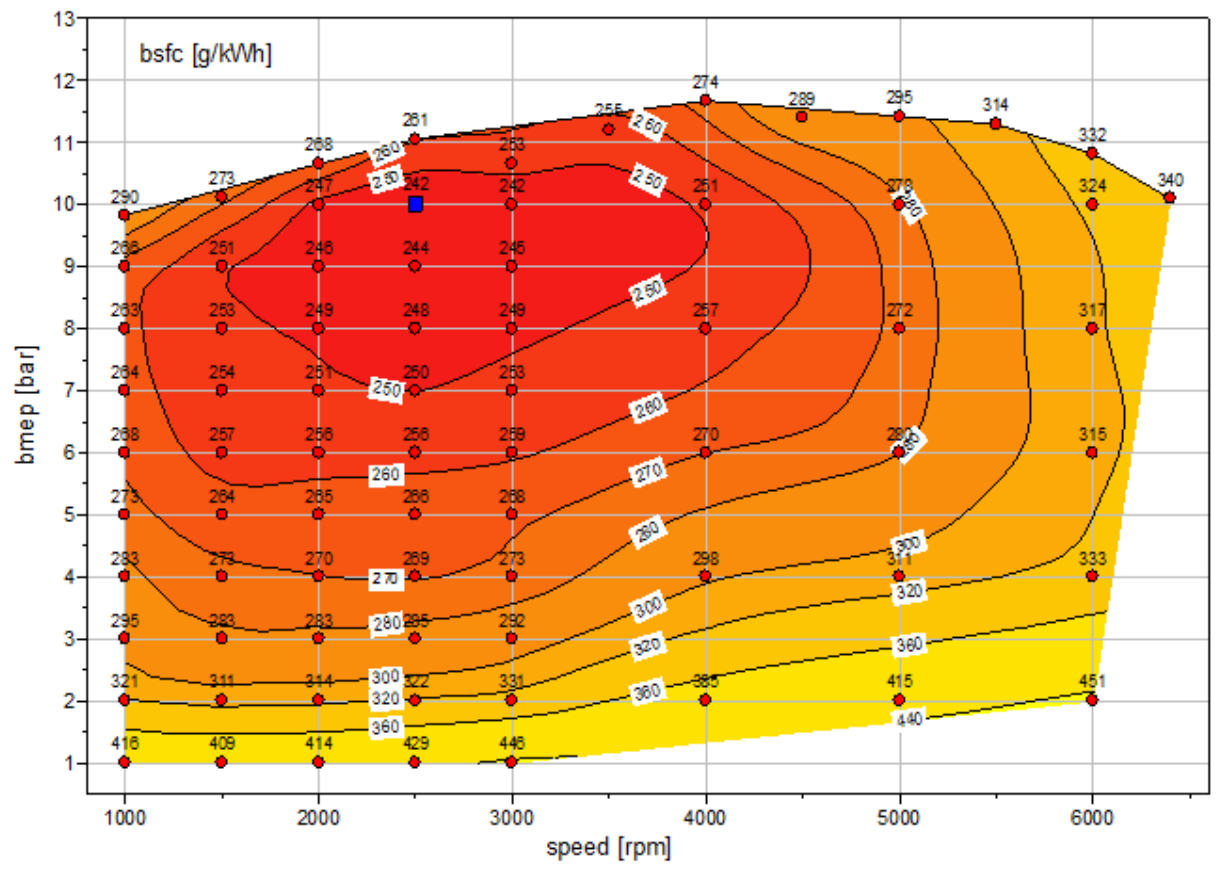

Figure 153. Engine 20 BSFC map

\subsubsection{Engine (eng21) 2L_cylinder_deac_engine21}

Engine 21 is a $2.0 \mathrm{~L}$, 4-cylinder, naturally aspired engine with DOHC, dual-cam VVT, DI and DEAC abilities. The engine was developed from engine 18. The VVT timing map of active cylinders is based on the cylinder IMEP of engine 18 [10]. Figure 154 shows the BSFC map for engine 21.

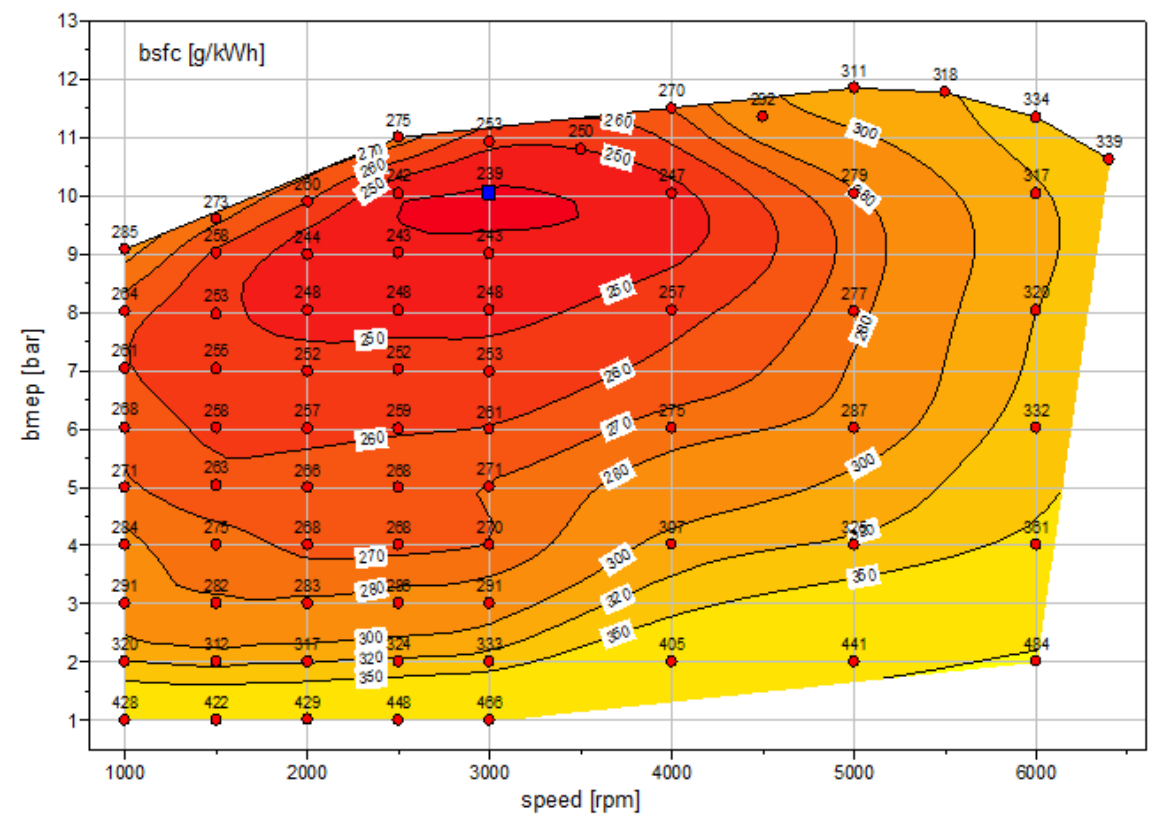

Figure 154. Engine 21 BSFC map 


\subsubsection{Engine (eng22b) $2 L \_e n g i n e 22 b \_A t k i n s o n \_2.5 L \_V V T \_C R 14$}

Engine $22 \mathrm{~b}$ is a $2.5 \mathrm{~L}$, 4-cylinder, Atkinson naturally aspired PFI engine with DOHC, dual cam VVT, and a compression-ratio of 14 . Figure 155 shows the BSFC map for engine $22 \mathrm{~b}$.

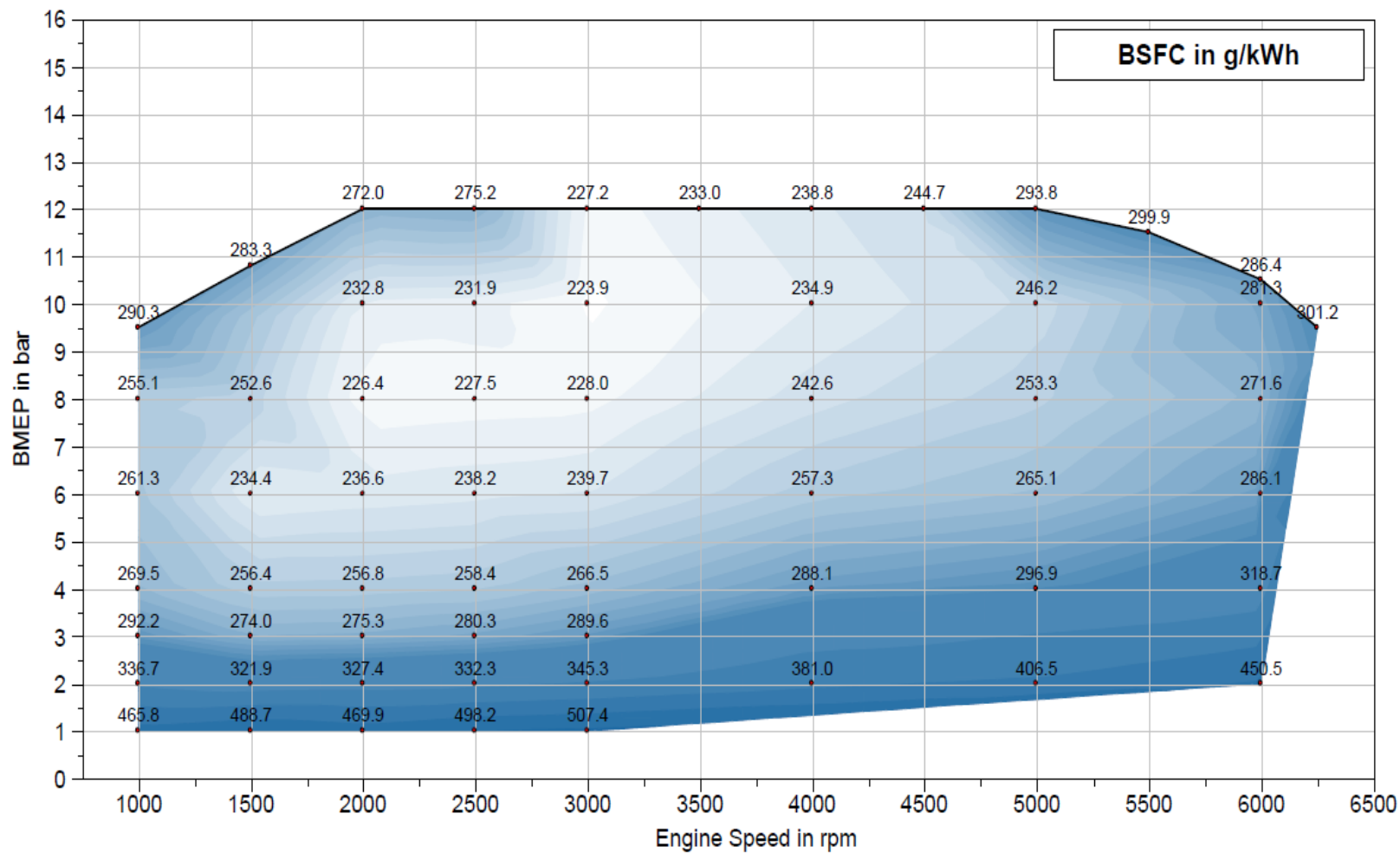

Figure 155. Engine 22b BSFC map

\subsubsection{Engine (eng23b) $2 L_{-}$engine23b_VVT_VVL_DI_cEGR_CR12}

Engine $23 \mathrm{~b}$ is a $2.0 \mathrm{~L}$, 4-cylinder, turbocharged DI engine with DOHC, dual cam VVT, intake VVL, and cooled EGR capabilities. The engine has a compression ratio of 12. Figure 156 shows the BSFC map for engine $23 \mathrm{~b}$. 


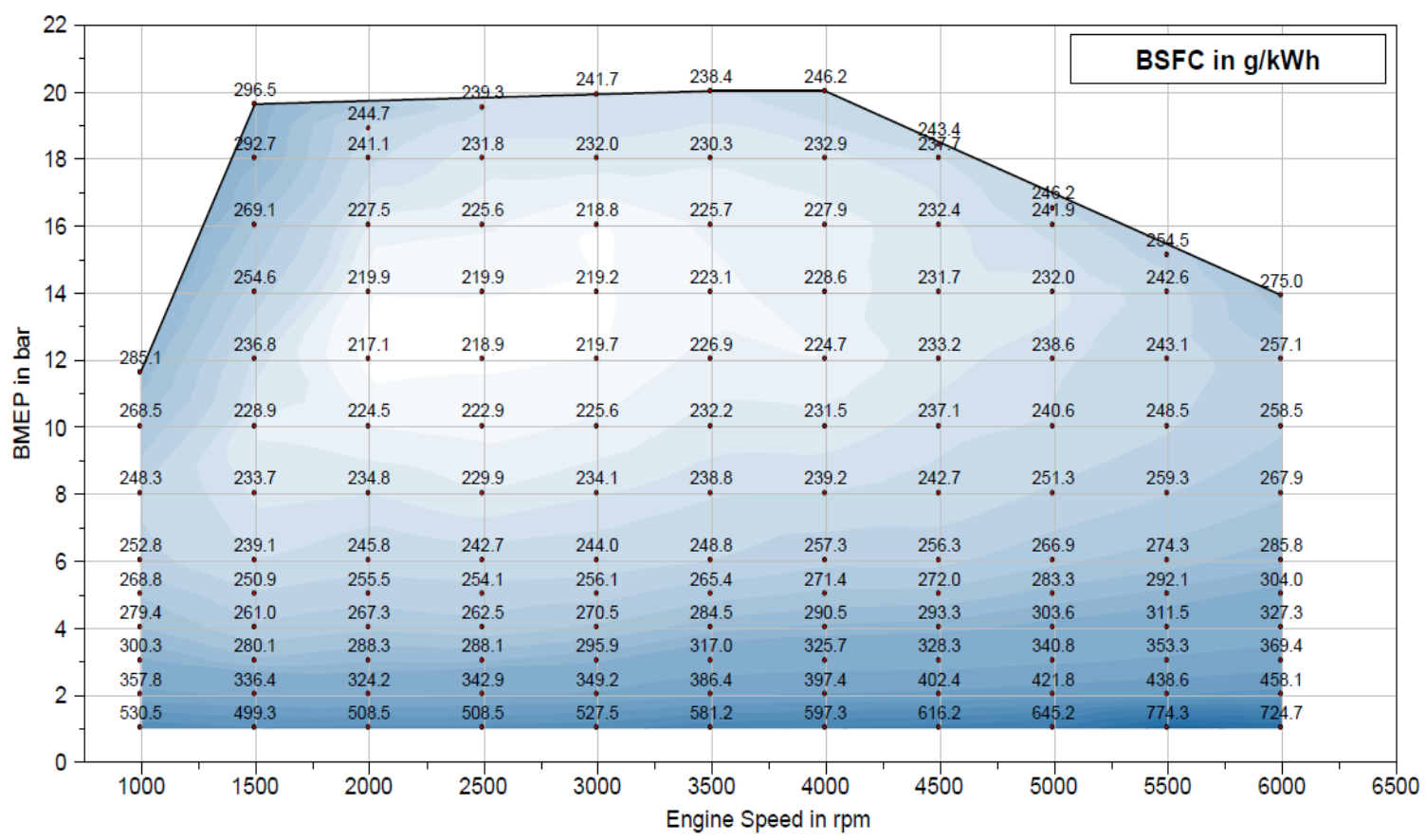

Figure 156. Engine 23b BSFC map

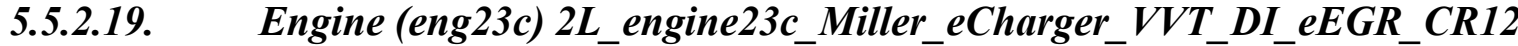

Engine $23 \mathrm{c}$ is a $2.0 \mathrm{~L}$, 4-cylinder, turbocharged DI engine with DOHC, dual cam VVT, and cooled EGR capabilities. The engine has a compression ratio of 12 . The turbocharging technology has an e-charger to enhance e-boost. Figure 157 shows the engine BSFC map for engine 23c.

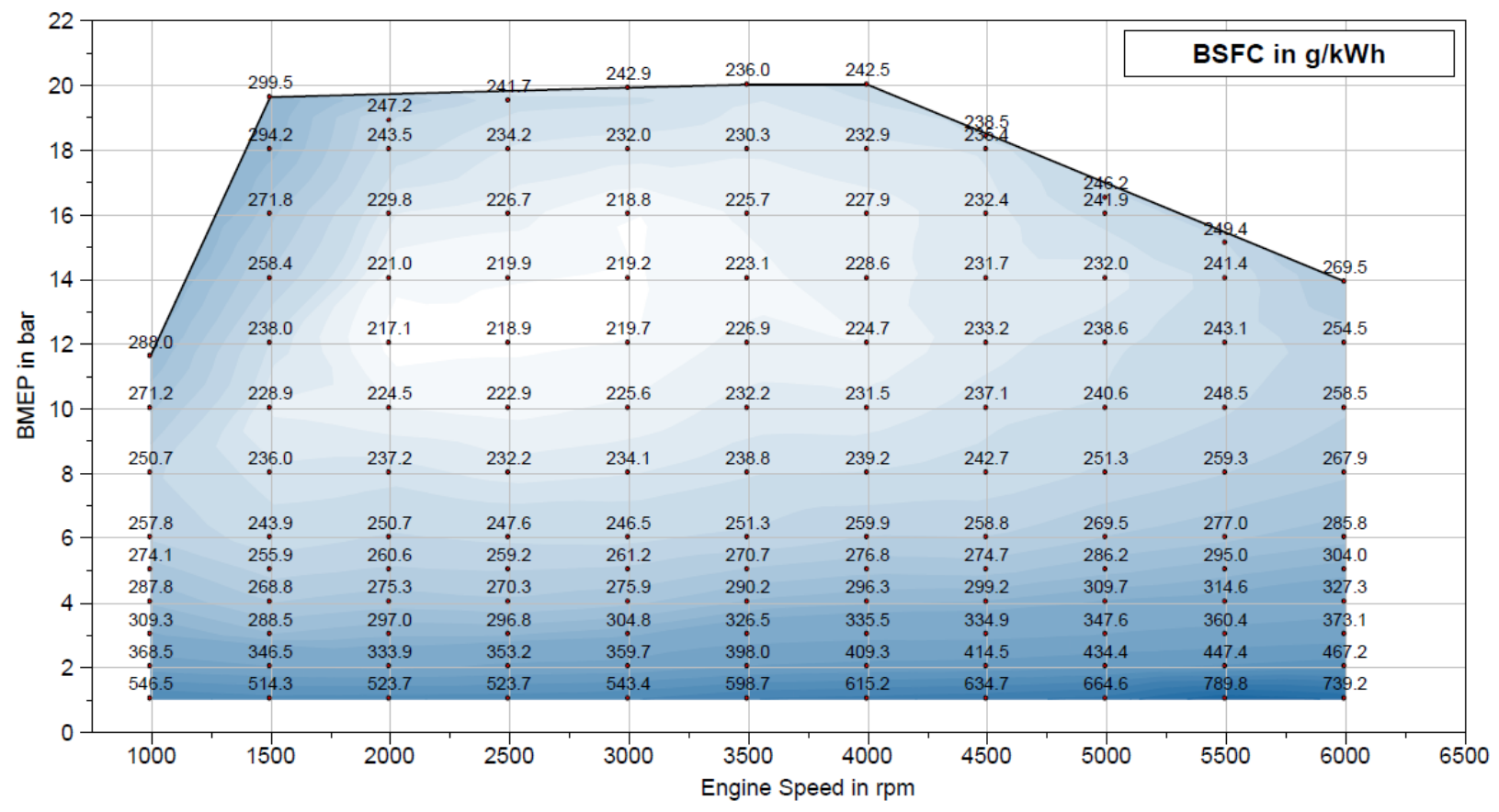

Figure 157. Engine 23c BSFC map 


\subsubsection{Engine (eng24) 2014_mazda_skyactiv_2p0L_USTier2}

Engine 24 is a Mazda SKYACTIV 2.0 L engine using Tier 2 fuel. The engine efficiency map has been provided by EPA [8]. Figure 158 shows the BSFC map for engine 24.

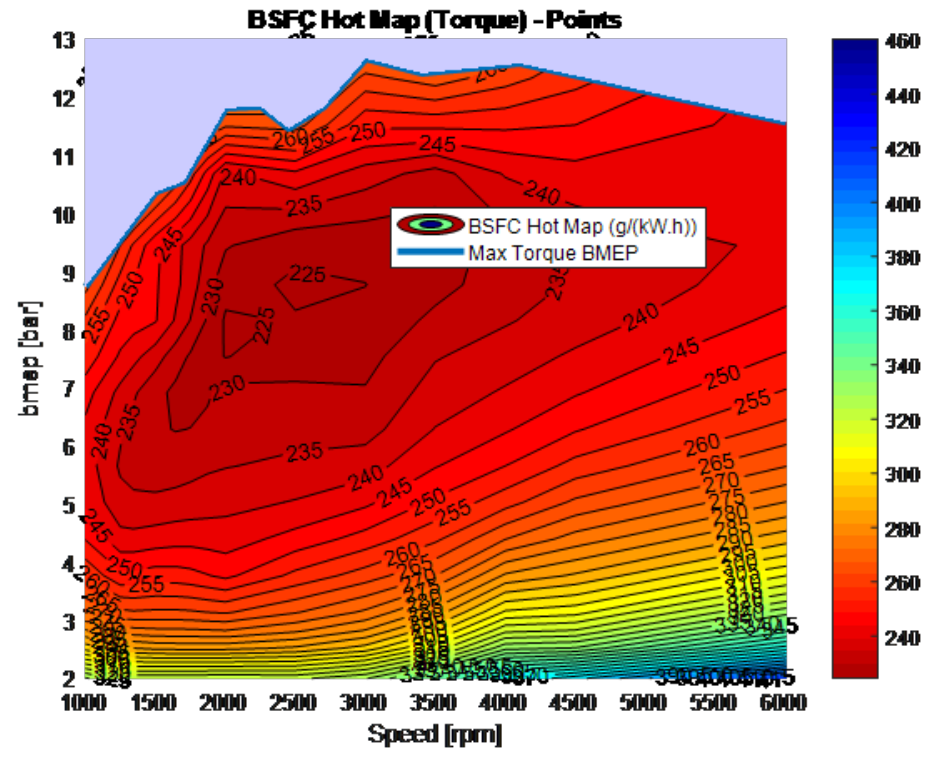

Figure 158. Engine 24 BSFC map

\subsubsection{Engine (eng25) future_mazda_skyactiv_2p0L_atkinson_cyl_deac}

Engine 25 is the future Atkinson engine with cooled EGR and DEAC. The data for the engine has been provided by EPA [34]. The engine is based on the Mazda SKYACTIV 2.0 L engine. EPA tested a GM 4.3 L EcoTec engine with cylinder deactivation to implement the DEAC abilities in the SKYACTIV engine [28]. Figure 159 shows the BSFC map for engine 25.

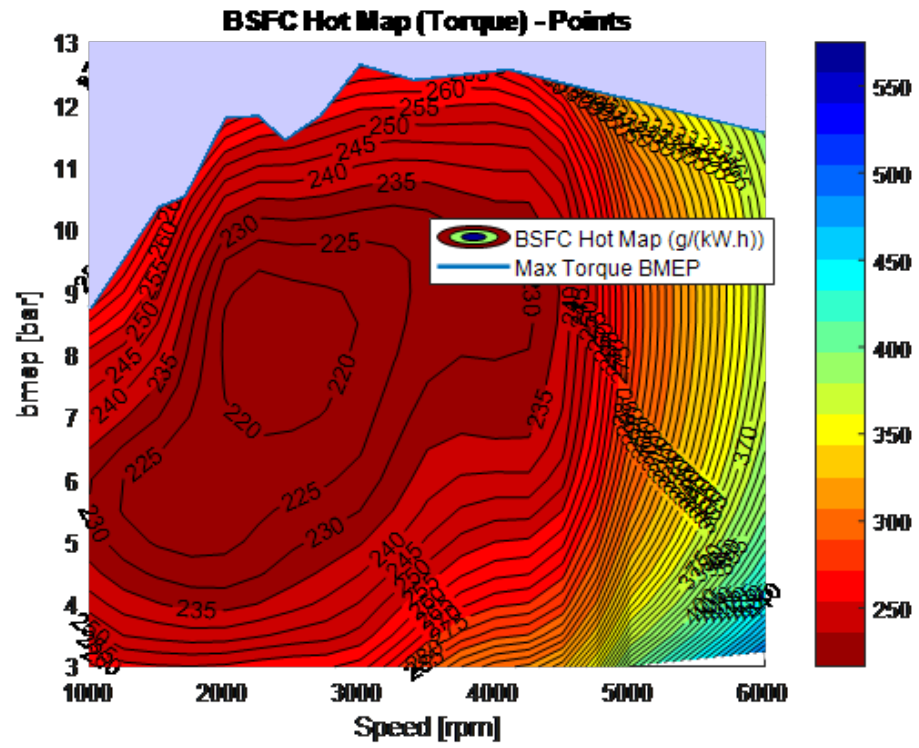

Figure 159. Engine 25 BSFC map 


\subsubsection{2. $\quad$ Engine (eng26) Atkinson}

Engine 26 is a $1.8 \mathrm{~L}, 4$-cylinder $73 \mathrm{~kW}$ full Atkinson engine. The data for the engine comes from Model Year 2010 Toyota Prius AMTL test data, but for this analysis the thermal efficiency was scaled up to match the Model Year 2017 Toyota Prius. Figure 160 shows the BSFC map for engine 26.

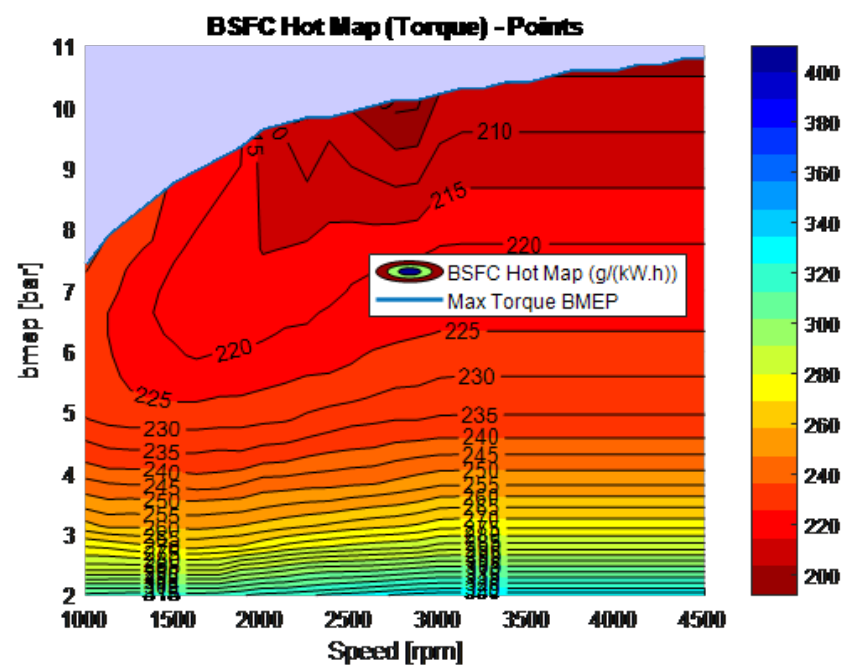

Figure 160. Engine 26 BSFC map

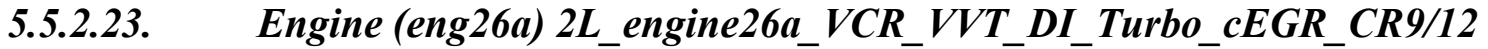

Engine 26a is a $2.0 \mathrm{~L}$, 4-cylinder, turbocharged DI engine with DOHC, dual cam VVT, and cooled EGR capabilities. The engine has a variable compression ratio of 9/12. Figure 161 shows the engine BSFC map for engine 26a. The BSFC map shows the fuel map resulting from merging the two separate maps of individual compression ratios.

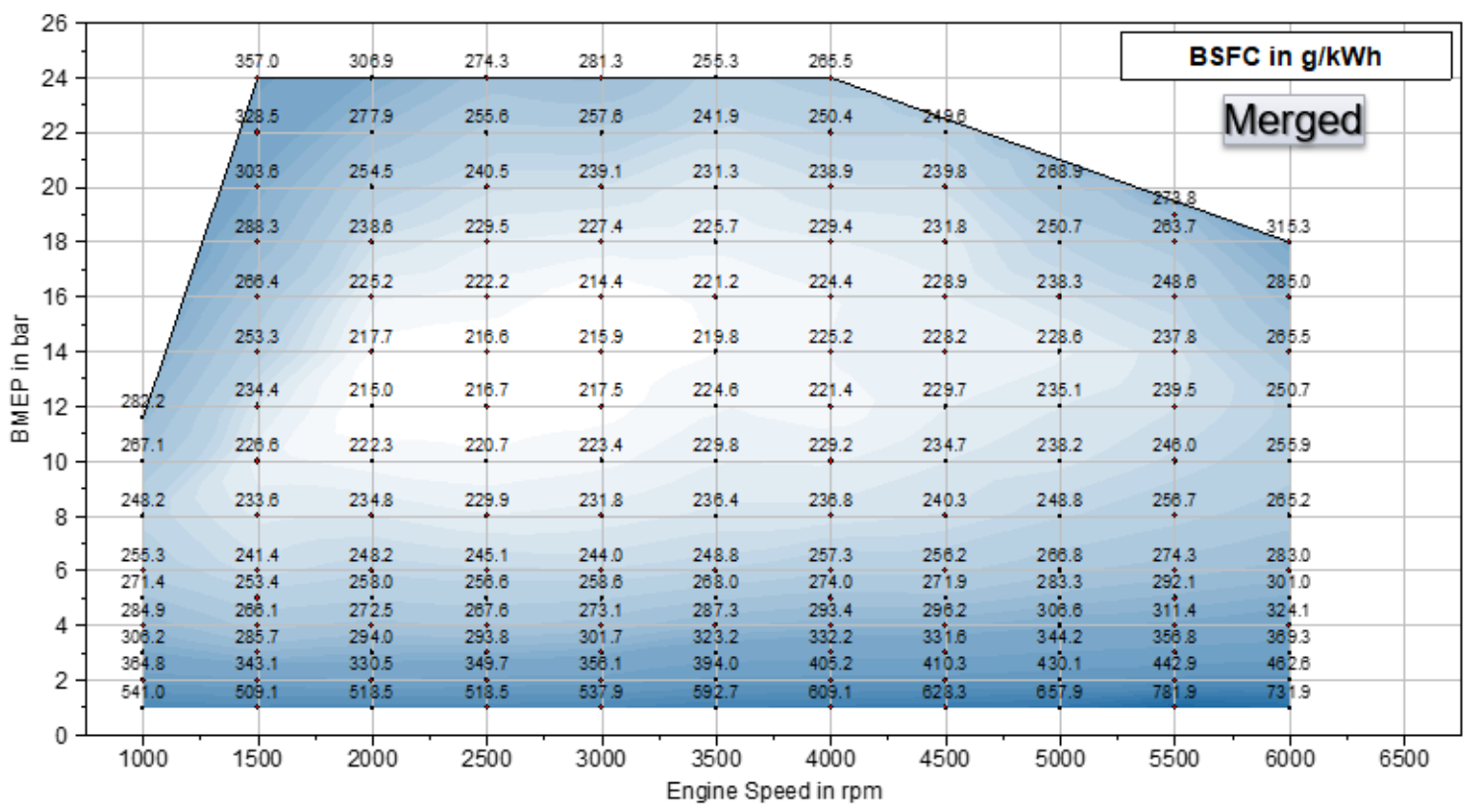

Figure 161. Engine 26a BSFC map 


\subsubsection{Incremental BSFC and Thermal Efficiency Difference of Engines}

Figure 162 shows the incremental differences (in percentage) in BSFC and thermal efficiency among the different engines.

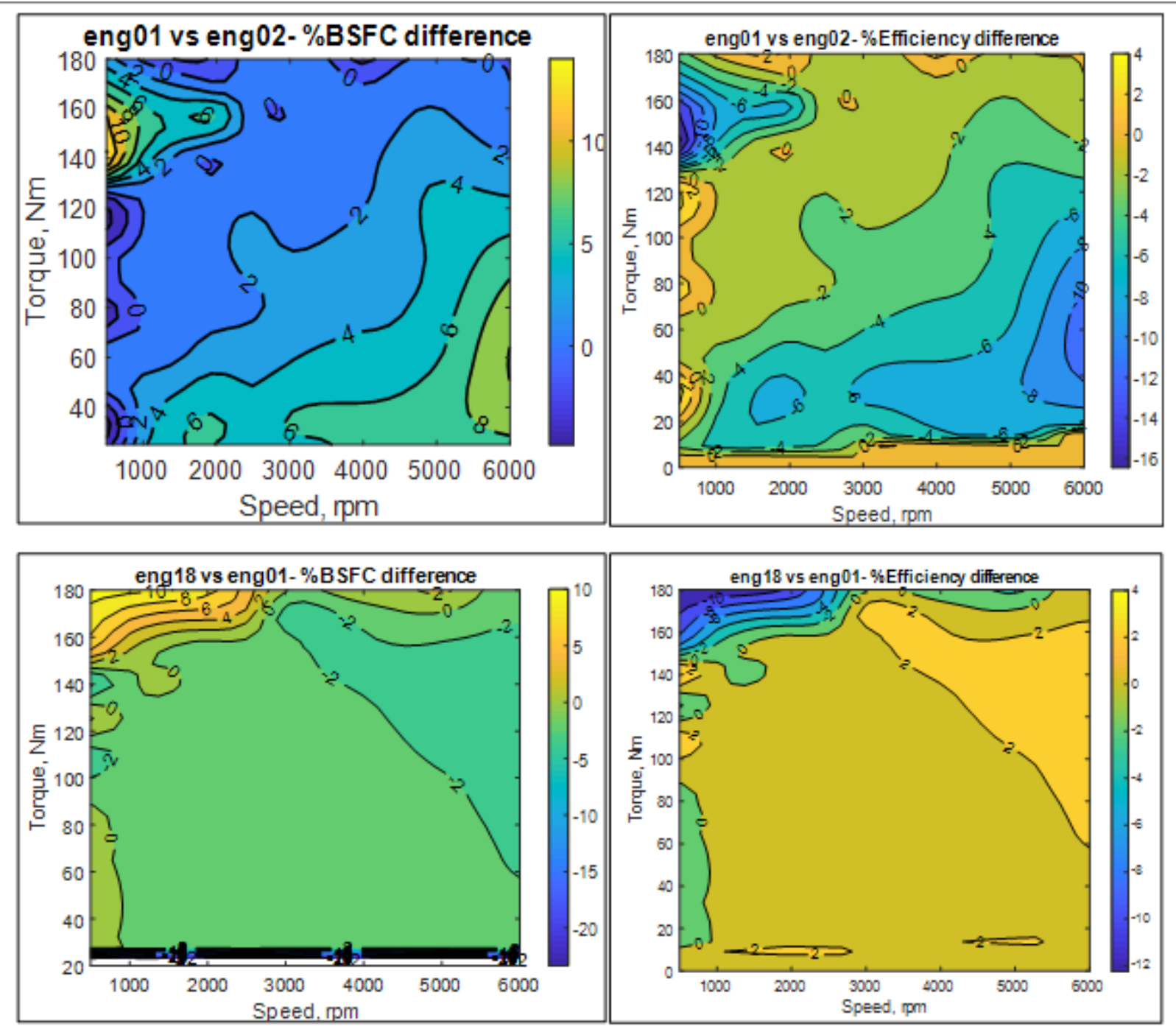




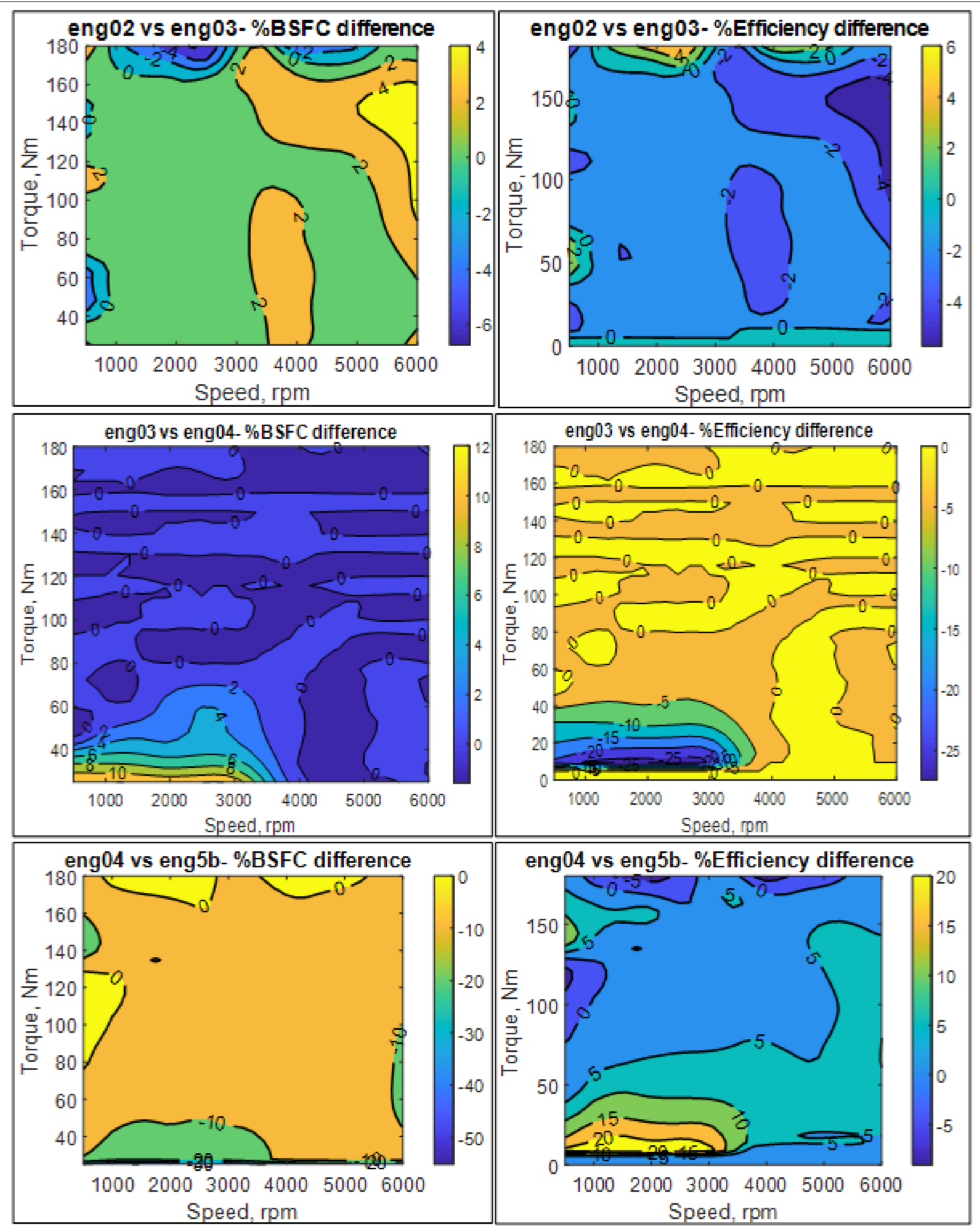




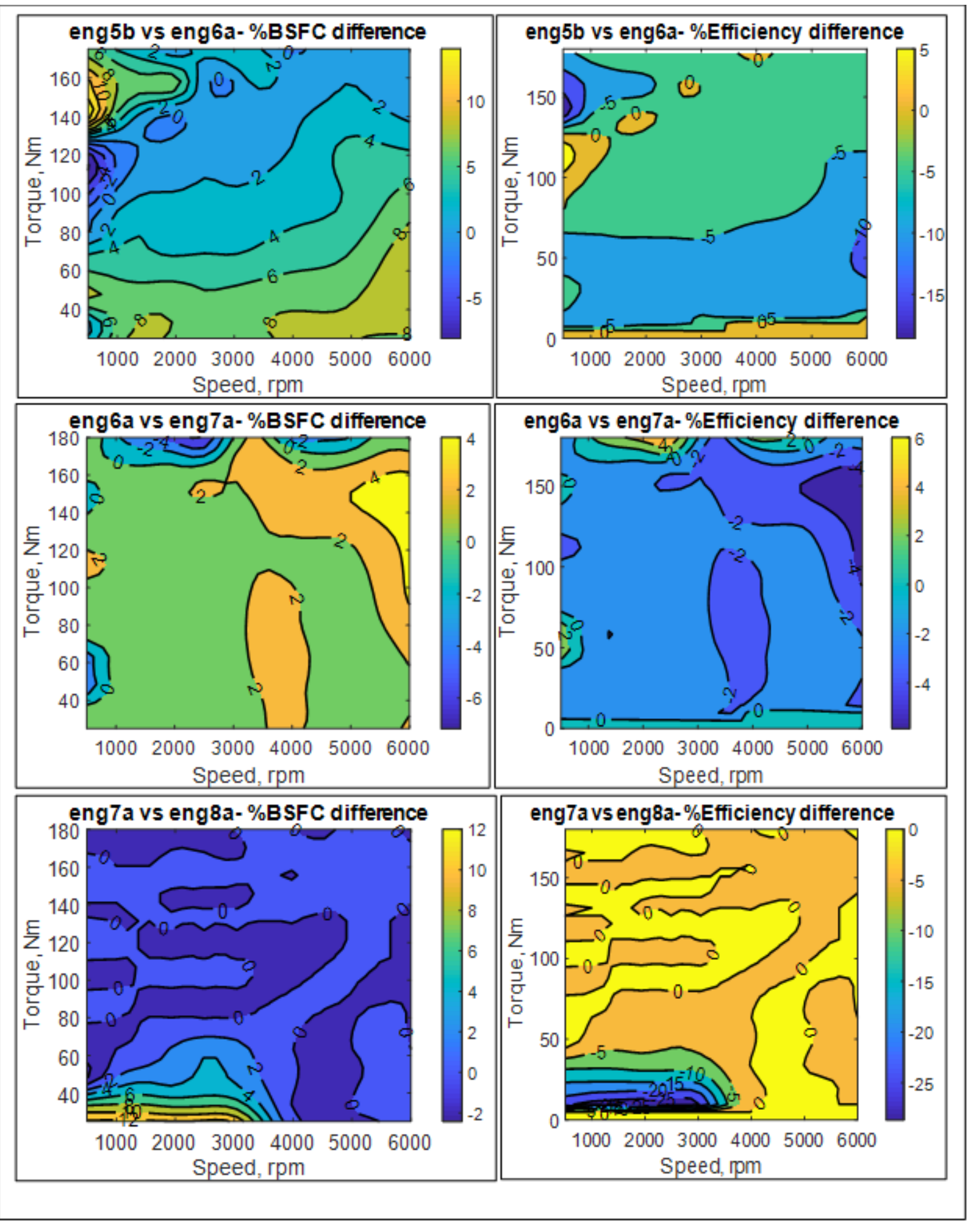




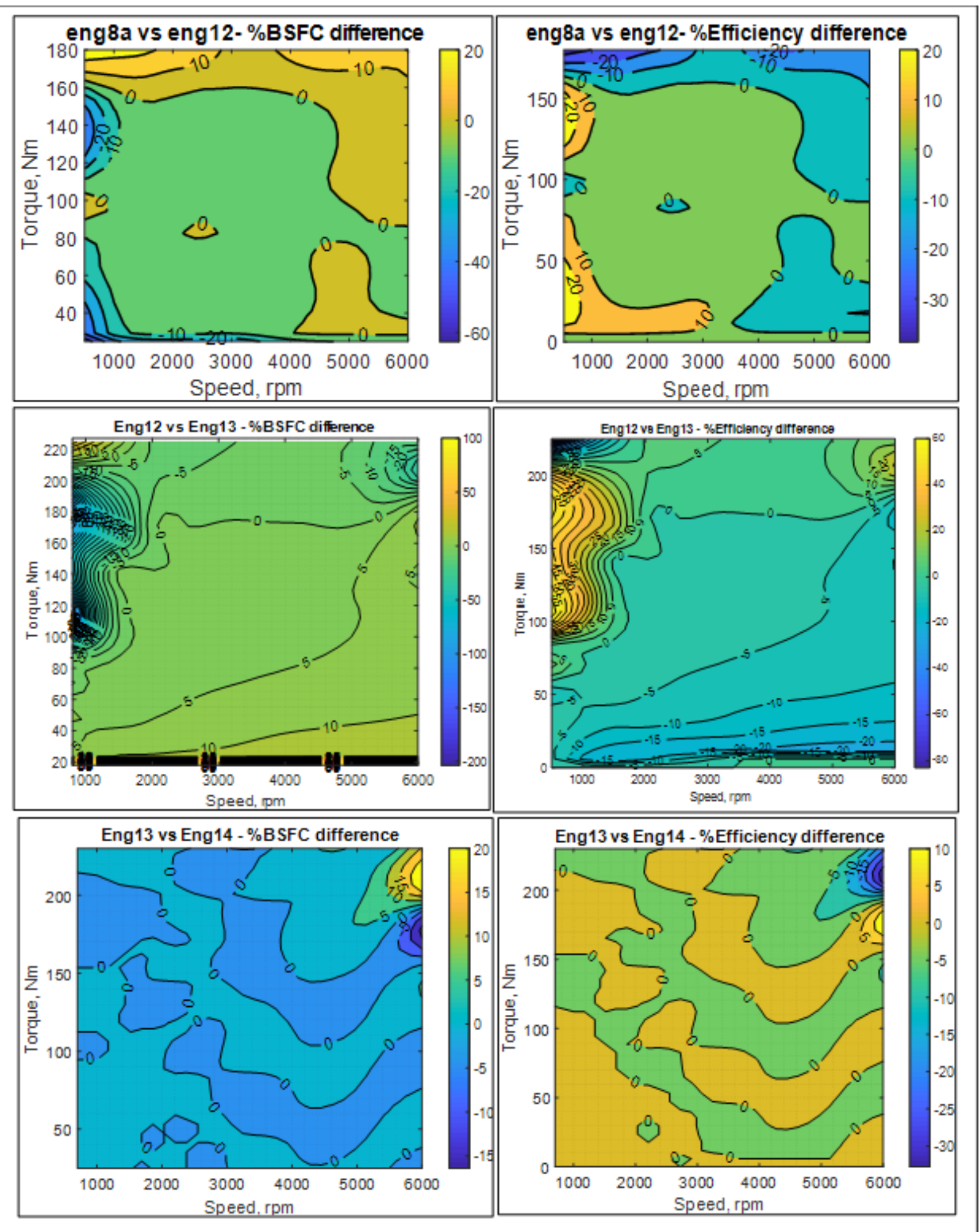



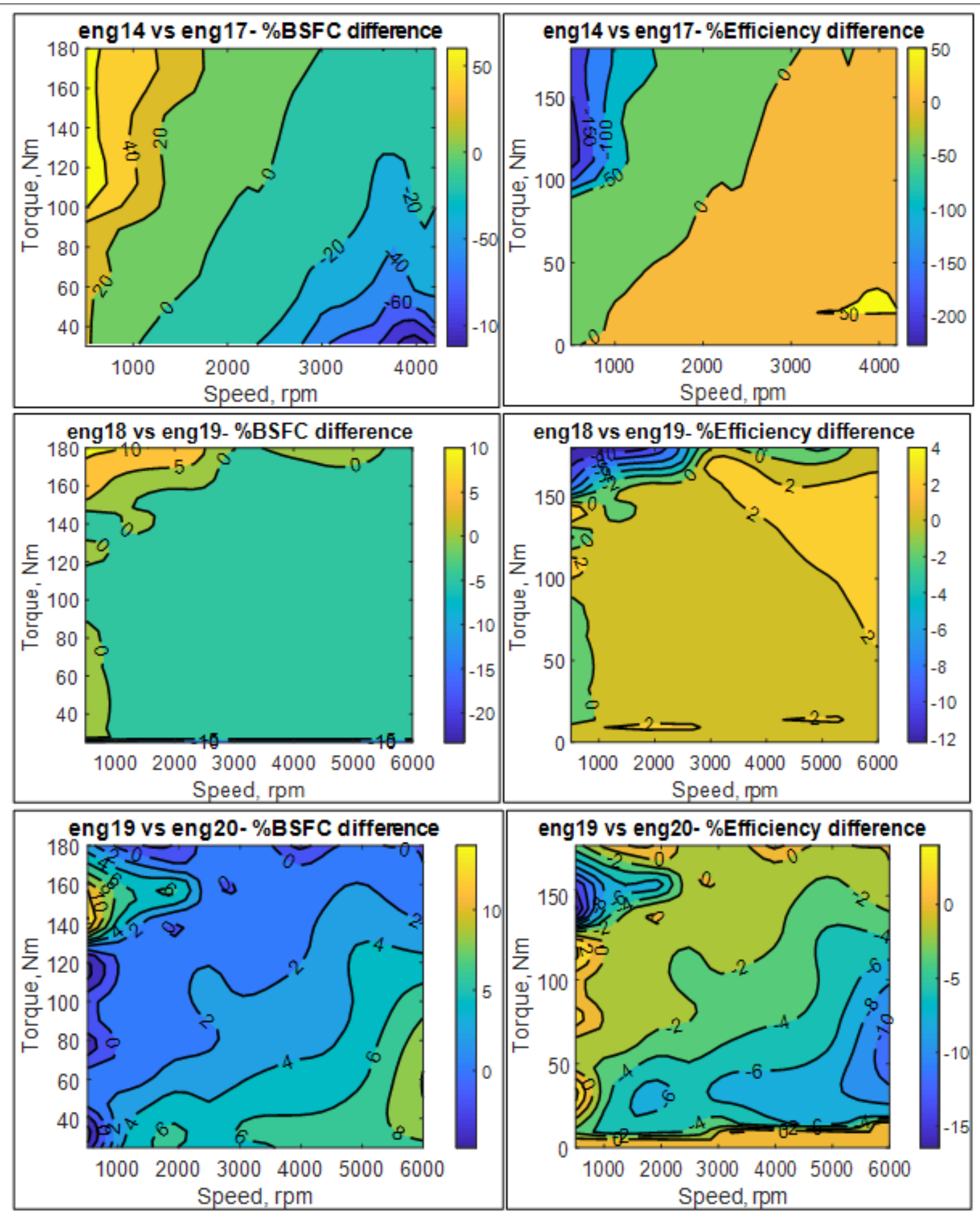


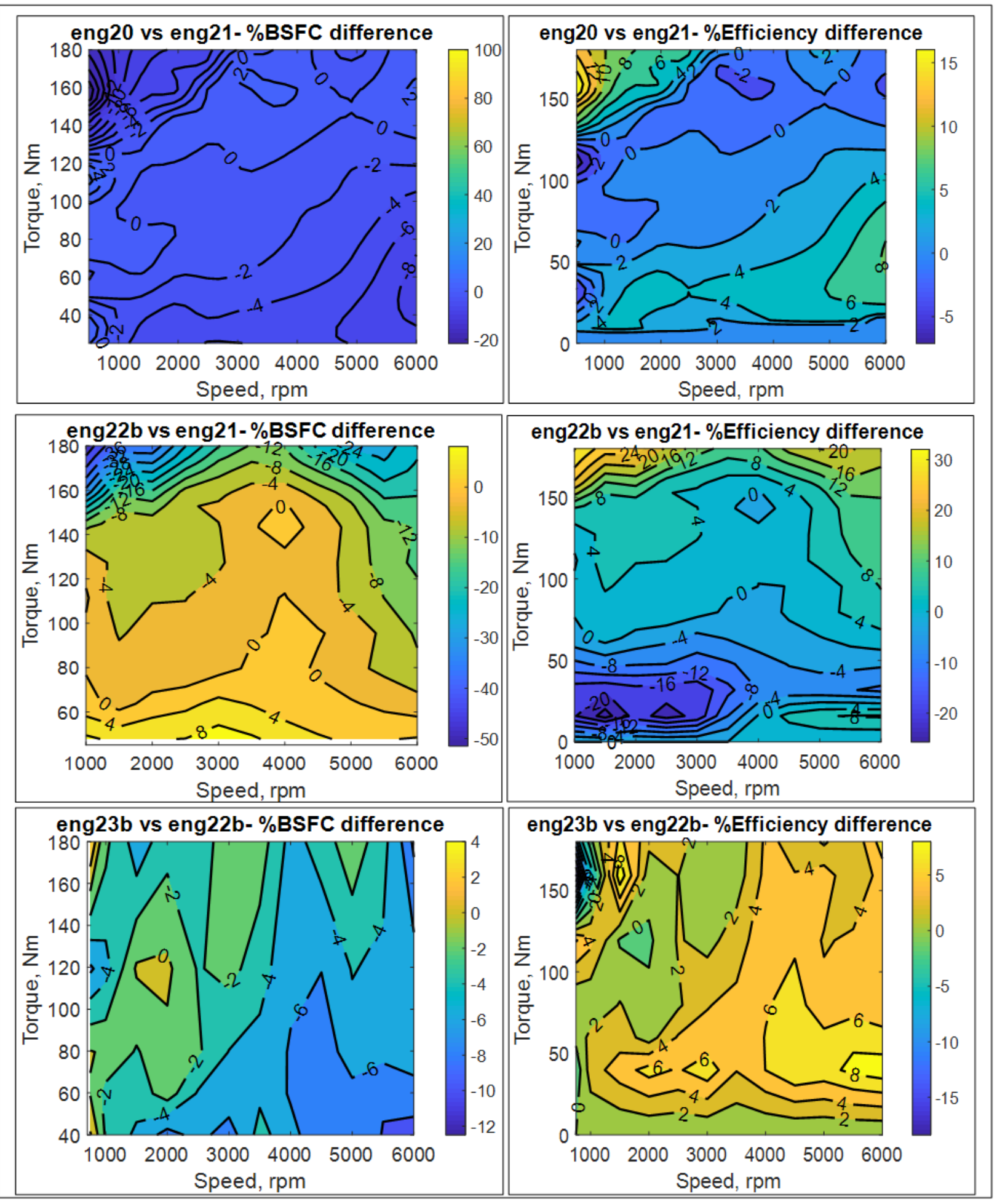

Figure 162. Incremental BSFC and efficiency differences among engines 


\subsubsection{Engine Efficiency Map Comparison With and Without DEAC Capability}

Figure 163 shows the engine efficiency difference between engine 1 and engine 19 (with DEAC capability added to engine 1).

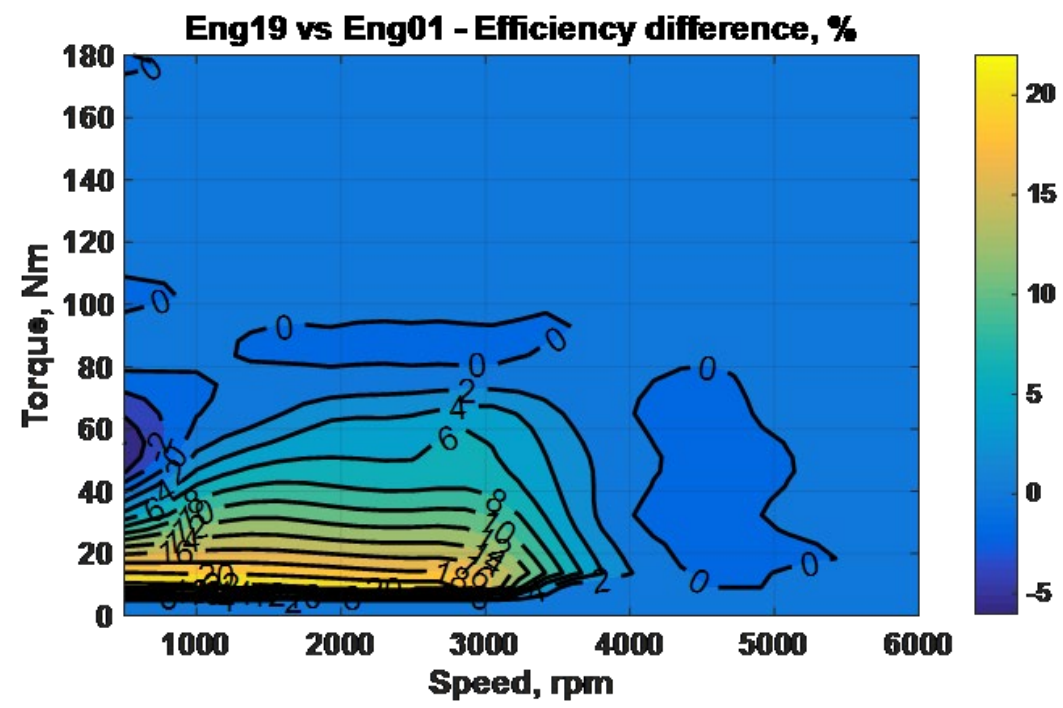

Figure 163. Engine efficiency difference between engine 19 and engine 1

Figure 164 shows the engine efficiency difference between engine 2 and engine 20 (with DEAC capability added to engine 2).

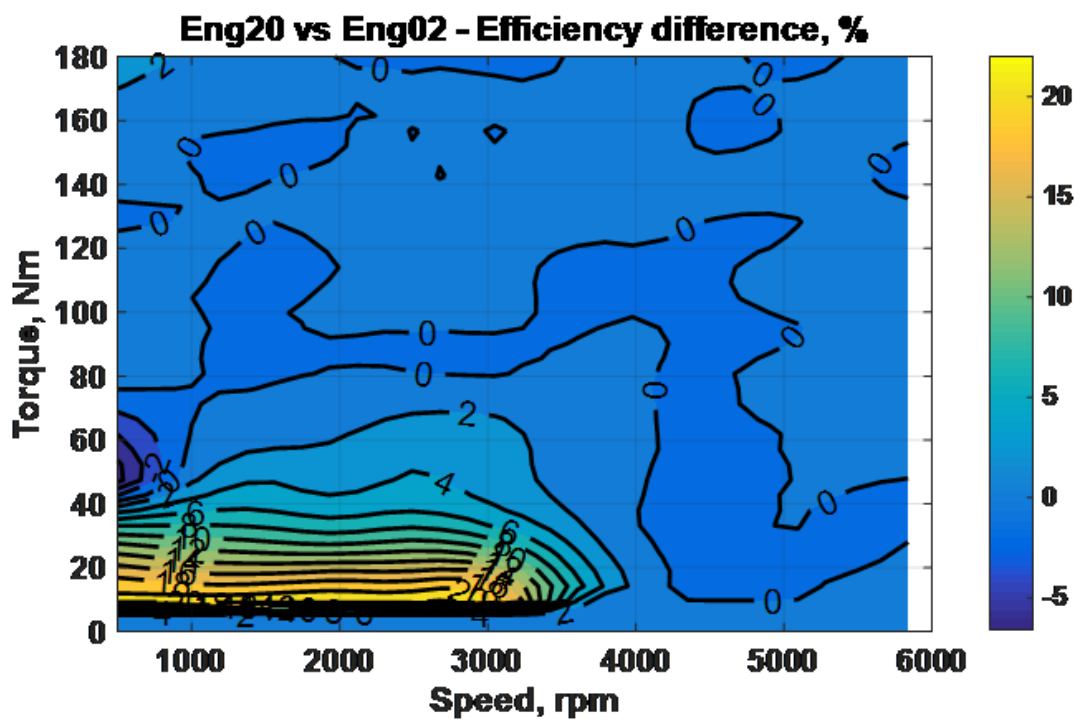

Figure 164. Engine efficiency difference between engine 20 and engine 2

Figure 165 shows the engine efficiency difference between engine 18 and engine 21 (with DEAC capability added to engine 18). 


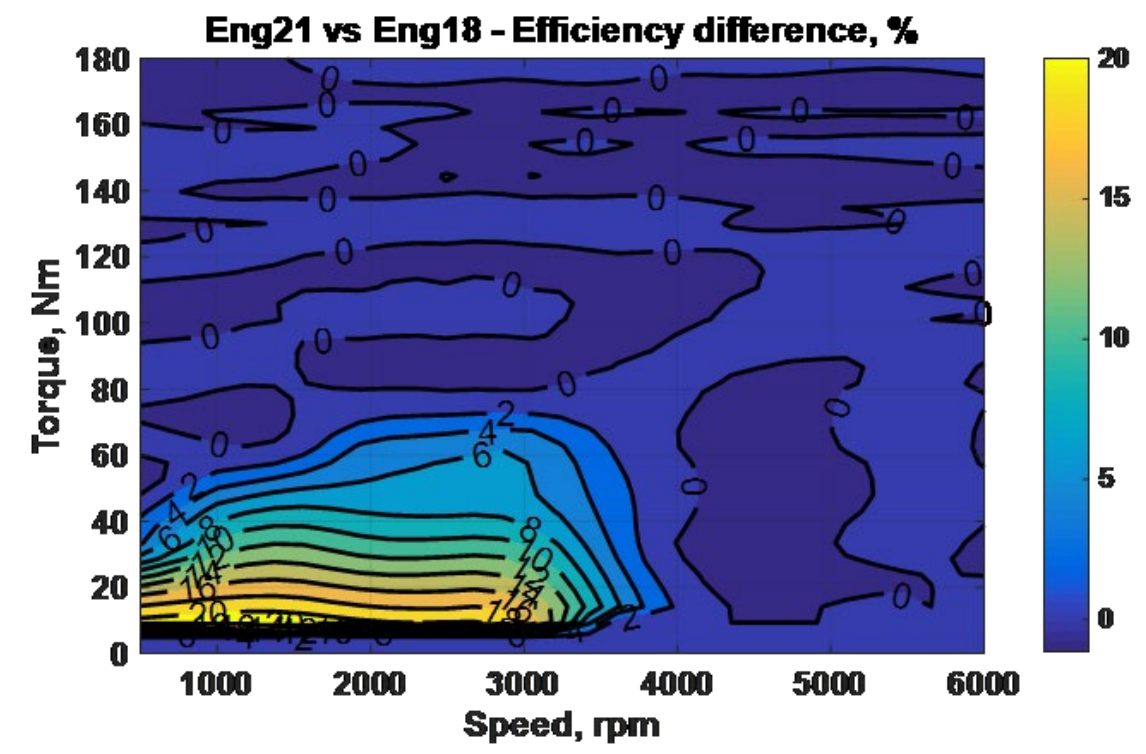

Figure 165. Engine efficiency difference between engine 21 and engine 18

\subsubsection{Idle Fuel Rate Update}

For the current set of runs, the specific values for the idle fuel flow rates have been provided by IAV. The specific values for the idle fuel flow rates are summarized in Table 25.

Table 25. IAV Idle Fuel Rate for All Engines

\begin{tabular}{|l|l|c|}
\hline \multicolumn{1}{|c|}{$\begin{array}{c}\text { Engine } \\
\text { List }\end{array}$} & \multicolumn{1}{|c|}{ Comments } & $\begin{array}{c}\text { Fuel Flow (kg/hr) } \\
\text { 650-700rpm } \\
\text { Warm Idle }\end{array}$ \\
\hline Eng1 & Gasoline, 2.0 I, 4 cyl, NA, PFI, DOHC, VVT & 0.364 \\
\hline Eng2 & VVL added to Eng1 & 0.355 \\
\hline Eng3 & DI added to Eng2 & 0.350 \\
\hline Eng4 & Cylinder deactivation ability added to Eng3 & $0.270 / 0.380$ \\
\hline Eng5b & Eng5a with valvetrain friction reduction (small friction reduction) & 0.339 \\
\hline Eng6a & Eng2 with valvetrain friction reduction (small friction reduction) & 0.315 \\
\hline Eng7a & Eng3 with valvetrain friction reduction (small friction reduction) & 0.308 \\
\hline Eng8a & Eng4 with valvetrain friction reduction (small friction reduction) & 0.216 \\
\hline Eng12 & Gasoline, 1.6 I, 4 cyl, turbocharged, DI, DOHC, VVT, VVL & 0.230 \\
\hline Eng12Deac & Cylinder deactivation ability added to Eng12 & 0.230 \\
\hline Eng13 & Eng12 downsized to 1.2 I & 0.200 \\
\hline Eng14 & Cooled external EGR added to Eng13 & 0.200 \\
\hline Eng18 & Gasoline, 2.0 I, 4 cyl, NA, DI, DOHC, VVT & 0.380 \\
\hline Eng19 & Cylinder deactivation ability added to Eng1 & $0.270 / 0.380$ \\
\hline Eng20 & Cylinder deactivation ability added to Eng2 & $0.270 / 0.380$ \\
\hline Eng21 & Cylinder deactivation ability added to Eng18 & $0.270 / 0.380$ \\
\hline Eng22b & 2.5liter Atkinson naturally aspirated & 0.364 \\
\hline
\end{tabular}




\begin{tabular}{|l|l|c|}
\hline $\begin{array}{c}\text { Engine } \\
\text { List }\end{array}$ & \multicolumn{1}{|c|}{ Comments } & $\begin{array}{c}\text { Fuel Flow (kg/hr) } \\
650-700 \mathrm{rpm} \\
\text { Warm Idle }\end{array}$ \\
\hline Eng23b & 2.0liter VVL Miller + VTG + EGR & 0.229 \\
\hline Eng23c & 2.0liter VVT Miller echarge + EGR & 0.229 \\
\hline Eng24 & Current SkyActiv 2.0L 93AKI & 0.3405 \\
\hline Eng25 & Future SkyActiv 2.0L CEGR 93AKI + DEAC & 0.4075 \\
\hline Eng26a & 2.0liter variable compression ratio + EGR & 0.229 \\
\hline
\end{tabular}

\subsection{Electric Machines}

The electric machine in belt-integrated starter generator (BISG) hybrid vehicles captures regenerative braking energy and provides minimal assist to the engine during high-transient operating modes. Because the electric machine is linked to the engine through a belt, its power is usually limited. A value of $10 \mathrm{~kW}$ peak power was assigned to the BISG electric machine for this study. The micro 12-V hybrid vehicles use a $5 \mathrm{~kW}$ peak power electric machine with no regenerative braking capabilities.

The maps below were developed assuming normal-temperature operating conditions. Electric machine inverter losses are included. The electric machine's power, like the engine's, is sized for the reference-sized powertrains. Table 26 details the electric machine efficiency map sources for the different powertrain configurations.

Table 26. Electric Machine Efficiency Map Sources for Different Powertrain Configurations

\begin{tabular}{|l|l|l|}
\hline \multicolumn{1}{|c|}{ Powertrain Type } & $\begin{array}{c}\text { Source of Efficiency Map for } \\
\text { Motor1 (Traction Motor) } \\
\text { Inverter }\end{array}$ & $\begin{array}{c}\text { Source of Efficiency Map for } \\
\text { Motor2 (Motor/Generator) + } \\
\text { Inverter }\end{array}$ \\
\hline Micro 12-V HEV, BISG & Camry EM1 data [6] & \\
\hline Parallel HEV & Sonata HEV data [23] & Camry EM2 data [6] \\
\hline $\begin{array}{l}\text { Split HEV and Blended } \\
\text { PHEV }\end{array}$ & Camry EM1 data [6] & Sonata HEV data [23] \\
\hline EREV PHEV & Camry EM1 data [6] & \\
\hline Fuel Cell HEV \& BEV & Chevrolet Bolt EM data [21] & \\
\hline
\end{tabular}

\subsubsection{Electric Machine Efficiency Maps}

Figure 166 shows the electric machine efficiency maps for different powertrains. 

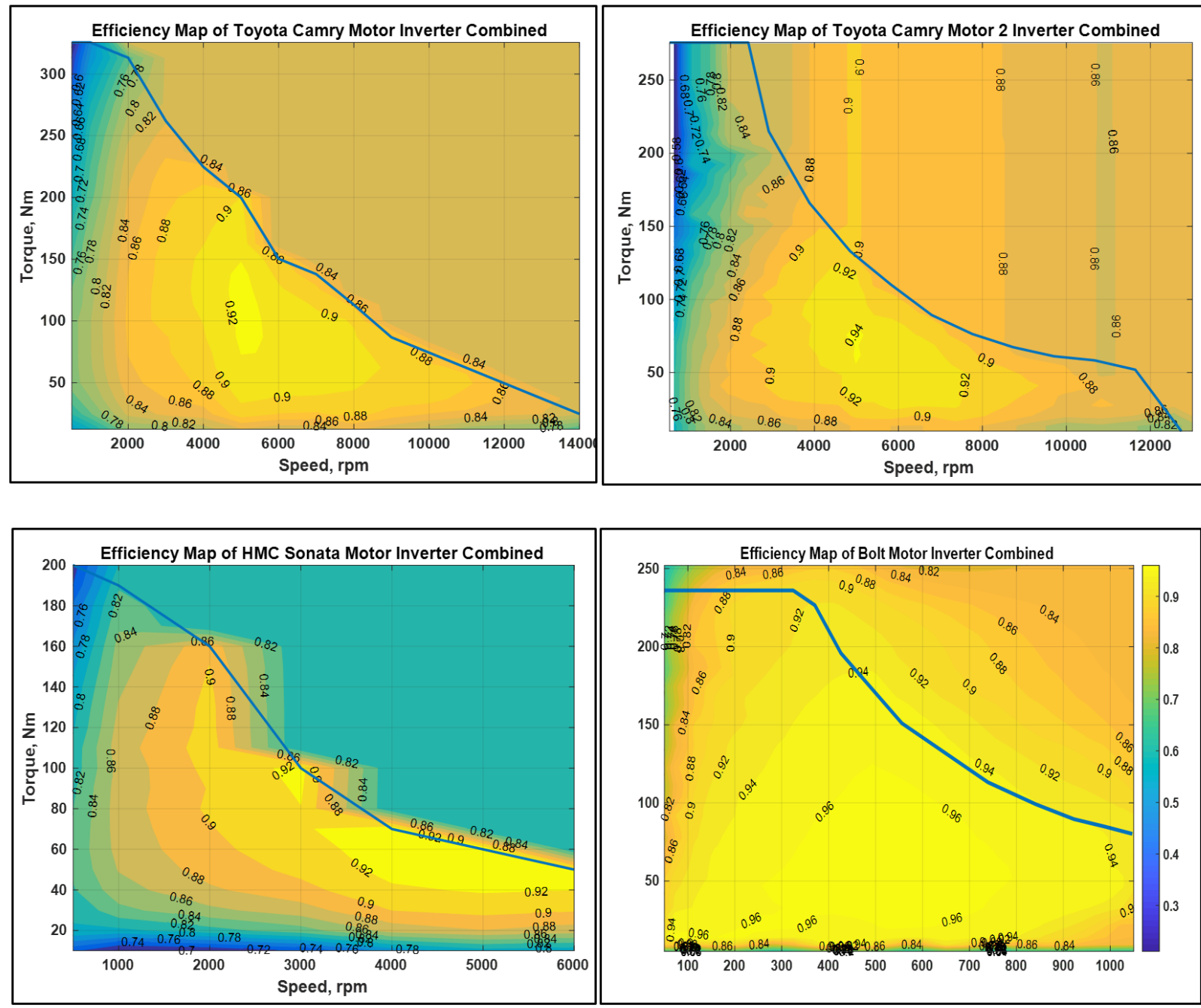

Figure 166. Electric machines efficiency maps for different powertrains

\subsubsection{Electric Machine Peak Efficiency Scaling}

For current set of runs, the peak efficiency of electric machines for different powertrains are scaled as shown in Table 1.

Table 27. Efficiency Scaling of Electric Machines

\begin{tabular}{|l|c|}
\hline \multicolumn{1}{|c|}{ Vehicle Powertrain } & Peak Efficiency Scaled (\%) \\
\hline $\begin{array}{l}\text { Micro HEV, Mild Hybrid BISG, Split } \\
\text { HEV, Par HEV }\end{array}$ & 96 \\
\hline $\begin{array}{l}\text { Split PHEV20, EREV PHEV50, Par } \\
\text { PHEV20, Par PHEV50 }\end{array}$ & 96 \\
\hline BEV and FCEV & 98 \\
\hline
\end{tabular}

A constant ratio was assumed between the continuous and peak torque curves, as follows: 
- 2 for micro-HEV, BISG, and CISG

- 2 for motor 1 and 1.5 for the motor 2 of the power-split HEV, blended PHEV and Voltec PHEV

- 1 for BEVs and fuel-cell HEV

\subsection{Fuel Cell System}

The fuel cell system was modeled to represent hydrogen consumption as a function of produced power. For the current analysis, Argonne implemented the latest values from DOE Hydrogen and Fuel Cells Program Record \#20005 [36]. According to that record, the current status of fuel cell system power density is $860 \mathrm{~W} / \mathrm{kg}$ with a peak system efficiency of $64 \%$. For the hydrogen storage weight, Argonne implemented a value of $0.044 \mathrm{~kg}$ per $\mathrm{kg}$ usable $\mathrm{H} 2$ fuel mass, based on the latest assumptions provided by HFTO [12].

The hydrogen storage technology selected is a high-pressure tank with, as noted, a specific weight of $0.044 \mathrm{~kg} \mathrm{H} 2 / \mathrm{kg}$, sized to provide a 360 -mile range on the combined cycle (UDDS + HWFET) based on adjusted energy values.

Figure 167 shows fuel cell efficiency vs. fuel cell power. The fuel cell efficiency curve was derived from 2017 Toyota Mirai vehicle benchmarking data in a technology assessment report [18].

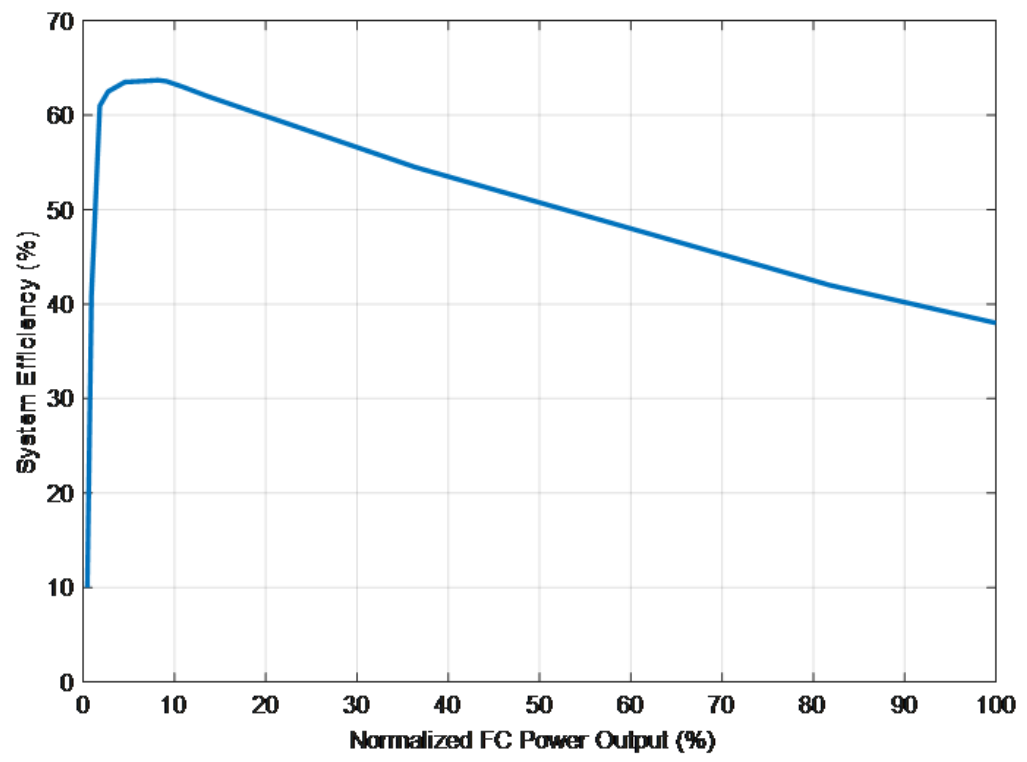

Figure 167. Fuel cell efficiency vs. power

\subsection{Energy Storage System}

Lithium-ion batteries are used for all hybrid powertrains. Different useable SOC ranges have been selected based on the powertrain configuration:

- $10 \%$ SOC range for micro and mild HEVs

- $20 \%$ SOC range for full HEVs

- $70 \%$ SOC range for PHEVs

- $90 \% \mathrm{SOC}$ range for BEVs 
Vehicle test data have shown that for the U.S. standard drive cycles and test conditions considered, battery cooling does not draw a significant amount of energy for most vehicle powertrain architectures [27]. The exception is high-energy PHEVs [17] and BEVs [14], for which an additional constant power draw is used for battery cooling.

For the current set of runs, the mass, capacities, and the voltages of the batteries across different vehicle classes and powertrains are computed using the $\mathrm{BatPaC}$ model described in Section 5.9.

\subsection{Battery Performance and Cost Model (BatPaC)}

$\mathrm{BatPaC}$, a lithium-ion battery performance and cost model for electric-drive vehicles, was developed by the Chemical Sciences and Engineering division at Argonne [3].

To accelerate the large-scale simulation process, a lookup table replaces the traditional BatPaC model initially developed in Microsoft Excel. ${ }^{\circledR}$ The lookup table is dependent on powertrain options and consists of two dimensions - pack power, and pack energy. The battery pack designs including number of cells, modules, cell chemistry and heat transfer fluid are fixed for each powertrain. Additional lookup tables for other $\mathrm{BatPaC}$ parameters (e.g., positive electrode thickness, negative electrode thickness, battery open circuit voltage, and battery pack volume) are also generated as constraints.

\subsubsection{Process}

The $\mathrm{BatPaC}$ model spreadsheet comes with a set of default specifications comparing seven different batteries for HEVs, PHEVs and BEVs (Table 28). For the current set of runs, BatPaC v4.0 - October 01, 2020 was used.

Table 28. BatPaC Input Details for BatPaC 4.0 October 01, 2020

\begin{tabular}{|l|l|c|}
\hline Key Inputs & Tab/Sheet & Row No. \\
\hline Vehicle type (micro HEV, HEV-HP, PHEV, EV) & Dashboard & E28 \\
\hline Battery energy storage, kWh & Dashboard & $42-44$ \\
\hline Pulse power requirement, kW & Dashboard & 33 \\
\hline Production volume, packs per year & Dashboard & 39 \\
\hline Cathode/anode combination & Dashboard & D9 \\
\hline
\end{tabular}

\subsubsection{Cathode/Anode Combination Selection}

A specific electrode combination is selected from the drop down menu on Dashboard E9 in the Chem Tab. The default values used in the calculations associated with the selected electrode are listed in Column D and $\mathrm{H}$. The user can override the default material property or price by entering new values in Column E and I. Figure 168 shows the BatPaC model input for cathode/anode selection.

\begin{tabular}{|c|c|c|}
\hline Chemistry & $\begin{array}{c}\text { Current } \\
\text { Selection }\end{array}$ & Choose \\
\hline Electrode Pair & NMC622-G & NMC622-G \\
\hline
\end{tabular}

Figure 168. BatPaC cathode/anode pair selection 


\subsubsection{2. $\quad$ Vehicle Pack Specifications Selection}

Vehicle and pack specifications are provided in the Dashboard tab, as shown in Figure 169.

\begin{tabular}{|c|c|c|c|c|c|c|c|}
\hline ery Design & $\begin{array}{c}\text { Current } \\
\text { Selection }\end{array}$ & Choose & & & & Ovenride & \\
\hline Vehicle Type & EV & EV & & \multirow{2}{*}{$\begin{array}{l}\text { Useable bustery } \\
\text { energy, \% of totat } \\
\text { (EV) }\end{array}$} & 85 & & \multirow{3}{*}{$\begin{array}{r}\text { Add Defa } \\
\text { Cells/Module } \\
\text { syste }\end{array}$} \\
\hline Coolant Type & EG-W & EG-W & & & & & \\
\hline \multirow[t]{2}{*}{ Calculate Fast Charging? } & No & No & & & & & \\
\hline & Battery 1 & Battery 2 & Battery 3 & Battery 4 & Battery 5 & Battery 6 & Battery 7 \\
\hline Target battery pack power at $20 \%$ SOC, $\mathbf{k W}$ & 180 & 220 & 260 & 300 & 120 & 400 & 360 \\
\hline Number of cells per module (total) & 12 & 12 & 12 & 12 & 14 & 12 & 18 \\
\hline Number of cells in parallel group in module & 1 & 1 & 1 & 1 & 1 & 1 & 2 \\
\hline Number of modules in row & 5 & 5 & 5 & 5 & 5 & 5 & 6 \\
\hline Number of rows of modules per pack & 4 & 4 & 4 & 4 & 2 & 4 & 4 \\
\hline Number of modules in parallel & 1 & 1 & 1 & 1 & 1 & 1 & 1 \\
\hline Number of packs manufactured per year & 100,000 & 100,000 & 100,000 & 100,000 & 100,000 & 100,000 & 100,000 \\
\hline Energy requirement for a UDDS cycle, Wh/mile & 250 & 250 & 250 & 250 & 250 & 250 & 350 \\
\hline \multicolumn{8}{|c|}{ Select capacity, energy, or vehicle range, but only one. } \\
\hline \multicolumn{8}{|l|}{ Pack capacity (Ah) } \\
\hline \multicolumn{8}{|l|}{ Pack energy (kWh) } \\
\hline Vehicle range (miles) & 180 & 220 & 260 & 300 & 100 & 270 & 300 \\
\hline
\end{tabular}

Figure 169. BatPaC Battery Design tab

The cell and module configurations can be defined for different battery packs. These parameters affect the cell dimensions and weight, the module, and the overall pack, including its voltage.

Fast-charging requirements could drive the battery cost by limiting the electrode thickness. Figure 170 shows the $\mathrm{BatPaC}$ Fast Charging tab. The additional cost of a fast charging constraint is displayed in Row 55. For this analysis run, the fast charge constraint was disabled by setting cell F10 to 0 .

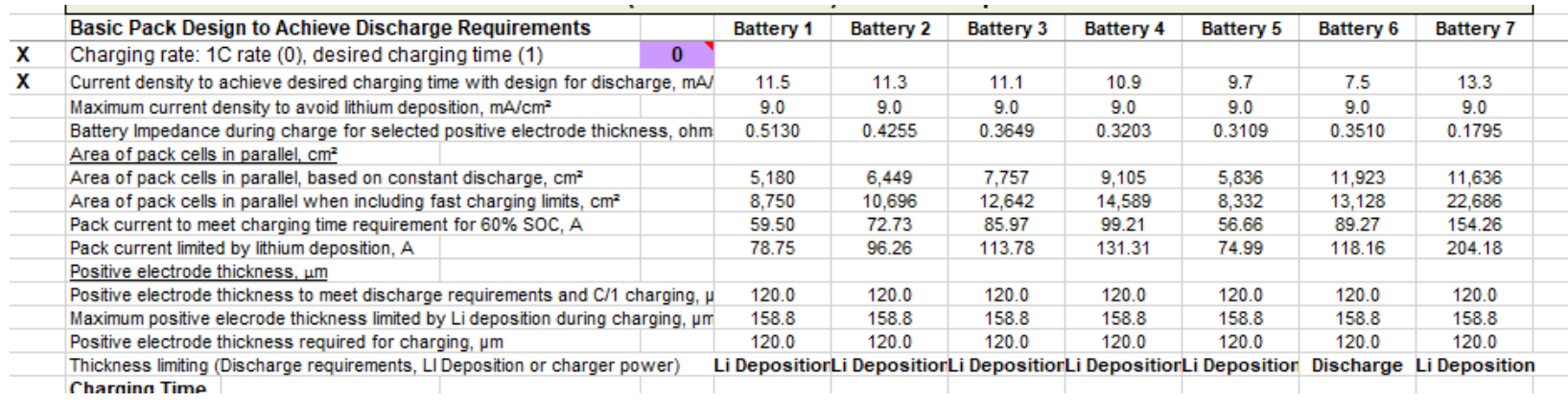

Figure 170. BatPaC Fast Charging tab

$\mathrm{BatPaC}$ provides a range of performance metrics and cost parameters for the different battery packs designed, some of which are summarized in Table 29.

Table 29. BatPaC Output Performance Metric

Performance Metrics

Cell, Module, and Pack Level

Dimensions

Mass

Volume

\section{Cost Parameters}

Cell, Module, and Pack

Cost of production

Cost breakdown 


\begin{tabular}{|l|l|}
\hline Voltage & \\
Wh/kg, Wh/liter & \\
Material inventory & \\
\hline
\end{tabular}

\subsubsection{BatPaC Examples from Existing Vehicles in the Market}

In order to validate $\mathrm{BatPaC}$ predictions, battery packs from current production vehicles across multiple vehicle classes and powertrains were benchmarked. The A2MAC1 data was then compared with the predicted battery pack designs from BatPaC, using the default assumptions (except the pack configuration).

\subsubsection{Full HEV}

The A2MAC1 battery cell teardown analysis reports for the following vehicles were used to evaluate the assumptions for full HEV:

- 2014-2018 Infiniti QX60

- 2016-2019 Prius Gen4

- 2016-2018 Toyota Rav4 Hybrid

- 2019 Toyota Camry Hybrid

- 2013-2016 Volkswagen (VW) Jetta Hybrid

- 2011-2019 Toyota Highlander Hybrid

- 2016-2019 Acura RLX Hybrid

The battery pack characteristics from HEV production vehicles [1] and $\mathrm{BatPaC}$ are shown in Table 30 and Table 31 , respectively.

Table 30. HEV Battery Pack Details

\begin{tabular}{|l|c|c|c|c|c|c|c|}
\hline Parameter & $\begin{array}{c}\text { 2014-2018 } \\
\text { Infiniti } \\
\text { QX60 }\end{array}$ & $\begin{array}{c}\text { 2016-2019 } \\
\text { Prius } \\
\text { Gen4 }\end{array}$ & $\begin{array}{c}\mathbf{2 0 1 6 - 2 0 1 8} \\
\text { Toyota } \\
\text { Rav4 } \\
\text { Hybrid }\end{array}$ & $\begin{array}{c}\mathbf{2 0 1 9} \\
\text { Toyota } \\
\text { Camry } \\
\text { Hybrid }\end{array}$ & $\begin{array}{c}\text { 2013-2016 } \\
\text { VW Jetta } \\
\text { Hybrid }\end{array}$ & $\begin{array}{c}\text { 2011-2019 } \\
\text { Toyota } \\
\text { Highlander } \\
\text { Hybrid }\end{array}$ & $\begin{array}{c}2014 \\
\text { Acura } \\
\text { RLX } \\
\text { Hybrid }\end{array}$ \\
\hline Power (kW) & 17 & 37.8 & 45.9 & 45.9 & 20 & 54 & 59 \\
\hline Energy (kWh) & 0.63 & 0.75 & 1.59 & 1.59 & 1.1 & 1.87 & 1.3 \\
\hline Cells/module & 40 & 28 & 34 & 34 & 15 & 30 & 12 \\
\hline Modules/pack & 1 & 2 & 1 & 1 & 4 & 1 & 6 \\
\hline $\begin{array}{l}\text { Number of } \\
\text { modules in } \\
\text { parallel }\end{array}$ & 1 & 1 & 1 & 1 & 1 & 1 & 1 \\
\hline $\begin{array}{l}\text { Total number } \\
\text { of cells }\end{array}$ & 40 & 56 & 34 & 34 & 60 & 30 & 72 \\
\hline Cathode type & NMC333-G & NMC333-G & NMC333-G & NMC333-G & NMC333-G & NMC333-G & NMC333-G \\
\hline $\begin{array}{l}\text { Cooling } \\
\text { medium }\end{array}$ & Air & Air & Air & Air & Air & Air & Air \\
\hline Cell mass (g) & 260 & 201 & - & 216 & 253 & - & - \\
\hline $\begin{array}{l}\text { Specific } \\
\text { energy }\end{array}$ & 22.4 & 32 & 34.2 & 34.2 & 20.8 & 27.5 & 33.2 \\
\hline
\end{tabular}




\begin{tabular}{|l|c|c|c|c|c|c|c|}
\hline \multicolumn{1}{|c|}{ Parameter } & $\begin{array}{c}\text { 2014-2018 } \\
\text { Infiniti } \\
\text { QX60 }\end{array}$ & $\begin{array}{c}\text { 2016-2019 } \\
\text { Prius } \\
\text { Gen4 }\end{array}$ & $\begin{array}{c}\text { 2016-2018 } \\
\text { Toyota } \\
\text { Rav4 } \\
\text { Hybrid }\end{array}$ & $\begin{array}{c}\text { 2019 } \\
\text { Toyota } \\
\text { Camry } \\
\text { Hybrid }\end{array}$ & $\begin{array}{c}\text { 2013-2016 } \\
\text { VW Jetta } \\
\text { Hybrid }\end{array}$ & $\begin{array}{c}\text { 2011-2019 } \\
\text { Toyota } \\
\text { Highlander } \\
\text { Hybrid }\end{array}$ & $\begin{array}{c}2014 \\
\text { Acura } \\
\text { RLX } \\
\text { Hybrid }\end{array}$ \\
\hline $\begin{array}{l}(\mathrm{Wh} / \mathrm{kg}) \text { at } \\
\text { pack }\end{array}$ & 28.3 & 33.44 & 46.5 & 46.5 & 53 & 68 & 39 \\
\hline $\begin{array}{l}\text { Battery pack } \\
\text { mass (kg) }\end{array}$ & 4.4 & 3.62 & - & 4.32 & 5 & - & - \\
\hline $\begin{array}{l}\text { Battery cell } \\
\text { capacity (Ah) }\end{array}$ & 144 & 204.4 & 244.8 & 244.8 & 220 & 288 & 260 \\
\hline $\begin{array}{l}\text { Battery pack } \\
\text { nominal } \\
\text { voltage (V) }\end{array}$ & & & & & & & \\
\hline
\end{tabular}

Table 31. BatPaC HEV Prediction

\begin{tabular}{|c|c|c|c|c|c|c|c|}
\hline Parameter & $\begin{array}{c}2014-2018 \\
\text { Infiniti } \\
\text { QX60 }\end{array}$ & $\begin{array}{c}\text { 2016-2019 } \\
\text { Prius } \\
\text { Gen4 }\end{array}$ & $\begin{array}{c}2016-2018 \\
\text { Toyota } \\
\text { Rav4 } \\
\text { Hybrid }\end{array}$ & $\begin{array}{c}2019 \\
\text { Toyota } \\
\text { Camry } \\
\text { Hybrid }\end{array}$ & $\begin{array}{c}2013-2016 \\
\text { VW Jetta } \\
\text { Hybrid }\end{array}$ & $\begin{array}{l}2011-2019 \\
\text { Toyota } \\
\text { Highlande } \\
\text { r Hybrid }\end{array}$ & $\begin{array}{c}2016-2019 \\
\text { Acura RLX } \\
\text { Hybrid }\end{array}$ \\
\hline Cell mass (g) & 144 & 176 & 416 & 416 & 144 & 544 & 224 \\
\hline $\begin{array}{l}\text { Specific energy } \\
(\mathrm{Wh} / \mathrm{kg})\end{array}$ & 28.13 & 31.875 & 48.125 & 48.125 & 37.5 & 52.5 & 31.875 \\
\hline $\begin{array}{l}\text { Battery pack } \\
\text { mfr. cost }(\$)\end{array}$ & 1492 & 1839 & 1669 & 1669 & 1863 & 1674 & 2233 \\
\hline $\begin{array}{l}\text { Total cost of } \\
\text { packs (\$/kwh) }\end{array}$ & 2368.25 & 2452 & 1049.7 & 1049.7 & 1693.64 & 895.2 & 1717.7 \\
\hline $\begin{array}{l}\text { Battery pack } \\
\text { mass }(\mathrm{kg})\end{array}$ & 22.4 & 28.8 & 33.6 & 33.6 & 28.8 & 35.2 & 41.6 \\
\hline $\begin{array}{l}\text { Battery pack } \\
\text { volume }\end{array}$ & 13 & 16 & 15 & 15 & 18 & 16 & 22 \\
\hline $\begin{array}{l}\text { Battery cell } \\
\text { capacity (Ah) }\end{array}$ & 4 & 4 & 13 & 13 & 5 & 17 & 5 \\
\hline $\begin{array}{l}\text { Battery pack } \\
\text { nominal } \\
\text { voltage }(v)\end{array}$ & 147 & 206 & 125 & 125 & 220 & 110 & 264 \\
\hline
\end{tabular}

\subsubsection{2. $\quad P H E V S$}

The battery cell teardown analysis reports from A2MAC1 and Total Battery Consulting (TBC) [29] were used to evaluate the assumptions for plug-in hybrids:

- 2016-2018 BMW X5 xDrive40e

- 2019 BMW i8

- 2016-2017 Mercedes GLE550e

- 2017-2018 Fusion Energi

- 2012-2015 Toyota Prius Plug-In 
- 2016-2018 Audi A3 e-tron

The battery pack characteristics from PHEV production vehicles [1] and BatPaC are shown in Table 32 and Table 35, respectively.

Table 32. PHEVs Battery Pack Details

\begin{tabular}{|c|c|c|c|c|c|c|}
\hline Parameter & $\begin{array}{c}2016-2018 \\
\text { BMW X5 } \\
\text { XDrive40e }\end{array}$ & $\begin{array}{l}2019 \text { BMW } \\
\text { i8 }\end{array}$ & $\begin{array}{c}\text { 2016- } \\
2017 \\
\text { Mercedes } \\
\text { GLE550e }\end{array}$ & $\begin{array}{c}2017-2018 \\
\text { Fusion } \\
\text { Energi }\end{array}$ & $\begin{array}{c}2012-2015 \\
\text { Toyota } \\
\text { Prius } \\
\text { Plug-In }\end{array}$ & $\begin{array}{c}\text { 2016-2018 } \\
\text { Audi A3 } \\
\text { e-tron }\end{array}$ \\
\hline Power (kw) & 83 & 105 & 90 & 68 & 60 & 75 \\
\hline Energy (kWh) & 9.2 & 11.6 & 8.7 & 7.6 & 4.4 & 8.7 \\
\hline Cell/ module & 16 & 96 & 20 & 21 & 14 & 96 \\
\hline Modules/pack & 6 & 1 & 6 & 4 & 4 & 1 \\
\hline $\begin{array}{l}\text { Number of modules in } \\
\text { parallel }\end{array}$ & 1 & 1 & 1 & 1 & 1 & 1 \\
\hline Total number of cells & 96 & 96 & 120 & 84 & 56 & 96 \\
\hline Cathode type & NMC333-G & NMC333-G & LFP-G & NMC333-G & NMC333-G & NMC333-G \\
\hline Cooling medium & EG-W & EG-W & EG-W & CoolA & CoolA & Liquid \\
\hline Cell mass $(\mathrm{g})$ & 703 & 824 & 631 & 690 & 720 & 690 \\
\hline $\begin{array}{l}\text { Specific energy } \\
(\mathrm{Wh} / \mathrm{kg}) \text { at pack }\end{array}$ & 85.7 & 102.6 & 76.3 & 65 & 58 & 74.4 \\
\hline Battery pack mass $(\mathrm{kg})$ & 105 & 113 & 133.67 & 123 & 76 & 117 \\
\hline $\begin{array}{l}\text { Battery cell capacity } \\
\text { (Ah) }\end{array}$ & 26 & 34 & 22 & 25 & 21 & 25 \\
\hline $\begin{array}{l}\text { Battery pack nominal } \\
\text { voltage }(v)\end{array}$ & 355 & 355 & 396 & 309.1 & 206 & 360 \\
\hline
\end{tabular}

Table 33. BatPaC PHEV Predictions

\begin{tabular}{|l|c|c|c|c|c|c|}
\hline Parameter & $\begin{array}{c}\mathbf{2 0 1 6 - 2 0 1 8} \\
\text { BMW X5 } \\
\text { xDrive40e }\end{array}$ & $\mathbf{2 0 1 9}$ BMW i8 & $\begin{array}{c}\text { 2016-2017 } \\
\text { Mercedes } \\
\text { GLE550e }\end{array}$ & $\begin{array}{c}\text { 2017-2018 } \\
\text { Fusion } \\
\text { Energi }\end{array}$ & $\begin{array}{c}\text { 2012-2015 } \\
\text { Toyota Prius } \\
\text { Plug-In }\end{array}$ & $\begin{array}{c}\text { 2016- } \\
\text { 2018 Audi }\end{array}$ \\
\hline Cell mass (g) & 528 & 660 & 528 & 492 & 444 & 492 \\
\hline $\begin{array}{l}\text { Specific energy at } \\
\text { pack (Wh/kg) }\end{array}$ & 97.5 & 101.67 & 73.33 & 104.17 & 90.83 & 102.5 \\
\hline $\begin{array}{l}\text { Battery pack mfr. cost } \\
(\$)\end{array}$ & 3842 & 4122 & 4331 & 3328 & 2488 & 3663 \\
\hline $\begin{array}{l}\text { Total cost of packs } \\
(\$ / \mathrm{kwh})\end{array}$ & 417.61 & 355.34 & 497.82 & 437.9 & 565.45 & 421.03 \\
\hline $\begin{array}{l}\text { Battery pack mass } \\
\text { (kg) }\end{array}$ & 94.8 & 114 & 118.8 & 73.2 & 48 & 85.2 \\
\hline $\begin{array}{l}\text { Battery pack volume } \\
(\mathrm{I})\end{array}$ & 42 & 50 & 54 & 41 & 30 & 42 \\
\hline
\end{tabular}




\begin{tabular}{|l|c|c|c|c|c|c|}
\hline Parameter & $\begin{array}{c}\text { 2016-2018 } \\
\text { BMW X5 } \\
\text { XDrive40e }\end{array}$ & 2019 BMW i8 & $\begin{array}{c}\text { 2016-2017 } \\
\text { Mercedes } \\
\text { GLE550e }\end{array}$ & $\begin{array}{c}\text { 2017-2018 } \\
\text { Fusion } \\
\text { Energi }\end{array}$ & $\begin{array}{c}\text { 2012-2015 } \\
\text { Toyota Prius } \\
\text { Plug-In }\end{array}$ & $\begin{array}{c}\text { 2016- } \\
\text { 2018 Audi } \\
\text { A3 e-tron }\end{array}$ \\
\hline $\begin{array}{l}\text { Battery cell capacity } \\
\text { (Ah) }\end{array}$ & 26 & 33 & 22 & 25 & 21 & 25 \\
\hline $\begin{array}{l}\text { Battery pack nominal } \\
\text { voltage }(\mathrm{v})\end{array}$ & 352 & 352 & 394 & 308 & 206 & 352 \\
\hline
\end{tabular}

\subsubsection{3. $\quad E V S$}

The battery cell teardown analysis from A2MAC1 and TBS for the following vehicles were used to evaluate the assumptions used for electric vehicles.

- 2019 BMW i3

- 2017-2019 Chevrolet Bolt

- 2019 Hyundai Kona Standard Range

- 2020 Nissan Leaf

- 2019 Audi e-Tron

The battery pack characteristics from BEV production vehicles [1] and $\mathrm{BatPaC}$ are shown in Table 34 and Table 35, respectively.

Table 34. Battery Pack Details for EVs

\begin{tabular}{|l|c|c|c|c|c|}
\hline \multicolumn{1}{|c|}{ Parameter } & $\begin{array}{c}\text { 2019 BMW } \\
\mathbf{i 3}\end{array}$ & $\begin{array}{c}\text { 2017-2019 } \\
\text { Chevrolet } \\
\text { Bolt }\end{array}$ & $\begin{array}{c}\text { 2019 Hyundai } \\
\text { Kona } \\
\text { Standard } \\
\text { Range }\end{array}$ & $\begin{array}{c}\text { 2020 Nissan } \\
\text { Leaf }\end{array}$ & $\begin{array}{c}\text { 2019 Audi } \\
\text { e-Tron }\end{array}$ \\
\hline Power (kW) & 125 & 160 & 100 & 110 & 300 \\
\hline Energy (kWh) & 42.2 & 60 & 39.2 & 40 & 95 \\
\hline Number cells/module & 12 & 32 & 90 & 8 & 12 \\
\hline Number modules/pack & 8 & 9 & 2 & 24 & 36 \\
\hline $\begin{array}{l}\text { Number of modules in } \\
\text { parallel }\end{array}$ & 1 & 3 & 2 & 2 & 4 \\
\hline Total number of cells & 96 & 288 & 180 & 192 & 432 \\
\hline Cell chemistry & NMC622-G & NMC622-G & NMC622-G & NMC532-G & NMC622-G \\
\hline Cooling medium & EG-W & EG-W & EG-W & EG-W & EG-W \\
\hline Cell Mass (g) & 2212 & 832 & 965 & 908 & 877 \\
\hline $\begin{array}{l}\text { Specific energy (Wh/kg) } \\
\text { at pack }\end{array}$ & 160 & 140.63 & 123.7 & 135.6 & 142 \\
\hline Battery pack mass (kg) & 275 & 426.64 & 317 & 295 & 681 \\
\hline Battery cell capacity (Ah) & 120 & 56 & 63 & 56.3 & 61.2 \\
\hline $\begin{array}{l}\text { Battery pack nominal } \\
\text { voltage (V) }\end{array}$ & 360 & 355 & 327 & 350 & 396 \\
\hline
\end{tabular}

Table 35. BatPaC EVs Prediction 


\begin{tabular}{|l|c|c|c|c|c|}
\hline \multicolumn{1}{|c|}{ Parameter } & $\begin{array}{c}\text { 2019 BMW } \\
\text { i3 }\end{array}$ & $\begin{array}{c}\text { 2017-2019 } \\
\text { Chevrolet } \\
\text { Bolt }\end{array}$ & $\begin{array}{c}\text { 2019 Hyundai } \\
\text { Kona } \\
\text { Standard } \\
\text { Range }\end{array}$ & $\begin{array}{c}\text { 2020 Nissan } \\
\text { Leaf }\end{array}$ & $\begin{array}{c}\text { 2019 Audi } \\
\text { e-Tron }\end{array}$ \\
\hline Cell mass (g) & 1730 & 840 & 880 & 910 & 890 \\
\hline $\begin{array}{l}\text { Specific energy at pack } \\
(\text { Wh/kg) }\end{array}$ & 172 & 167 & 138 & 150 & 166 \\
\hline Battery pack mfr. cost (\$) & 8023 & 11949 & 8707 & 9119 & 17583 \\
\hline $\begin{array}{l}\text { Total cost of packs } \\
(\$ / k W h)\end{array}$ & 190.12 & 199.15 & 222.12 & 227.98 & 185.08 \\
\hline Battery pack mass (kg) & 246 & 358 & 284 & 267 & 572 \\
\hline Battery pack volume (L) & 113 & 165 & 122 & 130 & 268 \\
\hline Battery cell capacity (Ah) & 118 & 56 & 58 & 57 & 59 \\
\hline $\begin{array}{l}\text { Battery pack nom. } \\
\text { voltage (V) }\end{array}$ & 360 & 360 & 338 & 351 & 405 \\
\hline
\end{tabular}

\subsubsection{Use of BatPaC in Autonomie}

For the current study, significant changes were made to the BatPaC inputs to Autonomie as different battery pack configurations were selected for different vehicle classes and powertrains, including numbers of the following:

- Cells per module

- Cells in parallel

- Modules in row

- Rows of modules per pack

- Modules per battery pack

- Modules in parallel

BatPaC provides Autonomie with the battery pack weight and cost as well as the cell capacity in ampere hours $(\mathrm{Ah})$ for different battery total energy and power requirements. Table 36 shows the $\mathrm{BatPaC}$ assumptions for micro HEVs across five different vehicle classes.

Table 36. BatPaC Assumptions for Micro HEV

\begin{tabular}{|l|c|c|}
\hline \multicolumn{1}{|c|}{ Vehicle Class } & Compact, Midsize & $\begin{array}{c}\text { Small SUV, Midsize } \\
\text { SUV, Pickup }\end{array}$ \\
\hline Cell chemistry & LFP-G & LFP-G \\
Cooling type & CoolA & CoolA \\
\hline Numbers of cells per module & 4 & 4 \\
Number of cells in parallel & 1 & 1 \\
Number of modules in row & 1 & 1 \\
Number of rows of modules per pack & 1 & 1 \\
Number of modules per battery pack & 1 & 1
\end{tabular}




\begin{tabular}{|l|c|c|}
\hline \multicolumn{1}{|c|}{ Vehicle Class } & Compact, Midsize & $\begin{array}{c}\text { Small SUV, Midsize } \\
\text { SUV, Pickup }\end{array}$ \\
\hline Cells per battery pack & 4 & 4 \\
Maximum thickness limit, um & 70 & 70 \\
Number of batteries produced per year & 100,000 & 100,000 \\
Nominal pack voltage & 13.1 & 13.1 \\
\hline
\end{tabular}

Table 37 below provides the BatPaC assumptions for mild hybrid BISG for five different vehicle classes.

Table 37. BatPaC Assumptions for Mild Hybrid BISG

\begin{tabular}{|l|c|c|}
\hline \multicolumn{1}{|c|}{ Vehicle Class } & Compact, Midsize & $\begin{array}{c}\text { Small SUV, } \\
\text { Midsize SUV, } \\
\text { Pickup }\end{array}$ \\
\hline Cell chemistry & LFP-G & LFP-G \\
Cooling type & CoolA & CoolA \\
\hline Numbers of cells per module & 14 & 14 \\
Number of cells in parallel & 1 & 1 \\
Number of modules in row & 1 & 1 \\
Number of rows of modules per pack & 1 & 1 \\
Number of modules per battery pack & 1 & 1 \\
Number of modules in parallel & 1 & 1 \\
Cells per battery pack & 14 & 14 \\
Maximum thickness limit, um & 70 & 100,000 \\
Number of batteries produced per year & 100,000 & 45.9 \\
Nominal pack voltage & 45.9 & \\
\hline
\end{tabular}

Table 38 below provides the BatPaC assumptions for full $\mathrm{HEV}$ s for five different vehicle classes.

Table 38. BatPaC Assumptions for Full HEVs

\begin{tabular}{|l|c|c|}
\hline \multicolumn{1}{|c|}{ Vehicle Class } & Compact, Midsize & $\begin{array}{c}\text { Small SUV, } \\
\text { Midsize SUV, } \\
\text { Pickup }\end{array}$ \\
\hline Cell chemistry & NMC622-G & NMC622-G \\
Cooling type & CoolA & CoolA \\
\hline Numbers of cells per module & 40 & 60 \\
Number of cells in parallel & 1 & 1 \\
Number of modules in row & 1 & 1 \\
Number of rows of modules per pack & 1 & 2 \\
Number of modules per battery pack & 1 & 2
\end{tabular}




\begin{tabular}{|l|c|c|}
\hline \multicolumn{1}{|c|}{ Vehicle Class } & Compact, Midsize & $\begin{array}{c}\text { Small SUV, } \\
\text { Midsize SUV, } \\
\text { Pickup }\end{array}$ \\
\hline Number of modules in parallel & 1 & 1 \\
Cells per battery pack & 40 & 60 \\
Maximum thickness limit, um & 70 & 70 \\
Number of batteries produced per year & 100,000 & 100,000 \\
Nominal pack voltage & 150 & 225 \\
\hline
\end{tabular}

Table 39 below provides the BatPaC assumptions for power-split PHEV 20 AER/par PHEV 20 AER for five different vehicle classes.

Table 39. BatPaC Assumptions for PHEV20 AER

\begin{tabular}{|l|c|c|}
\hline \multicolumn{1}{|c|}{ Vehicle Class } & Compact, Midsize & $\begin{array}{c}\text { Small SUV, } \\
\text { Midsize SUV, } \\
\text { Pickup }\end{array}$ \\
\hline Cell chemistry & NMC622-G & NMC622-G \\
Cooling type & EG-W & EG-W \\
\hline Numbers of cells per module & 20 & 24 \\
Number of cells in parallel & 1 & 1 \\
Number of modules in row & 2 & 2 \\
Number of rows of modules per pack & 2 & 2 \\
Number of modules per battery pack & 4 & 4 \\
Number of modules in parallel & 1 & 1 \\
Cells per battery pack & 80 & 96 \\
Maximum thickness limit, um & 70 & 70 \\
Number of batteries produced per year & 25,000 & 25,000 \\
Nominal pack voltage & 300 & 360 \\
\hline
\end{tabular}

Table 40 below provides the BatPaC assumptions for PHEV 50 AERs for five different vehicle classes.

Table 40. BatPaC Assumptions for PHEV50 AER

\begin{tabular}{|l|c|c|}
\hline Vehicle Class & Compact, Midsize & $\begin{array}{l}\text { Small SUV, } \\
\text { Midsize SUV, } \\
\text { Pickup }\end{array}$ \\
\hline Cell chemistry & NMC622-G & NMC622-G \\
Cooling type & EG-W & EG-W \\
\hline Numbers of cells per module & 24 & 25 \\
Number of cells in parallel & 2 & 2
\end{tabular}




\begin{tabular}{|l|c|c|}
\hline Vehicle Class & Compact, Midsize & $\begin{array}{l}\text { Small SUV, } \\
\text { Midsize SUV, } \\
\text { Pickup }\end{array}$ \\
\hline Number of modules in row & 2 & 2 \\
Number of rows of modules per pack & 4 & 4 \\
Number of modules per battery pack & 8 & 8 \\
Number of modules in parallel & 1 & 1 \\
Cells per battery pack & 192 & 200 \\
Maximum thickness limit, um & 70 & 70 \\
Number of batteries produced per year & 25,000 & 25,000 \\
Nominal pack voltage & 360 & 375 \\
\hline
\end{tabular}

Table 41 below provides the BatPaC assumptions for BEV 200 AERs for five different vehicle classes.

Table 41. BatPaC Assumptions for BEV200 AERs

\begin{tabular}{|l|c|c|}
\hline Vehicle Class & Compact, Midsize & $\begin{array}{l}\text { Small SUV, } \\
\text { Midsize SUV, } \\
\text { Pickup }\end{array}$ \\
\hline Cell chemistry & NMC622-G & NMC622-G \\
Cooling type & EG-W & 19 \\
\hline Numbers of cells per module & 16 & 4 \\
Number of cells in parallel & 4 & 5 \\
Number of modules in row & 5 & 4 \\
Number of rows of modules per pack & 4 & 20 \\
Number of modules per battery pack & 20 & 1 \\
number of modules in parallel & 1 & 380 \\
Cells per battery pack & 320 & 70 \\
Maximum thickness limit, um & 70 & 25000 \\
Number of batteries produced per year & 25000 & 356.3 \\
Nominal pack voltage & 300 & \\
\hline
\end{tabular}

Table 42 below provides the BatPaC assumptions for BEV 300 AERs for five different vehicle classes.

Table 42. BatPaC Assumptions for BEV300 AERs

\begin{tabular}{|l|c|c|}
\hline \multicolumn{1}{|c|}{ Vehicle Class } & Compact, Midsize & $\begin{array}{c}\text { Small SUV, } \\
\text { Midsize SUV, } \\
\text { Pickup }\end{array}$ \\
\hline Cell chemistry & NMC622-G & NMC622-G \\
\hline
\end{tabular}




\begin{tabular}{|l|c|c|}
\hline \multicolumn{1}{|c|}{ Vehicle Class } & Compact, Midsize & $\begin{array}{c}\text { Small SUV, } \\
\text { Midsize SUV, } \\
\text { Pickup }\end{array}$ \\
\hline Cooling type & EG-W & EG-W \\
\hline Numbers of cells per module & 19 & 21 \\
Number of cells in parallel & 4 & 4 \\
Number of modules in row & 5 & 5 \\
Number of rows of modules per pack & 4 & 4 \\
Number of modules per battery pack & 20 & 20 \\
number of modules in parallel & 1 & 1 \\
Cells per battery pack & 380 & 420 \\
Maximum thickness limit, um & 70 & 70 \\
Number of batteries produced per year & 25000 & 25000 \\
Nominal pack voltage & 356.3 & 393.8 \\
\hline
\end{tabular}

\subsubsection{1. $\quad$ Setting Assumptions in BatPaC for this analysis}

This section details how the BatPaC files are set up for different powertrains. There are different spreadsheets for different powertrains, and in each, the different columns correspond to different vehicle classes.

\section{Vehicle Type}

In the Dashboard tab of the BatPaC file, the vehicle powertrain type (microHEV, HEV-HP, PHEV, $\mathrm{EV}$ ) is selected from the dropdown menu in cell E28.

\section{Battery Design}

Vehicle Type

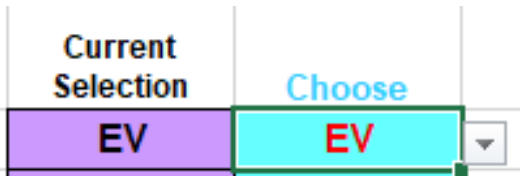

Figure 171. Setting vehicle type

\section{Battery Jacket Mass Parameter}

In the Battery Design tab, users can modify the battery jacket mass parameter (cell G176). The aluminum jacket for the pack has been replaced with stainless steel (new equation circled below in Figure 172) This parameter has been updated for full HEVs, PHEVs and BEVs. 


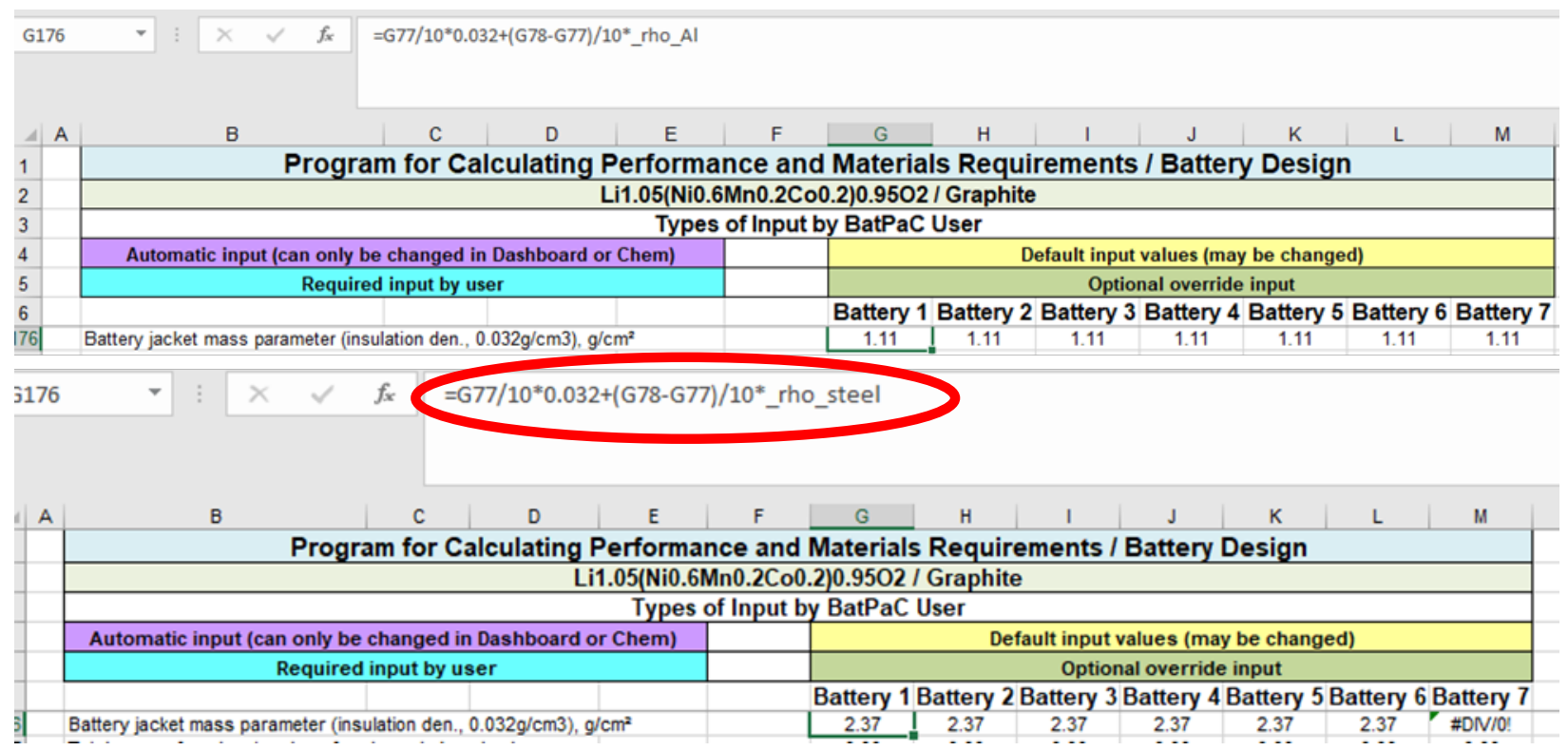

Figure 172. Battery jacket mass inputs for BEV200

\section{Battery Jacket Total Thickness}

In the Battery Design tab, users can modify the thickness of the battery jacket (cell G78). The default parameter is shown in the top picture of Figure 173. The new equation has been modified and is shown in the bottom picture of Figure 173 (circled). This parameter has been updated for full HEVs, PHEVs and BEVs.

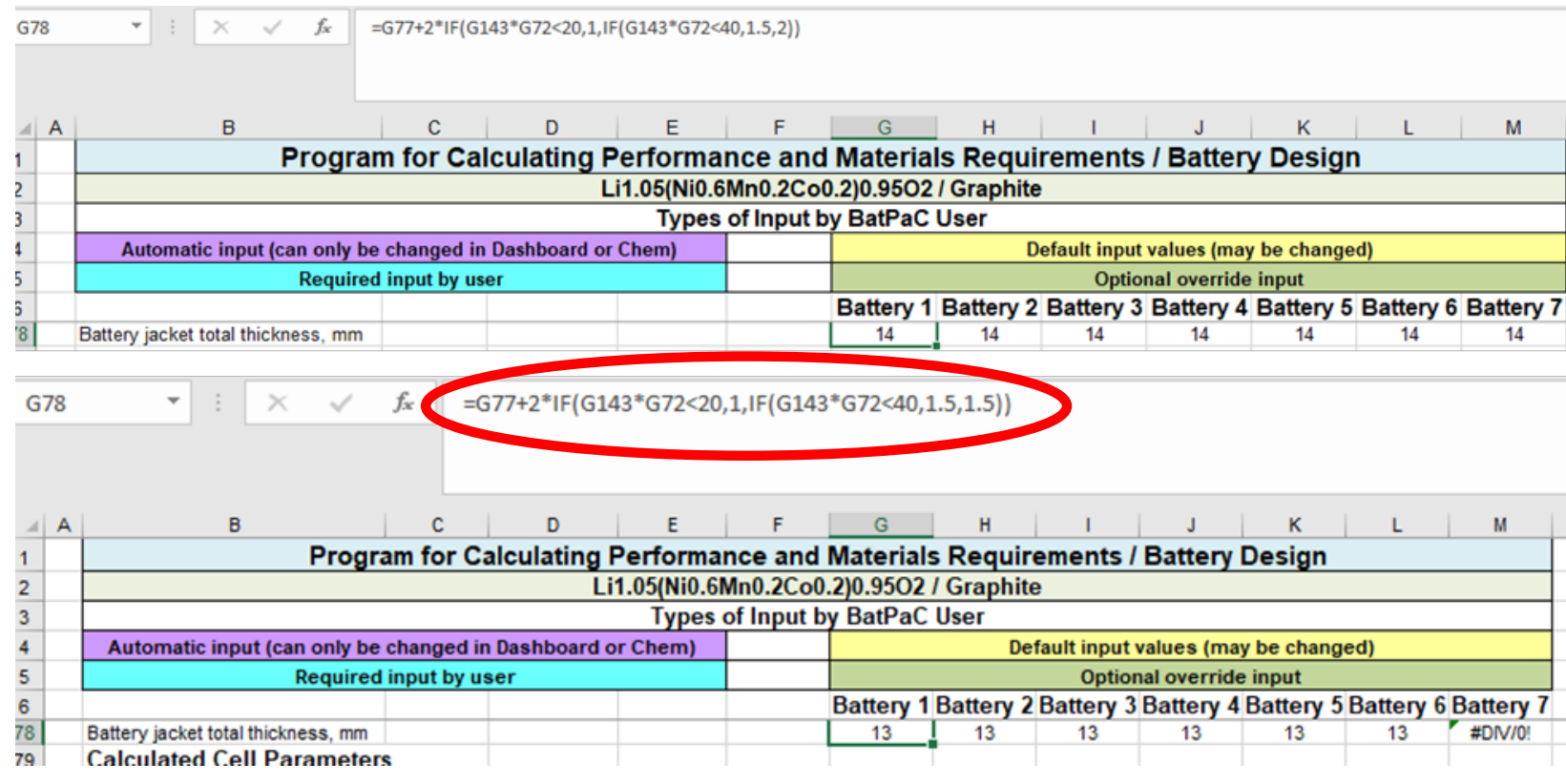

Figure 173. Battery jacket total thickness input for BEV200 


\section{Battery Pack Weight Multiplication Factor}

Argonne simulated existing vehicle battery packs in $\mathrm{BatPaC}$ using A2Mac1 teardown reports [1]. The details of the teardown validation are provided in Section 5.9.2. To better represent battery weight across multiple hybridization degrees, multiplication factors (Table 43) were applied to the battery pack weights to better match A2Mac1 teardowns. This parameter has been updated for full HEVs, PHEVs and BEVs.

\section{Table 43. Weight Multiplication Factor across Powertrains}

\begin{tabular}{|l|c|c|c|}
\hline Vehicle Powertrains & HEVs & PHEVs & BEVs \\
\hline Weight Multiplication Factor & 1.6 & 1.2 & 1 \\
\hline
\end{tabular}

\section{Cell Chemistry}

In the Dashboard tab, users can select the cell chemistry from the dropdown menu in cell E9. The default cell chemistry values can then be viewed in column $\mathrm{C}$ of the Chem tab. For example, Figure 174 shows the cell chemistry selection for BEV200. For this analysis, NMC622 was selected for full HEVs, PHEVs and BEVs as the cell chemistry is applicable to a wide range of vehicles based on the benchmarking detailes provided in Section 5.9.2.

\begin{tabular}{|c|c|c|c|c|}
\hline System ID & NMC622-G & ID \# & \multicolumn{2}{|c|}{25} \\
\hline \multicolumn{5}{|l|}{ Description } \\
\hline Property & Applied Value & BatPaC Default & Override & Notes \\
\hline Couple Name & \multicolumn{2}{|c|}{ Li1.05(Ni0.6Mn0.2Co0.2)0.9502 / Graphite } & & \\
\hline Positive electrode active material & NMC622 & NMC622 & & \\
\hline Positive electrode active material molecular weight & 94.4 & 94.39 & & \\
\hline Positive electrode active material capacity, mAh/g: & 180.0 & 180.00 & & \\
\hline \multicolumn{5}{|l|}{ Positive electrode composition, Weight \% } \\
\hline Positive electrode active material weight $\%$ & 96.0 & 96.0 & & \\
\hline Positive electrode carbon addtive weight $\%$ & 2.0 & 2.0 & & \\
\hline Positive electrode binder weight $\%$ & 2.0 & 2.0 & & \\
\hline Binder solvent for positive electrode & NMP & NMP & & \\
\hline Void, vol\% of positive electrode & 25.0 & 25.00 & & \\
\hline \multicolumn{5}{|l|}{ Material Density, $\mathrm{g} / \mathrm{cm}^{3}$} \\
\hline Positive electrode active material density, $\mathrm{g} / \mathrm{cm}^{3}$ & 4.65 & 4.65 & & \\
\hline Positive electrode carbon additive density, $\mathrm{g} / \mathrm{cm}^{3}$ & 1.83 & 1.83 & & \\
\hline Positive electrode binder density, $\mathrm{g} / \mathrm{cm}^{3}$ & 1.77 & 1.77 & & \\
\hline Maximum thickness limit for positive electrode, $\mu \mathrm{m}$ & 70 & 120 & & \\
\hline Minimum thickness limit for positive electrode, $\mu \mathrm{m}$ & 15 & 15 & & \\
\hline Positive electrode specific particle area "a", $\mathrm{cm}^{2} / \mathrm{cm}^{3}$ & 80,000 & 80,000 & & \\
\hline
\end{tabular}

Figure 174. Cell chemistry for BEV200

\section{Coolant Type}

In the Dashboard tab, the coolant type corresponding to the different powertrains are selected from the dropdown menu in cell E29. 


\section{Battery Design \\ Vehicle Type \\ Coolant Type \\ Calculate Fast Charging?}

\begin{tabular}{|c|c|}
$\begin{array}{c}\text { Current } \\
\text { Selection }\end{array}$ & Choose \\
\hline EV & EV \\
\hline EG-W & EG-W \\
\hline No & No \\
\hline
\end{tabular}

\section{Figure 175. Setting coolant type}

\section{Battery Design}

In the Dashboard tab, the battery design information (number of cells per module, number of cells in parallel, number of modules in row, number of rows of modules per pack, and number of modules in parallel) is set in cells D34:J38. The number of modules per battery pack and the cells per battery pack can be found in the Battery Design tab, in rows 72 and 74, respectively.

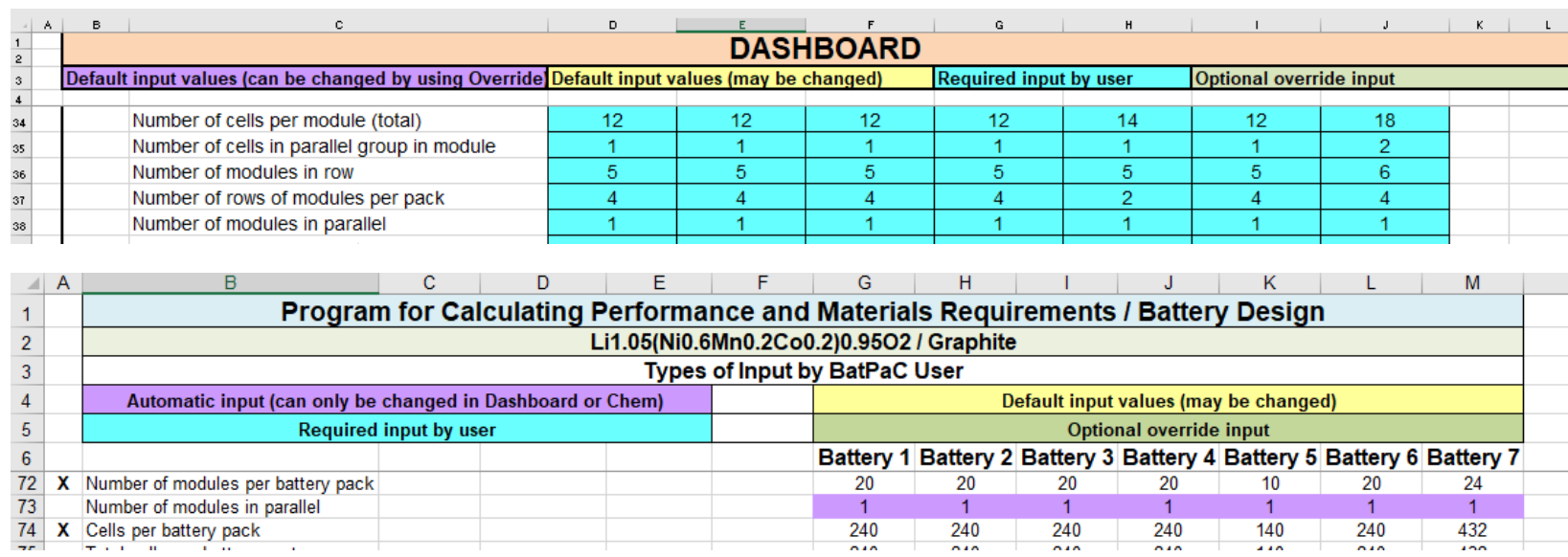

Figure 176. Battery design information

\section{Maximum Thickness Limit}

Based on the current status of the industry and feedback from the developers of the BatPaC model, a maximum thickness limit of $70 \mu \mathrm{m}$ is specified for all batteries. In the Dashboard tab, the maximum thickness value is placed in cell E14.

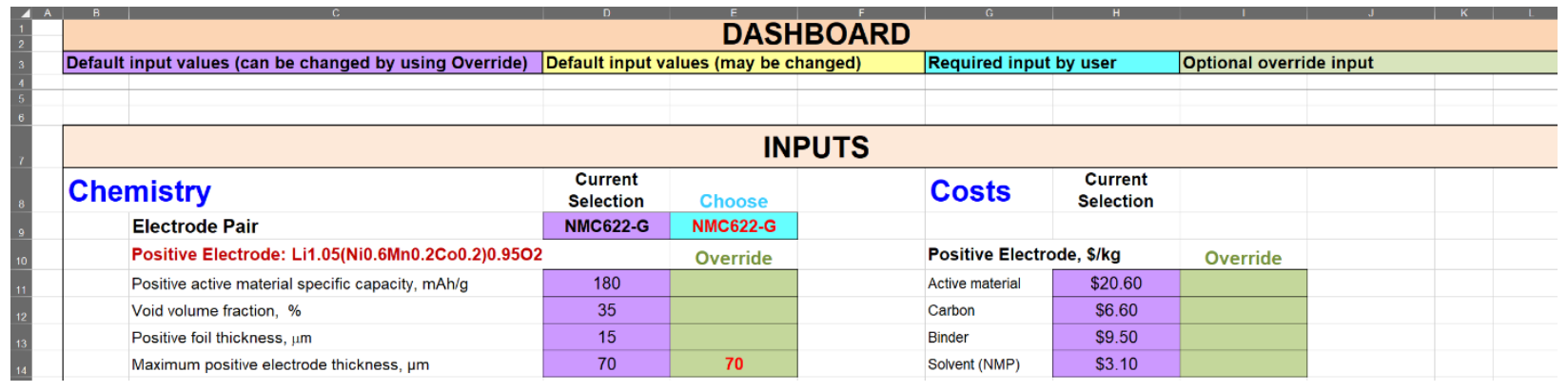

Figure 177. Setting maximum thickness 


\section{Number of Battery Systems Manufactured per Year}

In the Dashboard tab, the number of battery systems manufactured per year is placed in row 39. A constant production volume of 25,000 battery systems is assumed for PHEVs and BEVs, and a production volume of 100,000 battery systems is assumed for all HEVs, including micro HEV and mild hybrid BISG.

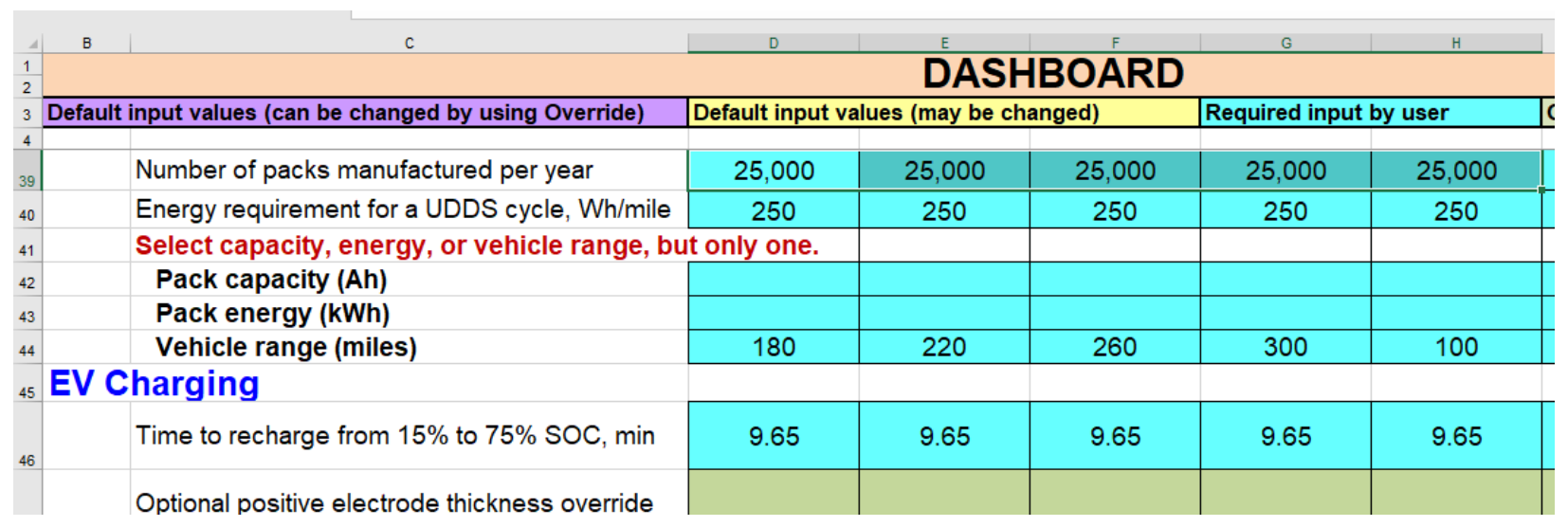

Figure 178. Battery systems manufactured per year

\section{BMU Model Updates}

The BMU model has been updated to represent the current state of battery systems manufactured for full HEVs, PHEVs and BEVs. The new model takes into consideration how the price of some of the individual components changes with the current/voltage requirements of the pack. The main updates include:

- A single contactor-main is needed if the total voltage of the pack is less than $100 \mathrm{~V}$, due to reduced shock hazard. Two are needed for higher voltages

- The cost of contactor-main now scales with the total current (linear interpolation)

- 10A contactor, $\$ 10 /$ unit

- $600 \mathrm{~A}$ contactor, $\$ 52.86$ /unit

- Interfaces between electronic system also scale with total current:

- $\$ 20$ for 50A max current

- $\$ 60$ for 500A max current

$\circ$ p-value changed from 0.9 to 0.95

- Auxiliary contactor and the charger fuse are not needed for PHEV, HEV-HP

- Removed multiplier of 1.5 for max current based on power

- Default number of cells per ASIC changed to 10

\subsubsection{2. $\quad$ Inputs to BatPaC from Autonomie}

The following inputs are sent to BatPaC for Autonomie simulations:

- $\quad$ Battery pack power $(\mathrm{kW})$

- Total battery pack energy $(\mathrm{kWh})$ 


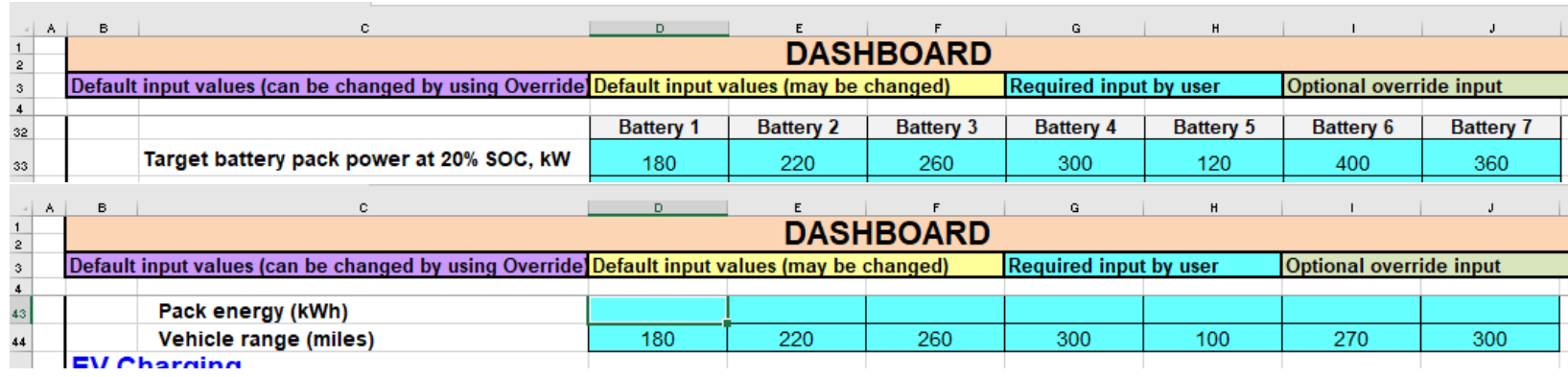

Figure 179. Autonomie inputs to BatPaC

\subsubsection{Outputs from BatPaC into Autonomie}

The following outputs are sent to Autonomie from BatPaC:

- Estimated total battery cost $(\$)$

- Total battery mass $(\mathrm{kg})$

- Battery pack capacity (Ah)

- Nominal battery system voltage (v)

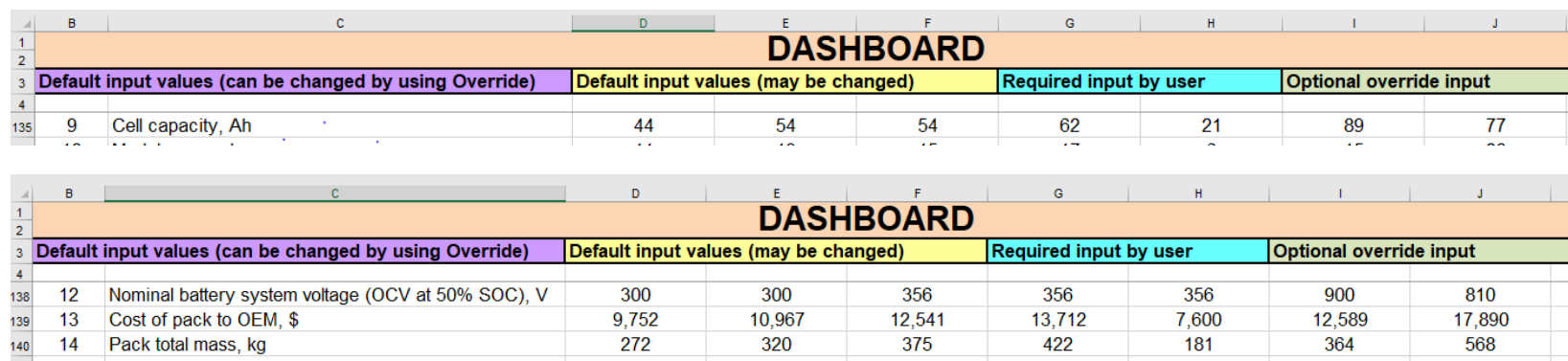

Figure 180. BatPaC Outputs to Autonomie

\subsubsection{BatPaC Lookup Tables}

\subsubsection{Micro $\mathrm{HEV}$}

For micro HEVs, the costs of additional pack level component (pack hardware, TMS, high voltage wiring, battery management unit) are subtracted.

Table 44 below summarizes the final lookup tables generated for micro-HEVs for BatPaC cost, mass and cell capacity. 
Table 44. BatPaC Lookup Table for Micro HEVs

\begin{tabular}{|c|c|c|c|c|c|c|c|c|c|c|c|c|}
\hline & \multirow{2}{*}{\multicolumn{2}{|c|}{ - }} & \multicolumn{10}{|c|}{ Energy, kWh } \\
\hline & & & & & & 0.8 & & 0 & & 1.2 & & .4 \\
\hline \multirow{8}{*}{ 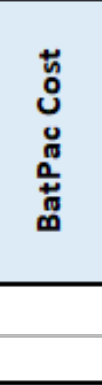 } & \multirow{6}{*}{$\begin{array}{l}3 \\
\frac{3}{4} \\
5 \\
\vdots \\
\vdots \\
0 \\
0\end{array}$} & 0.5 & $\$$ & 210 & $\$$ & 242 & $\$$ & 266 & & 301 & $\$$ & 356 \\
\hline & & 1.1 & $\$$ & 211 & $\$$ & 237 & $\$$ & 256 & & 278 & $\$$ & 300 \\
\hline & & 1.5 & $\$$ & 214 & $\$$ & 239 & $\$$ & 257 & & 277 & $\$$ & 298 \\
\hline & & 2.0 & $\$$ & 218 & $\$$ & 242 & $\$$ & 258 & & 279 & $\$$ & 299 \\
\hline & & 2.5 & $\$$ & 221 & $\$$ & 246 & $\$$ & 261 & & 282 & $\$$ & 301 \\
\hline & & 3.0 & $\$$ & 225 & $\$$ & 249 & $\$$ & 265 & & 285 & $\$$ & 304 \\
\hline & \multirow{2}{*}{\multicolumn{2}{|c|}{ 1010 }} & \multicolumn{10}{|c|}{ Energy, kWh } \\
\hline & & & \multicolumn{2}{|c|}{0.6} & \multicolumn{2}{|r|}{0.8} & \multicolumn{2}{|c|}{1.0} & \multicolumn{2}{|r|}{1.2} & \multicolumn{2}{|c|}{1.4} \\
\hline \multirow{6}{*}{ 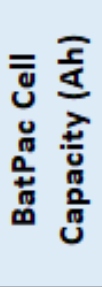 } & \multirow{6}{*}{$\begin{array}{l}3 \\
\frac{3}{2} \\
\vdots 0 \\
3 \\
0 \\
0\end{array}$} & 0.5 & \multicolumn{2}{|c|}{51.8} & \multicolumn{2}{|c|}{77.8} & \multicolumn{2}{|c|}{98.2} & \multicolumn{2}{|r|}{130.0} & \multicolumn{2}{|c|}{183.9} \\
\hline & & 1.1 & \multicolumn{2}{|c|}{48.2} & \multicolumn{2}{|r|}{69.0} & \multicolumn{2}{|c|}{83.6} & & 102.6 & \multicolumn{2}{|c|}{122.6} \\
\hline & & 1.5 & \multicolumn{2}{|c|}{47.4} & \multicolumn{2}{|c|}{67.4} & \multicolumn{2}{|c|}{81.1} & & 98.7 & \multicolumn{2}{|c|}{116.9} \\
\hline & & 2.0 & \multicolumn{2}{|c|}{46.9} & \multicolumn{2}{|c|}{66.5} & \multicolumn{2}{|c|}{79.7} & & 96.6 & \multicolumn{2}{|c|}{113.9} \\
\hline & & 2.5 & \multicolumn{2}{|c|}{46.7} & \multicolumn{2}{|c|}{65.9} & \multicolumn{2}{|c|}{79.0} & & 95.5 & \multicolumn{2}{|c|}{112.3} \\
\hline & & 3.0 & \multicolumn{2}{|c|}{46.5} & & 55.6 & & & & 94.7 & & 1.2 \\
\hline
\end{tabular}

The battery mass of micro HEVs differs across classes and performance categories and is defined in the assumptions sheet, shown in Table 45.

Table 45. Mass Assumptions for Micro HEVs

\begin{tabular}{|l|l|l|l|}
\hline \multirow{2}{*}{$\begin{array}{l}\text { Vehicle } \\
\text { Class }\end{array}$} & Performance & Battery Mass $(\mathbf{k g})$ \\
\cline { 3 - 4 } & Catery & Gasoline & Diesel \\
\hline Compact & Base & 15 & 20 \\
\hline Compact & Premium & 25 & 30 \\
\hline Midsize & Base & 18 & 20 \\
\hline Midsize & Premium & 28 & 30 \\
\hline Small SUV & Base & 18 & 20 \\
\hline Small SUV & Premium & 28 & 30 \\
\hline Midsize SUV & Base & 20 & 25 \\
\hline Midsize SUV & Premium & 30 & 35 \\
\hline Pickup & Base & 20 & 20 \\
\hline Pickup & Premium & 30 & 35 \\
\hline
\end{tabular}

\subsubsection{2. $\quad$ Mild Hybrid BISG}

For mild hybrid BISGs, mass and costs of additional pack level components (pack hardware, TMS, high voltage wiring, BMU) are subtracted.

Table 46 below summarizes the final lookup tables generated from BatPaC for mild hybrid BISG battery packs for cost, mass and cell capacity. 
Table 46. BatPaC Lookup Table for Mild Hybrid BISGs

\begin{tabular}{|c|c|c|c|c|c|c|c|c|c|c|c|c|c|}
\hline & \multirow{2}{*}{\multicolumn{2}{|c|}{ 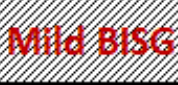 }} & \multicolumn{11}{|c|}{ Energy, kWh } \\
\hline & & & 0.30 & \multicolumn{2}{|c|}{0.40} & \multicolumn{2}{|c|}{0.60} & \multicolumn{2}{|c|}{0.81} & \multicolumn{2}{|c|}{1.00} & \multicolumn{2}{|c|}{1.20} \\
\hline \multirow{6}{*}{ 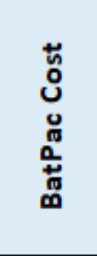 } & \multirow{6}{*}{$\begin{array}{l}3 \\
\frac{3}{2} \\
\vdots \\
\vdots \\
0 \\
0\end{array}$} & 5.00 & 315 & $\$$ & 326 & \$ & 347 & $\$$ & 368 & $\$$ & 386 & $\$$ & 405 \\
\hline & & 6.00 & 32 & $\$$ & 332 & \$ & 354 & $\$$ & 374 & $\$$ & 393 & $\$$ & 411 \\
\hline & & 7.69 & 33 & $\$$ & 342 & $\$$ & 363 & $\$$ & 384 & $\$$ & 403 & $\$$ & 421 \\
\hline & & 8.00 & 33 & $\$$ & 344 & $\$$ & 365 & $\$$ & 386 & $\$$ & 405 & $\$$ & 423 \\
\hline & & 9.00 & & $\$$ & 350 & $\$$ & 370 & $\$$ & 392 & $\$$ & 411 & $\$$ & 429 \\
\hline & & 10.00 & & $\$$ & 356 & $\$$ & 376 & $\$$ & 397 & $\$$ & 416 & $\$$ & 435 \\
\hline & \multirow{2}{*}{\multicolumn{2}{|c|}{10}} & \multicolumn{11}{|c|}{ Energy, kWh } \\
\hline & & & 0.30 & & .40 & & 60 & & 81 & & 00 & & 20 \\
\hline \multirow{8}{*}{ 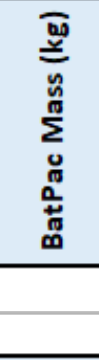 } & \multirow{6}{*}{$\begin{array}{l}\frac{3}{3} \\
\frac{1}{3} \\
\vdots \\
0 \\
0\end{array}$} & 5.00 & 2.7 & & 3.2 & & 1 & & 8 & & .5 & & .3 \\
\hline & & 6.00 & 2.9 & & 3.4 & & 4 & & 1 & & 8 & & .5 \\
\hline & & 7.69 & 3.2 & & 3.7 & & .7 & & .6 & & 3 & & .0 \\
\hline & & 8.00 & 3.3 & & 3.7 & & .7 & & .7 & & .4 & & .1 \\
\hline & & 9.00 & & & 3.9 & & 8 & & .8 & & 7 & & .4 \\
\hline & & 10.00 & & & 4.1 & & .0 & & .0 & & .9 & & .7 \\
\hline & \multirow{2}{*}{\multicolumn{2}{|c|}{ 1 }} & \multicolumn{11}{|c|}{ Energy, kWh } \\
\hline & & & 0.30 & & .40 & & 60 & & 81 & & 00 & & 20 \\
\hline \multirow{6}{*}{ 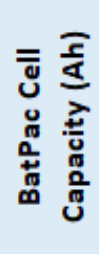 } & \multirow{6}{*}{$\begin{array}{l}3 \\
\frac{3}{2} \\
\vdots \\
\vdots \\
0 \\
0\end{array}$} & 5.00 & 6.56 & & 3.83 & & 20 & & .79 & & .15 & & .68 \\
\hline & & 6.00 & 6.56 & & 3.82 & & 17 & & .75 & & .08 & & .58 \\
\hline & & 7.69 & 6.55 & & 3.81 & & 15 & & .70 & & 01 & & .47 \\
\hline & & 8.00 & 6.55 & & 3.81 & & 14 & & .70 & & .00 & & .46 \\
\hline & & 9.00 & & & 3.80 & & 13 & & .68 & & .97 & & .42 \\
\hline & & 10.00 & & & 3.80 & & 12 & & .66 & & 95 & & .39 \\
\hline
\end{tabular}

The mild hybrid BISGs battery packs considered in the current analysis consist of $0.403 \mathrm{kWh}$ total energy with $7.69 \mathrm{~kW}$ power output. As a result, BatPaC provides a manufacturing cost of $\$ 342$ with a pack mass value of $3.7 \mathrm{~kg}$.

\subsubsection{Full Hybrid HEV}

Table 47 below summarizes the cost, mass and cell capacities generated for compact and midsize full hybrids from $\mathrm{BatPaC}$ using the assumptions specified in Table 47. 
Table 47. BatPaC Lookup Table for Full Hybrids (Compact/Midsize)

\begin{tabular}{|c|c|c|c|c|c|c|c|c|c|c|c|c|c|c|c|c|c|c|c|c|c|c|c|c|c|c|}
\hline \multirow{9}{*}{ 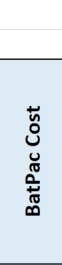 } & \multirow{2}{*}{\multicolumn{2}{|c|}{ HEV }} & \multicolumn{24}{|c|}{ Energy, kWh } \\
\hline & & & & 0.9 & & 1.0 & & 1.2 & & 1.4 & & 1.6 & & 1.8 & & 2.0 & & 2.2 & & 2.4 & & 2.6 & & 2.8 & & 3.0 \\
\hline & \multirow{7}{*}{ 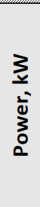 } & 10.0 & $\$$ & 1,054 & $\$$ & 1,064 & $\$$ & 1,083 & $\$$ & 1,102 & $\$$ & 1,120 & $\$$ & 1,138 & $\$$ & 1,156 & $\$$ & 1,174 & $\$$ & 1,192 & $\$$ & 1,210 & $\$$ & 1,227 & $\$$ & 1,245 \\
\hline & & 20.0 & $\$$ & 1,103 & $\$$ & 1,113 & $\$$ & 1,133 & $\$$ & 1,152 & $\$$ & 1,170 & $\$$ & 1,188 & $\$$ & 1,206 & $\$$ & 1,223 & $\$$ & 1,241 & $\$$ & 1,258 & $\$$ & 1,275 & $\$$ & 1,292 \\
\hline & & 30.0 & $\$$ & 1,158 & $\$$ & 1,166 & $\$$ & 1,184 & $\$$ & 1,202 & $\$$ & 1,221 & $\$$ & 1,240 & $\$$ & 1,258 & $\$$ & 1,275 & $\$$ & 1,292 & $\$$ & 1,309 & $\$$ & 1,326 & $\$$ & 1,343 \\
\hline & & 40.0 & $\$$ & 1,221 & $\$$ & 1,225 & $\$$ & 1,239 & $\$$ & 1,254 & $\$$ & 1,271 & $\$$ & 1,289 & $\$$ & 1,307 & $\$$ & 1,325 & $\$$ & 1,343 & $\$$ & 1,361 & $\$$ & 1,377 & $\$$ & 1,394 \\
\hline & & 60.0 & & & & & & & $\$$ & 1,376 & $\$$ & 1,386 & $\$$ & 1,399 & $\$$ & 1,413 & $\$$ & 1,429 & $\$$ & 1,445 & $\$$ & 1,461 & $\$$ & 1,478 & $\$$ & 1,495 \\
\hline & & 80.0 & & & & & & & & & & & $\$$ & 1,522 & $\$$ & 1,530 & $\$$ & 1,541 & $\$$ & 1,554 & $\$$ & 1,567 & $\$$ & 1,582 & $\$$ & 1,597 \\
\hline & & 100.0 & & & & & & & & & & & & & & & $\$$ & 1,668 & $\$$ & 1,674 & $\$$ & 1,684 & $\$$ & 1,695 & $\$$ & 1,707 \\
\hline \multirow{7}{*}{ 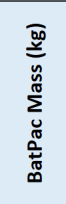 } & \multirow{7}{*}{ 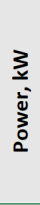 } & 10.0 & & 22.81 & & 23.30 & & 24.26 & & 25.24 & & 26.21 & & 27.19 & & 28.17 & & 29.16 & & 30.15 & & 31.14 & & 32.14 & & 33.14 \\
\hline & & 20.0 & & 23.94 & & 24.75 & & 26.23 & & 27.29 & & 28.24 & & 29.20 & & 30.15 & & 31.11 & & 32.06 & & 33.02 & & 33.98 & & 34.94 \\
\hline & & 30.0 & & 25.23 & & 25.83 & & 27.22 & & 28.61 & & 30.07 & & 31.51 & & 32.61 & & 33.55 & & 34.50 & & 35.44 & & 36.39 & & 37.34 \\
\hline & & 40.0 & & 27.22 & & 27.59 & & 28.67 & & 29.90 & & 31.19 & & 32.57 & & 33.95 & & 35.30 & & 36.68 & & 37.92 & & 38.86 & & 39.80 \\
\hline & & 60.0 & & & & & & & & 33.63 & & 34.48 & & 35.51 & & 36.68 & & 37.89 & & 39.11 & & 40.42 & & 41.66 & & 43.02 \\
\hline & & 80.0 & & & & & & & & & & & & 39.62 & & 40.34 & & 41.27 & & 42.28 & & 43.38 & & 44.53 & & 45.69 \\
\hline & & 100.0 & & & & & & & & & & & & & & & & 45.60 & & 46.24 & & 47.08 & & 47.98 & & 49.00 \\
\hline \multirow{7}{*}{ 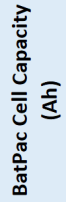 } & \multirow{7}{*}{ 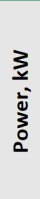 } & 10.0 & & 6.05 & & 6.73 & & 8.09 & & 9.45 & & 10.82 & & 12.19 & & 13.57 & & 14.96 & & 16.35 & & 17.75 & & 19.15 & & 20.55 \\
\hline & & 20.0 & & 6.02 & & 6.69 & & 8.04 & & 9.39 & & 10.74 & & 12.10 & & 13.45 & & 14.81 & & 16.17 & & 17.53 & & 18.90 & & 20.27 \\
\hline & & 30.0 & & 6.01 & & 6.68 & & 8.03 & & 9.37 & & 10.72 & & 12.06 & & 13.41 & & 14.76 & & 16.11 & & 17.47 & & 18.82 & & 20.18 \\
\hline & & 40.0 & & 6.01 & & 6.68 & & 8.02 & & 9.36 & & 10.70 & & 12.04 & & 13.39 & & 14.74 & & 16.08 & & 17.43 & & 18.78 & & 20.13 \\
\hline & & 60.0 & & & & & & & & 9.35 & & 10.69 & & 12.03 & & 13.37 & & 14.71 & & 16.05 & & 17.39 & & 18.74 & & 20.08 \\
\hline & & 80.0 & & & & & & & & & & & & 12.02 & & 13.36 & & 14.69 & & 16.03 & & 17.38 & & 18.72 & & 20.06 \\
\hline & & 100.0 & & & & & & & & & & & & & & & & 14.69 & & 16.03 & & 17.36 & & 18.70 & & 20.04 \\
\hline
\end{tabular}

Table 48 summarizes the cost, mass and cell capacities generated for full hybrid SUVs and pickups from $\mathrm{BatPaC}$, using the assumptions specified.

Table 48. BatPaC Lookup Table for Full Hybrids (SUVs/Pickups)

\begin{tabular}{|c|c|c|c|c|c|c|c|c|c|c|c|c|c|c|c|c|c|c|c|c|c|c|c|c|c|}
\hline & \multirow{2}{*}{\multicolumn{2}{|c|}{15}} & \multicolumn{23}{|c|}{ Energy, kWh } \\
\hline & & & 0.9 & & 1.0 & & 1.2 & & 1.4 & & 1.6 & & 1.8 & & 2.0 & & 2.2 & & 2.4 & & 2.6 & & 2.8 & & 3.0 \\
\hline \multirow{7}{*}{ 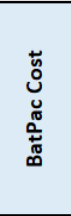 } & \multirow{7}{*}{ 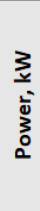 } & 10.0 & 1,233 & $\$$ & 1,243 & $\$$ & 1,262 & $\$$ & 1,281 & $\$$ & 1,299 & $\$$ & 1,318 & $\$$ & 1,336 & $\$$ & 1,354 & $\$$ & 1,372 & $\$$ & 1,389 & $\$$ & 1,407 & $\$$ & 1,424 \\
\hline & & 20.0 & 1,274 & $\$$ & 1,284 & $\$$ & 1,304 & $\$$ & 1,323 & $\$$ & 1,341 & $\$$ & 1,359 & $\$$ & 1,377 & $\$$ & 1,394 & $\$$ & 1,412 & $\$$ & 1,429 & $\$$ & 1,446 & $\$$ & 1,463 \\
\hline & & 30.0 & 1,321 & $\$$ & 1,329 & $\$$ & 1,346 & $\$$ & 1,365 & $\$$ & 1,383 & $\$$ & 1,402 & $\$$ & 1,420 & $\$$ & 1,438 & $\$$ & 1,455 & $\$$ & 1,472 & $\$$ & 1,489 & $\$$ & 1,506 \\
\hline & & 40.0 & 1,376 & $\$$ & 1,380 & $\$$ & 1,393 & $\$$ & 1,409 & $\$$ & 1,426 & $\$$ & 1,443 & $\$$ & 1,461 & $\$$ & 1,479 & $\$$ & 1,498 & $\$$ & 1,515 & $\$$ & 1,532 & $\$$ & 1,548 \\
\hline & & 60.0 & & & & $\$$ & 1,505 & $\$$ & 1,510 & $\$$ & 1,520 & $\$$ & 1,532 & $\$$ & 1,547 & $\$$ & 1,562 & $\$$ & 1,578 & $\$$ & 1,595 & $\$$ & 1,612 & $\$$ & 1,628 \\
\hline & & 80.0 & & & & & & & & & & $\$$ & 1,639 & $\$$ & 1,647 & $\$$ & 1,657 & $\$$ & 1,670 & $\$$ & 1,684 & $\$$ & 1,698 & $\$$ & 1,713 \\
\hline & & 100.0 & & & & & & & & & & & & & & $\$$ & 1,764 & $\$$ & 1,771 & $\$$ & 1,780 & $\$$ & 1,791 & $\$$ & 1,803 \\
\hline \multirow{7}{*}{ 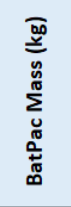 } & \multirow{7}{*}{$\begin{array}{l}3 \\
\frac{3}{4} \\
\overline{0} \\
\vdots \\
0\end{array}$} & 10.0 & 25.47 & & 25.96 & & 26.93 & & 27.92 & & 28.90 & & 29.89 & & 30.88 & & 1.88 & & 32.88 & & 33.88 & & 34.89 & & 35.90 \\
\hline & & 20.0 & 26.63 & & 27.45 & & 29.00 & & 30.02 & & 30.98 & & 31.94 & & 32.90 & & 3.86 & & 34.83 & & 35.79 & & 36.76 & & 37.73 \\
\hline & & 30.0 & 27.82 & & 28.53 & & 29.86 & & 31.40 & & 32.79 & & 34.26 & & 35.39 & & 6.34 & & 37.29 & & 38.24 & & 39.19 & & 40.15 \\
\hline & & 40.0 & 29.85 & & 30.28 & & 31.32 & & 32.59 & & 34.00 & & 35.32 & & 36.75 & & 8.23 & & 39.66 & & 40.75 & & 41.70 & & 42.64 \\
\hline & & 60.0 & & & & & 35.78 & & 36.34 & & 37.21 & & 38.26 & & 39.47 & & 0.71 & & 41.92 & & 43.28 & & 44.64 & & 45.93 \\
\hline & & 80.0 & & & & & & & & & & & 42.28 & & 43.05 & & 3.97 & & 45.06 & & 46.16 & & 47.34 & & 48.53 \\
\hline & & 100.0 & & & & & & & & & & & & & & & 18.25 & & 48.90 & & 49.74 & & 50.69 & & 51.67 \\
\hline \multirow{7}{*}{ 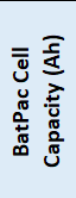 } & \multirow{7}{*}{ 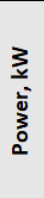 } & 10.0 & 4.03 & & 4.48 & & 5.39 & & 6.30 & & 7.21 & & 8.13 & & 9.05 & & 9.97 & & 10.90 & & 11.83 & & 12.76 & & 13.70 \\
\hline & & 20.0 & 4.01 & & 4.46 & & 5.36 & & 6.26 & & 7.16 & & 8.06 & & 8.97 & & 9.87 & & 10.78 & & 11.69 & & 12.60 & & 13.51 \\
\hline & & 30.0 & 4.01 & & 4.46 & & 5.35 & & 6.25 & & 7.14 & & 8.04 & & 8.94 & & 9.84 & & 10.74 & & 11.64 & & 12.55 & & 13.45 \\
\hline & & 40.0 & 4.01 & & 4.45 & & 5.35 & & 6.24 & & 7.13 & & 8.03 & & 8.93 & & 9.82 & & 10.72 & & 11.62 & & 12.52 & & 13.42 \\
\hline & & 60.0 & & & & & 5.34 & & 6.23 & & 7.12 & & 8.02 & & 8.91 & & 9.81 & & 10.70 & & 11.60 & & 12.49 & & 13.39 \\
\hline & & 80.0 & & & & & & & & & & & 8.01 & & 8.90 & & 9.80 & & 10.69 & & 11.58 & & 12.48 & & 13.37 \\
\hline & & 100.0 & & & & & & & & & & & & & & & 9.79 & & 10.68 & & 11.58 & & 12.47 & & 13.36 \\
\hline
\end{tabular}

\subsubsection{PHEV 20 AER (Power-Split/Parallel)}

Table 49 summarizes the cost, mass and cell capacities generated for compact and midsize PHEV20 AERs from $\mathrm{BatPaC}$, using the assumptions specified. 
Table 49. BatPaC Lookup Table for PHEV20 AERs (Compact/Midsize)

\begin{tabular}{|c|c|c|c|c|c|}
\hline & \multirow{2}{*}{\multicolumn{2}{|c|}{101}} & \multicolumn{3}{|c|}{ Energy, kWh } \\
\hline & & & 5.0 & 10.0 & 20.0 \\
\hline \multirow{11}{*}{ 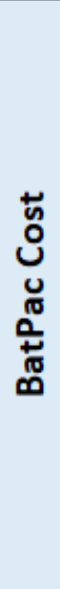 } & \multirow{11}{*}{ 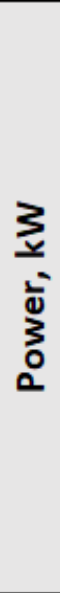 } & 30.0 & 2,591 & 3,212 & 4,372 \\
\hline & & 40.0 & 2,609 & 3,228 & 4,387 \\
\hline & & 60.0 & 2,657 & 3,260 & 4,416 \\
\hline & & 80.0 & 2,802 & 3,295 & 4,447 \\
\hline & & 100.0 & 2,870 & 3,335 & 4,478 \\
\hline & & 120.0 & 2,945 & 3,403 & 4,513 \\
\hline & & 140.0 & 3,071 & 3,515 & 4,548 \\
\hline & & 160.0 & 3,207 & 3,615 & 4,584 \\
\hline & & 200.0 & & 3,831 & 4,668 \\
\hline & & 240.0 & & 4,021 & 4,842 \\
\hline & & 280.0 & & 4,273 & 5,002 \\
\hline \multirow{11}{*}{ 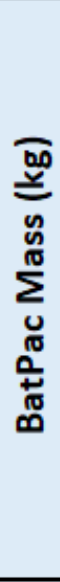 } & \multirow{11}{*}{ 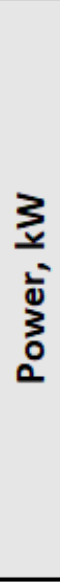 } & 30.0 & 50.0 & 79.2 & 143.9 \\
\hline & & 40.0 & 50.1 & 79.3 & 144.0 \\
\hline & & 60.0 & 50.8 & 79.5 & 144.2 \\
\hline & & 80.0 & 53.7 & 79.8 & 144.4 \\
\hline & & 100.0 & 56.5 & 80.1 & 144.7 \\
\hline & & 120.0 & 59.5 & 81.4 & 145.0 \\
\hline & & 140.0 & 63.0 & 92.1 & 145.2 \\
\hline & & 160.0 & 67.1 & 94.6 & 145.5 \\
\hline & & 200.0 & & 100.6 & 146.2 \\
\hline & & 240.0 & & 107.3 & 150.4 \\
\hline & & 280.0 & & 115.1 & 155.8 \\
\hline \multirow{11}{*}{ 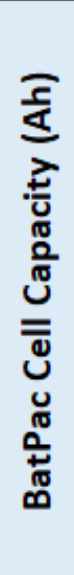 } & \multirow{11}{*}{ 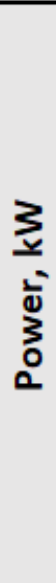 } & 30.0 & 16.8 & 33.5 & 67.1 \\
\hline & & 40.0 & 16.8 & 33.5 & 67.1 \\
\hline & & 60.0 & 16.8 & 33.5 & 67.1 \\
\hline & & 80.0 & 16.7 & 33.5 & 67.1 \\
\hline & & 100.0 & 16.7 & 33.5 & 67.1 \\
\hline & & 120.0 & 16.7 & 33.5 & 67.1 \\
\hline & & 140.0 & 16.7 & 33.5 & 67.1 \\
\hline & & 160.0 & 16.7 & 33.5 & 67.1 \\
\hline & & 200.0 & & 33.4 & 67.1 \\
\hline & & 240.0 & & 33.4 & 67.0 \\
\hline & & 280.0 & & 33.4 & 66.9 \\
\hline
\end{tabular}

Table 50 summarizes the cost, mass and cell capacities generated for SUV and pickup PHEV20 AERs from $\mathrm{BatPaC}$ using the assumptions specified. 
Table 50. BatPaC Lookup Table for PHEV20 AERs (SUVs/Pickup)

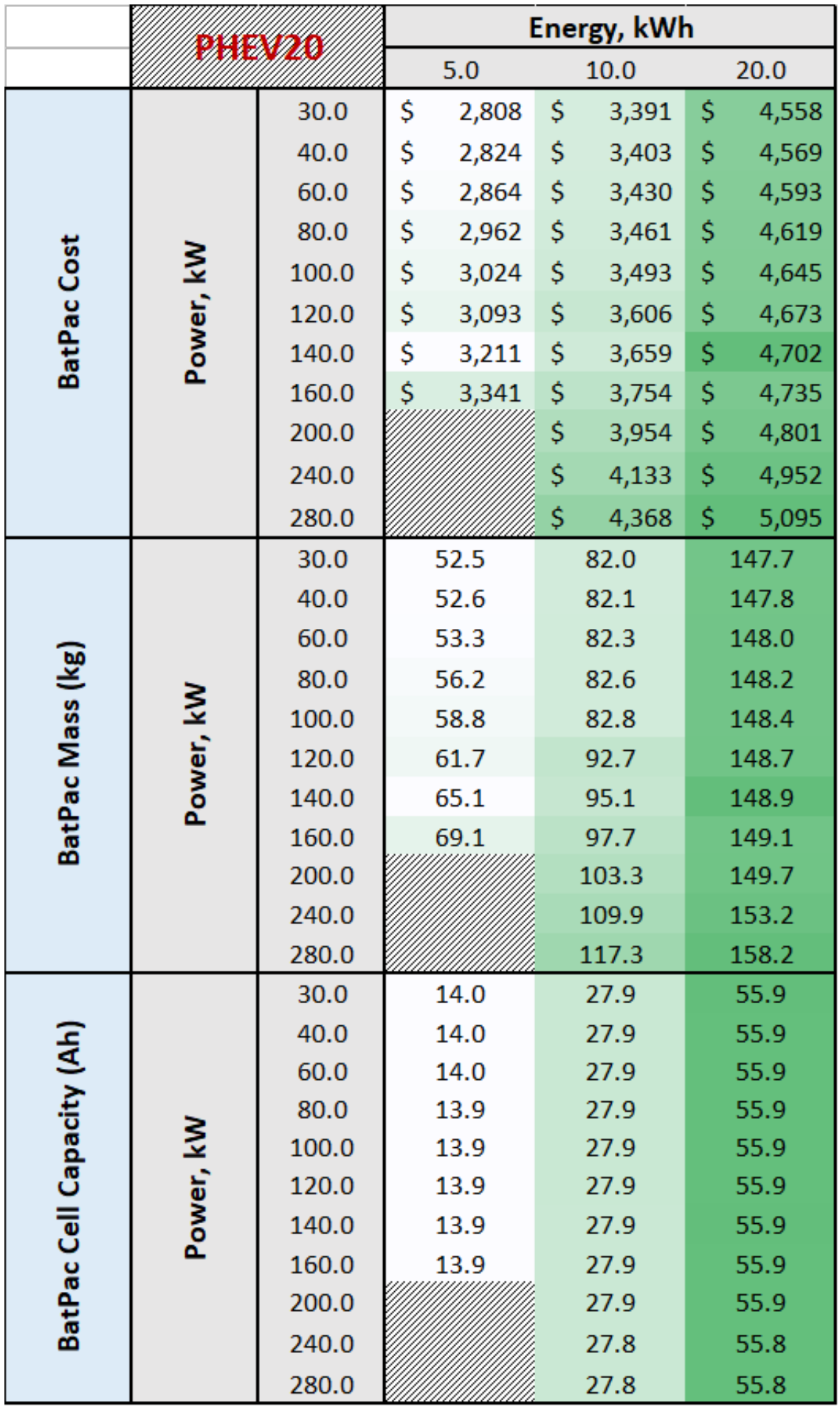

\subsubsection{PHEV50 AER (Voltec EREV/Parallel)}

Table 51 summarizes the cost, mass and cell capacities generated for compact and midsize PHEV50 AERs from $\mathrm{BatPaC}$ using the assumptions specified. 
Table 51. BatPaC Lookup Table for PHEV50 AERs (Compact/Midsize)

\begin{tabular}{|c|c|c|c|c|c|c|c|c|c|c|c|c|c|c|}
\hline & \multirow{2}{*}{\multicolumn{2}{|c|}{ 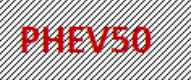 }} & \multicolumn{12}{|c|}{ Energy, kWh } \\
\hline & & & & 0.0 & & 0.0 & & 0.0 & & 0.0 & & 0.0 & & 50.0 \\
\hline \multirow{9}{*}{ 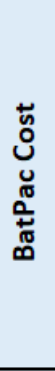 } & \multirow{9}{*}{ 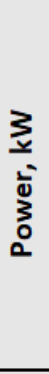 } & 60.0 & $\$$ & 4,192 & $\$$ & 5,327 & $\$$ & 6,383 & $\$$ & 7,434 & $\$$ & 8,454 & $\$$ & 9,483 \\
\hline & & 80.0 & $\$$ & 4,226 & $\$$ & 5,355 & $\$$ & 6,410 & $\$$ & 7,460 & $\$$ & 8,480 & $\$$ & 9,509 \\
\hline & & 100.0 & $\$$ & 4,260 & $\$$ & 5,384 & $\$$ & 6,437 & $\$$ & 7,487 & $\$$ & 8,507 & $\$$ & 9,535 \\
\hline & & 120.0 & $\$$ & 4,312 & $\$$ & 5,414 & $\$$ & 6,465 & $\$$ & 7,515 & $\$$ & 8,534 & $\$$ & 9,562 \\
\hline & & 140.0 & $\$$ & 4,365 & $\$$ & 5,445 & $\$$ & 6,494 & $\$$ & 7,542 & $\$$ & 8,561 & $\$$ & 9,589 \\
\hline & & 160.0 & $\$$ & 4,462 & $\$$ & 5,480 & $\$$ & 6,524 & $\$$ & 7,571 & $\$$ & 8,589 & $\$$ & 9,616 \\
\hline & & 200.0 & $\$$ & 4,667 & $\$$ & 5,551 & $\$$ & 6,586 & $\$$ & 7,629 & $\$$ & 8,645 & $\$$ & 9,672 \\
\hline & & 240.0 & $\$$ & 4,846 & $\$$ & 5,671 & $\$$ & 6,653 & $\$$ & 7,690 & $\$$ & 8,704 & $\$$ & 9,729 \\
\hline & & 280.0 & $\$$ & 5,081 & $\$$ & 5,815 & $\$$ & 6,724 & $\$$ & 7,754 & $\$$ & 8,764 & $\$$ & 9,788 \\
\hline \multirow{9}{*}{ 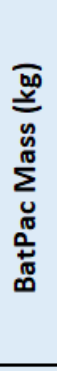 } & \multirow{9}{*}{ 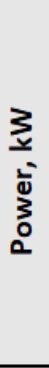 } & 60.0 & \multirow{2}{*}{\multicolumn{2}{|c|}{$\begin{array}{l}107.59 \\
107.99\end{array}$}} & \multicolumn{2}{|c|}{168.31} & \multicolumn{2}{|c|}{225.91} & \multicolumn{2}{|c|}{282.58} & \multicolumn{2}{|c|}{338.24} & \multicolumn{2}{|c|}{393.00} \\
\hline & & 80.0 & & & \multicolumn{2}{|c|}{168.65} & & 6.24 & \multicolumn{2}{|c|}{282.91} & & 8.57 & \multicolumn{2}{|c|}{393.32} \\
\hline & & 100.0 & \multicolumn{2}{|c|}{108.43} & \multicolumn{2}{|c|}{169.01} & & 6.58 & \multicolumn{2}{|c|}{283.24} & & 8.89 & \multicolumn{2}{|c|}{393.65} \\
\hline & & 120.0 & \multicolumn{2}{|c|}{109.54} & \multicolumn{2}{|c|}{169.38} & & 6.93 & \multicolumn{2}{|c|}{283.58} & & 9.23 & \multicolumn{2}{|c|}{393.98} \\
\hline & & 140.0 & \multicolumn{2}{|c|}{111.74} & \multicolumn{2}{|c|}{169.77} & & 7.29 & \multicolumn{2}{|c|}{283.93} & & 9.56 & \multicolumn{2}{|c|}{394.31} \\
\hline & & 160.0 & & 4.29 & & 0.18 & & 7.66 & & 4.28 & & 9.91 & & 94.65 \\
\hline & & 200.0 & & 0.56 & & 1.07 & & 8.43 & & 5.01 & & 0.61 & & 5.33 \\
\hline & & 240.0 & & 7.38 & & 3.57 & & 9.25 & & 5.76 & & 1.34 & & 96.04 \\
\hline & & 280.0 & & 5.21 & & 8.96 & & 0.14 & & 6.56 & & 2.09 & & 6.77 \\
\hline & & 60.0 & & 3.96 & & 7.93 & & 1.91 & & 5.88 & & 9.86 & & 3.84 \\
\hline & & 80.0 & & 3.96 & & 7.93 & & 1.91 & & 5.88 & & 9.86 & & 3.84 \\
\hline 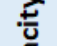 & & 100.0 & & 3.96 & & 7.93 & & 1.91 & & 5.88 & & 9.86 & & 3.84 \\
\hline$\pi$ & $\underline{\underline{y}}$ & 120.0 & & 3.96 & & 7.93 & & 1.91 & & 5.88 & & 9.86 & & 3.84 \\
\hline$\underline{=}$ & $\sum_{3}^{\bar{\nu}}$ & 140.0 & & 3.95 & & 7.93 & & 1.91 & & 5.88 & & 9.86 & & 3.84 \\
\hline U & ס & 160.0 & & 3.94 & & 7.93 & & 1.90 & & 5.88 & & 9.86 & & 3.84 \\
\hline క్ర & & 200.0 & & 3.93 & & 7.93 & & 1.90 & & 5.88 & & 9.86 & & 3.84 \\
\hline 高 & & 240.0 & & 3.92 & & 7.92 & & 1.90 & & 5.88 & & 9.86 & & 3.84 \\
\hline & & 280.0 & & 3.91 & & 7.90 & & 1.90 & & 5.88 & & 9.86 & & 3.84 \\
\hline
\end{tabular}


Table 52 summarizes the cost, mass and cell capacities generated for SUV and pickup PHEV50 AERs from $\mathrm{BatPaC}$ using the assumptions specified.

Table 52. BatPaC Lookup Table for PHEV50 AERs (SUVs/Pickups)

\begin{tabular}{|c|c|c|c|c|c|c|c|c|}
\hline & \multirow{2}{*}{\multicolumn{2}{|c|}{ 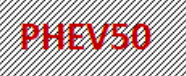 }} & \multicolumn{6}{|c|}{ Energy, kWh } \\
\hline & & & 10.0 & 20.0 & 30.0 & 40.0 & 50.0 & 60.0 \\
\hline \multirow{9}{*}{ 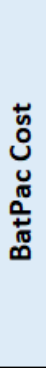 } & \multirow{9}{*}{$\begin{array}{l}\frac{3}{3} \\
\frac{\mathbf{0}}{3} \\
0 \\
0\end{array}$} & 60.0 & 4,248 & 5,385 & 6,441 & 7,493 & 8,513 & 9,543 \\
\hline & & 80.0 & 4,279 & 5,412 & 6,467 & 7,518 & 8,538 & 9,569 \\
\hline & & 100.0 & 4,314 & 5,439 & 6,494 & 7,544 & 8,564 & 9,594 \\
\hline & & 120.0 & 4,364 & 5,468 & 6,521 & 7,571 & 8,590 & 9,620 \\
\hline & & 140.0 & 4,416 & 5,498 & 6,549 & 7,597 & 8,616 & 9,646 \\
\hline & & 160.0 & 4,511 & 5,530 & 6,577 & 7,625 & 8,643 & 9,672 \\
\hline & & 200.0 & 4,714 & 5,600 & 6,637 & 7,681 & 8,698 & 9,724 \\
\hline & & 240.0 & 4,889 & 5,714 & 6,700 & 7,740 & 8,752 & 9,778 \\
\hline & & 280.0 & 5,122 & 5,856 & 6,769 & 7,801 & 8,812 & 9,835 \\
\hline \multirow{9}{*}{ 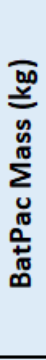 } & \multirow{9}{*}{$\begin{array}{l}\frac{3}{3} \\
\frac{\mathbf{0}}{0} \\
\frac{3}{0} \\
0\end{array}$} & 60.0 & 108.86 & 169.74 & 227.46 & 284.11 & 339.86 & 394.69 \\
\hline & & 80.0 & 109.24 & 170.07 & 227.78 & 284.42 & 340.17 & 395.00 \\
\hline & & 100.0 & 109.66 & 170.41 & 228.11 & 284.74 & 340.49 & 395.31 \\
\hline & & 120.0 & 110.79 & 170.77 & 228.44 & 285.07 & 340.80 & 395.63 \\
\hline & & 140.0 & 112.96 & 171.15 & 228.79 & 285.40 & 341.13 & 395.95 \\
\hline & & 160.0 & 115.47 & 171.54 & 229.14 & 285.74 & 341.46 & 396.27 \\
\hline & & 200.0 & 121.71 & 172.39 & 229.88 & 286.43 & 342.13 & 396.93 \\
\hline & & 240.0 & 128.50 & 174.86 & 230.67 & 287.16 & 342.83 & 397.61 \\
\hline & & 280.0 & 136.29 & 180.22 & 231.52 & 287.92 & 343.55 & 398.31 \\
\hline \multirow{9}{*}{ 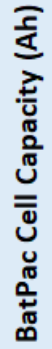 } & \multirow{9}{*}{ 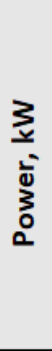 } & 60.0 & 13.41 & 26.82 & 40.23 & 53.64 & 67.06 & 80.49 \\
\hline & & 80.0 & 13.41 & 26.82 & 40.23 & 53.64 & 67.06 & 80.49 \\
\hline & & 100.0 & 13.41 & 26.82 & 40.23 & 53.64 & 67.06 & 80.49 \\
\hline & & 120.0 & 13.40 & 26.82 & 40.23 & 53.64 & 67.06 & 80.49 \\
\hline & & 140.0 & 13.39 & 26.82 & 40.23 & 53.64 & 67.06 & 80.49 \\
\hline & & 160.0 & 13.38 & 26.82 & 40.23 & 53.64 & 67.06 & 80.49 \\
\hline & & 200.0 & 13.37 & 26.82 & 40.23 & 53.64 & 67.06 & 80.49 \\
\hline & & 240.0 & 13.36 & 26.80 & 40.23 & 53.64 & 67.06 & 80.49 \\
\hline & & 280.0 & 13.36 & 26.78 & 40.23 & 53.64 & 67.06 & 80.49 \\
\hline
\end{tabular}

\subsubsection{6. $\quad B E V 200 A E R$}

Table 53 summarizes the cost, mass and cell capacities generated for compact and midsize BEV200 AERs from $\mathrm{BatPaC}$ using the assumptions specified. 
Table 53. BatPaC Lookup Table for BEV200 AERs (Compact/Midsize)

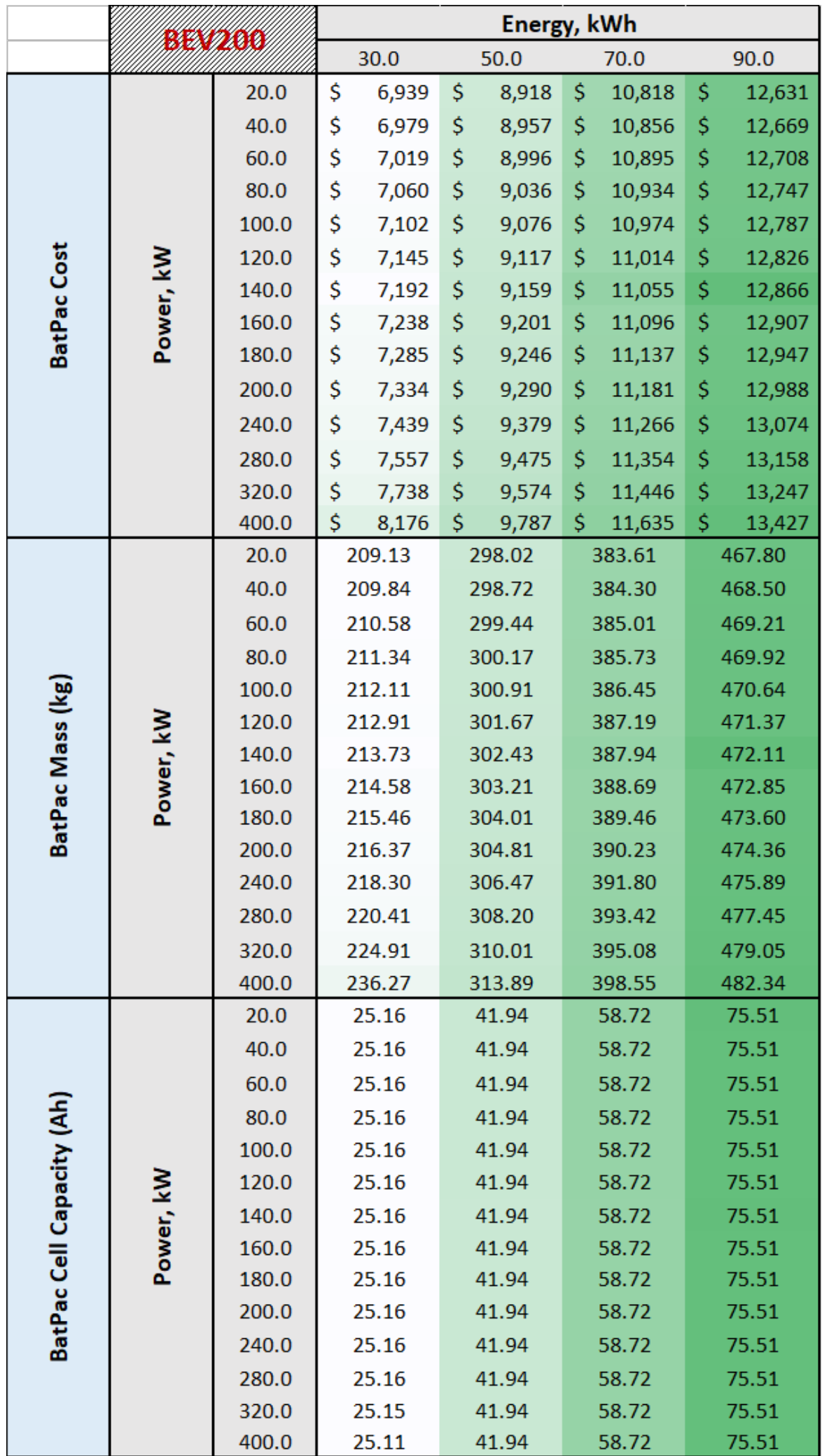


Table 54 summarizes the cost, mass and cell capacities generated for SUV and pickup BEV200 AERs from $\mathrm{BatPaC}$ using the assumptions specified.

Table 54. BatPaC Lookup Table for BEV200 AERs (SUVs/Pickup)

\begin{tabular}{|c|c|c|c|c|c|c|c|c|c|c|c|c|}
\hline & \multirow{2}{*}{\multicolumn{2}{|c|}{ 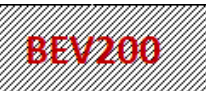 }} & \multicolumn{10}{|c|}{ Energy, kWh } \\
\hline & & & & 30.0 & & 50.0 & & 70.0 & & 90.0 & & 120.0 \\
\hline \multirow{14}{*}{ 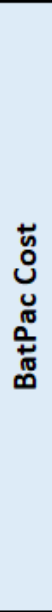 } & \multirow{14}{*}{$\begin{array}{l}\frac{3}{2} \\
\frac{1}{0} \\
3 \\
0 \\
0\end{array}$} & 20.0 & $\$$ & 7,323 & $\$$ & 9,312 & $\$$ & 11,220 & $\$$ & 13,039 & $\$$ & 15,761 \\
\hline & & 40.0 & $\$$ & 7,356 & $\$$ & 9,345 & $\$$ & 11,252 & $\$$ & 13,072 & $\$$ & 15,793 \\
\hline & & 60.0 & $\$$ & 7,390 & $\$$ & 9,378 & $\$$ & 11,285 & $\$$ & 13,105 & $\$$ & 15,826 \\
\hline & & 80.0 & $\$$ & 7,425 & $\$$ & 9,412 & $\$$ & 11,319 & $\$$ & 13,138 & $\$$ & 15,859 \\
\hline & & 100.0 & $\$$ & 7,461 & $\$$ & 9,446 & $\$$ & 11,352 & $\$$ & 13,171 & $\$$ & 15,892 \\
\hline & & 120.0 & $\$$ & 7,498 & $\$$ & 9,481 & $\$$ & 11,387 & $\$$ & 13,205 & $\$$ & 15,925 \\
\hline & & 140.0 & $\$$ & 7,535 & $\$$ & 9,516 & $\$$ & 11,421 & $\$$ & 13,239 & $\$$ & 15,959 \\
\hline & & 160.0 & $\$$ & 7,574 & $\$$ & 9,552 & $\$$ & 11,456 & $\$$ & 13,273 & $\$$ & 15,993 \\
\hline & & 180.0 & $\$$ & 7,614 & $\$$ & 9,589 & $\$$ & 11,491 & $\$$ & 13,308 & $\$$ & 16,027 \\
\hline & & 200.0 & $\$$ & 7,658 & $\$$ & 9,626 & $\$$ & 11,527 & $\$$ & 13,343 & $\$$ & 16,061 \\
\hline & & 240.0 & $\$$ & 7,746 & $\$$ & 9,702 & $\$$ & 11,599 & $\$$ & 13,413 & $\$$ & 16,130 \\
\hline & & 280.0 & $\$$ & 7,843 & $\$$ & 9,781 & $\$$ & 11,673 & $\$$ & 13,485 & $\$$ & 16,198 \\
\hline & & 320.0 & $\$$ & 7,998 & $\$$ & 9,865 & $\$$ & 11,749 & $\$$ & 13,558 & $\$$ & 16,269 \\
\hline & & 400.0 & $\$$ & 8,409 & $\$$ & 10,043 & $\$$ & 11,908 & $\$$ & 13,709 & $\$$ & 16,414 \\
\hline \multirow{14}{*}{ 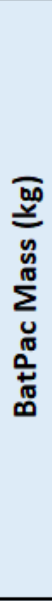 } & \multirow{14}{*}{$\begin{array}{l}\frac{3}{2} \\
\frac{1}{0} \\
3 \\
0 \\
0\end{array}$} & 20.0 & & 19.58 & & 09.68 & & 96.24 & & 480.35 & & 605.89 \\
\hline & & 40.0 & & 20.18 & & 10.26 & & 96.82 & & 480.93 & & 606.46 \\
\hline & & 60.0 & & 20.80 & & 10.87 & & 97.42 & & 781.53 & & 607.06 \\
\hline & & 80.0 & & 21.44 & & 11.48 & & 98.02 & & 482.13 & & 607.65 \\
\hline & & 100.0 & & 22.09 & & 12.11 & & 98.64 & & 82.74 & & 608.25 \\
\hline & & 120.0 & & 22.76 & & 12.74 & & 99.26 & & 483.35 & & 608.86 \\
\hline & & 140.0 & & 23.45 & & 13.39 & & 99.88 & & $\$ 83.97$ & & 609.47 \\
\hline & & 160.0 & & 24.17 & & 14.04 & & 00.52 & & 484.59 & & 610.09 \\
\hline & & 180.0 & & 24.90 & & 14.71 & & 01.16 & & 485.22 & & 610.71 \\
\hline & & 200.0 & & 25.67 & & 15.39 & & 01.81 & & 485.86 & & 611.33 \\
\hline & & 240.0 & & 27.29 & & 16.78 & & 03.13 & & 487.15 & & 612.59 \\
\hline & & 280.0 & & 29.06 & & 18.23 & & 04.49 & & 788.46 & & 613.87 \\
\hline & & 320.0 & & 32.55 & & 19.74 & & 05.88 & & 489.80 & & 615.16 \\
\hline & & 400.0 & & 43.51 & & 22.99 & & 08.80 & & 992.56 & & 617.81 \\
\hline \multirow{14}{*}{ 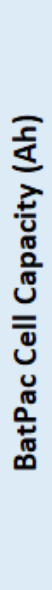 } & \multirow{14}{*}{$\frac{3}{3}$} & 20.0 & & 1.19 & & 35.32 & & 49.45 & & 63.58 & & 84.79 \\
\hline & & 40.0 & & 1.19 & & 35.32 & & 49.45 & & 63.58 & & 84.79 \\
\hline & & 60.0 & & 1.19 & & 35.32 & & 49.45 & & 63.58 & & 84.79 \\
\hline & & 80.0 & & 1.19 & & 35.31 & & 49.45 & & 63.58 & & 84.79 \\
\hline & & 100.0 & & 1.19 & & 35.31 & & 49.45 & & 63.58 & & 84.79 \\
\hline & & 120.0 & & 1.19 & & 35.31 & & 49.45 & & 63.58 & & 84.79 \\
\hline & & 140.0 & & 1.19 & & 35.31 & & 49.45 & & 63.58 & & 84.79 \\
\hline & & 160.0 & & 1.19 & & 35.31 & & 49.45 & & 63.58 & & 84.79 \\
\hline & & 180.0 & & 1.19 & & 35.31 & & 49.45 & & 63.58 & & 84.79 \\
\hline & & 200.0 & & 1.19 & & 35.31 & & 49.45 & & 63.58 & & 84.79 \\
\hline & & 240.0 & & 1.19 & & 35.31 & & 49.45 & & 63.58 & & 84.79 \\
\hline & & 280.0 & & 1.19 & & 35.31 & & 49.45 & & 63.58 & & 84.79 \\
\hline & & 320.0 & & 1.18 & & 35.31 & & 49.45 & & 63.58 & & 84.79 \\
\hline & & 400.0 & & 1.15 & & 35.31 & & 49.45 & & 63.58 & & 84.79 \\
\hline
\end{tabular}




\subsubsection{7. $\quad B E V 300 A E R$}

Table 55 summarizes the cost, mass and cell capacities generated for compact and midsize BEV300 AERs from BatPaC, using the assumptions specified in Section 5.9.3.

Table 55. BatPaC Lookup Table for BEV300 AERs (Compact/Midsize)

\begin{tabular}{|c|c|c|c|c|c|c|c|}
\hline & \multirow{2}{*}{\multicolumn{2}{|c|}{57}} & \multicolumn{5}{|c|}{ Energy, kWh } \\
\hline & & & 30.0 & 50.0 & 70.0 & 90.0 & 120.0 \\
\hline \multirow{14}{*}{ 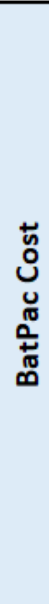 } & \multirow{14}{*}{$\frac{3}{3}$} & 20.0 & 7,323 & 9,312 & $\$ 11,220$ & 13,039 & 15,761 \\
\hline & & 40.0 & 7,356 & 9,345 & $\$ 11,252$ & 13,072 & 15,793 \\
\hline & & 60.0 & 7,390 & 9,378 & $\$ 11,285$ & 13,105 & 15,826 \\
\hline & & 80.0 & 7,425 & 9,412 & $\$ \quad 11,319$ & 13,138 & 15,859 \\
\hline & & 100.0 & 7,461 & 9,446 & $\$ 11,352$ & 13,171 & 15,892 \\
\hline & & 120.0 & 7,498 & 9,481 & $\$ 11,387$ & 13,205 & 15,925 \\
\hline & & 140.0 & 7,535 & 9,516 & $\$ \quad 11,421$ & 13,239 & 15,959 \\
\hline & & 160.0 & 7,574 & 9,552 & $\$ 11,456$ & 13,273 & 15,993 \\
\hline & & 180.0 & 7,614 & 9,589 & 11,491 & 13,308 & 16,027 \\
\hline & & 200.0 & 7,658 & 9,626 & 11,527 & 13,343 & 16,061 \\
\hline & & 240.0 & 7,746 & 9,702 & 11,599 & 13,413 & 16,130 \\
\hline & & 280.0 & 7,843 & 9,781 & $\$ 11,673$ & 13,485 & 16,198 \\
\hline & & 320.0 & 7,998 & 9,865 & $\$ 11,749$ & 13,558 & 16,269 \\
\hline & & 400.0 & 8,409 & $\$ \quad 10,043$ & $\$ 11,908$ & 13,709 & 16,414 \\
\hline \multirow{14}{*}{ 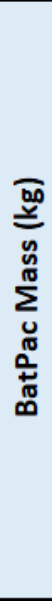 } & \multirow{14}{*}{$\frac{3}{3}$} & 20.0 & 219.58 & 309.68 & 396.24 & 480.35 & 605.89 \\
\hline & & 40.0 & 220.18 & 310.26 & 396.82 & 480.93 & 606.46 \\
\hline & & 60.0 & 220.80 & 310.87 & 397.42 & 481.53 & 607.06 \\
\hline & & 80.0 & 221.44 & 311.48 & 398.02 & 482.13 & 607.65 \\
\hline & & 100.0 & 222.09 & 312.11 & 398.64 & 482.74 & 608.25 \\
\hline & & 120.0 & 222.76 & 312.74 & 399.26 & 483.35 & 608.86 \\
\hline & & 140.0 & 223.45 & 313.39 & 399.88 & 483.97 & 609.47 \\
\hline & & 160.0 & 224.17 & 314.04 & 400.52 & 484.59 & 610.09 \\
\hline & & 180.0 & 224.90 & 314.71 & 401.16 & 485.22 & 610.71 \\
\hline & & 200.0 & 225.67 & 315.39 & 401.81 & 485.86 & 611.33 \\
\hline & & 240.0 & 227.29 & 316.78 & 403.13 & 487.15 & 612.59 \\
\hline & & 280.0 & 229.06 & 318.23 & 404.49 & 488.46 & 613.87 \\
\hline & & 320.0 & 232.55 & 319.74 & 405.88 & 489.80 & 615.16 \\
\hline & & 400.0 & 243.51 & 322.99 & 408.80 & 492.56 & 617.81 \\
\hline \multirow{14}{*}{ 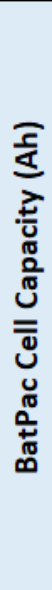 } & \multirow{14}{*}{$\frac{3}{3}$} & 20.0 & 21.19 & 35.32 & 49.45 & 63.58 & 84.79 \\
\hline & & 40.0 & 21.19 & 35.32 & 49.45 & 63.58 & 84.79 \\
\hline & & 60.0 & 21.19 & 35.32 & 49.45 & 63.58 & 84.79 \\
\hline & & 80.0 & 21.19 & 35.31 & 49.45 & 63.58 & 84.79 \\
\hline & & 100.0 & 21.19 & 35.31 & 49.45 & 63.58 & 84.79 \\
\hline & & 120.0 & 21.19 & 35.31 & 49.45 & 63.58 & 84.79 \\
\hline & & 140.0 & 21.19 & 35.31 & 49.45 & 63.58 & 84.79 \\
\hline & & 160.0 & 21.19 & 35.31 & 49.45 & 63.58 & 84.79 \\
\hline & & 180.0 & 21.19 & 35.31 & 49.45 & 63.58 & 84.79 \\
\hline & & 200.0 & 21.19 & 35.31 & 49.45 & 63.58 & 84.79 \\
\hline & & 240.0 & 21.19 & 35.31 & 49.45 & 63.58 & 84.79 \\
\hline & & 280.0 & 21.19 & 35.31 & 49.45 & 63.58 & 84.79 \\
\hline & & 320.0 & 21.18 & 35.31 & 49.45 & 63.58 & 84.79 \\
\hline & & 400.0 & 21.15 & 35.31 & 49.45 & 63.58 & 84.79 \\
\hline
\end{tabular}


Table 56 summarizes the cost, mass and cell capacities generated for SUV and pickup BEV300 AERs from $\mathrm{BatPaC}$ using the assumptions specified.

Table 56. BatPaC Lookup Table for BEV300 AERs (SUVs/Pickups)

\begin{tabular}{|c|c|c|c|c|c|c|c|c|c|c|c|c|c|c|c|c|}
\hline & \multirow{2}{*}{\multicolumn{2}{|c|}{ 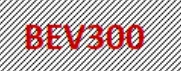 }} & \multicolumn{14}{|c|}{ Energy, kWh } \\
\hline & & & & 0.0 & & 50.0 & & 70.0 & & 90.0 & & 20.0 & & 40.0 & & 60.0 \\
\hline \multirow{14}{*}{ 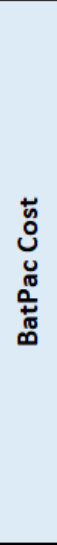 } & \multirow{14}{*}{ 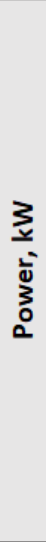 } & 20.0 & $\$$ & 7,570 & $\$$ & 9,565 & $\$$ & 11,478 & $\$$ & 13,302 & $\$$ & 15,977 & $\$$ & 17,758 & $\$$ & 19,481 \\
\hline & & 40.0 & $\$$ & 7,600 & $\$$ & 9,595 & $\$$ & 11,508 & $\$$ & 13,332 & $\$$ & 16,006 & $\$$ & 17,788 & $\$$ & 19,510 \\
\hline & & 60.0 & $\$$ & 7,631 & $\$$ & 9,626 & $\$$ & 11,538 & $\$$ & 13,362 & $\$$ & 16,036 & $\$$ & 17,817 & $\$$ & 19,539 \\
\hline & & 80.0 & $\$$ & 7,663 & $\$$ & 9,656 & $\$$ & 11,568 & $\$$ & 13,392 & $\$$ & 16,066 & $\$$ & 17,847 & $\$$ & 19,569 \\
\hline & & 100.0 & $\$$ & 7,695 & $\$$ & 9,688 & $\$$ & 11,599 & $\$$ & 13,422 & $\$$ & 16,096 & $\$$ & 17,877 & $\$$ & 19,599 \\
\hline & & 120.0 & $\$$ & 7,729 & $\$$ & 9,719 & $\$$ & 11,628 & $\$$ & 13,451 & $\$$ & 16,125 & $\$$ & 17,906 & $\$$ & 19,627 \\
\hline & & 140.0 & $\$$ & 7,763 & $\$$ & 9,749 & $\$$ & 11,659 & $\$$ & 13,482 & $\$$ & 16,155 & $\$$ & 17,936 & $\$$ & 19,658 \\
\hline & & 160.0 & $\$$ & 7,798 & $\$$ & 9,782 & $\$$ & 11,691 & $\$$ & 13,513 & $\$$ & 16,186 & $\$$ & 17,967 & $\$$ & 19,688 \\
\hline & & 180.0 & $\$$ & 7,835 & $\$$ & 9,815 & $\$$ & 11,723 & $\$$ & 13,545 & $\$$ & 16,217 & $\$$ & 17,997 & $\$$ & 19,719 \\
\hline & & 200.0 & $\$$ & 7,872 & $\$$ & 9,849 & $\$$ & 11,756 & $\$$ & 13,576 & $\$$ & 16,248 & $\$$ & 18,028 & $\$$ & 19,749 \\
\hline & & 240.0 & $\$$ & 7,952 & $\$$ & 9,918 & $\$$ & 11,821 & $\$$ & 13,640 & $\$$ & 16,309 & $\$$ & 18,088 & $\$$ & 19,809 \\
\hline & & 280.0 & $\$$ & 8,038 & $\$$ & 9,990 & $\$$ & 11,889 & $\$$ & 13,704 & $\$$ & 16,372 & $\$$ & 18,151 & $\$$ & 19,872 \\
\hline & & 320.0 & $\$$ & 8,186 & $\$$ & 10,064 & $\$$ & 11,956 & $\$$ & 13,770 & $\$$ & 16,437 & $\$$ & 18,215 & $\$$ & 19,935 \\
\hline & & 400.0 & $\$$ & 8,581 & $\$$ & 10,224 & $\$$ & 12,100 & $\$$ & 13,907 & $\$$ & 16,569 & $\$$ & 18,343 & $\$$ & 20,061 \\
\hline \multirow{14}{*}{ 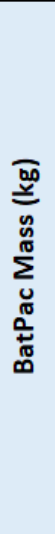 } & \multirow{14}{*}{ 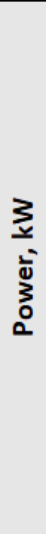 } & 20.0 & & 6.44 & & 317.31 & & 04.52 & & 89.17 & & 14.87 & & 97.40 & & 79.19 \\
\hline & & 40.0 & & 6.99 & & 17.84 & & 05.04 & & 189.68 & & 15.39 & & 97.92 & & 79.70 \\
\hline & & 60.0 & & 17.55 & & 18.38 & & 05.58 & & 90.22 & & 15.92 & & 98.45 & & 80.23 \\
\hline & & 80.0 & & 8.12 & & 18.94 & & 06.12 & & 90.76 & & 16.46 & & 98.99 & & 80.76 \\
\hline & & 100.0 & & 8.71 & & 19.50 & & 06.68 & & 91.31 & & 17.01 & & 99.53 & & 81.30 \\
\hline & & 120.0 & & 9.32 & & 20.08 & & 07.24 & & 91.86 & & 17.56 & & 00.08 & & 81.85 \\
\hline & & 140.0 & & 9.94 & & 20.66 & & 07.80 & & 192.42 & & 18.11 & & 00.62 & & 82.39 \\
\hline & & 160.0 & & 0.59 & & 21.25 & & 08.38 & & 92.98 & & 18.66 & & 01.18 & & 82.94 \\
\hline & & 180.0 & & 1.25 & & 21.86 & & 08.96 & & 93.55 & & 19.22 & & 01.73 & & 83.50 \\
\hline & & 200.0 & & 1.94 & & 22.47 & & 09.55 & & 94.12 & & 19.79 & & 02.29 & & 84.05 \\
\hline & & 240.0 & & 3.41 & & 23.73 & & 10.74 & & 95.29 & & 20.93 & & 03.42 & & 85.17 \\
\hline & & 280.0 & & 5.00 & & 25.04 & & 11.97 & & 96.48 & & 22.08 & & 04.56 & & 86.30 \\
\hline & & 320.0 & & 8.50 & & 26.41 & & 13.23 & & 197.68 & & 23.25 & & 05.72 & & 87.45 \\
\hline & & 400.0 & & 9.19 & & 29.34 & & 15.86 & & 00.18 & & 25.65 & & 08.07 & & 89.77 \\
\hline \multirow{14}{*}{ 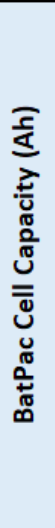 } & \multirow{14}{*}{ 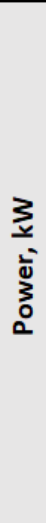 } & 20.0 & & 9.17 & & 31.95 & & 44.74 & & 57.53 & & 6.71 & & 9.51 & & 02.31 \\
\hline & & 40.0 & & 9.17 & & 31.95 & & 44.74 & & 57.52 & & 6.71 & & 9.51 & & 02.30 \\
\hline & & 60.0 & & 9.17 & & 31.95 & & 44.74 & & 57.52 & & 6.71 & & 9.50 & & 02.30 \\
\hline & & 80.0 & & 9.17 & & 31.95 & & 44.74 & & 57.52 & & 6.71 & & 9.50 & & 02.30 \\
\hline & & 100.0 & & 9.17 & & 31.95 & & 44.74 & & 57.52 & & 6.71 & & 9.50 & & 02.30 \\
\hline & & 120.0 & & 9.17 & & 31.95 & & 44.74 & & 57.52 & & 6.71 & & 9.50 & & 02.30 \\
\hline & & 140.0 & & 9.17 & & 31.95 & & 44.74 & & 57.52 & & 6.71 & & 9.50 & & 02.30 \\
\hline & & 160.0 & & 9.17 & & 31.95 & & 44.74 & & 57.52 & & 6.71 & & 9.50 & & 02.30 \\
\hline & & 180.0 & & 9.17 & & 31.95 & & 44.74 & & 57.52 & & 6.71 & & 9.50 & & 02.30 \\
\hline & & 200.0 & & 9.17 & & 31.95 & & 44.74 & & 57.52 & & 6.71 & & 9.50 & & 02.30 \\
\hline & & 240.0 & & 9.17 & & 31.95 & & 44.74 & & 57.52 & & 6.71 & & 9.50 & & 02.30 \\
\hline & & 280.0 & & 9.17 & & 31.95 & & 44.74 & & 57.52 & & 6.71 & & 9.50 & & 02.30 \\
\hline & & 320.0 & & 9.16 & & 31.95 & & 44.74 & & 57.52 & & 6.71 & & 9.50 & & 02.30 \\
\hline & & 400.0 & & 9.13 & & 31.95 & & 44.74 & & 57.52 & & 6.71 & & 9.50 & & 02.30 \\
\hline
\end{tabular}

\subsection{Accessory Loads}

The electrical and mechanical accessory base load is assumed to be constant over the drive cycles, with values varying by powertrain type. Derived from AMTL data, this value is used to represent the average accessory load consumed during the standard urban FTP and EPA's HWFET drive-cycle dynamometer testing. For the current set of runs, NHTSA has directed Argonne to vary the base 
accessory loads for different vehicle classes and performance categories across the different vehicle powertrains. Table 57 shows the base accessory load assumptions by vehicle class and performance category across the different vehicle powertrains.

Table 57. Base Accessory Load Assumptions

\begin{tabular}{|l|l|c|c|c|}
\hline \multirow{2}{*}{ Vehicle Class } & \multirow{2}{*}{$\begin{array}{c}\text { Performance } \\
\text { Category }\end{array}$} & \multicolumn{3}{c|}{ Vehicle Powertrain } \\
\cline { 3 - 5 } & Conventional & HEVs & PHEVs and BEVs \\
\hline Compact & Base & 250 & 275 & 375 \\
\hline Compact & Premium & 300 & 375 & 475 \\
\hline Midsize & Base & 250 & 275 & 375 \\
\hline Midsize & Premium & 300 & 375 & 475 \\
\hline Small SUV & Base & 300 & 325 & 425 \\
\hline Small SUV & Premium & 300 & 375 & 475 \\
\hline Midsize SUV & Base & 300 & 325 & 425 \\
\hline Midsize SUV & Premium & 350 & 375 & 475 \\
\hline Pickup & Base & 300 & 325 & 425 \\
\hline Pickup & Premium & 300 & 375 & 475 \\
\hline
\end{tabular}

5.11. Light-Weighting Technologies

For the current set of analysis runs, DOT/NHTSA proposed that light-weighting be applied to the glider weight as defined by the body and chassis only. For final rulemaking, the agencies decided to use the definition of the glider weight defined by the following systems:

- Body

- Chassis

- Interior

- Safety system

- Brake mechanism

- Steering system

- Mechanical accessories

- Electrical accessories

- Wheels

Light-weighting is applied across all glider systems except for those related to safety.

The secondary effects of light-weighting (such as downsizing) are taken into account as part of the vehicle sizing algorithm. To emphasize technology platform sharing in the study, vehicles with lower levels of mass reduction (5\% and $7.5 \%)$ inherit the sizing characteristics of the reference vehicle $(0 \%$ mass reduction). Vehicles with higher levels of mass reduction are resized to meet the vehicle technical specifications. The percentages of mass reductions selected for the study are as follows:

- Light-weighting level 0 (MR0): $0 \%$ (reference vehicle is sized)

- Light-weighting level 1 (MR1): 5\% (inherited from reference vehicle)

- Light-weighting level 2 (MR2): 7.5\% (inherited from reference vehicle)

- Light-weighting level 3 (MR3): 10\% (vehicle is sized) 
- Light-weighting level 4 (MR4): $15 \%$ (vehicle is sized)

- Light-weighting level 5 (MR5): 20\% (vehicle is sized)

- Light-weighting level 6 (MR6): 30\% (vehicle is sized)

\subsection{Aerodynamic Reduction Technologies}

Along with different levels of light-weighting reductions, five levels of aerodynamic reduction have been applied. The percentages of aerodynamic reduction selected for the study are as follows:

- Aerodynamic reduction level 0 (AERO0): 0\%

- Aerodynamic reduction level 1 (AERO1): 5\%

- Aerodynamic reduction level 2 (AERO2): $10 \%$

- Aerodynamic reduction level 3 (AERO3): 15\%

- Aerodynamic reduction level 4 (AERO4): $20 \%$

\subsection{Rolling Resistance Reduction Technologies}

For this study, three levels of rolling resistance reduction have been applied for each vehicle configuration. The percentages of rolling resistance reduction selected for the study are as follows:

- Rolling resistance-reduction level 0 (ROLL0): $0 \%$

- Rolling resistance-reduction level 1 (ROLL1): 10\%

- Rolling resistance-reduction level 2 (ROLL2): $20 \%$ 


\section{TEST PROCEDURE AND ENERGY CONSUMPTION CALCULATIONS}

All simulations were performed under hot conditions. Cold-start penalties were assessed after the simulations, based on test data collected at AMTL and EPA published values. A two-cycle test procedure, based on the urban dynamometer driving schedule (UDDS) and highway fuel economy driving schedule (HWFET) drive cycles [30], was used.

\subsection{Conventional Vehicles}

The conventional vehicle test procedure follows the current EPA two-cycle test procedure (U.S. Environmental Protection Agency, 2018).

The urban cycle for a non-hybrid vehicle (Figure 181) has four parts:

1. Bag 1: cold start

2. Bag 2: stop and go

3. Engine $\mathrm{OFF}$

4. Bag 3: hot start

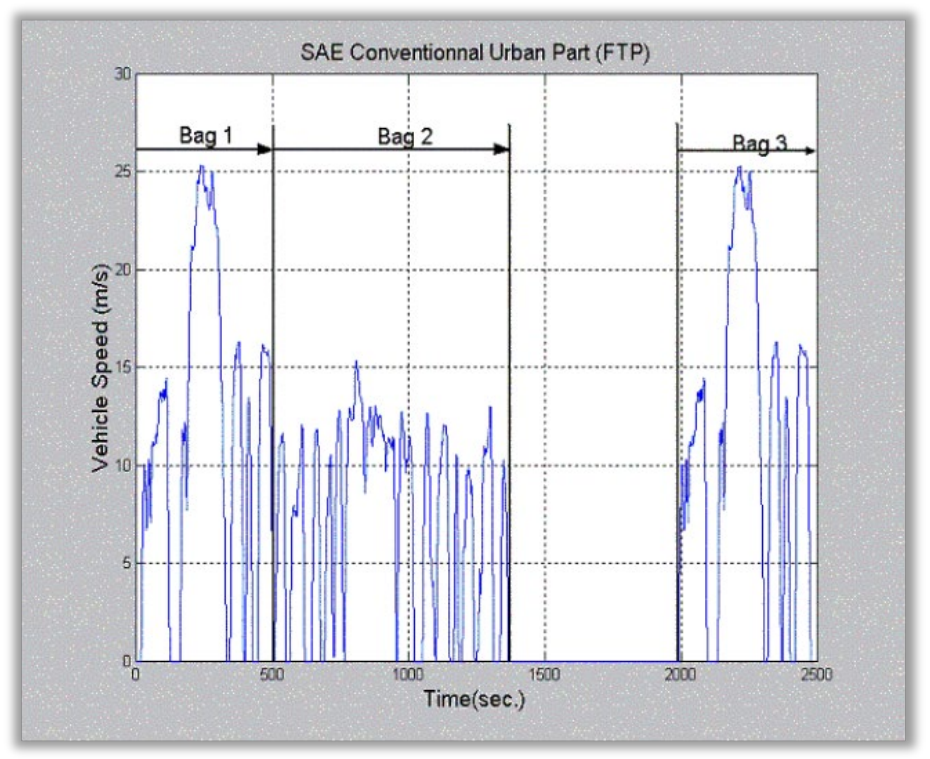

Figure 181. The urban cycle for a non-hybrid vehicle

The highway cycle for a non-hybrid vehicle has only one part, the HWFET (Figure 182). 


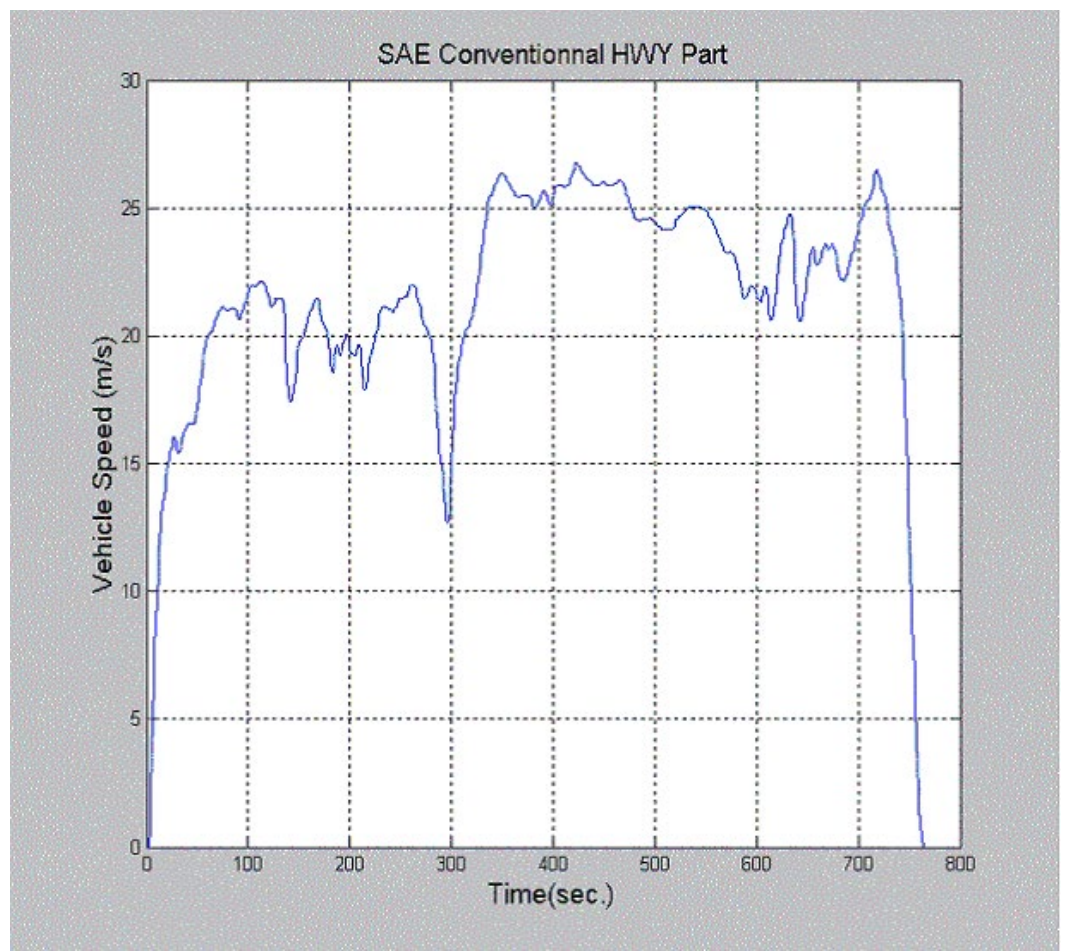

Figure 182. The highway cycle for a non-hybrid vehicle

\subsection{Hybrid Electric Vehicles}

The HEV procedure is similar to the conventional vehicle procedure except that the drive cycles are repeated until the initial and final battery SOCs are within a tolerance of $0.5 \%$ (see Figure 183 and Figure 184), based on SAE J1711 procedure [7]. This procedure is used for mild hybrid BISG, split HEV, par HEV and FC HEV vehicle powertrains.

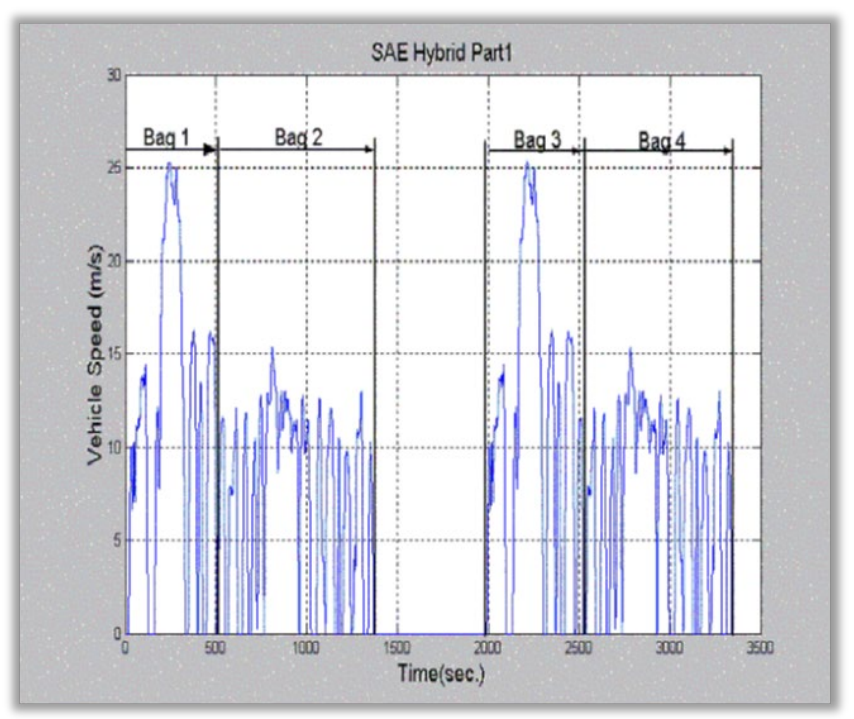

Figure 183. The urban cycle for a hybrid vehicle 


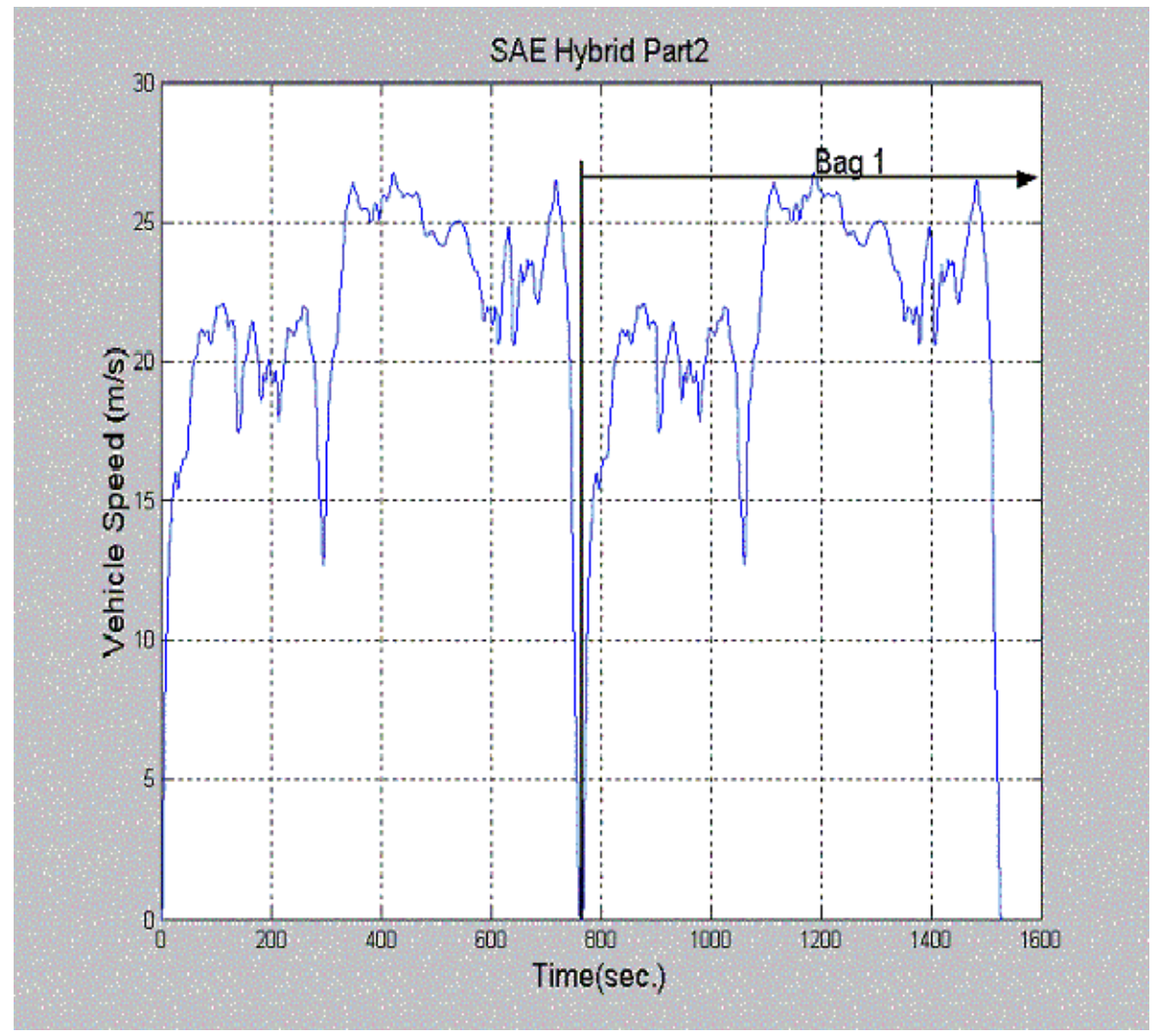

Figure 184. The highway cycle for a hybrid vehicle (only the results from the second cycle were used)

\subsubsection{Fuel Consumption}

For the urban cycle, fuel consumption was computed using Equation 1:

(1) Fuel Consumption $=0.43 \frac{V_{\text {Fuel }}^{1}+V_{\text {Fuel }}^{2}}{\text { Dist }_{1}+\text { Dist }_{2}}+0.57 \frac{V_{\text {Fuel }}^{3}+V_{\text {Fuel }}^{Z}}{\text { Dist }_{3}+\text { Dist }_{Z}}$

Where

$V_{\text {Fuel }}^{y}=$ Volume of fuel from Bag y

Dist $_{y}=$ Distance driven by the vehicle for the Bag y part of the cycle

$Z=$ Bag 2 for a non-hybrid vehicle and Bag 4 for a hybrid.

The same equation was used to compute the gas-equivalent fuel consumption as well as the SOCadjusted fuel consumption by replacing $V_{F u e l}$ with the corresponding physical quantity.

The highway cycle results were the same as for an urban cycle, except for the hybrid case, in which only the results from Bag 1 were used to compute the values:

(2) Fuel Consumption $=\frac{V_{\text {Fuel }}^{2}}{\text { Dist }}$ 


\subsubsection{Combined Fuel Consumption}

The combined fuel consumption is a weighted value lying between the urban and highway cycles:

(3) $F C^{\text {combined }}=0.55 \times F C^{\text {urban }}+0.45 \times F C^{\text {highway }}$

\subsection{Plug-in Hybrid Electric Vehicles}

This section describes the methodology currently implemented in Autonomie for PHEVs. This procedure is used for split PHEV20, EREV PHEV50, par PHEV20 and par PHEV50 vehicle powertrains. The implementation is based on the $\mathrm{J} 1711$ procedure. The procedure is divided into several phases, as described below.

\subsubsection{1. $\quad$ Charge Sustaining on the UDDS Cycle}

- Set battery SOC to charge sustaining (CS) value.

- Run UDDS.

- 10-minute soak with the key OFF.

- Run UDDS.

- Assume the cycle charge is balanced. Display warning if it does not meet $1 \%$.

\section{Weightings and Cold Factor Correction}

The following equations show the cold compensation:

(4) $M_{0-505}^{*}=\frac{M_{0-505}}{1-C F_{75 F}}$

Where

$M_{0-505}=$ fuel mass consumed during the time window between 0 and $505 \mathrm{sec}$

$C F_{75 F}=$ cold-factor correction at $75^{\circ} \mathrm{F}$

$M_{0-505}^{*}=$ cold-corrected mass of fuel

(5) $\operatorname{Vol}_{0-505}^{*}=\frac{M_{0-505}^{*}}{\delta_{\text {gasoline }}}$

Where

$V_{o l} l_{0-505}^{*}=$ volume of fuel consumed during the time window between 0 and $505 \mathrm{sec}$, and $\delta_{\text {gasoline }}=$ density of gasoline .

One can then calculate FC ${ }^{\text {UDDS }}$, the fuel consumed on the UDDS cycle:

(6) $F C^{U D D S}=0.43 \times\left(\frac{V_{0 l_{0-505}^{*}+V_{0 l} l_{506-1372}}}{D_{0-505}+D_{506-1372}}\right)+0.57 \times\left(\frac{V_{0 l_{1972-2477}+V o l_{2478-3340}}}{D_{1972-2477}+D_{2478-3340}}\right)$ 


\subsubsection{2. $\quad$ Charge Sustaining on the HWFET Cycle}

- Set battery SOC to CS value.

- Run HWFET.

- Wait four seconds.

- Run HWFET.

- Assume the cycle is charge balanced.

- Perform calculations on the second HWFET cycle.

$$
F C^{H W F E T}=\frac{V^{H o l_{765-1529}}}{D_{765-1529}}
$$

Where

$\operatorname{Vol}_{765-1529}=$ volume of fuel consumed during the time window between 765 and 1,529 sec

$D_{765-1529}=$ distance traveled during the time window between 765 and $1,529 \mathrm{sec}$

$F C^{H W F E T}=$ highway fuel consumption

\subsubsection{3. $\quad$ Charge Depleting on the UDDS and HWFET Cycles}

- The charge-depleting (CD) calculations are identical for the UDDS and HWFET cycles.

- Set battery SOC to full charge test initial SOC.

- Run UDDS (HWFET).

- $\quad 10$-minute soak with the key OFF (15-second pause with key ON).

- Run UDDS (HWFET).

- 10-minute soak with the key off (15-second pause with key ON).

- Repeat until SOC reaches the CD/CS crossover point, and the last cycle is completed.

- Round down the number of cycles unless the CD range is less than one cycle. In that case, round up the number of cycles. At least on CD cycle is required to run the analysis.

\section{Cold Weighting Calculation}

The user specifies the number of cycles over which to apply the cold correction factor:

(8) $N_{\text {cold }}=\min \left(N_{\text {cold }}^{\text {user }}, N_{c d}\right)$

(9) $N_{\text {hot }}=N_{c d}-N_{\text {cold }}$

Where

$N_{\text {cold }}=$ number of cold cycles

$N_{\text {hot }}=$ number of hot cycles

$N_{\text {cold }}^{\text {user }}=$ number of user-specified cold cycles

$N_{c d}=$ total number of CD cycles

$$
M_{c d}=\left[\frac{\alpha_{c o l d} M_{c d-c o l d}^{1}}{1-C F_{75 F}}, \cdots, \frac{\alpha_{c o l d} M_{c d-c o l d}^{N_{c o l d}}}{1-C F_{75 F}}, \alpha_{\text {hot }} M_{c d-h o t}^{1}, \cdots, \alpha_{\text {hot }} \mathrm{M}_{c d-h o t}^{N_{h o t}}\right]^{T}
$$


Where

$M_{c d-c o l d}^{1}=$ mass of fuel consumed during the first cold CD cycle

$M_{c d-c o l d}^{N_{\text {cold }}}=$ mass of fuel consumed during the last cold CD cycle

$C F_{75 F}=$ cold-start fuel economy penalty at $75^{\circ} \mathrm{F}$

$M_{c d-h o t}^{1}=$ mass of fuel consumed during the first hot CD cycle

$\mathrm{M}_{c d-h o t}^{N_{\text {hot }}}=$ mass of fuel consumed during the last hot CD cycle

$\alpha_{\text {cold }}=$ user-specified cold weighting factor (default value $\left.=0.43\right)$

$\alpha_{\text {hot }}=$ user-specified hot weighting factor (default value $\left.=0.57\right)$

$M_{c d}=$ column vector of cold-corrected fuel mass

$$
\operatorname{Vol}_{c d}=\frac{M_{c d}}{\delta_{\text {gasoline }}}
$$

Where

$V o l_{c d}=$ column vector of cold-corrected fuel volumes

Note that each element in the $\operatorname{Vol}_{c d}$ vector is divided by its respective distance:

(12) $\quad F C_{c d}=\frac{V o l_{c d}}{D_{u d d s}}$

Where

$F C_{c d}=$ column vector of cold-corrected fuel consumption

The net battery energy used was calculated for each cycle using the open-circuit voltage and the current:

$$
\text { for } i=1, \cdots, N_{c d} ; E_{c d}^{i}=\int_{(i-1) T_{u d d s}}^{(i) T_{u d d s}+t} V_{o c}(\tau) \times I(\tau) d \tau
$$

Where

$E_{c d}^{i}=$ net battery energy used during the $i^{\text {th }} \mathrm{CD}$ cycle

$T_{u d d s}=$ duration of the UDDS cycle + soak time or $($ HWFET $+15 \mathrm{sec})$

$i=$ index of the CD cycle

$N_{c d}=$ total number of CD cycles

$V_{o c}=$ open-circuit voltage as a function of time during the cycle

$I=$ battery current as a function of time during the cycle

$$
E_{c d}=\left[E_{c d}^{1}, \cdots, E_{c d}^{N_{c d}}\right]^{T}
$$

Where

$E_{c d}=$ column vector of net battery energy used on each cycle 
Note that each element in the $E_{c d}$ vector is divided by its respective distance:

$$
E C_{c d}=\frac{E_{c d}}{D_{u d d s} \times \eta_{c h g}^{e s s} \times \eta_{\text {charger }}}
$$

Where

$E C_{c d}=$ column vector of electrical-energy consumption in AC-Joules (wall outlet)

$D_{u d d s}=$ distance traveled on a UDDS (or HWFET $\left.-D_{H W F E T}\right)$ cycle

$\eta_{c h g}^{e s s}=$ user-definable efficiency of the battery during charging $($ default value $=0.99)$

$\eta_{\text {charger }}=$ user-definable efficiency of the charger (wall or in-vehicle) $($ default value $=0.88)$

$$
\begin{gathered}
\text { for } i=1, \cdots, N_{c d} ; \mu_{i}=\mu\left(i \times D_{u d d s}^{i}\right)-\mu(i-1) \times D_{u d d s}^{i} \\
\mu_{c d}=\left[\mu_{1}, \cdots, \mu_{N_{c d}}\right]
\end{gathered}
$$

Where

$\mu_{c d}=$ row vector of utility factors

$\mu_{1}=$ utility factor on the first CD cycle

$\mu_{i}=$ utility factor on the $i^{\text {th }} \mathrm{CD}$ cycle

$\mu_{N_{c d}}=$ utility factor on the last CD cycle

$\mu=$ fleet mileage fraction utility factor as a function of distance

$$
F C=\mu_{c d} F C_{c d}+\left(1-\sum_{i}^{N_{c d}} \mu_{i}\right) F C_{c s}
$$

Where

$F C=$ fuel consumed on the city or highway portion of the PHEV procedure

$$
E C=\mu_{c d} E C_{c d}
$$

Where

$E C=$ electrical energy consumed during the city or highway portion of the PHEV procedure

\subsection{Electric Vehicles (BEVs)}

For BEVs, the SAE J1634 test procedure is followed (Light Duty Vehicle Performance and Economy Measure Committee, 2017): start the battery at full SOC and run until minimum SOC is reached. The electric consumption is then computed as:

$$
\text { Electric Consumption }=\frac{\int V_{\text {oc }} \times I_{\text {ess }}}{\eta_{\text {ess }} \eta_{\text {charger }}}
$$

Where

$\eta_{\text {ess }}=$ efficiency of the battery while charging

$\eta_{\text {charger }}=$ average efficiency of the charger while charging 
$V_{o c}=$ open-circuit voltage as a function of time over the cycle

$I_{e s s}=$ current as a function of time over the cycle

The SAE J1634 multi-cycle test (MCT) consists of repeated UDDS and HWFET cycles combined with constant-speed driving phases. The drive cycles are used to determine the energy consumption and range associated with specific and established driving patterns. The constant-speed driving schedules in the middle and the end of the test are intended to: 1) reduce test duration by depleting the battery more rapidly than the established certification drive schedules, 2) improve the robustness of the energy determination by minimizing the impact of drive style variation, and 3) prevent inconsistent triggering of EOT criteria that can occur at high power-demand points when a BEV is following a dynamic drive schedule at low states-of-charge.

Figure 185 illustrates how multiple drive cycles (UDDS, HWFET and constant-speed cycle) are combined in the combo MCT test.

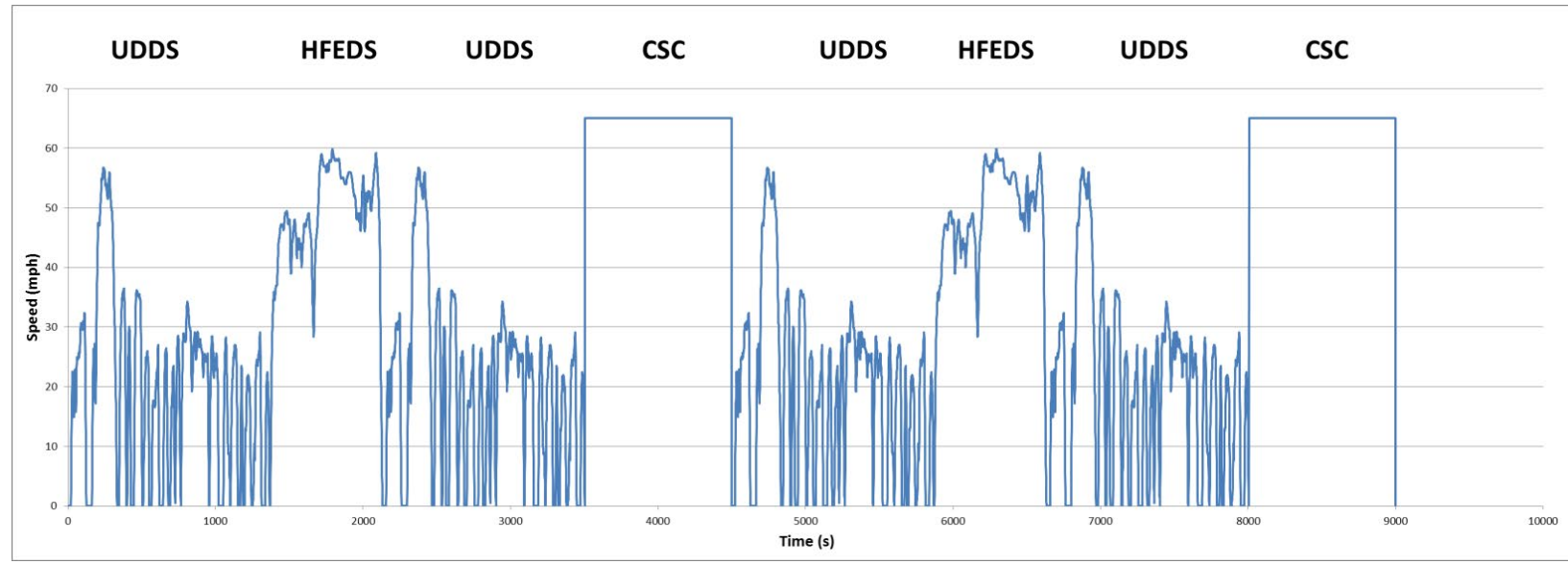

Figure 185. Multi-cycle test

The multi-cycle test enables the determination of the cycle-specific range as well as the measurement of the cycle-specific energy consumption. The range of the driving cycle is determined using both the energy consumption of the cycle and the usable battery energy of the vehicle.

\subsection{Cold-Start Penalty}

The EPA database of MY 2020 light-duty vehicles [31] was analyzed for fuel economy values reported for the different bags. Following an average of the ratio of Bag 3 fuel economy to Bag 1 fuel economy, an appropriate cold-start penalty was selected to be implemented. Figure 186 shows the ratio of Bag 3 fuel economy to Bag 1 fuel economy across different engine technologies for MY 2020 vehicles. 

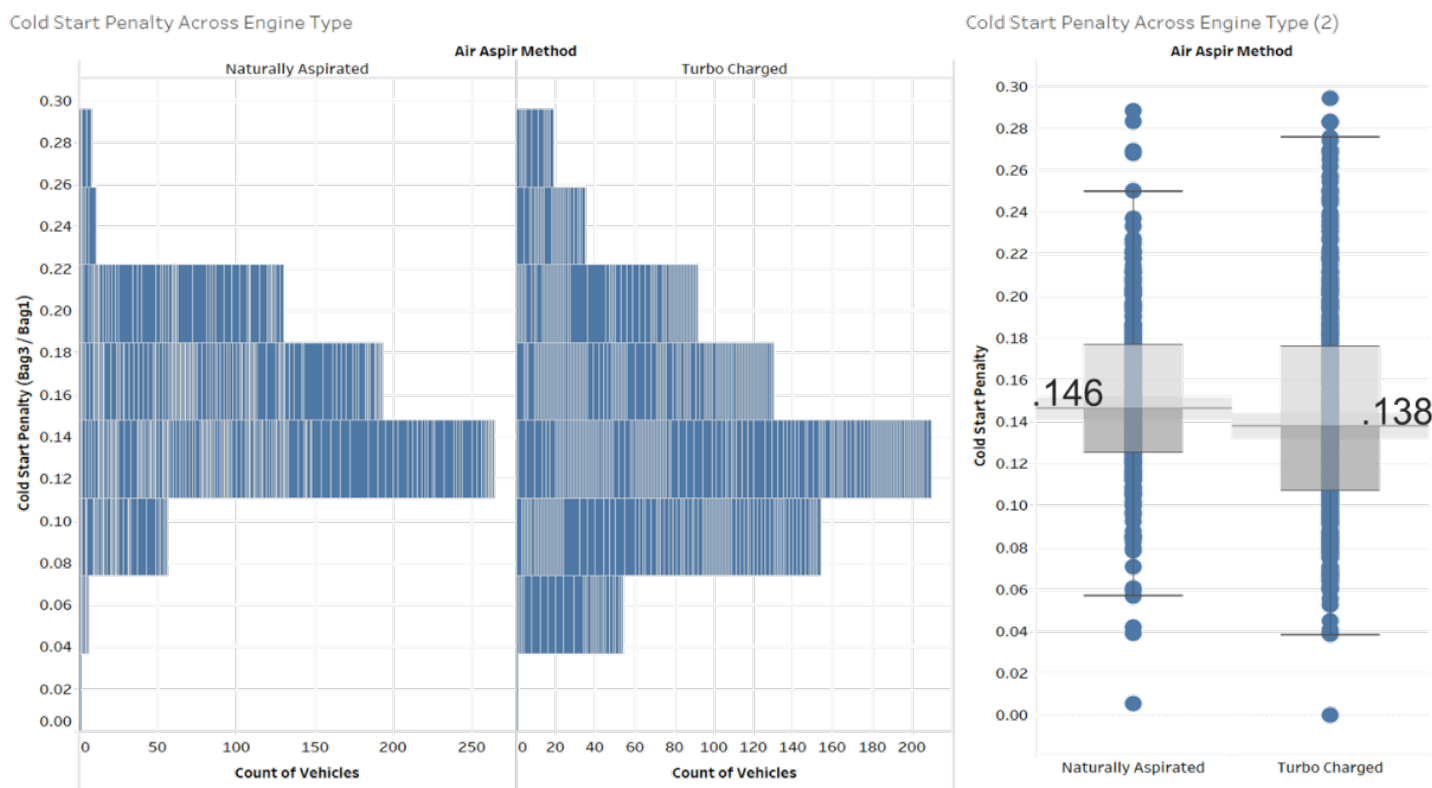

Figure 186. Cold-start penalty on Bag 1 across different engine types

The figure shows that different engine aspiration methods influence the cold-start penalty on Bag 1 . Therefore, we decided to separate out the cold-start penalty on Bag 1 fuel economy associated with the different engine types. As with the Bag 1 cold-start penalty, we evaluated the effect of the additional penalty on Bag 2, as shown in Figure 187.
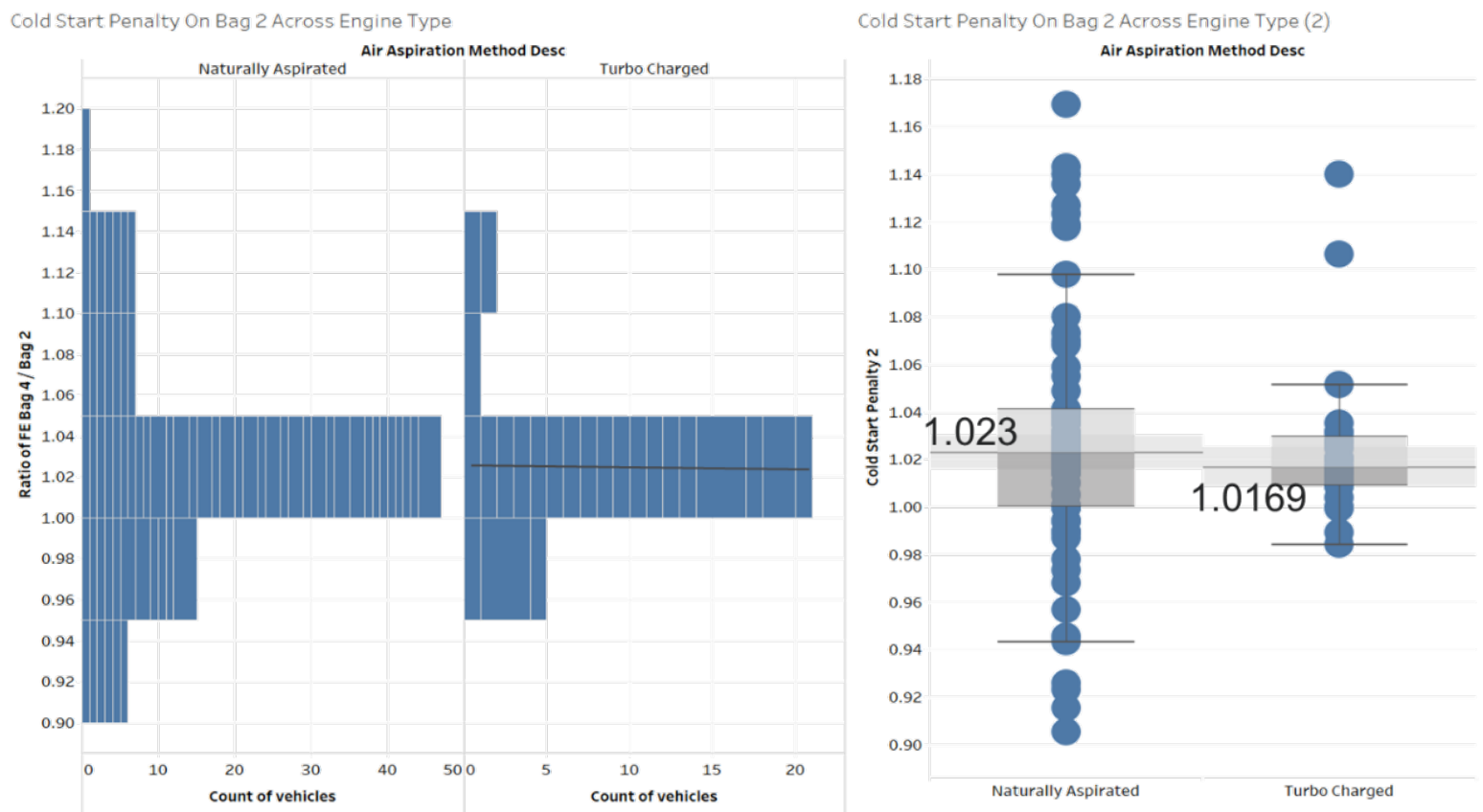

Figure 187. Cold-start penalty on Bag 2 across engine types 
As with the case of the Bag 1 cold-start penalty, Figure 187 shows the influence of the different engine types on the additional cold-start penalty on Bag 2. Based on our detailed analysis, we determined that we will use the combinations of cold-start penalties in FY21 runs for light-duty vehicles shown in Table 58.

\begin{tabular}{|c|c|c|}
\hline $\mathrm{NA} / \mathrm{TC}^{\mathrm{a}}$ & Bag & Penalty (\%) \\
\hline & Bag 1 & 14.6 \\
\hline & Bag 2 & 2.3 \\
\hline & Bag 1 & 13.8 \\
\hline & Bag 2 & 1.7 \\
\hline
\end{tabular}

${ }^{\mathrm{a}} \mathrm{NA}=$ naturally aspirated; $\mathrm{TC}=$ turbo-charged .

The cold-start penalty was applied to fuel consumption during the FTP for conventional vehicles, HEVs, and PHEVs; 0\% was applied for BEVs. 


\section{VEHICLE SIMULATION SETUP PROCESS}

Argonne's large-scale simulation process was developed to run a very large number of vehicle simulations quickly and effectively, allowing Argonne to quickly respond to DOT requests by simulating any technology combination in any vehicle class. The following subsections describe the steps in the process.

\subsection{Powertrain Template}

Powertrain templates contain basic information such as name, class, and technology, as well as component information such as battery technology, engine technology, and transmission type. To automate the differentiated process of the different powertrain options, three different templates are defined: conventional, parallel hybrids, and other hybrids (power-split HEV/PHEV, EREV, fuel cell, and $\mathrm{BEV})$.

The template contains seven tabs: Vehicle, Parameter, Control, Sizing, Run, Translation, and Assumptions. In each tab, columns outline vehicle configurations.

\subsubsection{Vehicle Tab}

The Vehicle tab of the template defines the initialization files, the component models required for each vehicle, and the vehicle configuration selected. The initialization files selected will depend on the CAFE decision tree selected and the technological combination nominated for that vehicle. Figure 188 shows the Vehicle tab of the conventional template.

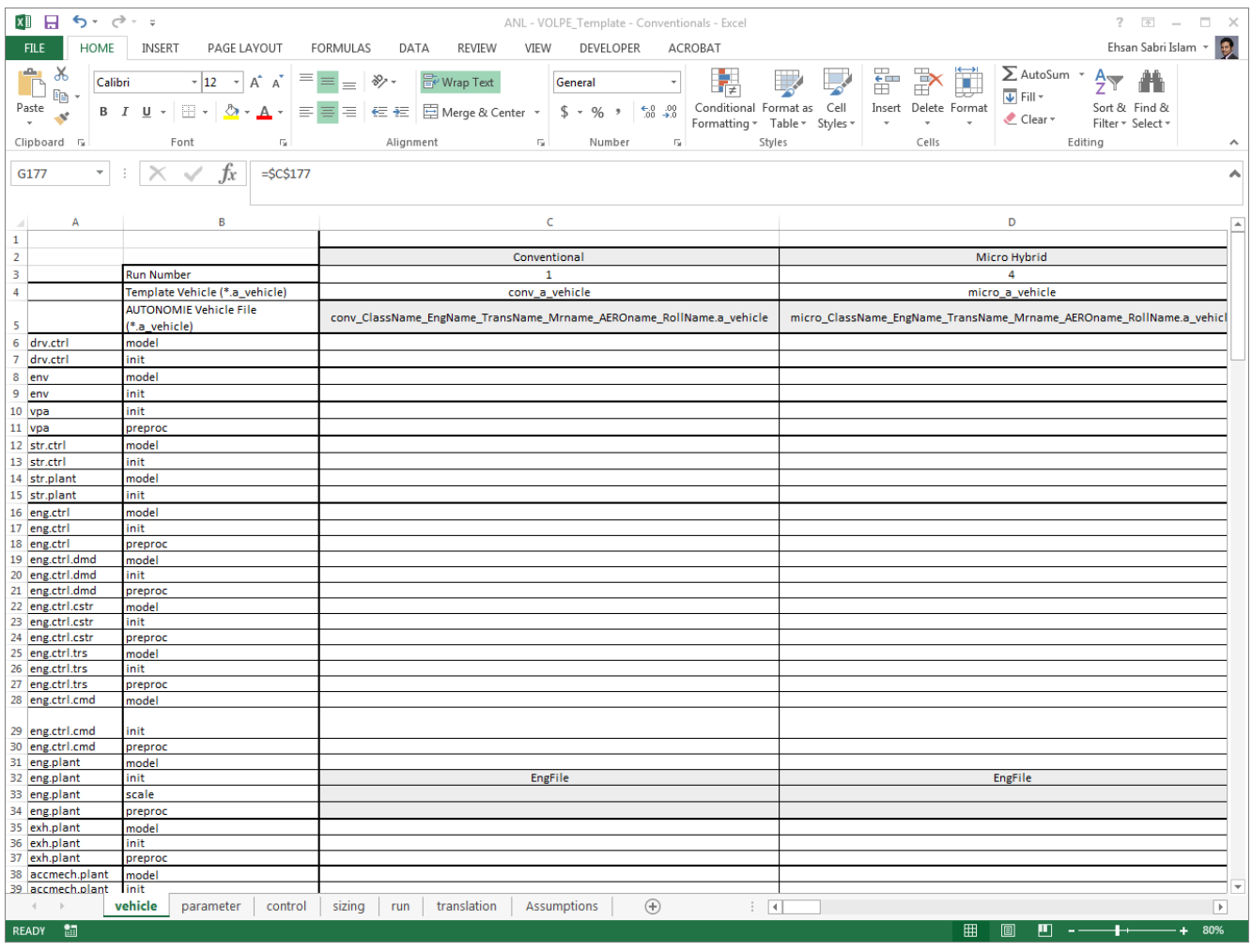

Figure 188. Vehicle tab of conventional template 


\subsubsection{Parameter Tab}

The Parameter tab of the template defines the values of the components specific to the vehicle designated (e.g., power, weight, performance constraints). Figure 189 shows the Parameter tab of the conventional template.

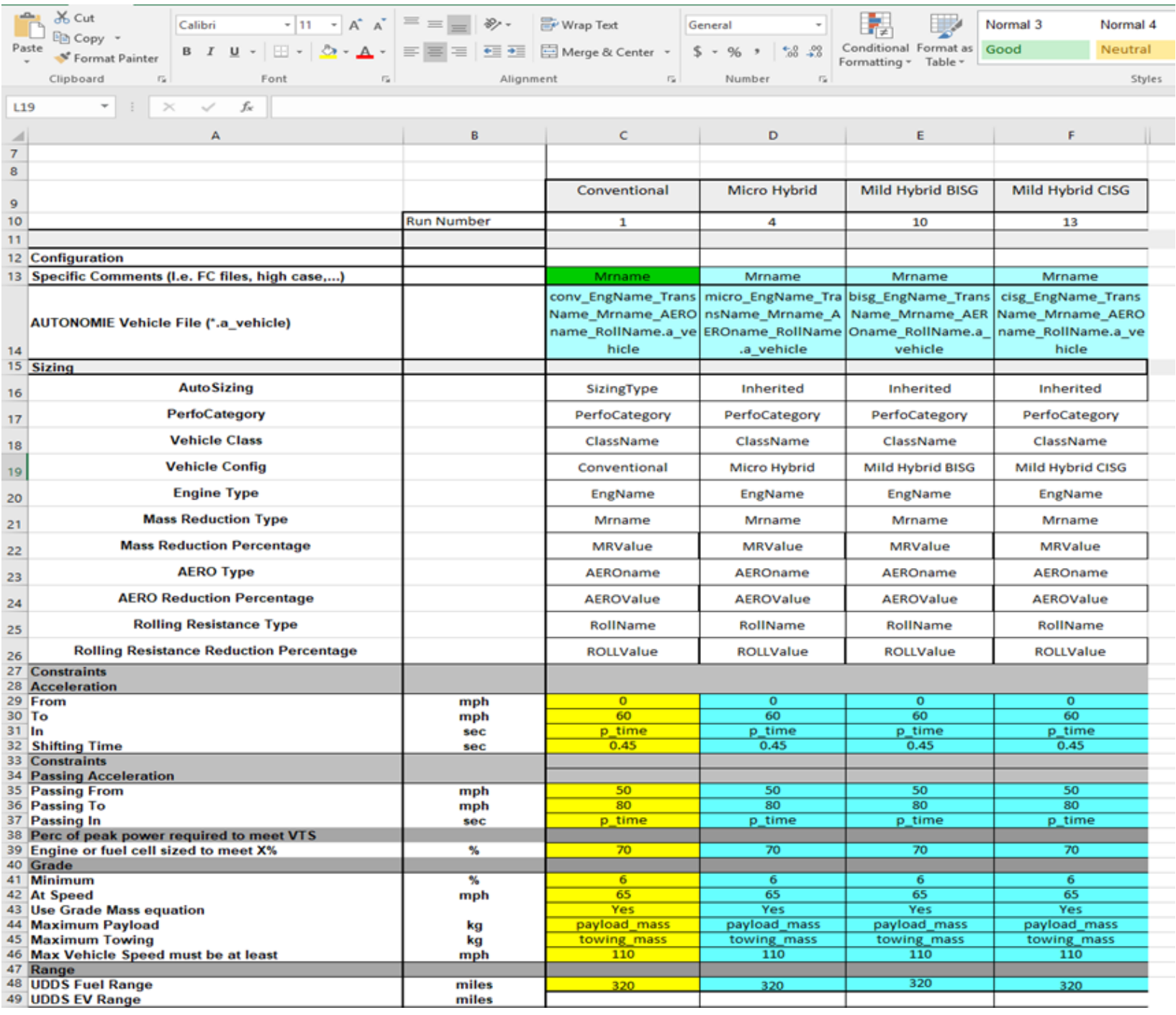

Figure 189. Parameter tab of conventional template 


\subsubsection{Control Tab}

The control tab selects the appropriate controller for the designated vehicle. Figure 189 shows the Control tab of the hybrid template.

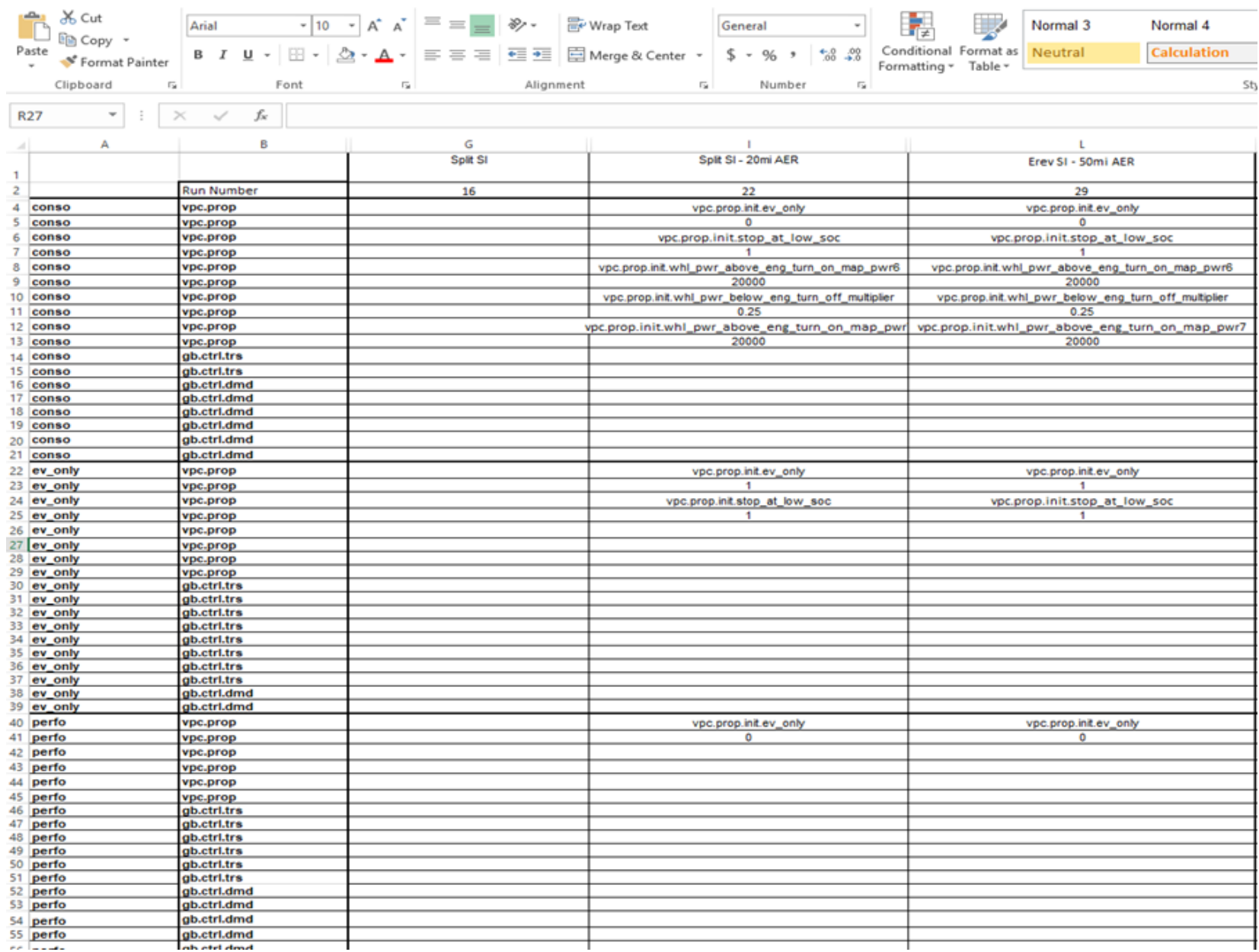

Figure 190. Control tab of hybrid template 


\subsubsection{Sizing Tab}

The Sizing tab selects the appropriate sizing rule to size each component to match the required vehicle technical specifications. Figure 191 shows the Sizing tab of the hybrid template.

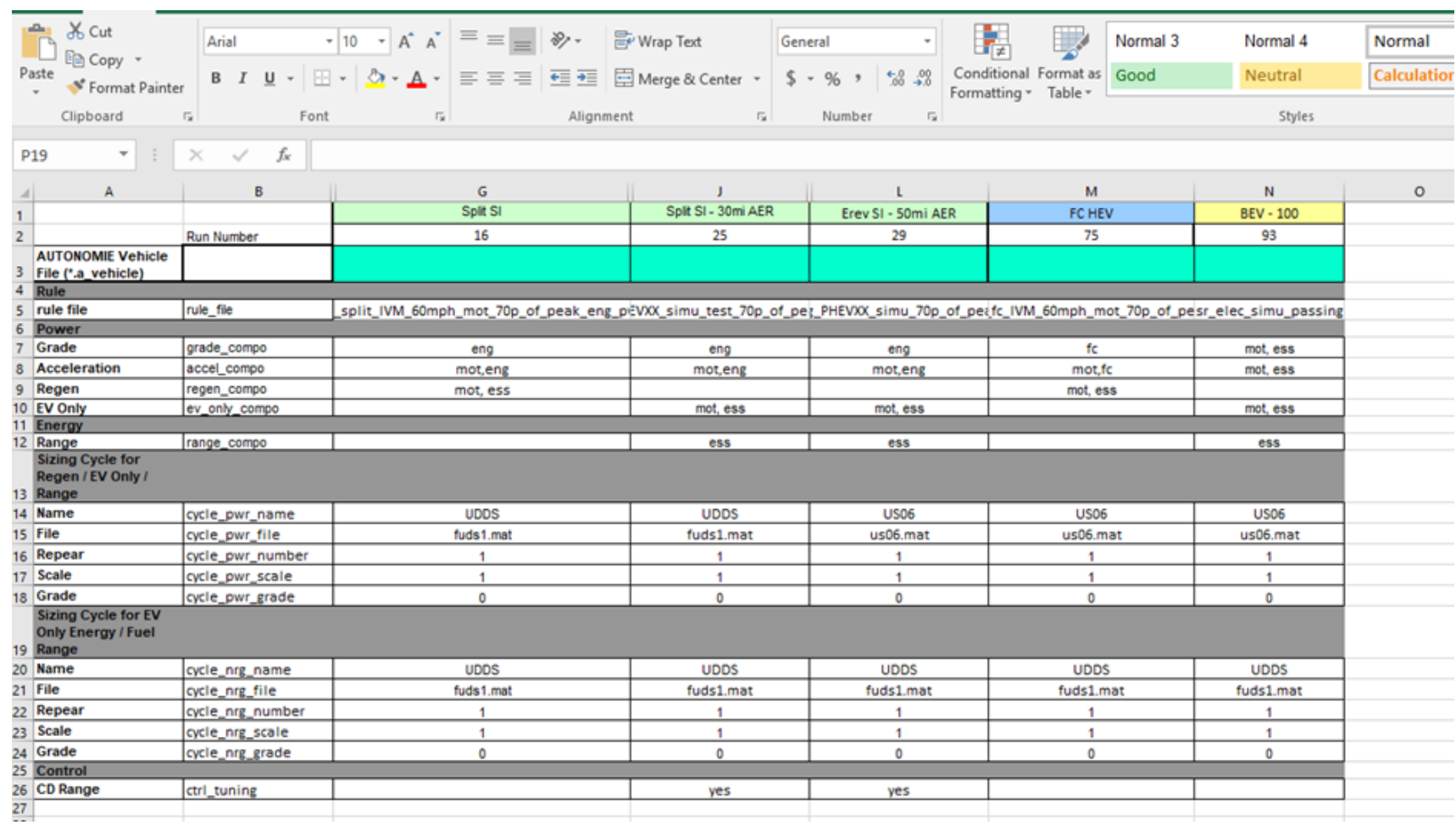

Figure 191. Sizing tab of hybrid template 


\subsubsection{Run Tab}

The Run tab selects the drive cycle/procedure to be simulated for each powertrain options. Figure 192 shows the Run tab of the hybrid template.

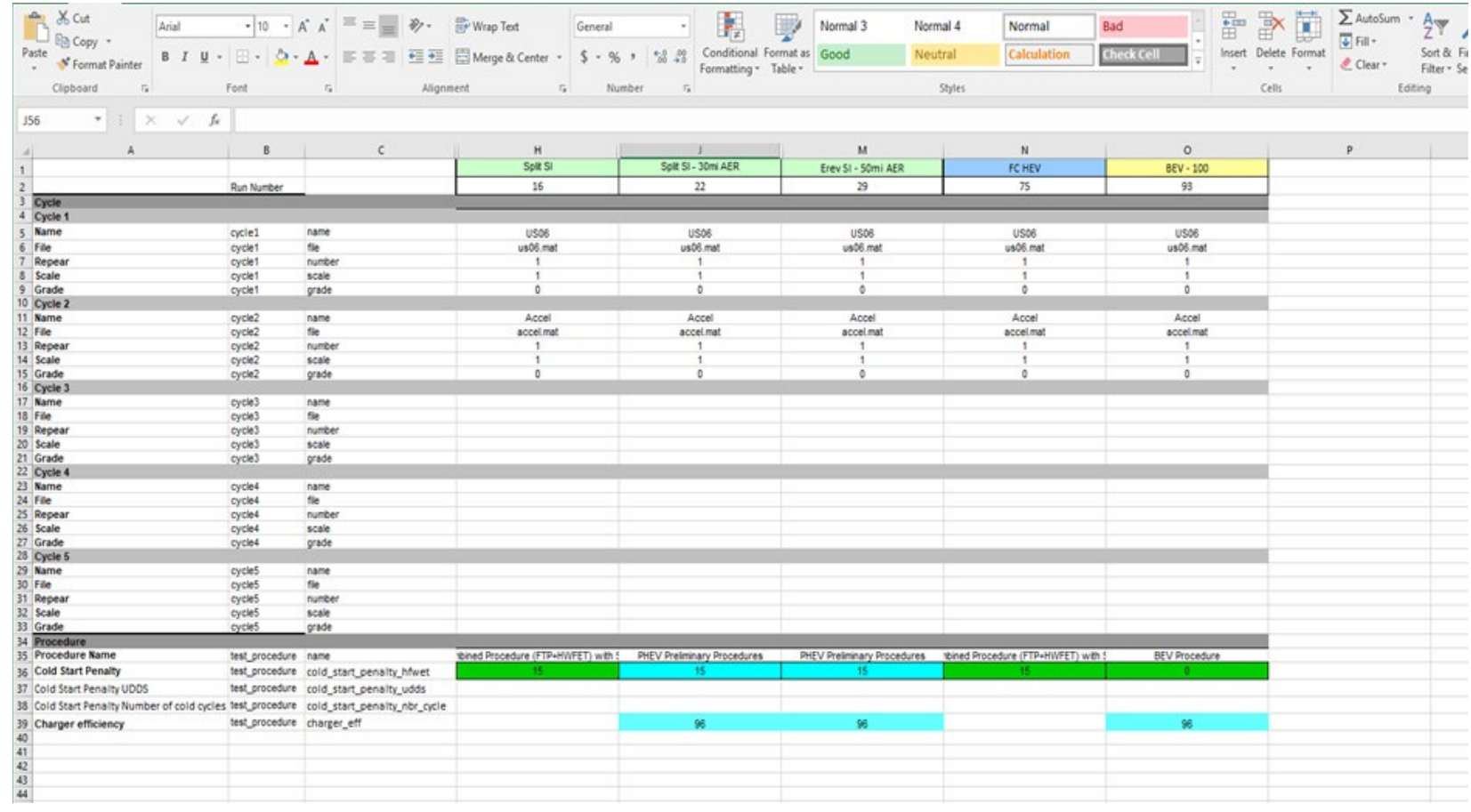

Figure 192. Run tab of hybrid template 


\subsubsection{Translation Tab}

The Translation tab, shown in Figure 193, translates and transfers inputs into Autonomie to build the vehicle model.

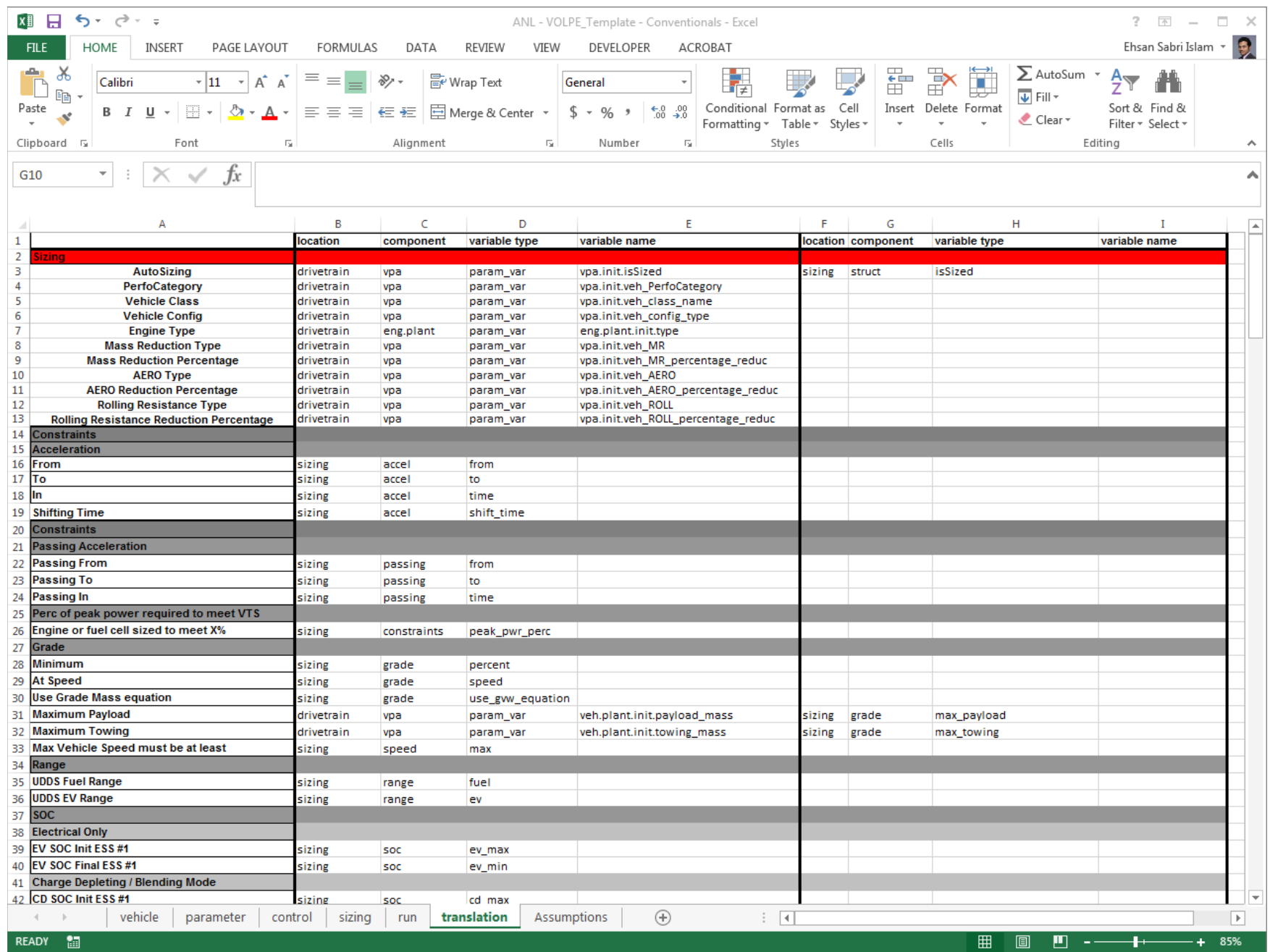

Figure 193. Translation tab of template

\subsection{Multi-Vehicle Template Expansion and Duplication}

After the large-scale simulation process defines the list of all component and vehicle inputs, a multiplier code expands the reference/template file into as many as needed to define the vehicle's technological combinations based on the decision tree inputs. This step stores all of the template files in the folders for each vehicle class and performance category. Figure 194 shows the vehicle template files for the different vehicle classes and performance categories in their respective folders. 


\begin{tabular}{|c|c|c|c|c|c|c|c|}
\hline \multicolumn{2}{|l|}{ Name } & ^ & \multicolumn{2}{|c|}{ Date modified } & \multicolumn{2}{|c|}{ Type } & Size \\
\hline \multicolumn{2}{|l|}{ 听 } & NV_eng01_5AU_MRO_AERO0_ROLLO & \multicolumn{2}{|c|}{ 11/8/2017 7:01 PM } & \multicolumn{2}{|c|}{ Microsoft Excel W... } & $125 \mathrm{~KB}$ \\
\hline 1] Compag & \multicolumn{2}{|l|}{ Name } & & \multicolumn{2}{|l|}{ Date modified } & Type & Size \\
\hline 风 Compad & \multicolumn{3}{|c|}{ 1]. Compact_Perfo_CONV_eng01_5AU_MRO_AEROO_ROLLO } & \multicolumn{2}{|c|}{ 11/9/2017 1:02 AM } & Microsoft Excel W... & $125 \mathrm{~KB}$ \\
\hline termpoy & \multicolumn{3}{|c|}{ 1. Compact Perfo CONV ena01 5AU MRO AEROO ROLL1 } & \multicolumn{2}{|c|}{ 11/9/20171:02 AM } & Microsoft Excel W... & $125 \mathrm{~KB}$ \\
\hline तi:c & \. Compa & \multicolumn{4}{|l|}{ Name } & Date modified & Type \\
\hline (1) Compad & Compa & \multicolumn{4}{|c|}{ 凶] Midsize_NonPerfo_CONV_eng01_SAU_MRO_AEROO_ROLLO } & 11/7/2017 10:49 PM & Microsoft Exc \\
\hline 风] Compad & Compa & \multicolumn{4}{|c|}{ (].] Midsize_NonPerfo_CONV_eng01_SAU_MRO_AEROO_ROLL1 } & 11/7/2017 10:49 PM & Microsoft Exc \\
\hline (1) Compad & 1. Compa & \multicolumn{4}{|c|}{ [] Midsize_NonPerfo_CONV_eng01_SAU_MRO_AEROO_ROLL2 } & 11/7/2017 10:49 PM & Microsoft Exc \\
\hline (1) Compad & ब. Compa & \multicolumn{4}{|c|}{ 凶] Midsize_NonPerfo_CONV_eng01_SAU_MRO_AERO1_ROLLO } & 11/7/2017 10:49 PM & Microsoft Exc \\
\hline बit Compad & ] Compa & \multicolumn{4}{|c|}{ \] Midsize_NonPerfo_CONV_eng01_5AU_MRO_AERO1_ROLL1 } & 11/7/2017 10:49 PM & Microsoft Exc \\
\hline (1) Compad & (1) Compa & \multicolumn{4}{|c|}{ ג] Midsize_NonPerfo_CONV_eng01_5AU_MRO_AERO1_ROLL2 } & 11/7/2017 10:49 PM & Microsoft Exc \\
\hline बi Compad & (1) Compa & \multicolumn{4}{|c|}{ 【] Midsize_NonPerfo_CONV_eng01_SAU_MRO_AERO2_ROLLO } & 11/7/2017 10:49 PM & Microsoft Exc \\
\hline बi Compad & [1] Compa & \multicolumn{4}{|c|}{ (1] Midsize_NonPerfo_CONV_eng01_5AU_MRO_AERO2_ROLL1 } & 11/7/2017 10:49 PM & Microsoft Exc \\
\hline बi Compad & [1] Compa & \multicolumn{4}{|c|}{ (1) Midsize_NonPerfo_CONV_eng01_5AU_MRO_AERO2_ROLL2 } & 11/7/2017 10:49 PM & Microsoft Exc \\
\hline (]) Compad & (1) Compa & \multicolumn{4}{|c|}{ ג] Midsize_NonPerfo_CONV_eng01_SAU_MRO_AERO3_ROLLO } & 11/7/2017 10:49 PM & Microsoft Exc \\
\hline 冈 Compad & 1] Compa & \multicolumn{4}{|c|}{ 1] Midsize_NonPerfo_CONV_eng01_SAU_MRO_AERO3_ROLL1 } & 11/7/2017 10:49 PM & Microsoft Exc \\
\hline & ג] Compa & \multicolumn{4}{|c|}{ (1] Midsize_NonPerfo_CONV_eng01_5AU_MRO_AERO3_ROLL2 } & 11/7/2017 10:49 PM & Microsoft Exc \\
\hline 1: Compad & D. Compa & (1) Midsize_NonPerfo_CONV_eng0 & $5 \mathrm{AU}_{-}$ & RO_AERO4_ROLLO & & 11/7/2017 10:50 PM & Microsoft Exc \\
\hline A: Compad & (] Compa & (1) Midsize_NonPerfo_CONV_eng0 & $5 \mathrm{AU}_{-}$ & 20_AERO4_ROLL1 & & 11/7/2017 10:50 PM & Microsoft Exc \\
\hline ब Con & Compa & (1) Midsize_NonPerfo_CONV_eng0 & $5 \mathrm{AU}_{-}$ & RO_AERO4_ROLL2 & & 11/7/2017 10:50 PM & Microsoft Exc \\
\hline a] Compad & acc & ब] Midsize_NonPerfo_CONV_eng0 & $5 A U_{-}$ & R1_AEROO_ROLLO & & 11/7/2017 10:50 PM & Microsoft Exc \\
\hline बit Combad & i. Compa & (1) Midsize_NonPerfo_CONV_eng0 & $5 \mathrm{AU}_{-}$ & 21_AERO0_ROLL1 & & 11/7/2017 10:50 PM & Microsoft Exc \\
\hline 32,490 items & 凶it Compa & ג] Midsize_NonPerfo_CONV_eng0 & $5 \mathrm{AU}_{-}$ & 21_AERO0_ROLL2 & & 11/7/2017 10:50 PM & Microsoft Exc \\
\hline & 34,110 item & (1]. Midsize_NonPerfo_CONV_eng0 & $5 \mathrm{AU} \mathrm{U}_{-}$ & R1_AERO1_ROLLO & & 11/7/2017 10:50 PM & Microsoft Exc \\
\hline & & (1]. Midsize_NonPerfo_CONV_eng0 & $5 \mathrm{AU}_{-}$ & R1_AERO1_ROLL1 & & 11/7/2017 10:50 PM & Microsoft Exc \\
\hline & & (1) Midsize_NonPerfo_CONV_eng0 & $5 \mathrm{AU}_{-}$ & 21_AERO1_ROLL2 & & 11/7/2017 10:50 PM & Microsoft Exc \\
\hline & & (1]. Midsize_NonPerfo_CONV_eng0 & $5 \mathrm{AU}_{-}$ & 21_AERO2_ROLLO & & 11/7/2017 10:50 PM & Microsoft Exc \\
\hline & & $\begin{array}{l}\text { 1.: Midsize NonPerfo CONV ena0 } \\
32,490 \text { items }\end{array}$ & & AERO2 ROLL1 & & 11/7/2017 10:50 PM & Microsoft Exc \\
\hline
\end{tabular}

Figure 194. Multiple vehicle template files

\subsection{Vehicle Assumptions Definition}

An automated process has been developed to read and write vehicle attributes and weight assumptions into template files to minimize manual inputs. Figure 195 shows the vehicle assumption inputs for different vehicle attributes and weights for different vehicle classes and performance categories. The code reads the different vehicle attributes and weights and updates the template file for each vehicle class and performance category folder. 


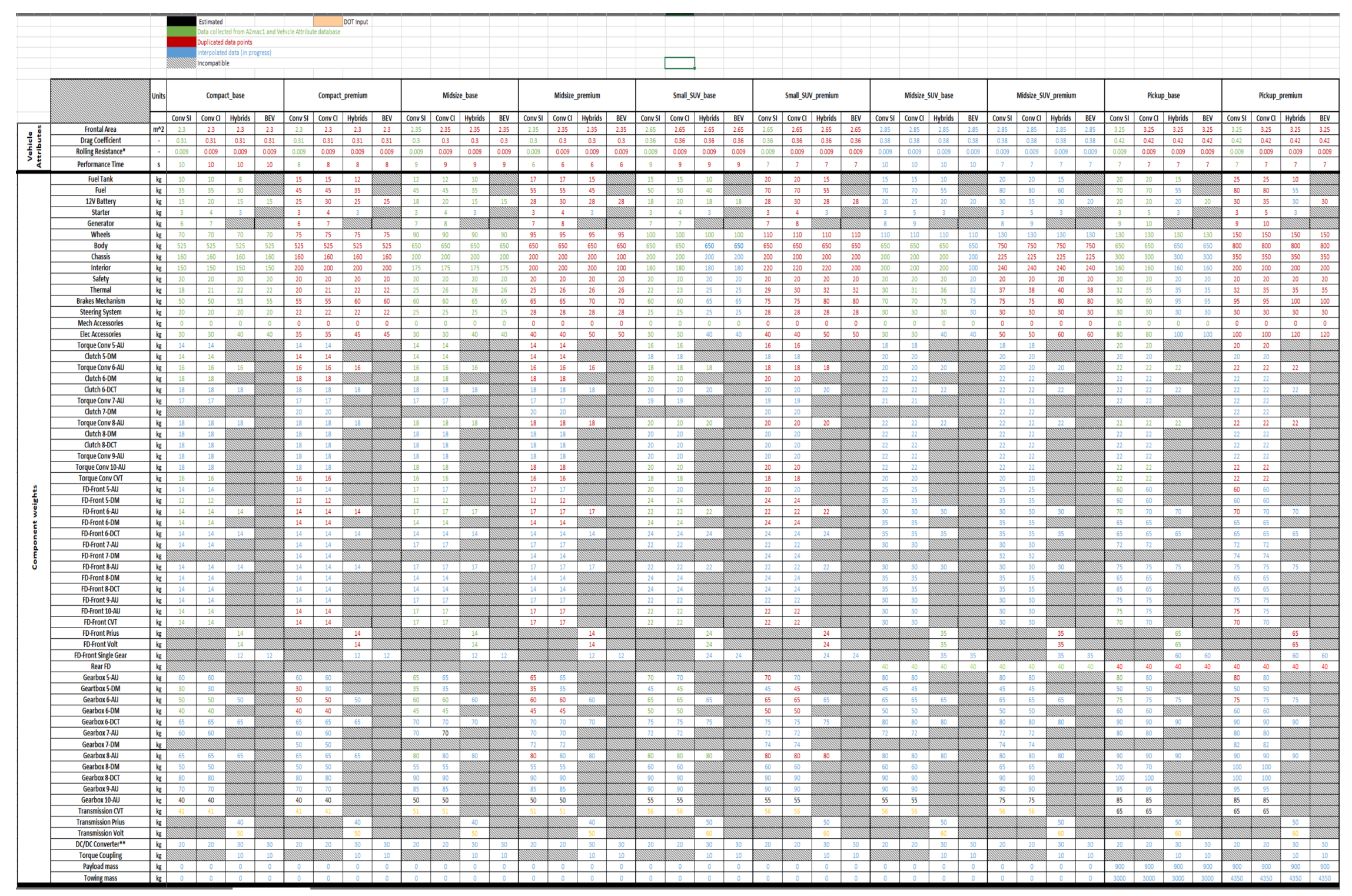

Figure 195. Main vehicle assumption inputs 


\section{VEHICLE SIZING PROCESS}

A unique approach to sizing the vehicle powertrain components ensures platform sharing among vehicles. While the reference baseline vehicles are sized to meet specific sets of performance criteria, the rest of the vehicles inherit their properties from the baseline reference vehicle and may have variations in performance. This approach represents real-world platform sharing (i.e., OEMs have a limited number of engines that are used across classes). A vehicle with inherited properties is referred to as an inherited vehicle.

\subsection{Inheritance}

The process of inheritance retrieves the values of different vehicle parameters of interest (engine power, engine weights, etc.) from the respective baseline reference vehicle and updates the inherited vehicle with the inherited value.

\subsubsection{Conventional Powertrain (Conventional/Micro-12V/Mild Hybrid BISG)}

Figure 196 shows the conventional powertrain inheritance flowchart for the range of vehicles and the baseline vehicles from which they inherit.

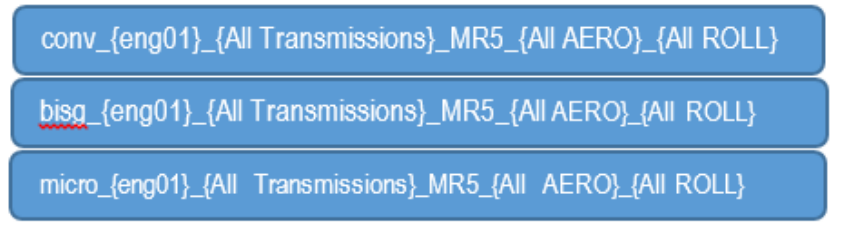

conv_\{eng01\}_\{All Transmissions\}_MR6_\{All AERO\}_\{All ROLL $\}$

bisg_\{eng01\}_\{All Transmissions\}_MR6_\{All AERO\}_\{All ROLL\}

micro_\{eng01\}_\{All Transmissions\}_MR6_\{All AERO\}_\{All ROLL $\}$

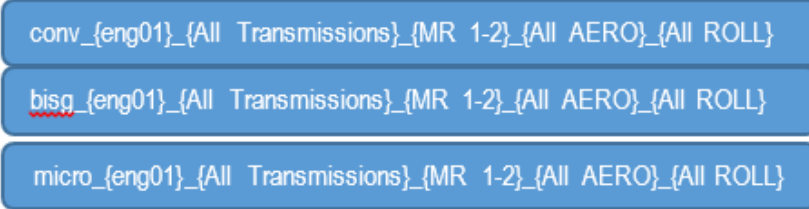

conv_\{eng01\}_\{All Transmissions\}_MR3_\{All AERO\}_\{All ROLL\}

bisg_\{eng01\}_\{All Transmissions\}_MR3_\{All AERO\}_\{All ROLL\}

micro_\{eng01\}_\{All Transmissions\}_MR3_\{All AERO\}_\{All ROLL $\}$

conv_\{eng01\}_\{All Transmissions $\}$ MR4_\{All AERO\}_\{All ROLL $\}$

bisg_\{eng01\}_\{All Transmissions\}_MR4_\{All AERO\}_\{All ROLL\}

micro_\{eng01\}_\{All Transmissions\}_MR4_\{All AERO\}_AAll ROLL\}

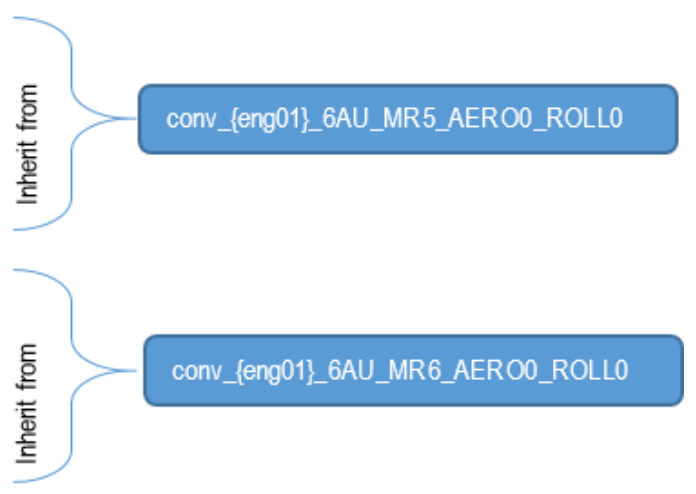

conv_\{eng01\}_6AU_MR0_AERO0_ROLLO

conv_\{eng01\}_6AU_MR3_AERO0_ROLL0

conv_\{eng01\}_6AU_MR4_AERO0_ROLL0

Figure 196. Conventional powertrain inheritance flowchart for eng01 
For a given engine, all transmissions, AERO and ROLL combinations would inherit the engine power and mass from conventional/6AU/AERO0/ROLL0 for a given MR combination. The micro-hybrid and mild-hybrid powertrains inherit from conventional as well.

For each conventional inherited vehicle, the algorithm in Figure 197 is implemented.

Get engine power and engine mass of sized vehicle
Update engine power of inherited vehicle

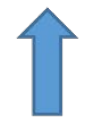

Move to the next vehicle to be inherited
Re-compute vehicle mass with updated engine mass

Figure 197. Inheritance algorithm for conventional vehicle

\subsubsection{Hybrid Powertrains (Split HEV/Split PHEV/EREV PHEV/Fuel Cell HEV/BEV)}

Figure 198 shows the hybrid powertrain inheritance flowchart for the range of vehicles studied and the respective baseline vehicle to inherit from for each of the respective hybrid powertrains.

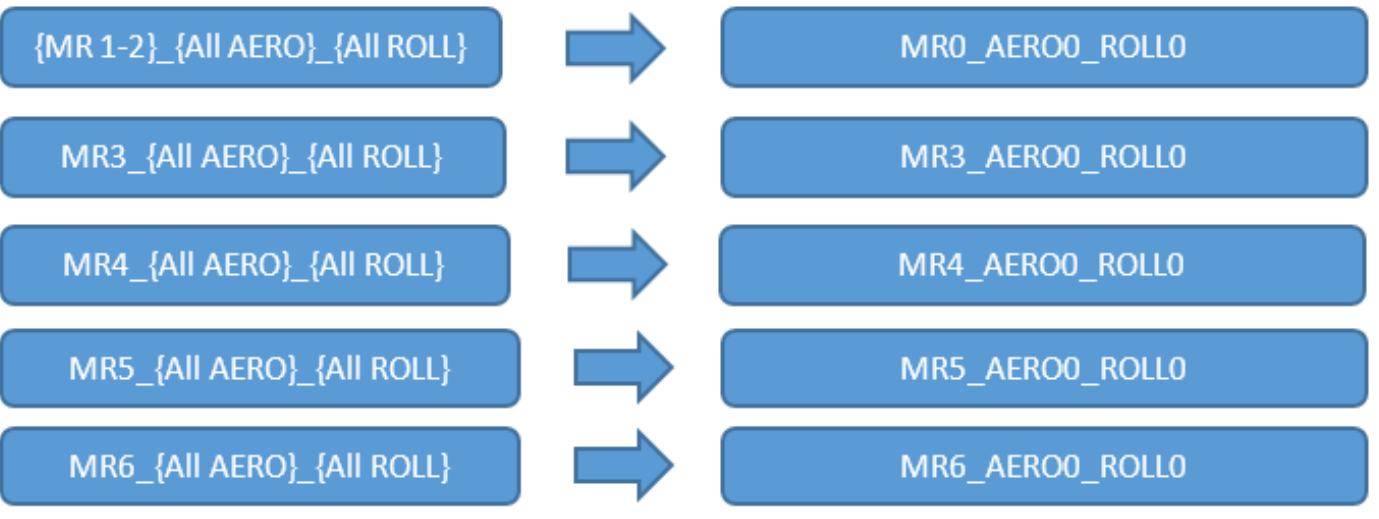

Figure 198. Hybrid powertrain vehicle inheritance flowchart 


\subsubsection{1. $\quad$ Inheritance for Battery-Electric Vehicles}

For each inherited BEV, the algorithm in Figure 199 is implemented.
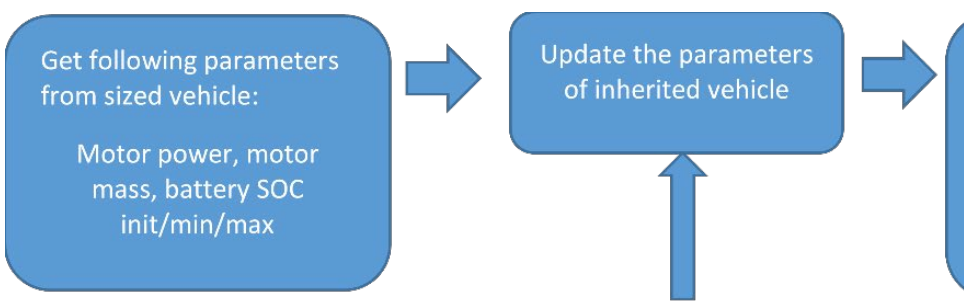

Resize the battery using the regular sizing logic

Re-compute vehicle mass

with updated battery and motor mass

Move to the next

vehicle to be inherited

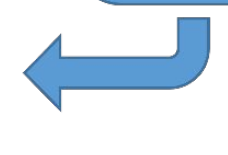

Figure 199. Inheritance algorithm for battery-electric vehicles

\subsubsection{Inheritance for Fuel-Cell HEVS}

For each inherited fuel-cell HEV, the algorithm shown in Figure 200 is implemented.
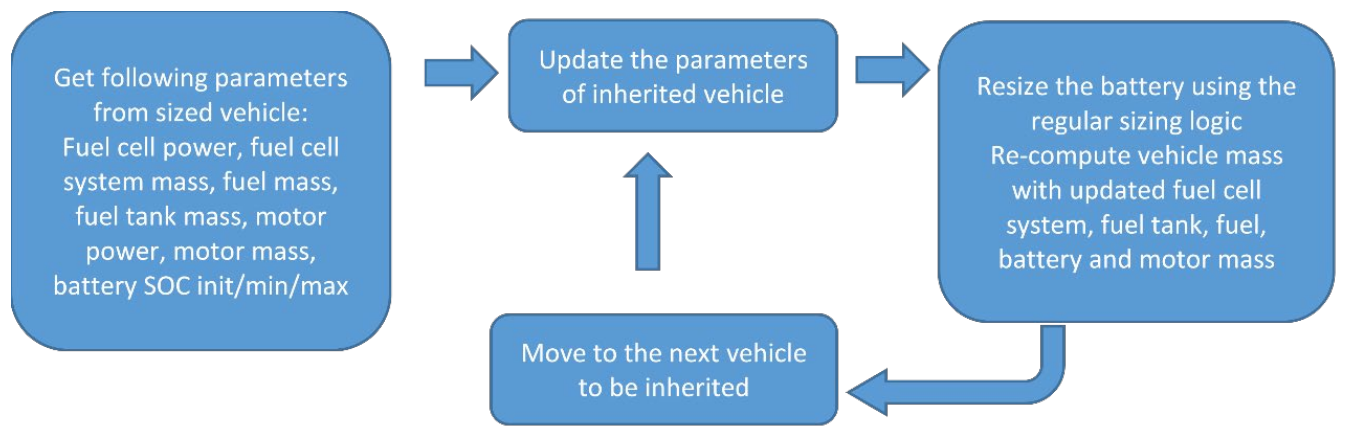

Figure 200. Inheritance algorithm for fuel-cell $\mathrm{HEVS}$

\subsubsection{Inheritance for EREVS}

For each inherited EREV, the algorithm shown in Figure 201 is implemented.
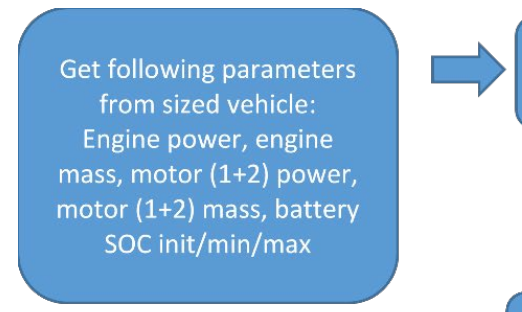

Update the parameters of inherited vehicle

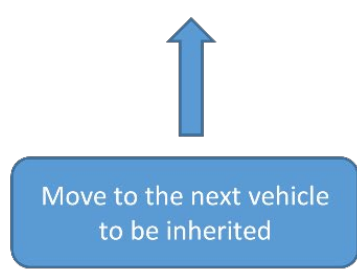

Resize the battery using the

regular sizing logic

Re-compute vehicle mass

with updated engine, motor

$(1+2)$, and battery mass

Figure 201. Inheritance algorithm for EREVS 


\subsubsection{Inheritance for Split PHEVS}

For each inherited split PHEV, the algorithm in Figure 202 is implemented.
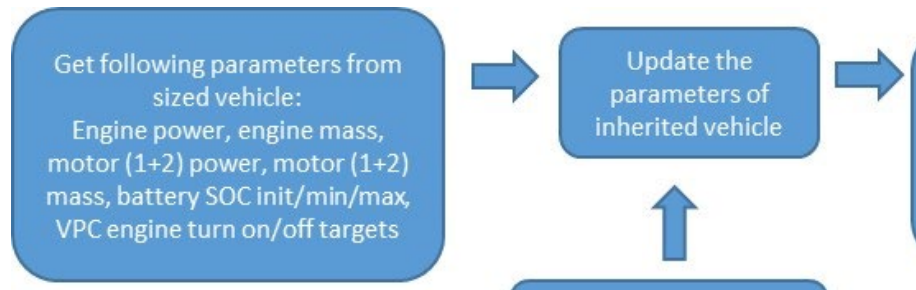

Resize the battery using the regular sizing logic

Re-compute vehicle mass

with updated engine, motor

$(1+2)$, and battery mass

Move to the next vehicle to be inherited

Figure 202. Inheritance algorithm for split PHEVS

\subsubsection{Inheritance for Split HEVS}

For each inherited split HEV, the algorithm shown in Figure 203 is implemented.
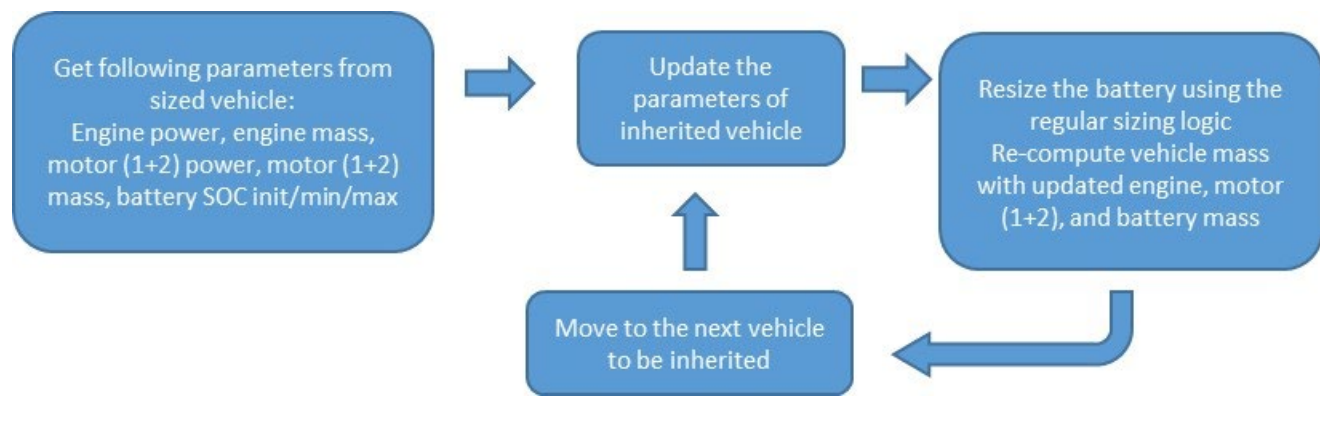

Figure 203. Inheritance algorithm for split HEVS

\subsubsection{Parallel Hybrid Powertrains}

\subsubsection{Inheritance for Parallel HEVS}

The flowchart in Figure 204 shows the range of inherited parallel hybrid vehicles and the reference baseline vehicles that the vehicles inherit from.
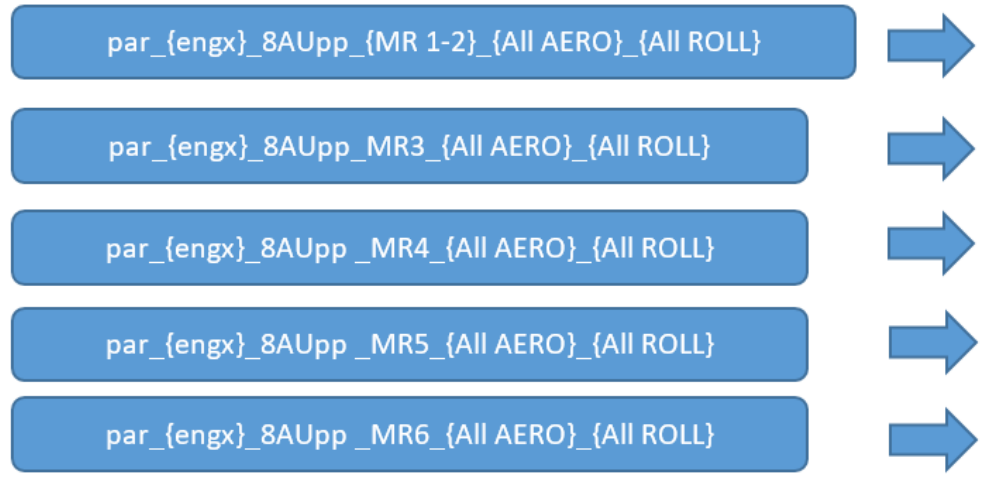

par_\{engx\}_8AUpp_MRO_AEROO_ROLLO

par_\{engx\}_8AUpp_MR3_AEROO_ROLLO

par_\{engx\}_8AUpp_MR4_AEROO_ROLLO

par_\{engx\}_8AUpp_MR5_AEROO_ROLLO

par_\{engx\}_8AUpp_MR6_AEROO_ROLLO

Figure 204. Parallel HEVs: Inheritance from reference baseline vehicles 
For each inherited parallel HEV, the algorithm in Figure 205 is implemented.
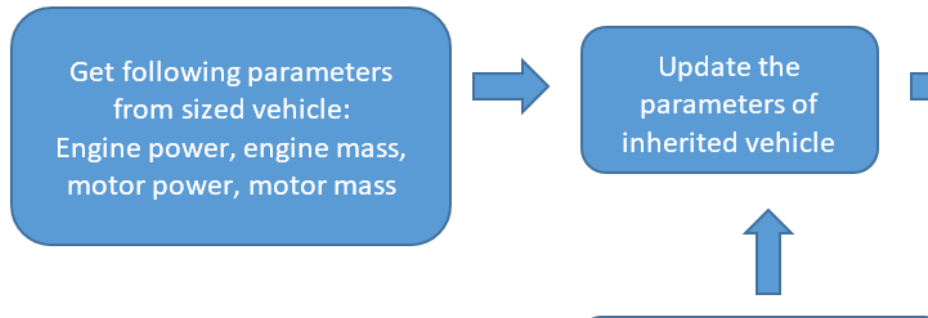

Move to the next vehicle to be inherited

Figure 205. Inheritance algorithm for parallel HEVs

\subsubsection{2. $\quad$ Inheritance for Parallel PHEVS}

The flowchart in Figure 206 shows the range of inherited parallel hybrid vehicles and the reference baseline vehicles that the vehicles inherit from.
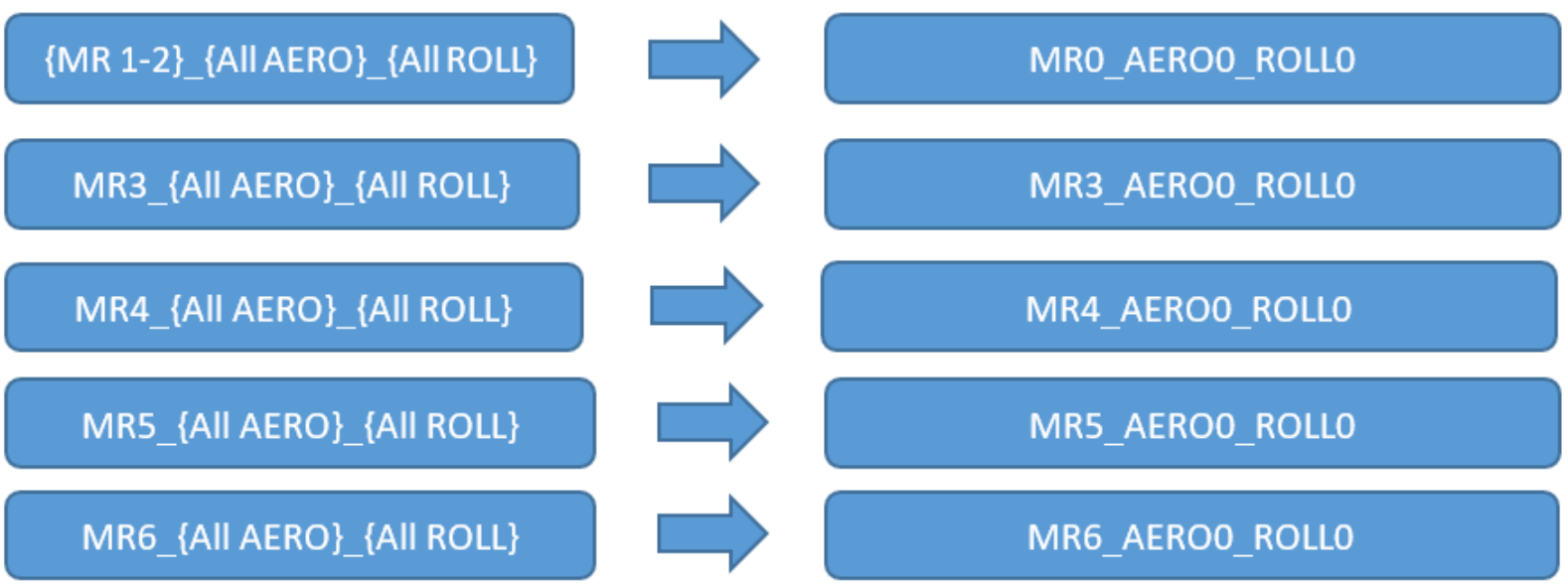

Figure 206. Parallel PHEVs: Inheritance from reference baseline vehicles

For each inherited parallel HEV, the algorithm in Figure 207 is implemented.

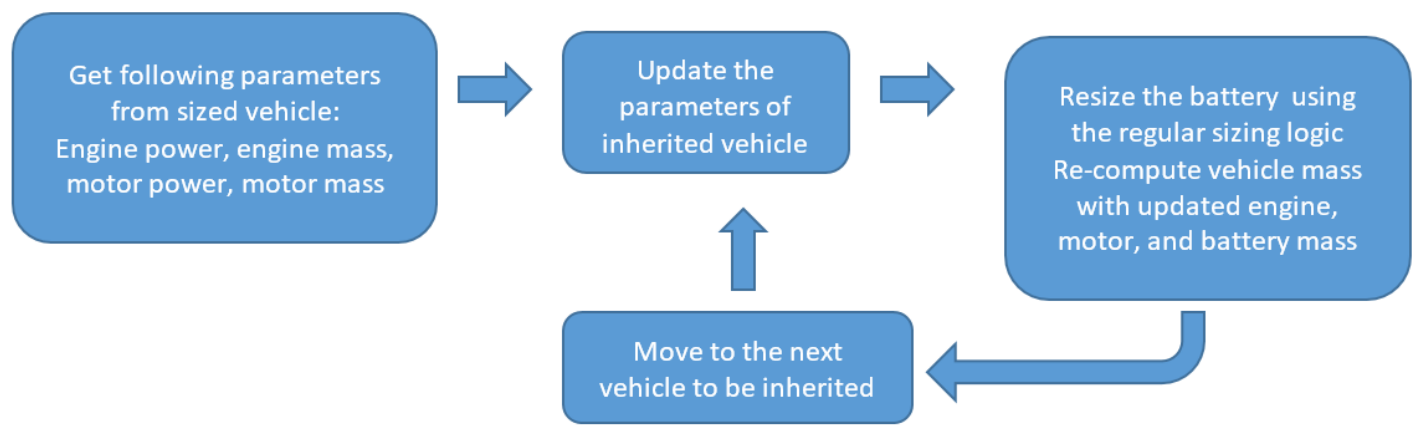

Figure 207. Inheritance algorithm for parallel HEVs 


\subsection{Vehicle Technical Specifications}

Each vehicle class and performance type has specific vehicle performance requirements. Table 59 shows the details of the different vehicle classifications and the corresponding performance times used to size the vehicles. The vehicles can exceed specific performance targets (e.g., 0-60 mph time) owing to other constraints (e.g., EV range and EV mode).

Table 59. Vehicle Class Performance Times

\begin{tabular}{|l|l|c|c|}
\hline $\begin{array}{c}\text { Vehicle } \\
\text { Class }\end{array}$ & \multicolumn{1}{|c|}{ Performance Category } & $\begin{array}{c}\mathbf{0 - 6 0} \text { mph Time } \\
(\mathbf{s})\end{array}$ & $\begin{array}{c}\text { 50-80 } \mathbf{~ m p h ~ T i m e ~} \\
\text { (s) }\end{array}$ \\
\hline Compact & Non-Performance (Base) & 10 & 10 \\
\hline Compact & Performance (Premium) & 8 & 8 \\
\hline Midsize & Non-Performance (Base) & 9 & 9 \\
\hline Midsize & Performance (Premium) & 6 & 6 \\
\hline Small SUV & Non-Performance (Base) & 9 & 9 \\
\hline Small SUV & Performance (Premium) & 7 & 7 \\
\hline Midsize SUV & Non-Performance (Base) & 10 & 7 \\
\hline Midsize SUV & Performance (Premium) & 7 & 7 \\
\hline Pickup & Non-Performance (Base) & 7 & 7 \\
\hline Pickup & Performance (Premium) & 7 & 10 \\
\hline
\end{tabular}

Along with the initial vehicle acceleration time to $60 \mathrm{mph}$, all vehicles are sized to meet the following requirements at minimum:

- Maximum grade (gradeability): $6 \%$ at $65 \mathrm{mph}$ at gross vehicle weight (GVW).

- Maximum vehicle speed: $>100 \mathrm{mph}$

- Payload: $900 \mathrm{~kg}$ (pickup base/premium only)

- Towing: 3,000 kg (pickup base) and 4,350 kg (pickup premium)

These requirements are a good representation of the current American automotive market and of American drivers' expectations. The relationship between curb weight and GVW for current technologyconfiguration-powertrain combinations is modeled from the existing vehicles in the market, and it forms the basis for estimating the GVWs of future vehicle scenarios. Figure 208 shows the gross vehicle weight rating (GVWR) vs. curb weight relationship. 


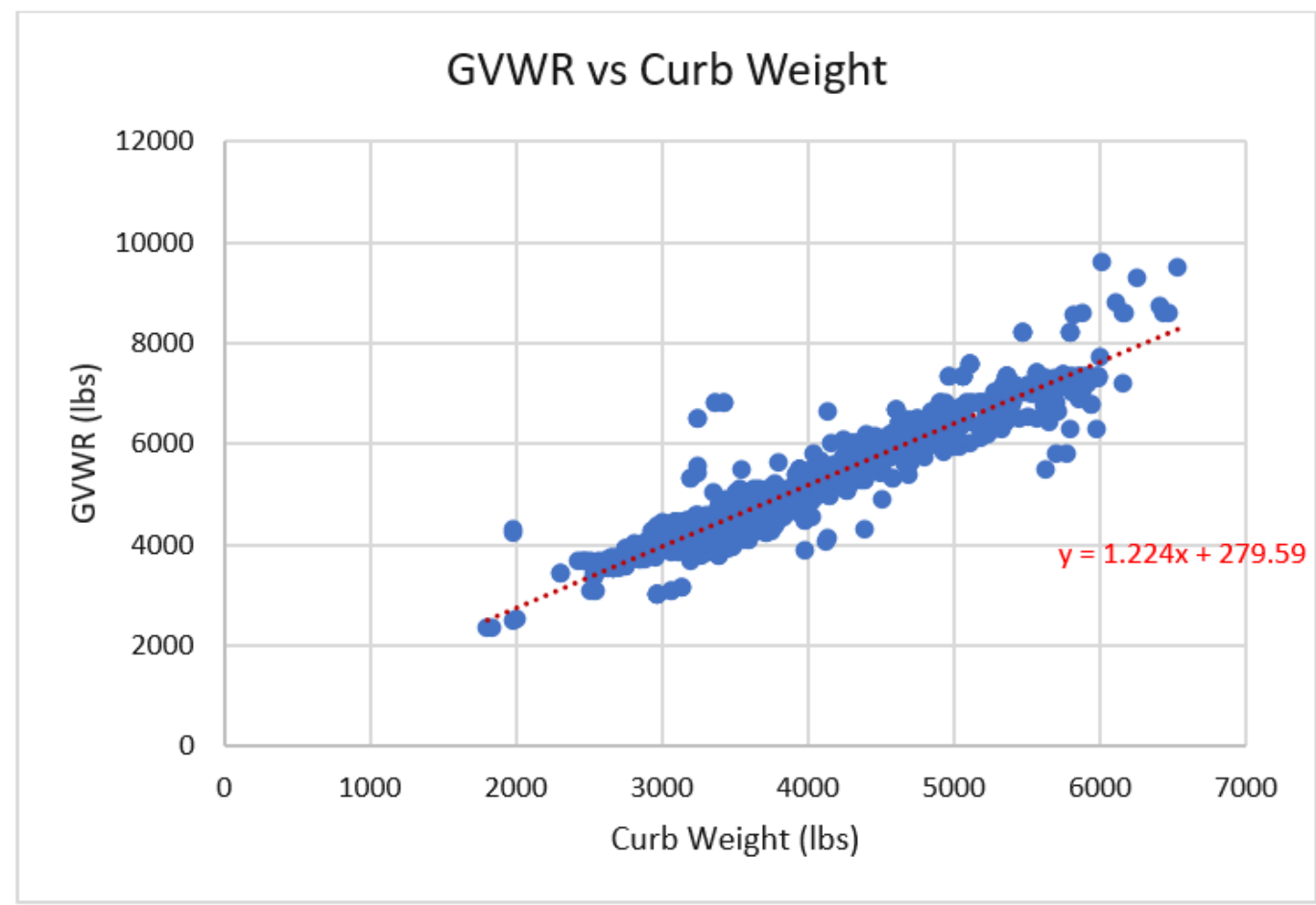

Figure 208. Curb weight vs. GVWR

Using the equation derived from the figure above, the relationship is determined as follows:

GVWR $=1.224 \times($ curb weight $)+279.59$

\subsection{Vehicle Powertrain Sizing Algorithms}

Improperly sizing components will lead to differences in energy consumption and will influence the effectiveness results. Therefore, we have developed several automated sizing algorithms to provide a fair comparison between technologies. Algorithms have been defined for powertrains (e.g., conventional, power-split, series, electric) and applications (e.g., HEV, PHEV).

All algorithms are based on the same concept: The vehicle is built from the bottom up, meaning each component assumption (e.g., specific power, efficiency) is taken into account to define the entire set of vehicle attributes (e.g., weight). This process is iterative, as the main component characteristics (e.g., maximum power, vehicle weight) are modified until all vehicle technical specifications are met. The transmission gear span or ratios are currently not modified to be optimized with specific engine technologies. On average, the algorithms take between five and 10 iterations to converge.

\subsubsection{Conventional Vehicle Sizing Algorithm}

A conventional vehicle is mainly defined by its internal combustion engine (ICE): Its ability to follow a cycle or meet acceleration performance is directly linked to its power density. Therefore, the sizing algorithm focuses on calculating the mechanical power needed to meet the requirements. Figure 209 illustrates the steps in the sizing process. After a default vehicle is created, a simulation determines the engine peak power and vehicle weight. 
First, the desired power to meet the gradeability and acceleration performance requirements is estimated, and engine power is updated with the maximum value.

Then, the sizing enters an acceleration loop to verify the performance (e.g., initial vehicle movement to $60 \mathrm{mph}$ ). The definition of initial vehicle movement (IVM) is that the vehicle must move $1 \mathrm{ft}$ (about $1 / 3 \mathrm{~m}$ ) before the clock starts to record the performance time. This metric provides a more consistent result and removes phenomena that are difficult to model at initial acceleration - such as tire and clutch slip-from consideration.

Finally, acceleration performance for passing (i.e., time to accelerate from 50 to $80 \mathrm{mph}$ ) is measured with the vehicle's updated parameters. At the end, the times to reach the targets (0-60 mph and 50-80 $\mathrm{mph}$ ) are compared with the simulated data, which is the main condition to exit the routine. Figure 209 shows the detailed steps of the conventional-vehicle sizing algorithm.

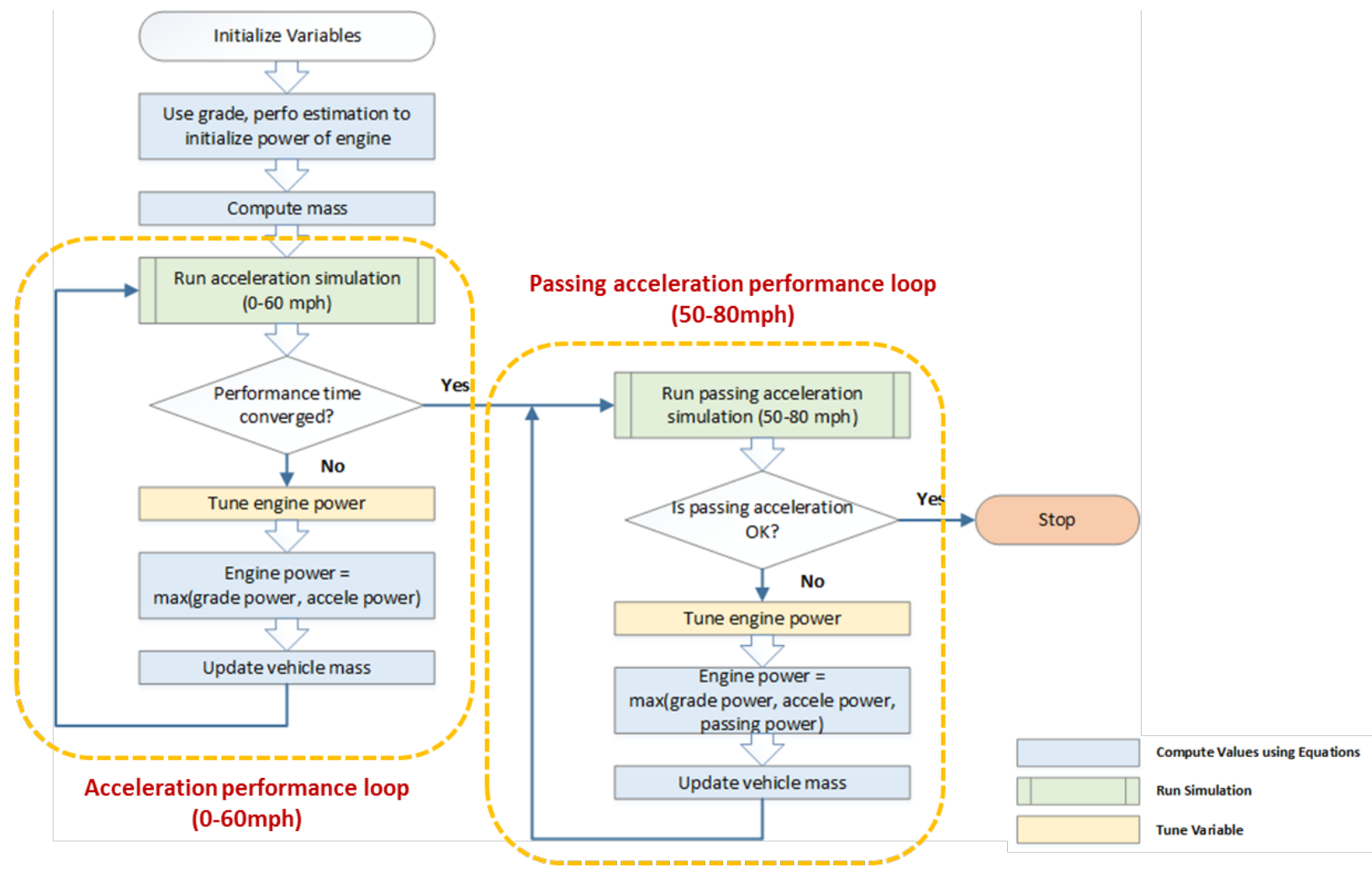

Figure 209. Conventional powertrain sizing algorithm 


\subsubsection{Split HEV Sizing Algorithm}

Figure 210 shows the detailed steps of the split HEV sizing algorithm.

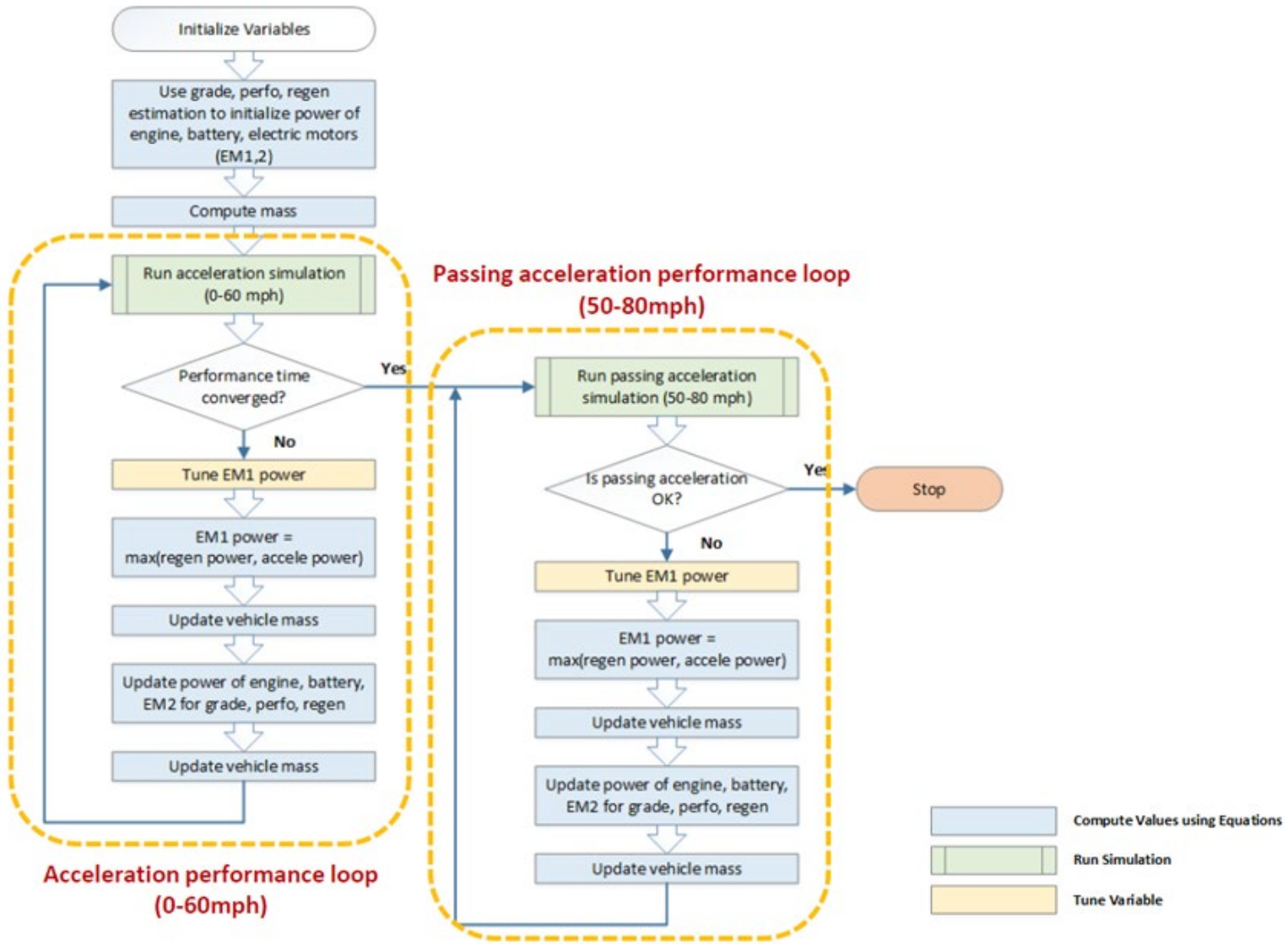

Figure 210. Split hybrid electric powertrain sizing algorithm

The main algorithm for split-HEV is as follows:

- The engine is sized to achieve at least $70 \%$ of the peak power required to meet VTS (acceleration performance or gradeability).

- The battery and electric machine (EM1) powers are sized for maximum regenerative braking.

- The vehicle weight is updated based on the engine peak power, electric machines $(E M 1,2)$ peak power, and battery power.

- The electric machine (EM2) is sized as follows:

- Start ICE at Vmax ( $57 \mathrm{mph}$ for UDDS cycle). ICE should be ON (i.e. EM2 peak power for engine start at top speed on UDDS cycle).

- Control maximum power of engine at Vspd $=0$ (i.e., EM2 peak power for engine control on performance).

- Control ICE at maximum grade (i.e., EM2 continuous power for engine control on grade, engine power fraction going through electro-mechanical power path). 


\subsubsection{Parallel HEV Sizing Algorithm}

Figure 211 shows the detailed steps of the parallel HEV (par HEV) sizing algorithm.

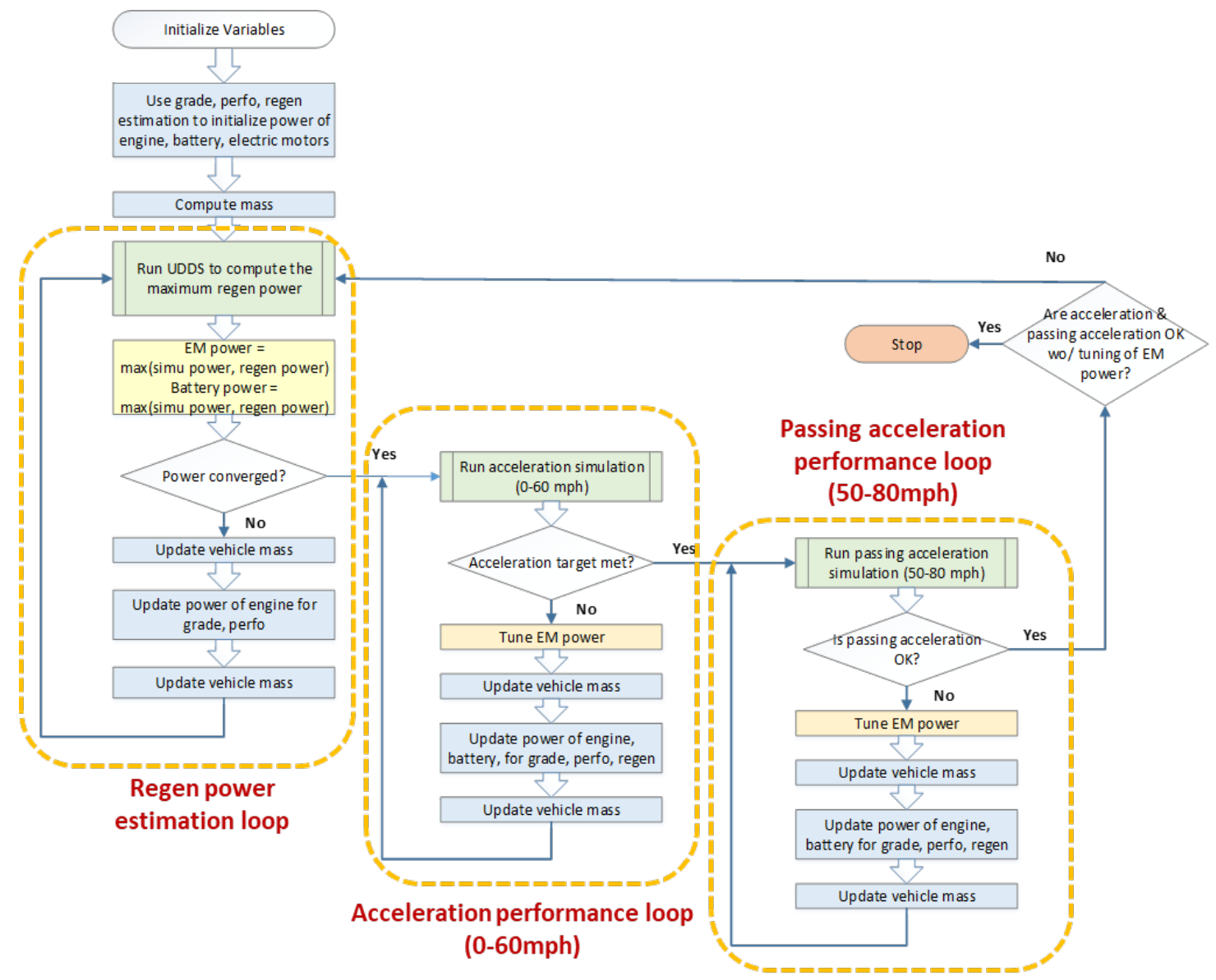

Figure 211. Parallel hybrid electric powertrain sizing algorithm

The main algorithm for parallel HEV sizing is as follows:

- The engine is sized to achieve at least $70 \%$ of the peak power required to meet VTS (acceleration performance or gradeability).

- The battery and electric machine (EM1) powers are sized for maximum regenerative braking.

- The vehicle weight is updated based on the engine peak power, electric machine (EM1) peak power, and battery power. 


\subsubsection{Parallel PHEV Sizing Algorithm}

Figure 212 shows the detailed steps for the parallel PHEV (par PHEV20) sizing algorithm.

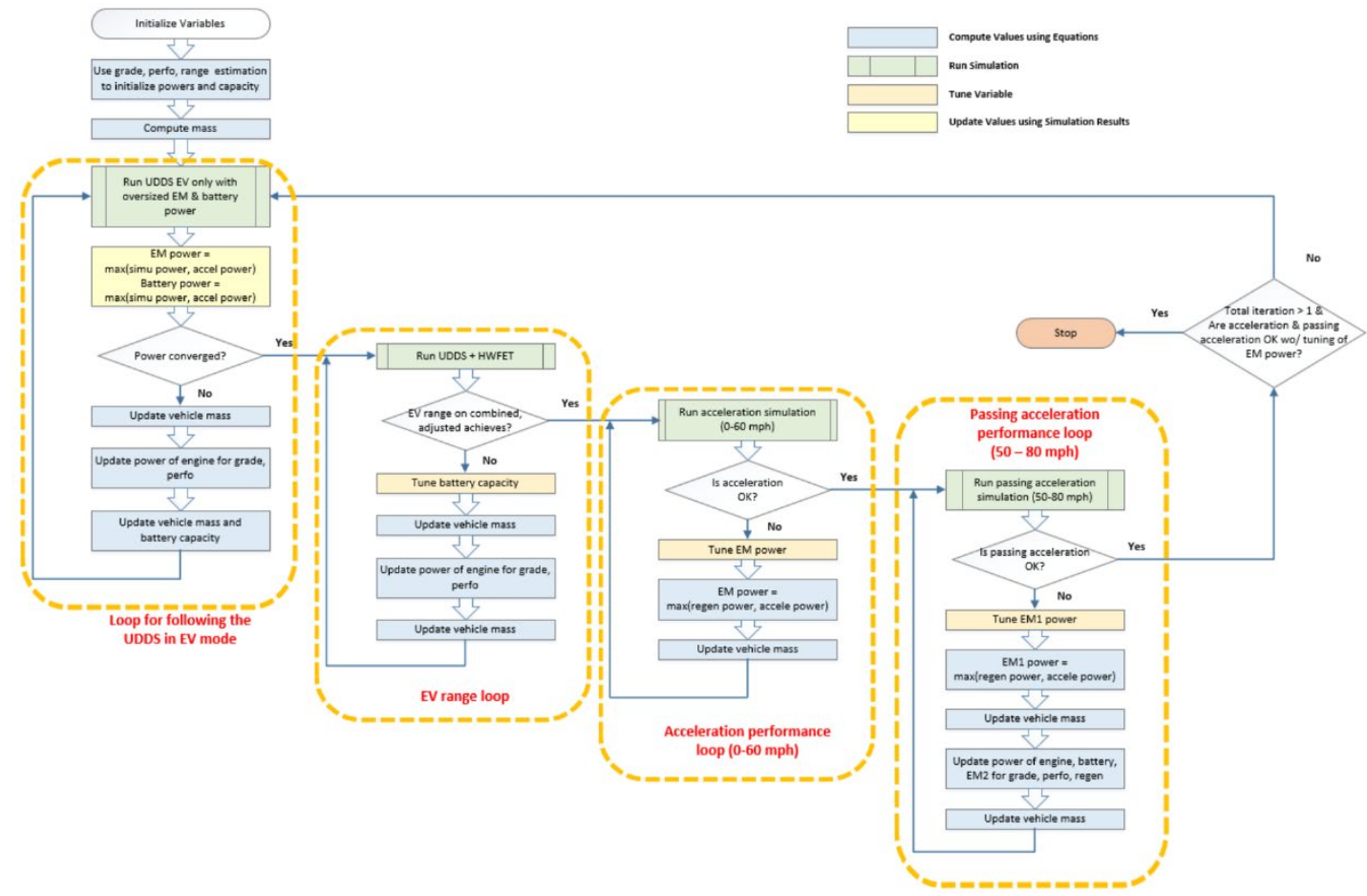

Figure 212. Parallel plug-in hybrid (Par PHEV20) electric vehicle powertrain sizing

Figure 213 shows the detailed steps for the parallel PHEV (par PHEV50) sizing algorithm.

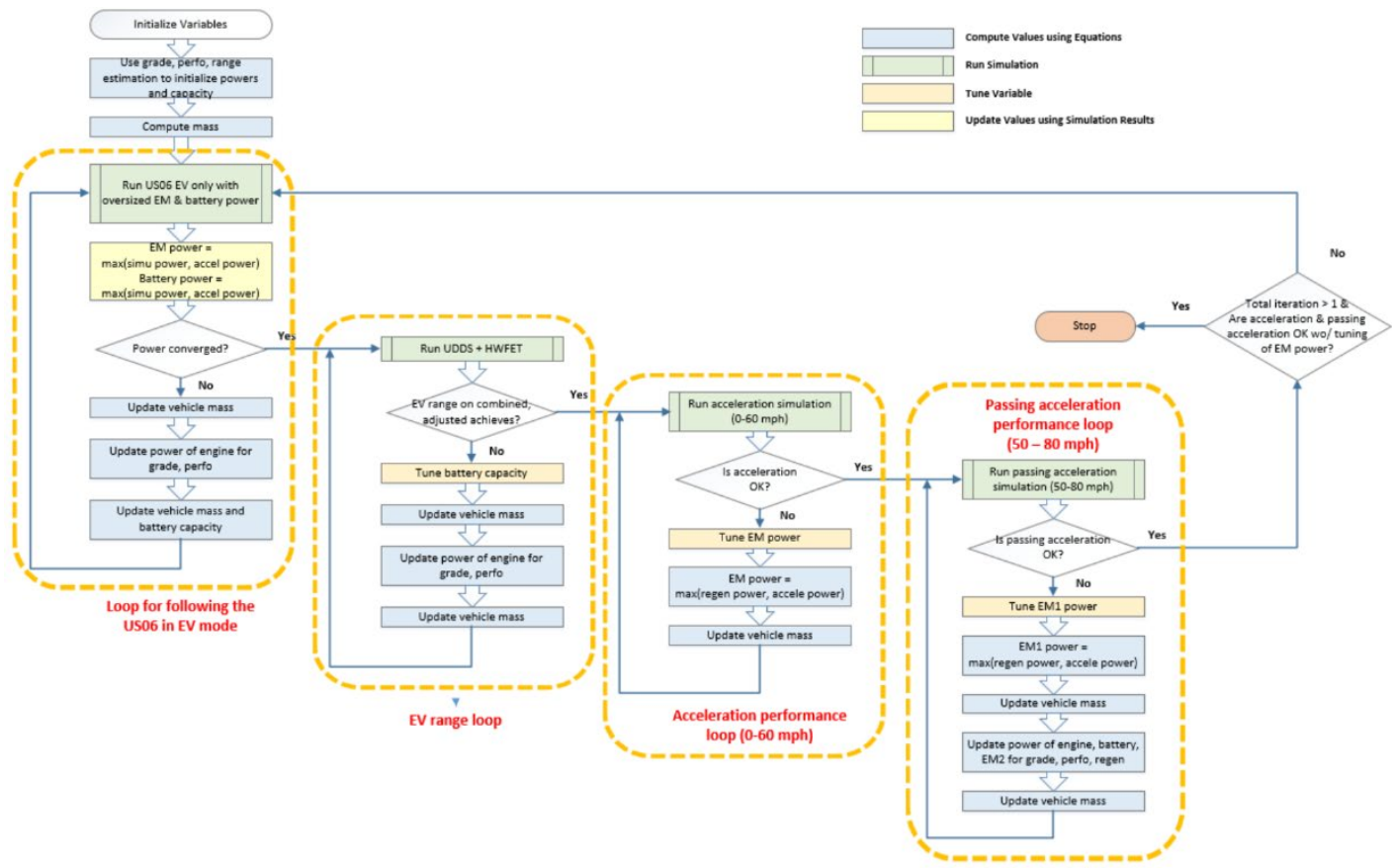

Figure 213. Parallel plug-in hybrid (Par PHEV50) electric vehicle powertrain sizing 
The main sizing algorithm for parallel PHEV sizing is as follows:

- The engine is sized to achieve at least $70 \%$ of the peak power required to meet VTS (acceleration performance or gradeability).

- The battery energy is sized to achieve the specified AER on the combined cycle (UDDS + HWFET), on the basis of adjusted energy values.

- The battery and electric machine (EM) powers are sized to follow the UDDS cycle in EV mode for Par PHEV20 and US06 cycle in EV mode for Par PHEV50 at low SOC (beginning of CS mode) or to meet the requirement of acceleration performance.

- The vehicle weight is updated based on the engine peak power, electric-machine (EM) peak power, and battery energy.

\subsubsection{Split-PHEV Sizing Algorithm}

Figure 214 shows the detailed steps of the split-PHEV (split PHEV20) sizing algorithm.

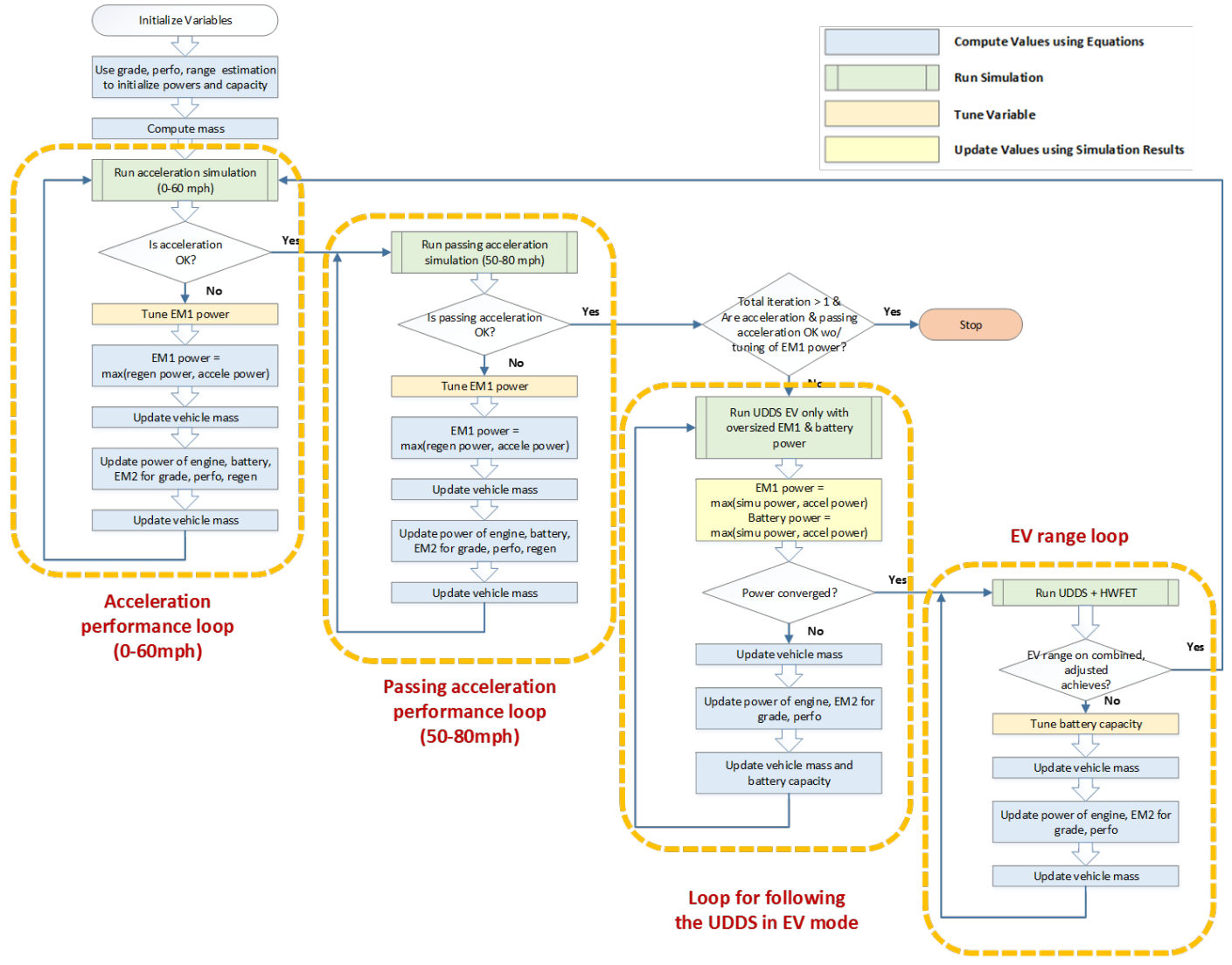

Figure 214. Split PHEV sizing algorithm

The main sizing algorithm for split-PHEV sizing is as follows:

- The engine is sized to achieve at least $70 \%$ of the peak power required to meet VTS (acceleration performance or gradeability).

- The battery energy is sized to achieve the specified AER on the combined cycle (UDDS + HWFET), on the basis of adjusted energy values. 
- The battery and electric machine (EM1) powers are sized to follow the UDDS cycle in EV mode at low SOC (beginning of CS mode) or to meet the requirement of acceleration performance.

- The vehicle weight is updated based on the engine peak power, electric-machine $(E M 1,2)$ peak power, and battery energy.

- The electric machine (EM2) is sized as follows:

- Start ICE at Vmax ( $\sim 57 \mathrm{mph}$ for UDDS cycle). ICE should be ON (i.e. EM2 peak power for engine start at top speed on UDDS cycle).

- Control maximum power of engine at Vspd $=0$ (i.e., EM2 peak power for engine control on performance).

- Control ICE at maximum grade (i.e., EM2 continuous power for engine control on grade, engine power fraction going through electro-mechanical power path).

\subsubsection{Voltec PHEV (Extended Range) Vehicle Sizing Algorithm}

Figure 215 shows the detailed steps for the Voltec PHEV vehicle sizing algorithm, used for the EREV PHEV50 vehicle powertrain.

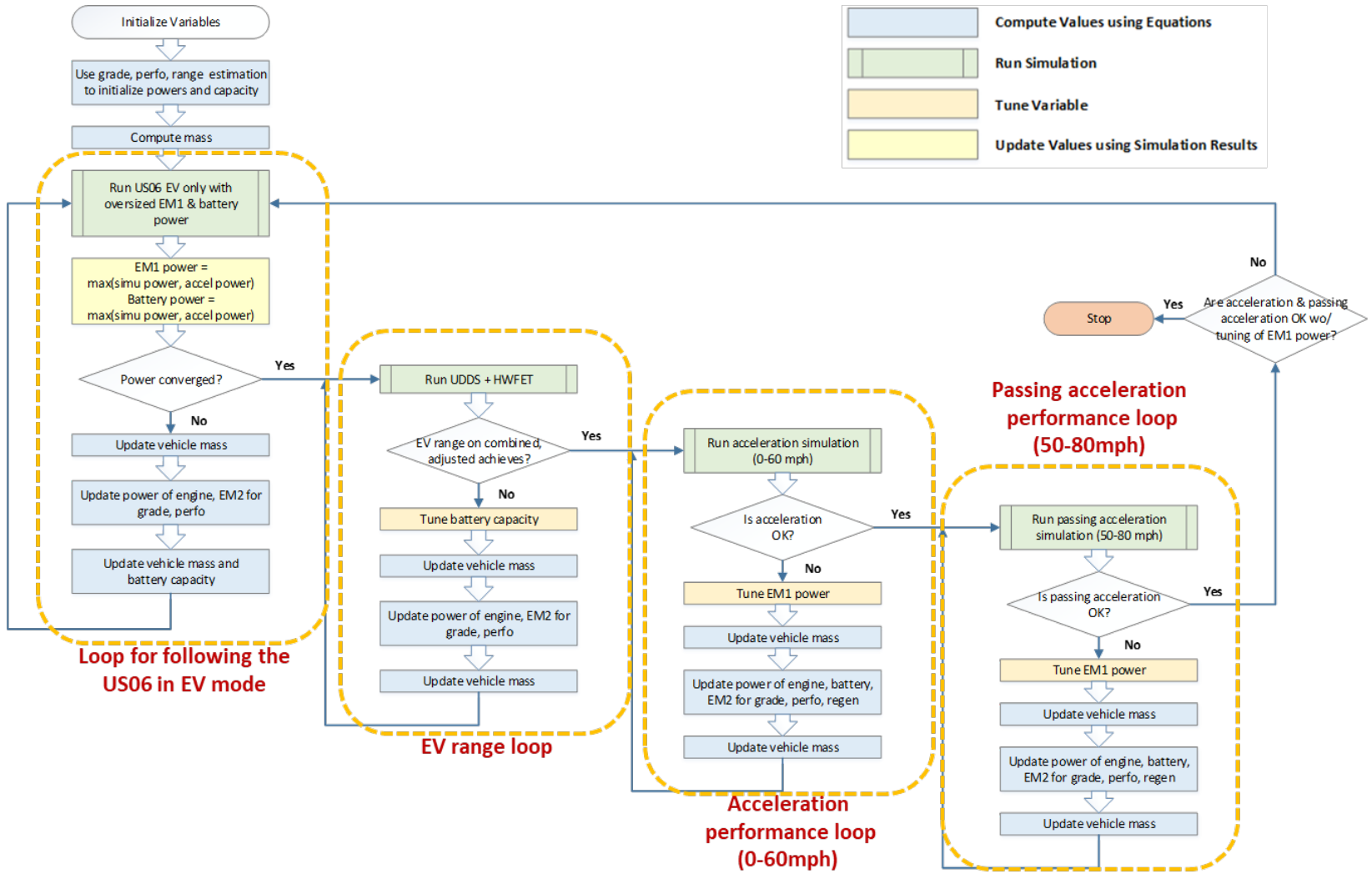

Figure 215. Voltec PHEV vehicle sizing algorithm

The main sizing algorithm for Voltec extended-range PHEV sizing is as follows:

- The engine is sized to achieve at least $70 \%$ of the peak power required to meet VTS (acceleration performance or gradeability).

- The battery energy is sized to achieve the specified AER on the combined cycle (UDDS + HWFET), on the basis of adjusted energy values. 
- The battery and electric machine (EM1) powers are sized to follow the US06 cycle in EV mode at low SOC (beginning of CS mode) or to meet the requirement of acceleration performance.

- The vehicle weight is updated with respect to the engine peak power, electric-machine $(\mathrm{EM} 1,2)$ peak power, and battery energy.

- The electric machine (EM2) is sized as following:

- Start ICE at Vmax ( 57 mph for UDDS cycle). ICE should be ON (i.e., EM2 peak power for engine start at top speed on UDDS cycle)

- Control maximum power of engine at Vspd $=0$ (i.e., EM2 peak power for engine control on performance)

- Control ICE at max grade (i.e., EM2 continuous power for engine control on grade, engine power fraction going through electro-mechanical power path).

\subsubsection{BEV Sizing Algorithm}

Figure 216 shows the detailed steps of the BEV sizing algorithm, used for BEV200 and BEV300 vehicle powertrains.

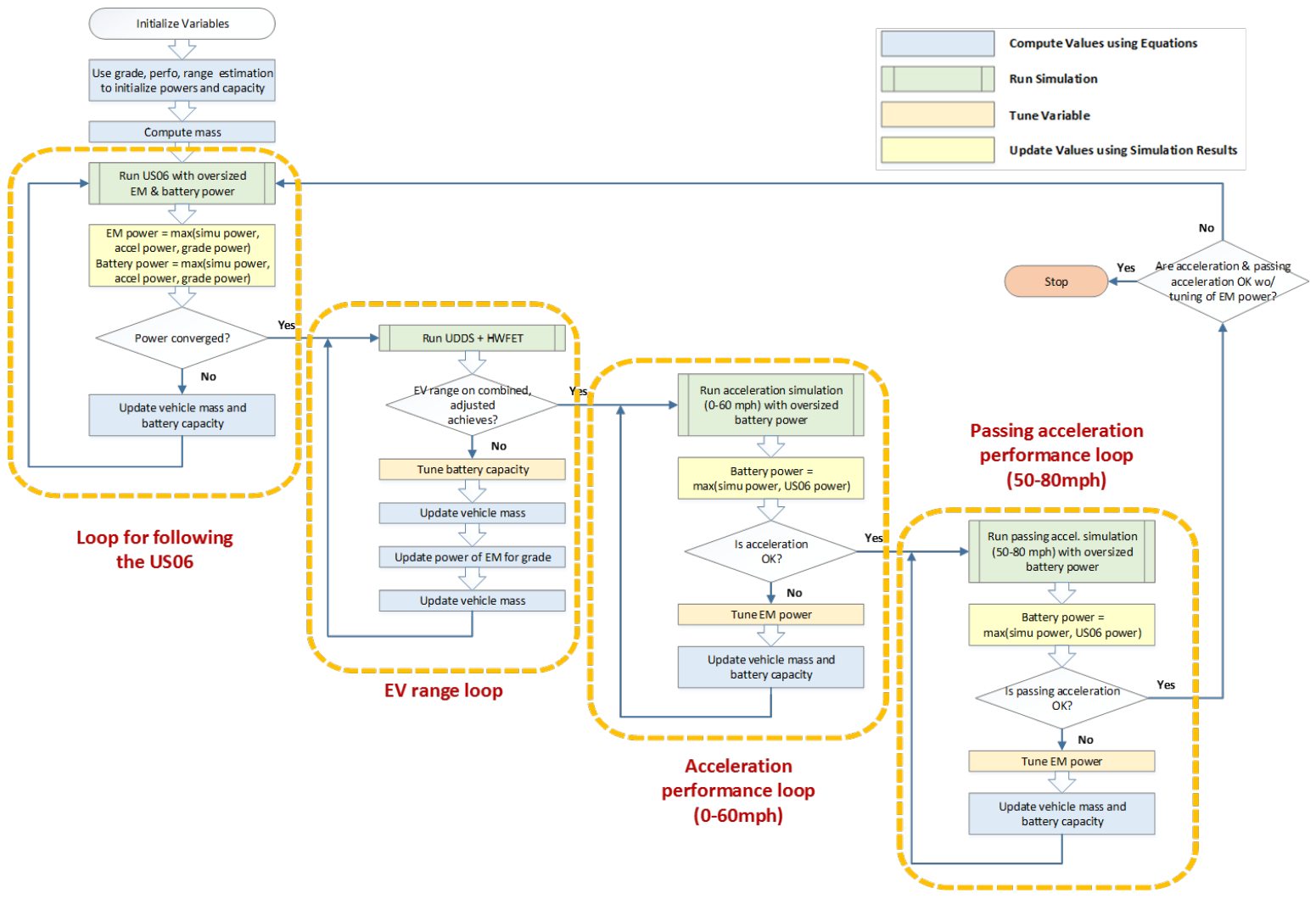

Figure 216. BEV sizing algorithm

The main sizing algorithm for BEV sizing is as follows:

- Battery and electric machine (EM) powers are sized to be able to follow the US06 cycle at low SOC (beginning of CS mode) or to meet the requirement of acceleration performance.

- The battery energy is sized to achieve the specified AER on the combined cycle (UDDS + HWFET), on the basis of adjusted energy values.

- The vehicle weight is a function of the electric-machine (EM) peak power and battery energy. 


\subsubsection{Fuel Cell Series HEV Sizing Algorithm}

Figure 217 shows the detailed steps of the fuel cell HEV sizing algorithm.

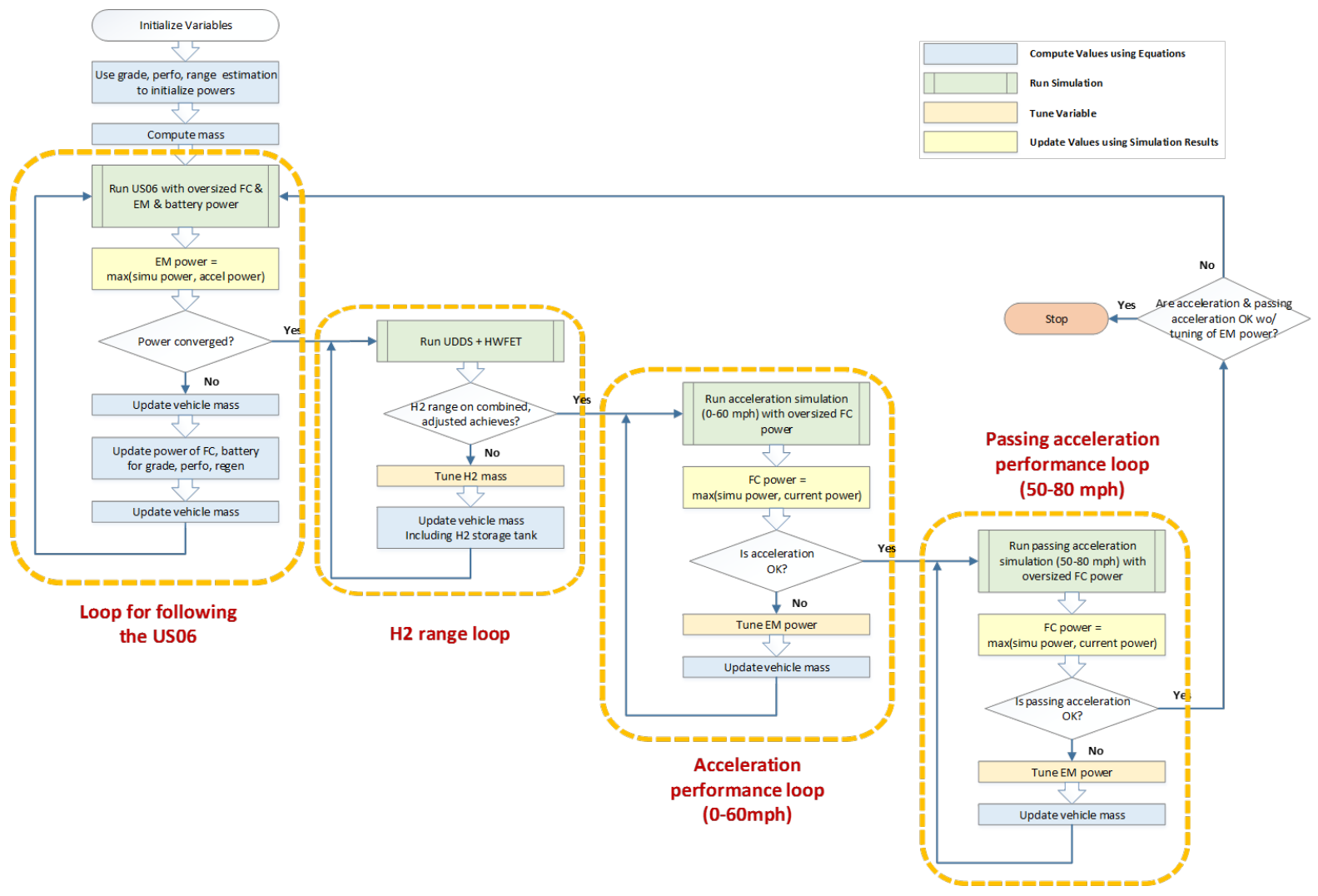

Figure 217. Fuel cell series HEV sizing algorithm

The main sizing algorithm for fuel-cell HEV sizing is as follows:

- The fuel cell is sized to achieve at least $70 \%$ of the peak power required to meet the VTS (acceleration performance or gradeability).

- The hydrogen storage capacity is sized to achieve the specified AER on the combined cycle (UDDS + HWFET), on the basis of adjusted values.

- The vehicle weight is a function of the fuel-cell peak power, electric-machine peak power, and battery cell number.

\subsubsection{Selection of Drive Cycles for Electric Machine Sizing for PHEVs}

The selection of drive cycles for electric machine sizing for PHEVs is based on an analysis of existing vehicles in the market. Figure 218 shows the relationship of electric machine sizes to battery energy for PHEV20s. 
Electric Machine vs. Battery Energy (PHEV 25 miles or less)

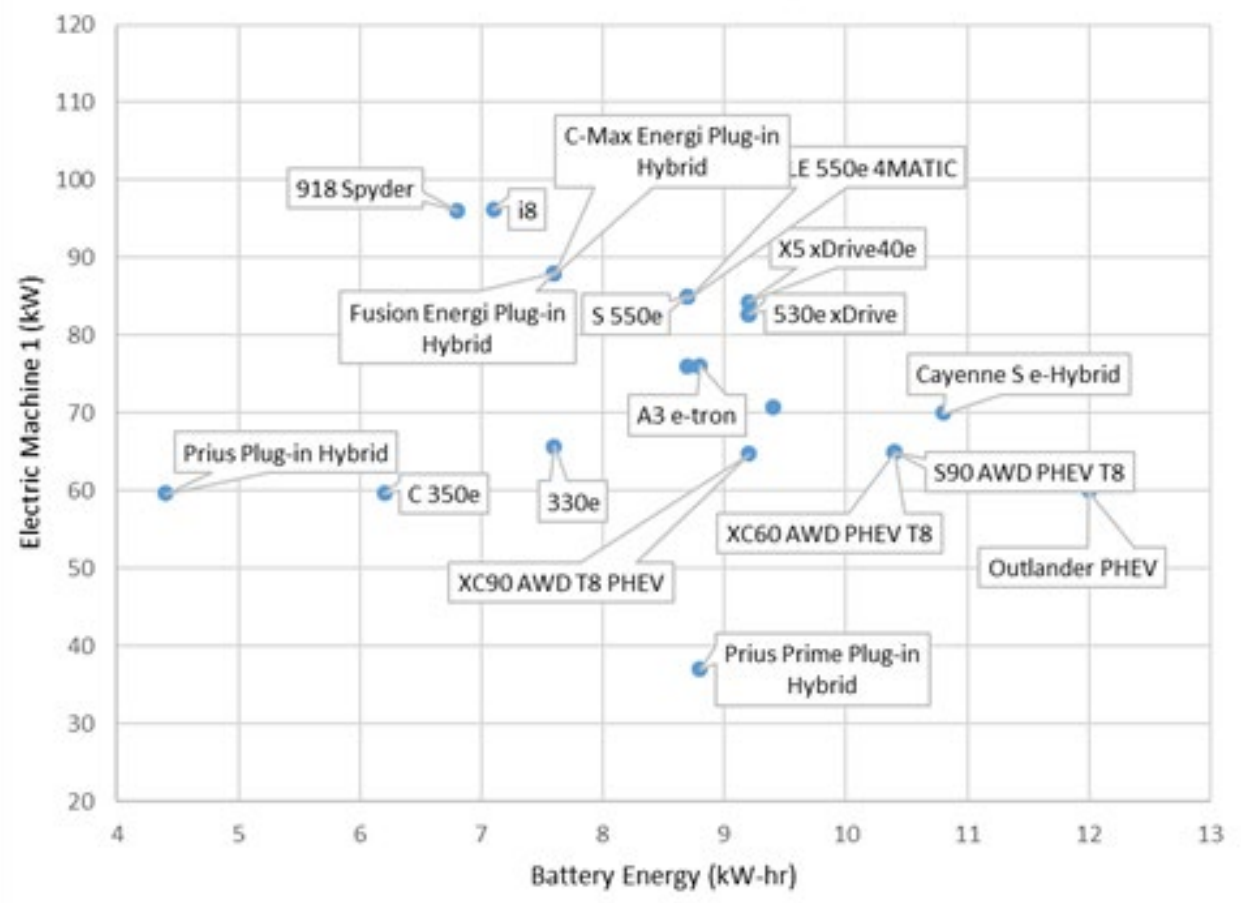

Figure 218. Electric machine (kW) vs. battery energy (kWh) for PHEV20s

Figure 219 shows the relationship of electric machine sizes to battery energy for PHEV50s.

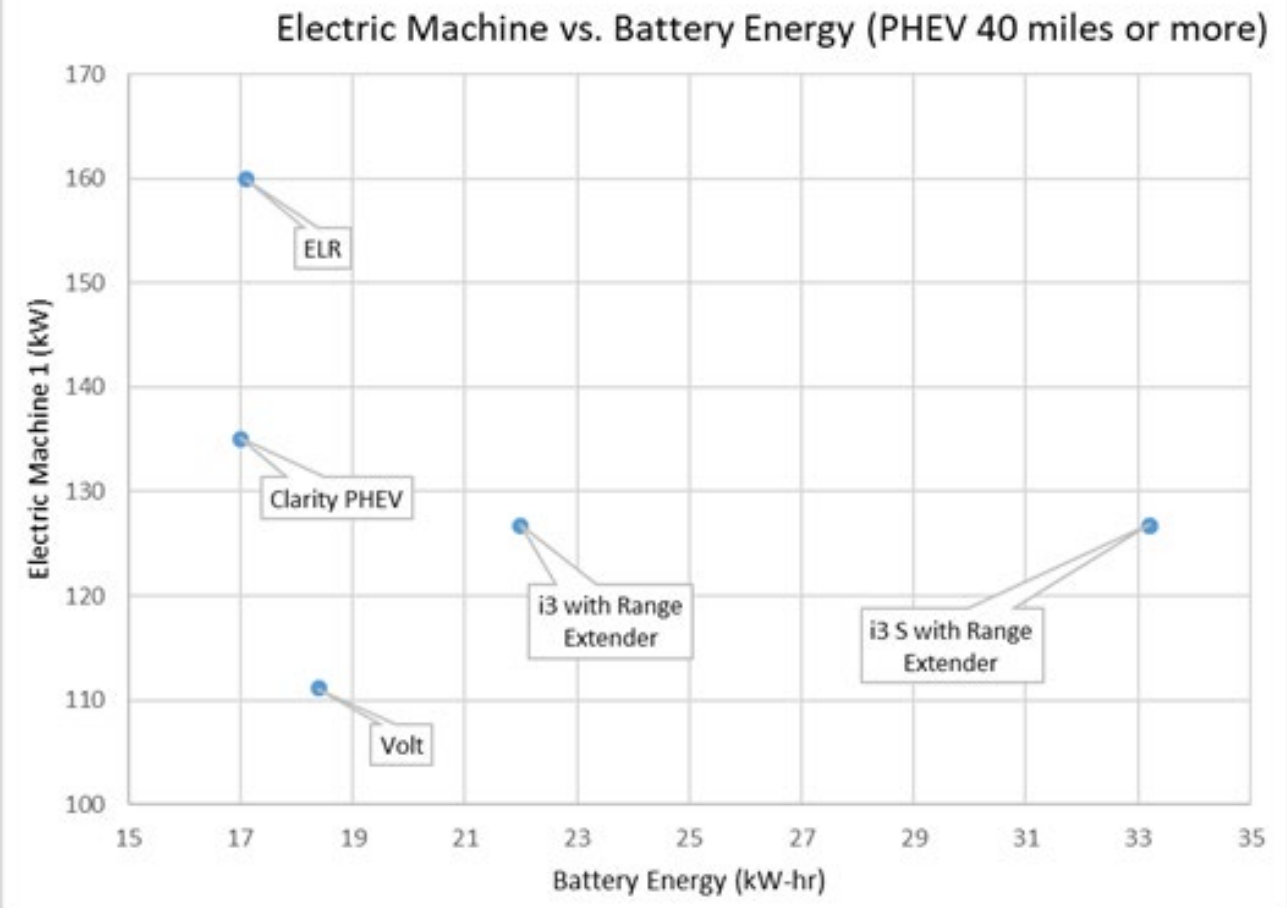

Figure 219. Electric machine (kW) vs. battery energy (kWh) for PHEV50s 
Figure 220 shows the relationship of electric machine sizes to battery energy for BEVs.

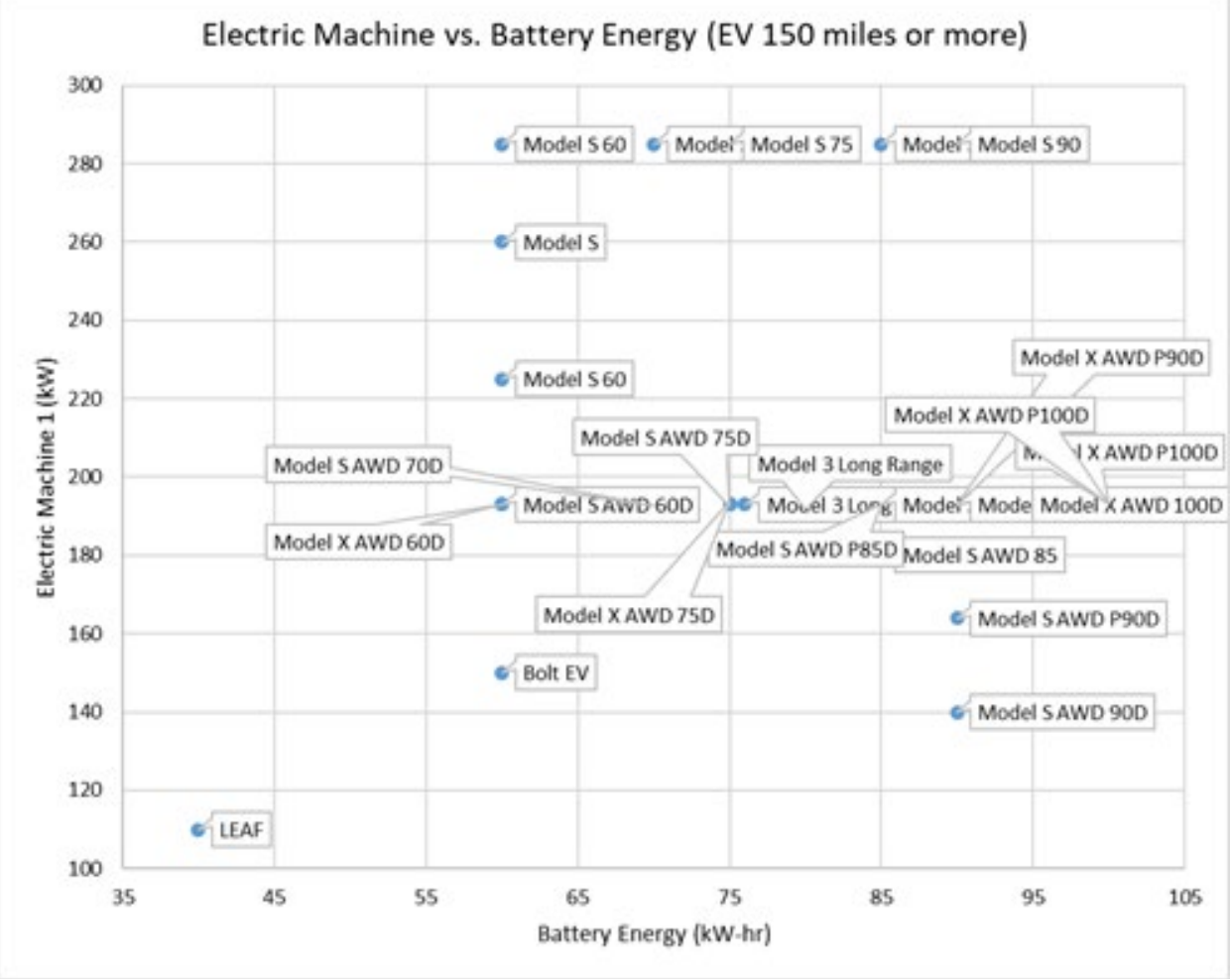

Figure 220. Electric machine $(\mathrm{kW})$ vs. battery energy (kWh) for BEVS

From the analyses, it can be seen that the low-range PHEVs usually have an electric machine power requirement of less than $100 \mathrm{~kW}$, and the higher-range PHEVs have more than $100 \mathrm{~kW}$ (in line with BEVs). The more aggressive power requirement can be established from following the US06 cycle in EV mode rather than UDDS, which is applicable to low-range PHEVs.

\subsection{Determining the Relationship Between Engine Displacement and Number of Cylinders}

As part of the current analysis, we also evaluated different engine displacements available across the number of engine cylinders to update the relationship used in previous analysis runs. We further evaluated the influence of major manufacturers on engine displacement. Figure 221 shows the relationship between the number of engine cylinders and engine displacement for existing vehicles in the U.S. market for MY20 vehicles from EPA test car data [33]. 


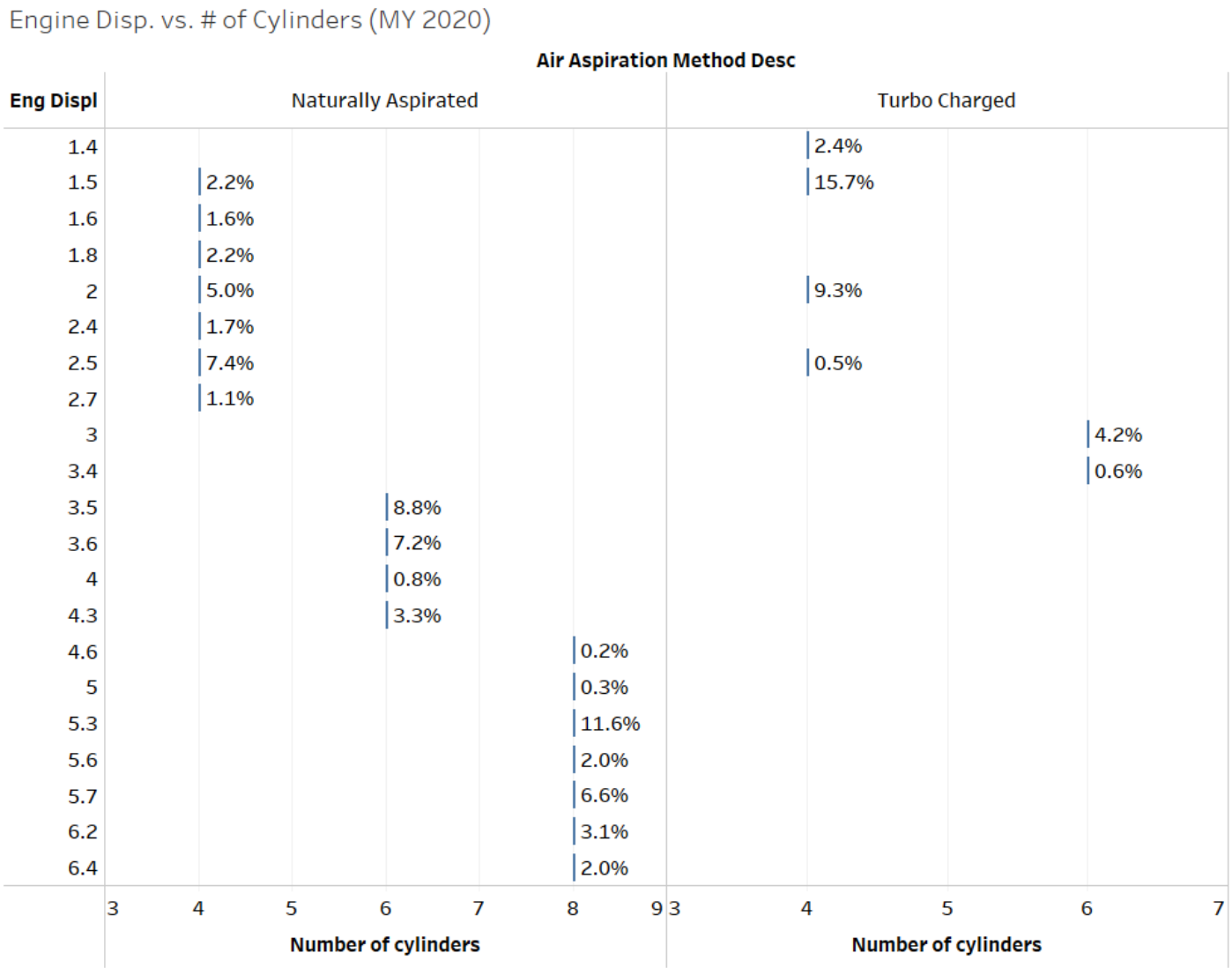

Figure 221. Relationship between engine displacement and number of engine cylinders

Using this relationship, thresholds were created to define the number (and type) of engine cylinders for given engine displacements across different engine configurations. These 15 different engine displacements cover about $93.2 \%$ of the conventional market in MY20. The thresholds are shown in Table 60

Table 60. Thresholds for Engine Displacement vs. Number of Engine Cylinders

\begin{tabular}{|l|l|l|c|}
\hline \multicolumn{1}{|c|}{$\begin{array}{c}\text { Number and Configuration of } \\
\text { Engine Cylinders }\end{array}$} & \multicolumn{2}{|c|}{ Engine Displacement (L) } & \multirow{2}{*}{ Total } \\
\hline \multirow{2}{*}{ 4-cylinder, in-line (14) } & NA & $1.5,1.6,1.8,2,2.5$ & \multirow{2}{*}{6} \\
\cline { 2 - 3 } & TC & $1.4,1.5,2$ & 5 \\
\hline \multirow{2}{*}{ 6-cylinder, V6 } & NA & $3 . .5,3.6,4,4.3$ & \multirow{2}{*}{4} \\
\cline { 2 - 3 } & TC & 3 & 4 \\
\hline 8-cylinder, V8 & NA & $5.3,5.6,5.7,6.2$ & \\
\hline
\end{tabular}


The flowchart in Figure 222 shows the detailed method used to calculate the engine displacement and number of cylinders from the initial engine size derived from the engine map.
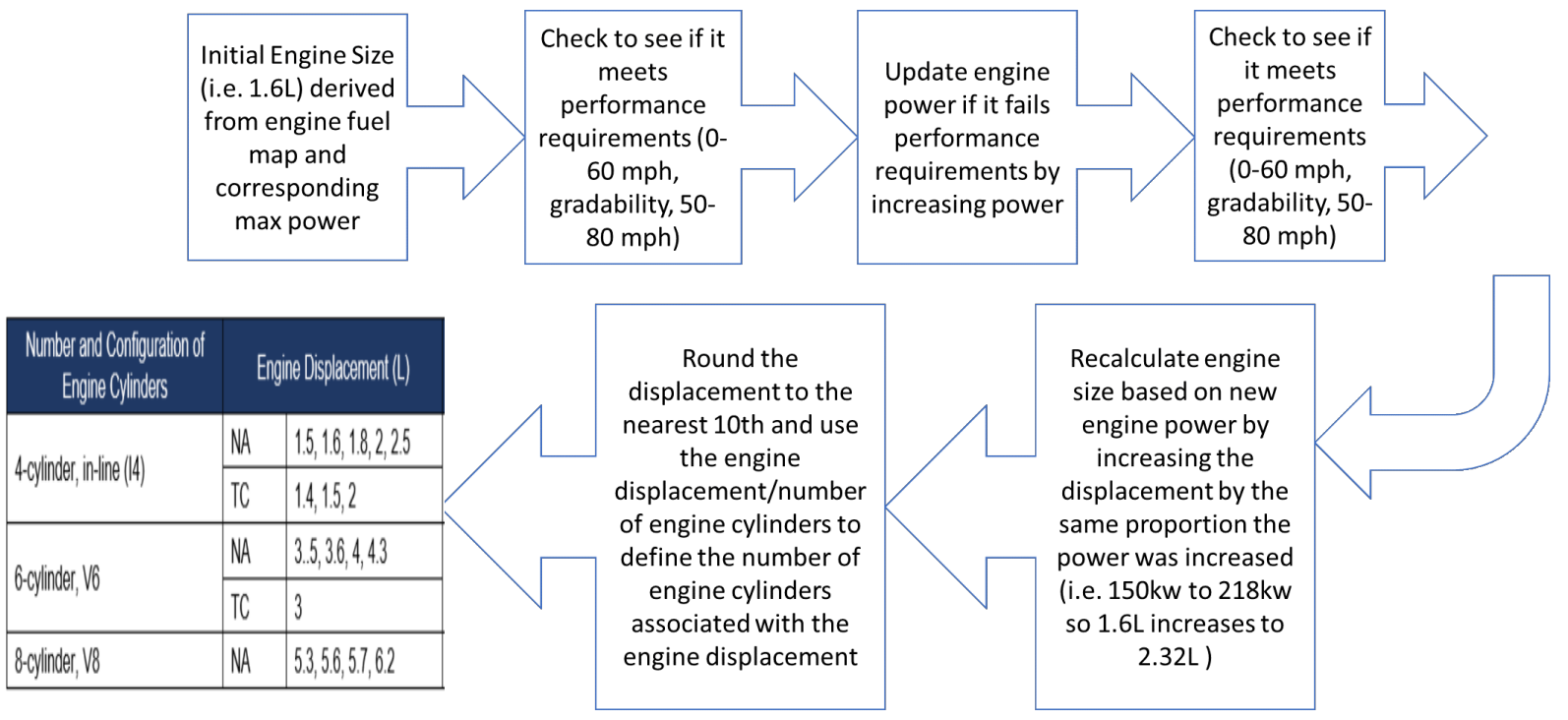

Figure 222. Method for computing engine displacement and number of cylinders 


\section{DISTRIBUTED COMPUTING PROCESS}

At this stage of the large-scale simulation process, all the vehicles are created and ready to be sized and simulated in Autonomie. Running 1.2 million vehicles requires more than 5 million simulations, from sizing algorithms - imposing recurrence and iteration/looping - to vehicle simulation on cycles and combined or PHEV procedures.

With a multitude of technology combinations to simulate, and the objective of providing direct inputs from Autonomie to the CAFE model, the usual computing resources are no longer practical. Running all of the simulations on one computer would take several months or years to complete. However, thanks to advances in distributed computing, simulation time can be greatly reduced. Among the computing resources available at Argonne is a cluster of several thousand worker nodes dedicated to the Vehicle \& Mobility Systems Group. A larger high-performance computing facility will be used in the future to further accelerate the simulations.

\subsection{Setup}

Autonomie is used as the simulation framework, synchronized by a cluster head node computer. The head computer extracts the data describing the different technology pathways from the vehicle files and distributes it, as diagrammed in Figure 223. An algorithm optimizes the distribution of jobs for vehicle simulations.

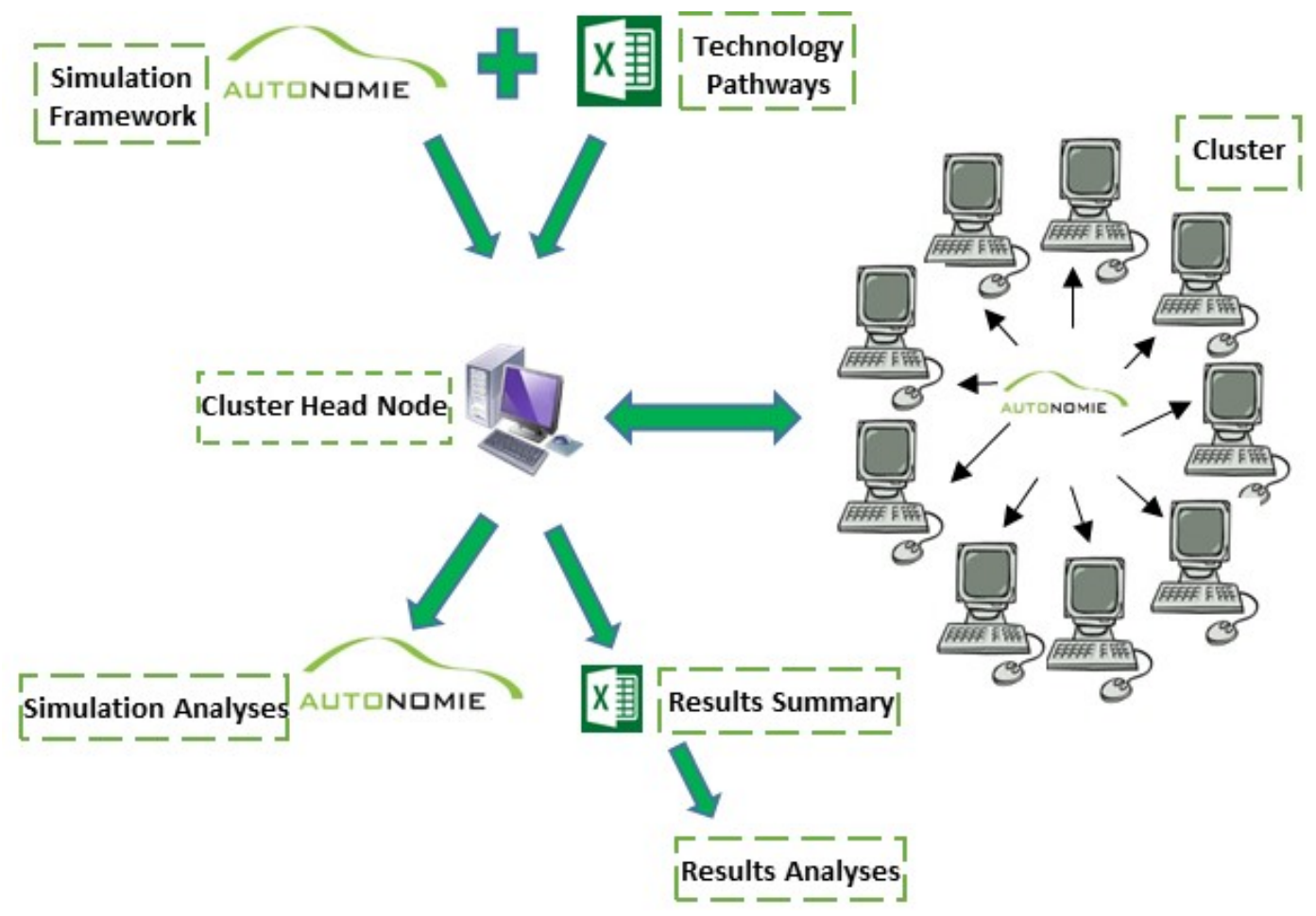

Figure 223. Distributed computing process 


\subsection{Distributed-Computing Flexibility}

One of the biggest advantages of distributed computing is that it facilitates the quick reruns of simulations that occur many times during any study. Experience enabled Argonne to develop a new process: an ultimate large-scale simulation process that is functional, smooth, and flexible, with the ability to easily and quickly add and rerun as many vehicles and new technologies as needed. The generic process can automatically handle additional technologies without any code modification. As a result, the CAFE model's future technological needs can be easily and quickly integrated at any time, and new runs can be implemented to directly feed the model for CAFE rulemaking analyses. 


\section{VEHICLE SIMULATION PROCESS}

Once the vehicles are sized or inherited to meet the desired vehicle technical specifications, they are simulated following the appropriate standard driving cycles and run procedures. It is important to properly store individual results as structured data because they will be reused to support database generation and easy browsing. Figure 224 shows the folder organization for each individual simulation.

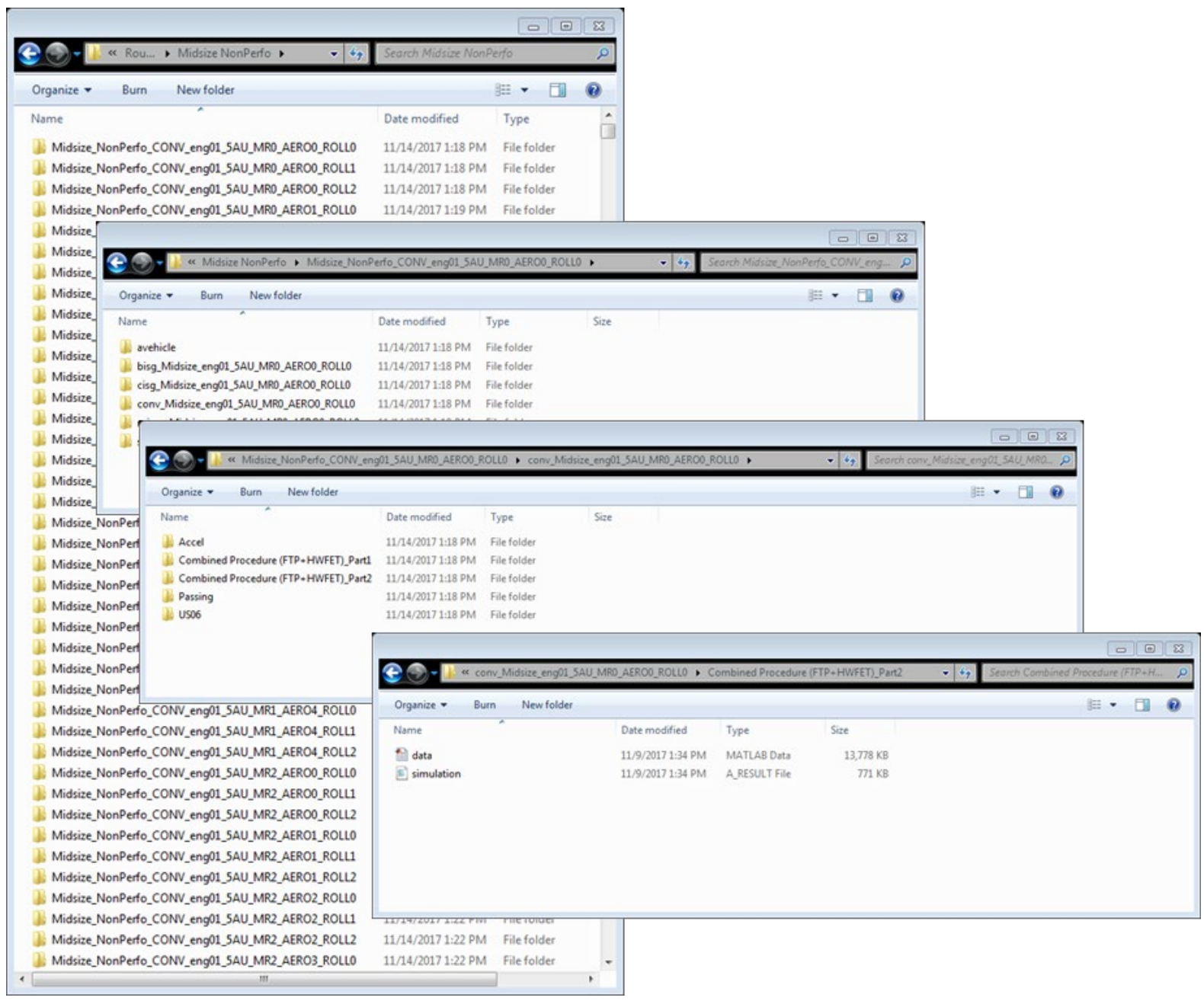

Figure 224. Vehicle simulation folder organization

Each folder contains the results for one combination and characterizes one branch/path of the tree. Folders can contain up to five directories, depending on the vehicle technology and the type of run performed. Results are divided into directories representing the cycle or procedure simulated. For example, the combined procedure for conventional vehicles has two folders, containing the FTP and HWFET runs, and the PHEV procedure has four folders, for the FTP and HWFET runs plus the CS and $\mathrm{CD}$ modes. The last directory is the sizing structure (performance test). 


\subsection{Data.mat File}

Data.mat is the results file containing all of the vehicle parameters and the time-based signals. A sample of signals and parameters included in data.mat is shown in Figure 225.

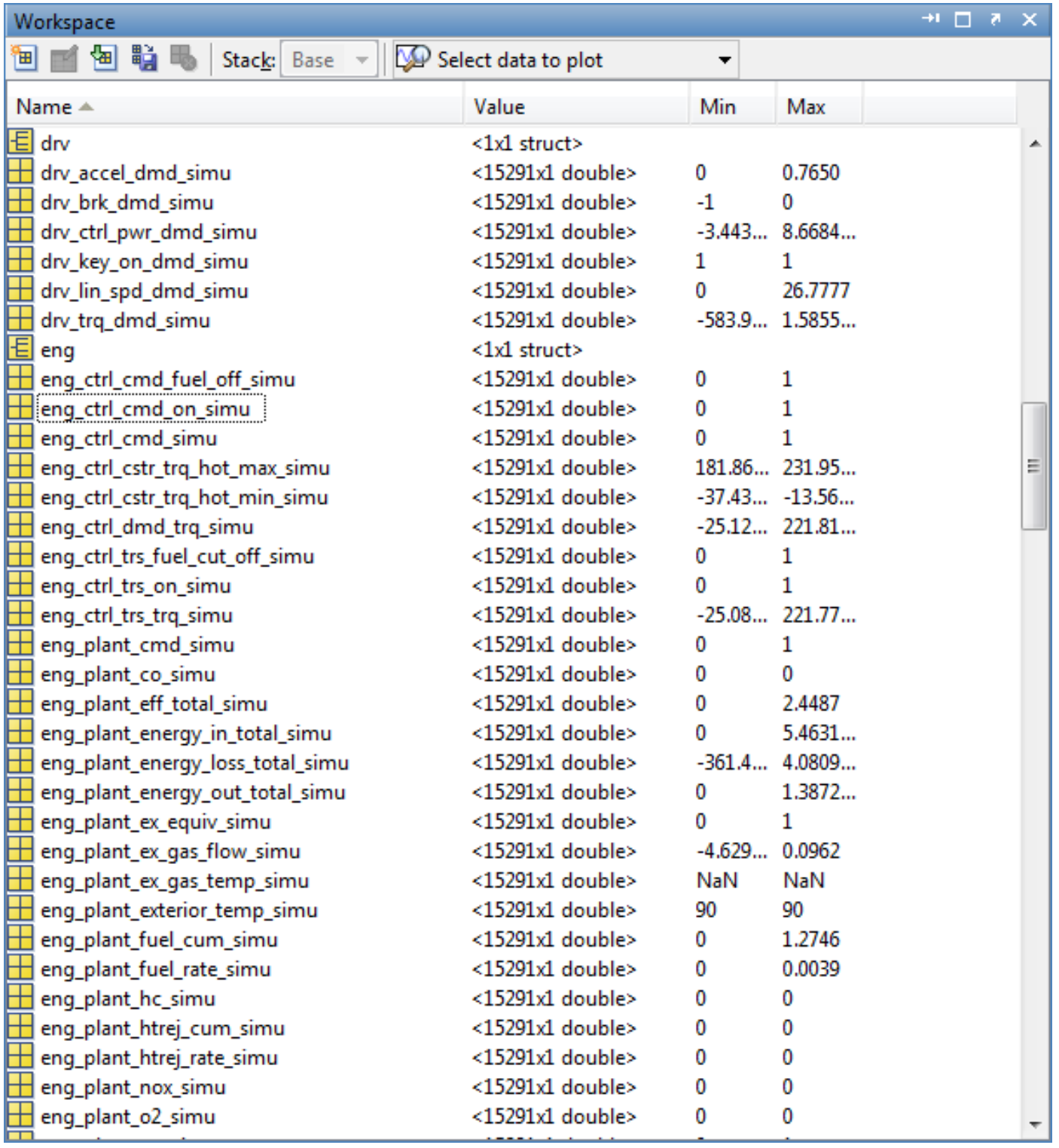

Figure 225. Sample Autonomie result (data.mat) 


\subsection{XML Results File}

As shown in Figure 226, simulation. a result is an XML version of the results file that includes the main simulation inputs and outputs. This file is later used to generate the complete database.

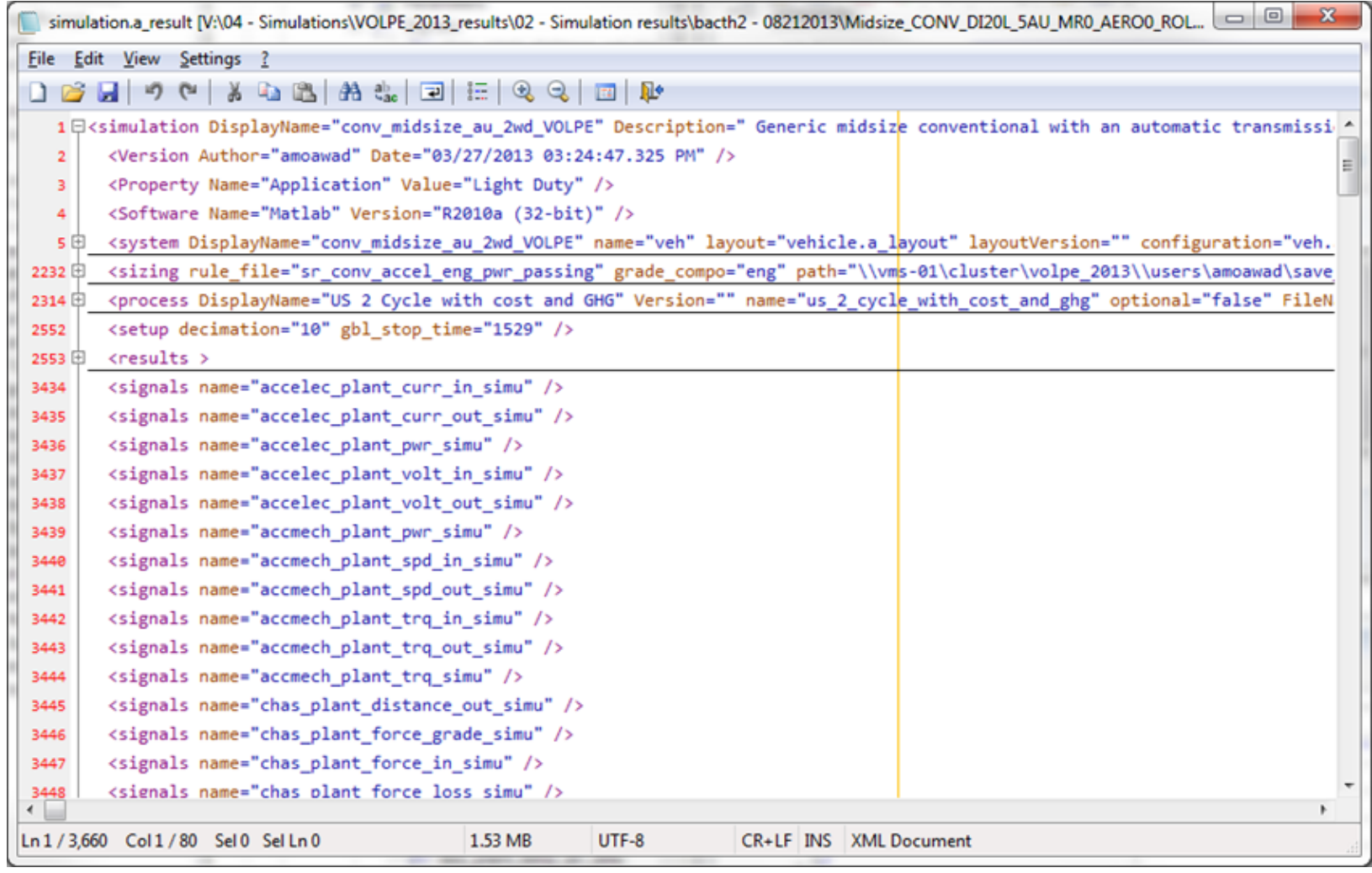

Figure 226. XML Autonomie results file (simulation.a_result) 


\section{SIMULATION RESULTS ANALYSIS AND DETAILED VALIDATION PROCESSES}

Analysis of the simulation results database is performed using Tableau and MATLAB. This section highlights some examples of these analyses with the objective of automatically detecting potential outliers so as to analyze them in depth.

\subsection{Engine Operating Points Across Transmissions}

Evaluating the engine density (i.e., operating points) across different transmission types and numbers of gears would help us evaluate the impact of improved transmissions. The particular vehicle selected for this analysis is as follows:

- Vehicle class: Midsize

- Performance category: Non-performance

- Engine: Engine 01

- Mass reduction: MR Level 0 (MR0)

- Aerodynamic reduction: AERO Level 0 (AERO0)

- Rolling-resistance reduction: ROLL Level 0 (ROLL0)

As expected, the average engine speed decreases with more gears and increased gear span.

\subsubsection{Manual Transmission}

Figure 227 shows the engine operating points for 5- and 6-speed manual transmissions during UDDS and HWFET cycles.
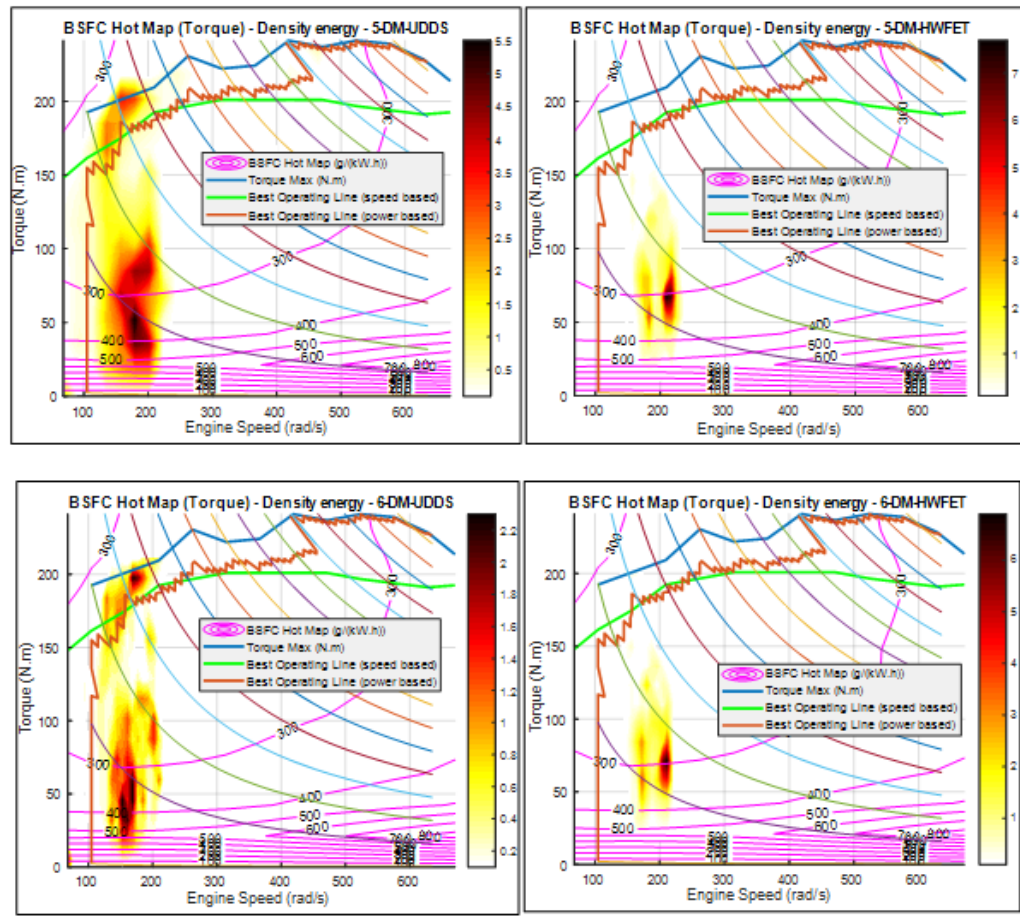

Figure 227. Engine operating points for 5- and 6-speed manual transmissions 


\subsubsection{Automatic Transmission}

Figure 228 shows the engine operating points for automatic transmission types with different numbers of gears.
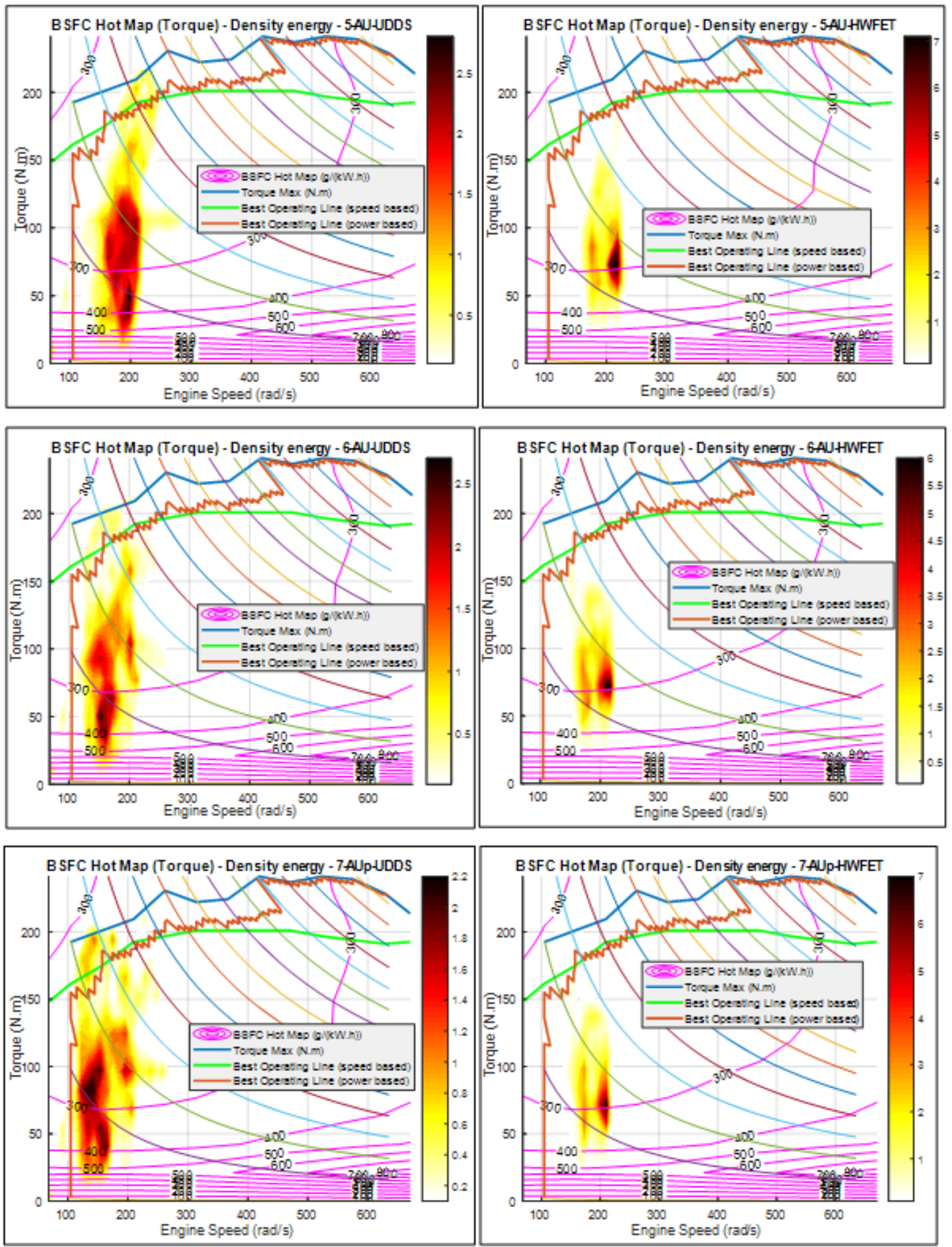

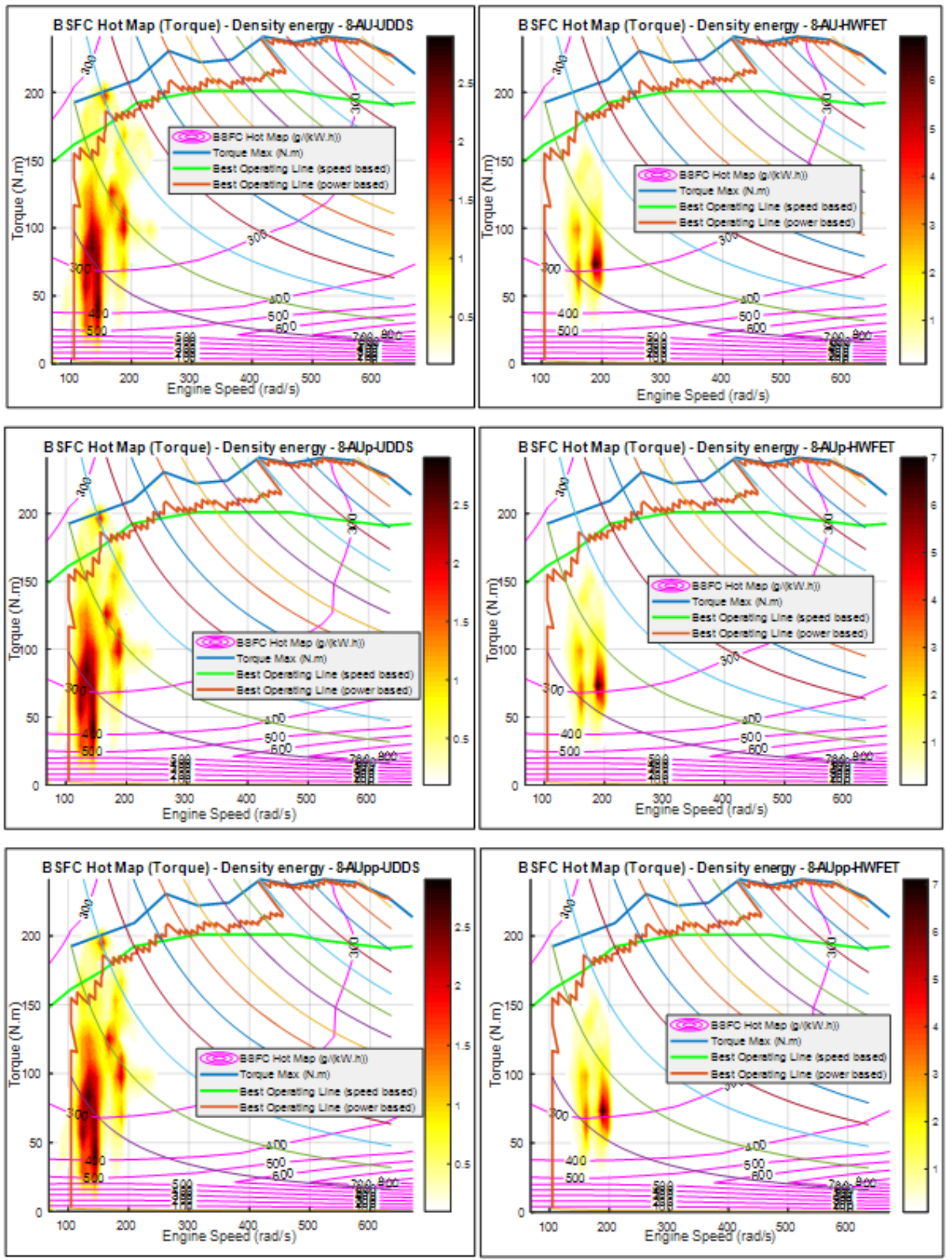

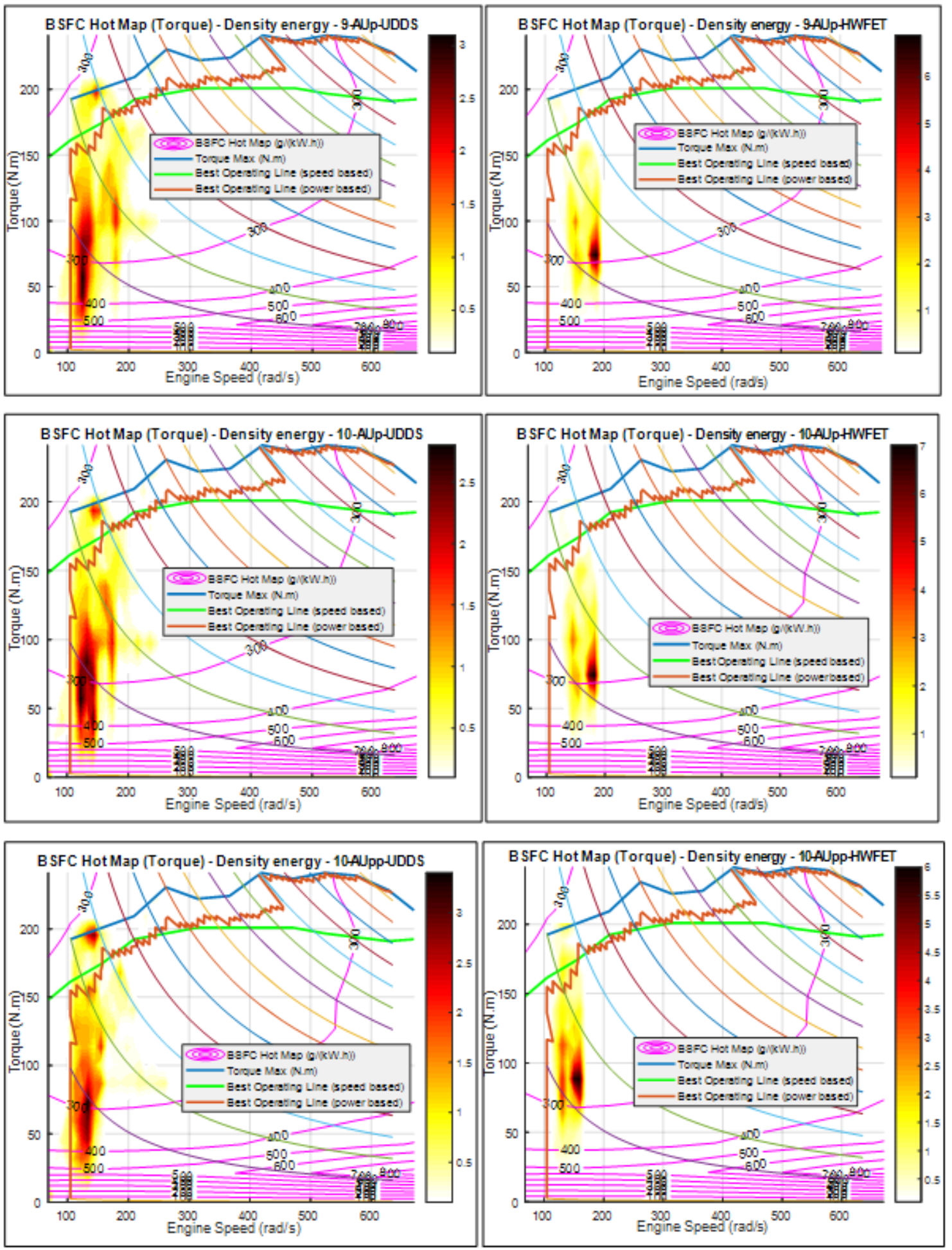

Figure 228. Engine operating points for automatic transmissions 


\subsubsection{Dual Clutch Transmissions}

Figure 229 shows the engine operating points for 6- and 8-speed DCTs.
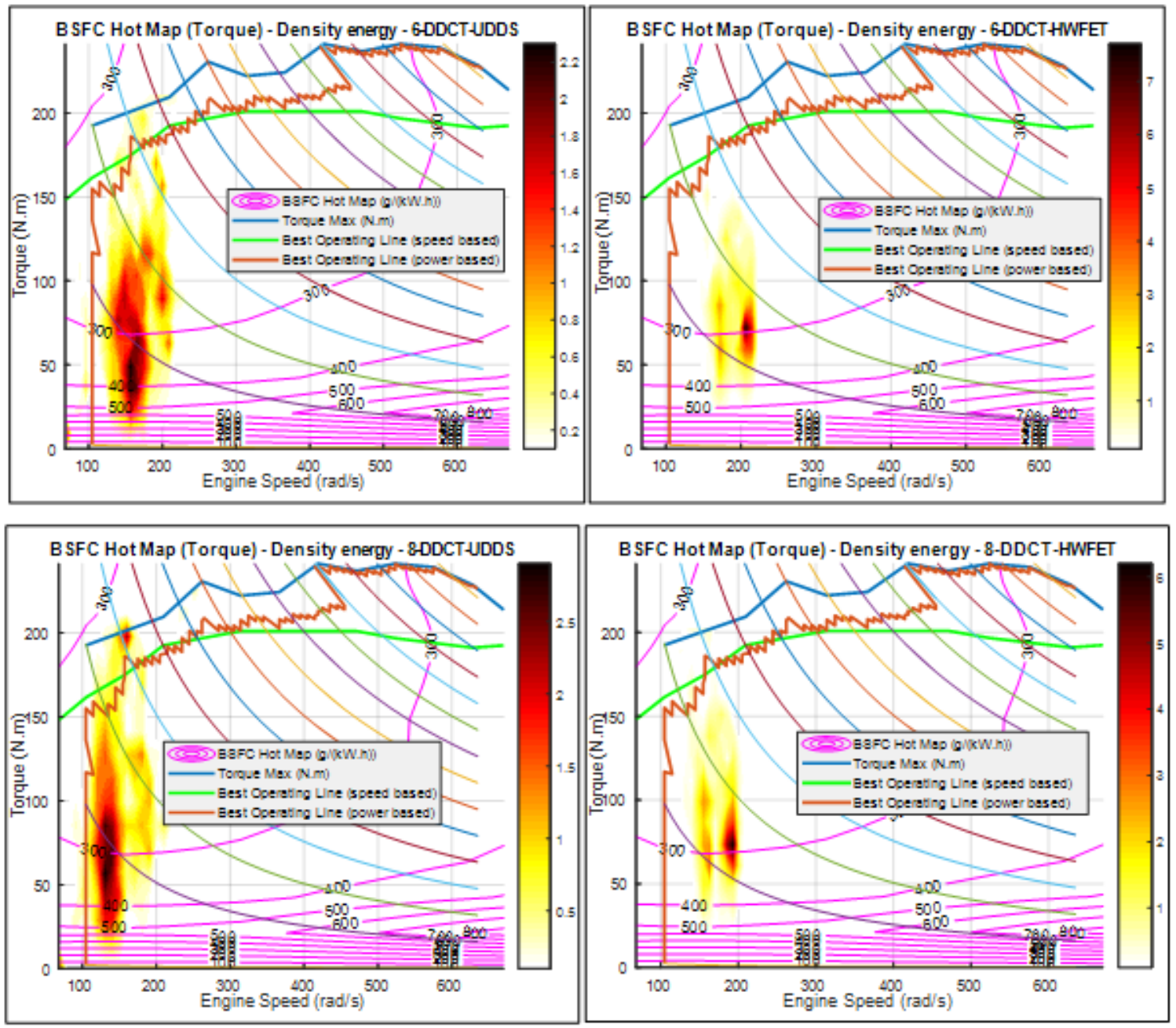

Figure 229. Engine operating points for 6- and 8-speed DCT transmissions 


\subsubsection{CVT transmission}

Figure 230 shows the engine operating points for two CVT types.
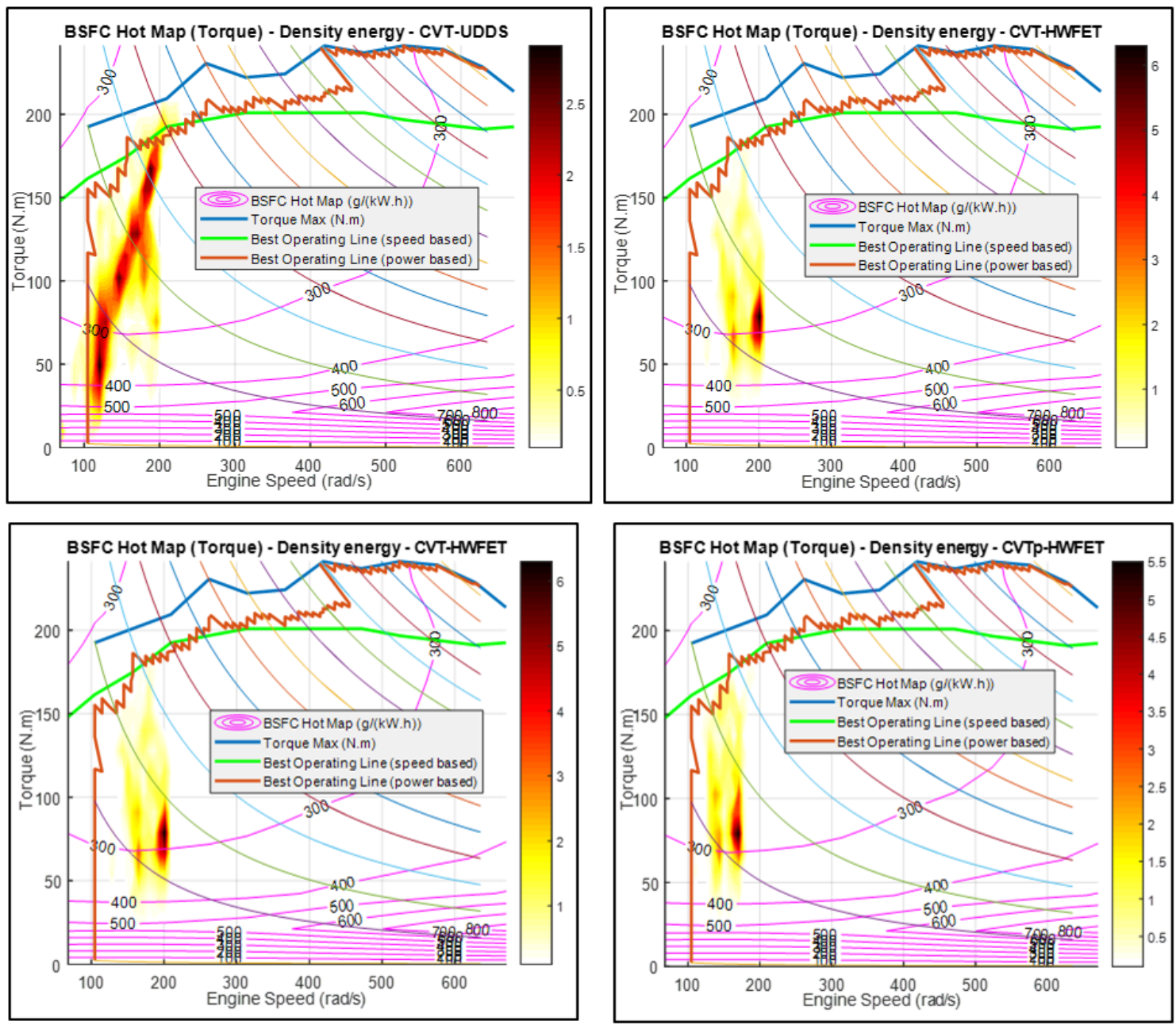

Figure 230. Engine operating points for CVTs

\subsection{Powertrain Efficiency Analysis}

Evaluating powertrain efficiency across different transmission types and numbers of gears as well individual powertrains would help us evaluate the impact of improved technologies. In this section, the particular vehicle combination selected for this analysis is as follows:

- Vehicle class: Midsize

- Vehicle powertrain: Conventional

- Performance category: Non-performance

- Aerodynamic reduction: AERO Level 0 (AERO0)

- Rolling-resistance reduction: ROLL Level 0 (ROLL0) 
Figure 231 shows the powertrain efficiency values for both UDDS and HWFET cycles for different engine types with automatic transmission and different numbers of gears.
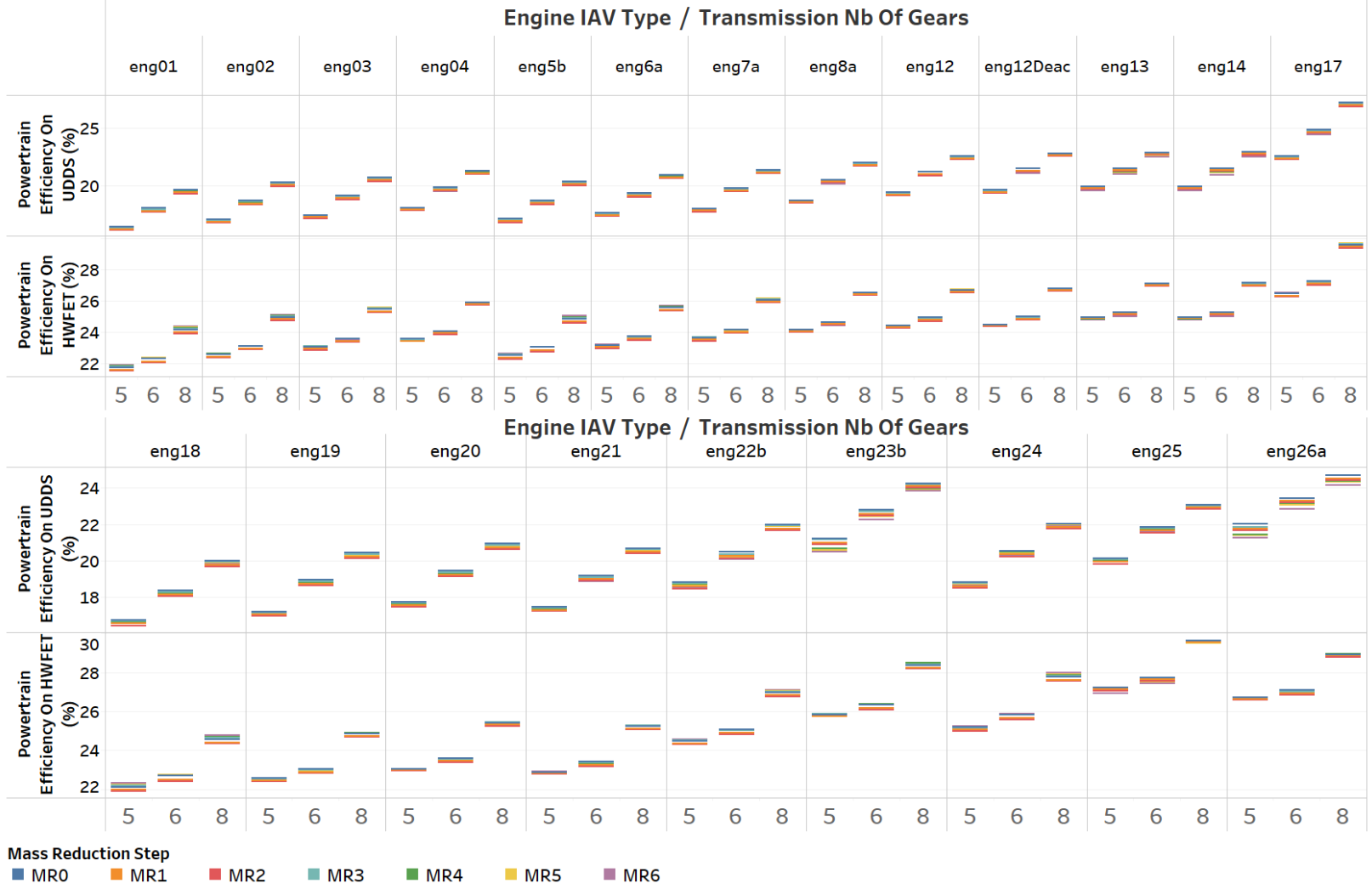

Figure 231. Powertrain efficiency values of different engine types with automatic transmissions and different numbers of gears

It can be seen that with an increasing number of gears, the powertrain efficiency increases for both UDDS and HWFET. This increase is more pronounced with engines demonstrating higher efficiencies (for example, eng01 vs. eng26a). 
Figure 232 shows the powertrain efficiency values for UDDS and HWFET for different engine types with automatic $+(\mathrm{AU}+)$ transmissions with different numbers of gears.
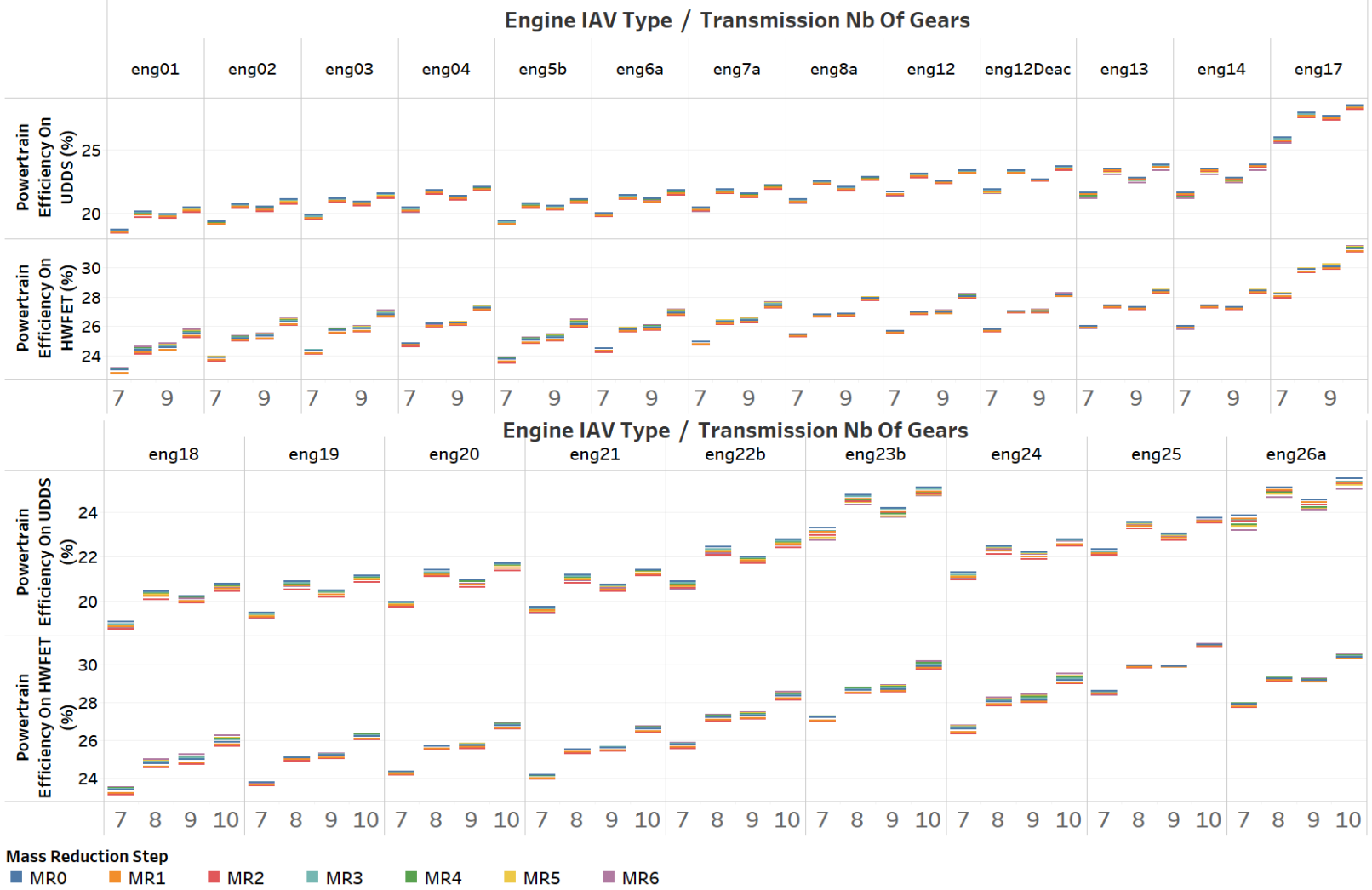

Figure 232. Powertrain efficiency values of different engine types with $A U+$ transmissions with different numbers of gears

From the figure, it can be seen that going from 8-speed to 9-speed AU+ causes powertrain efficiency to decrease slightly for UDDS, due to increased shifting, as well as a drop in gearbox efficiency.

However, for HWFET runs, powertrain efficiency increases with the number of gears. 
Figure 233 shows the powertrain efficiency values for different engine types with automatic++ $(\mathrm{AU}++)$ transmissions and different numbers of gears.

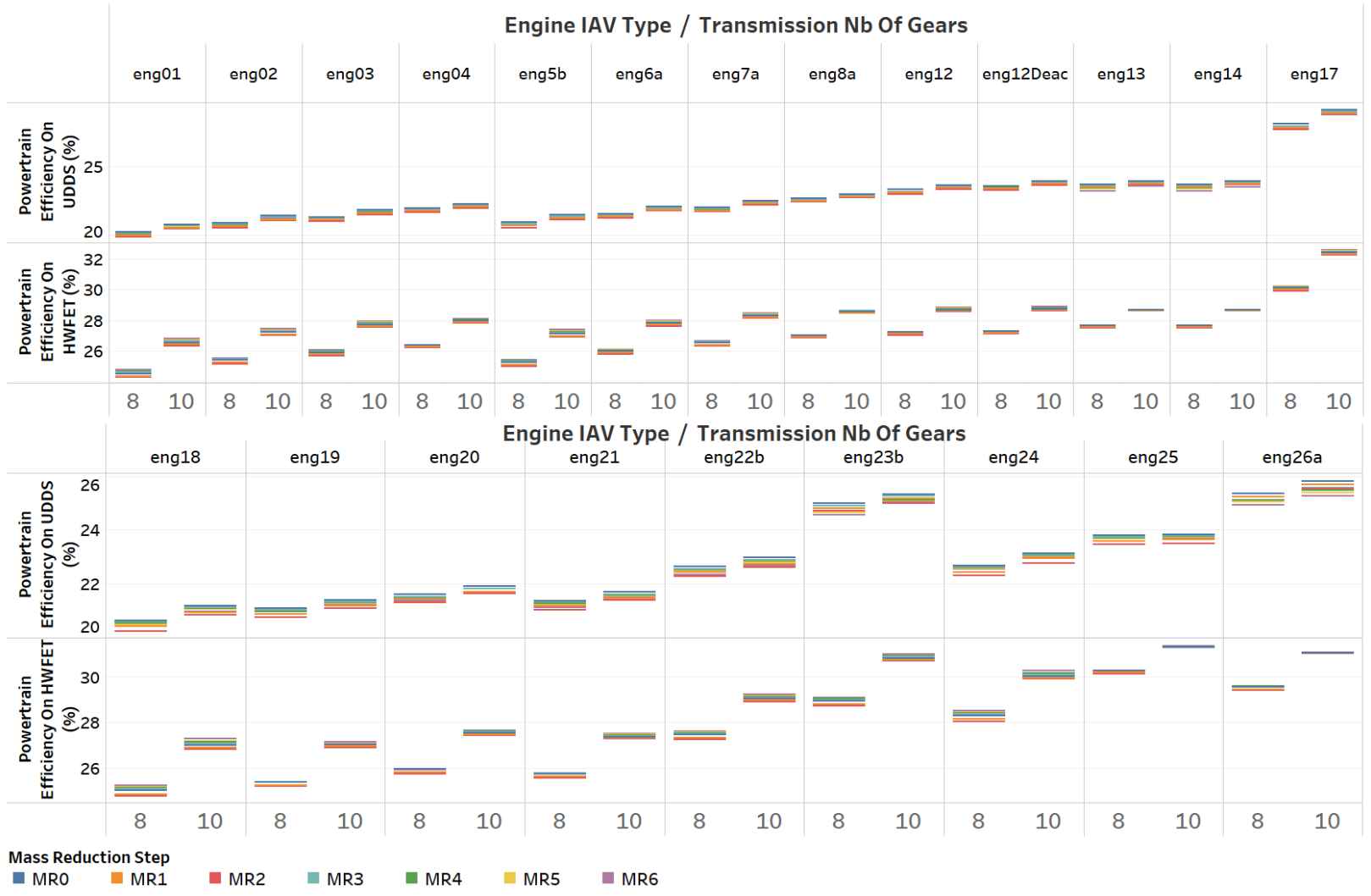

Figure 233. Powertrain efficiency values of different engine types with $A U++$ transmissions with different numbers of gears

For AU++ transmission, powertrain efficiency increases with an increased number of gears for UDDS and HWFET runs. Values also improve for engines demonstrating higher technology effectiveness (for example, eng01 vs. eng26a). 
Figure 234 shows the powertrain efficiency values for the different engine types with DCTs and different numbers of gears.

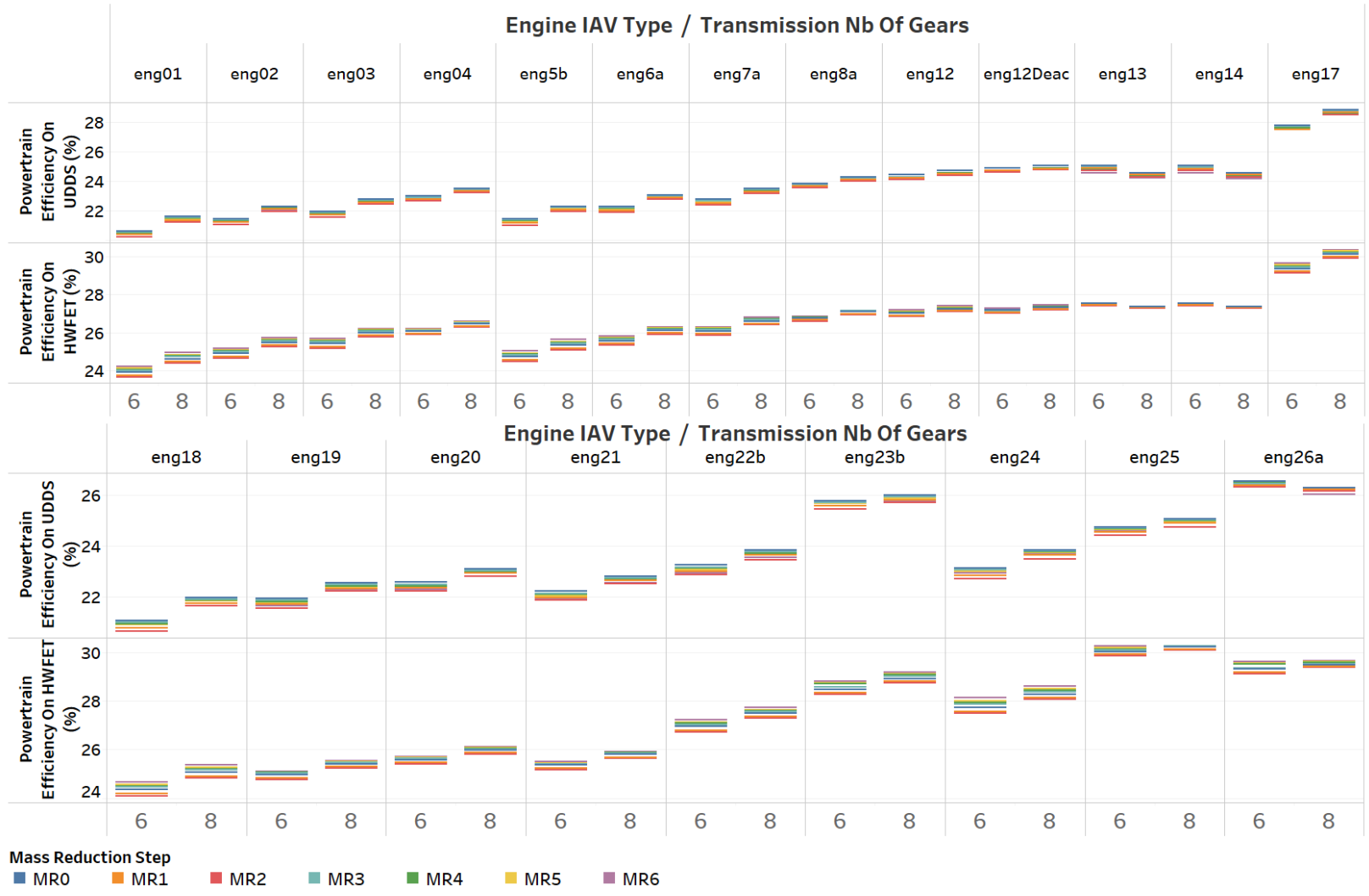

Figure 234. Powertrain efficiency values of different engine types with DCTs with different numbers of gears

The powertrain efficiency of DCT transmissions increases with the number of gears for both UDDS and HWFET runs. Values also improve for engines demonstrating higher technology effectiveness (for example, eng01 vs. eng26a). 
Figure 235 shows the powertrain efficiency values for different engine types with manual transmissions and different numbers of gears.

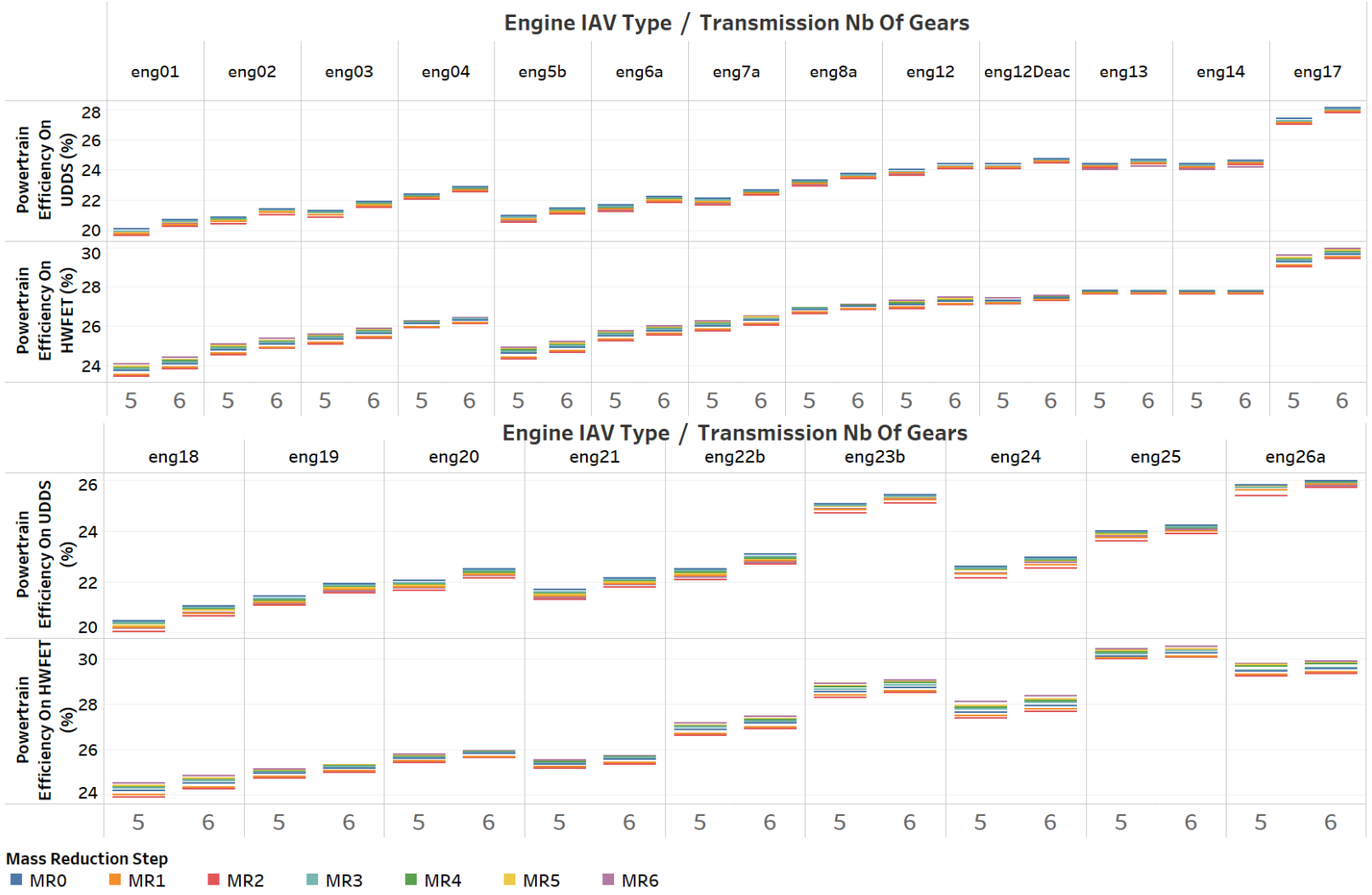

Figure 235. Powertrain efficiency values of different engine types with manual transmissions with different numbers of gears

It can be seen that with increasing number of gears, the powertrain efficiency increases for both UDDS and HWFET. This increment is further increased with engines demonstrating higher efficiencies (for example, eng01 vs. eng26a). 
Figure 236 shows the powertrain efficiency values for different engine types with CVTs.
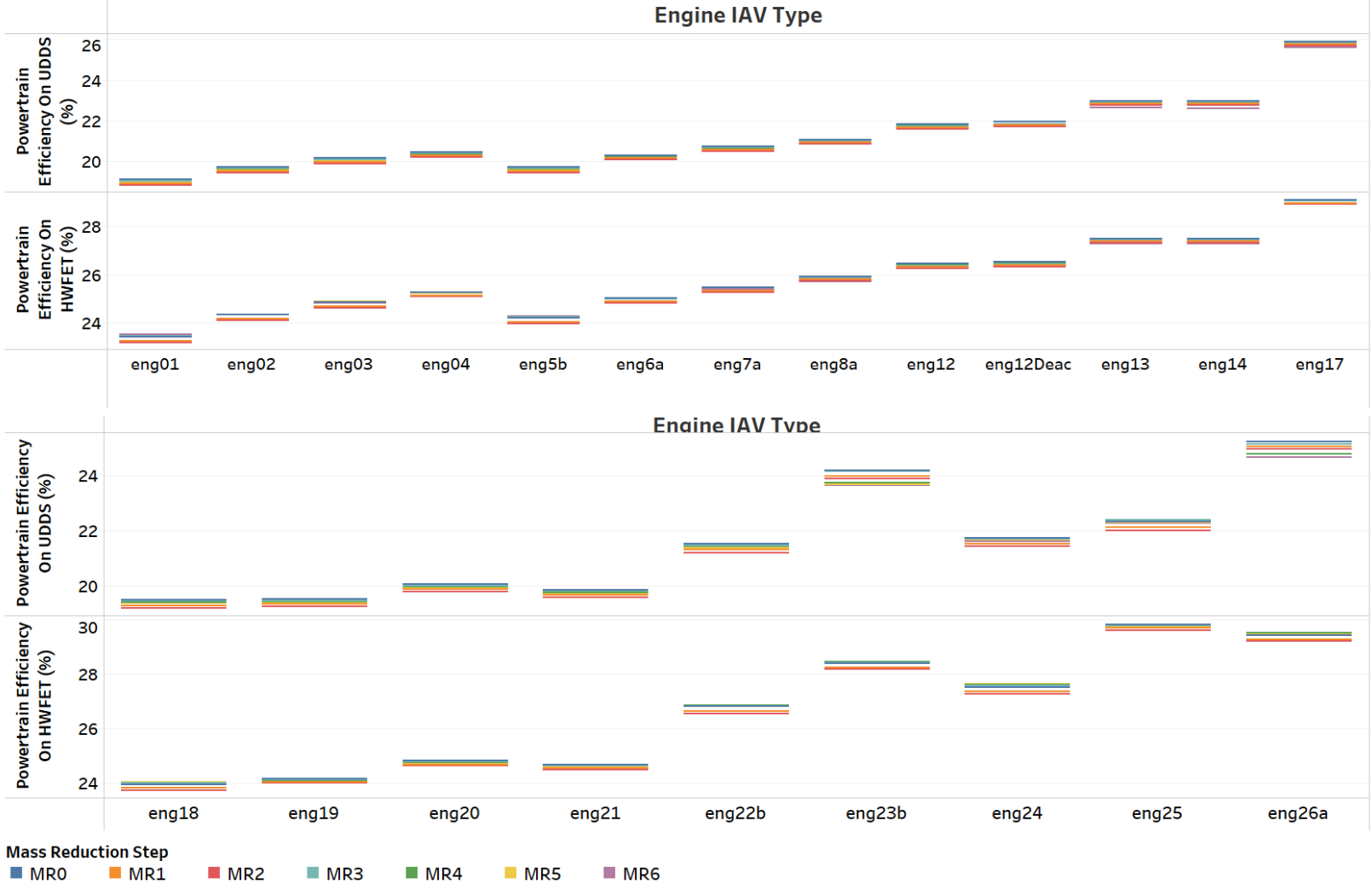

Figure 236. Powertrain efficiency values of different engine types with CVT

For CVTs, the powertrain efficiency increases with higher engine efficiencies (for example, eng01 vs. eng26a). 
Figure 237 shows the powertrain efficiency values for different engine types with CVT+.
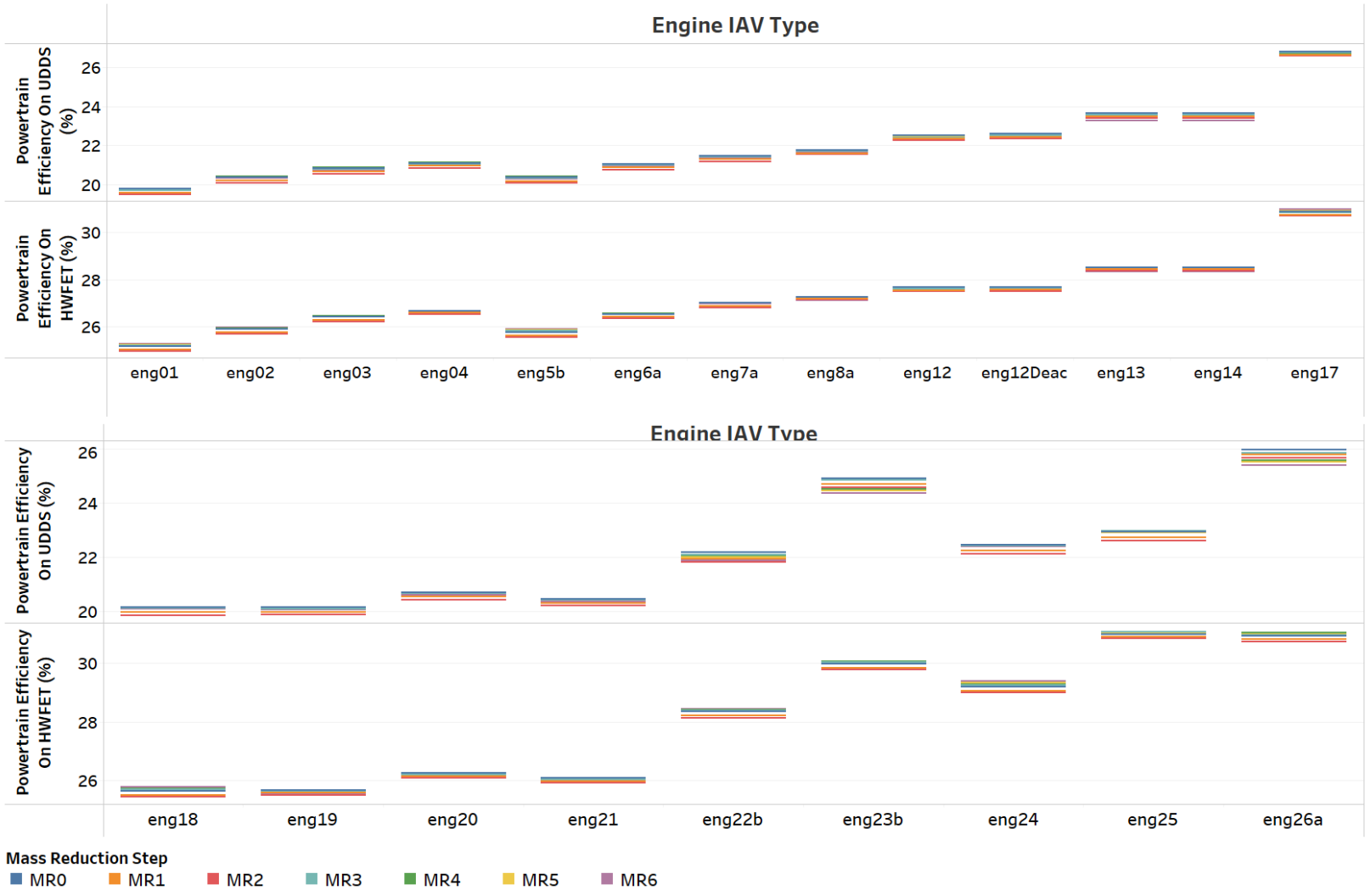

Figure 237. Powertrain efficiency values of different engine types with CVTps

As with CVTs, for CVT + the powertrain efficiency increases with higher engine efficiencies (for example, eng01 vs. eng26a).

\subsection{Total Number of Shifting Events with Different Transmission Types and Numbers of Gears}

The total number of shifting events (upshift + downshift) determines the drive quality, so it is important to check the total number of shifting events across transmissions and numbers of gears against vehicle test data. Throughout this analysis process, the following vehicle was used:

- Vehicle class: Midsize

- Performance category: Non-performance

- Powertrain type: Conventional

- Engine: Engine 01

- Aerodynamic reduction: AERO Level 0 (AERO0)

- Rolling-resistance reduction: ROLL Level 0 (ROLL0) 


\subsubsection{Automatic Transmission Types}

Figure 238 shows the total number of shifting events for automatic (AU), automatic+ (AUp), and automatic++ (AUpp) transmissions with different numbers of gears during a UDDS cycle.

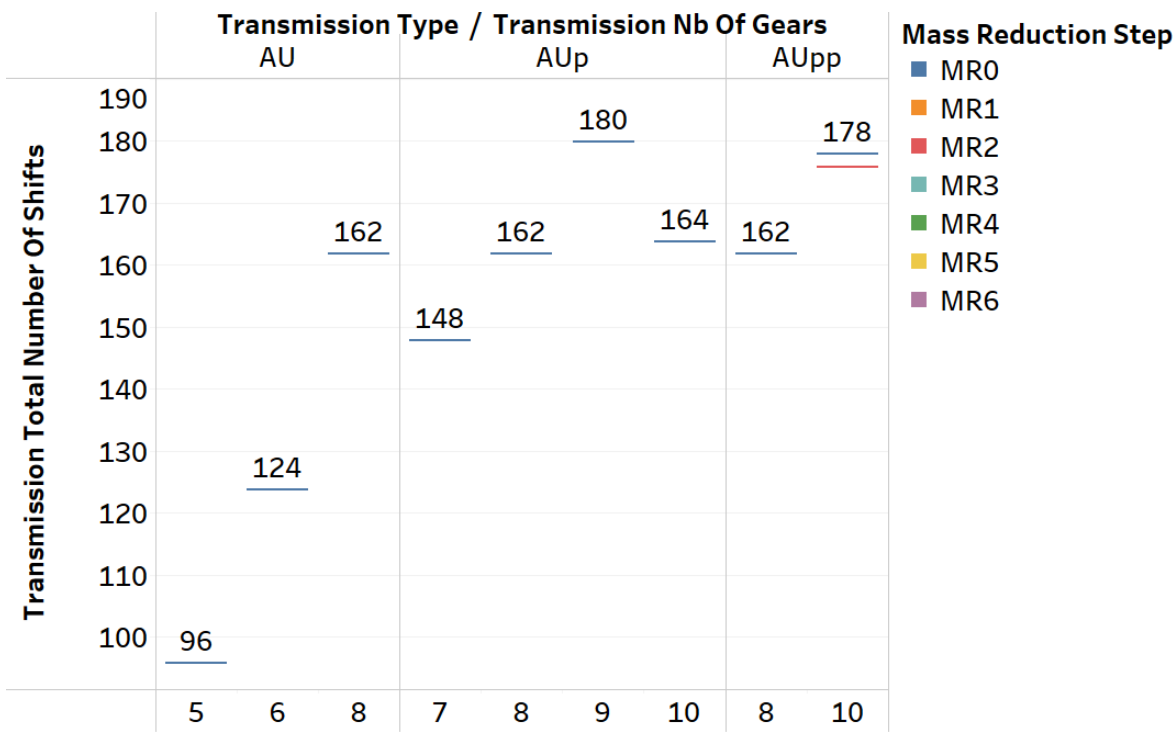

Figure 238. Total number of shifting events for AU/AU+/AU++ transmissions during UDDS cycle

For UDDS runs, the total number of shifting events increases with increasing number of gears. However, for 10-speed transmissions, the total number of shifting events is similar to that of an 8-speed transmission, due to the implementation of gear-skipping methods.

Figure 239 shows the total number of shifting events for automatic (AU), automatic+ (AUp), and automatic ++ (AUpp) transmissions with different numbers of gears during a US06 (a high acceleration aggressive driving schedule) cycle.

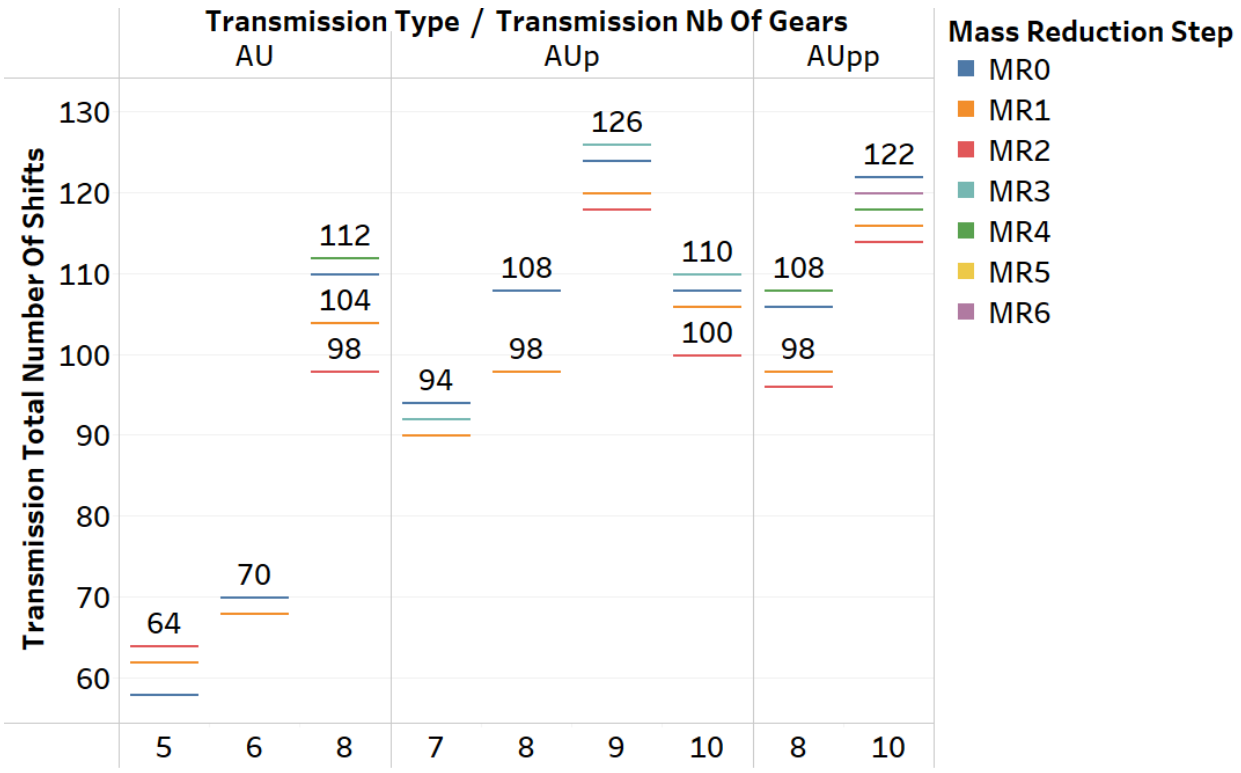

Figure 239. Total number of shifting events for AU/AU+/AU++ transmissions during US06 cycle 
For US06 runs, the total number of shifting events increases with increasing number of gears, again with the exception of 10-speed transmissions due to the implementation of gear-skipping methods. The total number of shifting events for US06 runs is lower than for UDDS runs.

\subsubsection{Manual Transmission Types}

Figure 240 shows the total number of shifting events for manual (DM) transmissions with different numbers of gears during a UDDS cycle.

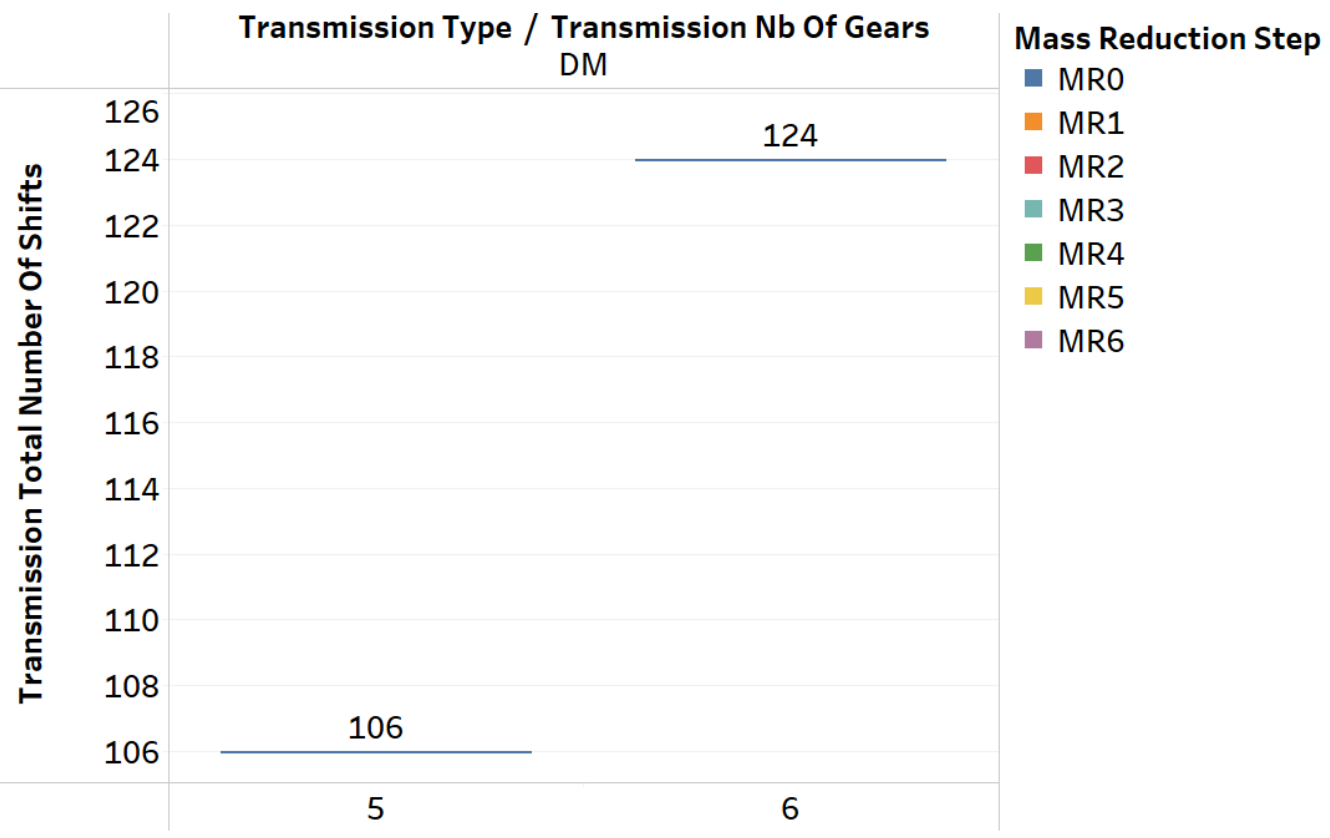

Figure 240. Total number of shifting events for DM transmissions during UDDS cycle

For DM runs, the total number of shifting events increases with increasing number of gears. The total number of shifting events for a 5-speed DM is higher than that of automatic transmission types, due to the difference in gear span and final drive ratio. The total number of shifting events for a 6-speeed DM is the same as for a 6-AU.

Figure 241 shows the total number of shifting events for manual (DM) transmissions with different numbers of gears during a US06 cycle. 


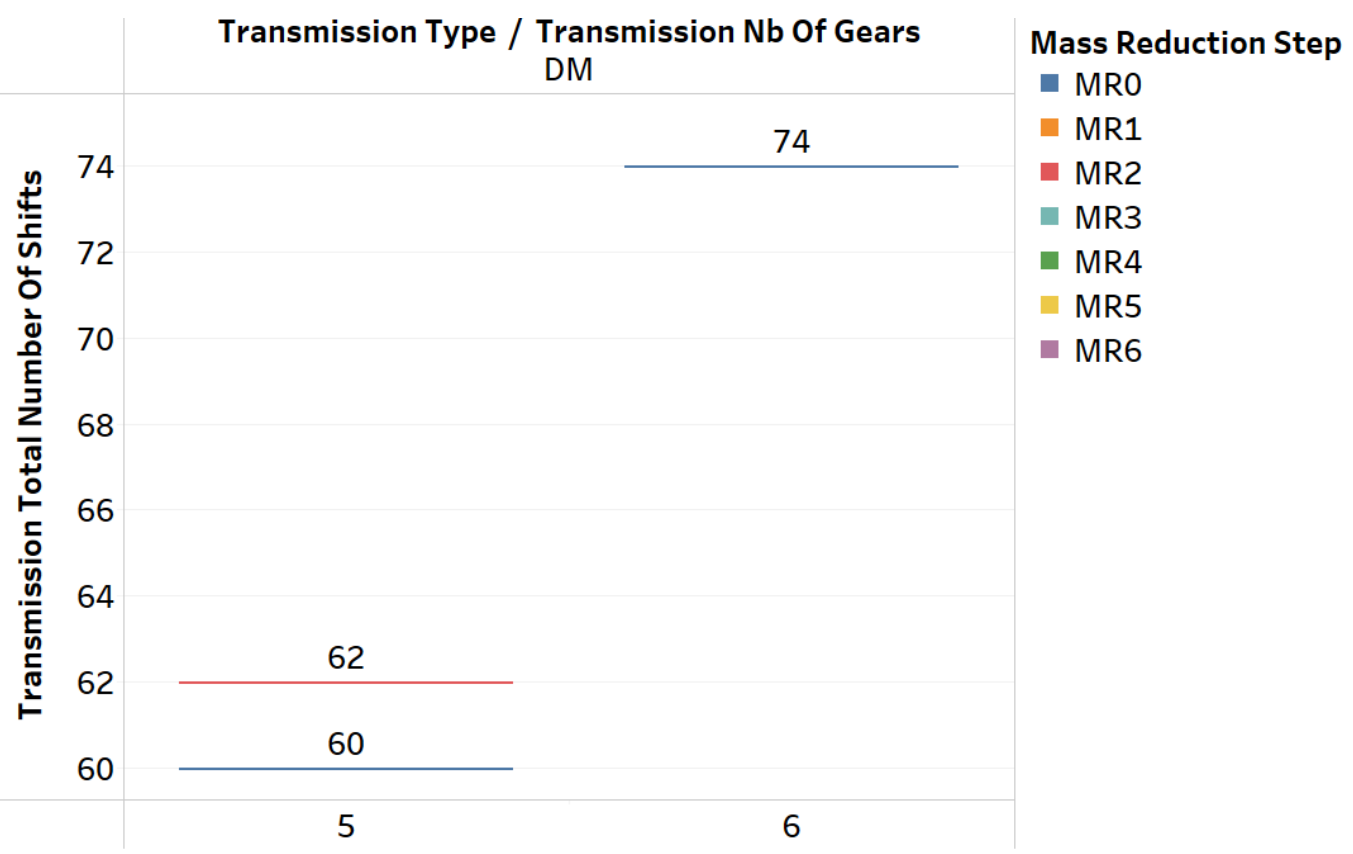

Figure 241. Total number of shifting events for DM transmissions during US06 cycle

As in UDDS runs, the total number of shifting events for DM transmissions during a US06 cycle run is comparable to that of $\mathrm{AU}$ transmissions.

\subsubsection{Dual Clutch Transmission Types}

Figure 242 shows the total number of shifting events for DCTs with different numbers of gears during a UDDS cycle.

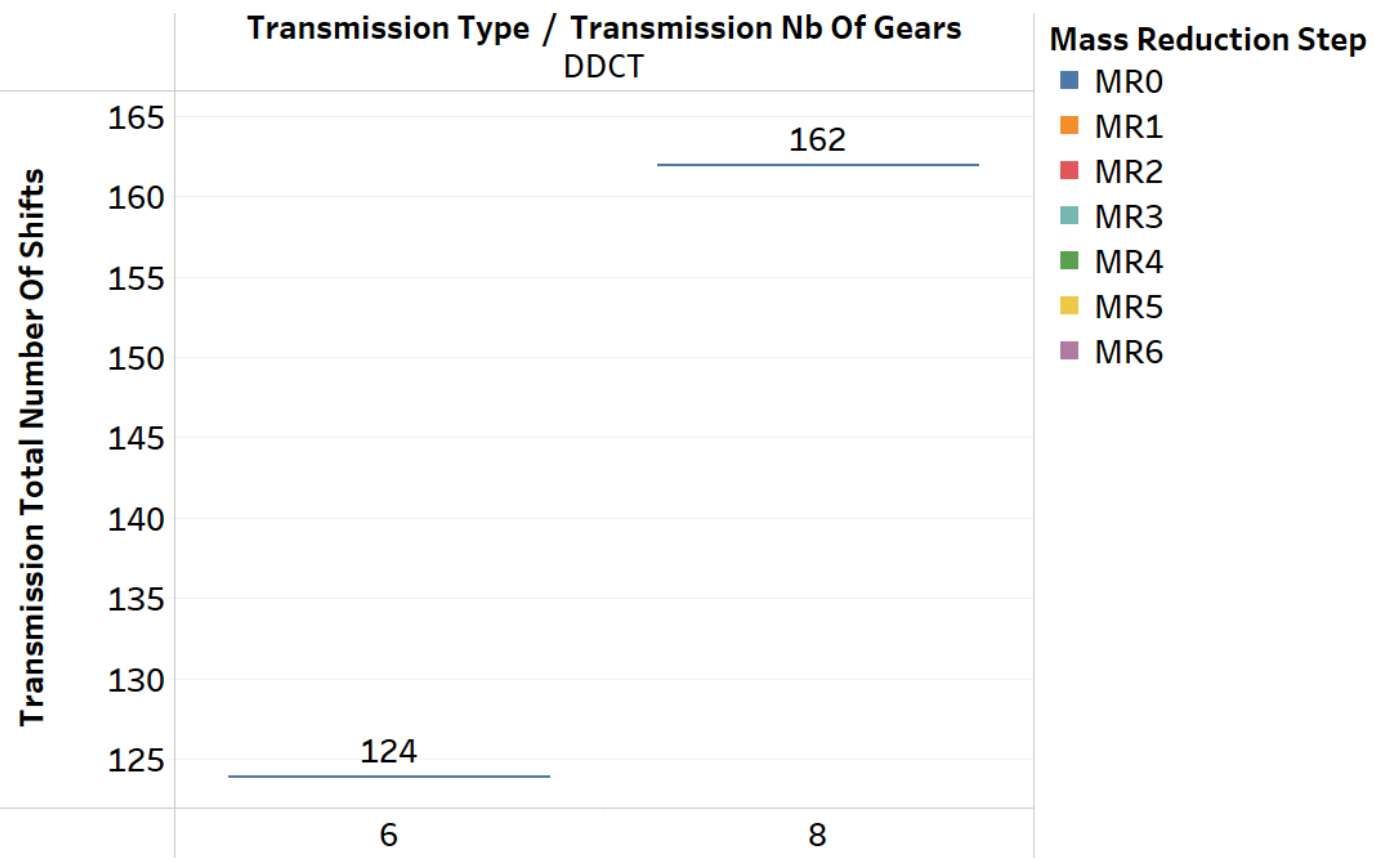

Figure 242. Total number of shifting events for DCTs during UDDS cycle 
For DCT transmissions, the total number of shifting events increases with an increasing number of gears in UDDS runs, as was observed for AU/DM transmission types.

Figure 243 shows the total number of shifting events for DCTs with different numbers of gears during a US06 cycle.

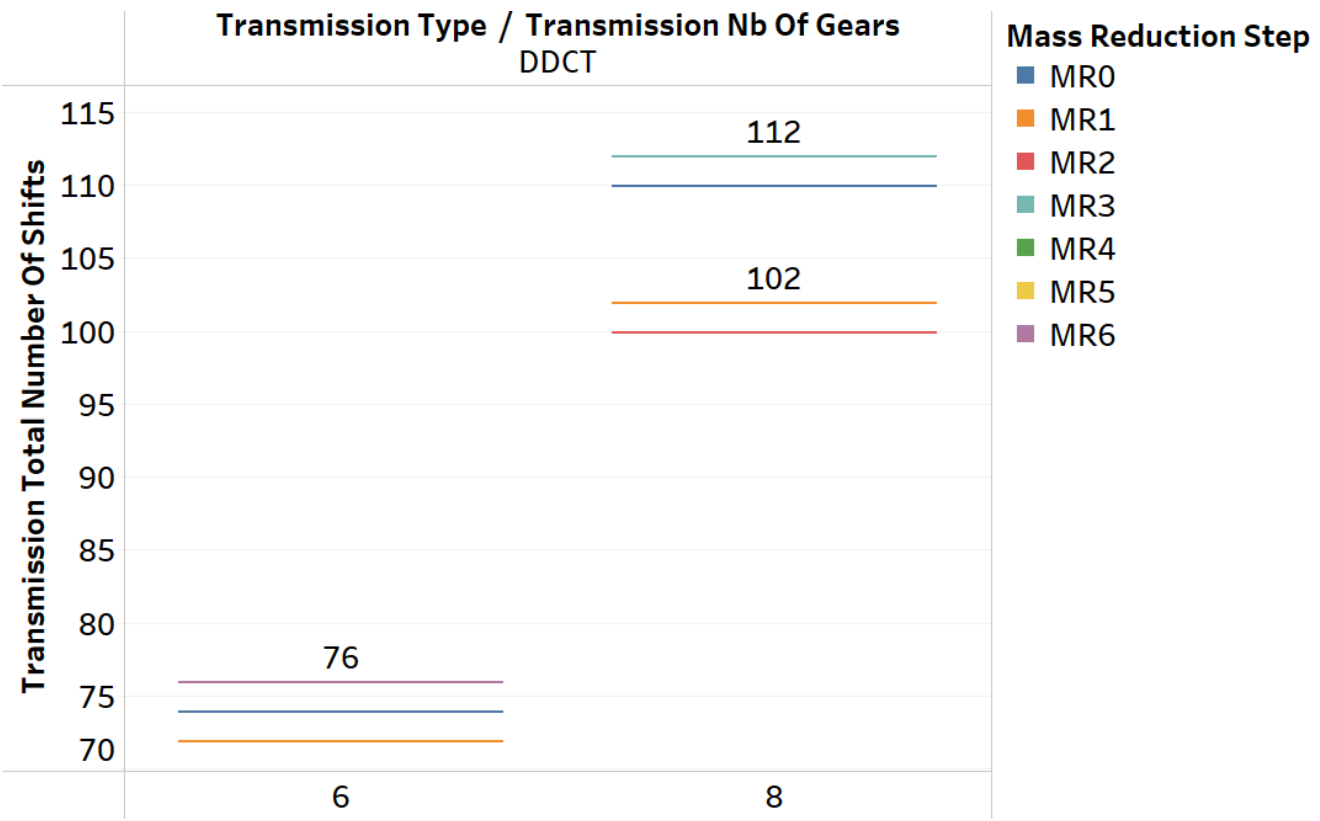

Figure 243. Total number of shifting events for DCTs during US06 cycle

\subsection{Engine Power Inheritance Validation}

As part of the vehicle inheritance validation, the engine power of the inherited vehicles was analyzed in comparison to baseline vehicles sized for the conventional powertrain. Figure 244 shows the engine power of the conventional vehicles in the compact vehicle class (non-performance category) in response to the mass reduction step.
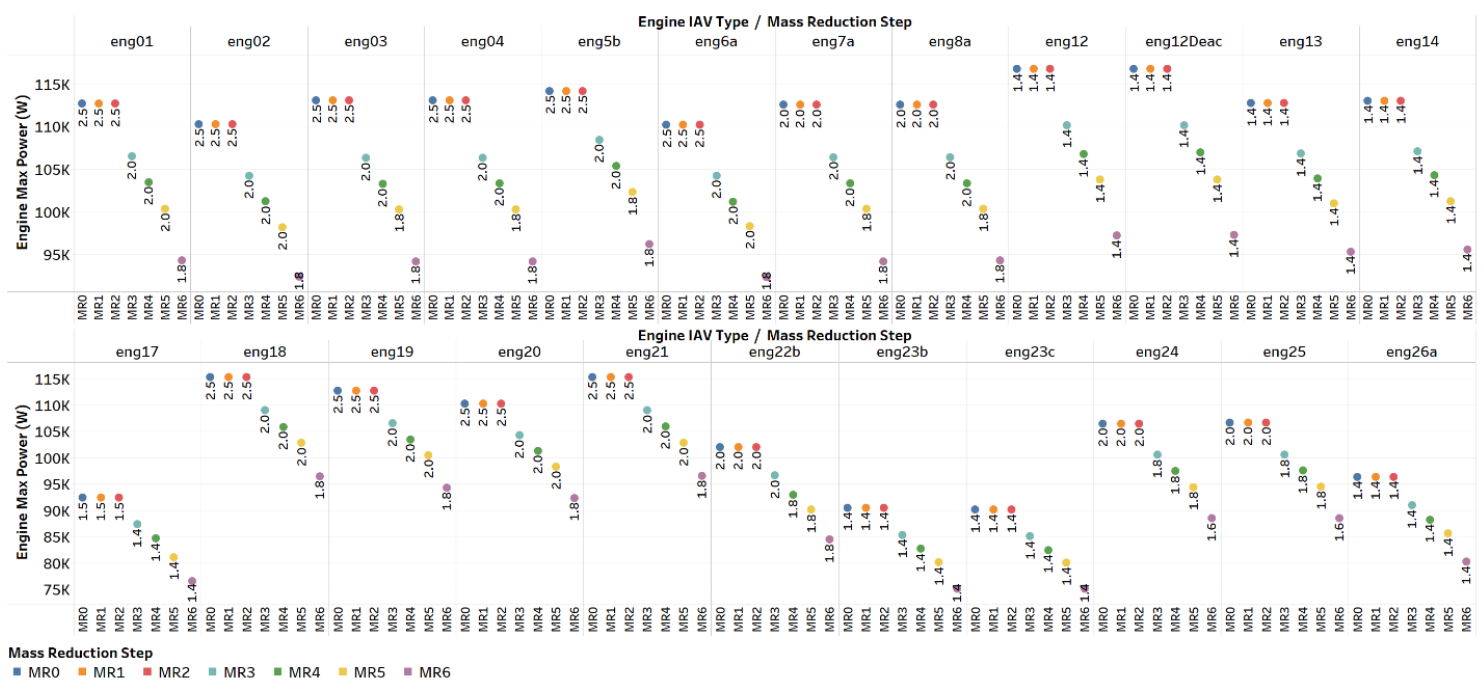

Figure 244. Engine power vs. mass reduction step (compact, non-performance) 
Figure 245 shows the engine power of the conventional vehicles in the compact vehicle class (performance category) in response to the mass reduction step.

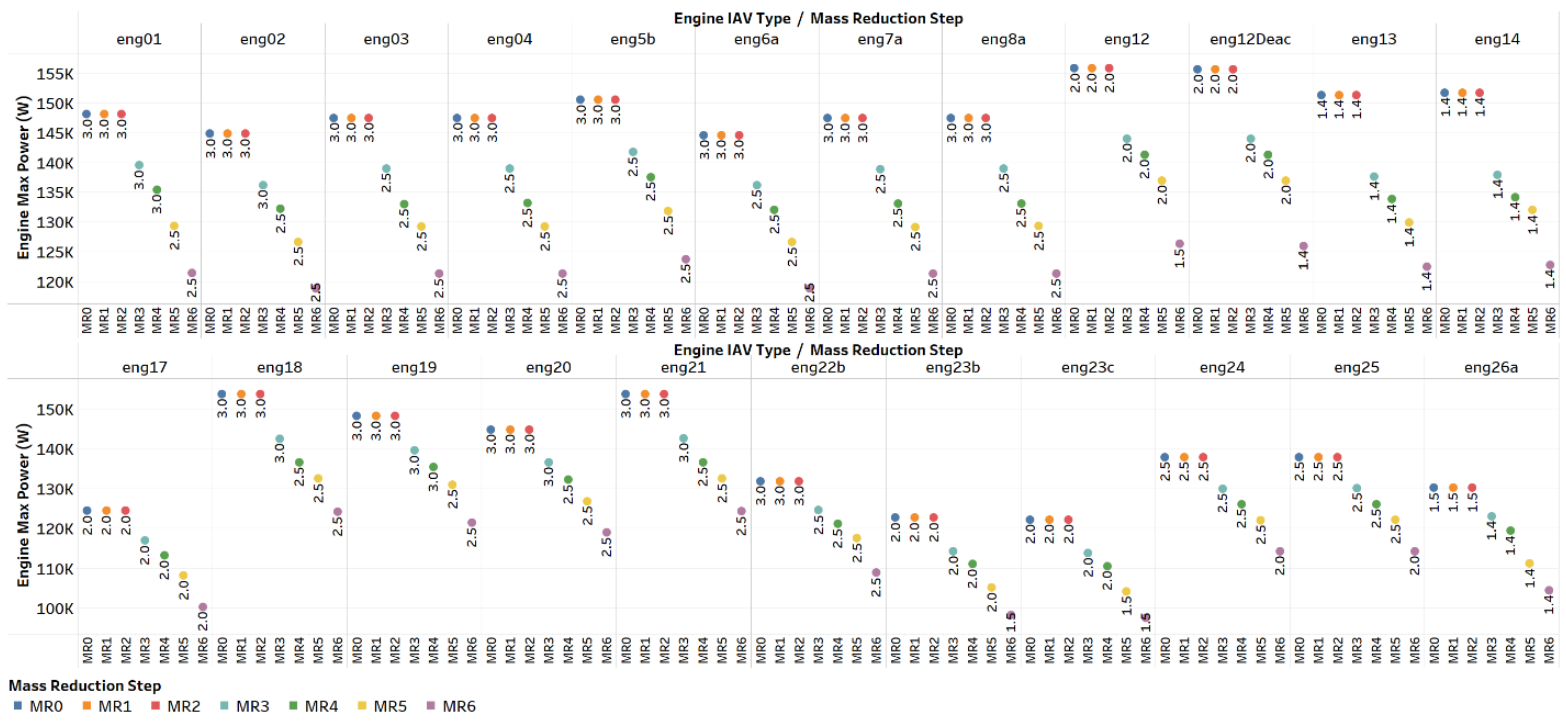

Figure 245. Engine power vs. mass reduction step (compact, performance)

Figure 246 shows the engine power of the conventional vehicles in the midsize vehicle class (nonperformance category) in response to the mass reduction step.

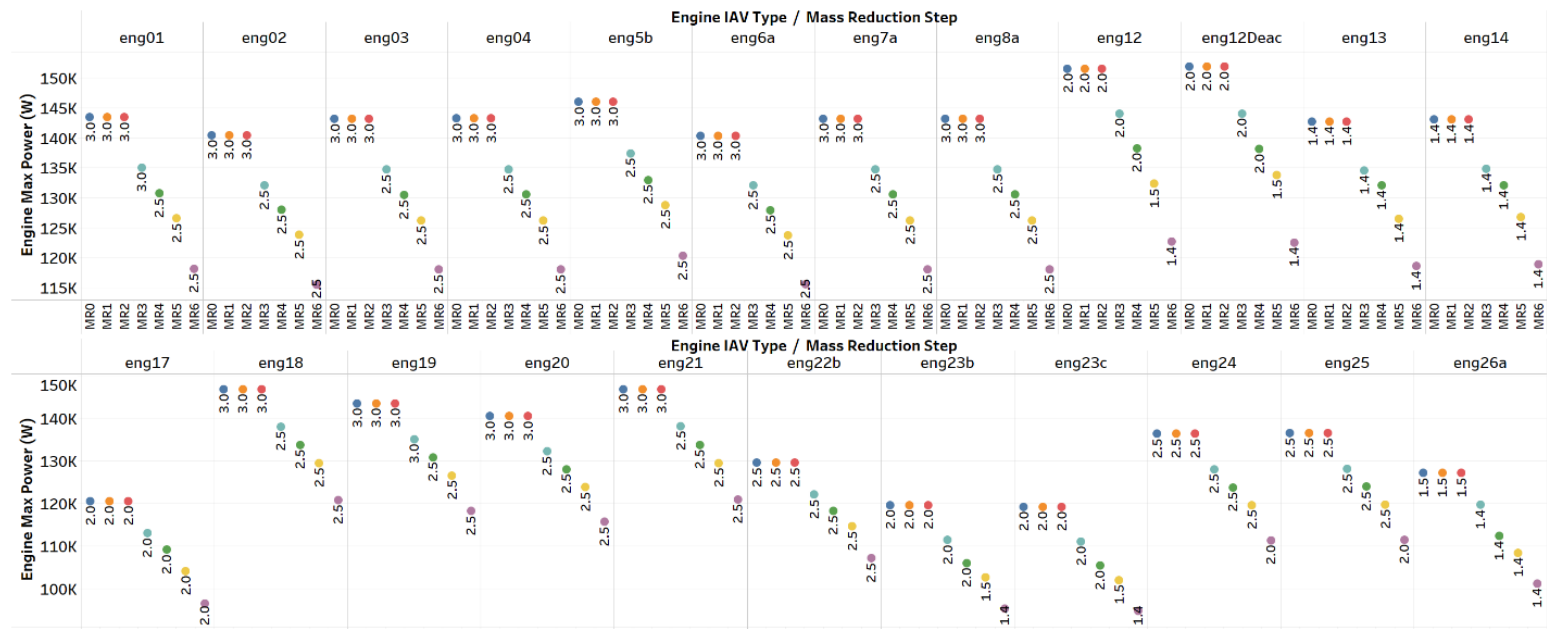

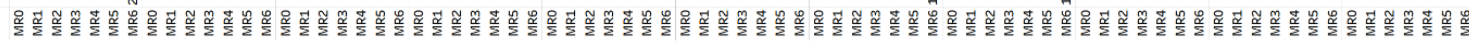
Mass Reduction Step
| MR0 | MR1 | MR2 | MR3 | MR4 | MR5 | MR6

Figure 246. Engine power vs. mass reduction step (midsize, non-performance) 
Figure 247 shows the engine power of the conventional vehicles in the midsize vehicle class (performance category) in response to the mass reduction step.

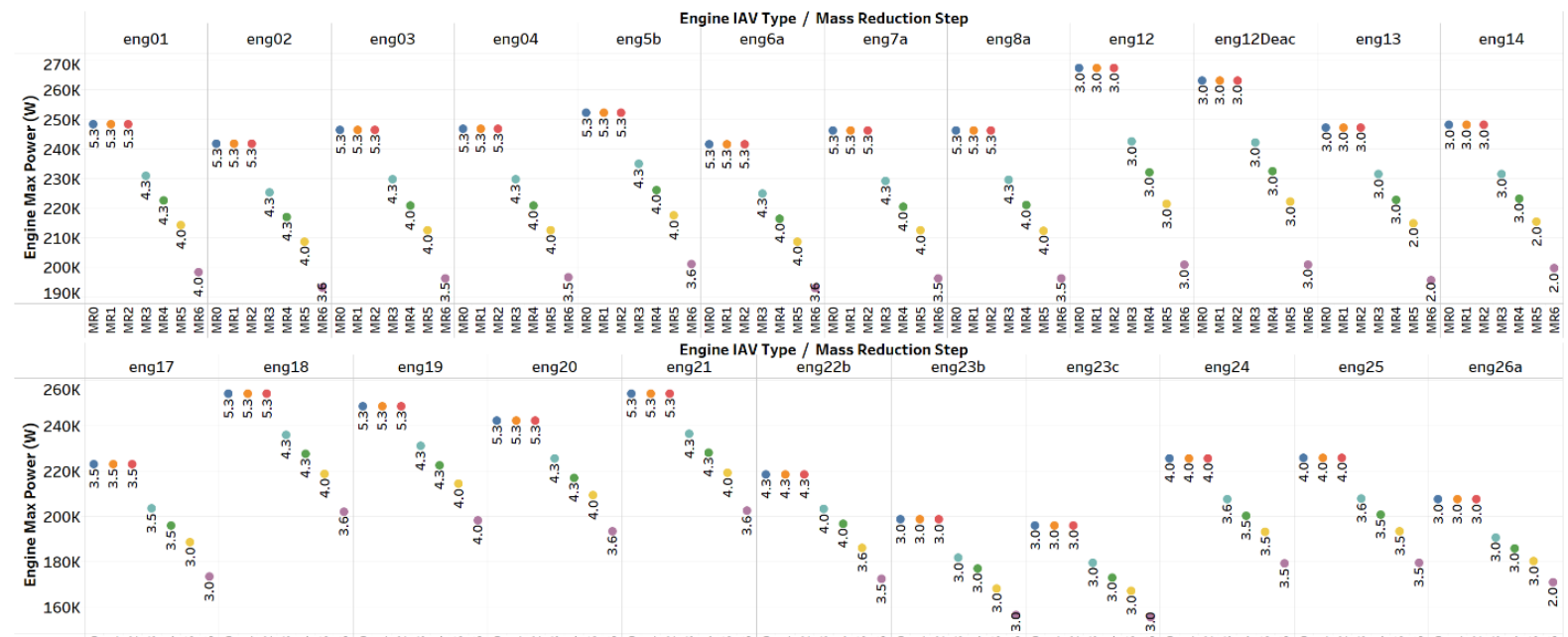

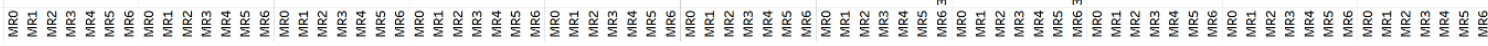

Mass Reduction Step
= MRO = MR1 | MR2 $=$ MR3 $=$ MR4 $=$ MR5 $=$ MR6

Figure 247. Engine power vs. mass reduction step (midsize, performance)

Figure 248 shows the engine power of the conventional vehicles in the small SUV vehicle class (nonperformance category) in response to the mass reduction step.

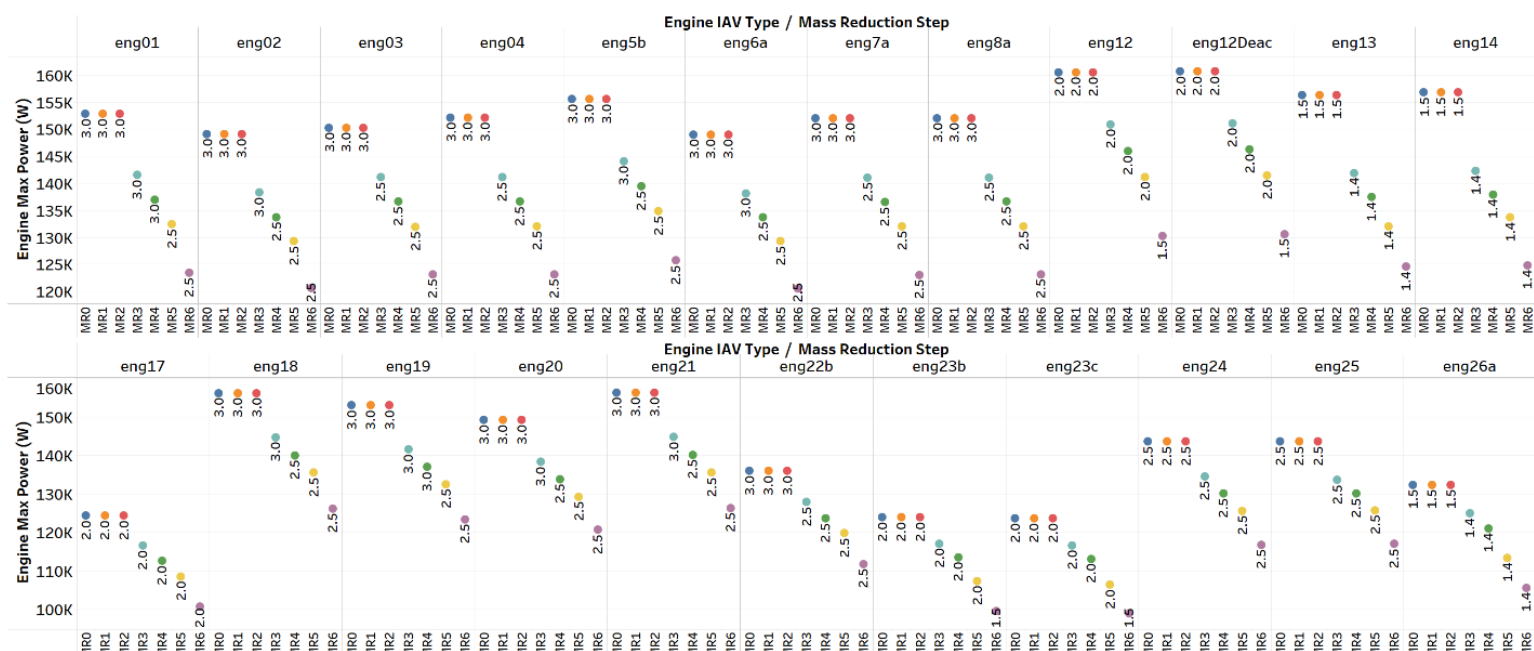

Mass Reduction Step
| MRO || MR1 || MR2 || MR3 || MR4 || MR5 || MR6

Figure 248. Engine power vs. mass reduction step (small SUV, non-performance) 
Figure 249 shows the engine power of conventional vehicles in the small SUV vehicle class (performance category) in response to the mass reduction step.

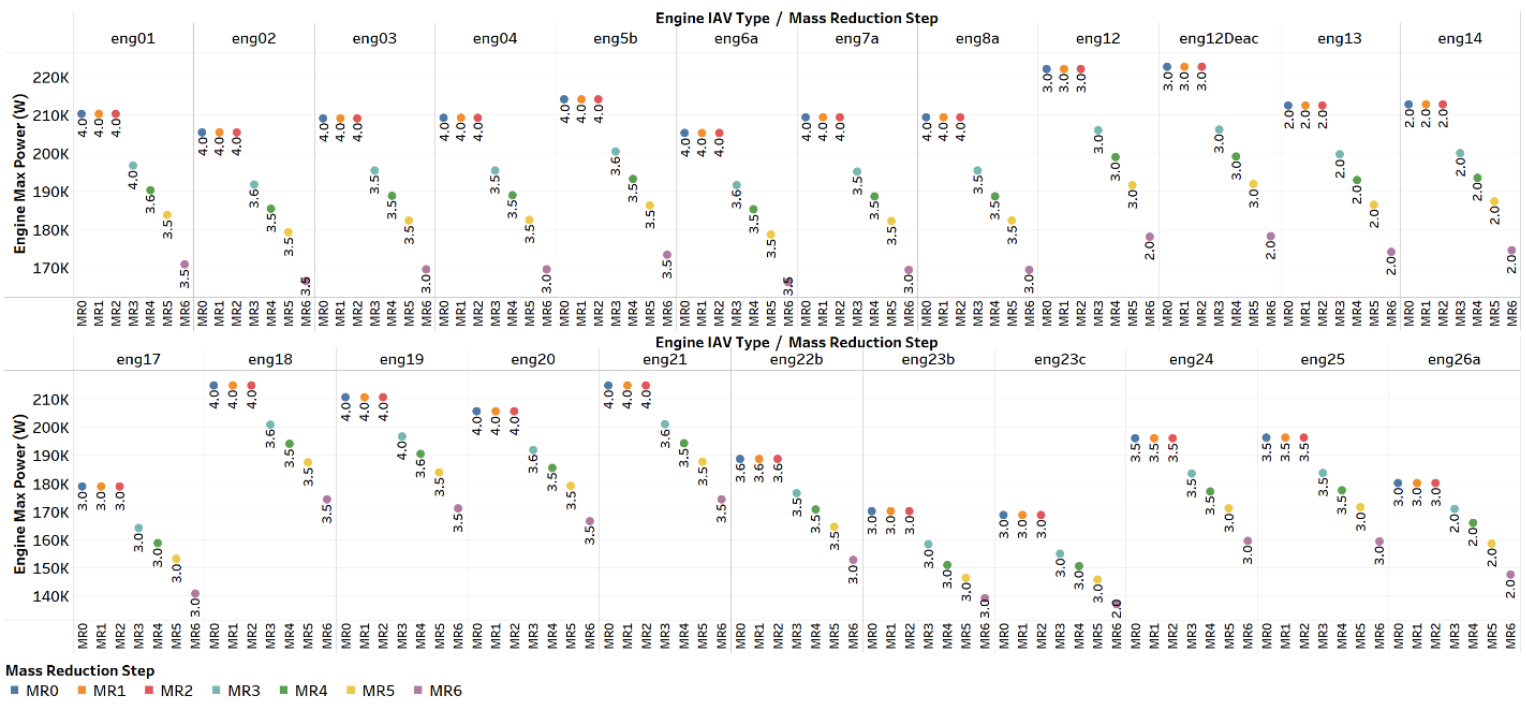

Figure 249. Engine power vs. mass reduction step (small SUV, performance)

Figure 250 shows the engine power of the conventional vehicles in the midsize SUV vehicle class (non-performance category) in response to the mass reduction step.
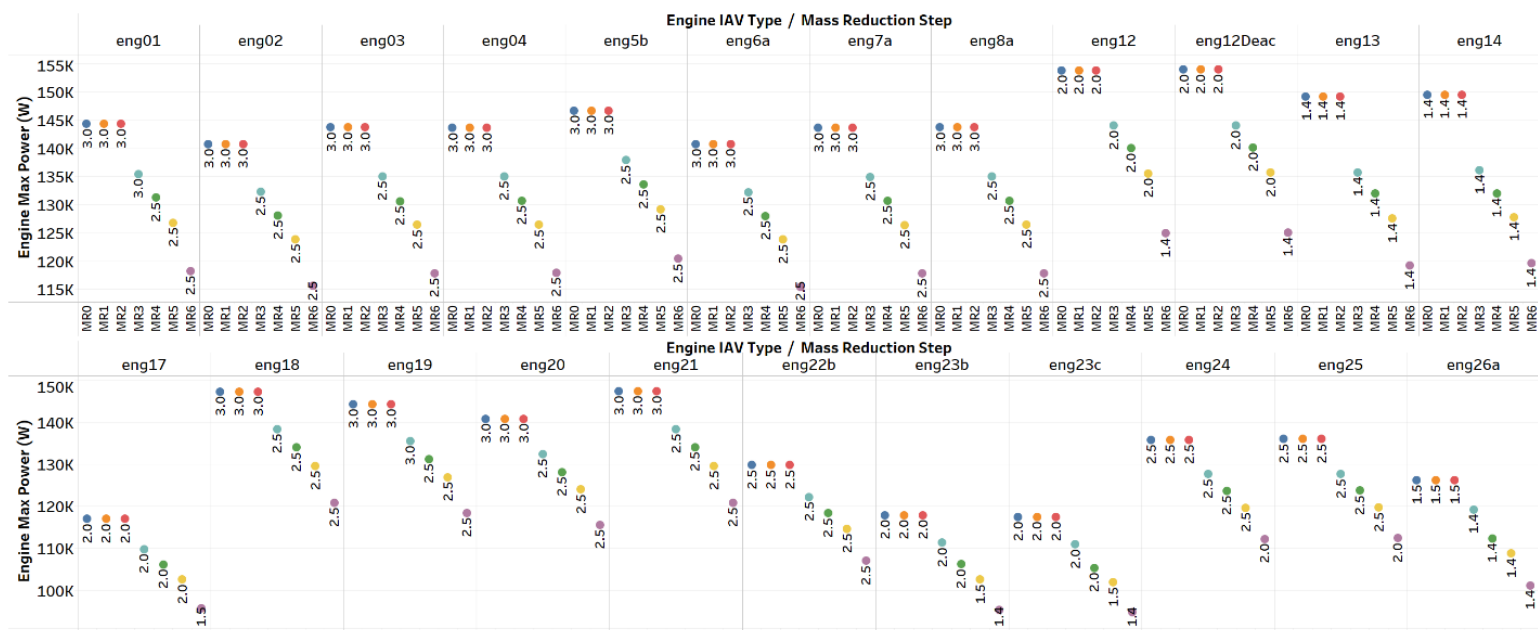

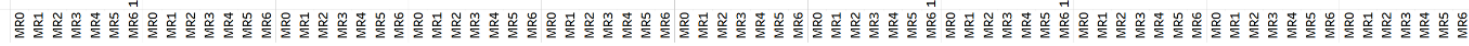
Mass Reduction Step
| MRO $=$ MR1 $\mid$ MR2 $=$ MR3 $=$ MR4 $=$ MR5 $=$ MR6

Figure 250. Engine power vs. mass reduction step (midsize $S U V$, non-performance) 
Figure 251 shows the engine power of the conventional vehicles in the midsize SUV vehicle class (performance category) in response to the mass reduction step.

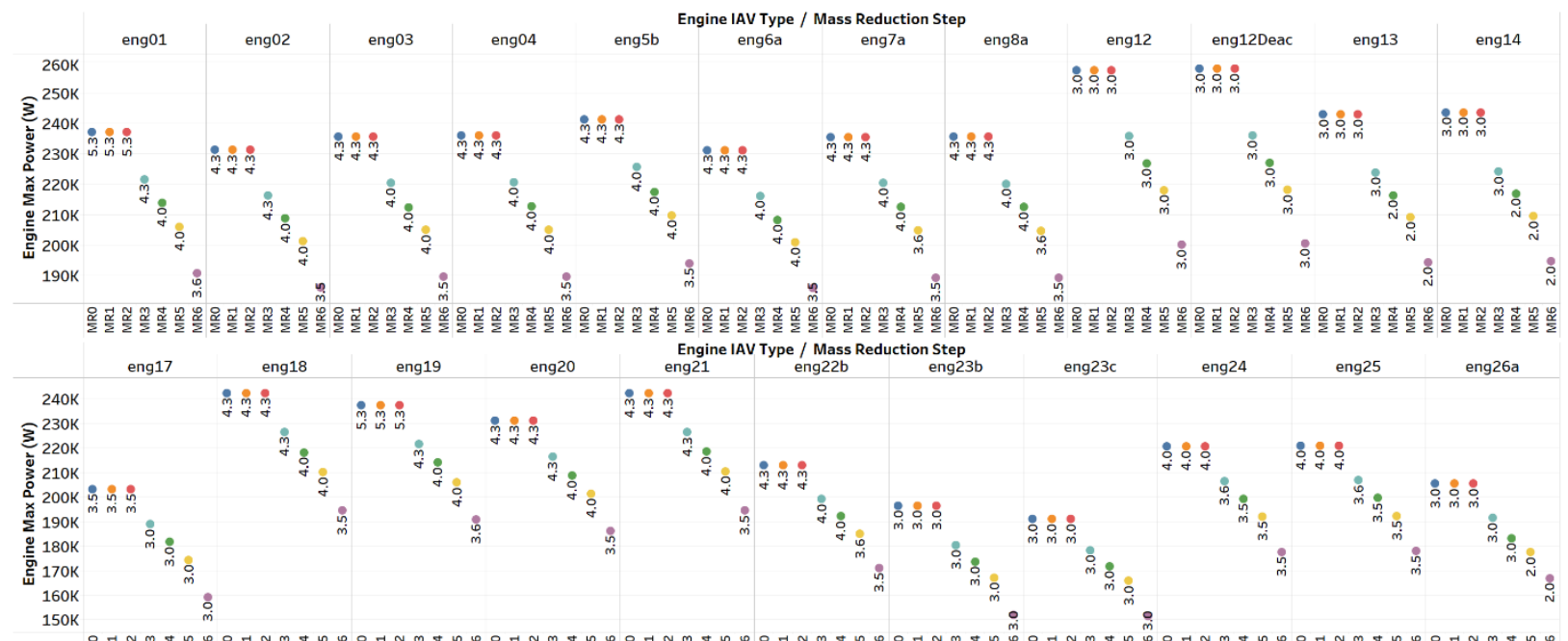

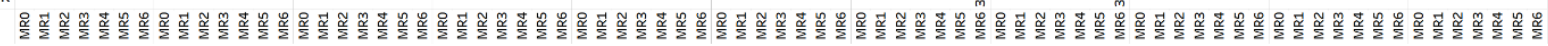

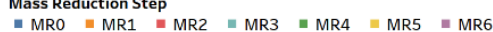

Figure 251. Engine power vs. mass reduction step (midsize SUV, performance)

Figure 252 shows the engine power of the conventional vehicles in the pickup vehicle class (performance category) in response to the mass reduction step.

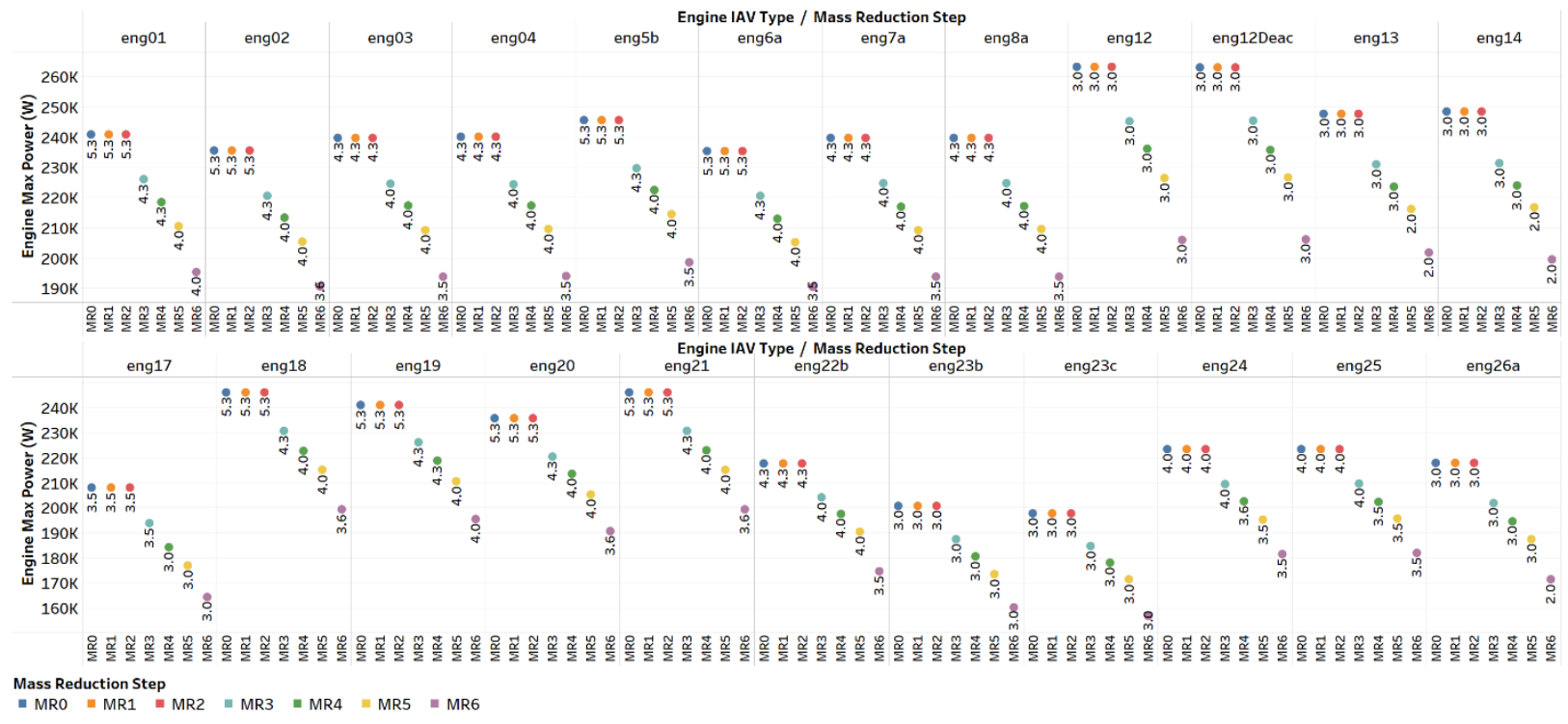

Figure 252. Engine power vs. mass reduction step (pickup, non-performance) 
Figure 253 shows the engine power of conventional vehicles in the pickup class (performance category) in response to the mass reduction step.

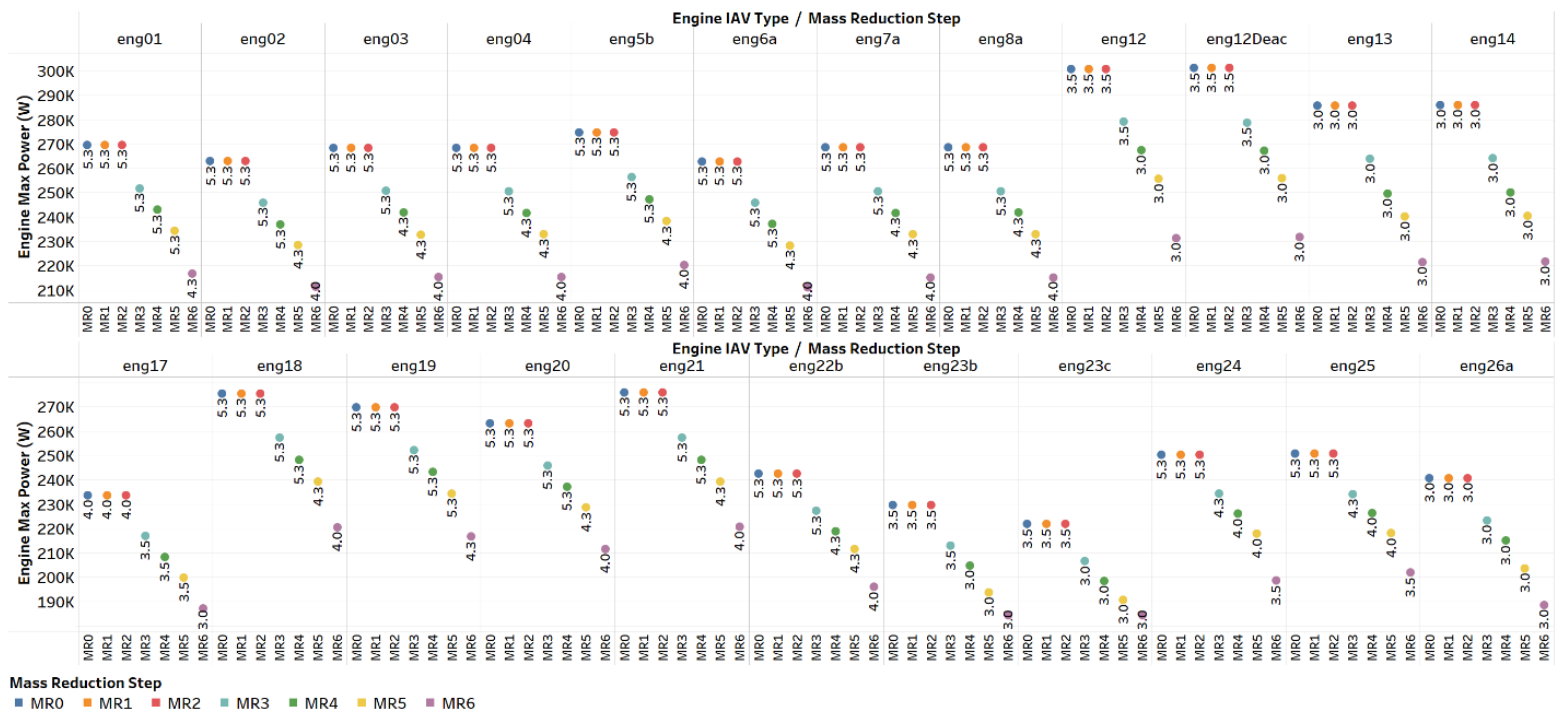

Figure 253. Engine power vs. mass reduction step (pickup, performance)

\subsection{Validation Against Vehicle Technology Database}

The vehicle simulation results are further validated against existing vehicles in the market using an internal vehicle technology database from Argonne. Fuel economy and acceleration times across vehicle classes and performance categories were analyzed. The vehicle simulation results and the vehicles from the vehicle technology database represent all combinations of conventional powertrains.

\subsubsection{BEV Weight, Range and Battery Energy Analysis}

Figure 254 shows the motor-specific power vs. vehicle acceleration time for existing BEVs in the market compared with simulated BEV200 vehicles used in this analysis. 


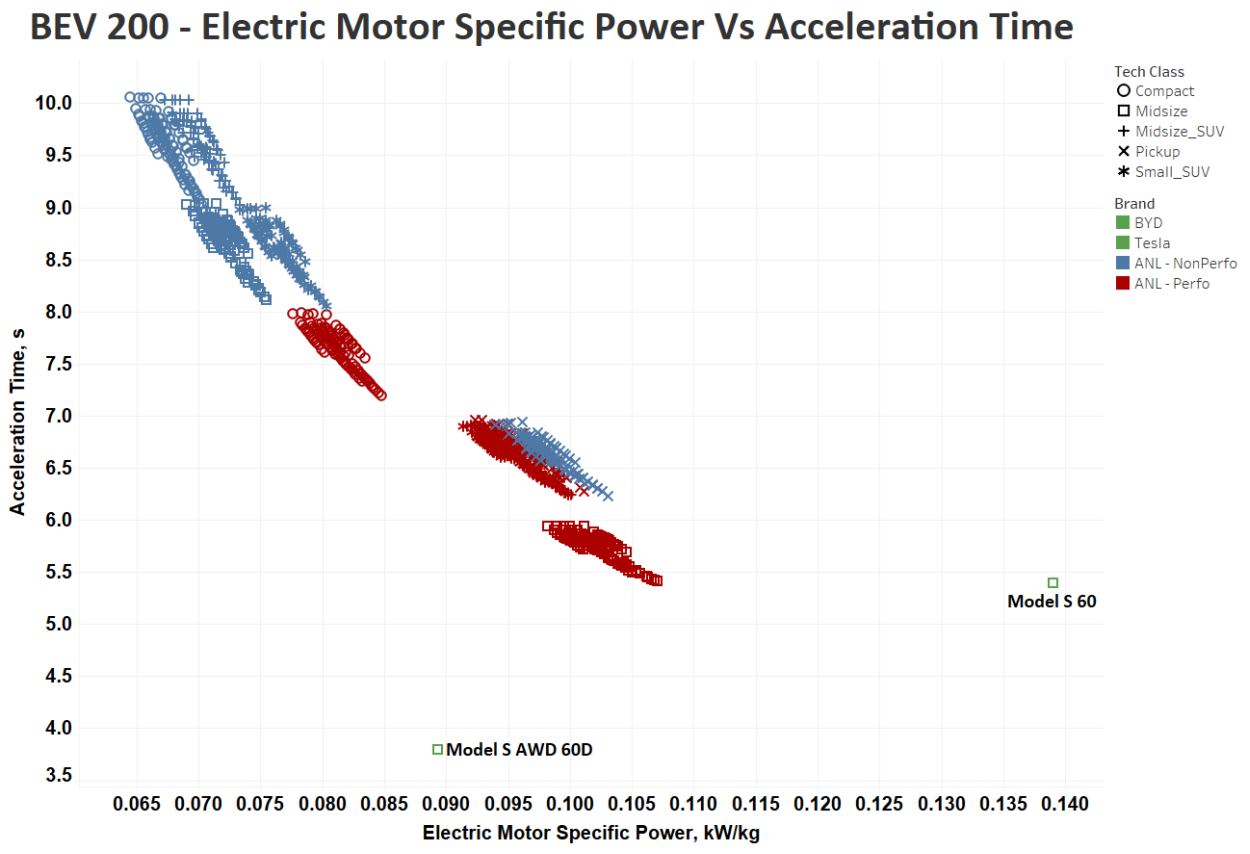

Figure 254. Motor-specific power vs. acceleration time (BEV200)

Figure 255 shows the total battery energy vs. all-electric range for existing BEVs in the market compared with simulated BEV200 vehicles used in the analysis.

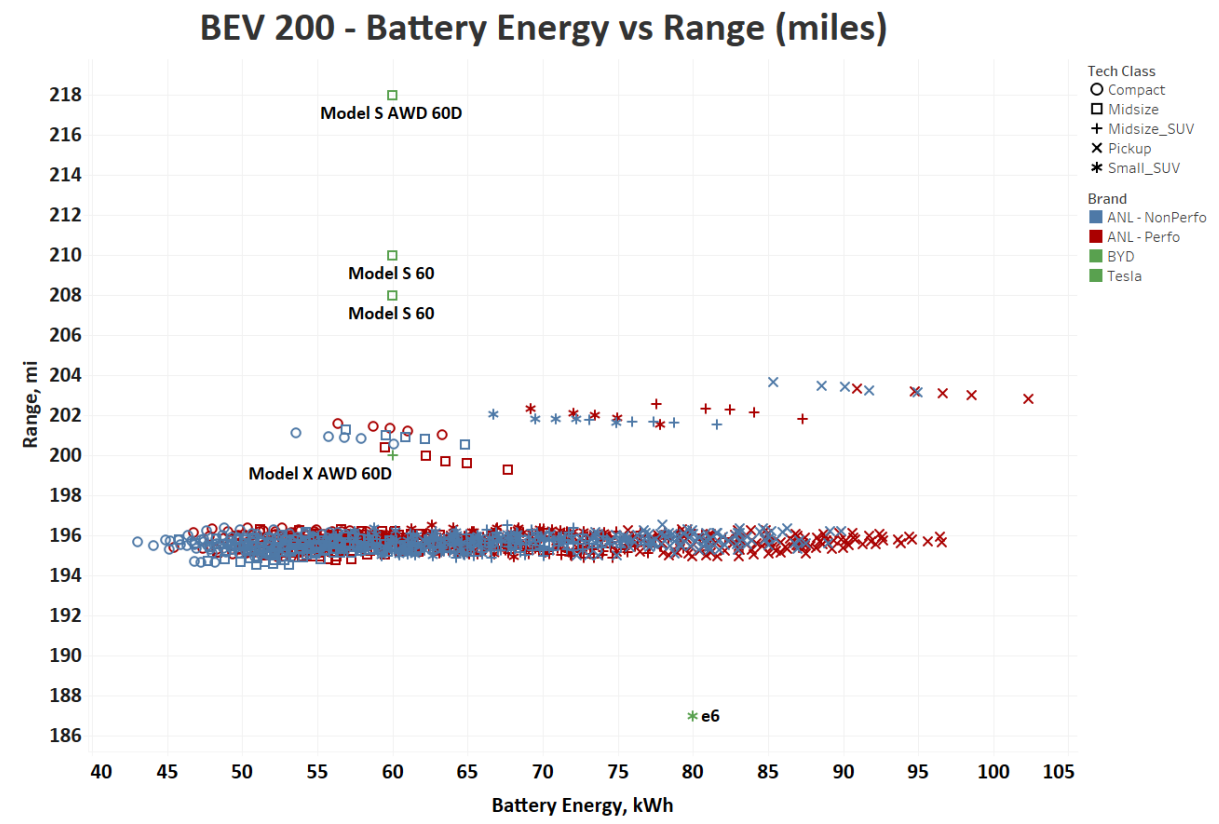

Figure 255. Battery energy vs. combined range (BEV200)

Figure 256 shows the total battery energy vs. all-electric range for existing BEVs in the market compared with simulated BEV300 vehicles used in the analysis. 


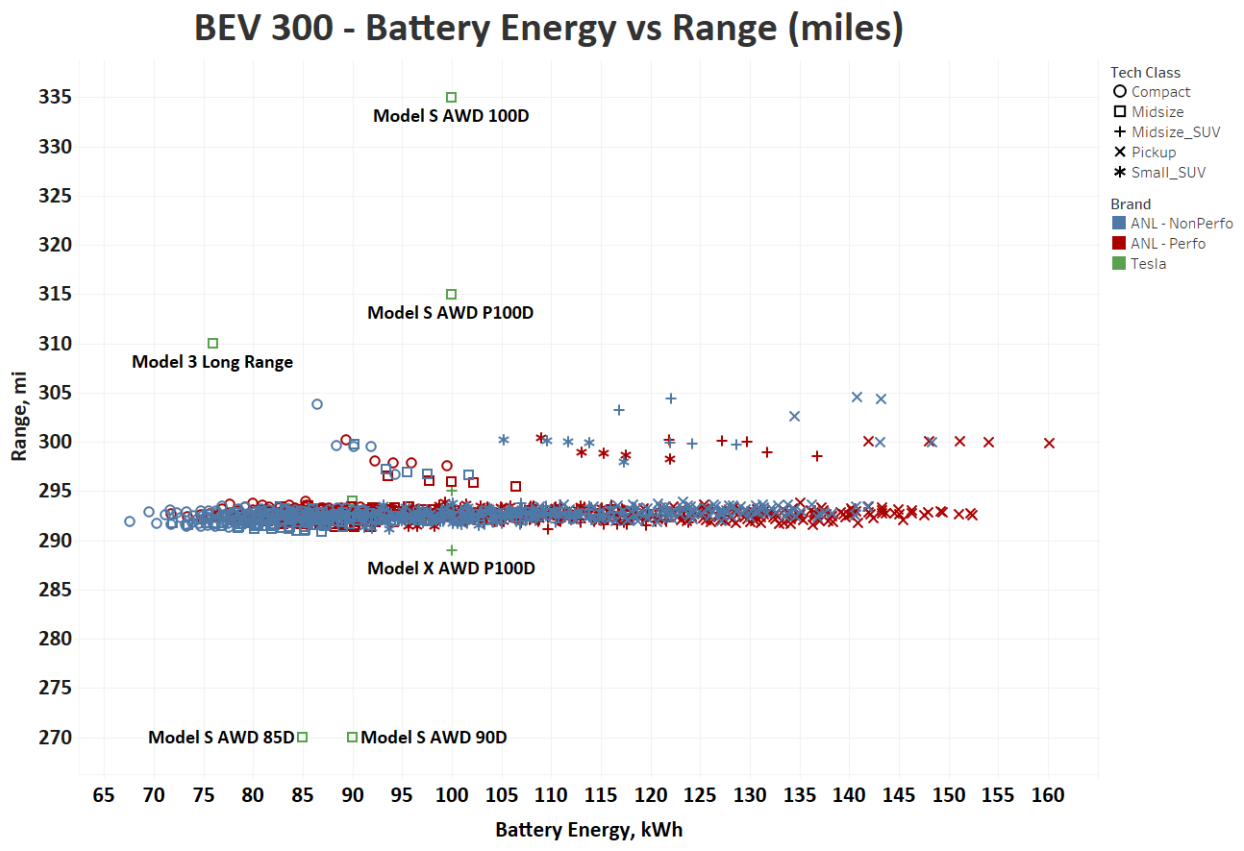

Figure 256. Battery energy vs. combined range (BEV300)

Figure 257 shows the motor specific power vs. vehicle acceleration time for existing BEVs in the market compared with simulated BEV300 vehicles used in this analysis.

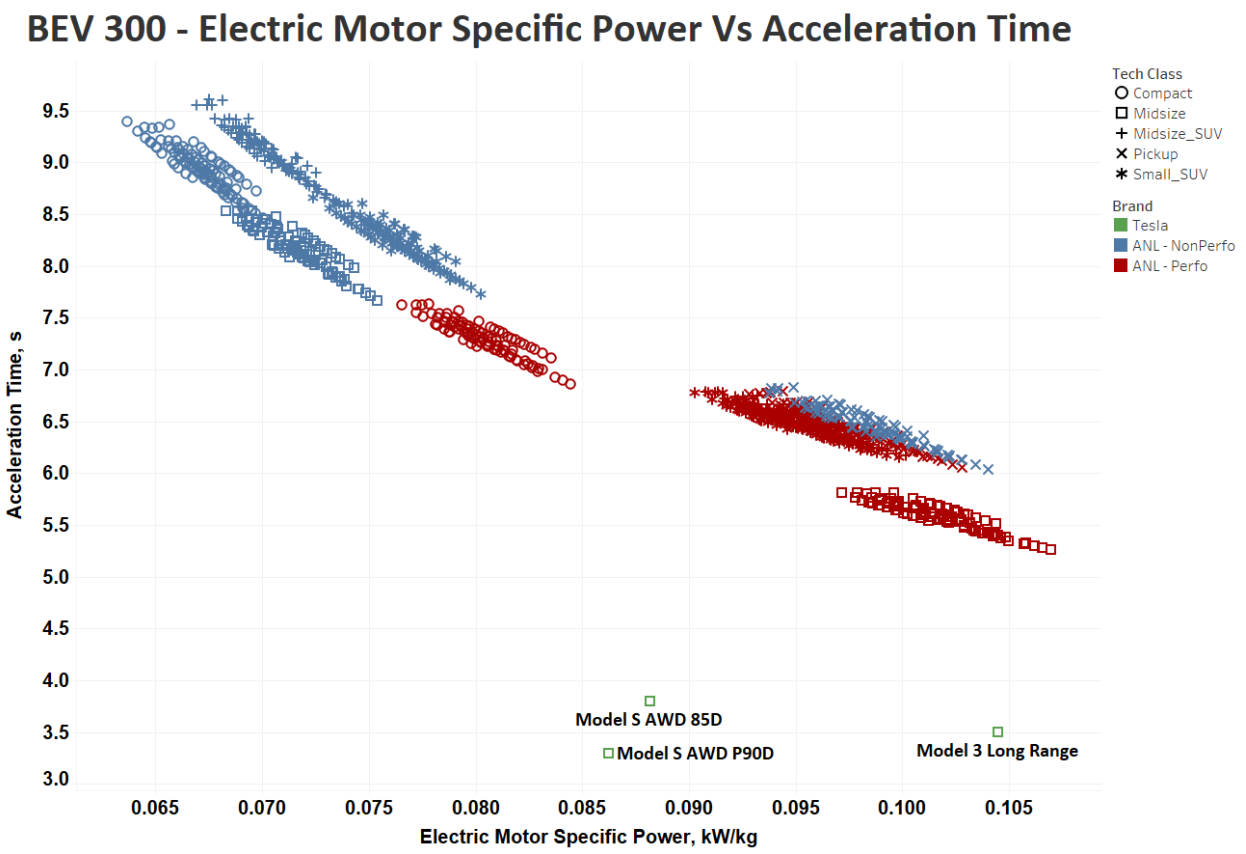

Figure 257. Motor-specific power vs. acceleration time (BEV300)

Figure 258 shows the distribution of total battery energy vs. vehicle curb weight for existing BEVs in the market compared with simulated BEV200 vehicles used in the analysis. 


\section{BEV 200 - Battery Energy vs Vehicle Curb Weight}

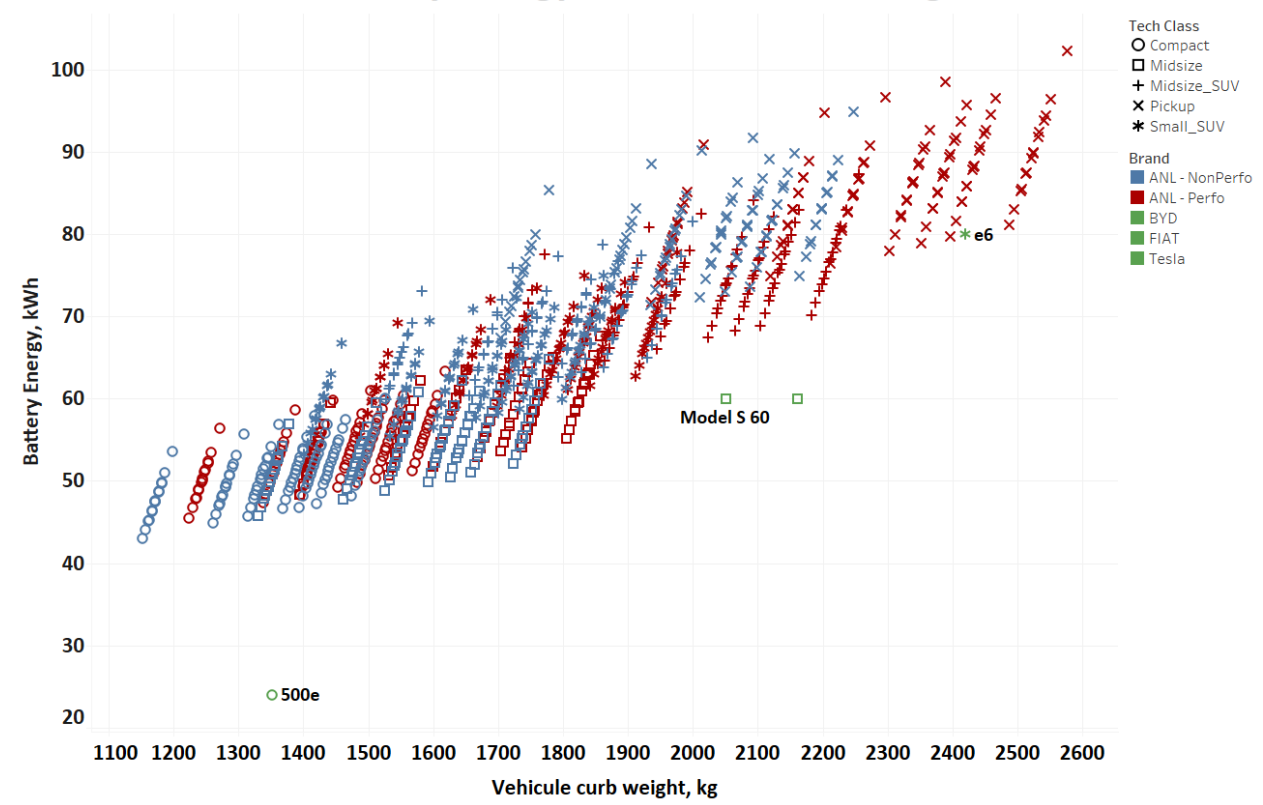

Figure 258. Battery energy vs. vehicle curb weight (BEV200)

Figure 259 shows the distribution of total battery energy vs. vehicle curb weight for existing BEVs in the market compared with simulated BEV 300 vehicles used in this analysis.

\section{BEV 300 - Battery Energy vs Vehicle Curb Weight}

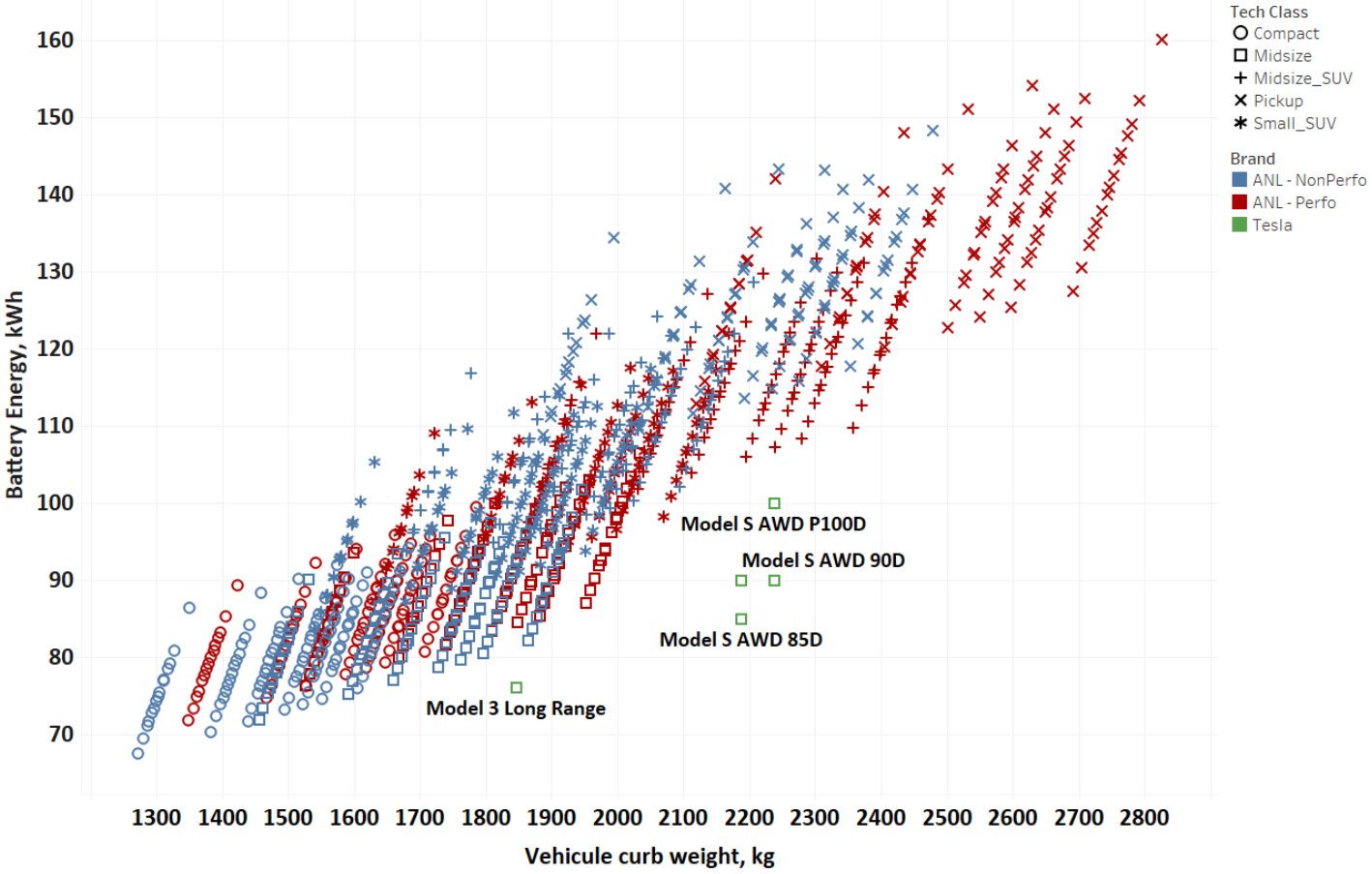

Figure 259. Battery energy vs. vehicle curb weight (BEV300) 


\subsection{Machine Learning for Outlier Detection}

A random sample consensus algorithm (RANSAC) method was used to validate the simulation results using machine learning. The technique involves iteratively estimating the parameters and best model that fit sufficiently many points (supposed inliers). Outliers will not fit the true model within some error (maximum deviation) attributed to the effect of noise.

For example, Figure 260 shows that most simulation values fit the model within the given confidence interval bands.

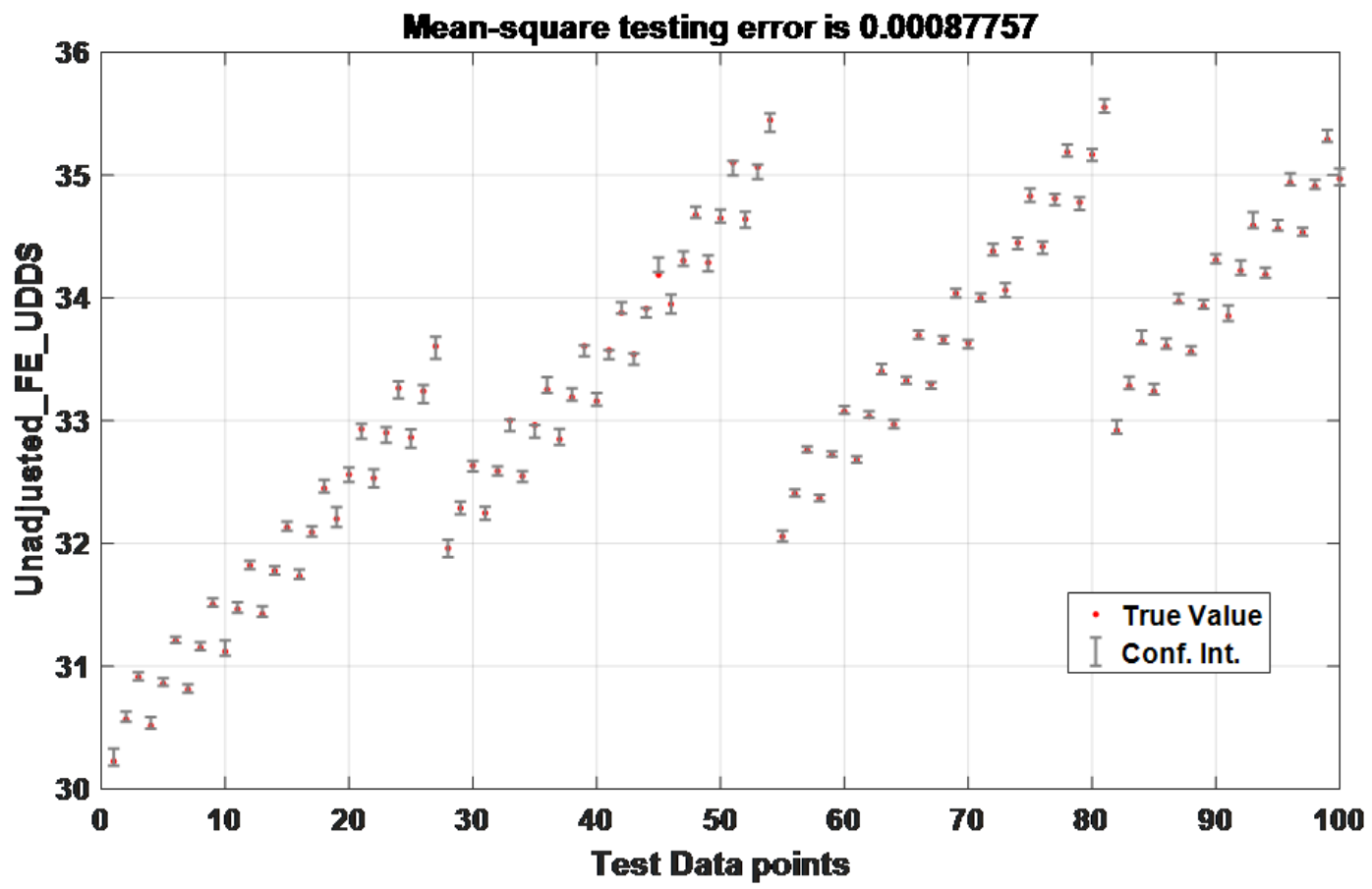

Figure 260. Confidence interval bands of simulation results

Figure 261, however, shows a situation where some engineering sense is required to conclude that the point detected by the RANSAC method to be an outlier is actually not an outlier. The RANSACgenerated model is a reasonable approximation of the simulation results. 


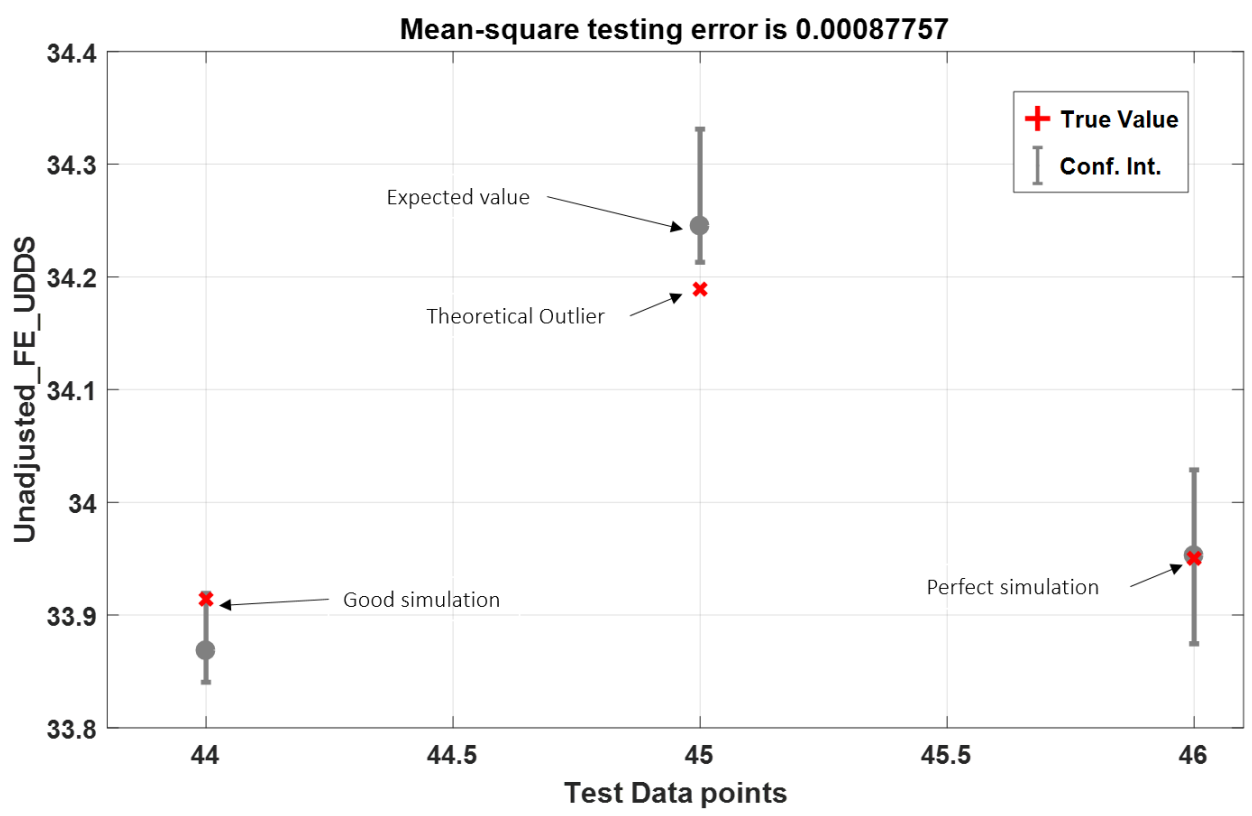

Figure 261. RANSAC validation method

\subsection{Statistical Methods}

Most statistical tests rely on the assumption of normality, so it is crucial to first determine whether the generated data is normally distributed. For results validation, the quantile-quantile (QQ) plot is a simple way to graphically confirm whether the data come from a normal distribution. Figure 262 shows the QQ plot of the simulation results.

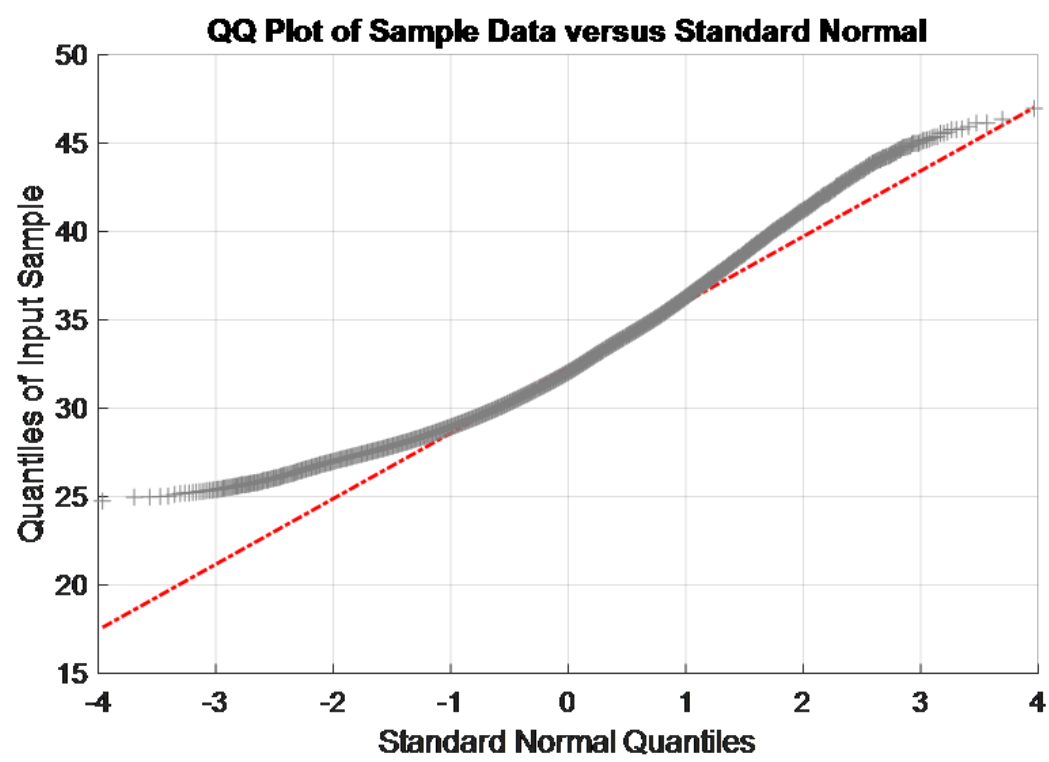

Figure 262. QQ plot to confirm the normal distribution of results 


\section{REFERENCES}

1. A2Mac1 Automotive Benchmarking. 2020. https://a2mac1.com.

2. Autonomie. "Model Validation." https://www.autonomie.net/currentprojects/ModelValidation.html.

3. Battery Performance and Cost (BatPaC) model. 2017. Argonne National Laboratory. https://www.anl.gov/cse/batpac-model-software.

4. Bonkoski, P., and H. Schilling. 2014. GT Power Study - BSFC Maps of Multiple Engine Concepts. Presented at ANL Project Final Deliverables Meeting, Northville. https://www.nhtsa.gov/staticfiles/rulemaking/pdf/cafe/140227_ANL_GTStudy_FinalDelivery.pdf

5. Burress, T. 2012. Benchmarking of Competitive Technologies. Washington, D.C.: Presented at the 2012 U.S. DOE Hydrogen and Fuel Cells Program and Vehicle Technologies Program Annual Merit Review and Peer Evaluation Meeting. https://www.energy.gov/sites/prod/files/2014/03/f10/ape006_burress_2011_o.pdf.

6. Burress, T. A., C. L. Coomer, S. L. Campbell, L. E. Seiber, L. D. Marlino, R. H. Staunton, and J. P. Cunningham. 2008. Evaluation of the 2007 Toyota Camry Hybrid Synergy Drive System. https://doi.org/10.2172/928684.

7. Duoba, M. 2009. Argonne Facilitation of PHEV Standard Testing Procedure SAE J1711. Presented at 2009 DOE Hydrogen Program and Vehicle Technologies Program Annual Merit Review and Peer Evaluation Meeting, Washington D.C.

8. Ellies, B., C. Schenk, and P. Dekraker. 2016. "Benchmarking and Hardware-in-the-Loop Operation of a 2014 MAZDA SkyActiv 2.0L 13:1 Compression Ratio Engine." SAE Technical Paper 2016-011007. https://doi.org/10.4271/2016-01-1007.

9. General Motors. "General Motors Voltec Technology." https://gmauthority.com/blog/gm/generalmotors-technology/gm-electric-vehicle-technology/general-motors-voltec-technology/.

10. IAV Inc. 2016. Responses and Updates to IAV Engine Maps.

11. Ide, T., A. Udagawa, and R. Kataoka.1995. "Simulation Approach to the Effect of the Ratio Changing Speed of a Metal V-Belt CVT on the Vehicle Response." Vehicle System Dynamics 24:4-5,. 377-388 https://doi.org/10.1080/00423119508969098.

12. Islam, E. S., A. Moawad, N. Kim, and A. Rousseau. Energy Consumption and Cost Reduction of Future Light-Duty Vehicles through Advanced Vehicle Technologies: A Modeling Simulation Study Through 2050. 2020. https://doi.org/10.2172/1647165.

13. Jeong, J., N. Kim, K. Stutenberg, and A. Rousseau. 2019. "Analysis and Model Validation of the Toyota Prius Prime." SAE Technical Paper 2019-01-0369. https://doi.org/10.4271/2019-01-0369.

14. Jeong, J., W. Lee, N. Kim, K. Stutenberg, and A. Rousseau. 2017. "Control Analysis and Model Validation for BMW i3 Range Extender." SAE Technical Paper 2017-01-1152. https://doi.org/10.4271/2017-01-1152.

15. Kim, N, H. Lohse-Busch, and A. Rousseau. 2014. "Development of a model of the dual clutch transmission in Autonomie and validation with dynamometer test data." International Journal of Automotive Technology 15: 263-271. http://dx.doi.org/10.1007/s12239-014-0027-5. 
16. Kim, N., A. Rousseau, and H. Lohse-Busch. 2014. "Advanced Automatic Transmission Model Validation Using Dynamometer Test Data." SAE Technical Paper 2014-01-1778. https://doi.org/10.4271/2014-01-1778.

17. Kim, N., S. Choi, J. Jeong, R. Vijayagopal, K. Stutenberg, and A. Rousseau. 2018. "Vehicle Level Control Analysis for Voltec Powertrain." World Electric Vehicle Journal 9: 29. http://dx.doi.org/10.3390/wevj9020029.

18. Lohse-Busch, H., K. Stutenberg, S. Ilieve, and M. Duoba. 2018. Laboratory testing of a 2017 Ford F150 3.5L V6 EcoBoost with a 10-speed transmission. National Highway Traffic Safety Administration. https://www.nhtsa.gov/sites/nhtsa.gov/files/documents/812520.pdf.

19. Miller, M., Holmes, A., Conlon, B., and Savagian, P., "The GM "Voltec" 4ET50 Multi-Mode Electric Transaxle," SAE Int. J. Engines 4(1):1102-1114, 2011, https://doi.org/10.4271/2011-01-0887

20. Meng, Y., M. Jennings, P. Tsou, D. Brigham, D. et al. 2011. "Test Correlation Framework for Hybrid Electric Vehicle System Model." SAE Int. J. Engines 4(1):1046-1057. https://doi.org/10.4271/201101-0881.

21. Momen, F., K. Rahman, Y. Son and P. Savagian. 2016. "Electrical propulsion system design of Chevrolet Bolt battery electric vehicle" 2016 IEEE Energy Conversion Congress and Exposition (ECCE): 1-8. https://doi.org/10.1109/ECCE.2016.7855076.

22. National Highway Traffic Safety Administration (NHTSA). 2016. Transmission Data Reporting Summary.

23. Olszewski, M. 2011. Oak Ridge National Laboratory Annual Progress Report for the Power Electronics and Electric Machinery Program. https://doi.org/10.2172/921775.

24. Pachernegg, S. 1969. "A Closer Look at the Willans-Line." SAE Technical Paper 690182. https://doi.org/10.4271/690182.

25. Pasquier, M., M. Duoba and A. Rousseau. 2001. Validating simulation tools for vehicle system studies using advanced control and testing procedure. Presented at the 18th International Electric Vehicle Symposium EVS18. Berlin, Germany. https://www.autonomie.net/docs/6\%20$\% 20$ Papers/Validation/validating_simulation_tools.pdf.

26. SAE International. 2017. Battery Electric Vehicle Energy Consumption and Range Test Procedure J1634_202104.https://www.sae.org/standards/content/j1634_202104/.

27. Son, H., N. Kim, S. Ko, A. Rousseau, and H. Kim. 2015. "Development of Performance Simulator for a HEV with CVT and Validation with Dynamometer Test Data." World Electric Vehicle Journal 7: 270-277. http://dx.doi.org/10.3390/wevj7020270.

28. Stuhldreher, M. 2016. "Fuel Efficiency Mapping of a 2014 6-Cylinder GM EcoTec 4.3L Engine with Cylinder Deactivation.” SAE International Technical Paper 2016-01-0662. https://doi.org/10.4271/2016-01-0662.

29. TBC. Total Battery Consulting - Reports \& Consulting. 2019. https://totalbatteryconsulting.com/ 30. U.S. Environmental Protection Agency. 2018. Retrieved from https://www.epa.gov. 
31. U. S. Environmental Protection Agency. 2019a. "2013 GM 6T40 Transmission - Test Data Package Dated 05-09-19.” Benchmarking Advanced Low Emission Light-Duty Vehicle Technology: Transmission Test Data. https://www.epa.gov/vehicle-and-fuel-emissions-testing/benchmarkingadvanced-low-emission-light-duty-vehicle-technology.

32. U. S. Environmental Protection Agency. 2019b. "2014 FCA HFE 845RE Transmission - Test Data Package - Dated 04-09-19.” Benchmarking Advanced Low Emission Light-Duty Vehicle Technology: Transmission Test Data. https://www.epa.gov/vehicle-and-fuel-emissionstesting/benchmarking-advanced-low-emission-light-duty-vehicle-technology.

33. U. S. Environmental Protection Agency. 2016. "Data on Cars Used for Testing Fuel Economy." https://www.epa.gov/compliance-and-fuel-economy-data/data-cars-used-testing-fuel-economy.

34. US Environmental Protection Agency. 2016. Process for Generating Engine Fuel Consumption Map (Future Atkinson engine with cooled EGR and cylinder deactivation). https://www.epa.gov/sites/production/files/2016-11/documents/procs-gen-eng-fuel-cons-map-futatkinson-eng.pdf.

35. USCAR Transmission Working Group. 2015. "Inputs to U.S. DRIVE Target Setting Process."

36. U.S. Department of Energy. 2020. DOE Hydrogen and Fuel Cells Program Record \#20005: Automotive Fuel Cell Targets and Status. https://www.hydrogen.energy.gov/pdfs/20005-automotivefuel-cell-targets-status.pdf.

37. Van der Sluis, F., T. van Dongen, G. van Spijk, A. van der Velde, et al. 2007. "Efficiency Optimization of the Pushbelt CVT." SAE Technical Paper 2007-01-1457. https://doi.org/10.4271/2007-01-1457.

38. Wileman, C. (2021, July). Light-duty vehicle transmission benchmarking, 2017 Ford F-150 with 10R80 and 2018 Honda Accord with Earth Dreams CVT (Report No. DOT HS 813 163). National Highway Traffic Safety Administration. 


\section{Argonne $\mathbf{A}$}

\section{Energy Systems Division}

Argonne National Laboratory

9700 South Cass Avenue, Bldg. 362

Lemont, IL 60439-4854

www.anl.gov

Argonne National Laboratory is a U.S.

Department of Energy Laboratory

managed by UChicago Argonne, LLC 